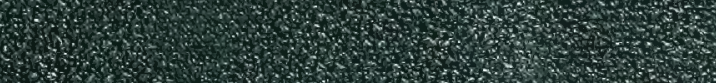

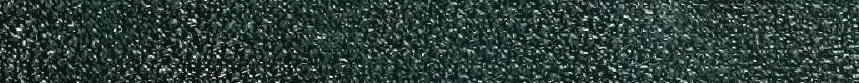

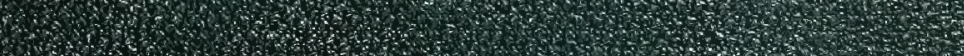
4.

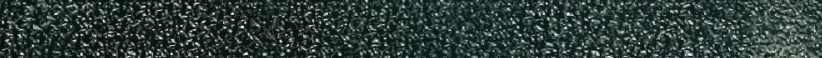

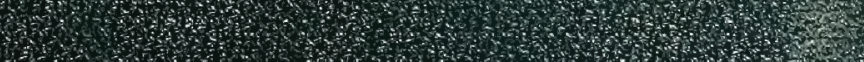

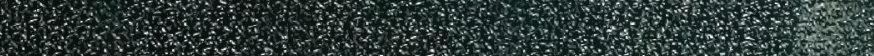

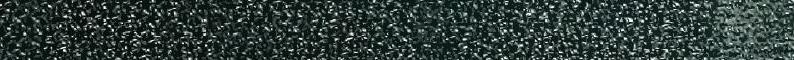

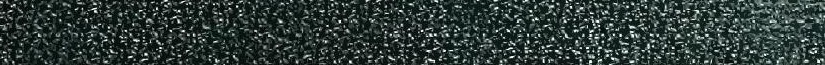

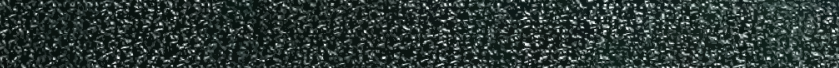

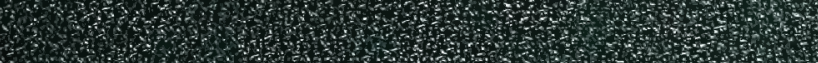
6.5.7.

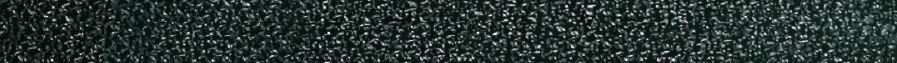

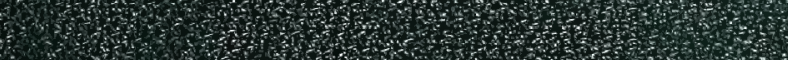

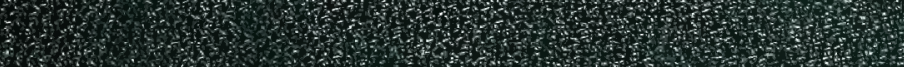

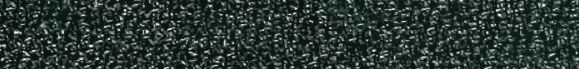

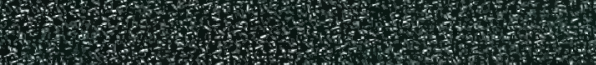
(5)

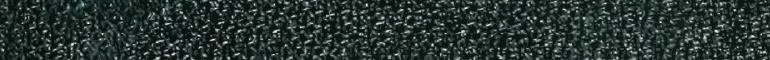

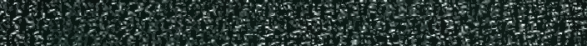

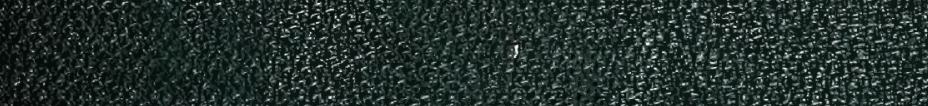

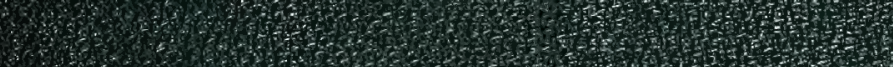

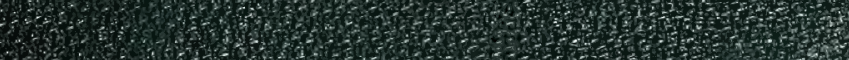

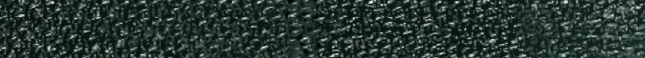

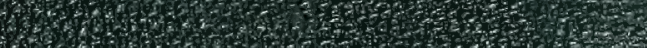

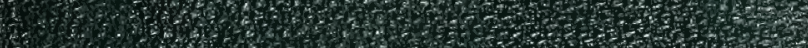

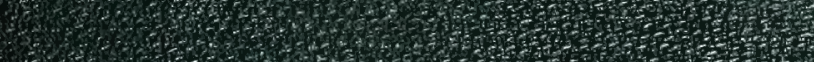

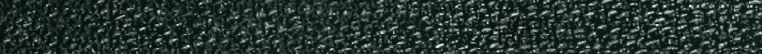

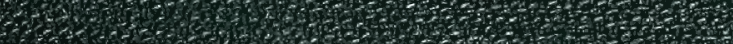

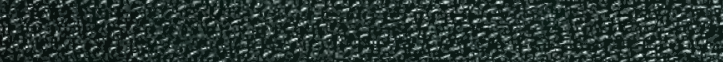

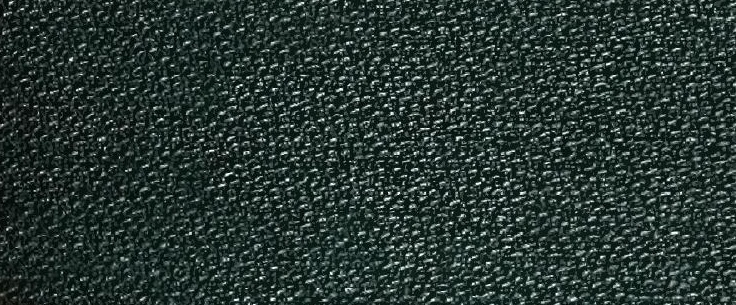

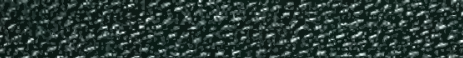

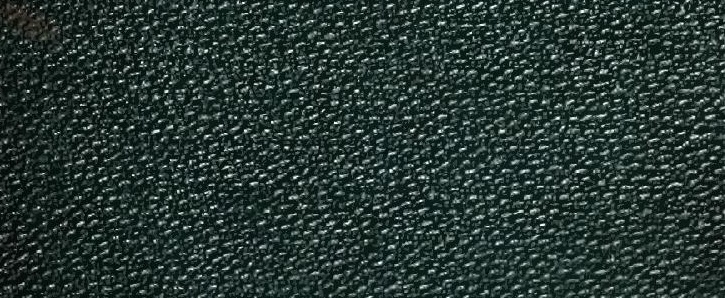


3
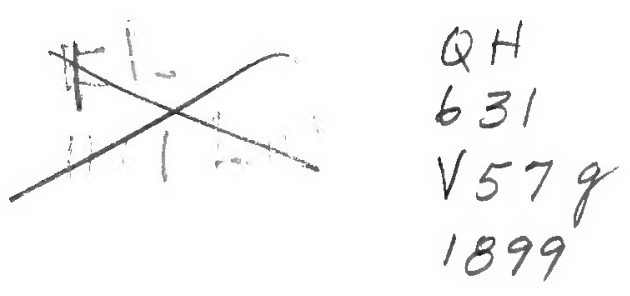

CORNELL UNIVERSITY.

THE

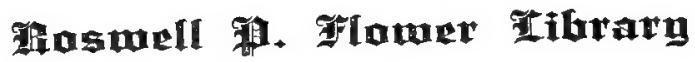

THE GIFT OF

ROSWELL P. FLOWER

FOR THE USE OF

THE N. Y. STATE VETERINARY COLLEGE.

$\mathbf{1 8 9 7}$ 


\section{QH 631. Cornell University Library}

General physiology; an outline of the sc 


\section{Cornell University Library}

The original of this book is in the Cornell University Library.

There are no known copyright restrictions in the United States on the use of the text. 


\section{GENERAL PHYSIOLOGY}


S 


\title{
GENERAL PHYSIOLOGY
}

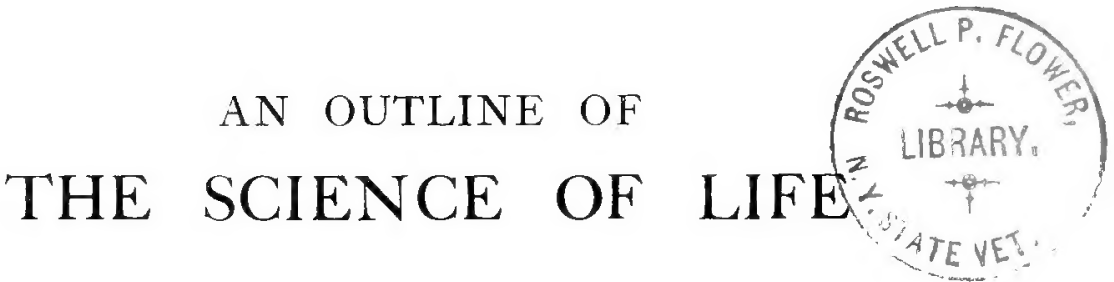

\author{
BY MAX VERWORN, M.D., Ph.D. \\ A.r. PROFESSOK OF P'PYSIOLOGY \\ 1P THE MEDICAL FACULTY OF THE UNIVERSITY OF JWNA
}

TR.ANSLATED FROM THE SECONL GERMAN HITION

$$
\text { AND EDITEW, BY }
$$

FREDERIC S. LEE, Ph.D.

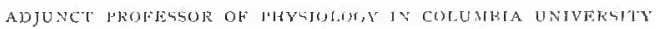

WIH TIVO HUNDKED AND EIGHTY-FIVE JILUSTK-ITIONS

\section{zoñon}

MACMILLAN AND CO., LimiteI)

NEW YORK: THE MACMILLAN COMPANY 
QA

Richard Clay and SONS, Limited

631

LADON AND BUNGAY.

$\checkmark 57 \mathrm{~g}$

1899 
To

THE MEMORY OF

\title{
JOHANNES MÜLLER
}

THE MASTER OF HIS SCIENCE

THESE PAGES ARE

\author{
羿edicatè
}

BY A PHYSIOLOGIST 


(1)




\title{
PREFACE TO THE FIRST EDITION
}

\author{
The elementary constituent of all \\ living substance and the substratum of \\ all elementary vital phenomena is the \\ cell. Hence, if the task of physiology \\ lies in the explanation of vital pheno- \\ mena, it is evident that general physio- \\ logy can be only cell-physiology.
}

Modern physiology has arrived at a point in its development where it must constantly extend its inquiries to the cell, the elementary substratum of all life that exists upon the earth's surface. It appears more and more clear that the general problems of life are cell-problems. This fact suggested to me the idea of examining from the cell-physiological standpoint these general problems, and the facts, theories, and hypotheses of the nature of life-subjects which thus far had never received comprehensive treatment-and thus outlining a field in which the various branches of special physiology might unite. In the present book, therefore, I have made an attempt to treat general physiology as general cell-physiology.

In dedicating this effort to the memory of Johannes Müller, I would express the obligation that we all owe to the work of our great master in physiology. But, more than all else, I would indicate Müller's comparative-physiological standpoint, a standpoint that I have always strongly endeavoured to maintain in my own work. The comparative method of dealing with physiological problems, which Mïller's researches made so extremely fruitful, was unfortunately laid aside after his death, as physiology dealt more and more with the special problems of the human body. But it is now being shown constantly that the amount of material available for work in this latter field is too small in view of the variety of problems. Hence, if wrong and false generalisations 
are to be avoided, and the science is to be allowed free development, it appears to me indispensable to return to Muiller's method. For this reason I have dedicated the following pages to the memory of that great physiologist.

The plan of the present book first assumed fixed form during a journey which I made in the year 1890 to different points on the Mediterranean Sea and the Red Sea for the purpose of making comparative-physiological researches. After my return my university lectures in Jena gave me an opportunity to present the collected material in connected form. But the greater part of the labour remained to be performed, and in the summer of $1892 \mathrm{I}$ began the writing of the book. Although for nearly ten year's I have been busy with the problems of general physiology and have endeavoured to contribute something to their solution, so much labour has been associated with the collection, examination, selection, completion, and arrangement of the much scattered material, that the book has progressed slowly-and with varied feelings on my part. I have often wondered whether the result would accord with the enthusiasm and love with which the task was undertaken. Only the criticism of my colleagues can decide this. It is not to be expected that a book which brings together for the first time in a unified form a mass of material hitherto regarded as heterogeneous, shall upon its first appearance pretend to completeness. I cherish no illusions that I have succeeded more than approximately. I am fully aware that many faults and errors must have crept in, and these $I$ beg my colleagues in friendliness to correct.

It has afforded me especial satisfaction that one of my American colleagues, Professor Frederic S. Lee, of New York, in an address. before the New York Academy of Sciences (94), has developed simultaneously and independently the same ideas regarding the claims of modern physiology as are presented in detail by myself in the first chapter of this book. These ideas have also been expressed by me elsewhere, especially in an article in the Mrrnist (Chicago, '94).

If a book is to reach a wide circle of readers, its language must be neither too technical nor too prosaic. I have endeavoured to comply with this requirement. I wished to write something that would appeal first to my fellow physiologists, and offer them. besides certain new facts and ideas, a summary of our scatt(rexl knowledge. But at the same time I wished the work to give to any interested scientific reader, whether a student of medicine, 
philosophy, botany, or zoology, an outlook over the problems, facts, theories, and hypotheses of life; in other words, I wished to give him an introduction to general physiology, and thus afford him an idea of the important theoretical basis of his study. It is not easy to adapt oneself to these diverse aims. How far I have succeederl in doing this, only the judgment of the reader can decide. I bespeak his indulgent criticism.

I gratefully acknowledge my obligations to all my friends who have taken active part in the planning, developing, and completing of my task, and especially to Mr. Gustav Fischer, who has shown great liberality in the publication of the book.

THE AUTHOR.

Londox, November 4, 1894. 


\section{PREFACE TO THE SECOND EDITION}

In offering the second edition of this work to the public, I feel it obligatory upon me to express my warmest thanks for the extremely favourable reception given the book upon its first appearance, by readers and especially by critics. I have been pleasantly surprised to realise-as I have been made to realise by personal talks, by letters, and particularly by the criticism of professional journals both at home and abroad-that the subject of general physiology excites active interest and receives abundant recognition in the circles not simply of theoretical natural science, but of practical medicine. It gives me much satisfaction to perceive in this a sign that the practical medicine of the day acknowledges the profound importance of a knowledge of the general physiology of cell-life for an understanding of the physiological and pathological phenomena exhibited in the cellcommunity of the human body. I am encouraged in this riew by the gratifying fact that cell-physiological researches have increased greatly in number during the last few years. In thisccond edition I have endeavoured to note the more important of the later results. Unfortunately, because of lack of space, I have been obliged to treat many of these with more brevity than I clesired, and to curtail the amount of attention giren in the first edition to some of the older work. But by the introduction of a considerable number of new figures, and the replacement of certain faulty ones by better, I trust that the whole has been made more comprehensible. I cannot expect the present edition to be free from errors and faults; but I trust that every critic will recognise the great difficulties involved in the treatment of such a large amount if material, and will be indulgent towards mistakes. I am sincerely grateful to my critics for having called attention to crrors in the first edition. So 
far as these were errors of fact, I have endeavoured to correct them; so far as the points raised were based upon differences in conception, or points of view, I have conscientiously tried to allow them their full value. Translations of the book into English and Italian are in course of preparation, and a Russian edition has recently appeared. Since the latter was published wholly without my knowledge, and has not been seen by either my publisher or myself, I am forced to disclaim all responsibility for it.

I cannot forbear expressing my warmest thanks to Dr. Gustav Fischer, for the pains taken by him in issuing the present edition.

\section{THE AUTHOR.}

UNIVERSITY OF JENA :

The Physiological Instutute, June, 1897. 


\section{PREFATORY NOTE TO THE ENGLISH TRANSLATION}

The first comprehensive treatment of general phrsiology was contained in Claude Bernard's now classic Legons sur les pthénonines de la vic communs aux animaux et aur végétaux, which was published in 1878-79. Since that time the only adequate work upon the subject has been Professor Verworn's Allgemeine Physiologi. The first edition of this book appeared in 1894. This wis followed in 1897 by a second and revised edition. The work has been welcomed by European and American biologists, who have felt the need of a review and summary of the rapidly accumulating details of cell-physiology, and its ability and suggestiveness have been widely recognised. Many of the special views of the author have encountered opposition-a fact that perhaps is indicative of their value-yet, however much we may agree or disagree with him upon special points, we all must acknowledge his breadth and be grateful to him for presenting such a wealth of facts, and for pointing out so clearly the possibilities of research. With Professor Verworn's consent I have undertaken the task of translating and editing the book; first, with the hope that in its English form it may enable English-speaking biologists and general scientific readers to realize more fully than before the wide scope of the science of Physiology; and, secondly, because the book presents in a form convenient for the use of students suggestive and stimulating discussions of vital physiological questions.

\section{FREDERIC S. LEE.}

Columbra Untvikstey, New Y'urk,

1 Itwh 1, 1898. 


\section{CONTENTS}

\section{CHAPTER I}

THE AIMS AND METHODS OF PHYSIOLOGICAI RESEARCH

I. The Problem of Physiology

II. 'The History of Phystological Research a , 6

A. The Earliest Times. B. The Period of Galen. C. The Period of Harvey. D. The Period of Haller. E. The Period of Johannes Müller

III. The Method of Physiologrcal Research

A. The Past Achievements of Physiological Research. B. The Relation of Psychology to Physiology. 1. The Question of the Limits of a Knowledge of Nature. 2. Physical World and Mind. 3. Psycho-monism. C. Vitalism. D. Cell-Physiology

\section{CHAPTER II}

\section{LIVING SUBSTANCE}

I. The Composition of Livisi: Substance

A. The Individualisation of Living Substance. 1. The Cell as an Elementary Organism, 2. General and Special Cell-constituents. 3. Multinucleate Cells and Syncytia. B. The Morphological Nature of Living Substance. 1. The Form and Size of the Cell. 2. Protoplasm. " The Solid Constituents of Protoplasm. b. The Ground-substance of Protoplasm. 3. The CellNucleus. a. The Form of the Nucleus. b. The Substance of the Nucleus. ". The Structure of the Nucleus. C. The Physical Properties of Living Substance. 1. The C'onsistency of Living Substance. "2. The Specific Gravity of Living Substance. 3. The Optical Properties of Living Substance. D. The Chemical Properties of Living Nubstance. 1. The Organic Elements. 2. The 
Chemical Compounds of the Cell. a. Proteids. b. Carbohydrates. c. Fats. d. 'The Inorganic Constituents of Living Substance.

e. The Distribution of Substances in Protoplasm and Nucleus.

II. Living and Lifeless Substance.

A. Organisms and Inorganic Bodies. 1. Structural Differences.

2. Genetic Differences. 3. Physical Differences. 4. Chemical Differences. B. Living and Lifeless Organisms. 1. Life and Apparent Death. 2. Life and Death.

\section{CHAPTER III}

\section{ELEMENTARY VITAL PHENOMENA}

I. The Phenomena of Metabolism

A. The Ingestion of Substances. 1. Food-stuffs. 2. The Mode of Food-Ingestion by the Cell. B. The Transformation of Ingested Substances. 1. Extracellular and Intracellular Digestion. 2. Ferments and their Mode of Action. 3. Assimilation and Dissimilation. ". Assimilation. b. Dissimilation. C. The Output of Substances. 1. The Mode of Output of Substances by the Cell. 2. Secretions and Excretions. a. Secretions. b. Excretions.

II. The Phenomena of Form-Changes

A. Phylogenetic Development. 1. Heredity. 2. Adaptation. B. Ontogenetic Development. 1. Growth and Reproduction. 2. The Forms of Cell-division, $a$. Direct Cell-division. b. Indirect Cell-division. 3. Fertilisation. 4. The Development of the Multicellular Organism.

III. The Phenomena of Transformation of Energy

A. The Forms of Energy. B. The Introduction of Energy into the Organism. 1. The Introduction of Chemical Energy. 2. The Introduction of Light and Heat. C. The Production of Energy by the Organisn. 1. The Production of Mechanical Energy. a. Passive Movements. b. Movements by Swelling of the Cellwalls. $\therefore$ Movements by Change of the Cell-turgor. d. Movements by Change of the Specitic Gravity of the C'ell. e. Morements by Secretion. $f$. Movements by Growth. q. Movements hy Contraction and Expansion. 2. The Production of Light. 3. The Production of Heat. 4. 'The Production of Electricity.

\section{CHAPTER IV}

\section{THE GENERAL, CONDITIONS OF LIFE}

I. The Present Conditions of Life upon the Earth's Surface A. The General External Conditions of Life. 1. Food. 2. Water. 3. Oxygen. 4. Temperature. 5. Pressme. B. The General Internal Conditions of Life.

II. The Origin of Life upon the Farth

A. Theories concerning the Origin of Life upon the Earth. 1. The Ductrine of Spontaneous Generation. 2. The Theory of Cosmozoa. 
3. Preyer's Theory of the Continuity of Life. 4. Pfligger's Ideal.

B. Critical. 1. Ftemity or Beginning of Living Substance.

2. The Descent of Living Substance.

III. The History of Death .

A. The Phenomena of Necrobiosis, 1. Histolytic Processes. 2. Metamorphic Processes. B. The Causes of Death. 1. External and Internal Causes of Death. 2. The Question of Physical Immortality.

\title{
CHAPTER V
}

\author{
STIMULI AND THEIR ACTIONS
}

I. The Nature of Stmulation

A. The Relation of Stimuli to Vital comditions. 1. The Varieties of the Stimulus. 2. The Intensity of the Stimulus. 3. Trophic Stimuli. B. The Irritability of Living Substance. 1. The Conception of Irritability and the Nature of Reactions. "2. The Duration of Reactions. 3. The Conduction of the Stimulus.

II. The Phenomena of Cell-Stimulation

A. The Actions of the Various Stimuli. 1. The Actions of Chemical Stimuli. " . The Phenomena of Excitation. b. The Phenomena of Depression. 2. The Actions of Mechanical Stimuli. *. The Phenomena of Excitation. b. The Phenumena of Depression. 3. The Actions of Thermal Stimuli. $a$. The Phenomena of Excitation. b. The Phenomena of Depression. 4. The Actions of Photic Stinuli. a. The Phenomena of Excitation. b. The Phenomena of Depression. 5. The Actions of Electrical Stinuli. a. The Phenomena of Excitation. b. The Phenomena of Depression. B. The Directive Effects of Unilateral Stimulation. 1. Chemotaxis. 2. Barotaxis. 3. Phototaxis. 4. Thermotaxis. 5. Galvamotaxis. C. The Phenomena of Orer-Stimulation. 1. Fatigue and Exhaustion." ". Excitation and Depression. 3. Death by Over-stimulation.

\section{CHAPTER VI \\ THE MECHANISH OF LIFE}

I. The Vital Process . .

A. The Metabolism of Biogens. 1. Biogens. 2. Bintonus. B. The Action of Stimuli upon the Metabolism of Biogens. 1. Changes of Biotonus upon Total Stimulation. 2. The Interference of Reactions. 3. Polar Changes of Biotonus and the Mechanism of Axial Orientation upon Trilateral Stimulation.

II. The Mechanics of Celt-Life . .

A. The Rôle of the Nucleus and the Protoplasm in the Life of the Cell. 1. 'The Theory of the Dominance of the Nucleus in the Cell. 2. Nucleus and Protoplasm as Links in the Metabolic Chain of the Cell. B. Derivation of the Elementary Vital Phenomena from the Metabolism of the Cell. 1. The Mechanics of 
Cell-metabolism. $\quad \alpha$. Scheme of Cell-metabolism. $\quad b$. The Mechanics of the Ingestion and Output of Substances. 2. The Mechanics of Changes of Cell-form. a. Growth as the Fundamental Phenomenon of Change of Form. $b$. Developmental Mechanics, c. Structure and Liquid. $d$. The Mechanics of Hereditary Transmission. 3. The Mechanics of the Transformation of Energy by the Cell. a. The Circulation of Energy in the Organic World. b. The Principle of the Transformation of Chemical Energy in the Cell. \%. The Source of the Energy of Muscle. $d$. Theory of the Movements of Contraction and Expansion.

iII. The Constitutional Relations of che Cell-Community .

A. Independence and Dependence of the Cells. B. Differentiation and Division of Labour among the Cells. C. Centralisation of Administration. 


\title{
GENERAL PHYSIOLOGY
}

\author{
CHAPTER I
}

\section{THE AIMS AND METHODS OF PHYSIOLOGICAL RESEARCH}

IN every department of human culture a survey of its aims and its achievements is desirable. Such a survey is, in a certain sense, a map; at any moment it can serve for orientation, and can be combined with similar maps of other departments to form a harmonious and comprehensive idea of the world.

This desire is warranted especially in the natural sciences, the enormous development of which has influenced so powerfully the civilisation of the present century.

Mankind has two potent needs, to the satisfying of which it is the purpose of science to contribute: a practical need, which is manifested in a search after a fitting and agreeable adaptation of the external conditions of life-the great development of modern technique and medicine bears witness to the efficiency of science in this respect ; and a theorctical need, which increases with civilisation and is manifested in a craving for causality or, in other words, a search after a harmonious idea of life and the world. Both needs are powerful, although they differ in intensity in accordance with individuality. Mankind has the right to demand of natural science that it shall never lose sight of its purpose and shitl not mistake its attitude toward the other aspects of human life, a danger that, with the enormous extension of specialisation, is now growing imminent.

One-sided specialisation is continually falling into this error. It leads far into barren fields, gradually ceases to recognise neighbouring territory, and at last becomes incapable of co-labouring in the general tasks of science. It scarcely needs mention that it would be a mistake to lay aside specialisation altogether. Broadminded specialisation is one of the chief factors in the advance of knowledge; without it, no general knowledge can be acquired. But a difference exists between special researches carried out for 
the purpose of helping to solve a problem that concerns a practical or a theoretical need of life, and those that deal with chance or any external circumstance for the purpose of seeing what, if anything, will be the outcome. The former constitute true research, the latter, pastime. One-sided specialisation considers none of the great needs of mankind, and brings science finally to the unenviable standpoint of the famulus Wagner.

It is absolutely essential to the advance of a science that in its special researches it keep clearly in view its general aim, its great problem ; investigation then becomes systematic. This is possible only when the investigator possesses such a survey as is referred to above.

Such an outlook over aims, paths, and achievements, in place of a mass of disconnected facts, is required, not by the individual investigator alone, but by every cultured man who would learn from science what is of value for the practical or theoretical needs of his life; for science serves life, not life science.

\section{The Problem of Phystologiy}

THE ancient Greeks associated with the word " $\phi v \sigma \iota s$ " the conception of all living nature, a significance that finds expression in its purest form in the Homeric poems. Since that time the idea expressed by the word has undergone many changes. The original significance soon gave place to a more general one, and at the height of Grecian culture the Ionic philosophers, the oldest natural philosophers of Greece, were called " $\phi v \sigma \iota$ 'o $\lambda$ oyou," the conception of $\phi u ́ \sigma \iota s$ being extended to all nature. Later, with the separation of physics as an independent science in its present sense, the conception became again narrow, but different from the original one. being limited to non-living nature and thus possessing a significance the exact opposite of the original one.

If the word $\phi v i \iota s$ be conceived in its proper original sense, the term "Physiology" expresses fully the essence of the science to which the term is now applied, and it is unnecessary to replace it with the later word "Biology," with which at present very different ideas are associated.

Physiology is the science of the phenomena of living nature, and, accordingly, its task is the investigation of life.

In spite of the apparent simplicity of its task, the science has already laboured for centuries upon this problem. A little consideration will make its difficulties evident. It is only necessary to attach ideas to the expressions "life" and "investigation," which in this combination appear at first as empty words.

We will consider first the subject-matter of physiology, namely, life. The untrained person associates usually with this word a 
mass of ideas that concern phenomena of a secondary nature, because he thinks only of the remote results, constantly observed in daily life, of primary vital phenomena. With him life is characterised by various occupations, labours, pleasures, walking, travelling, reading, speaking, eating, drinking, etc., one or another activity appearing as the essential part of his own life according to his vocation and individuality: to one person, life is labour, to another a constant festivity. But the various occupations of daily life are combinations of a few primary vital phenomena. If the development of the conception of life be followed back to early antiquity, when mankind had no presentiments of all the occupations that accompany a highly developed culture, when he was unacquainted with fire, when he did not know how to make even the most primitive tools, the conclusion is reached that the conception sprang from the combination of a number of simple phenomena, which early man discovered by self-observation, especially those phenomena that are associated with evident movements, such as locomotion, breathing, nutrition, the heart-beat, and others. In fact, it is not difficult to analyse into their primary constituents the complex occupations of our present life, and to recognise that its diversity is produced by various combinations of a few elementary phenomena, such as nutrition, respiration, growth, reproduction, movement, and the production of heat. If life be thus conceived as a sum of certain simple phenomena, the task of physiology is to determine, investigate, and explain the latter.

It must be remembered, however, that such a conception of life is limited to the vital phenomena of human beings, while the field of life is far greater. Animals and plants likewise exhibit vital phenomena, and it may be asked whether these latter are the same as or different from the phenomena that prevail among men. It is evident that all living organisms must be included in the sphere of physiological investigation, the flower and the worm equally with man. Hence the first duty of physiology is to mark out the field of the living, to determine what is living and what is not living -an undertaking that is more difficult than it appears.

The conception of life has not always been the same. It has experienced fundamental changes in the course of the development of the human species. Formed first with respect to mankind, it was early extended to other objects. With primitive races, the conception was much wider than at present, and they termed living what is no longer regarded as such. With them stars, fire, wind and waves were beings endowed with life and mind, and they were personified in the image of man. The remains of these ideas are still found in the mythology of the classic and modern races. In the course of time the distinction between living and lifeless has been made constantly sharper, but even to-day a child regards a steam engine as a living animal. The child is guided more or 
less consciously by the same criterion as the primitive races, who from the fact of motion, considered as living the dancing flame of a fire or a moving wave. In fact, of all vital phenomena, motion is the one that gives most strongly the impression of living.

It may be said that only primitive races and children are misled by the criterion of motion, and that the civilised and adult man, who is versed in a knowledge of life, is capable of deciding easily in any given case between the living and the lifeless. But this is not always true. For example, are dried grains living or lifeless? Is a lentil that has lain unchanged in a chest for years living? Scientific men themselves are not agreed upon this point. The lentil, when dry, does not show phenomena of life, but, if placed in moist earth, it can at any moment be induced to do so. It then sprouts and grows into a plant.

The decision between the living and the lifeless becomes, however, much more difficult with objects that are not commonly seen in daily life, e.g., certain microscopic things. Long observation and very detailed investigation are frequently required in order to determine whether certain bodies that are found in a liquid by microscopic examination are living or not. If a drop of the dregs be taken from a bottle of weissbeer and examined with the micriscope, it will be found that the liquid contains innumerable small pale globules, often clinging together in groups of two or three, completely at rest so long as they are observed, and showing no trace of movement or other change. Very similar small globules. may be observed with a microscope in a drop of milk. The two. kinds of globules can be distinguished from one another by strong magnifying powers only. No trace of vital phenomena can be found in either by the most patient and continued microscopic examination, yet the two objects are as widely different as a liring organism and a lifeless substance; for the globules from the beer are the so-called yeast-cells (Saccharomyces circtisic), the active agent in the fermentation of the beer and fully developed, unicellular, living organisms, while the globules from the milk are lifeless droplets of fat, which, by their abundant presence and their reflection of light from all sides, give to the milk its white colour. As a counterpart to these two objects, we may consider a third. In the body-cavity of the frog on either side of the spinal column between the transverse processes of the vertebræ there lie small, yellowish-white masses. If a bit of the contents of one of these be removed with a knife and placed with a drop of water upon a slide, and the whole be covered with a cover-gliss, there may be seen with strong powers of the microscope a mass of minute granules and short rods of different sizes, which are trembling and dancing in constant motion, the smaller particles very actively, the larger ones more slowly. Every untrained person, brought before these three preparations and asked which of the three objects 
appears to him living and which lifeless, would invariably pronounce the yeast-cells and the fat-droplets lifeless, the dancing granules living; but the latter are nothing more than minute calcareous crystals, so light that they are put into trembling motion passively by the excessively delicate motion that the particles of every liquid possess. The manifestation of motion, which, because we see no external source, we are inclined to ascribe to an internal cause, here misleads to the assumption of life. Such examples may be found in unlimited number.

Hence, under certain circumstances it is not at all easy to distinguish the living from the lifeless, and it is accordingly clear that the first duty of physiology must be to inquire after the criteria of such a distinction, i.e., mentally to circumscribe the isubject-matter, life, in relation to non-living nature.

Not less great are the difficulties that we meet when we consider the second idea that is included in the task of physiology, that of investigation. What is meant by investigation or explanation?

Civilised man appears to be distinguished essentially from primitive races by a great desire, namely, that of seeking after the "causes of phenomena, or, in other words, a craving for causality. This longing in all things to ask "why," from a pure desire for knowledge apart from any practical aim, appears to be an acquisition of civilisation, and its origin and development can be seen clearly in children of a certain age. When we have discovered a cause for any phenomenon, the craving for causality in that respect is satisfied; we have investigated and explained the phenomenon. This is true of investigation in all departments of science, of historical and philological science as well as that of nature, in so far as the development of the science has progressed beyond the stage characterised by the mere accumulation of facts. But when we have discovered the immediate cause of any phenomenon, we have satisfied the craving for causality only relatively, for the cause itself is a phenomenon that must be explained. Thus gradually and systematically we put individual phenomena and series of phenomena into causal connection with one another, and constantly reduce larger and larger groups to their causes. Ultimately, however, the question arises how far this reduction may be carried successfully. Is there a final cause for the phenomena, or may the reduction be continued to infinity?

In all fields of non-living nature, especially in physics and chemistry, investigation has shown that all phenomena thus far known and investigated may be reduced in the last instance to a single common cause, namely, the movement of very small material elements. The whole physical world is conceived as consisting of separate, indivisible, extremely small, elementary particles called 
atoms, and the various motions of the atoms, which fill universal space, are regarded as producing all phenomena in nature.

If it be the task of physiology to explain the occurrence of vital phenomena, i.e., to investigate their causes, it then becomes a question whether, in living nature likewise, all phenomena can be reduced to the motions of atoms, or whether it is necessary to take refuge in another principle. Next to determining the boundaries of the field of investigation, the chief task of physiology lies in answering this question.

Since early times mankind has been conscious of the great gap that exists between two groups of vital phenomena, the physical and the mental. Hence the above question is a double one. If it be possible actually to reduce the physical phenomena of life to the same elementary causes as the phenomena of the lifeless world, the result will not necessarily hold good for psychical phenomena, and the relations between the physical and the psychical must be analysed. If it be impossible to trace psychical phenomena to the same ultimate cause as the events of the physical world, another explanation must be sought, and the important question will then arise whether psychical phenomena can be explained at all. But if it be allowed that they can be brought into causal relations with the phenomena of the physical world, the question will still remain, What are atoms? The question of the possibility of answering this will then arise. If it can be answered, will our craving for causality then be satisfied?

The investigation of life is thus confronted with a multitude of questions, which tax to the uttermost the capabilities of the human mind.

\section{The History of Physiological Research ${ }^{1}$}

An examination of the history of physiological research is not only interesting, but important for a correct judgment of the present condition of physiology and the future course which it has to take in order to accomplish its established purpose.

\section{A. THE EARLIEST TIMES}

The earliest traces of naive physiological ideas are lost in the impenetrable obscurity of prehistoric times. A picture of them has been handed down in the mythology of the early civilised races. This represents a condition in which all knowledge and all formation of ideas are grouped about the veneration of higher

1 The account of the earlier epochs in the development of physiology is based upon the following works: K. Sprengel, I'ersuch ciner pragmatischen Geschichte der Aramikunde; H. Haeser, Lehrbuch der Geschichte der Wedicin. In his Elemente der allgemeinen Physiologie, Preyer girus a short sketch of the history of physiology based upon the latter book. 
beings. The primitive worship of the early races and the knowledge associated with it may be regarded as an indivisible whole, from which, in the course of the hundreds and thousands of years, theological, philosophical, scientific, and medical conceptions gradually and slowly have been crystallised out as independent groups of ideas.

The early notions of life were very naïve and crude. All that moved was living and was endowed with mind. The property of motion was the criterion of life. Wind, water, fire and stars were personified. Meteorites which moved through the air, called "bætyli," were regarded by the Phonicians as endowed with mind, and were believed to be healing, while Sûsruta, the author of the Yajurveda, the most ancient Indian work upon the art of healing, represented all motile bodies as living, in distinction from nonmotile, or lifeless, bodies. The art of healing, which was almost wholly a doctrine of drugs, and in primitive ages was developed especially upon the Pontus, where witcheraft flourished and where Hecate was reverenced, was crudely empirical, was in league with magic and mystery, and wholly lacked a physiological basis.

In these earliest times only one class of phenomena received detailed consideration, namely, the higher psychical phenomena, which reveal man's life most directly to himself. A doctrine of the mind was developed even in ancient Egypt, probably under Indian influence, which had for its basis the dualism of body and mind, and reached its culmination in the idea of the passage of the mind after the death of the body into other bodies. Later, this notion was transplanted to Greece by the Greek philosophers, especially Pythagoras. In general, from the earliest times onward, the phenomena of mental life served as a peculiar stimulus for priests and philosophers, the earliest theorisers, and in antiquity. of all fields of investigation, psychology was cultivated the most.

While physiological notions were scarcely influenced by medicine until long after Hippocrates, in Greece they were enriched in a significant manner by the first blooming of philosophy as a distinct discipline independent of the priesthood. The oldest Greek philosophers, the Ionic "physiologists," the Eleatics, as well as the Atomists and the independent thinkers of the same time, whose aim was the development of a cosmology, were forced in the pursuit of this aim to reflect upon the origin of living nature. Whatever judgment may be passed upon the unbridled character of the speculations of these ancient thinkers, the correctness of their notions regarding many of the phenomena of life will always remain a very surprising fact. Among many of these early philosophers it is singular to meet with ideas which, after more than two thousand years, have again become current and are reckoned among the most important foundations of the present science of life. This is particularly true of opinions concerning the origin and development of 
the organic world. The notion of the derivation of man from animallike ancestors originally inhabiting the water, is found clearly expressed by Anaximander (b. about 620 B.c.); and Heraclitus (about 500 B.C.) had an idea of the significance of the struggle for existence (Ép of living things is the clearest and most surprising. According to him, plants appeared first, then the lower animals, and from them the higher animals and, finally, men were developed by a process of perfection. The effective principle in this perfecting process he perceived in the fact that ill-adapted individuals are destroyed in the struggle for life, while those that are capable of living produce offspring. Almost twenty-five hundred years elapsed before this simple conception of the descent and natural selection of organisms, clearly expressed by Empedocles, was empirically grounded by Darwin and was established as the natural explanation of the otherwise marvellous multiplicity of organic forms.

Many ideas, more or less correct, regarding special physiological phenomena are found also among the early Greek philosophers. But these scattered truths are mingled with so many fantastic and purely arbitrary notions that, from their associations, they lose their real value. No coherent, systematic observations or reflections concerning vital phenomena exist before Aristotle.

From the side of practical medicine, likewise, the investigation of life experienced no considerable advance, even when medical art, hitherto without a critic, was placed by Hippocrates (460-377 B.C.) upon a sound basis.

A physiological doctrine appeared first among the followers of Hippocrates, probably under the influence of Plato's philosophy, and it was soon perfected and controlled all the medical ideas of that time. This is the doctrine of the spirits $(\pi \nu \in \hat{v} \mu a)$, in the main thought of which can be found the first germ of a fundamental physiological truth. This doctrine asserts that the pnexm", an excessively subtile material agent, is attracted by the human lungs, passes from the lungs into the blood, and is distributed by the latter throughout the body. All vital phenomena depend upon the action of this agent. This conception, which, naturally, was adorned with all sorts of absurd accompaniments, suggests strongly our modern ideas concerning the rôle of oxygen in the organism.

\section{B. THE PERIOD OF (XALEN}

The first intimation of an attempt to explain vital phenomena appears in the carly Hippocratic doctrine of the moma. This was expanded, especially in the Alexindrian school, by Herophilus (about 300 B.C.) and Erasistratus (d. 280 B.C.), the latter of whom distinguished a $\pi \nu \in \hat{v} \mu \alpha \zeta \omega \tau \iota \kappa o ́ v$ (vital splirits) in the heart and a

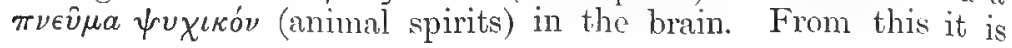


evident that the problem of physiology, the explanation of vital phenomena, had already begun more or less clearly to be recognised. Hitherto, individual physiological facts had been observed, and physiological questions had been discussed incidentally. But now, the more clearly the problem of physiology began to be formulated, the more the treatment of physiological questions began to assume the character of scientific investigation.

Aristotle (384-322 B.C.), the great polyhistor of antiquity, established the preliminary conditions for this advance by accumulating a vast mass of material in the form of facts. The significance of Aristotle's relation to physiology does not lie in explaining vital phenomena-very often his explanations are uncritical, and, moreover, they do not appear prominent in his work-but rather in observing and recording a great number of physiological phenomena. In the midst of this material by the side of striking and acute researches, there occurs, as might have been expected, much erroneous observation; such, for example, is the origin of eels and frogs from mud by spontaneous generation. Nevertheless, his recorded observations form the basis of the new stage of development into which physiology passed after Aristotle, and which is characterised by the clear recognition of the physiological problem and its vast importance in practical medicine.

After Aristotle, by his systematising work, had laid a broad empirical foundation for natural science, the doctrine of the pneuma received a wider extension among the later pneumatic physicians, especially through the efforts of Athenaeus and Aretaeus (both about 50 A.D.). It is in the nature of this doctrine, that it must endeavour to comprehend and explain the phenomena of life from a single point of view; and, accordingly, we find now for the first time a clear, conscious recognition of the physiological problem and a systematic comprehension of physiological phenomena. The man who first clearly perceived the nature and significance of physiology was Galen (131-about 200 A.D.). Galen saw that practical medicine could not thrive unless it were based upon a very detailed knowledge of the normal vital phenomena of the human body. The investigation of the vital functions of the body must be the first pre-requisite of an art of healing. This practical aim was the first incentive to the development of physiology, and controlled the science almost exclusively until the eighteenth century. Galen was also the first to recognise clearly the importance of a knowledge of the anatomy of the body in an understanding of the functions of its parts, and laid great value upon the dissection of animals; he himself dissected pigs and monkeys especially. Moreover, he perceived the importance of animal experimentation in the investigation of physiological phenomena; and, although the experimental method did not assume under him that exact form and that fundamental 
importance which many centuries later Harvey knew how to give it, Galen himself practised vivisection upon pigs and monkeys.

Along with general recognition of his immortal service, Galen has often been reproached with the charge that he was not content with collecting physiological facts, making observations and devising experiments, but that he felt strongly the necessity of arranging his collected material into a complete and comprehensive system of physiology, in which he allowed hypothesis and philosophical speculation a place that exact investigation ought to have filled. Nothing can be more unjust than this reproach. If Galen had been satisfied with ascertaining disconnected physiological facts, physiology and with it all medicine would not have been advanced one step farther than Aristotle had already brought them. Galen's greatest importance lies in the union of scraps of physiological knowledge into a coherent system. Isolated observations obtain value only in connection with other facts, and only a survey of the relations of facts makes possible further systematic progress. It is only natural that, in this first attempt to put together the material of physiological observation into a coherent picture of the life of the human body, recourse must now and then be had to hypothesis, even much bold hypothesis. The single fault from which Galen's system suffers is not its binding cement of philosophical speculation, but the peculiar dualism that misled him, in accordance with which, in explaining vital phenomena, he strove to give at the same time a place both to the rigid idea of necessity, which sprang from his exact scientific investigations, and to teleology, which was derived from the Aristotelian philosophy. Nevertheless, in a just estimation of his time, when Aristotelian ideas had already begun a universal sway that was to last more than a thousand years, Galen can scarcely be reproached for this, the less when it is recalled that the teleological idea of a final purpose in all things appears here and there in modern natural science even to-day, quite independent of philosophy.

Galen's system is based upon the doctrine of the spirit= ( mncuma $^{2}$ ). The causes of all the vital phenomena of the human bucly, which is composed of the four fundamental juices, viz.: the blowd, the phlegm, the yellow and the black gall, are the three different forms

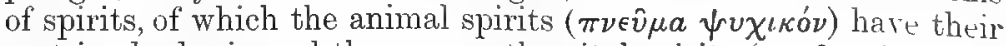
seat in the brain and the nerves, the rital spirits ( $\pi \nu \in \hat{\nu} \mu a \zeta \omega \tau \iota \kappa o ́ v)$

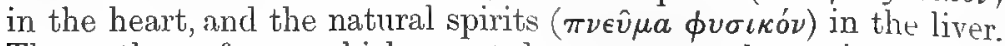
These three forms, which must be regenerated continually by the receipt of vital spirits from the air, are the agencics that maintain the functions of the respective organs. The body possesses many functions, but they may be arranged, according to. the forms of the spirits, into three classes, and each function is

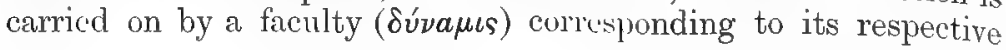


pneuma. The psychical (animal) functions comprise thinking, feeling, and voluntary motion; the sphygmical (vital) functions, the heart-beat, the pulse, and the production of heat; the physicul (natural) functions, nutrition, growth, secretion, and reproduction with its related activities. The blood is formed in the liver, and the veins arise there. Through the veins the blood goes to the right ventricle of the heart, where the useful part is separated from the useless; the former is carried to the left ventricle, while the latter goes through the pulmonary artery to the lungs. In the lungs, the useless part is regenerated by the spirits and made useful. It is remarkable with what prophetic gift Galen pointed to a constituent of the air as the spirits, the nature of which he could not yet divine. He expresses clearly the supposition that it will be possible at some time to isolate that constituent of the air that forms the spirits. More than fifteen hundred years elapsed before Galen's supposition was confirmed by the discovery of oxygen by Priestley and Lavoisier. The blood, regenerated by the receipt of spirits in the lungs, flows through the pulmonary veins into the left heart, whence, together with the rest of the useful blood, it is carried by the aorta and its branches throughout the whole body. Galen's views upon the nervous system are equally interesting. In the brain and the spinal cord are the origins of the sensory and the motor activities of the nerves. The motor nerves act by pulling like a string upon the motor organs. In the special physiology of nerves, Galen investigated particularly the action of the vagus and the intercostal nerves upon respiration and the action of the heart, and he cut the spinal cord transversely and longitudinally-experiments which show how deeply he had penetrated into an understanding of the mutual relations of the individual organs of the body.

Galen's physiological system was for that time a monumental work, and the fact that Galen's views continued for thirteen hundred years as the unassailable code of medicine is surely not. to be ascribed simply to the decay of the ancient culture and to the complete barrenness of the middle ages in scientific matters. The development of physiological investigation took not a single step forward during the middle ages. The Arabians, who had come to possess the ancient culture, were, indeed, prominent as physicians, but Islam forbade them alike independent investigation and philosophical thought. Even Avicenna (Ibn Sina, 980-1037), who was the most prominent of the Arabian physicians and showed philosophical tendencies, performed no original work. With slight changes his system was the system of Galen, whose glory he obscured by his own powerful authority in the civilised world of that time. Moreover, the many famous medical schools which arose at that time in Italy, France, and Spain trained many able physicians, but did not advance beyond Galen's ideas, not- 
withstanding the fact that here and there an isolated physiological observation was made. This condition of stagnation continued until into the sixteenth century.

\section{C. 'THE PERIOD OF HARVEY}

An independent advance in physiology is first met with in the sixteenth century. One of the first to abandon Galen's system was Paracelsus (1493-1541), who developed a complete system of nature. It was permeated with theosophical notions, a tendency that appeared still stronger in his followers and drove them wholly over to mysticism. Nevertheless, it contained many original, although frequently absurd, ideas. Paracelsus opposed the weak echoes of Galen's system and its outgrowths which had appeared during the middle ages, and this was at that time an important advance. The foundation of his system is the unity of nature. Nature is a unit, the macrocosm. In man as the centre of nature all forms of existence are containcd. Hence, man is to be regarded as a microcosm. Nature, however, must not be considered as complete but as for ever becoming. The more special aspects of his system are arbitrary and unimportant, and, as is usual in such cases, this first beginning of independent investigation was comparatively crude; before all other things it lacked a purely empirical and experimental basis.

At the same time, in France and in Italy a freer tendency began to appear in the medical schools. Fernelius (1497-1558) had many new ideas, although they were based wholly upon Galen's system. From the various forms of Galen's pneuma he separated the anima. The former consists of the most subtile material substance; the latter is the soul, which is to be recognised only by its effects. He advanced the further idea that the phenomena within the organism depend finally upon certain mysterious causes.

Special physiological investigation received $i n$ impulse from the great anatomical discoveries in the schools of France and Italy, where knowledge of the anatomy of the human body was placed upon a wholly new and strictly empirical basis by Tesalius, Eustachio, Faloppio, and others. Researches upon the structure of the heart and the course of the vessels were the most fruitful for physiology. The doctrine of the circulation of the blood, as founded by Galen, underwent fundamental changes. By proving the imperviousness of the interventricular septum, Servetu (1511-1553) refuted Galen's idea that the blood goes from the right ventricle of the heart directly into the left ventricle. His followers, Colombo (d. 1559) and Cesalpino (1519-1603), added to this new facts upon the circulation of the blood in the lungs; and Argentieri (1513-1572), who opposed the doctrine of the animal 
spirits, and happily thought to put in its place heat as the cause of vital phenomena, emphasized the fact that the nutrition of the whole body is provided for by the blood alone. By these special investigations into the physiology of the blood, the path was made easy to the greatest discovery of this period, that of the circulation of the blood by Harvey (1578-1657). The importance of Harvey's discovery lies in the fact that he first established the physiological connection of the arteries and veins peripherally, and the passage of the blood from the arteries into the venous trunks and thence to the heart; he thus laid a basis for the fact that all the blood passes through the heart and moves in a closed circuit through the whole body. He added to this a great number of special facts concerning the mechanism of the circulation, all of which-and herein lies the great significance of his work-rested upon keen observation and an exact experimental basis. Following the exact tendencies of his time-the time which brought forth also a. Copernicus, a Galileo, a Bacon, and a Descartes-Harvey, by his brilliant discovery, raised the experimental method again to an honourable position in physiology, after it had remained in complete oblivion for thirteen centuries. The spirit of the conscientious investigator and great logical acuteness characterise Harvey's personality and stamp him as the first real physiologist after the long night of the middle ages. A second doctrine "de generatione animalium," stands with equal honour by the side of his doctrine of the circulation of the blood. In this he put forward the dictum, "omne vivum ex ovo," which has since obtained vast significance in the science of life, and in the various forms in which it has been expressed in recent times controls all modern physiological views of organic reproduction.

Of the adherents of the great theosophical school which Paracelsus instituted, one only is important in the history of physiology, namely, van Helmont (1577-1644), since, in spite of the mysticism that characterised the whole theosophical tendency, he made thoroughly accurate observations. Starting with the Paracelsian doctrine of the unity and the constant development of nature, he conceived all natural bodies to be composed of matter and "archeus" (energy). Things exist and live only in this combination. As a result of it, all things are living. There are, however, different grades of life, and the so-called lifeless bodies exist at the lowest grade. Among van Helmont's special physiological ideas, his chemical doctrine of ferments is especially interesting. He rejects Galen's idea that digestion goes on in the stomach through the action of heat, and puts in its place the correct conception, that digestion is performed by a "ferment" associated with the gastric juice.

The philosophical systems of Francis Bacon (1561-1626) and Descartes (1596-1650) exercised a great influence upon the further 
development of physiology. Bacon's monistic philosophy, which, because of its vigorous accentuation of the inductive method of investigation, has become the basis of all modern natural science, inaugurated the great series of new and exact physiological observations, founded upon experiment, which has continued from that time to enrich our knowledge of vital phenomena. The philosophy of Descartes, although purely dualistic, was full of importance for the physiology of the senses and the theory of knowledge, because of the theory of sense-perception which formed its starting-point. Descartes was the first to maintain the proposition that the only thing in the universe of which we have certain knowledge is subjective psychical sensation. Mind, sensation, thought, is the only fixed point from which the universe can be surveyed and discovery proceed. "Cogito, ergo sum." Senseperception, therefore, gives us no information concerning things, for it is deceptive; and things, i.e., bodies, are in reality wholly different from what they appear through our sense-organs to be. These propositions are capable of the widest application. Further, they are so well grounded and so precisely and clearly expressed, and give so admirable a basis for a philosophical system, that one must wonder how it is possible for Descartes, who was usually a clear and consistent thinker, to be so inconsistent as to arrive finally at the complete dualism of body and mind. It is a temptation to believe that he maintained ultimate consistency secretly: but that, for practical reasons, he allowed the pressure of the ecclesiastical conditions of his time to give this unexpected turn to his philosophy, while content with the feeling that the unpredjudiced thinker would note and correct the evident incongruity. Of the greatest physiological importance, however, is his clear discernment in his dualism that the bodies of animals and men act wholly like machines and move in accordance with purely mechanical laws. But here again dualism comes in as a disturbing element, for Descartes ascribes the impulse to all movement to the soul, which, from its seat in the only unpaired organ of the brain, the pituitary gland, controls the individual parts of the body. Nevertheless, the general physiological ideas of Descartes have been of great value to physiology, and the gifted thinker also marle many very important special physiological observations, which markedly advanced our knowledge of physiological optics and acoustics.

Descartes' notion that, as regards its vital activities, the human body is to be regarded as a complicated machine, was especially fruitful for physiology in the ingenious application which Borelli (1608-1679) made of it in the science of animal movement. Borelli undertook for the first time to reduce the movements of the organic motor apparatus to purely physical principles, and thus laid the foundation of our present mechanics of animal motion. 
The chief result of this undertaking found expression in the inauguration of a peculiar school founded upon Borelli's doctrine, the iatromechenical school (called also iatrophysical and iatromathematical), which played a considerable rôle in the further development of physiology, since it endeavoured to explain other vital phenomena of the animal body upon purely physical principles. At the same time, some of Borelli's followers, especially Glisson, by regarding contractility as a property residing within musclesubstance itself, became the precursors of the later doctrine of the irritability of muscle.

Almost contemporaneous with the founding of the iatrophysical school there arose another school, the iatrochemical, which for a time flourished by the side of the former. Its founder was Sylvius (1614-1672). Dissatisfied with the narrowness of the iatrophysicists, but recognising the importance of their principle in explaining vital phenomena, Sylvius emphasized the chemical side in addition to the physical, and in accordance with this elaborated chiefly the physiology of digestion and respiration by extending van Helmont's doctrine of the ferments. In the theory of respiration also, Mayow (1645-1679) expressed very pertinent thoughts upon the analogy between respiration and combustion.

At this time physiology derived considerable assistance, the value of which for physiological investigation, however, has not been completely taken advantage of even to the present day, from the invention of the compound microscope and the microscopic discoveries made by means of it by Leeuwenhoek (1632-1723), Malpighi (1628-1694) and Swammerdamm (1637-1685). The knowledge of the physiology of reproduction and development, nore than all else, was thus markedly advanced. The first microscopic discoveries in this field naturally led to many excusable errors. When, for example, aqueous infusions of decomposable substances were made and the appearance of Infusoria in immense numbers was observed in them, spontaneous generation from lifeless substances was believed to have taken place, contrary to Harvey's dictum, "omne vioum ex ovo," but in accordance with Aristotle's earlier assumption even for higher animals. On the other hand, Harvey's dictum became the starting-point of important discoveries; for Malpighi followed the development of ova with the microscope, while Leeuwenhoek's pupil, Ludwig van Hammen, discovered spcrmatozoa, the importance of which Leeuwenhoek immediately recognised.

These and a great number of special physiological discoveries which active investigation brought forth give to the period of the seventeenth and eighteenth centuries after Harvey's appearance the character of the dawn of exact investigation in physiology, just as the influence of exact methods pervaded and animated all science of that period. Yet, as is constantly happen- 
ing in the history of science, systems appeared at that time il evidence of a reaction against excessive specialisation, which fell into the opposite extreme of lacking all exact foundation and resting upon pure speculation. Boerhaave (1668-1738) skilfully avoided this pitfall in his eclectic system, which was put together from the various dogmas of his time and assumed as the source of all vital phenomena a "principium nervosum" in the form of a very subtile fluid. But the systems of Hoffmann (1660-1742) and of Stahl (1660-1734) did not escape it. Hoffmann's "mechanicodynamical" system is purely teleological, and arose under the influence of the philosophy of Leibnitz. Hoffmann regarded the ether as the ultimate cause of all vital phenomena; its movement follows mechanical principles, but it receives its immediate impulse from the idea of the purpose of its own existence, that resides in every ether-atom. But Stahl's "animistic system," which combated Hoffman's doctrines, rests still more upon a speculative basis. At the foundation of Stahl's system there lies a dualism of body and mind, according to which the body in its activities follows mechanical laws, but is animated and preserved from decay and destruction by the "anima." Upon the nature of the anima Stahl expresses himself in very uncertain and contradictory terms. In spite of its unsupported speculations and its many contradictions, anımism obtained numerous adherents, which may be explained by the facts that the innumerable details acquired by the many special researches of the period were not properly sifted, and there was no coherent understanding of vital phenomena.

\section{THE PERIOD OF HALLER}

Haller (1708-1777) responded in a genuinely scientific manner to. the need of a unitary arrangement of the details, and, as it had once been with Galen and, later, with Harvey, a new epoch in the development of physiological investigation dates from his appearance. As Galen had first recognised the practical significance of physiology and had made the knowledge of rital phenmmena the basis of practical medicine, and as Harvey by the introduction of exact experimental investigation had created the fruitful method, the employment of which in the sixteenth and seventeenth centuries called forth the enormous mass of individnal discoveries, so Haller for the first time brought together as a whole all the extensive material of facts and theories in his "Elcmento physiologian corporis humani." $\mathrm{He}$ thus made physiology an independent science, which was not simply to serve practicil purposes in the interest of the art of healing but also to musue purely theoretical aims for itself alone.

In this circunstance lies Haller's great importance in the development of physiology. The gromping of a heterogeneuns. 
mass of facts into a closed and intelligible whole is always stimulating and fruitful, and this explains the immense authority and powerful influence which Haller exercised in the development of physiological investigation. His uwn physiological researches, however, while very conscientious and exact, as, e.g., those upon the respiratory movements and the theory of irritability, contain no epoch-making discoveries, and some of them even had the misfortune to play an obstructive role in the further development of the science. This is especially true of two doctrines which he advocated-the so-called theory of preformation, and the theory of irritability.

The theory of preformution (theory of incasement) arose in connection with the microscopic observations upon the development of the ovum which were made in the seventeenth century. When it was seen how from a single small egg a complete animal was developed by the gradual maturing of one organ after another, the idea arose that all organs appearing in the course of development and, in brief, the whole animal, are preformed or already enclosed as such within the egg, and are made risible to the eye only by a process of growth and unfolding; that, therefore, the human egg or, as some believed, the spermatozoon, is a minute but a completely formed homunculus. The necessary consequence of this idea was the assumption that at the creation of the world all coming generations were contained, already preformed, in the egg of each animal. The preposterousness of this riew led a young physician, Caspar Friedrich Wolff (1733-1794) to maintain a new theory in opposition to that of preformation. Wolff"s "thcoria generationis," which later became the basis of all our modern ideas of the development of organisms, denied incasement and put in its place 'pigenesis. This asserted that all organs of the body are formed one after another in the course of development, in other words, that they originate as entirely new parts and have nerer pre-existed as such in the egg. Haller could not accept the idea of epigenesis, but opposed it energetically; and, supporting the dogma of preformation with his whole authority, he retarded progress in the doctrine of animal development for more than half a century.

Haller's theory of irritability influenced the development of physiology in a somewhat different manner. Haller's own researches in this direction were experimental and very exact, and materially advanced the general theory of irritability ; but they were misinterpreted in various respects and extended by his followers, and formed the chief starting-point of a doctrine that confused all physiology down to the middle of the present century, and even now emerges again here and there in varied form. "This is the doctrine of rital force. The fact of the irritability, or the direct excitability, of muscles had been emphasized by the earlier iatrophysicists, especially by Glisson (1597-1677). Haller took up the 
question, and adderl the experimental proof of the fact that the muscle-fibre possesses the property of contracting upon stimulation independently of nervous influence, a quality which he sharply distinguished as irritability from the sensibility belonging to nerves. This sharp distinction affirmed a difference between the excitation of nerve and that of muscle which did not correspond wholly to reality, and awoke in many of Haller's adherents and follower's the need of demonstrating irritability to be a uniform phenomenon.

This was attempted most successfully by an Englishman, John Brown (1735-1788), a gifted but careless thinker. Brown recognised in general a single excitability common to the nervous and muscular system, which system he regarded as a unit. The capacity of becoming excited by stimuli is possessed by all living nature, and is, indeed, the fundamental characteristic by which living beings, animals and plants, are distinguished from lifeless. Regarding the nature of excitability, Brown, like all other physiologists of the time, had little to say.

The hopes of the iatromechanics and iatrochemists of being able completely to resolve vital phenomena into physics and chemistry were not fulfilled. In irritability there existed a phenomenon which, as was believed, distinguished all organisms from lifeless bodies, and appeared to mock at a physico-chemical explanation. The unexplained conception of irritability, therefore, in union with the dynamical systems of Hoffmann and Stahl still prevailing, became the starting-point of vitalism or the doctrine of vitul force, which in its most complete form asserted a distinct dualism of living and lifeless nature. This theory appeared first in France, especially in the School of Montpellier, and later in Germany, and its hazy notions of vital force soon controlled all physiology. In France vitalism was founded by Bordeu (1722-1766), developed further by Barthez (1734-1806) and Chaussier (1746-1828), and formulated most distinctly by Louis Dumas (1765-1813). The vitalists soon laid aside more or less completely mechanical and chemical explanations of vital phenomena, and introduced, as an explanatory principle, an all-controlling, unknown and inscrutable "force hyperméchanique." While chemical and phrsical forces are responsible for all phenomena in lifeless bodies. in liring organisms this special force induces and rules all vital actions. In Germany vitalism did not reach this degree of clearness. Its founder, Reil (1759-1813), differed from the French vitalists, and in his treatise "Ueber die Lebensh'r ft." expressed fairly clearly the view that the phenomena of living organisms are chemico-physical in nature, but that principles are at the same time in control which are conditioned exclusively in organisms by the characteristic form and composition of living substance. Later ritalists, howerer, attempted no analysis of vital force; they employed it in a wholly mystical form as a convenient explanation of all sorts of vital phe- 
nomena, and they distinguished several varieties. The "nisus formativus," e.g., or peculiar "formative effort," offered a simple explanation of the forms of organisms, accounting for the facts that from the egg of a fowl a fowl and no other species always developed. and that the offspring of dogs are always dogs. In place of a real explanation a simple phrase, such as "formative effort," or "vital force," was satisfactory, and signitied a mystical force belonging to organisms only. Thus it was easy to "explain" the most complex vital phenomena.

But some investigators were not content with this kind of explanation, and, while indifferent to the doctrine of vital force, continued to search for a chemico-physical explanation of vital phenomena. They received a strong stimulus from the new discoveries of Galvani (1737-1798), who proved that electricity is produced by the living animal body, especially by the nerves. Naturally the value of this fact was very soon overestimated, and under the ban of the prevalent philosophy of nature, particularly as a result of the researches of Ritter (1776-1810) and partly also those of Alexander von Humboldt (1769-1859) and others, who extended Galvani's experiments, the idea arose and later became very popular, that the galvanic current is the cause of all vital phenomena, and even that all phenomena of all nature may be explained in general by galvanic polarity.

The great chemical discoveries of the previous century alsu influenced the development of physiology. Vegetable physiology was especially advanced by Ingenhouss (1730-1799), who developed the theory of the consumption of carbonic acid by plants. The discovery of axygen by Priestley (1733-1804) and Lavoisier (17431794), which was so momentous for physiology, bore its first fruits when Girtanner (1760-1800) showed that venous blood receives oxygen in the lungs from the inspired air. Thus the old doctrine of the pneuma, which controlled physiological ideas for centuries, was justified in modern form, and at the same time the ingenious idea of Mayow, who had compared respiration to a process of combustion, was raised to the rank of a fundamental physiological fact.

Besides the physical and chemical discoveries of that time, those in analomy led also to important physiological results. Most prominent among these was the fundamental law of special nervephysiology, announced by Charles Bell (1774-1842), and later proved experimentally by Johannes Miiller, which affirms that the posterior roots of the spinal nerves are sensory (conducting centripetally), while the anterior roots are motor (conducting centrifugally).

Finally, in microscopy Spallanzani (1729-1799), and later especially Treviranus, obtained the distinction of having disproved experimentally by careful researches the theory of the 
spontaneous generation of animalcules in putrid infusions; they showed that these lowest of all living things develop only from germs which are to be found everywhere in the air and the water, and that even here Harvey's dictum "cmne vivum ex ovo" admits of no exception.

England and France produced the most of these exact researches, while in Germany the most prominent thinkers, such as Oken, were swept on by the philosophy of nature with its powerful tendency toward pure speculation in the fields of natural science.

\section{E. THE PERIOD OF JOHANNES MÜLLER}

Juhannes Müller ${ }^{1}(1801-1858)$ is one of those monumental figures that the history of every science brings forth but once. They change the whole aspect of the field in which they work, and all later growth is influenced by their labours.

Like the other investigators of his time Muiller was a vitalist, but his vitalism had an acceptable form. To him vital force was something different from the forces of Jifeless nature, but its administration rigorously followed physico-chemical laws, so that his whole endeavour was to explain vital phenomena mechanically. In doing this he went over the whole field of vital activities uniformly, neglecting no part, and by his own investigations, which were always original, he laid the foundations upon which we work to-day. He always kept his attention directed towards the whole; he never undertook special investigations which would not help him to solve some large general problem. His ingenuity-and it is this that is so much missed in the more recent physiology - was expressed in the manner in which he attacked problems. He did not recognise one physiological method alone, but employed boldly every mode of treatment that the problem of the moment demanded. Physical, chemical, anatomical, zoological, microscopic and embryological knowledge and methods equally were at his disposal, and he employed all of these whenever it was necessary for the accomplishment of his purpose at the time.

The philosophy of nature experienced its most luxuriant growth during this time under the influence of the ideas of Schelling and Hegel, and with its unbridled speculation, which lacked all basis of fact, seriously threatened scientific investigation. But it exercised only the most beneficent effect upon the rigorously critical mind of Müller. He recognised in the ambitious tendencies of the natural philosophers a germ of truth, and under its influence fashioned his own manner of scientific investigation into a genuinely philosophical type. While keeping constantly in view the large

1 The most inportant estimate of Johannes Miiller is to be found in the memorial address upon him given by du Bois-Reymond ('59). 
problems and the goal of science, he regarded critically the special methods and questions only as means to an end, as means for arriving at a harmonious comprehension of nature. Throughout his whole life he remained steadily true to this philosophical conception of science, which he had set forth with energy in his inaugural address, "Von dem Beduirfniss der Physiologie nach einer philosophischen Naturbetrachtung." It is remarkable that, notwithstanding the unalloyed admiration aroused by the figure of Miiller, the later physiology has often wholly neglected this element. This is particularly noticeable in two fields in which from his youth up he took the most active interest, - that of psychology, and that of comproative physiology.

Psychology is avoided by the physiology of to-day almost with fear, an attitude that is in peculiar contrast with that of Muiller. He regarded physiology as essential to advance in psychology by empirical methods, and in his examination for the doctorate he defended the thesis, "Psychologus nemo nisi physiologus." Undoubtedly, the science of psychology ought not to be considered as simply a part of physiology. But the achievements of physiology in the field of the nervous system and the sense-organs are of so fundamental significance for psychology, that it may be said that the former science is more nearly related than any other to the latter. Müller's own labours show very clearly with what success physiology is capable of handling psychological problems, for scarcely any physiological discovery has a more important bearing upon all psychology and the theory of knowledge-although unfortunately it is not generally appreciated-than the doctrine of the specific energy of the nerves or organs of the special senses. This doctrine affirms that different stimuli of whatever kind, when applied to the same sense-organ, e.g. the eye, are capable of calling forth only one and the same kind of sensation, namely, that sensation that is mediated by the sense-organ in question under the influence of its natural stimulus, in the case of the eye, light. Vice. i.r's organs, calls forth entirely different sensations according to the nature of the organ upon which it works. This doctrine is founded upon two fundamental facts: first, that in reality the external world is not what it appears to us to be when perceived through the spectacles of our sense-organs; and, second, that by the path of our sense-organs we cannot arrive at an adequate knowledge of the world. Besides this fundamental proposition, however, Miuller discovered many other important psychological facts, which he has presented in his works; "Zur vergleichenden Physiologie des Gesichtssinnes des Menschen und der Thicre," "Ueber" die phantastischen Gesichtserscheinungen," and the section "Vom, Seelenleben" in his "Handbuch der Physiologie des Menschen."

Müller's teacher Rudolphi had said: "Comparative anatomy is 
the surest support of physiology; without it physiology is scarcely conceivable." Müller was incited by this idea, and the result was the foundation of a wholly new science in his comparatie physiology. Throughout his whole life he defended the position expressed in the words, "Physiology can be only comparative," and amiong the very large number of his physiological works there are few in which the comparative principle is not more or less clearly expressed.

He presented the results of his own investigations together with practically all the physiological knowledge of his time in his "Handbuch der Physiologie des Menschen." This work stands tolay unsurpassed in the genuinely philosophical manner with which the material, swollen to vast proportions by innumerable special researches, was for the first time sifted and elaborated into a unitary picture of the mechanism within the living organism. In this respect the "Handbuch" is to-day not only unsurpassed, but unequalled. Naturally many of its details are incorrect according to present ideas; later researches performed with a more perfect technique have greatly extended and transformed some departments; even many of Müller's general physiological ideas such as that of vital force, have been completely abandoned br the later physiology; nevertheless, it remains that of all the numerous handbooks that have since appeared none has reached that of the great master as regards the mode of dealing with the material. Must of the later Hand-books, Text-books, Elements, etc., although intended almost exclusively for the use of students, do not take the trouble to point out even briefly the aims, the problem and the purpose of physiological science, let alone giving to the matter as a whole a philosophical treatment in Müller's sense. Such a lack must be regarded as a serious detriment by thinking students who do not learn simply by rote. Only a very few text-books form an exception to this, as, e.g., Brucke's admirable "Vorlesungen vibe" Physiologie."

The tireless physiological activity of Miiller, which won for him the fame of being the greatest physiologist of all time, did not prevent him from giving himself up in the later years of his life with equal enthusiasm to morphology, especially zoology, comparative anatomy, and paleontology, and of acyuiring the name of the greatest morphologist of his time. Su many-sicled ancl comprehensive was he that by his own fundamental labours h. mastered two large sciences, either one of which a single persun is at present hardly able to surcey maided.

It is no wonder that so large a ralm could not be held together as a unit after the death of its rulcr. Like Alexander's unirersal (mpire, it became divided into many small territories, each one of which controlled itself; and with the present boundary of seimece it would be diffieult to find a worthy sucessor to Müller, eren 
if he were endowed with the latter's superhuman power of labour.

Morphology had become independent long before Muiller. Soon after his death the course of physiology became divided and directed along purely chemical and purely physical paths.

Movement in the chemical direction was guided by Wöhler (1800-1882) and Liebig (1803-1873). In the year 1828 Wöhler gave the theory of vital force its death-wound by his epoch-making synthesis, out of purelyinorganic substances, of urea, a body produced in nature only by organisms. It had been believed that substances that were produced by the organism were produced only through the activity of vital force; but here for the first time a very characteristic material product of the animal body was manufactured artificially in the chemical laboratory. This synthesis was soon followed by others. Justus von Liebig established new views regarding the metabolism of organisms; and later Voit, Pfliiger, Zuntz, and others, advanced the theory of metabolism further, though not in entire agreement with one another. Physiological chemistry became more and more independent, partly under the influence of Mulder and Iehmann, who first made a survey of the field, and especially under that of Kühue, who by his original methods and investigations, particularly upon the chemico-physiological relations of the proteids, diffused new light and expressed his conception of the science in his text-book. Finally, most recently, through the labours of Hoppe-Seyler, Hammersten, Bunge, Halliburton, Baumann, Kossel, and others, physiological chemistry as an independent science has quite cut itself loose from physiology, to the detriment of the latter.

E. H. Weber (1795-1878), Volkmann (1801-1877), Ludw1g (1816-1895), Helnholtz (1821-1894), du Bois-Reymond (18181896), Marey, and others, led the movement in the physical direction. Ludwig mechanically transmitted the rhythmic changes of pressure of the pulse to a moving writing-lever, and made them record themselves upon the smooth surface of paper moved at a uniform rate (Fig. 1). He thus surpassed all others in creating a method of the greatest value in the investigation of the purely physical activities of the animal body. This graphic method proved so extremely fruitful that it found wide employment in physiology. It was used for the graphic representation of muscle-contraction, of respiratory movements, of the heart-beat, etc. In France, Marey developed it to unexpected completeness; so that now it serves as the most important method of investigation in all researches that deal with the phenomena of macroscopic movement. One other method became fundamentally important in physical physiology, namely, that of the comprehensive and ingenious technique of galvanic stimulation, which was created by E. du Bois-Reymond's classic researches upon the general physics of muscle and nerve. 
By the perfection of this technique du Bois-Reymond made the galvanic current of all stimuli the most convenient to employ and
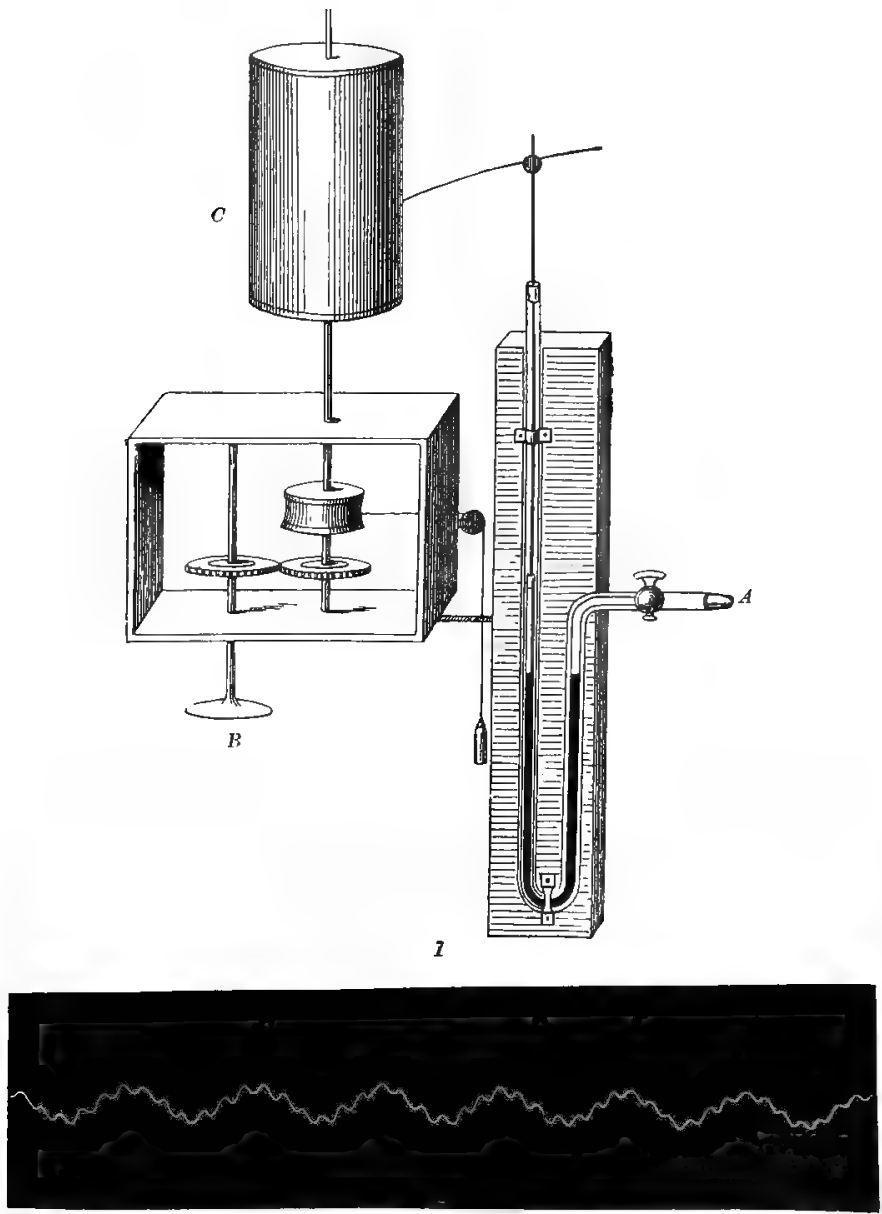

II

FrG. 1.-I. Ludwig's kymograph. One limb of the manometer is connected with an artery at A; the blood-pressure is tramsmitted to the column of mereury (ropresented in black), thence to the flont upon the mercury in the uther limb, and puts this Huat with its writing-lever in motion. The writing-lever inscribes it muvuments upu the drum, $C$, which is kept in constant rotation by a clock-work, B. (From Brilcke). 11. l'ulse-curve from a rablit, The $r$ small waves represent the variations in blood-pressure that constitute the pulse; the large wives, the variations that the blood-pressure undergous as the result of respiration.

the most capable of fine gradation and (asy localisation for nerves and muscles: for these reasons it nuw holds the first places in stimulation-experiments. 
The wide applicability of this ingenious physical method is due to the perfection of the technique of vivisection on the part of the great French physiologists, Magendie (1783-1855) and Claude Bernard (1813-1878). Claude Bernard guided operative physiology to its highest development, without at the same time becoming narrow. He was a philosophical investigator who in his researches kept in view the general problems of life. It is no wonder, therefore, that all French physiology of to-day must be considered as of Claude Bernard's school.

In comparison with the chemical and physical features of physiology, after Johannes Müller's death other features receded into the background, or were entirely neglected. Psychological research was advanced especially by discoveries regarding the physiology of the sense-organs, in which the ingenious investigations of Helmholtz and Hering led to most important results, and the physiology of the central nervous system of higher vertebrates, knowledge of which was perfected by the epoch-making labours of Flourens (1794-1864), Hitzig, Munk, Goltz, Horsley, and others. Preyer's endeavour to follow the development of the psychical phenomena of human beings through the early years of life has been followed by a few others. At first little attention was paid to the general questions of physiology. Lotze's Allgemeine Physiologie des hörperlichen Lebens (1851) was purely speculative, and treated physiological questions from the standpoint of philosophy; nevertheless, it necessarily would have proved a valuable stimulus to the experimental physiology of that time in the investigation of important questions, if in exact science interest in general problems had been greater. Although the striking works of Charles Robin, Chimie, anatomique et physiologique (1853) and Anatomie et physiologie cellulaire (1873), presented a coherent summary of the anatomy and physiology of the cell, unfortunately they were little appreciated from the physiological side. So also the cell-pathological researches and ideas of Rudolf Virchow (Cellularpathologie, 1858), which quite overturned medical ideas, until very recently and in spite of their showing very clearly the enormous practical importance of general physiological researches upon the cell, have had scarcely the slightest influence upon the development of physiology, because the latter science was captivated by questions of a more special kind. More attention was excited by Claude Bernard's Lecons sur les phénomènes de la vie. communs aux animaux et aux végétaix (1878), which treated a number of general questions concerning life in a classic manner, although somewhat unequally. Preyer endeavoured to discuss the questions of general physiology more uniformly in his Elemente der allgemeinen Physiologie (1883), but unfortunately the book contains only a schematic summary of the subject. Finally, the researches of the histologists and the zoologists afforded many 
contributions to the physiology of the cell, and in our own time, from this side especially, the physiology of reproduction, fertilisation, development, and heredity has been taken away from physiology proper, and developed into a fruitful and independent subject. $^{1}$

The comparctive methon has not been employed in physiology since Johannes Muiller's time, unless the few researches that have been conducted upon other animals than the usual dogs, rabbits, and frogs are to be considered as comparative.

Plant physiology, however, has developed quite independently into a flourishing seience; and the distinguished labours of Hofmeister, Nägeli, Sachs, Pfeffer, Strasburger, Berthold, and others have made this in recent times the most complete branch of physiology. This is due partly to the fact that all vital relations are much simpler and more easily surveyed in plants than in animals, and partly to the fact that plant physiology has made use of certain acquisitions of science that have thus far found little or no application to the physiology of animals.

There are three of the greatest discoveries of this century, from the further expansion of which physiology is justified in still expecting great results.

One of these is the law of the conservation of energy, which was definitely expressed by Robert Mayer (1814-1878), and was established most comprehensively by Helmholtz. IIodern chemical investigations had led to a recognition of the law of the conservertion of matter, by showing that the quantity of matter, of atoms, in the universe is constant, and that the smallest atom cannot by any agency be destroyed or recreated. The law of the conservation of energy expresses the same fixedness for the sum of the energy of the universe. Energy, like matter, can be neither destrored nor recreated; when it seems to appear or disappear, it merely passes from one form into another. Among the recognised forms of energy two varieties are distinguished: cnergy of motion, or kinetic energy, when power is in action, i.e. is producing motion; and encrgy of position, or potential cuergy, when it is latent but under certain condition can come into action. Thus, c.g., the potential energy that was produced in the Carboniferous age by transformation of the kinetic energr of the sun's rays through the activity of plants and was stored up as chemical affinity in vast strata of coal, passes over into heat upon combustion of the coal. The heat is transformed by steam engines which are heated by the coal, into the energ? if

1 Résumés of what has been accomplished in this field are given by the following books: Die. Zelle und lie Gewebe, hy O. Hertwig (1892) [authorised English translation, The Cell: Outlimes of General Anatomy and Physiolony, 1895]; Gesrmmelte Abhandlungen über Enlmichlungsmechanik, hy W. Roux (1895); La structury dil protoplasma ef les thcories sur l'hérédité, ete, by Ives Delage (1895); [and The Cell in Developument ont Inhritrenre, by F. B. Wilson (1896)]. 
mechanical work, and this can be changed by means of a dynamo into electricity and be made to serve finally for the production of the electric light. Thus we perform daily the remarkable experiment of re-transforming, after millions of years, into its original form, the kinetic energy of the sun's rays which the plants of the Carboniferous age employed for storing up carbon, and thus illuminating our nights with the radiance of the sun that shone upon the surface of the earth in immemorial times ( $C f$. Bunge).

The application of the law of the conservation of energy to the energetics of organisms was attenuted by Robert Mayer, and has since been undertaken many times. By the calorimetric researches also of Dulong, Helmholtz, Rosenthal, Rubner, and others, the proof has been afforded experimentally that this law is as true in living nature as in lifeless. But our knowledge is extremely scanty concerning the mode of action of energy in the various performances of the body, concerning the transformations undergone by the energy in its path through the living substance. In this respect plant physiology, which is indebted especially to the striking researches of Pfeffer upon the energetics of the plant-cell for important discoveries and suggestions, is relatively farther advanced than animal physiology. In this subject of the energetics of living substance the future offers a wide field of labour, which is full of reward.

The second of the great discoveries, which also has yielded chiefly to plant physiology its most important results, but has not yet been employed at its full value in the science of the physiology of animals, is the fact that organisms are composed of cells. The beginnings of the cell-theory are to be found in botanical studies. The microscopists of the seventeenth and eighteenth centuries, especially Malpighi, Treviranus, Mohl, and Meyen found that plants are composed of small microscopic chambers, or cells, and elongated tubes which have liquid contents. The elongated tubes soon proved themselves to be structures that arise from series of cells by a dissolution of the transverse walls. Brown found next a more solid nucleus as a wide-spread structure in the liquid cellcontents. But schleiden first put into general form the idea that all plants are composed of cells, and he distinguished as an essential constituent of the cell-contents, besides the cell-sap and nucleus, the semi-liquid motile plant-slime, which was termed by Mohl protoplasm. In the meantime the wide occurrence of cells in the animal kingdom had become recognised, and, soon after Schleiden, Schwann founded the cell-theory for the animal kingdom by showing that animals are composed of cells or cell-products, and in their development progress from forms that contain only a few similar cells. Later, embryology established the fact that in general all organisms are developed from a single cell, the eggcell, into a cell-community which nay bcome large and powerful, 
and in which the various parts, tissues, and organs consist of specific forms of cells. Although this knowledge carried with it the fact that the cell is the element of the living organism and the place where the life-processes occur, nevertheless, the cell, except in botany and embryology, has not yet been made a subject of special physiological study. We shall see presently that precisely in this direction is to be expected an essential advance in the physiology of the future.

The third discovery, which thus far has not been fruitful in physiology, is that of descent in the organic world. The theory of descent, sketched in its outlines by Lamarck, and firmly founded by Darwin upon the principle of selection, has produced a great revolution in all morphological research, and impressed upon modern morphology its characteristic stamp. The theory shows that all the varied forms of organisms stand in genetic relationship to one another by descent, and that ultimately all have been derived from the simplest organisms. The theory of selection ascribes the enormous variety of forms to natural selection conditioned by the struggle for existence; in this struggle only those individuals of a generation survive that are best adapted to existing external conditions-in other words, those that are best fitted to live. Thus, after an oblivion of more than two thousand years the ancient idea of Empedocles of the descent and gradual change of the organic world by selection has celebrated its resurrection in the present century by the empirical foundationwork of Darwin. Embryology, so far as it relates to the development of form in organisms, has flourished to an unexpected degree from the powerful stimulus given it as the result of Darwin's theory, especially by Haeckel and his pupils, but so far physiology has not availed itself of the evolution idea. The evolution of vital activities, the origin and development of the many functions possessed by the individual parts of the living body, is thus far almost a terra incognita. During the last few decades but one physiological problem of evolution, the problem of heredity, has been very actively discussed, and this almost exclusively from the zoological side. But the point has now been reached where experimental physiology alone is able to bring about further advance.

\section{The Method of Physiological. Research}

- It has been learned that the problem of physiology lies in the explanation of vital phenomena, and it has been seen, in its main features, how physiological research has developed in the course of history. It is now incumbent upon us to summarise with reference to the development of science what physiology has 
already accomplished in the direction of its established goal, and to inquire by what path it may reach this goal.

\section{A. 'THE PAST ACHIEVEMENTS OF PHYSIOLOGICAL RESEARCH}

The aim of Physiology is to explain vital phenomena, i.e., to discover their elementary causes, to put them into causal relation with one another, to see whether their elementary causes are the same as those of the phenomena of inorganic nature. What has been accomplished in this direction?

The answer brings little encouragement, for, when the various branches of physiology are carefully reviewed, it is found that thus far practically nothing has been learned beyond the gross mechanical and chemical activities of the vertebrate body. The causes upon which these activities depend are, for the most part, a complete puzzle.

We know that respiration depends upon the laws of aerodynamics; by the rhythmic diminution and increase of pressure of the air in the lungs, as a result of the contraction and relaxation of the respiratory muscles, the air streams passively in and out; oxygen is removed from it by the red corpuscles of the blood and is chemically united with the substance of the corpuscles. But we have scarcely an idea as to how the contraction of the respiratory muscles comes about, or what events call forth the change of form, termed contraction and relaxation, and the performance of work in the individual muscle-cells.

We know that the circulation of blood in our bodies follows the laws of hydrodynamics, that it is conditioned by the rhythmic variation of differences of pressure within the vascular system, which are brought about by the contraction and relaxation of the heart-muscle. We have here again exactly the same problem as in respiration, for, although Engelmann has recently proved that the causes of the rhythmic contractions of the cardiac muscle lie in the living substance of the muscle-cells, as to the manner in which the contractions come about physiology has enlightened us very little.

We know that the digestion of the ingested food takes place strictly in accordance with chemical laws; the chemical substances secreted by the gland-cells of the digestive canal transform the food chemically, exactly as we can imitate the processes by the help of those digestive secretions outside the body in the testtube. But physiological chemistry leaves still unexplained how the gland-cells come to secrete their specific substances, why the cells of the salivary glands produce only ptyalin, and the cells of the gastric glands only pepsin, although the same food is brought to both by the blood.

We know that in resorption the food-stuffs, changed chemically 
by the digestive juices, are taken up through the cells of the intestinal wall into the body. Wo know, moreover, that a great part of the ingested fat, after being divided into microscopic globules, is taken into the protoplasmic bodies of the intestinal epithelium-cells by their own activity, while the same cells do not take up other particles of equal microscopic size, such as granules of pigment. But Physiology has not yet learned how this selective faculty of the intestinal epithelium-cells is to be explained mechanically.

We have seen how in the development of the human body the succession of definite morphological stages up to the complete man, which previously was so mysterious, may be understood naturally from the fundamental law of biogenesis. But it is still a much-debated question how in this development of the cells that arise from the segmentation of the egg some become gland-cells, others nerve-cells, and others epidermis-cells.

We have learned that the movements of the skeletal bones, the arms, the legs, and the joints, follow purely mechanical and mathematical laws, especially the laws of the action of levers. But the action of the skeletal muscles which causes the movement of the skeletal bones is the same puzzle that is mentioned above, namely, the contraction of the muscle-cells.

From the law of the conservation of energy we know that the heat and the electricity produced by the living body are derived from chemical changes which the ingested food undergoes in the body-tissues. But we do not know at all with what chemical processes the cells of the various tissues are concerned in the production of this heat and electricity.

We know, finally, that the higher sense-organs of man are constructed in accordance with the principles of physical apparatus; the eye, e.g., according to the principle of a camera obscura, so that a reduced inverted image of an object in the external world is formed upon its background according to the laws of the refraction of light. but it is a constant puzzle as to what occurs in this process in the retinal cells and how from them by the mediation of the optic nerves the ganglion-cells in the brain are excited to produce in us the idea of the image.

This enumeration might be long continued, but what has been said suffices for the recognition of a general fact. Everywhere, to whatever branches of physiology we may turn, wherever the gross activities of the body are traced to the activity of the individual cells, we always come upon an unsolved problem. The pessimist, indeed, might be led to maintain with Bunge ('94): "All processes in the organism which may be explained mechanically are no more phenomena of life than are the movements of the leaves and branches of a tree that is shaken by the stomi, or the movement of the pollen that the wind wafts from the male poplar to the 
female." But, if we despair of a chemico-physical explanation of vital phenomena, nothing remains but to take refuge again in the long-buried doctrine of vital force. In fact, very recently this idea has again appeared in various places, notably in the writings of Hanstein, Kerner, Bunge, Rindfleisch, and other men of science.

: We inight, however, be much more inclined to despair if we should look at the field of psychical phenomena. In the physiology of the brain and the sense-organs, indeed, much has been cleared up concerning the physical relations of certain psychical processes. But the old riddle of the causal relations between body and mind, which occupied so fully the thinking intellect even in earliest times, remains apparently wholly untouched by natural science.

Under such disheartening conditions the investigator is constantly oppressed by the questions: Are there limits to our knowledge of vital phenomena? If so, where do these limits lie? Or are we ipon a false path? Was our attitude of inquiry into nature a mistaken one, so that we have not understood her answer!

\section{B. THE RELATION OF PSYCHOLOAY TO PHYSIOLOGY}

\section{The Question of the Limits of a Knowledge of Nature}

Are there limits to our knowledge of nature? And if so, where do they lie? These questions have repeatedly arisen in the present generation, which is proud of its achievements in natural science, and have been treated in various ways. We can most fittingly consicler them in connection with the well-known address of $\mathbf{E}$. du Bois-Reymond ('84), "Ueber die Grenzen des Naturerkennens," in which the recently deceased author, who was a master of language among German naturalists, discussed this theme in his accomplished style.

With the lack of philosophical methods of thought which unfortunately is so wide-spread in the science of to-day, the most remarkable ideas upon the basis of our knowledge of nature are often met with. This circumstance unfortunately justifies speculative philosophy in looking with contempt upon science, its rival in the recognition of truth. It is, therefore, necessary to examine these questions somewhat carefully, and, first, to inquire concerning the limits of knowledge, not only in organic, but in all nature.

Modern science, especially physics and chemistry, is here the leader, and endeavours to reduce all the phenomena of the physical world to motions of atoms. Accordingly, du BoisReymond, in order to obtain a fixed pint upon which to base his considerations, defines a knowledge of nature as follows: "A knowledge of nature-more accurately expressed, scientific know- 
ledge or knowledge of the physical world, with the aid and in the sense of theoretical natural science-is the reduction of changes in matter to the motions of atoms, which motions are accomplished by the intrinsic forces of the atoms independently of time; in other words, it is the resolution of natural events into the mechanics of atoms." Recent science has, in fact, succeeded in showing in gross outline how natural phenomena may be derived from definite motions of atoms. We know that in all bodies the atoms are moving, in gaseous bodies very actively, in liquids more slowly, in solids very little. We know that light, heat and electricity depend upon regular, excessively rapid vibrations of atoms; that sound is caused by definite modes of atomic vibration: and that chemical changes of bodies are conditioned likewise by characteristic movements and rearrangements of atoms.

Following a fanciful conceit of Laplace, who imagines a human mind perfected to the highest degree and possessing such a knowledge of atomic motions as we have in astronomy of the motions of the stars, du Bois-Reymond continues: "If we were to imagine all changes in the physical world to be resolved into the motions of atoms, which are due to constant intrinsic atomic forces, the universe would be known in the scientific sense. The condition of the world at any period of time would appear as the immediate result of its condition during the previous period and the immediate cause of its condition during the following period. Law and chance would be merely other names for mechanical necessity. A stage in the knowledge of nature can be conceived in which the whole world-process would be represented by one mathematical formula, by one immeasurable system of simultaneous differential equations. from which could be deduced the place, direction of movement, and velocity of every atom of the universe at every moment."

The human mind is only " a feeble image," it is true, of such a mind fancied by Laplace, but it differs from the latter only in degree, and in the achievements of the latter we can perceive the ideal which the human mind in its development is constantly approaching. Let us imagine for once that we had reached this ideal and were in possession of the "world-formula." What would then be gained?

In order to explain a definite phenomenon of nature, we would need only to introduce into the world-formula certain values resulting from observation, and by computation we would be able to prove the phenomenon in question to be a necessary consequence of our known observations. Our craving for causality would perhaps be captivated for awhile by this play, but soon it would become free again and would call to us with louder and louder voice. So far so good; we can now understand all phenomena of the physical world in their causal relations to cach other; we can explain them as perfectly definite motions of atoms; but what is an atom? 
Here, according to du Bois-Reymond, we stand at one limit of our knowledge of nature.

What an atom is, i.e., what matter endowed with energy is, the world-formula does not explain. If we ask how we arrive at the conception of an atom, we find that we conceive it as an excessively small, indivisible, elementary part of a body, derived by continued division of the body; but if a body be continually divided until its atoms are reached, nothing but body is obtained. Atoms are bodies, and have the general characteristics of bodies. We cannot, therefore, expect to obtain by division something that elucidates the nature of the body. When we explain an unknown phenomenon by the motions of atoms, we merely resolve it into unknown phenomena. What an atom is, we do not learn, for it has only the properties which we attribute to it on the basis of the sense-perception of what large bodies show us, i.c., it is hard, impenetrable, possesses form, and moves. But we obtain not the slightest information regarding the nature of the matter that is endowed with energy, ice, that of which the physical world consists. Our craving for causality remains, therefore, in this respect unsatisfied, and as the result of our analysis we find ourselves at the first limit of our knowledge.

But this is not the only limit. If, again, we possessed "astronomical knowledge" of the physical world, as du Bois-Reymond expresses it, i.e, the same mathematically exact knowledge of the motions of atoms that we have of the motions of the heavenly bodies, we would then, indeed, understand all phenomena of the physical world, but we would not understand how consciousness arises, how in general a psychical phenomenon, even the very simplest, comes to be. If we had, e.g., astronomical knowledge of our brain, we would know the position and motion of every atom at every moment; we could also follow definitely the specific physical changes, rearrangements, and motions of atoms inseparably associated with specific psychical phenomena, and "it would be," as di Bois-Reymond says, " of unbounded interest, if with our mental eye turned inward we could observe the cerebral mechanics of an arithmetical problem, like the mechanics of a calculating machine; or if we could know what dance of the atoms of carbon, hydrogen, nitrogen, oxygen, phosphorus and other elements, corresponds to the delight of musical sensation, what whirl of such atoms to the acme of sense-enjoyment, what nulecular storm to the frantic pain resulting from maltreatment of the nervus trigcminus." $=t^{2} t^{+1}=\mathrm{che}$

We could know all these if we possessed "astronomical knowledge" of the brain. We could thus convince ourselves by selfobservation that consciousness is inseparably associated with atomic motion. But with all this it would remain for ever concealed from us how consciousness arises, how the simplest psychical 
phenomenon comes to be. However carefully we might follow the motions of individual atoms in the brain, we would see only motions, collisions, and again motion. Thus, it is evident that a mechanical explanation of consciousness, of psychical phenomena, from the motions of atoms is an impossibility for us, and we find ourselves at a second limit of our knowledge of nature, which appears not less impassable than that of a knowledge of matter and energy.

But supposing the first to be passed, and the riddle of matter and energy to be solved, how would it be with the second limit? Would it be passed at the same time or would it still be impassable? We can evidently imagine consciousness, or rather the simplest form of mind, to be inherent in the nature of an atom, and, therefore, to be known when the nature of matter is known. In fact, this idea would be the only one that could be adopted by a monistic science, which seeks to explain all phenomena by one principle; and Haeckel especially, who is an energetic advocate of monism among men of science, has always maintained it. du Bois-Reymond alludes to such a possibility only briefly when he says: "Finally, the question arises, whether the two limits of our knowledge of nature may not perhaps be the same, i.e., whether, if we understand the nature of matter and energy, we may not also understand how under certain conditions matter may have sensa tions, desires, and thoughts. This idea is, of course, the simplest one, and according to the known principles of investigation is preferable to its opposite, according to which, as before said, the world appears doubly inconceivable. But it lies in the nature of things that we cannot elucidate this point, and all further words concerning it are idle." Therefore, "as to the riddle of matter and energy and their conceptions," du Bois-Reymond decides upon complete renunciation and proclaims to science not only a temporary "ignoramus," but an eternal and demonstrative "ignorubimus."

\section{Physical World and Mind}

We have followed du Bois-Reymond's course of thought thus in detail, in order to show that the knowledge assumed by him as the starting-point of his considerations very soon encounters obstructions, in view of which the world appears incomprehensible. But eternal renunciation falls heavily upon the indefatigable thinker, and he is bound to ask whether this assumed path of knowledge is a right one, whether the definition of a knowledge of nature as a resolution into the mechanics of atoms is correct or justified. We will, therefore, test this basis of our considerations and inquire what knowledge is.

For this purpose we will take the conception "knowledge" in 
its widest and most general sense. One indispensable condition of the conception is the assumption that something exists. If we make this assumption, if we have something real or actual, a fixed point, then knowledge is simply the causal reduction of all phenomena to this reality. We have a measure for knowledge in the satisfying of our craving for causality; and the latter will necessarily be satisfied, when once we have placed all phenomena in causal relation to the one reality.

Nevertheless, an objection may here be raised. Let us suppose that we have succeeded in reducing all phenomena to the one reality. (This reality appears in the different philosophical systems under very different names, such as God, thing-in-itself, the unknown, etc.-the terms are equivalent and without material significance.) The question would then arise, whether our craving for causality would be satisfied, or whether it would not force us still farther to ask, What is this thing which exists, this reality, the unknown, the thing-in-itself, God, or whatever it is termed? In the latter case, here, again, would be a linit to our knowledge. If we understand it rightly, however, this limit would be a logical error, a false conclusion. Our craving for causality arose and became established in the course of evolution by the continual reduction of effects to causes, and it is easily possible that in the present case it would continue for awhile from inertia to hold before us the question, why? But it is evident that we would thus be guilty of an error of reasoning; for, if all phenomena were reduced to the one reality, it would be a complete contradiction to wish to know that reality in terms of non-reality. The demand that, after complete knowledge of the world, we must know the world still more involves an evident absurdity. Hence, the above objection is only an apparent one.

We assume, therefore, the desire to reduce all phenomena to that which is real. Then the question arises, What is real?

Here we come in contact with a mistaken view which is especially wide-spread in science and has been faithfully handed down from primitive time as an heirloom from the childhood of the human mind. This is the view that the physical world existing outside of us and independent of our own mind is real, and that, accordingly, we must reduce all phenomena to its laws. The impossibility of such an undertaking is plainly shown in the above argument of du Bois-Reymond. Yet a great many men of science-among those who, like du Bois-Reymond, have reflected upon the limitations of human knowledge, we need mention only the gifted botanist Nägeli (77) - have held it to be possible that even psychical phenomena may be resolved into the processes of matter. Hence it is useful to clarify our ideas as to what matter really is.

At first sight bodies appear to us as actual objects outside of 
our own minds. Any doubt as to the existence of a physical world outside of mind, will appear absurd to one who has not reflected upon it: a body, e.g., a stone, a tree, a man, which we look upon, really exists, no one will deny this; we actually see the body, others see it; and we say it exists. We are right; without a doubt it exists, but it does not exist outside our mind; for, when we examine carefully the grounds for speaking as we do, we find that what we believe we see or feel as a body outside our mind is actually something quite different.

Let us prove this. We have created our knowledge of a physical world by means of sense-perception. The question as to what can and does give us this knowledge is, therefore, one belonging to the physiology of the senses. Now the physiology of the senses shows that all that comes in through the door of our senses affords us, simply and solely, sensations. The many features that constitute the image of a body, e.g., a piece of gold, are so many different sensations, e.g., a yellow colour, hardness, weight and coldness. Persons with an innate defect in a sense, in whom a certain group of sensations is not mediated, e.g., persons born blind, have, therefore, an idea of the physical world that is wholly different from that of normal persons. This is clearest in those interesting cases in which persons who are born blind and have constructed their physical world solely by means of the senses of touch, hearing, smell, taste, etc., have been made to see by surgical operations. If objects that such persons have often had in their hands be brought for the first time before their eyes without their examining them by the other senses, e.g., by touching, they do not recognise them: a ball appears to them as something wholly new, and only when they touch it do they realise to their surprise its identity. At that moment a new world begins to arise in them. The physical world depends, therefore, wholly upon the development of our sense-organs; to animals with senseorgans developed differently from ours it must appear very different, in proportion as they receive other sensations. With our death, with the destruction of the senses and the nervous system, the physical world in its previous form disappears.

These facts are of far-reaching significance. They show that what appears to us as matter is in reality our own sensations, or ideas, our own mind. When I see a body or perceive it by means of my other senses, in reality I have not a body outside of myself but only a number of sensations in my mind. Beyond these I know nothing concerning it and can only form hypotheses.

It is necessary that we accustom ourselves to this fundamental truth, and that we get rid of the error of the existence of a physical world outside of mind. In order to facilitate this let us consider the consequences of this truth.

If the physical world is only my own sensation, or, better, since 
it involves a complexity of sensations, my own idea, I must assume that a reason for this idea exists. Hence the question arises, What is the thing outside of my mind that produces in me through the scnses this idea? In other words, what is the external world?

This question contains an error. As is well known, natural science has shown that every phenomenon in the physical world has as its cause another physical phenomenon. This is only an expression of the law of cause and effect, i.f., the law of causality. Hence the cause of my sensation of the physical is another sensation or idea, which is located not outside of but within my mind. This is nothing but a paraphrase for the fact that our conception of causality has arisen out of a combination of separate experiences, which our mind has obtained by observation of the regular sequence of its own elements, its sensations and ideas. In other words, causality itself, like all other sensations, ideas, conceptions, or whatever we may term it, exists only in our own mind. If, therefore, the cause of my idea of the physical is located within, the supposition of a reality without is wholly unjustified.

Various philosophers have, in fact, endeavoured to base the reality of an external world upon the causality of phenomena. But both rest upon the same error, and the argument presents the rare spectacle of an attempted proof of something by means of that which is to be proved.

It is not to be denied that to every one who follows this line of thought for the first time the above result must appear paradoxical, and he will immediately raise the objection that besides himself many other men exist, possessing minds and capable of making exactly the same assertions concerming themselves and their own minds. But here the delusion is again evident. To me, other men are bodies, I perceive in them nothing else. Hence they are only my idea. And when they tell me that they have a mind like myself, that they likewise feel and think, it is true; but what they say to me, their speech, their movements, are only physical phenomena and, therefore, only my own ideas According to our scientific mode of expression, their mind has its seat in their brain, but if, by a surgical operation upon a living man, I am ever enabled to examine the brain, I learn that nothing is to be found there but physical elements. I am thus forced to the conclusion that what $I$ regard as the mind of another is also only my own idea. In short, whatever path I take, I come constantly to the conclusion that all that seems to be outside of me, whether it be a lifeless body, a living man or a human mind, is in reality only my own mind. Beyond my own mind I cannot go. My own individuality, indeed, is only an idea of my mind, and, therefore, I cannot finally say, the world is my idea, but I must say the world is an idea, or a sum of ideas, and what appears to me as my 
individuality is only a part of this complex of ideas, just as is the individuality of other men and the whole physical world.

Although this reasoning will appear to every one at first sight strange and unusual, it is by no means new. More than two hundred years ago Descartes made the fundamental fact, that the whole physical world is only an idea, the starting-point of his philosophy. Later, Berkeley and, still later, Fichte and Schopenhauer employed it as the basis of their systems, which were widely different in other respects. More recently among men of science Mach ('86) has adopted a similar view as the nucleus of his views regarding the theory of knowledge. It is to be hoped that this monistic conception will gain ground more and more in science; it alone holds strictly to experience, it is not hypothetical, and it necessarily sets aside the ancient doctrine of the dualism of the body and the mind, a doctrine that reached its highest development in the Egyptian theory of the wandering of the soul and has continued through the whole history of philosophy.

\section{Psycho-monism}

When the history of the problems that have kept the human intellect busy during the long course of its evolution is studied, it is found that many problems that perplexed the ancients have continued unchanged and unsolved down to the present day; others have been solved; while still others that have been prominent even for centuries have afterwards disappeared without finding a solution. The ancient question of the squaring of the circle, over which many a brain has puzzled in vain, that of perpetual motion, which since early times has been prominent in physics, and many others, have quite disappeared, although no one has ever squared the circle and no one has constructed a machine for perpetual motion. If it be asked how it happens that this is so, the answer is, because it is recognised that the basis of these supposed problems is false, and they are, therefore, insoluble. If the attempt be made to divide all the numbers of a series by 2 without a remainder, it is found impossible to do it. So it is with the above problems, which for centuries have harassed one generation of thinkers after another.

So it is also with the attempted explanation of psychical by physical events. It still engages unremittingly the attention of those who are not pleased with having limitations to their conception of the world, yet no one, however earnest his thought, comes nearer a solution. Only gradually will the conviction force its way, that this problem, like those above mentioned, will always resist solution because the question is falsely put.

That the attempted explanation is wrong is at once clear from 
the preceding considerations. It was found that the sole reality that we are able to discover in the world is mind. The idea of the physical world is only a product of the mind, and with the alteration of an old sentence of the sensualists, it can be said: Nihil est in universo, quod non antea fuerit in intellectu. But this idea is not the whole of mind, for we have many mental constituents, such as the simple sensations of pain and of pleasure, that are not ideas of bodies. The task of psychology, i.e., the investigation of mind, consists in the analysis of all mental constituents. By investigating the contents of mind, by decomposing the higher psychical phenomena, the more complex groups and series of ideas, into their simple constituents, psychology arrives, finally, at the most primitive psychical phenomena, the psychical elements, and in the same degree discovers the laws of the arrangement of these elements into the higher groups and series of ideas. Just as in mathematics the endless variety of numbers is formed according to laws out of the numerical unit, so psychology reduces the endless variety of psychical phenomena to their formation, according to laws, out of the psychical elements. But the idea of matter, or, better, of an atom, is not a psychical element, it is a great complex of highly developed ideas. An atom is nothing but a thing possessing all the properties of a body, such as hardness, impenetrability, form, and extension, all of which presuppose very complex psychical processes. The endeavour of natural science to reduce the phenomena of the physical world to the mechanics of atoms is justifiable; it is an endeavour to derive the phenomena of large bodies from the properties of their material parts. But the attempt to reduce to the motions of atoms all psychical phenomena, not only ideas of the physical world but others, such as simple sensations, is precisely as absurd as the endeavour to reduce all numbers in the numerical series to 2 instead of to the numerical unit, for the complex notion of the atom is not a unit, not a psychical element. Herein lies the fallacy of the problem, and hence, as the history of human thought has shown so strikingly, all attempts to explain the psychical by the physical must fail.

The actucel problem is precisely the reverse. It consists not in explaining psychical by physical phenomena, but rather in reducing to its psychical elements physical, like all other psychical, phenomena.

In natural science the view is frequently met with, that knowledge of the world falls into two sharply separated categories, namely, metaphysics and science. Metaphysics is left to philosophy, and science is limited to the investigation of the physical world. But the fact is often overlooked or intentionally neglected, that every process of knowledge, including scientific knowledge, is merely a psychical event, that science also deals with " metaphysics," as in accordance with an ancient and unfortunate manner of expression it is customary to term it, and even 
that science cannot exist without metaphysics. This fact cannot be banished by the well-known method of the ostrich.

It this appears to be a contradiction to contrast nature ( $\phi \dot{v} \sigma \iota s$ ) with something "beyond "nature $(\mu \epsilon \tau \grave{a} \tau \dot{\eta} \nu \phi \dot{v} \sigma \iota \nu)$. There is but one world, whether this be termed nature, mind, reality, or anything else. It follows also that there is but one kind of knowledge, and not two. As soon, therefore, as the question arises of principles and bases of knowledge, all artificial boundaries disappear. We should not be deceived. The goal toward which the human mind is striving in its theoretical investigations is not simply at knowledge of the lifeless physical world or of living bodies, or of this or that psychical phenomenon, but it is a knowledge of the world. A division of labour among investigators, however, should not only not be deprecated, but, because of the excessive multitude of phenomena, should be encouraged; nevertheless, the purely supplementary purpose of such a division must be kept in mind, and the artificial boundaries between the various fields of labour must not be confounded with the natural boundaries. A severe blow will be inflicted upon the coming centuries, if the gulf between philosophy and science widens constantly from both sides; if, upon the one side, confused speculation, and, upon the other, narrow specialisation constantly prevail and prevent a mutual approach toward a beneficent common labouring-ground. Science cannot make salutary advances without a philosophical workingplan, and we see in the history of science that great discoveries have been made, not by restricted specialisation, but by investigators working philosophically, i.e., systematically, methodically, and cognisant of their aim. But philosophy can obtain really important results just as little by a purely speculative method, by not keeping close to established facts and not submitting its speculations to the severe criticism of experience. The history of science proves that true advance comes only by thoughtful investigation. The above theoretical considerations regarding knowledge ought to give us a basis for investigation such as every thinking investigator sooner or later must make for himself, and upon which he must build broadly and freely in order that his labours may be fruitful.

The most important result afforded by the above considerations is the monistic standpoint, in accordance with which the world appears as unitary, and the dualism of the physical world and mind as an illusion. The fact, which appears so remarkable from other standpoints, is, therefore, not surprising, that the laws that control the physical world and those that control mental phenomena are completely identical. This appears necessarily so when we find that the phenomena of the physical world are arranged according to space, time and causality, and when we recognise therein the logical principles of our own thought; the 
laws of the physical world are the laws according to which our own psychical phenomena occur, because the physical world is only our own idea. All science, therefore, is in this sense psychology.

We will now summarise our considerations regarding investigation. We started out with the question, whether there are impassable limits to a knowledge of the world. If we understand by knowledge the reduction of phenomena to the motions or the mechanics of atoms, limits do, indeed, exist. For not only is the atom, and hence matter, yet to be explained, but, as du BoisReymond's clever undertaking has shown very clearly, it is impossible to reduce psychical phenomena to the mechanics of atoms. If, however, we conceive knowledge in a more general and the only justified sense, namely, the reduction of phenomena to the elements of reality, we find that no limits exist, for the sole reality is our mind and all phenomena are only its contents; explanation, therefore, consists simply in the reduction of all psychical phenomena to their elements. In this sense, all science, and in general all knowledge, is in the end psychology. We thus come to the only consistent standpoint, namely, monism, the unitary view of the world, which seeks to derive all phenomena from a single cause. From this standpoint we see why we meet with limits when we define knowledge to be the reduction of phenomena to the mechanics of atoms. An atom is not an element of reality but a complex idea, hence all phenomena are not reducible to atoms; just as in a series of numbers the element of which is the unit 1, all are reducible to the common unit but not to a number more complicated than $1, e . g ., 2$. It is thus evident that a limit can no more exist to the investigation of physical, than to that of psychical phenomena; for, since bodies, in other words, atoms or matter, are only ideas, in other words, psychical phenomena, they may be reduced to the same psychical elements as ideas.

\section{VITALISM}

We will now turn again to the consideration of vital phenomena. The above reflections have shown the possibility of reducing all phenomena, physical, as well as psychical, to a common cause. The question that led to those reflections, whether vital phenomena are based upon the same causes as those of non-living nature, would be answered affirmatively if we were to go back to final causes-and it has been found that no insurmountable boundaries limit research. If, however, we confine ourselves to the special field of physiology, the investigation of the physical phenomena of life, we know that natural science has shown that the phenomena 
of lifeless bodies may be derived from the properties of the smallest material elements, atoms endowed with energy, and the question then takes the form: May the phenomena of living bodies also be reduced to the same factors?

Vitalısm says, no; a special force, vital force, prevails in organisms and induces vital phenomena. Vital force is limited to the world of living matter and is not identical with the chemicophysical forces of lifeless nature.

These words contain the essence of vitalism. It is interesting to inquire upon what the hypothesis of vitalism rests, and what justification it has. In the above review of the history of physiological research, the history of the doctrine of vital force was outlined. It was there seen that the conception arose in connection with the phenomena of irritability, and that it has always been vague and has served chiefly for convenience. This indistinctness regarding it is the chief difficulty in the way of its critical elucidation. If the conception could be sharply defined, it could be treated more easily.

The claim for a vital force rests solely upon the fact that thus far it has not been possible to reduce certain vital phenomena to chemico-physical principles. Indeed, when the achievements of physiological research were summarised above, the discouraging fact became apparent that the vital phenomena that have been explained are only the gross physical and chemical activities of the body, and that whenever the attempt has been made to show their deeper causes, unsolved problems have always opposed it. Bunge ('94) even asserts: "The more we strive to investigate vital phenomena exhaustively, in many directions, and fundamentally, the more we come to perceive that events which we believed we could explain physically and chemically are excessively intricate and for the present mock at all mechanical explanation."

Although the fact is to be little doubted, that thus far, many, especially the elementary and general vital phenomena have defied all chemico-physical explanation, the assertion is not, therefore, logically justified, that these phenomena do not follow chemico-physical laws at all, but that a special vital force exists and causes them. Moreover, there are facts that speak against the existence of such a force.

In spite of all endeavours thus far, the vitalists have not succeeded in establishing the existence of any special force in organisms, i.e., they have not been able to characterise such a force from its effects, as physics and chemistry have done for the forces of inorganic nature. With regard to none of the actions of the body supposed to be due to a vital force, have they been able to contradict the assertion that such actions are only the expression of complex chemico-physical relations. E.g., it was long believed 
that certain substances found exclusively in the living organism cannot be produced chemico-physically, but arise only through the working of the vital force. But, as has been seen (p. 23), in the year 1828, Wöhler weakened this once so powerful support for the vitalistic creed by manufacturing synthetically in his laboratory urea, a body which ordinarily is produced only in the metabolism of the living organism. $\mathrm{He}$ obtained this from ammonium cyanate, $\left(\mathrm{NH}_{4}, \mathrm{CNO}\right.$, which is isomeric with urea $\left(\mathrm{NH}_{2}\right)_{2} \mathrm{CO}$, i.e., possesses the same number of the same atoms in a different arrangement. But ammonium cyanate is manufactured out of purely inorganic materials. This synthesis of urea has been followed by others of equal importance, all of which go to show that characteristic substances belonging to the organism may be compounded artificially. The assumption of a special vital force for their production in the organism is thus rendered superfluous. Of course it has not yet been possible to manufacture a great number of substances belonging to the animal and the plant body. Indeed, the most important of these substances, proteids, have thus far resisted artificial production in the laboratory, but the reasons for this are evident. The exact chemical composition of proteids is not yet known; we know what atoms are contained in them, but we have no idea how these atoms are joined together. Accordingly it is evident that success in the attempt to manufacture proteids artificially ought not yet to be expected. A second reason is that thus far we have no correct idea of the chemico-physical conditions under which these substances arise in the organism; and chemistry has recently shown very clearly that the inauguration of a chemical process depends not only upon the presence of the necessary substances, but also upon the fulfilment of certain other conditions. Thus, it has been found that many chemical transformations that do not take place in a large space, occur very easily in capillary spaces under otherwise like conditions, and vice versa. An example long known is the union of oxygen and hydrogen in the spongy-platinum of Döbereiner's lamp. It is known that hydrogen streaming out of a tube into the air does not spontaneously unite with the oxygen of the air; but, if it pass into the fine pores of platinum-sponge, the union takes place at once, and the hydrogen burns with a luminous Hame to form water. Such processes are termed condensations. It is known also that many chemical processes take place only when the substances involved are present in large quantity -a phenomenon which as mass-effect plays an important rôle not only in the laboratory but also, as already demonstrated, in the animal body. Recently, Preyer and Wendt ('91) have suggested the presence of condensation-processes and the absence of mass-effect in capillary spaces as a reason why in the organism, where capillary spaces exist in the cells and their 
contents, certain chemical transformations take place differently from their customary manner in mass.

Another consideration, which renders the assumption of a special vital force still more untenable, is the following. The calorimetric researches of the present time show that in the adult animal which is in complete metabolic equilibrium, i.e., which gives off from its body as excretions exactly as many atoms as it takes in as food, complete dynamic equilibrium also exists, i.e., exactly the same quantity of energy that enters the body with the food as chemical tension leaves the body during the vital activity of the animal. Hence all the energy that is transformed by the body in the performance of its work must be derived solely from the energy that comes in with the food. If this were not so, we would be led to absurd conclusions; for, if the activities of the body were supplied from a fund of special energy, a "vital force," we would be obliged to assume, not only that the latter is continually constructed in the body out of nothing in order continually to maintain the work of the body, but also that the potential of the food being superfluous is continually disappearing in the body. At the present day no true man of science can agree with such an idea. Johannes Muiller was a vitalist, and, although the law of the conservation of energy was unknown to him, he felt and endeavoured to avoid this difficulty by assuming that vital force works according to chemico-physical laws. By such an assumption a specific force, different from chemico-physical forces, is in principle laid aside, for vital force is then only a collective term for the complicated chemico-physical relations upon which vital phenomena depend. In fact, many scientists conceive the term in this sense only, and, if Muiller had been acquainted with the law of the conservation of energy, he would surely hare aroided the expression "vital force."

Since the middle of the present century the old conception of vital force has disappeared completely from physiology. Hence it appears strange to hear at the present time here and there the catch-words of that doctrine. A careful examination of this reappearance shows, however, that the old words are now employed in a very unfortunate connection, that their sense has been completely changed, and that, when "vitalism" and "neovitalism" are now spoken of, something wholly different from the old doctrine of vital force is meant. In general, among the phenomena of the newer vitalism two groups may be distinguished, which may be termed mechanical and psychical vitalism. ${ }^{1}$

Mcchanical ritalism is the view that vital phenomena depend at bottom upon the agency of physical and chemical forces; but that in living organisms these forces are linked together into such a peculiar and thus far unexplored complex that for the present it

1 Cf. Verworn $(96,2)$. 
must be contrasted with all the forces of inorganic nature as a specific vital force, characterising the actions of living organisms only. In other words, by vital force is meant simply the special aspect of chemico-physical forces that lies at the basis of vital phenomena. It is evident that no objection can be brought against the facts upon which this idea is based. But it is another question whether the terms "vital force" and "vitalism" are justified in this case. The new idea' has nothing to do with the old vitalism, which assumed a "force hypermechanique" as the cause of vital phenomena. A return to the ill-reputed word, which arouses a well-defined prejudice, is simply to give up the advantage afforded / by the hard-earned conviction of the unity of cause in all nature.

Psychical vitalism, as defended by Bunge ('94), and essentially, although more poetically than exactly, by Rindfleisch ('95), is something wholly different. It is properly not a physiological but rather a philosophical doctrine, which springs from a correct appreciation of the inadequacy of materialism, and it is to be regretted that it employs the extremely unsuitable names "vitalism" and "neovitalism."

We will consider somewhat more fully the vitalistic standpoint of Bunge. Bunge expresses the vitalistic creed unambiguously in the statement: "If the opponents of vitalism maintain that absolutely no other factors are present in living nature than simply and solely the forces and substances of inanimate nature, I must take issue with their doctrine." Nevertheless, from his further deductions it appears equally evident that his vitalism is no vitalism at all. Bunge's vitalism in reality is essentially a philosophical idealism arising from considerations similar to those expressed above regarding the theory of knowledge. Bunge is guilty of the one inconsistency, however, that he ascribes mind to organic, but not to inorganic, nature; and it is this inconsistency that leads him to profess vitalism, for to him mind is the element that distinguishes the phenomena of the living physical world from those of the lifeless. It is a cause of personal satisfaction that one of our most prominent physiologists has energetically defended views similar to those to which my own general considerations have led, and, therefore, I venture to present herewith the passage in question from the introduction of Bunge's book; it contains more profound reasoning than is. usually realised. Relative to Johannes Miiller's law of the specific energy of the special senses, Bunge says: "I mean the simple law that one and the same stimulus, one and the same event in the external world, one and the same thing-in-itself, acting upon the different sense-nerves always causes ("discharges") different sensations, and that different stimuli acting upon the same sensenerve always cause the same sensation; in other words, that events in the external world have nothing in common with our 
sensations and ideas, that the external world is for us a book with seven seals, and that the only things immediately accessible to our observation and knowledge are the conditions and events of our own consciousness.

"This simple truth is the greatest and deepest that the human mind has ever conceived. And it leads us also to a complete understanding of that which constitutes the essence of vitalism. The essence of vitalism does not consist in our being satisfied with a word and foregoing the thought. It consists in our taking the only right path of knowledge, proceeding from the known, the inner world, to explain the unknown, the outer world. Mechanism, which is nothing but materialism, takes the reverse and wrong path, it proceeds from the unknown, the outer world, to explain the known, the inner world."

We have seen that if we would explain the phenomena of the world in their entirety we must go back to elements that are very different from atoms; that, however, when we confine ourselves to physical phenomena, we find no difference between the factors that work in lifeless and those that work in living bodies. Logic demands that every body, whether living or lifeless, must be subject to the general laws of bodies, which physics and chemistry reveal. It is evident that these two sciences are not yet completed, and that in the future many of their essential views will undergo profound changes. But so much is certain: an explanatory principle can never hold good in physiology with reference to the physical phenomena of life that is not also applicable in chemistry and physics to lifeless nature. The assumption of a specific vital force in every form is not only wholly superfluons, but inadmissible.

\section{CELL-PHYSIOLOGI}

How does it happen that after the ill-reputed idea of the existence of a vital force has been regarded for decades as definitely set aside, modern science turns again to this outlawed word as a motto, in spite of the great variety of its significations? Why have such words as "vital force" and "vitalism" been able to exert in recent times an influence upon investigators such as Hanstein ('80), Kerner ('87), Bunge ('94), Rindfleisch ('88, '95), and others? It is not difficult to discover the reason. It is the same that in Haller's time gave birth to the idea of vital force, namely, the inability to explain vital phenomena mechanically, $i . \ell^{\text {. }}$ to reduce them to chemico-physical principles. This condition has existed during recent decades also, but it was largely neglected so long as the attention was occupied more with the epoch-making physiological discoveries of Ludwig, du BoisReymond, Helmholtz and others. We are becoming more con- 
scious of it now, when the brilliant discoveries of the great physiologists of the present century have been followed to their ultimate consequences, when the mechanics of the grosser actions of the body are essentially known, when research is engaged in extending into their details the results obtained in the old direction, and when by the old methods nothing essentially new and pre-eminent is being discovered. There is also another favouring circumstance. The science of to-day is in great part still under the ban of that potent spell with which du Bois-Reymond benumbed and discouraged ambitious minds, when by proclaiming his "ignorabimus" he placed research in an attitude of eternal renunciation, the necessity of which is acknowledged the more willingly since it was urged by such an authority and in so convincing a form. This renunciation, combined with the fact that great difficulties stand in the way of solving certain problems of life by the methods hitherto in use, is sufficient to explain psychologically an inclination toward coquetting with vitalism, whether the latter appears in its ancient or in its modern attire.

Nevertheless, eternal renunciation falls heavily upon the human mind, and even du Bois-Reymond did not accept it easily. From this natural aversion to such a conclusion we may suppose that the standpoint of renunciation toward vital problems is not inherent in the human mind and is not justified. The above considerations vindicate this supposition, and, moreover, the standpoint is denied in practice by most investigators. If, therefore, it is not the correct one, and if, nevertheless, the physical phenomena of life are based upon mechanical processes, it only remains to adopt another course.

We have arrived at a turning-point in physiology, a turningpoint that was never more apparent. The appearance of neovitalism is a sign of it. The old spirit of vital force is reappearing to many men of science to-day, just as in history, previous to great changes, significant spirits have appeared to clairvoyants.

It is not difficult to see what characterises this turning-point. When we inquire what we have attained in physiology, we find that we have become acquainted chiefly with the gross chemical and physical phenomena of the body-thanks to the ingenious methods of investigation and the weighty discoveries of the investigators of our time, which are often characterised by an exactness such as is found elsewhere only in the discoveries of physics. We know the laws of the activity of the heart, the movement of the blood, the exchange of air in the lungs, the contraction of muscle, the conduction of nerves; we know the actions of the sense-organs, how the digestive juices act upon the food, and the special anatomical basis of many psychical phenomena. But all these are only the mass-effects of large parts of the body, 
they are not the end-results of vital activity. All that we are now accomplishing by means of the special methods that were created by the great masters of physiology for this purpose, is essentially only an extension of our present knowledge into finer details, and its application to analogous conditions. Every glance into physiological literature proves this. Every new number of the journals shows it. At present there is no dominating tendency in physiology, such as was the physical tendency a short time ago. A new great discovery is made along the present path only rarely, in spite of a frequently marvellous employment of ingenuity and knowledge, and yet the real riddles of life are not yet solved. We would not go so far as Bunge goes, and maintain that all phenomena which thus far have been explained mechanically are not vital phenomena at all; but there can be no doubt that thus far we have not been able to explain the general, the elementary, vital phenomena. This impotence of the physiology of to-day in the presence of the simplest vital processes points. plainly to the fact that the methods that have explained the mechanics of gross and special physiological activities, however ingeniously they were devised for that purpose, fail for other purposes, for the investigation of the elementary and general activities.

In order to solve the elementary general problem we must take a wholly different path. There is only one such path, and it was. clearly indicated when the facts in the history of physiological research were summarised. Consideration of the individual functions of the body urges us constantly toward the cell. The problem of the motion of the heart and of muscle-contraction resides in the muscle-cell: that of secretion in the gland-cell; that of food-reception and resorption in the epithelium-cell and the white blood-cell; that of the regulation of all bodily activities. in the ganglion-cell. The cell-theory has long shown that the cell is the structural element of the living body, the elementary organism in which the vital processes have their seat. Anatomy, embryology, zoology, and botany have long recognised the significance of this fact, and the great achievements of these sciences are a brilliant proof of the fruitfulness of the cell-method of investigation. But only very recently has the simple and plainly logical consequence begun to be recognised that, if physiology considers its task to be the investigation of rital phenomena, it must investigate them in the place where they have their seat, i.e. in the cell. If it is not to be content with extending still farther the present knowledge of the gross activities of the human body, but would rcally explain elementary and general vital phenomena, it must assume the character of ccll-physiology.

It might appear paradoxical that thirty-five years after Rudolf Virchow ('58) expounded, in his Cellularpathologie, the cell principle as the basis of all organic investigation-a basis upon 
which all our medical ideas now rest-physiology is beginning to develop from a science of the organ into that of the cell. But we can recognise in this only the normal course of development, which first takes into consideration the gross activities of the organs and then pushes gradually deeper and deeper until it arrives at the cell. At all times anatomy has been the forerunner of physiology; and it must be so in order to smooth the way. Just as anatomy began with the organs of the body and only in the present century has reached the smallest elements of the organs, the cells, with the delicate morphological investigation of which the brilliant advance of modern anatomy is consummated, so physiology necessarily began with the study of the functions of the large and obvious organs, and not till the present time has it been able to attack the vital phenomena of the cell. We would be guilty of gross ingratitude if we were to underestimate the eminent importance of past physiological research, upon the results of which we more or less constantly build. Its aims and ideas are destined to lead us still farther, and its methods are indispensable. Yet, in judging the course of physiological research, we cannot forget one factor which controls the development of every science, the psychological factor of fashion. The course of every science depends upon the powerful influence of great discoveries. Wherever we look at the history of investigation, we find that imposing discoveries, such as are represented in physiology by the work of Ludwig, Claude Bernard, du Bois-Reymond, Liebig, Pasteur, Koch, and others, divert interest from other fields and cause many investigators to labour on in the same direction with the same methods, especially when the methods prove so unusually fruitful as in the cases mentioned. Thus, definite fields of work in connection with epoch-making achievements immediately become the fashion, while interest flags in other fields. In the course of time equalisation takes place, for every field is limited and in time becomes exhausted. We have evidently arrived at such a period in physiology; the science of the physiology of the organ has passed the culminating-point of its development. In the course of time cell-physiology also will become exhausted, and other aims and methods, such as the state of the problem at the time demands, will succeed it in the incessant evolution.

For the present, cell-physiology has before it an unbounded field of labour. There are, of course, investigators who, although convinced of the pressing necessity of a cell-physiology, and realising that the cell as the seat of the vital processes must constitute the object of research, nevertheless doubt whether we are at all able to get at the vital mysteries in the cell. It can, therefore, reasonably be asked that a way and methods be shown by which a cell-physiology may be founded. Doubt of the practicability of this undertaking springs chiefly from a fact which unfortunately has 
characterised physiology since the death of Johannes Muiller, and to which attention has already been directed, namely, the entire lack of a comparative physiology. The science has not yet entered upon this important heritage from Müller, our greatest master. How few objects of research the physiology of to-day possesses-- the dog, the rabbit, the guinea-pig, the frog, and a few other higher animals. How little known are the many splendid objects offered to the observant eye by the enormous number of lower animals. And it is precisely among these objects that there are to be found such as are fitted in a surprising degree for the cell-physiological solution of elementary physiological questions.

It is quite true that if one attempts to treat the problems of digestion, resorption, and motion, from the standpoint of cellphysiology on men or on the higher animals solely, he will soon run against more or less serious technical difficulties in the investigation of the living gland-cell, the intestinal epithelium-cell, and the muscle-cell. Nevertheless, the admirable investigations of Heidenhain upon secretion, the formation of lymph and resorption, have shown what result the cell-physiological method has been able to achieve even here. Such systematic histological experiments, in which the living cell, while its connection with the body is still intact, is put under definite conditions, and the final results are then investigated after the sudden death of the animal for the purpose of drawing conclusions regarding the events that take place during life under the corresponding conditions, will still without doubt yield much of value. In the tissue-cells the conditions are relatively favourable for chemical investigation; at least in many cases chemistry is capable of investigating metabolism in large living cell-complexes, and drawing from them conclusions regarding the life of the individual cells. In fact, we are indebted to this phase of chemistry for very decided light upon animal metabolism. But, naturally, in the animal body little opportunity is afforded for employing pure tissues, i.e., complexes of similar cells, as objects of research, and the uncertainty of the significance of the results increases enormously in proportion to the morphological complication of the object. Moreover, investigations on tissue-cells are limited by the fact that frequently, at least in warm-blooded animals, the tissues offer serious obstacles to the employment of methods such, e.g., as that of microscopic experiment during normal life. The free-living cells in the organism, such as the white blood-corpuscles, offer considerably fewer difficulties in this respect; and thus it has come about that in very recent times we have obtained very detailed knowledge of the vital phenomena of leucocytes, especially through the labours of Metschnikoff. Massart, Leber, Buchner, and many others.

If, however, the comparative-physiological standpoint, which Johannes Müller always defended energetically, be adopted, an 
unbounded field for cell-physiological investigation is revealed. The comparative method demonstrates one fact of fundamental importance, namely, that the elementary vital phenomena belong to every cell, whether it be from a tissue of the higher animals, the lower animals, the plants, or free-living, an independent unicellular organism. Every one of these cells exhibits in its individual form general vital phenomena. Realising this, it is only necessary for the investigator to select from the variety of species the objects best fitted for each special research, and these obtrude themselves upon him in due form, if he possesses some knowledge of the animal and plant world. It is no longer necessary for him to cling to the tissue-cells of the higher vertebrates alone, which can be employed for microscupic experiments alive and under normal vital conditions only in rare and exceptional cases, and which, as soon as they are separated from the tissue, are under abnormal conditions and rapidly die or give reactions that may lead to false conclusions. Much more favourable in this respect are the tissue-cells of many invertebrates, cold-blooded animals, or plants, which can be investigated more readily under approximately normal conditions, although frequently they also do not endure long-continued study. But the free-living unicellular organisms, the Protista, appear to be the most favourable objects for cell-physiological purposes. They seem to have been created by nature for the physiologists, for, besides their great capacity of resistance, of all living things they have the invaluable advantage of standing nearest to the first and simplest forms of life; hence they show in the simplest and most primitive form many vital phenomena that by special adaptation have developed to great complexity in the cells of the cell-community.

Naturally it has been maintained that exactly the reverse is true, that those forms of cells that are adapted to very special functions in the cell-community of higher animals afford far more favourable objects for the investigation of the phenomena in question than unicellular organisms. Thus, it has been urged that the cross-striated muscle-cell is decidedly more fitted for the investigation of contraction than the amœba-cell, because in the latter all the phenomena of life are not separated, but are united with the same substratum. However logical this assertion may appear at first sight, upon careful consideration it proves to be little applicable. In the first place, it is a great error to assume that the various phenomena of life are inseparably united in one cell in unicellular organisms alone. This is equally true of every tissue-cell, whether it is adapted to a specific purpose or exhibits prominently to external observation a single vital phenomenon. Every cell, wherever it is, performs all the elementary functions of life. Without being nourished, without respiring, and without excreting, the muscle-cell can execute its 
movements no more than can the amcba. There is no cell whatever that plays one role alone, for it is inherent in the nature of the vital process to exhibit different phases. Hence it is quite wrong to regard the inauguration of the act of contraction in the muscle-cell as something simpler than that in the amoba. But further, microscopic study teaches that contraction in the crossstriated muscle-cell is associated with a substratum morphologically much more complex than that in the amoba. The differentiation of various kinds of elements in the former, regarding the significance of which we have scarcely any idea, is very astonishing in comparison with the naked amœba with its single cell-body. Moreover, the history of research has shown satisfactorily that in spite of the overwhelming amount of labour that has been employed for centuries upon the investigation of contractile phenomena in muscle, up to the present time we have hardly gone beyond conjecture of the most general kind in the solution of the problem. Hence we are not only justified in employing, but even compelled to employ the simpler forms of contractile substance as an important research-object in the investigation of the problem of contraction, and an analogous requirement is demanded for the investigation of other problems. It is obvious that in the study of an elementary vital phenomenon, even among unicellular organisms, objects must always be chosen in which the phenomenon is exhibited sufficiently clearly. For the investigation of secretion cells must be chosen in which the act of secretion is readily accessible; just as in the study of contraction only those in which contractile movements are directly visible. Further, it will be necessary to treat the vital phenomena in the various kinds of cells comparatively, for comparative cell-physiology alone is able to separate the special and unessential from the general and essential. Hence it would be a mistake to neglect the tissue-cells while studying unicellular organisms. Not rarely opportunities are offered where for one consideration or another the tissue-cells or whole masses of tissue of plants or animals are to be preferred; where, indeed, as in many special problems of physiology, the tissue-cell becomes the real object of investigation. It would not be in place here to present a one-sided or schematic view, or to lay down general rules. In every individual case the choice of object is to be determined solely by the problem. One thing only should always be kept in view, namely, the investigation of the life of the cell.

Morphology, the forerunner of all physiology, has smoothed the way for physiological research. We know to-day the structure of cells in minute detail, whether they are free-living or united into tissues, and we are indebted directly to histological research for much important information and many valuable suggestions respecting the vital phenomena, especially of tissue-cells, 
such as the cells of the central nervous system, glands, and muscles.

We need not be embarrassed in the employment of experimental physiological methods upon the cell, for, with the overwhelming variety of forms in existence, more than one can always be found that are equally fitted for the purpose, and upon which widely different, special methods may be advantageously used.

To begin with the simplest method, simple microscopic observation may be employed very conveniently with the free-living cell, and under certain circumstances with the tissue-cell also. Observation alone has led to a fair knowledge of the visible vital phenomena of the cell, and has been used in the detailed investigation of some of them. Among the most prominent acquisitions by this simple method may be mentioned the extremely valuable facts concerning the more detailed phenomena of fertilisation, segmentation, and reproduction which Flemming, Buitschli, van Beneden, the brothers Hertwig, Strasburger, Boveri, Heidenhain, and many others have discovered in recent years, partly on living cells, and partly on cells that have been preserved in certain stages.

$V$ ivisection-operations upon the cell may also be performed under the microscope to the same extent and with greater systematic exactness than they are performed macroscopically upon higher animals. Several investigators, such as Gruber, Balbiani, Hofer, and others have already employed this operative method with great success, and a number of researches have shown how fruitful it is for the treatment of general physiological problems. By this method also Roux, Chabry, the brothers Hertwig, Driesch, and others have carried out their striking experimental investigations upon the development of animals.

Further, a great variety of studies can be made upon the effects of different kinds of stimuli upon the vital phenomena of the cell in its various forms; in this field a comprehensive mass of facts has already been accumulated. A large number of researches upon unicellular organisms have shown that the reactions that appear in the cell upon the employment of chemical, mechanical, thermal, photic, and galvanic stimuli, are of the greatest importance in a knowledge of vital phenomena. By these researches it has been made possible in recent years to recognise more and more clearly the general laws of excitation and depression of vital processes and their results, and also to approach nearer an understanding of the phenomena of inhibition, which hitherto have been so obscure.

Finally, vital phenomena in the cell can be approached chemically, by both macrochemical and microchemical methods. Large masses of unicellular organisms, such as yeast-cells, leucocytes, and spermatozoa, and no less combinations of cells, such as the tissues, form excellent objects for macrochemical investigation. We are 
indebted to researches upon such objects as these for the most important portion of our knowledge of the chemical composition and metabolism of the cell. A great variety of favourable researchobjects are also found for microchemical investigation, although thus far, since the methods are still little developed, only the very first beginning in this direction has been made. The labours of Miescher, Kossel, Lilienfeld, Loew and Bokorny, Zacharias, Schwarz, Löwitt, and others, have already proved that the microchemical investigation of the cell has before it a rich future.

It is, however, superfluous to enumerate single methods which can be employed in cell-physiology. All methods that the special research at the moment demands are useful. Physiology must return constantly to the standpoint that made so fruitful the labours of Johannes Müller. Throughout his whole life, Müller defended practically and theoretically the view that there is not a sirgle physiological method, but that every method is right that leads to the goal. He always selected the method in accordance with the problem of the moment, never, as often happens to-tay, the problem in accordance with the method. The problem, not the method, is indivisible; for the solution of the problem the physiologist must employ, as the special purpose demands, alike chemical, physical, anatomical, embryological, zoological, botanical, mathematical, and philosophical methods; but all should lead to one goal, the investigation of life. 


\section{CHAPTER II}

\section{LIVING SUBSTANCE}

GaLen, the father of physiology, recognised clearly that an exact. knowledge of the anatomical relations of an organ is a pre-requisite to an explanation of its vital phenomena; and modern physiology down to the present clay, to its great advantage, has maintained this position. In every physiological investigation a knowledge of the material substratum, the vital phenomena of which is to be examined, must be considered as the first pre-requisite. This is true no less for general, than for special, physiology. Therefore, a consideration of living substance, i.e., its composition and its differences, in comparison with lifeless substance, must form the starting-point of general physiology.

\section{The Composition of Living Substance}

The attempt to explain the mystery that surrounds living substance, the substance that nourishes itself, breathes, moves, grows, reproduces, and develops, has exerted from the earliest times a peculiar stimulus upon the minds of inquiring thinkers. The ancients naively believed that they were able to explain the substance of living bodies by the intermixture of certain materials. Thus, Hippocrates believed that the normal human body consists of blood, phlegm, and bile, which are mixed together in certain proportions. In the middle ages, when people endeavoured to solve the riddle of nature by the great power of alchemy, they thought that they were upon the track of the secret of living substance. How strong this delusion was is shown by the many attempts of the middle ages to produce living substance artificially. The ardent expectation with which the mediæval alchemist in the sombre dusk of his laboratory, surrounded by skilled workers and strange apparatus, hoped every moment to see the homunculus arise complete from the retorts or crucibles is a very characteristic feature of the developmental stage of science during these centuries. But, however proud we may be of our modern science, we have no right 
to look with scorn upon those attempts of the middle ages, when we realise that from that time even to the most recent period the attempts have been continued to produce artificially not man himself, but the simplest forms of living substance. Yet all these attempts resemble the endeavour of a man to put together a complicated clock-work without knowing its essential parts. However simple the problem of the artificial production of living substance appeared to the middle ages, the progress of sober thought and critical investigation has shown constantly how far we are yet removed from a knowledge of the intimate composition of such substance. How is it possible to produce chemically a substance the chemical composition of which is not at all known? Modern research has been directed, therefore, more and more toward an examination of the composition of living substance. It has penetrated deeply, and continues to penetrate, into the morphological, physical and chemical relations, and the intimate structure of living matter.

\section{A. THE INDIVIDUALISATION OF LIVING SUBSTANCE}

\section{The Cell as an Elementary Organism}

When the organic world inhabiting the surface of the earth is examined, it is found that living substance does not form a single coherent mass, but that it is divided into separate organic individuals. It is not wholly easy to define the conception of the organic individual; yet many investigators, in recent times particularly Haeckel ('66), have endeavoured to give it a generally valid form. It arose in early times by a process of abstraction from ideas of man and the higher animals, which appear as unitary living beings independent of one another. But, as with all such early conceptions which spring from a limited circle of experiences and later come to cover a larger circle, the conception of the individual in its original form has become too narrow and requires an extension.

The original idea upon which the conception of individuality was based, was that of indivisibility. According to this an individual was a unitary whole, which was incapable of division without losing its characteristic properties. So long as none but men, vertebrates and perhaps insects were in mind this definition held good, for a man, a vertebrate or an insect cannot be divided into several independent individuals. But difficulties appear when we descend lower in the animal series or attempt to apply the conception to plants.

In fresh-water ponds and lakes there exists a peculiar representative of the great group of Cnidaria, the fresh-water polyp Hydra. This small animal, about one centimetre long, with its slender tube-like body bearing sevcral long thread-like tentacles that serve for catching prey (Fig. $2, A$ ), began to attract the at- 
tention of observers soon after the discovery of the microscope. It was found that this remarkable creature could be divided by a cross-cut into two halves, each one of which could transform itself again into a complete, but correspondingly smaller individual. The anterior half, bearing the tentacles, simply closes up the wound and attaches itself again at its posterior end, while from the posterior half new tentacles soon sprout out from the edges of the wound, and in a short time both pieces have become complete Hydras. The halves can be divided still further, and the animal can even be cut into a large number of small pieces, each one of which can transform itself into a complete individual. The unitary individual has thus been divided into two or even several individuals. If, therefore, indivisibility alone be the standard of

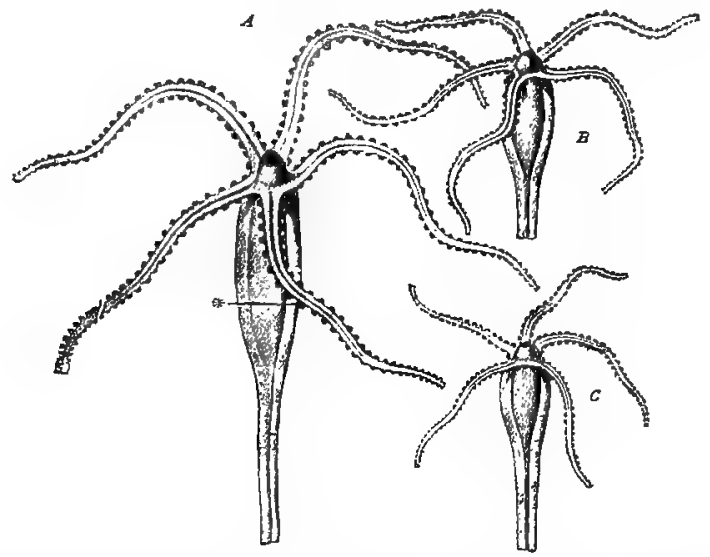

Fic. 2.-Hydia fusca, a fresh-water polyp; $A$, cut across at * $B$ and $C$, the two picces, which have become regenerated into two complete individuals.

individuality, $H y d r a$ is not an individual, for it can be divided without the loss, by the pieces, of the characteristics of the original animal; and the same is true of every tree and every shrub.

The criterion of the individual is, therefore, not to be found in indivisibility, but rather in undividedness or unity. So long as Hydra was undivided, it was an individual, a whole, a unit. By the division, however, the original individual came to an end and from it two new units arose which, so long as they are not further cut into pieces, represent complete individuals. Hence the fact of unity alone is decisive in defining the conception of individuality, if the latter is to be stated in such general terms that it holds good for all special cases. An organic individual would accordingly be merely a unitary mass of living substance.

But in this very general form the definition is too broad. According to it a small particle of living substance, cut off from the 
living cell under the microscope, would be an individual. Such a particle, however, cannot be so considered when it is seen how every minute mass of living substance, which has not the value of the cell, sooner or later invariably perishes. The capability of selfpreservation may, therefore, be added to the conception of the individual and the latter may be defined as follows: An onganic individual is a unitary moss of living substance which under definite external vital conditions is capable of self-preservation.

This definition applies to all single, free-living organisms which are spatially separated from one another and are not artificially divided, in other words to all organisms in the form in which they occur in nature. But it includes more than single organisms; it includes groups of organisms, each one of which is separated from the others by space, but which together form a unit. An example
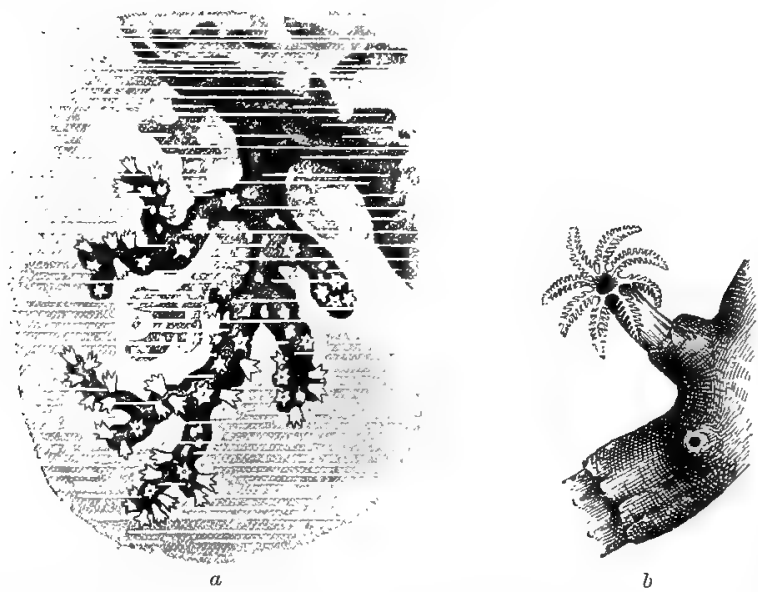

Fra. 3.-Eucorallium rubrum, the precious coral. a, A coral stem containing many individuals; $b$, a single individual highly magnified. (After Haeckel.)

of this is a community of ants. The community represents a single individual in so far as it is a unitary whole in which the single parts work together like the parts of an organism. But it consists of many single individuals, males, females, workers, and soldiers. It is thus seen that individuality may be of very different grades. It seems advantageous to distinguish the grades of individuality by terming the more comprehensive form an individual of a higher order, and the forms composing it individuals of a lower order. The condition in the coral-stem is like the relation between the ant-community and the individual ants. The coral-stem (Fig. 3, $a$ ) is an individual of a higher order, the single coral-polyp (Fig. 3, $b$ ) an individual of a lower order. The sole difference between this 
case and that of the community of ants is that here the individuals of the lower order are in physical connection with one another.

It will be advantageous to look about the organic world and see what different grades of individuality are to be found. The community, the colony, is evidently the highest grade, for a sum of communities is not a new and higher unit. The next lower stage in the community is the person. The coral-colony can be regarded in a certain sense as a person which consists of single organs; this relation, however, is clearer in another group of Coelenterata, the Siphonophora. The Siphonophora represent persons which consist
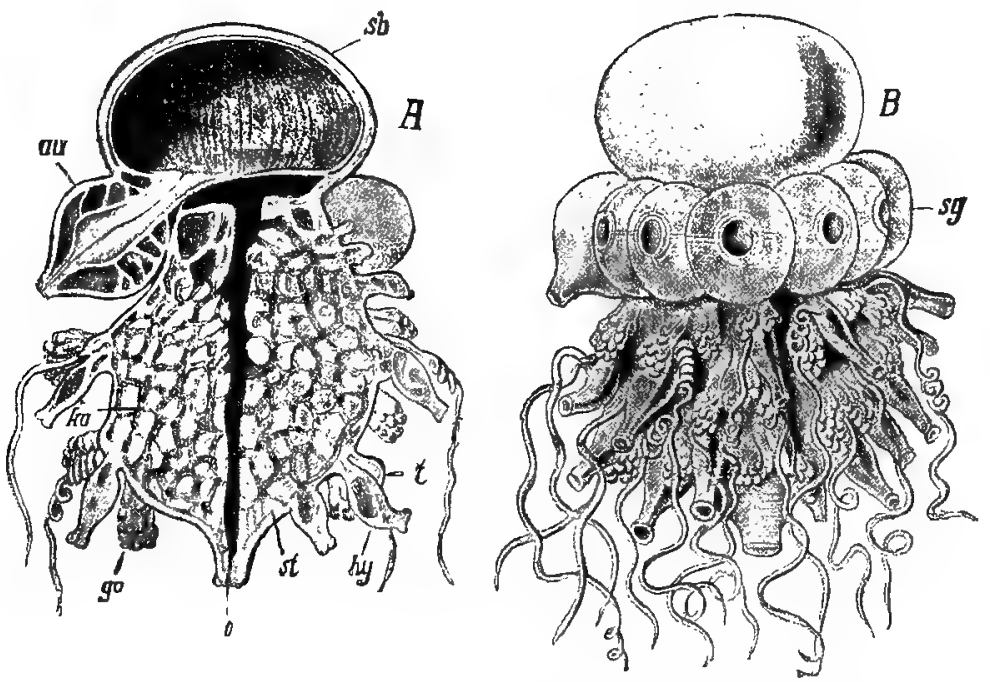

Frg. 4.-Stephalia corona, a Siphonophore. $A$, Longitudinal section; $B$, external view; $s b$, swimbladder; $s g$, swimming-bells; $g o$, sexual glands; $h y$, gastric tubes; $o$, chief gastric tube; $t$, tentacles. All the organs are single individuals. (After Haeckel.)

of a number of variously developed organs. Some of these organs are for purposes of movement, others for nutrition, others for reproduction, others for protection of the whole body, and all are grouped in regular order about a longitudinal axis (Fig. 4). But all the organs are single individuals, for the embryology of the Siphonophora shows that they all arise from morphologically homologous parts by budding; and that in certain cases single individuals, as, e.g., the swimming-bells, can separatethemselves from the stem and lead an independent existence as medusæ. It is seen, therefore, that the person of the Siphonophora can be considered as a colony of single organs, and that the stage of individuality of the person includes the lower stages of individuality of the organs. Careful dissection of an organ, e.y., a human arm, 
shows that it is composed of various constituents, which are termed tissues. The arm contains muscle-tissue, nerve-tissue, bone-tissue, etc.; the characteristic of the organ is its composition out of one or more tissues. The next lower stage of individuality, therefore, is the tissue. Certain organisms consist of but a single tissue, in which all the constituents are alike. Such free-living tissues are widely represented among the Algct. Eudorina elegans, e.g., is a small transparent ball of jelly, in which many spherical particles lie embedded, which upon close examination prove to be bits of living substance separated from one another. These single minute particles of living substance are termed cells. In this particular case each cell has two delicate flagella, by the movement of which the whole mulberry-mass of jelly is driven about in

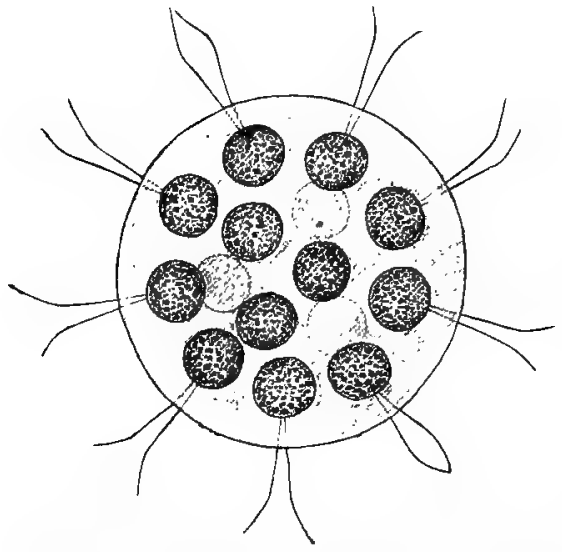

Fia. 5.-Eudorina elegans, a colony of Flagellata. The single individuals lie embedded in a common ball of jelly. the water (Fig. 5). Every such flagellate cell is an independent individual, and continues to live when separated from the ball of jelly, which happens, e.g., spontaneously in reproduction. It is seen, therefore, that the tissue contains within itself the single cell. The tissue is a colony of cells. In the cell the lowest stage of individuality has been reached. The cell is, indeed, composed of various constituents, of a soft ground-substance, the protoplasm, and a more solid cell-nucleus embedded in it; but in no case can these two constituents be separated without the death of both. Many experiments have shown that protoplasm is incapable of self-preservation without the cell-nucleus, and the nucleus similarly incapable without the protoplasm. Hence, according to the above definition of individuality, neither of the two represents an individual. In all nature no organism is known which represents a lower stage of individuality than the cell. As Bruicke ('61) says, the cell is the "elementary" organism."

Apparently in contradiction with this idea is the fact, recently established by many experiments, that under certain conditions the cell can be artificially divided into pieces which continue to live and even reproduce. If, c.g., a free-living infusorian cell, such 
as the delicate Stentor Roeselii (Fig. 6, A), which lives in fresh water and is especially adapted for this experiment, be divided into two parts in such a manner that each possesses a piece of the long rod-like nucleus, the same phenomenon appears as in Hydra: the two pieces regrow into small complete Stentors (Fig. 6, $B$ and $C$ ) and continue to live in all respects normally. In such an experiment the cell, an individual of the lowest order, has become divided into two individuals, and can even be divided into more, if the operation be performed so that each piece possesses some protoplasm as
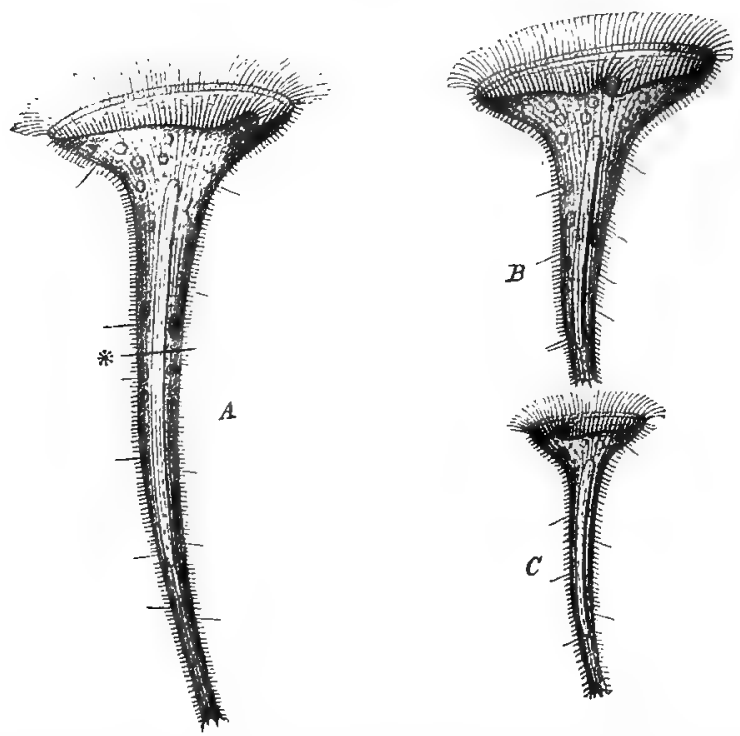

FIr. 0.-Stentor Roeselii, a trumpet-shaped infusorian; $A$, cut across at $* ; B$ and $C$, the two pieces, which have become regenerated into complete Stentors. The clear extended mass in the interior is the nucleus.

well as a piece of the nucleus. This fact is of fundamental importance, and we shall have occasion to recall it frequently. In the present case it stands only apparently in contradiction with the idea of the cell as the elementary individual; for by the cutting operation there are obtained, not new stages of individuality, but complete Stentors, i.e., individuals of the value of a cell. In all such divisions of cells, wherever protoplasm and nucleus are present in the pieces, the latter have the value of cells; in the process we do not go below the cell. If, however, the cut be made so that one piece contains protoplasm and nucleus, and the other only protoplasm, the former continues to live and represents a complete cell, while the latter, possessing no longer the individuality of a 
cell, invariably perishes. In every case, therefore, the cell remains the elementary organism.

If the above considerations be summarised, it is found that five stages of individuality can be distinguished in the organic world, and can be characterised as follows:

1. Individuals of the first order are cells. They represent elementary organisms that are not composed of lower units capable of life. An example is the unicellular, ciliate infusorian Stentor (Fig. 6).

2. Individuals of the second order are tissues. The tissues are associations of individuals of the first order, each one of which is like the others. An example is the flagellated spherical alga, Eudorina (Fig. 5).

3. Individuals of the third order are organs. The organs are associations of various kinds of individuals of the second order. An example is Hydra (Fig. 2), the body of which consists of only two layers of tissues.

4. Individuals of the fourth order are persons. The persons are associations of various individuals of the third order. An example is man, whose body consists of various organs united.

5. Individuals of the fifth order are communities. The communities are associations of individuals of the fourth order. Examples are communities of ants and bees.

This scheme requires one more remark. It shows that erery individual of a higher order consists of an assemblage of individuals of the next lower order, but the constituents of an individual of the higher order are not always real individuals, i.e., they are capable of self-preservation when living in union with, but not when separated from, their fellows; in other words, they are only virtual individuals. A person or individual of the fourth order, for example a man, consists of single organs, which are equal to individuals of the third order. These organs, however, are virtual, not real, individuals, for they perish when separated from their fellows. It is the same with individuals of all orders. E.g., the cell of an animal tissue, if separated from its fellows, is in itself incapable of life; in the tissue, therefore, it is only a virtual individual. In other cases, however, the constituents of an individual of a higher order, when separated from their fellows, can become real individuals of the next lower order, as is shown, e.g., by $E^{\prime} u d o r i n a$, in which the single cells when separated are in themselves capable of life.

From these considerations the important facts follow that in the ond all living individuals of whatever order either are composed of cells as the elementary structural components or are themselves 
frec-living cells. The cell must, therefore, be the seat of those events the expression of which is life.

In opposition to this conclusion, the attempt has lately been made by Altmann ('90) to demonstrate a still lower stage of individuality than the cell, and thus to contradict the view that cells are the elementary organisms. It has long been known that roundish granules of different sizes are of wide occurrence within cells, lying in an apparently homogeneous ground-substance; they have been termed elementary granules, granula, or microsomes (Fig. 7). In many cases only a few such granules are present in the cell, in other cases the whole cell is thickly filled with them, so that the groundsubstance between them almost disappears. Altmann considers these granules to be the true elementary organisms, and terms them "bioblasts." He believes that they represent in the cell the true living elements which are the seat of the vital phenomena. The cell itself, according to Altmann, is to

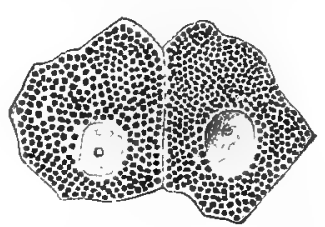

FIG. 7.-Liver-cells containing granules. (After Altmann.) be considered as a colony of bioblasts, hence not as an elementary organism but as an individual of a higher order. Of course single bioblasts cannot be kept alive when separated from the other bioblasts of the cell. Nevertheless, according to Altmann, there are in nature free-living bioblasts, namely, the Bacteria. The great horde of Fungi or Bacteria, as Altmann says, represent nothing but free-living elementary organisms, which as regards individuality are equal to the granules or bioblasts that in part constitute the cell-contents.

But one searches in vain in Altmann's works for an adequate confirmation of the hypothesis that the bioblasts are the elementary organisms. On the contrary, it is not difficult to perceive the untenableness of such a view. The majority of investigators have not accepted it, and Altmann's attempt must be regarded as wholly unsuccessful.

The following seem to be the two most important considerations which render the hypothesis of granules untenable. In the first place, Altmann brings together under the conception of the granule all sorts of different elements, which can by no means be homologised with each other. Lately, indeed, he has given up the idea that the chlorophyll bodies, which give the green colour to plant-cells, are granules, but the conception still contains the most heterogeneous elements. Thus, he considers as granules not only the minute grey particles that occur wide-spread in the most various free-living and tissue-cells, and differ greatly in chemical composition and significance for the cell-life, but he includes the fine granules of colouring matter in pigment-cells, which give to the tissues in which they lie their characteristic colour; the fine 
lamellar particles in the yolk of eggs; and even the small oildroplets and fat-globules that occur in various tissue-cells, especially in the liver and the cells of subcutaneous connective tissiue. Moreover, particles of ingested food, transformed foodconstituents, undigested food-stuffs, and products of cellular metabolism, i.e., substances that are playing or have played the most various roles in cell life, are put into the same category, and are considered as elementary organisms. In the second place, Altmann does not prove for one of all these forms that they show general vital phenomena, nor would success in such an attempt be expected, especially in the case of an oil-droplet or a pigmentgrain lying within the cell. Nevertheless, such proof must be furnished if the term "elementary organism" is to be allowed. Regarding Altmann's belief that bacteria must be considerer as free-living granules, not only is there no evidence for this. view, but lately the striking investigations of Bütschli ('90) have afforded proof that bacteria are complete cells, and hence urganisms that Altmann considers colonies of bioblasts.

These considerations suffice to overthrow Altmann's idea. And, in general, it seems entirely inadmissible to regard as elementary organisms structures that have no analogies with free-living organisms. If this be allowed, the conception of the organic individual collapses, for it is then not justifiable to consider any one portion of the living substance more than another as constituting the elementary organism. The term can be applied with equal justice to an atom of oxygen or carbon, or any other atom that takes a direct part in the life-process. There would be as many elementary organisms as organic elements. How to define an organism or an organic individual is one question, what in general to call living is another. The latter will be discussed later. As to the former, if the conception of the organic individual is not to be given up, it must be regarded as an unconditional requirement. that the organism be characterised by the presence of all those vital phenomena that have to do with self-preservation. Only the cell fulfils this condition; it is, therefore, the individual of the lowest. order and the elementary organism.

\section{General and Special Cell-constituents}

The thought that the enormous number of phenomena constituting life are associated in all their essentials with the microscopic bit of living substance that constitutes the living cell is an irresistible stimulus to research. Hence, from the time when the significance of cells as elementary organisms became first recognised until now, a host of investigators have busied themselves with the detailed study of the coll and its constituents. Thanks to this, our knowledge of cell-morphology has 
been extended from year to year, and the conception of the cell has been made constantly more precise.

The conception of the nature of the cell has not been always the same. As we have seen, ${ }^{1}$ the cell idea originated as a result of the microscopic observation of plants. The microscopists of the seventeenth and eighteenth centuries found that plant-tissue contained, besides long tube-like structures, small chamber-like elements set off from one another by walls, and containing liquid. Because of their similarity to the large cells of honeycomb these small structures received the name of "cells." Thus, at that time the cell was regarded as a simple droplet of liquid enclosed by a wall or membrane. The characteristic thing which led to the giving of the name "cell," a term very fitting for plant-cells, was the "cell-membrane," without which a chamber, vesicle, or cell was not possible. This idea continued to prevail even when Schleiden discovered, in addition to the cell-liquid or cell-sac, a slimy semiliquid mass, the "plant-slime," or, as Mohl called it, the "protoplasm," and when by Schwann the cell idea was extended to the elementary parts of animal tissues.

The fundamental work of Max Schultze ('61, '63) gave to the cell idea an entirely different meaning. The study of the Rhizopoda, those one-celled organisms whose naked protoplasmic bodies are capable of extending their viscous body-substance at any desired spot into fine threads and networks, led Schultze to the view that the essential part of the cell cannot be the cellmembrane, for the very numerous species of Rhizopoda have throughout life no cell-membrane; but that it is the substance which earlier had been termed "sarcode" by Dujardin ('41) in naked fresh-water Rhizopoda and Infusoria. A comparison of Rhizopod $a$ and plant-cells afforded Schultze the proof that sarcode, the substance of the Rhizopoda, is completely identical with protoplasm, the viscous contents of plant-cells; and thus he founded the theory of protoplasm, according to which the essential constituent of the cell is the protoplasm. The idea that the cell is a simple bit of protoplasm has proved brilliant in results, in opposition to the old view of the necessity of the cell-membrane. Not only have an enormous number of cells that lack a membrane become known among the numerous unicellular Rhizopoda (to which belong the Polythalamia or Foraminifera having calcareous shells, the Kadiolaria having silicious shells, and the Amoboe in which a shell is wholly wanting), but it has also been observed that in the development of many plants and animals one-celled stages occur as eggs, which are entirely devoid of a membrane. Hence, since Max Schultze's establishment of the protoplasm theory, the idea that the cell-membrane is a general cell-constituent has completely disappeared. 
Schultze's definition, however, does not include all the essential or general cell-constituents. In 1833 Brown had discovered in protoplasm a further specific structure, the cell-nucleus, which, by its refractive power, could be distinguished clearly as a spherical granule from the protoplasm enclosing it. Schleiden (38) took up this discovery of Brown and demonstrated the cell-nucleus to be a wide-spread constituent of the cell in many plants; but he was misled in his theory of phytogenesis into considering the nucleus as the element from which the cell first arises in the course of the individual development of the plant. Since that time constantly more attention has been given to the nucleus. It was found not only in plant-cells, but after Schwann's labours ('39) also in the most diverse animal cells. But, especially when by means of certain colouring-matters, such as carmine, hæmatoxylin, etc., it was stained, and thus was made clearly visible in the protoplasm in which it was embedded, the view was gradually adopted that it represents a very characteristic constituent of the cell; and soon the question arose whether cells ever exist without nuclei, or whether the nucleus is a general and, like the protoplasm, essential constituent of the cell.

Among the unicellular free-living Rhizopoda, to which Max Schultze had called attention, Haeckel ('70) found a considerable number in which no trace of a nucleus was to be demonstrated, and which, since they appeared to consist of a simple bit of protoplasm and thus were the lowest and simplest conceivable organisms, he termed Monera. Another group in which no nucleus could be demonstrated was that of the micro-organisms, the Bacteria, which likewise have excited the greatest interest in recent times. They are the smallest of all existing living beings, and, although they possess a fixed unchangeable form, they reveal no trace of differentiation in their apparently wholly uniform protoplasmic bodies. If we except the red blood-corpuscles of warm-blooded animals, which likewise show no differentiation of their bodysubstance into two separate parts, protoplasm and nucleus, but which, as has been demonstrated, develop from actual nucleated cells, the two groups of the Monera and the Bacteria remain as the sole apparently non-nucleated cells.

But with the recent wonderful development of the technique of microscopic staining the conception of the Moncra as nonnucleated cells has gradually changed. By the employment of the newer, complicated staining-methods constantly more of the organisms which Haeckel described as Monerca are being recognised as nucleated cells: in many of them even a large number of small nuclei have been demonstrated; and Gruber ('88) has found forms in which the nuclear substance is distributed through the whole protoplasm in innumerable, extremely minute granules (Fig. 8). Thus the number of the 
original Monerce is constantly diminishing, and the few that cannot yet be obtained for fresh investigation are now also regarded by most investigators as nucleated cells in which the earlier incomplete technique was not able to demonstrate nuclei, just as was the case with the others that are now recognised as nucleated.

The Bacteria have defied much longer than the Monera attempts to find in them a differentiation corresponding to the nucleus and protoplasm of other cells. All imaginable methods of staining and the strongest microscopic powers were not able to demonstrate the two different kinds of living substance within their minute and apparently completely homogeneous bodies. This state of our knowledge continued until a very few years ago, in spite of the great advance that bacteriology made. Recently, however, Buitschli ('90) succeeded in discovering a fine structure in the bodies of Bacteria. He found that by the use of very strong magnifying powers and not too strong illumination certain specific staining-reagents, which, as, e.g., hæmatoxylin, colour only the nuclear substance and not the protoplasm, make visible two different substances in the

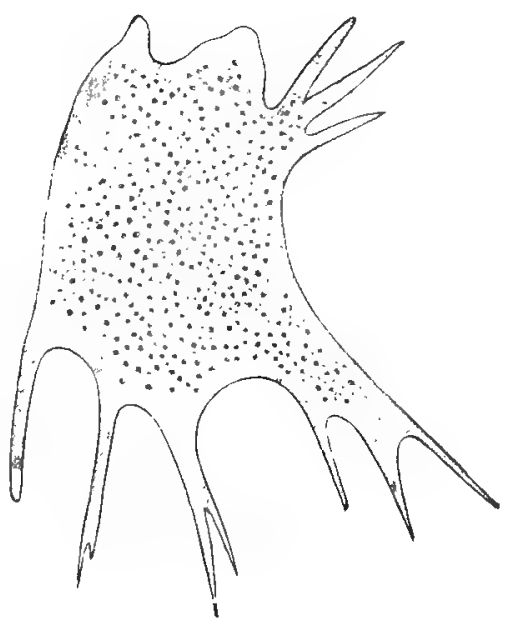

FIo. 8.-Pelomyxa pallida. A rhizopod containing very finely-divided nuclear substance (After Gruber.) bodies of Bacteria; one of these is stained intensely, the other not at all. The quantitative relations of the two substances are characteristic: the volume of the stained substance is usually greater than that of the unstained, but the relative arrangement of the two is different in different species. In one species, as, e.g., Bacterium lineola (Fig. $9, a)$, the stained substance lies in the middle, and the unstained substance forms a delicate peripheral layer about it; in others, especially the corkscrew-like forms of Spirillum, such as Spirillum undula (Fig. 9, b), which is common in stagnant water, the unstained substance is accumulated at one end or both ends of the elongated body, and the latter consists otherwise wholly of stained substance. This differentiation of the body-substance into two portions, one of which is stained and the other unstained by specific staining-reagents, appears to correspond entirely to the division of the living substance into nucleus and protoplasm that 
characterises all other cells. There are also among animals varieties of cells that show quite the same quantitative relation of the two substances to each other; an example of such is afforded by the sperm-cells, or spermatozoa, which consist of a large quantity of nuclear substance and a very small quantity of protoplasm.

Thus, from the present state of our knowledge, it appears that among the organisms now living upon the earth there are no cells in which a separation of two different substances is not present, but that every cell possesses a nucleus in addition to the protoplasm. It is, of course, another question whether during the evolution of living substance upon the earth organisms may not have existed at some earlier time, in which the whole body consisted of a single homogeneous substance, and no separation into different substances had yet taken place. If such organisms ever
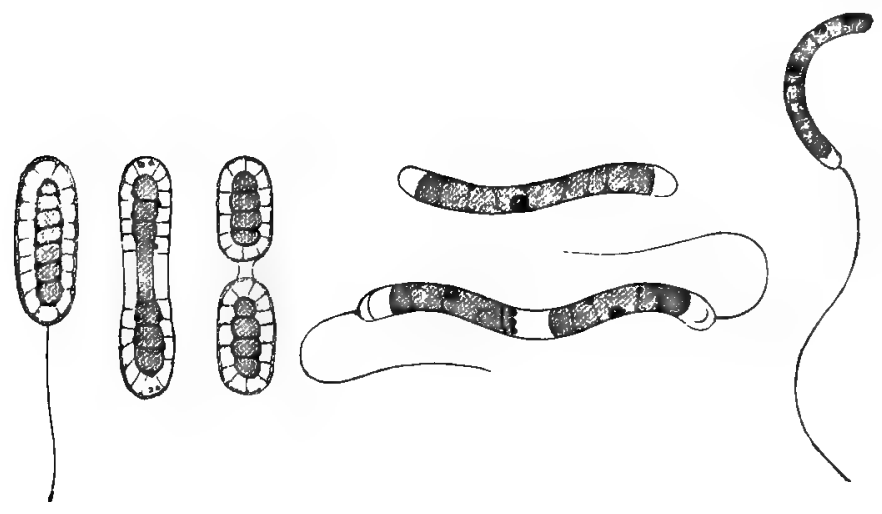

$a$

Fia. 9.-Structure of various Bacteria. (After Bütschli.) a, Bacterium lineola, normal and undergoing division. $b$, Spirillum unclula. c, Bacterium from stagnant water.

existed, they could be ranked in comparison with real cells as cytodes, as Haeckel terms non-nucleated elementary organisms. Notwithstanding them, it must be granted that there belongs to the conception of the cell at present, not only a single homogeneous mass, the protoplasm, but also a substance differing from it, the nuclear substance. Accordingly, Max Schultze's morphological definition would be widened as follows: The cell is a bit of protoplasm containing a distinct nucleus.

If the protoplasm be examined with strong magnifying powers, in many cells other distinct constituents besides the nucleus are found embedded in the protoplasmic ground-substance. In many cells oil-droplets occur, in others pigment-granules, in plant-cells starch-grains, etc.; but all these bodies do not occur in every kind of cell : they are special, not general, cell-constituents. It appeared 
recently, however, as if, in addition to the two previously known general constituents, the protoplasm and the nucleus, a third exists, the polar corpuscle, central corpuscle, or centrosome.

The centrosome (Fig. 10) has become known in detail only very recently. It had, indeed, been noticed when the peculiar phenomena of nuclear division in cell-multiplication were investigated twenty years ago; but not until later was it recognised by van Beneden ('83, '87) and Boveri ('87, '88, '90) as an important element in the cell, which reproduces like the nucleus in the increase of cells by division. van Beneden came to believe that the centrosome, like the nucleus and the protoplasm, is a general cell-constituent. This idea was supported by

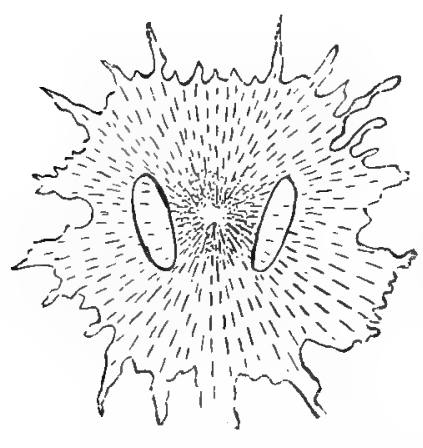

$\omega$
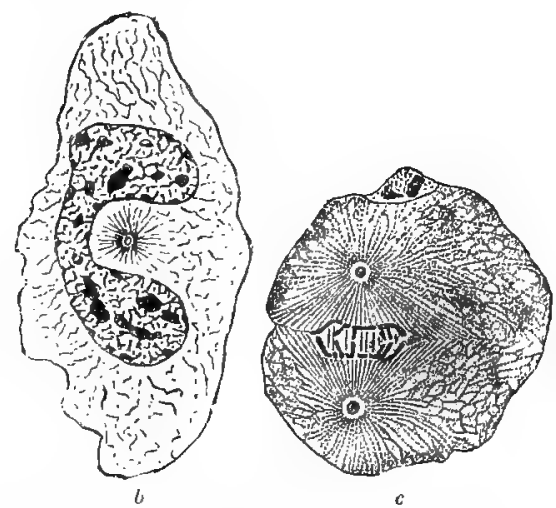

FIG, 10.-a, Pigment-cell from the pike; the centrosome with its protoplasmic radiation lies between the two nuclei. (After Solger.) $b$, Leucocyte from the larva of a salamander; the centrosome with the aster lies at the right of the dumb-bell-shaped nucleus. (After. Flemming.) $c$, Egg-cell in the act of dividing; there is a distinct protoplasmic radiation about each of the two centrosomes. (After Boveri.)

the observations of Flemming, Solger, Heidenhain, and others, who found one or more centrosomes in other kinds of cells, such as leucocytes, pigment-cells, epithelium-cells, etc., and even when they were not undergoing division. Nevertheless, in a great number of cells it has not been possible up to the present time to demonstrate such a body. Perhaps this is due to its nature. It is a granule that is very difficult to find in protoplasm on account of its minuteness, and no structure whatever has been proved in it by the help of the microscope. Moreover, as a rule it is not stained by the usual staining-reagents. The endeavours of $M$. Heidenhain to find for it specific staining-media, such as exist for the nucleus, have not yet led to wholly satisfactory results. Its presence is clearly revealed by the protoplasmic radiations by which in certain conditions of the cell it is surrounded. In the division of the cell the protoplasm arranges itself around the 
centrosome in the form of a radiating aster; the centrosome forms the middle point of the star-shaped figure (Fig. 10), and is easily discovered by this peculiar investment.

While many investigators, led by van Beneden, are inclined to regard the centrosome as a specific constituent of the cell, since it is always to be found in the protoplasm apart from the nucleus, o. Hertwig ('92) upholds the view that it belongs to the nucleus as a part of the nuclear substance, and passes from it into the protoplasm only during the activity of the former in fertilisation
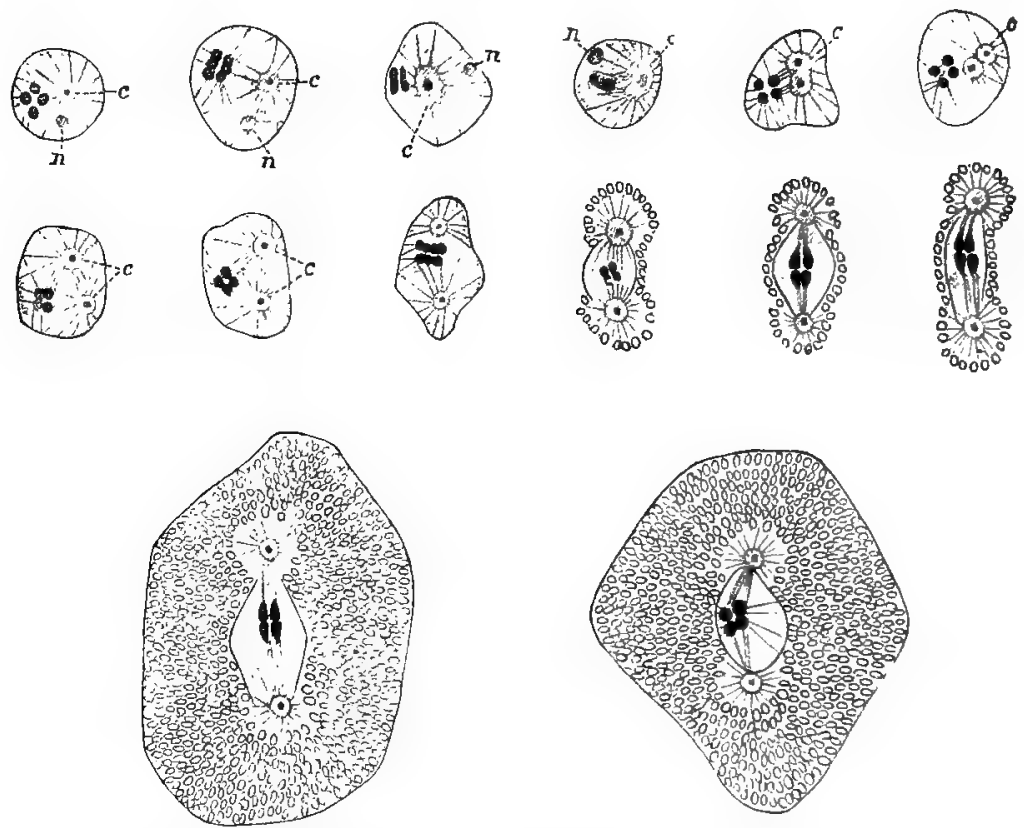

Fia. 11.-Division and withdrawal of the centrosome in the nucleus of the spermatozoa of A scaris megalocephala; the two upper rows show successive stages of the nucleus ( $n$, nucleolus; i, centrosome); below, two spermatozoa after the withdrawal of the centrosome from the nucleus. (After Brauer.)

and division, retreating afterwards again to the nucleus as a part of the substance of the latter during the resting-condition of the cell. That this view of Hertwig is applicable in certain cases has been shown recently by the striking investigations of Brauer $(93,2)$ upon the development of the spermatozoa of the threadworm, Ascaris megalocephala. Brauer was able to determine that in these cells the centrosome is contained within the resting nucleus, and in certain cases even undergoes division there; later it wanders out into the protoplasm and there produces the protoplasmic radiation which surrounds it during cell-division (Fig. 11). 
On the other hand, in the large majority of cases it remains continually, even during the resting-stage of 'the cell, outside the nucleus. Hence, as Heidenhain ('94) and Boveri ('95) remark, there is ground for considering the centrosome a constituent neither of the nucleus nor of the protoplasm; it must rather be regarded as an independent constituent of the cell. Since, however, many forms of cells, especially among unicellular organisms, are known in which thus far it has not been possible to discover a centrosome, it is not justifiable at present to regard it as a general cell-constituent. Among the vital phenomena of the cell, it is known thus far to share only in reproduction and fertilisation.

In accordance with these considerations the protoplasm in its entirety and the nucleus with its differentiations can be contrasted as the sole general cell-constituent, in distinction from all special constituents, such as the cell-membrane, starch-grains, pigmentgrains, oil-droplets, chlorophyll-bodies, centrosomes, etc.

\section{Multinucleate Cells and Syncytia.}

Five stages of individuality have been distinguished sharply from one another in organic nature; it must be remembered, however, that no sharp limits are to be found in the living world. Cells were distinguished as elementary organisms from the next higher stage of individuality, tissues; and it might seem as if no sharper boundary exists than that between the single cell and the tissue, which consists of a number of similar cells, and as if it would be very casy to distinguish the two stages of individuality from one another. In reality this is not so. There are individual organisms in which a distinction, as to whether they are elementary organisms ur tissues, does not reaclily appear; and such cases, like many others in which boundaries are to be established in nature, show that the fixing of sharp limits and definitions must contain finally a more or less arbitrary element, that, in-

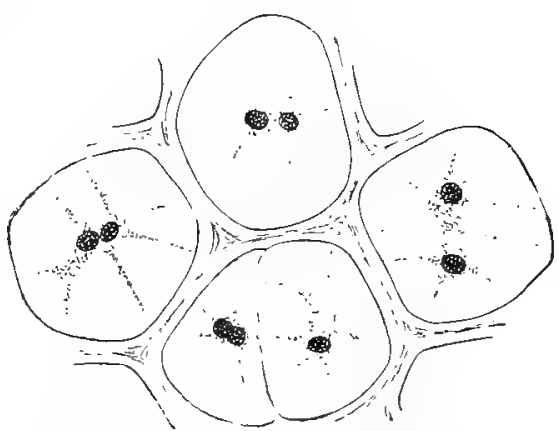

FIg. 12.-Cartilage; the cellw contain two nuclei. deed, all limits and definitions are only psychological helps toward knowledge.

The transition-forms between typical cells and genuine tissues are numerous. They consist of a unitary protoplasmic mass containing more than the one nuclens that is characteristic of the cell-type. Double-nucleated cells are found in many tissues, such 
as cartilage (Fig. 12). Many epithelial cells (Fig. 13, a) contain more than two nuclei; and the large ciliate infusorian, Opalina (Fig. 13,b), which lives parasitically in the intestine of the frog; contains a considerably larger number. Forms with innumerable nuclei are to be found among the marine algæ: e.g., in the thin lamellar protoplasmic layer of Caulerpa (Fig. 14), a giant cell of the shape and size of a leaf, there lies an immense number of nuclei, all of which together with the protoplasm are moving in a constant slow stream between the cell-walls, i.c., the two surfaces of the leaf.

All these organisms containing several nuclei can be separated as multinucleate cells from multicellular tissues by the fact that in the former the protoplasmic territory immediately surrounding the individual nuclei is not sharply defined from the neighbouring protoplasm, but together with all the rest of the protoplasm constitutes a unitary mass which appears as a whole shut off from the

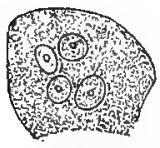

$a$

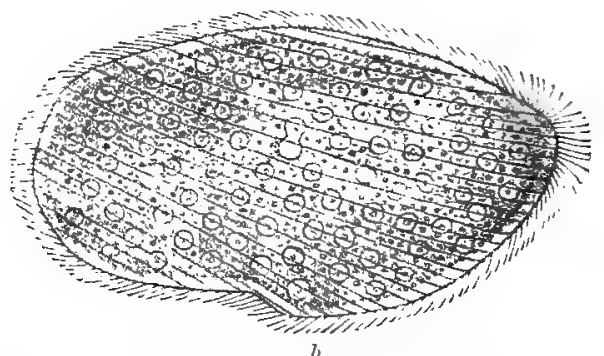

b

Fig. 13.-a, Epithelium-cell, containiug several nuclei from the urinary bludder of man. (After Virchow.) b, Opaline ranarum, a unicellular ciliate infusorian, containing mans nuclei, from the intestine of a frog. (After Zeller.)

outside by a definite surface, while in the tissue erery individual protoplasmic territory which belongs to a nucleus is sharply separated from all the rest. The multinucleate cell, therefore, represents one cell, which is characterised as a whole by a definite form of surface; the tissue, however, consists of a sum of single cells, each one of which has its own sharply defined form.

The distinction between multinucleate cells and genuine tissucs becomes more difficult in the case of certain low organisms, the Myxomycetes, which have frequently been claimed by the botanists as plants and by the zoologists as animals, and which in many respects are of great interest. They are sometimes seen in leafy forests, upon mouldy leaves or decaying tree-trunks, as white, yellow, or brownish-red networks: they often spread themselves out for several decimetres upon objects by means of their delicate arborescent strands (Fig. 15, I). Detailed examination shows that these networks, which sometimes torm thicker, lumpy masses of the same appearance, are of a soft slin!y consistency. If such a 
network be observed some hours after it is first seen, or upon the next day, it is found that not only its place but its shape has been completely changed; and if a small piece be cut off from it, laid upon a glass plate and kept in a moist place, it can be seen how the whole mass begins to flow slowly and to send out delicate processes in this and that direction, which branch in an arborescent manner and flow together into a network. In short, it is seen that the whole network is living. Myxomycetes consists of com-

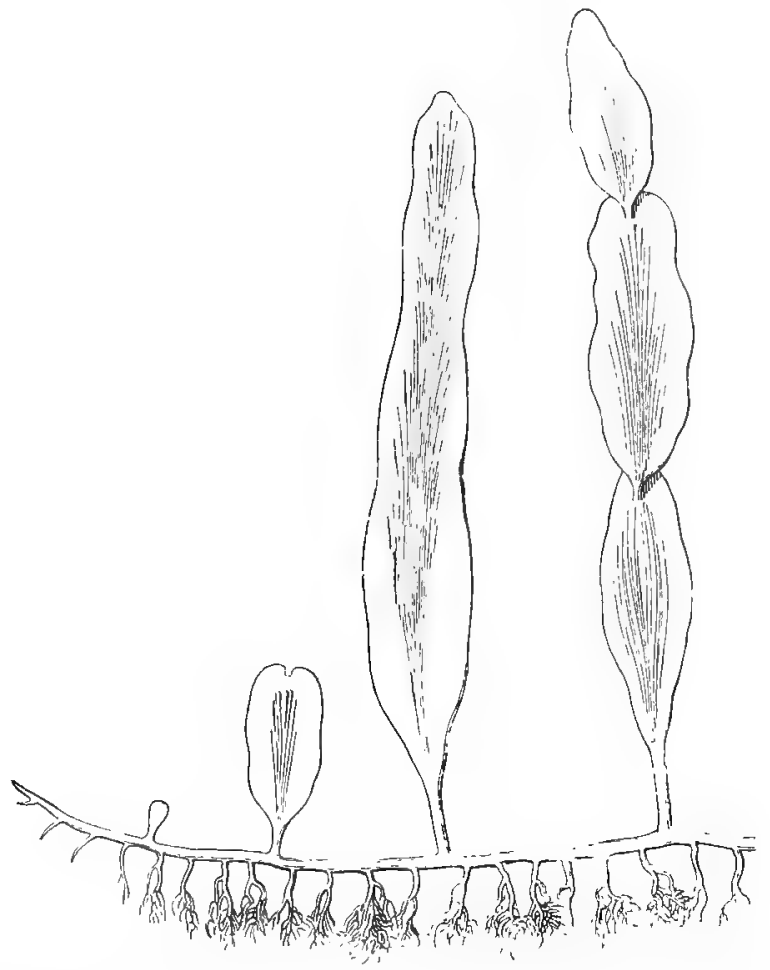

I'sc. 14.-Caulerpa, a leaf-shaped marine alga; the single leaves aro thin, protoplasmic lamellat enclosed between two flat cellulose walls and containing numberless small nuclei ; natural size. (After Reinke.)

pletely naked protoplasm. Microscopic examination and staining reveal in the fine strands of its plasmodia a large number of nuclei, which are continually being dragged along by the slowly flowing protoplasm and which roll over and under one another, plainly showing that they possess no fixed position but change their places constantly and irregularly in the unitary protoplasmic mass. Here individual cell-territories are not marked off within the protoplasmic body. According to the above criterion, therefore, we would be obliged to regard the plasmodia as multinucleate 
cells. But the origin of the Myxomycete plasmodia renders such a view uncertain. Myxomycetes reproduces by spores, i.e., by small microscopic capsules, the shells of which burst and give exit in each case to a small naked cell, which is capable of changing its shape and is provided with one nucleus (Fig. 15, $a, b, c$ ). A very large number of the spores always coexist and many separate cells creep out at the same time. These cells soon creep together, coalesce, and thus form a larger, unitary, protoplasmic mass, which contains a number of nuclei (Fig. $15 e, f)$. The mass grows by it: own nutritive efforts, the nuclei multiply by division, and thus
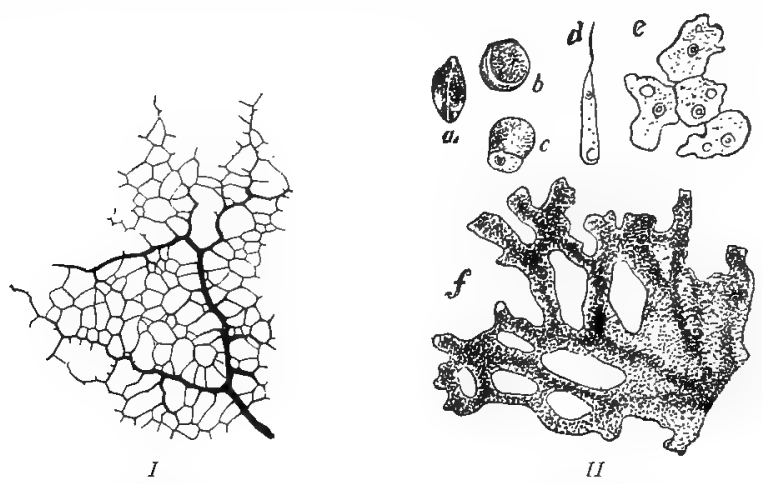

FIG. 15.-I, Aethalium septicum; a piece of a reticulate Myxomycete plasmodium, natural size. $1 I$, Chondrioderma difforme; $f$, piece of a plasmodium ; $a$, a spore, $b$, the same, swelling, $c$, the contents of the spore is creeping out; $d$, the spore has changed into a flageliated cell; $e$, the flagellated cells have transformed themselves into amobs, which are creeping together again to form a plasmodium. (II After Strasburger.)

arises finally the large, reticulate plasmodium. This plasmodium, therefore, although representing a unitary protoplasmic mass containing many nuclei and without cell-boundaries, has arisen from many single cells. Hence, strictly, it is not proper to consider the plasmodium of Myxomycetes as a multinucleate cell: at the same time it is not justifiable to speak of it as a genuine tissue, for no cell-boundaries are marked out for the single nuclei. A special name, therefore, has been created for these intermediate stages between the single cell and the tissue, and they have been called syncytia.

\section{B. THE MORPHOLOGICAL NATURE OF LIVING SUBSTANIE}

\section{The Form and Size of the Cell}

The chief fact that has stoor in the way of a consistent extension of the cell-theory and onc that still presents at first the greatest difficulties to all who study the finer structure of organisms, is the astonishing variety of forms in which the elementary constituent of prganisms appears. The forms of the different 
cells are so manifold that it is often difficult for the inexperienced observer to realise that they are all different modifications of one and the same type. In contradistinction to this endless variety there exists a wide-spread constancy in the form of one and the same kind of cell, so that the cells of any particular tissue of the human body, e.g., the liver, the skin, the bone, or the blood, are always to be recognised at once as such, i.e., as liver-, skin-, bone-, or blood-cells. A few examples will best illustrate the great differences in the forms of cells.

There are many cells that possess no constant form, but change their shape continually, and hence are termed amoboid cells. All amœboid cells have a naked protoplasmic body, upon the surface
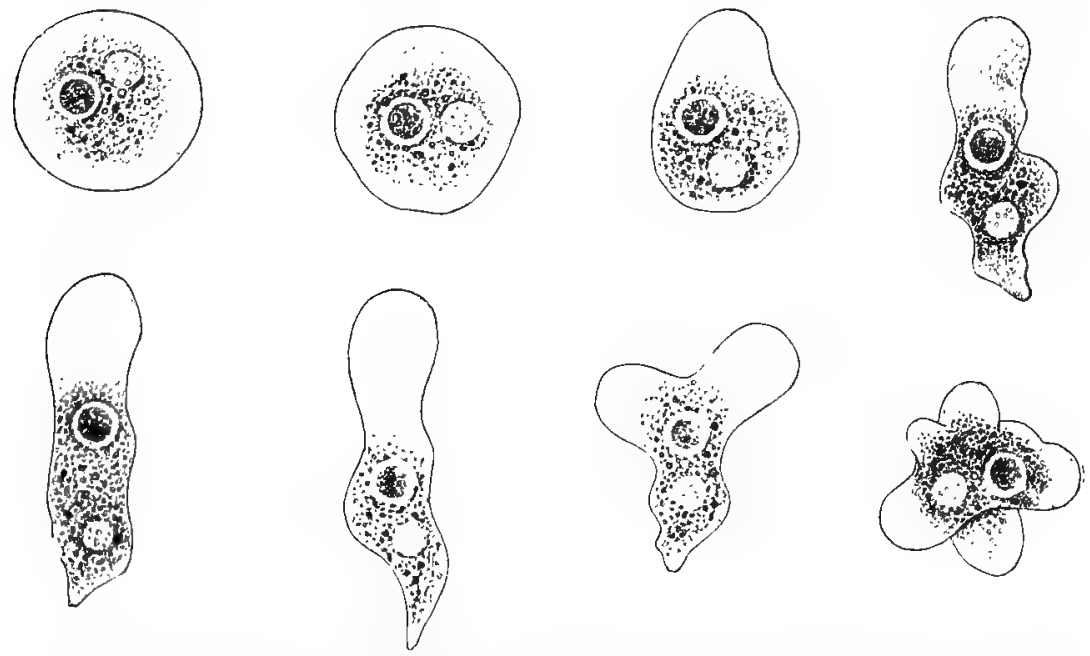

Fig. 16.-Amaba, showing successively different shapes in creeping; the hyaline exoplasm flows constantly forward; in the middle and behind lies the granular endoplasm, containing the (darker) nucleus and the (lighter) vacuole.

of which projections of the body-substance constantly appear and disappear, and thus a new shape is constantly being assumed. In different kinds of cells these projections or pseudopodia have different forms. Most fresh-water Amcebce. (Fig. 16) and the eggcells (Fig. 17, a) of many animals are characterised by broad, lobate or finger-shaped pseudopodia; leucocytes (Fig. 17, b), or colourless blood-cells, by pointed and divided pseudopodia; and many Rhizopoda (Fig. 17, c) and pigment-cells (Fig. 17, d) by threadlike and reticulate pseudopodia flowing into one another.

But by far the majority of cells possess a constant form, whether the protoplasm is enclosed in a membrane or not. The simplest form of cell that can be regarded as the type of the elementary 
organism is the spherical form, as it appears, for example, in many egg-cells (Fig. 18, a). From this type deviations in all sorts of directions occur. When the cells are united with other similar ones, as is the case in every tissue, their form is modified by the pressure which they receive from the surrounding cells. $\AA$ cell which in itself is spherical must, therefore, in a tissue take on a polyhedral form according to simple mechanical laws, just as

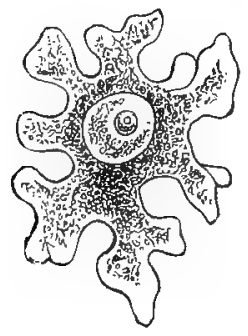

ct

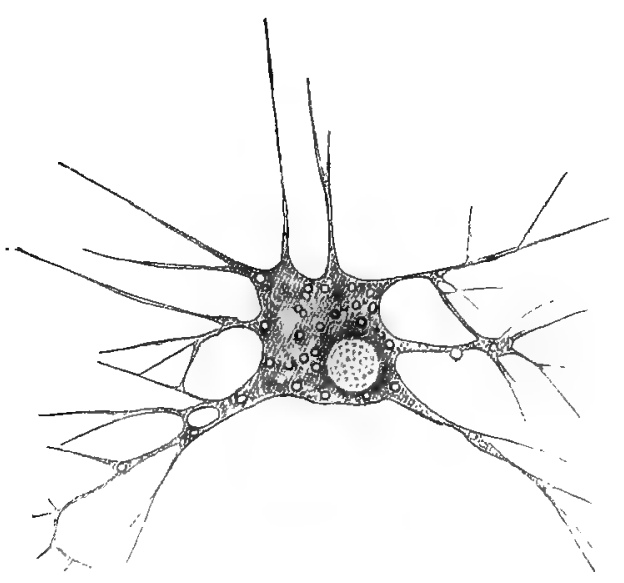

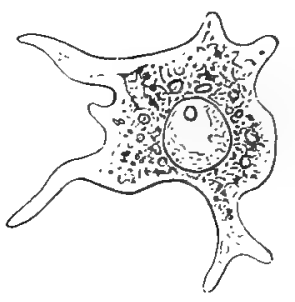

$b$

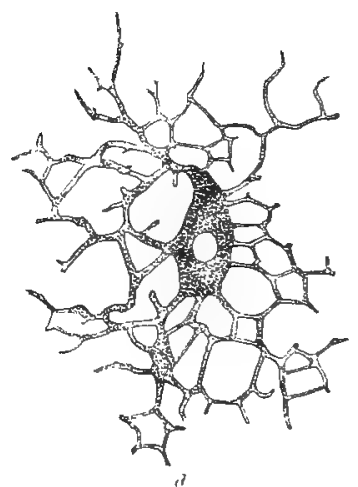

FIa. 17.- $a$, Egg-cell of a calcareous sponge, (After Haeckel,) b, Blood-cell of a crab. (After Haeckel.) c, Biomyxa vagans, a fresh-water rhizopod. $d$, Pigment-cell from the tail of a
tadpole.

peas lose their spherical shape, and become polyhedral when they are crowded thickly in a bottle and are made to swell. In fact the polyhedral shape of cells occurs very frequently in tissues, especially in epithelium-cells of the skin (Fig. 18, b) and glandcells. Further, one essential factor in causing a deviation from the spherical type is the formation of permanent processes upon the surface. In this way permanent forms of cells often occur of the shape that amoboid cells show temporarily. The green 
alga Euastrum (Fig. 19, a) represents such a cell with lobate processes, and the ganglion-cells in the central nervous system

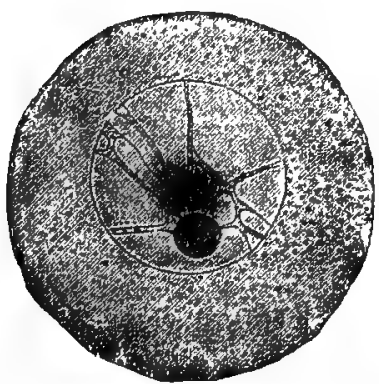

a.

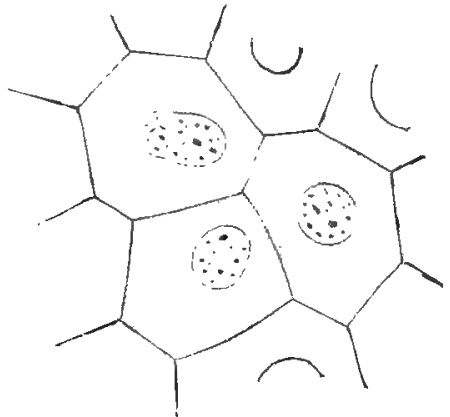

$b$

FIG. IS.- a Egg-cell from the opary of a sea-urchin. (After Hertwig.) b, Epidermis-cells from the frog.

of man, the brain and spinal cord, which give origin to the nervefibres, possess constant processes which appear exactly like the

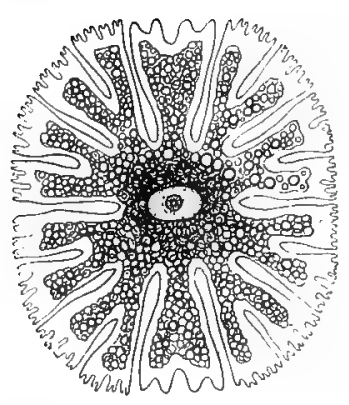

(l)

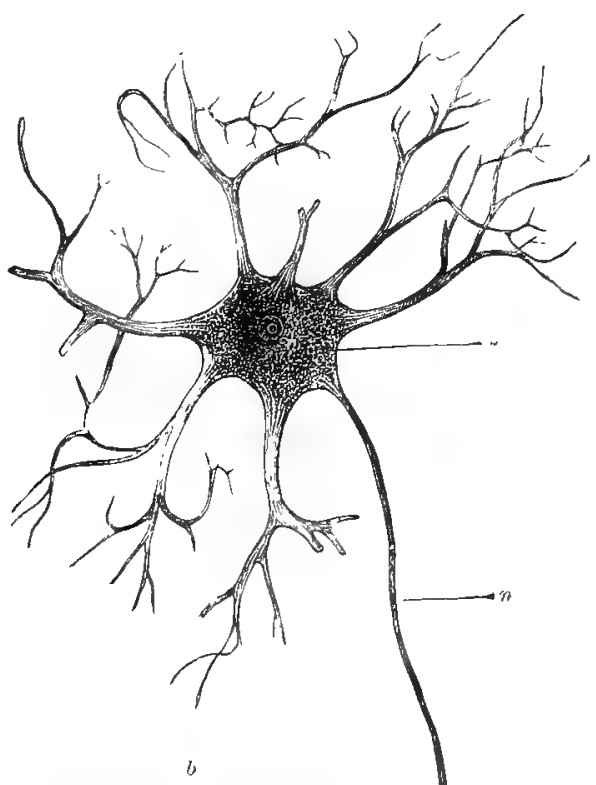

FIG. 19.- $九$, Euastinu, a unicellular alga from the group of the Hrinidiccea. (After Haeckel.) b, Ganglion-cell from the human spinal cord. (After Gegenbaur.) :, Cell-body ; $n$, nerve-process (axis-cylinder process).

pseudopodia of many rhizopod cells (Fig. 19, b). Other cells, the ciliated cells, have upon their sur- 
face motile but permanent processes, of the shape of eye-lashes. These ciliated cells are very wide-spread; they occur not only in tissues as ciliated epithelium-cells (Fig. 20, $\alpha$ ), but also free-living and constituting the great host of the Ciliatr, or ciliate Infusoria,
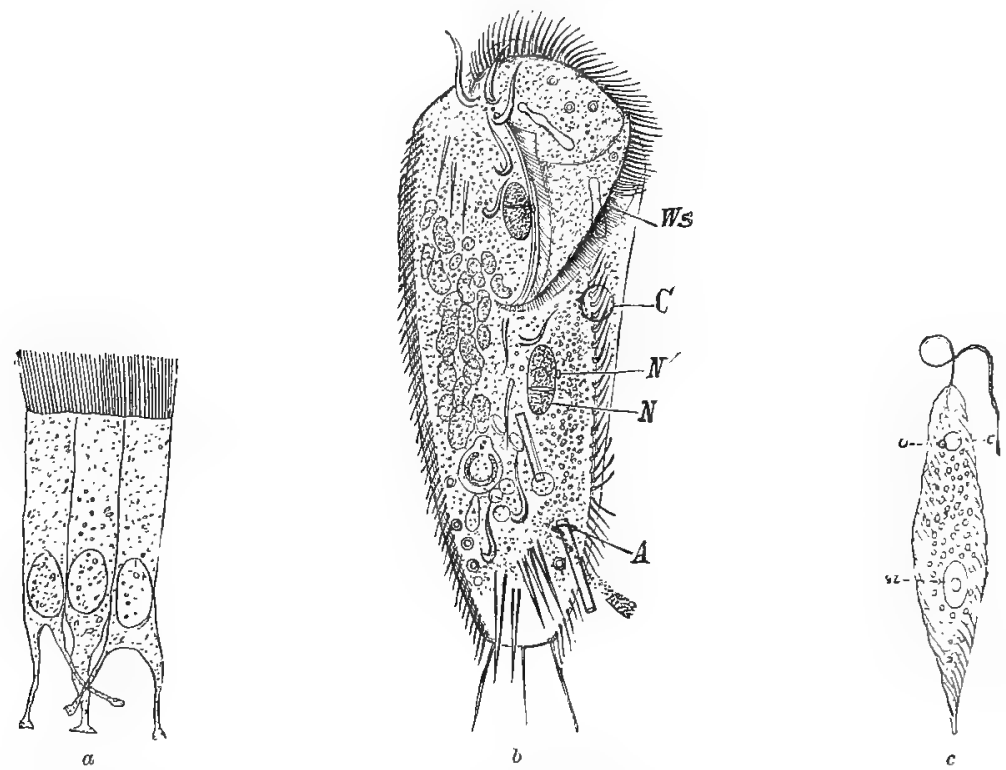

FIG. 20.- a, Ciliated epithelium-cells. (After Schiefferdecker.) b, Stylonychiamytilus, a ciliate-in. fusorian cell possessing variously differentiated cilia; $W Z$, mouth-region; $C$, contractile vacuole ; $N$, macronucleus ; $N$, micronucleus ; $A$, anal opening. (After Stein.) $c$, Buglenn viridis, a flagellate-infusorian cell possessing a single flagellum. $n$, Nucleus; 0 , eje-spot; $c$, vacuoie. (After Stcin.)

and the Flagellata, or flagellate Infusoria, according as the onecelled body possesses many similar'or variously differentiated cilia (Fig. 20,b), or only one flagellum or several (Fig. 20, c). Finally, there are cells that deviate from the type by being enormously extended in one direction, so that they appear as slender, bandor thread-like forms. Extremes in this direction are smooth and cross-striated muscles-cells (Fig. 21, a) and many spermatozoa (Fig. 21, b).

In contrast to the astonishing variety of form, it is surprising that the size of cells varies only within relatively narrow limits. It is a very noteworthy fact that by far the majority of all cells are microscopic. The size of organisms varies within very wide limits, from the extreme minuteness of a bacterium, measuring only a few thousandths of a millimetre, up to the enormous mass of an elephant or the huge spread of an American mammothtree. But large organisms are never found consisting of a single 
cell. Only a very few varieties of cells having a compact protoplasmic body reach a diameter of a few millimetres, and these are amceboid, their surface changing continually and their substance being in constant streaming motion. The fact that compact cells whose radii are approximately equal in all dimensions and whose protoplasm is not constantly streaming never surpass the size of a few millimetres, has only apparent exceptions. The bird's egg might be regarded as an exception. It is well known that the egg of a fowl before it has left the body represents a single cell; an ostrich egg would, therefore, be a single, compact giant cell, which apparently would contradict the above rule. This exception, however, as has been said, is only apparent, for the really active or living protoplasm of the eggcell has a very small bulk and in the form of an extremely thin and delicate lamella is laid over the rest of the mass, which latter consists of inactive egg-yolk, the food-material for the further developing and reproducing cell. Hence there is here, not a solid compact mass of living substance, but merely a thin lamella. Such an extension in one or two dimensions exists also in all other cells that exceed the usual size-e.g., the cross-striated muscle-cells of the leg-muscles, which are often more than a decimetre in length; ganglion-cells, which are extended into nerve-fibres more than a metre long; and the leaf-shaped cells of Caulerpa. In all these cases it appears that the ratio of the mass to the surface. of the cell never exceeds a certain valus. As will be seen later, this phenomenon is deeply grounded in the nature of living substance, and the formation of a large and massive organism is possible only by the employment of very small autonomous elements, such as the cells.

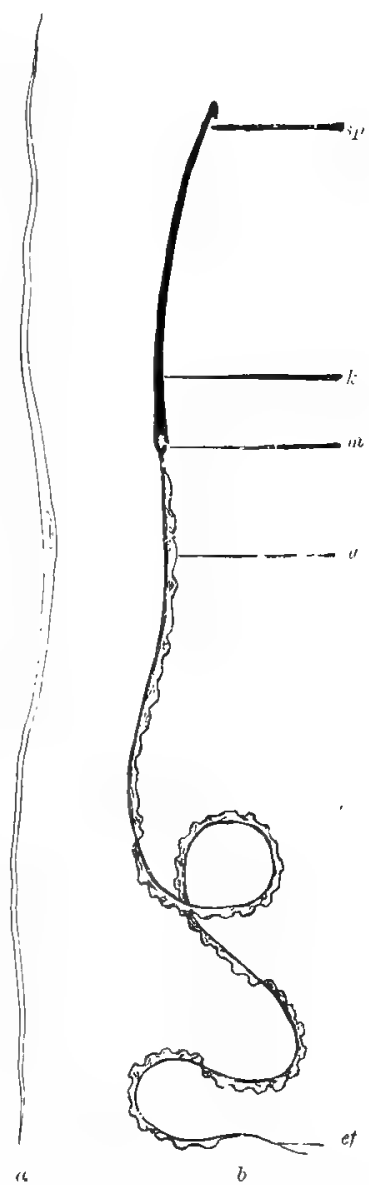

FIG. 21. $-a$, A smooth muscle-cell. (After Schiefferdecker.) b. Spermatozoon of Salamandra macilata. (After Hertwig.) $k$, Head; $s p$, tip ; $m$, middle-piece; $v$, undulating membrane; $e j$ endthread. 


\section{Protoplasm}

The mistake has frequently been made of considering protoplasm. a chemically unitary substance. This idea involves a double error, for, first, the conception of protoplasm, as created by the earlier cellinvestigators, was not a chemical but a morphological conception, and, secondly, it applied to the whole contents of the cell, with the exception of the nucleus. The cell-contents is, however, in neither the chemical nor the morphological sense a unitary substance, but is a mixture of many morphological constituents; and it must constantly be borne in mind that, since it is impossible to separate one or another constituent as accessory, the limitation of the term
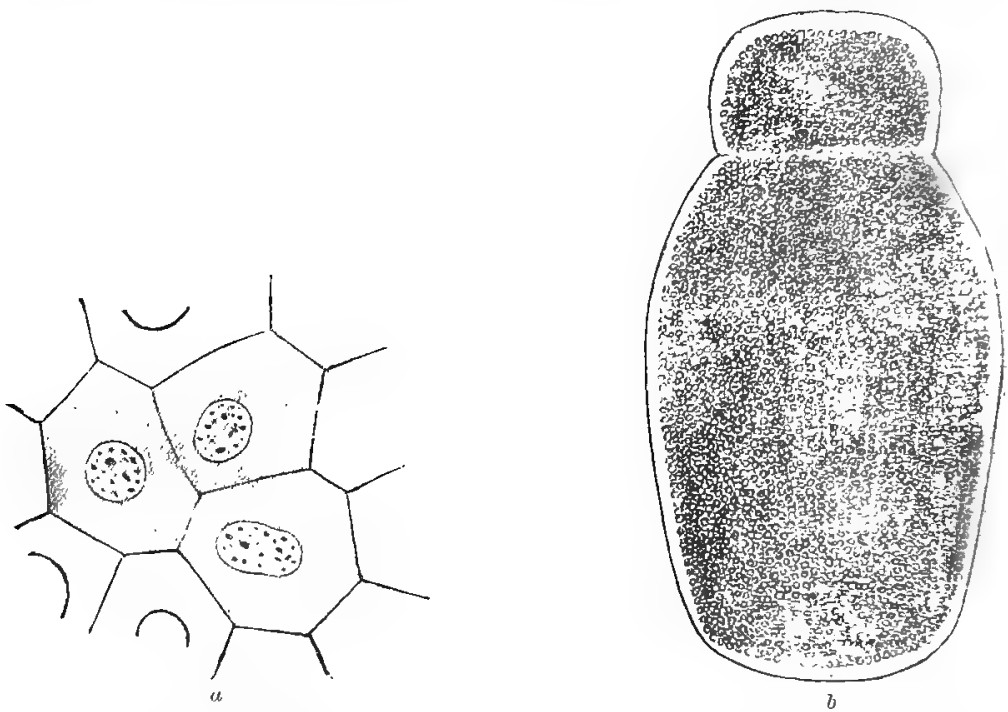

Fio. 22,-a, Epidermis-cells from the frog; the living substance appears completely hyaline. $b$. Clepsidrina blattcarun, a unicellular gregarine from the intestine of a cockroach; the protoplasm
is entirely filled with granules.

protoplasm to certain constituents of the cell is wholly inadmissible and leads to evil consequences. The conception of protoplasm, therefore, should be maintained under all circumstances strictly in its original sense as a comprehensive morphological conception; protoplasm is a sum, a mixture, of very different morphological elements. Even if by degrees its individual constituents become known morphologically and chemically, the comprehensiveness of the term will not thereby be set aside. Whatever significations the various substances may have in the vital process of the cell is a wholly different question, and does not affect the conception of protoplasm. 
When the contents of protoplasm are investigated, upon superficial examination two groups of constituents may be distinguished, namely, various well-defined bodies, such as grains, droplets, etc., and a uniform, semi-liquid, apparently homogeneous groundsubstance, in which the former, like the nucleus, lie embedded. But, while in many cells the ground-substance contains only a few solid bodies, as, e.y., in many epithelium-cells (Fig. 22, $\alpha$ ), in others it can scarcely be seen because of the abundant granular constituents, as is frequently the case in many plant-cells, and especially in certain parasitic unicellular organisms, the Gregcriner (Fig. $22, b)$.

\section{a. The Solid Constituents of Protoplasm}

The solid constituents of protoplasm are material elements of very various natures; they are special constituents, and do not occur in all cells. Among them occur bodies that are of the highest significance for the life of the cell in which they are contained, that impress upon the cell a characteristic feature; and also elements that play no róle whatever in the vital process, such as the indigestible residue of food. There are found, further, foodconstituents which are not yet changed, other substances which have been regularly transformed from the food by the vital process or have been formed anew, and, finally, in many cells independent organisms which live continually in then as symbionts or parasites and under certain circumstances play a definite rôlc in the life-process of the cells.

Among the solid protoplasmic constituents which are especially significant in the life of the cell, and which, therefore, can be considered as organs of the cell, or, better, since we understand by organ a structure composed of many cells, as cellorganoids, the chlorophyll-bodies of plantcells are especially important. These small, usually roundish, sometimes bandshaped bodies, which lie embedded in the ground-substance of the protoplasm (Fig. $23, a$ ), give to the plant-cell and thus to the whole plant its magnificent green
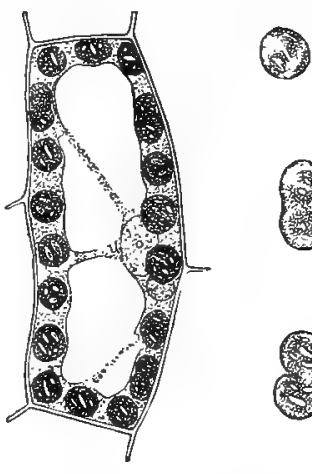

(6

Fir. 23.- $a$, A plant-cell containing chlorophyll-bodies. b, A chlorophyll-body undergoing division. (After Sachs.) colour, for their delicate albuminoid bodies are saturated with an intensely green colouring-matter. The chlorophyll-bodies are of the greatest importance for the plant-cell, for in them occurs a considerable part of its characteristic vital process. Other organoids, which in many cases ar" likewise of great importance for the cell-life, are the drops of liquid, or vacuoles, as they are 
commonly but inappropriately termed. Of the vacuoles two kinds may be distinguished. Some collect only occasionally in the protoplasm in a place where a substance lies that attracts water. Others are permanent, structures, and are present frequently in such great numbers that the mass of the protoplasm is small in proportion to them and merely forms thin walls for them; the protoplasm then presents a frothy appearance, as, e.g., in many plant-cells (Fig. 24, a) and Radiolaria (Fig. 24, b). Among the constant vacuoles that serve as cell-organoids there are the socalled contractile or pulsating vacuoles, drops of liquid that dis-

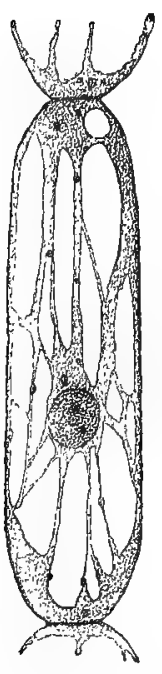

$a$

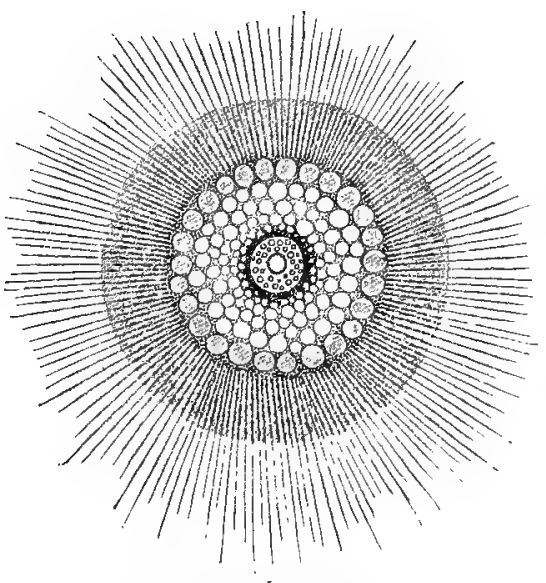

b

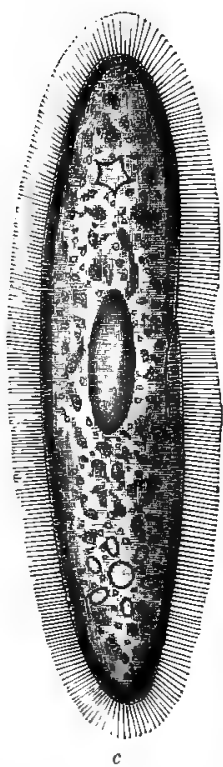

F'IG. 24.-a, Plant-cell from a stamen-hair of Tradescantia. (After Strasburger.) b, Thalassicolla nucleata, a radiolarian cell. c, Paramacium auretia, a ciliate-infusorian cell, which contains within the protoplasm at each end a pulsating vacuole.

appear and appear again at the same spot, usually rhythmically, while the liquid rhythmically mixes with the protoplasm and again accumulates. Many of these pulsating vacuoles have special efferent canals and a constant wall, as is the case in many unicellular free-living organisms, especially the ciliate Infusoria (Fig. $24, c)$.

In addition to such constant elements, in many cells solid constituents are inet with that are present as such only temporarily. Here belong especially the food-bodies that are found in cells that nourish themselves by taking in solid food-constituents. Unicellular naked organisms, such as Amoba, white blood-cells, 
infusorian cells, and others, not rarely show in their bodycontents small Algce, Bacteric, and Infusoria, which they have taken up from the outside (Fig. 25, I), and which sometimes are scarcely to be distinguished from other solid constituents of the protoplasm. These food-organisms become gradually digested and disappear.

There appear also frequently in the cell-body as products of digestion, both in the cells that ingest solid, and those that ingest only liquid food, definite granules, usually roundish, and varying greatly in nature (Figs. 7 and 22,b), which Altmann has grouped in part under the name granula, and which, as has already been
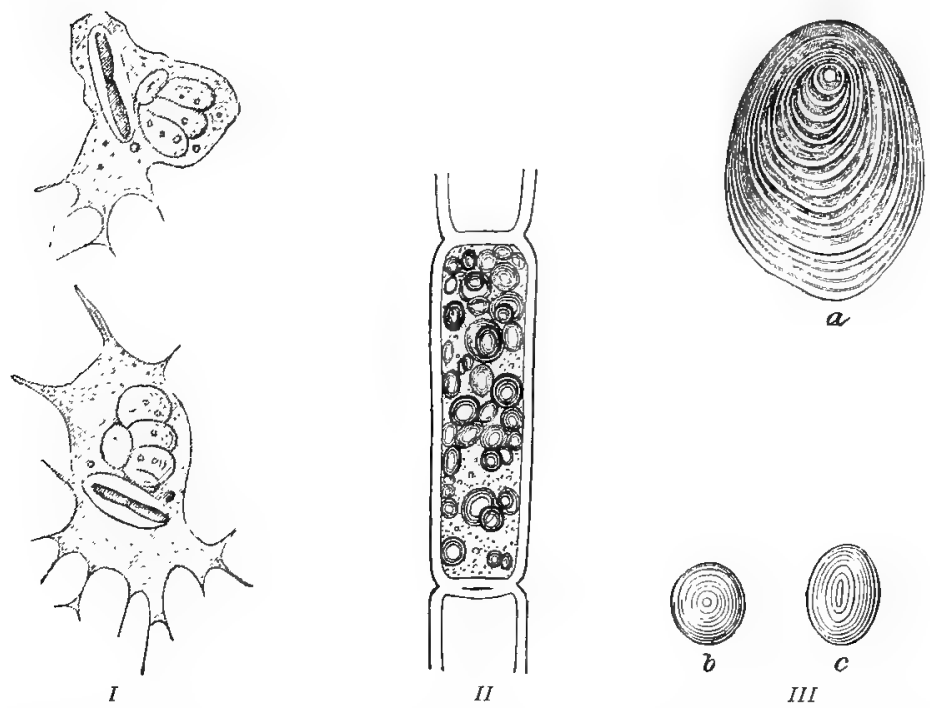

FIa.25.-I, Frog's leucocytes, or white blood-cells, each containing a bacterium. (After Metschnikoff.) II, A plant-cell containing starch-grains. III, Starch-grains isolated- $a$, from the potato; $b$, from corn ; $c$, from the pea.

seen, he regards as elementary organisms, the ultimate living elements of the cell. The composition and significance of most of these metabolic products of living substance which in the form of granules help to constitute the protoplasmic body, is not yet known. But some are characterised very exactly and are easily recognised, such as the concentrically stratified starch-granules in plant-cells (Fig. 25, II and III), the fat-droplets in the cells of the lacteal glands, the glycogen-granules in liver-cells, the pigmentgramules in the pigment-cells of the skin of many coloured animals (Fig. 17, d), the aleurone-grains, consisting of proteid, in the cells of sprouting plant-seeds, the crystals of calcium oxalate in plantcells, of calcum guanin in pigment-cells, and many others, specia] mention of which would lead us too far. 
In the contents of many cells there occurs a fourth group of solid elements, which either do not take part at all in the lifeprocess of the cell, or have ceased to do so. These are indigestible bodies which are taken in occasionally, such as sand-grains (Fig. 26), which are met with in many Amoebce, the indigestible residue of food-stuffs, such as shells, skeletons, and the capsules of food-organisms, and excretory substances, which remain for some time in the cell-body as useless by-products or as end-products of metabolism, to be excreted later.

Finally, among the solid elements of the protoplasm in certain cells, especially in aquatic animals, there occur not rarely symbiotic or parasitic unicellular organisms which strictly do not belong to the protoplasm of the cell in question, but in individual cases play an important rôle in the life of their host. Among such symbiotic organisms are especially many algæ, the Zooxanthellce and the

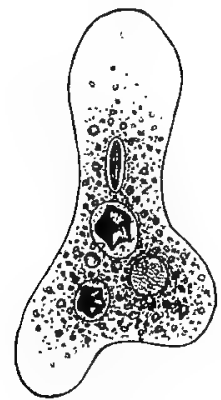

Fig. 26.-Amabca-cell containing in its protoplasm one diatom shell and two sand-grains.

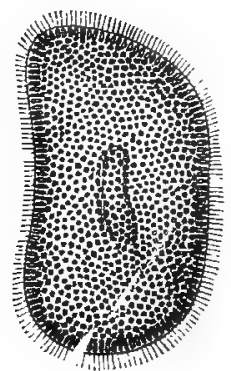

FIG. 27.-Paramacium busaria, a ciliateinfusorian cell, the exoplasm of which is filled with small parasitic alga-cells (Zoochlorell( $\left.{ }^{*}\right)$.

Zoochlorelle, the nature of which as independent organisms has been for a long time in dispute. They occur abundantly in the cells of lower animals, particularly in many Infusoria and Radiolaric, to which by the activity of their chlorophyll-bodies they furnish oxygen, so that as regards respiration their hosts are largely independent of the oxygen of the medium in which they live (Fig. 27).

We shall not enumerate exhaustively the solid components that are to be met with in cells. Such a list would fill many pages. It is only important here to understand how different in nature are the various solid constituents of protoplasm that may occur in individual cells, and how unjustified is the idea of the unitary character of protoplasm. We will now leave the solid elements, and turn to the consideration of the homogeneous ground-substance. 


\section{b. The Ground-substance of Protoplasm}

As already noted, the ground-substance of protoplasm, in which the granules, etc., are embedded, appears upon superficial examination completely homogeneous. This can be seen best in cells that contain only a few solid constituents stored in their groundsubstance; it is especially evident in many Anocbce, which are free-living cells possessing naked protoplasmic bodies that creep about at the bottom of stagnant water, constantly changing their form, and represent the lowest and simplest organisms inhabiting the surface of the earth. These interesting elementary organisms usually form upon their surface pseudopodia which are wholly free from granules, broad, finger-shaped, or lobate, and appear completely hyaline and structureless (Fig. 16, p. 75, and Fig. 28). In fact, in the Amoboe the hyaline protoplasm not rarely is completely structureless. All investigations up to the present time which have been undertaken with the best microscopic methods agree in this.

But this actual homogeneity of the groundsubstance of protoplasm is not the rule; on the contrary, the employment of high magnifying powers shows that by far the majority of cells possess in reality in their apparently homogeneous ground-mass an extremely fine and characteristic structure.

Remak ('44) observed that not only nervefibres but also the ganglion-cells of the central nervous system possess a very fine fibrous or fibrillar structure-an observation that was confirmed and extended by a large number of investigators, especially by Max Schultze ('71). A striated structure was later found in the protoplasm of various other cells, gland-cells,

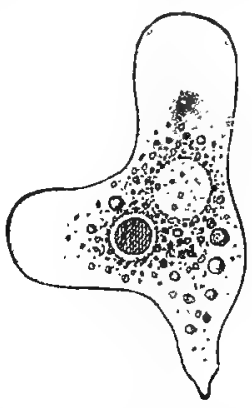

FIG. 28. -An amoeba-cell containing completely hyaline and homogeneous pseudopodial protoplasm. In the endoplasm by the side of the nucleus lies a pale contractile vacuole (droplet of liquid). epithelium-cells, muscle-cells, etc., and thus the idea was formed by various investigators that a fibrillar structure is wide-spread in protoplasm; this view is still defended today, especially by Flemming, Ballowitz, and Camillo Schneider.

But this theory early underwent a modification. Beginning in 1867, Frommann especially endeavoured to show by a long series of researches that the finer structure of the protoplasm of all cells is not properly fibrillar, but reticular; this view was adopted almost at the same time by Heitzmann, and soon obtained wide acceptance. According to this idea, protoplasm forms a network, or, better, a meshwork, the nodal points of which appear as individual granules. The whole meshwork of the cell is open to the 
outside, and between its threads exists a liquid, which, however, is different from the liquid of the medium in which the cell lives, i.e., the water, the body-juices, etc. It is difficult to understand why, as the adherents of the theory of the reticular structure of protoplasm hold to be possible, the internal cell-liquid in cells that possess no membrane, such as the leucocytes of the blood and Amcoba, the reticular structure of which has been described by Heitzmann in great detail, does not continually mix with the surrounding medium in spite of its great proportion of water. But attempts to stain such living protoplasmic masses by certain staining-solutions show clearly that the staining-fluid does not penetrate into the living protoplasm. This and similar difficulties which arise in connection with the idea of protoplasm as a meshwork open upon all sides have led many investigators to take a very sceptical attitude toward the theory of a reticular structure, although in various ways the reticular appearance of the protoplasm of many cells has been confirmed.

The striking researches with which in recent years Buitschli $(92,1)$ has been surprising the scientific world, have completely clarified our ideas upon the real nature of the protoplasmic structures so much observed. The protoplasm of a cell that contains so many vacuoles or droplets of liquid that its contents have a foamy appearance, presents with high powers of the microscope a picture, not of many vacuoles or bubbles pressed tightly together, but of a network, the threads of which form the cross-sections of the thin walls of the vacuoles. This is due to the fact that with strong powers surfaces only, and never bodies, are seen. The microscope shows only optical cross-sections of bodies. But the optical cross-section of a foam is a network. This fact led Biitschli to the conviction that the finer reticular appearance of protoplasm which appears homogeneous by feeble magnification, as has been observed in so many cells, is merely the optical expression of an extremely finely vacuolated foam-structure. In order to confirm this idea, Buitschli endeavoured artificially to produce microscopic foams of a fineness equal to the hypothetical protoplasmic structures, and he succeeded in this in a most gratifying manner. $\mathrm{He}$ employed for his experiments oil which was very finely rubbed up with potash or cane-sugar. Small droplets of this oil-mixture, when placed upon a slide with a drop of water, covered with a cover glass, and observed under a microscope, immediately took on an extremely fine foam-structure. This was due to the fact that the particles of potash or sugar, which were finely divided in the oil-droplet, attracted particles of water; the latter passed from the outside through the oil by diffusion, accumulated as extremely fine droplets closely about the former, and transformed the oil into a very fine foam. The oil-foams obtained in this way show such a remarkable similarity to the structure of protoplasm that they can 
scarcely be distinguished from the latter. From the accompanying figures (Fig. 29, $a$ and $b$ ), which are taken from Biitschli, the identity in structure of the two objects may be recognised at a glance. After the very careful and comprehensive investigations, the results of which Buitschli has published in his book, doubt can no longer exist that the problematic fine structure of protoplasm is in reality foam-structure, which depends upon the presence within a uniform ground-mass of a large number of extremely fine
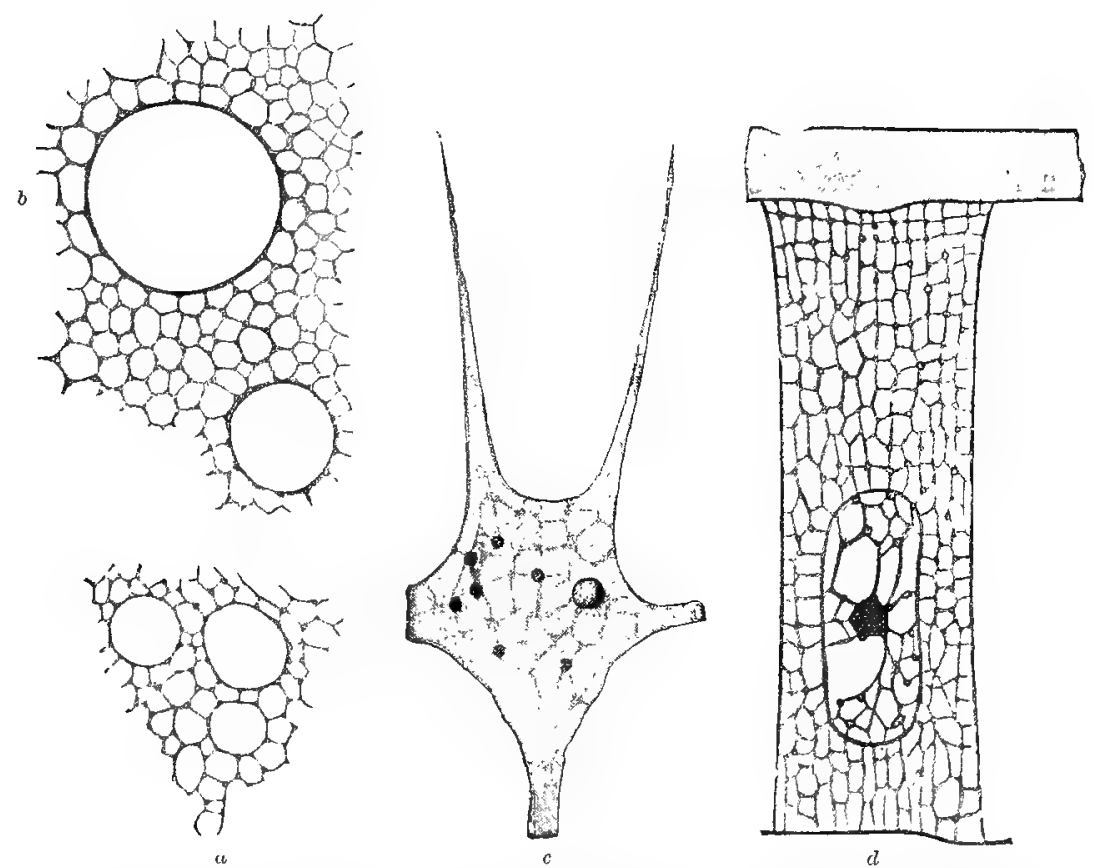

FIG. 29.-a, Foam-structure in the intracapsular protoplasm of Thalassicolla mucleata. b, Foam from olive oil and cane-sugar. c, Protoplasmic structure in a pseudopodial extension of a foraminifer-cell (Miliola). $l$, Protoplasnic structure of an epidermis-cell of an earthworm. (After Bütschli.)

vacuoles, lying almost at the limit of microscopic visibility, and so close together that their walls consist of relatively thin lamellæ. Further, Buitschli has demonstrated this foam-structure in so many wholly different forms of cells (Fig. 29, a, c, d) that its wide distribution can be disputed no longer.

As the result of these recent investigations the following picture can be formed of the finer morphological structure of protoplasm. Protoplasm consists of a ground-mass, in many cases completely homogeneous, in most cases very finely foam-like or honey-comblike, in which lies embedded a greater or less quantity of very 
various solid elements, or granules. In the foan-like protoplasm the granules always lie at the corners and angles where the foamvacuoles come together, never in the liquid of the bubbles themselves.

We have already spoken ${ }^{1}$ of the idea of Altmann, who regards the granules as the sole elementary parts of protoplasm, and the intermediate substance between the granules as non-living. In the light of Biitschli's investigations this view appears all the more untenable.

\section{The Cell-Nucleus}

In recent years the cell-nucleus has become a favourite object of morphological investigation. And here a psychological phenomenon is to be noted, which has constantly repeated itself in the history of the human mind, since mankind began to reflect upon things - this is the tendency toward exaggeration. The earlier investigators of protoplasm, especially Max Schultze, had convinced themselves that protoplasm shows important vital phenomena, and at once by cxcessive generalisation the view was promulgated that protoplasm is the sole bearer of vital phenomena, while the nucleus possesses an accessory significance. Since then it has been recognised that the nucleus participates prominently in certain vital phenomena; several investigators have shown that it plays a very important rôle in reproduction, fertilization, secretion, etc. Immediately the original view of the all-importance of the protoplasm has by an extreme reaction become exchanged for its opposite, that of the all-importance of the nucleus. As will be seen in a later section, here, as so often, the truth lies between the two. But every reaction is exaggerated. Opinions, like a pendulum, go first to the two extremes, and only after some time does the proper mean come to be maintained. Biology is indebted to these investigations upon the nucleus for the fact that our knowledge of it has been greatly extended. ${ }^{2}$

\section{a. The Form of the Nuclers}

The form of the nucleus is very different in different cells.

The first conception of the nucleus was formed from cells in which within a circumscribed protoplasmic mass a single, more or less spherical nucleus exists, which as regards its refractive power and its consistency differs essentially from the surrounding protoplasm. It was found later that the nucleus stands in sharp con-

1. Cf. p. 63, et folg.

3 A. Zimmermann has recently made a comprehensive survey of the results of research upon the nuclens, especially in plant-rells, in his book, Die Morphologie und Physiologie des pflanzlichen Zellkernes: Lim liritische Litteraturstudie. Jena,
1896 . 
trast tı the protoplasm by its characteristic behaviour toward certain reagents, especially colouring-matters. Nuclear masses possessing these characteristics are the most wide-spread in the urganic world. The nuclei of a majority of free-living and most tissue-forming cells among animals and plants are of this type. In it the relation of the volume of the nucleus to that of the protoplasm varies greatly. There are cells in which a relatively small nucleus is surrounded by a large mass of protoplasm, as, e.g., many Horcminifera, while in other cells, the mass of the protoplasm, in comparison with the nucleus, is extremely small, as in most spermatozoa. From the type of the single, more or less spherical nucleus deviations in very different directions occur. First, as regards the number of nuclei: As has already been seen, there are organisms that consist of a unitary protoplasmic mass in which lie embedded a large number of nuclei, such as multinucleate cells and syncytia. In such cases the number of the nuclei can be si) great and their size so excessively small that, as Gruber ('88) has observed in certain Thizopoda from the harbour of Genoa, especially Pelomyxa pallida, the nuclei lie distributed through the whole protoplasm as a fine powder (Fig. 30). With such a division of the nuclear mass as is present in multinucleate forms, the nuclear surface naturally is considerably larger than with the

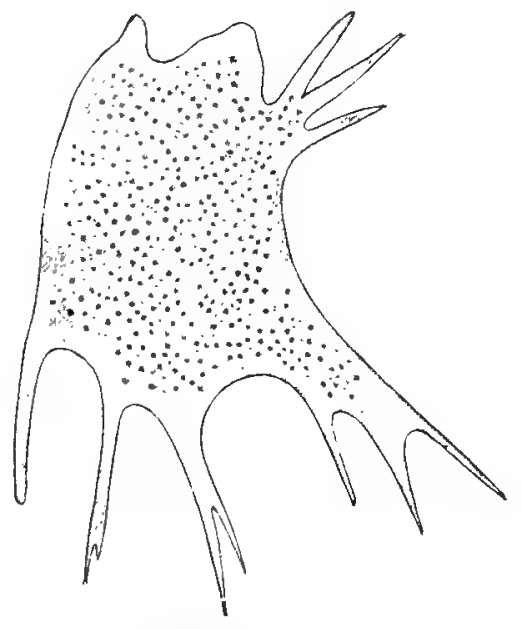

Fia. 30.-Pelowzyra pallide, a rhizopod-cell from the harbour of Genoa, containing finely. divided nuclear substance in the protoplasm. (After Gruber.) same quantity contained in a single large nucleus - a fact that is particularly important from the physiological point of view.

The same principle of surface-enlargement is seen also in the rlifferentiation of the form of the single nucleus. The most manifold and extreme deviations from the typical spherical form occur. Rod-shaped, band-shaped (Fig. 31, a, ) and moniliform (Fig. 31, b) nuclei are very common among ciliate Infiusorit. Going still further, the same principle leads to star-shaped and branched nuclei, which are found in certain cells in the bodies of insects, and reach their highest development in antler-like branched forms in the cells of the spinning-glands of many caterpillars (Fig. 31, c). It seems noteworthy that it is the nuclei of secreting cells, i.e., cells 
characterised by lively activity, in which the principle of surfaceenlargement by branching is especially expressed.

\section{b. The Substance of the Nucleus}

As regards the nature of the substance of the nucleus, exactly the same is true as in the case of the protoplasm. The nucleus is no more a unitary substance than is the protoplasm. It is a morphological structure, an organoid of the cell, which consists of several

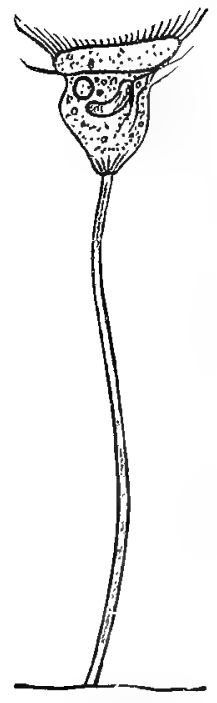

$a$
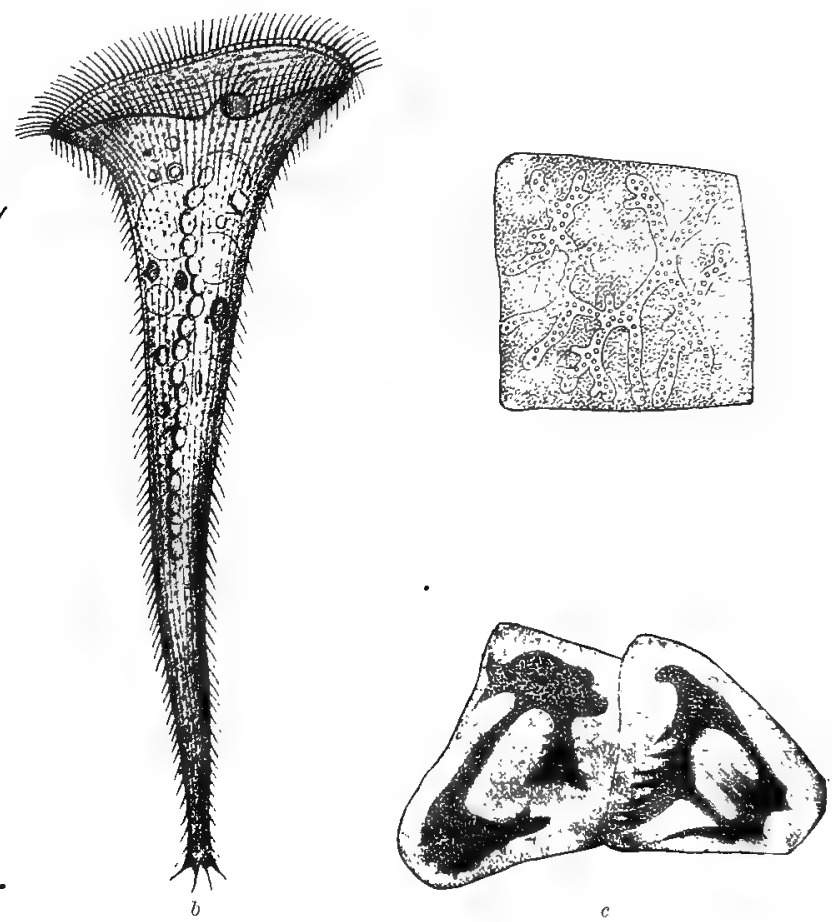

FiG. 31.-Cells containing different forms of nuclei. $a$, Vorticella, a ciliate infusorian, possessing a rod-shaped nucleus. $b$, stento", a ciliate infusorian, possessing a moniliform nucleus. $c, c$, Cells of the spinning-glands of the caterpillar possessing antler-like branched nuclei. "(After
Korschelt.)

different constituents that may be distinguished from one another microscopically more or less clearly, and all of which are not present in all cells at all times. Because of the exceeding minuteness of the objects, it is often difficult sharply to characterise the individual constituents. Therefore, their identity in two separate species is not always beyond doubt, and extended investigations are still needed before it will be known clearly what constituents 
of one nucleus correspond exactly to those of another. Nevertheless, a number of constituents, which apparently are wide-spread, are even now fairly well characterised. The following substances occur most constantly:-

1. The nuclear sap constitutes the liquid ground-substance, in which the solid nuclear constituents are contained (Fig. 32). M. Heidenhain, Reinke, and Korschelt have lately demonstrated that in many cells, even during life, it presents an extremely finely granular appearance.

2. The achromatic nuclear substance forms in the ground-substance a supporting-structure of fine threads, which are characterised, like the nuclear sap in which they are suspended, by not staining with the typical nuclear stains, such as the carmine stains, haemotoxylin, etc.

3. The chromatic nuclear substance is distinguished from the achromatic by its property of staining with these reagents. It is contained in the strands of the achromatic substance, as a rule in the form of small granules and irregular particles, and upon its
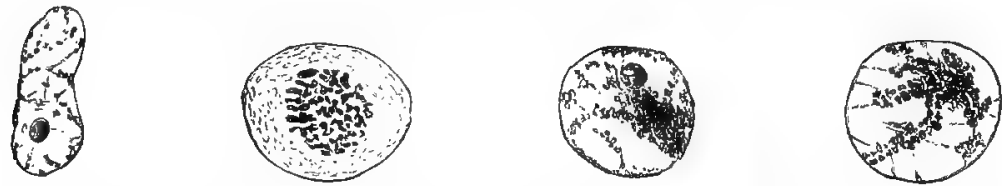

FId. 32.-Various nuclei from the mother-cells of the spermatozoa of the thread-worm of the horse, (After Hertwig.)

staining-power chiefly rests our knowledge of the finer structure of the nucleus.

4. The nucleolus is a homogeneous granule which is present comparatively rarely in nuclei; it consists of a strongly refractive substance which appears to be closely related to the chromatic substance. Since, as a rule, the substance of the nucleoli may be stained by the nuclear stains like the chromatic substance, the nucleolus has been considered by many investigators as a special accumulation of chromatic substance-a view which, however, because of the different relations of the two substances toward certain chemical reagents, cannot strictly be maintained.

All of these substances, to which with advancing knowledge of the nucleus others will perhaps be added, are present in very different quantities in different cells. A substance that is abundant in one nucleus may be insignificant in another, and it even appears as if certain substances can be wholly wanting in certain nuclei. In many cases the nuclear substances are surrounded and marked off from the protoplasm by a special nuclear membrane, which, however, like the cell-membrane in relation to the cell, is not a general constituent of the nucleus. 
Recently, Zacharias ('81-'87) and Frank Schwarz ('87) have endeavoured to replace the customary names of the individual substances by other names. Thus, the chromatic substance has been termed nuclein, the achromatic substance linin, the nucleolar substance paranuclein or pyrenin, the nuclear sap paralinin, and the substance of the nuclear nembrane amphipyrenin. The adoption of these names is not recommended, for they may so easily be confounded with chemical notions as to lead to the error of seeming to deal with chemical entities, while the nuclear substances in question are purely morphological. If the tern nuclein were to be employed in a chemical sense, the chromatic nuclear substance would be placed in a chemical contrast with the other nuclear substances that does not really exist, for the majority of other nuclear substances likewise belong chemically to the socalled nucleins, representing different kinds of the latter. Therefore it is more fitting to employ the original names above mentionerl for the morphological nuclear constituents, and not to confuse the latter with chemical substances.

One more phenomenon relative to the differentiations of the individual substances is of interest. This is the fact that, of the substances that occur together within the nucleus in most cells, some have become differentiated in many cells into separate masses within the protoplasm, so that two entirely different forms of nucleus occur side by side within the same cell. This condition is almost universally realised in ciliate Infusoria, which possess, in addition to a larger nucleus, the macronucleus, which in some species and at certain periods seems to consist chiefly of chromatic substance, one, several, or often a great number of the so-called accessory nuclei, or micronuclei, which likewise in some species and at certain periods seem to consist mostly of achromatic substance. The claim of the two elements in the infusorian cell to be regarded as two different nuclear substances is based upon the phenomena which, according to the striking investigations of $\mathrm{R}$. Hertwig ('88-'89), appear in the conjugation of two individuals. Here the chief nucleus goes to pieces completely in the protoplasm, and after conjugation a new rudiment of it is differentiated from the substance of the accessory nuclei. While in the ciliate Infusoric the two forms of nucleus remain throughout life, in the Diflugice of the Phizopoda a localised differentiation of two nuclei appears only during the period of conjugation and gives place afterwards to the minucleated condition. ${ }^{1}$

\section{c. The structure of the Nucleus}

It has been seen that the achromatic substance forms in the ground-mass of the granular nuclear sap' a supporting-structure, in

${ }^{\prime}$ Cff. Verworn ('91), 1). 
the strands and nodal points of which the chromatic substance and the nucleoli lie embedded in precisely the same manner as the solid elements, the granules, etc., lie in the alveolar walls of the protoplasm. Indeed, as Bütschli has shown, the similarity of the relation even goes so far in individual cases that the achromatic substance in the nucleus shows precisely the same alveolar structure that the ground-mass of the protoplasm as a rule possesses (Fig. 33).

All these structures are characteristic only of the so-called resting-stage of the cell. As soon as the latter prepares to multiply by division, very peculiar and very complex changes in the structure of the nuclear substance appear; these will be considered in detail in another chapter.

\section{THE PHYSICAL PROPERTIES OF LIVING SUBSTANCE}

\section{The Consistency of Living Substance}

Although the earlier investigators of the cell, such as Schleiden, Mohl and others, as the result of direct observation, considered the contents of the cell to be liquid, and compared its consistency with that of slime, later the idea found wide acceptance that protoplasm is at bottom a solid substance. This idea arose from purely theoretical considerations. Bricke ('61), especially, thought that the cell-contents cannot be liquid, for the reason that vital phenomena cannot possibly be associated with a liquid substratum, but presuppose a definite organisation, and the latter is not compatible with the nature of a liquid. Bruicke's view soon obtained many adherents, and appeared to be supported particularly by the theory of the reticular structure of protoplasm, as maintained by Frommann and Heitzmann. It was believed that the solid supporting-structure, with the organisation of which vital phenomena are associated, was represented by the network. It has turned out, however, that the supposed reticular structure is an optical delusion, and thus this basis for the view of the solid consistency of protoplasm has been taken away. In reality, with the present methods of microscopic investigation, only a strongprejudice in favour of other and untenable theories can overlook the fact that, with the exception of individual differentiations in certain cells, protoplasm behaves physically like a liquid.

The idea that vital phenomena can be associated with a solir 
substratum only is not only unjustified, but even untenable. Not only is it unsupported on any acceptable ground, but it even contradicts facts that may easily be observed. E.g., it is quite impossible to understand how protoplasm in the more or less stiff condition of a framework or network can be capable of streaming and flowing, as can be observed so easily in certain plant-cells and in Amcebe. It is impossible for a solid network to flow in such a manner that the individual particles of its mass mix continually with one another, as may be seen so clearly in Amoba. If at first sight the theory of the solid consistency may not be incompatible with the behaviour of cells that possess a constant form, it is absolutely so with the phenomena exhibited by naked protoplasmic masses.

Hence various investigators, especially Berthold ('86) and Biitschli $(' 92,1)$, have recently defended strongly the idea of the

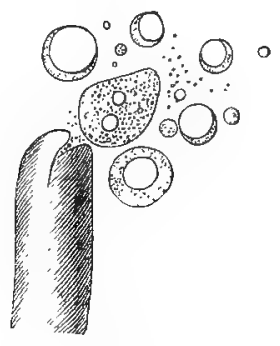

a

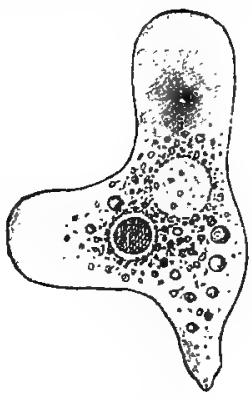

b

FIG. 34.- a, Vaucheria tube cut open at the upper end the protoplasm is flowing out and taking the form of globules. (After Pfeffer.) b, Amoba-cell containing a pale vacuole and various small fat-droplets.

liquid nature of the cell-contents, and no investigator who is familiar with the phenomena need hesitate to accept this view. Observation of a few facts is convincing of its truth.

The phenomena of movement, already mentioned, are the strongest proof of the liquid nature of protoplasm. In the protoplasnic strands of plant-cells and in the pseudopodia of Rhizopoda the living substance may be seen flowing like the water of a quiet stream, now slower, now faster, and in different places at unequal rates, so that, as can be observed easily in the constituents enclosed within the ground-mass, the granules, fat-droplets, etc., the particles continually mingle with one another. How would it be possible for a stiff ground-mass to flow like water in a stream?

Another thing that throws light upon the liquid consistency of protoplasm is the fact that protoplasmic masses, when oozing out of the cell after its walls have been crushed or cut, form drops and globules. The formation of such drops and globules can be observed very beautifully in the protoplasm of the alga Veucheria 
(Fig. 34, a). They can be observed also in the streaming protoplasmic strands of the uninjured plant-cell, when the electric current is sent through them. The protoplasm then collects at once into globules and small spindle-shaped masses, which, if the current be interrupted, become again extended and united, their substance flowing on (Fig. 35). The same can be seen in the pseudopodial filaments of many marine Rhizopoda upon shaking them strongly or continually (Fig. 36), and likewise in many other objects.

A third phenomenon that points to the liquid consistency of protoplasm, and one that can be observed in very different forms of cells, is the assumption of the globule- or drop-shape by accumulations of liquid enclosed within the protoplasm, such as the so-called vacuoles, and the fat- and oil-droplets, which appear here and there, increase in size, and under certain circumstances disappear (Fig. 34, b). Were the ground-mass of protoplasm stiff, it would be incomprehensible that these droplets of liquid of very different sizes always assume the spherical form and preserve it during their growth, as oil-droplets do. In such cases a spherical form is mechanically possible only when the surrounding medium exercises upon all sides equal pressures and yields equally, i.e.,
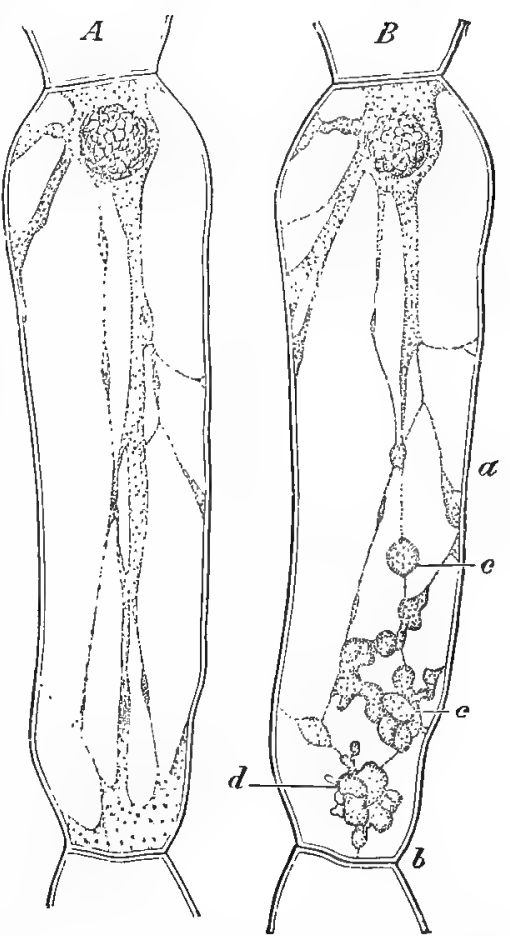

Fia. 35.-Tradescantia, Cell of a stamen-hair. $A$, Containing quietly streaming protoplasm. $B$, The same cell stimulated by an induced current. The protoplasm in the strands has become rounded into single globules $(c, d)$. (After Kihne.) when it is itself a liquid.

Innumerable phenomena of this kind may be cited, which are compatible only with the liquid nature of protoplasm. But those mentioned suffice completely to show that vital phenomena can very well be associated with a liquid substratum. Of course the liquid and the solid conditions of a body cannot be separated from one another by a sharp limit, but are united by imperceptible transitions. According to our present physical ideas the difference between the gaseous, liquid and solid conditions of a body depends solely upon the fact that in the first the molecules are in rapid 
motion, in the second they are moving somewhat more slowly, and in the last still more slowly. Since, therefore, only a gradual. difference exists, it is impossible to establish a sharp limit. In living substance also there are different grades of mobility among the particles, i.e., in one case the substance is like thin, in another case like thick liquid. In general, it possesses the consistency and mobility of raw white of egg, but it may be firmer, and certain constant differentiations of protoplasm may possess even the consistency of a soft jelly approximating a solid condition, without losing, however, the power of shifting its particles. Such a condition exists in muscle-fibres, flagella, cilia, the nucleus, and upon the surface of many protoplasmic masses that do not possess a
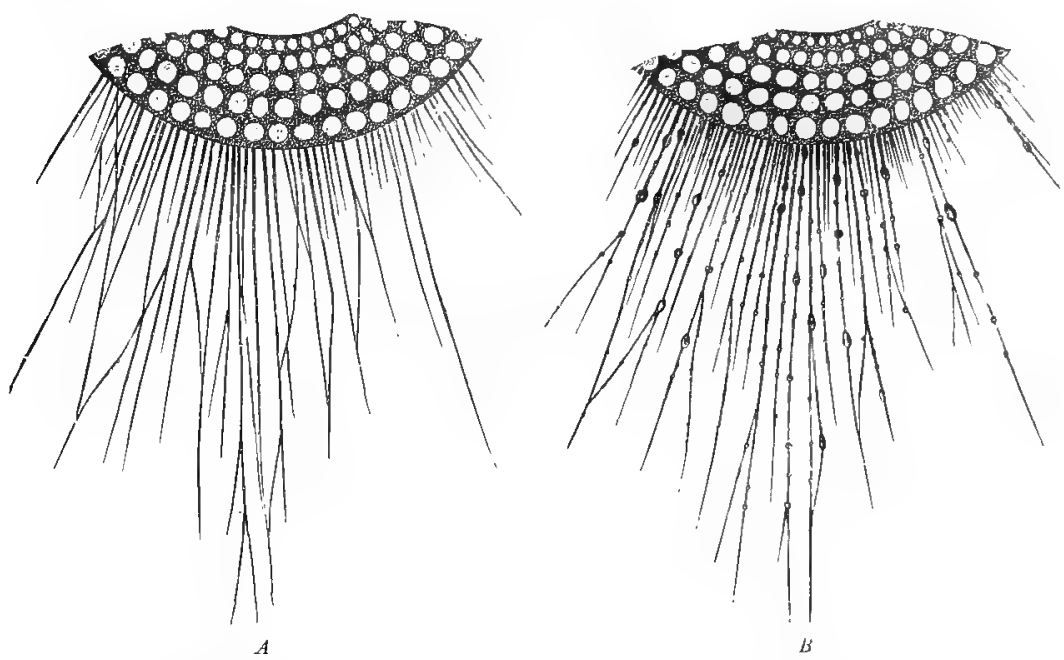

Fia. 36.-Orbitolites. Piece of the many-chambered calcareous disc, bearing outstretched pseudopodial filaments. $A$, Undisturbed. $B$, By strong shaking the protoplasm of the pseudopodia has been stimulated to form globules and spindles.

membrane, such as infusorian cells. The term solid is applicable to such cases only, if at all. But these cases of a more viscous consistency are always locally restricted within the cell; the rest of the cell-contents is always a thinner liquid.

Finally, it should not be forgotten that within the liquid there may be deposited all sorts of solid elements of very various consistencies, and that, therefore, the whole constitutes, not a homogeneous liquid, but a mixture, or, as Berthold terms it, an emulsion. For this reason it appears inadmissible to speak of an "aggregate condition " of protoplasm, as many observers do. Strictly speaking, the term "aggregate condition" can apply only to a homogeneous. substance, not to a mixture containing substances that possess in themselves very various aggregate conditions. 
The liquid nature of living substance is its most important physical characteristic. It requires that in its physical relations living substance must obey the laws of liquids. Accordingly, and in opposition to the idea that vital phenomena are associated only with a solid organisation, it will be seen that such phenomena may be understood only upon the supposition that their substratum is in a condition in which the particles are more or less capable of shifting. The structures that have a rigid consistency, like tendons, connective-tissue fibres, cell-membranes, and the groundsubstance of bone and of cartilage, show no active vital phenomena, and the old dictum, "Corporce non agunt nisi soluta," although its universality may be attacked here and there, applies perfectly to living substance.

\section{The Specific Gravity of Living Substance}

Among the physical properties of living substance its specific gravity possesses an important value for the understanding of certain vital phenomena. If cells of different kinds or pieces of tissue as pure as possible be allowed to fall into distilled water, it is observed that usually they sink to the bottom. It follows, therefore, that the cell-contents, as a whole, is in general heavier than water, i.e., possesses a specific gravity greater than 1. Very recently Jensen ('93, 1) has made a careful determination of the specific gravity of the one-celled ciliate infusorian, Paramcecium aurelic, in the following manner. It is well known that the specific gravity of a liquid can be raised by the addition of soluble salts, and can be graduated very finely by increase of the concentration. Jensen placed Paramcecia in a weak solution of potassium carbonate, the strength of which he raised until they no longer sank to the bottom, but remained suspended in the solution-a sign that the solution possessed the same specific gravity as the bodies of the Paramocict. Then the specific gravity of the solution was determined by means of an areometer. It was thus found that the cell-body of Paramcecium possesses a specific gravity of approximately. 1225. In general, the specific gravity of living substance cannot be much greater than this. So far as our knowledge at present extends, it is always a little greater than 1 .

But there are certain cases in which the aggregate weight of the cell deviates from this general principle, in which the specific gravity of the cell as a whole is less than 1 . These cases can be understood at once, if it is recalled that protoplasm is not a homogeneous substance. E.g., in the case of cells in which fat-droplets are stored in the ground-substance of the protoplasm it is possible that, although the ground-substance is heavier than water, the cell as a whole possesses a less specific gravity, since the accumulation of fat, which is considerably lighter than water, reaches such an 
extent that it overcomes the weight of the rest of the protoplasmic body. Such cases are realised in the fat-cells of the subcutaneous connective tissue in man and many animals; if such tissue be thrown into water, it floats upon the surface. For this reason fleshy men, in swimming, have to make less effort to maintain themselves above the water than thinner persons. Other substances in the cell-body can play the same rôle as fat, particularly bubbles of gas, which under certain circumstances can lower the specific gravity of the whole body of the cell enormously - a phenomenon that occurs in many shell-bearing fresh-water Rhizopoda (Arcella, Diffugici).

It follows from this fact that by the accumulation of lighter or heavier substances the cell under certain circumstances can actively diminish or increase its specific gravity, and, therefore, can actively rise or sink in water without employing locomotive organs. Under many conditions, e.g., when the vital conditions become unfavourable in the place where the organism lives, such a power is of great importance for the life of the organism. In all cases, however, where cells are found that are lighter than water, certain elements only are lighter, the whole protoplasm never. The ground-mass of the protoplasm appears always to be slightly heavier than water.

\section{The Optical Properties of Living Substance}

In most cases protoplasm is entirely colourless or grey; in thin layers free from solid contents it is transparent, in thick layers opaque. It refracts light somewhat more strongly than water.

As regards details, the various forms of living substance behave differently according to the condition of their constituents. Some solid elements, such as fat-droplets, drops of water, and chlorophyll grains, can be intensely coloured, so that the cells in which they are present in great quantities appear yellow, red, green, etc., as, e.g., in plant tissues. The power of refracting light also differs with the individual constituents, that of water-droplets in the vacuoles is less, that of fat-droplets greater than that of the ground-substance. It would carry us too far to examine all the individual cases, but it is of interest to consider somewhat in detail the behaviour of one form of living substance, viz., the so-called contractile substance, i.e, amœboid protoplasm, cilia, and muscle-fibres, which execute definite changes of form, called contractions.

In the first half of the century Boeck found that certain elements of the cross-striated muscle-fibre are doubly refractive, i.e., are able to divide a ray of light into two rays, which are transmitted with different velocities. Later, Bricke, especially, investigated this property in detail. Still later, Engelmann ('75) 
observed that not only the discs of cross-striated muscle, but in general all fibrous contractile substances, such as those of smooth and cross-striated muscle-cells, the contractile fibres or myoids of the infusorian body, and the cilia and flagella of all ciliated cells, exhibit positive uniaxial double refraction, in such a way that their optical axis coincides with the direction of the fibres. This fact indicates that the molecular structure of all these fibrous tissues must be different in the direction of the fibres from that in other directions-an inference that is important for the understanding of the phenomena of contraction in these objects. Engelmann has not been able to find double refraction in the naked contractile protoplasm of Rhizopoda, e.g., Amoeba. He observed it only in the straight, radiating pseudopodia of Actinosphcerium Eichhornii, a delicate fresh-water rhizopod; but here it belonged most probably, not to the contractile protoplasm, but to the stiff rays that occur as supporting-organs in the axis of the pseudopodia, and apparently have nothing to do with the contraction.

\section{THE CHEMICAL PROPERTIES OF LIVING SUBSTANCE}

\section{The Organic Elements}

Of all the natural sciences, chemistry, in dealing with the atoms, penetrates deepest into the composition of the physical world. It must hence be employed in elucidating the composition of living substance, and thereby completing the preparation for an understanding of vital phenomena. It is well known that chemistry has arrived at the point at which it recognises the vast variety of substances in the physical world to be composed of the atoms of a small number of relatively simple substances, which thus far it has not succeeded in decomposing. But, although by means of its analytical methods the division of the sixty-eight chemical elements has so far not been accomplished, and their composition out of still simpler substances cannot yet be proved experimentally, no chemist entertains longer any doubt that in reality these elements are not final units. Accordingly, many attempts have been made to arrange them in a genetic relation to one another, and to establish the relationship that is expressed in the analogies of the chemical behaviour of individual elements and their compounds, as a natural relationship arising by the direct derivation of one from another. Especially Mendelejeff, Lothar Meyer, and, most recently, Gustar Wendt and Preyer, arguing chiefly from the relations of the atomic weights of the elements and the similarity of certain elements as regards their own behaviour towards one another and the behaviour of their compounds, have attempted this with success; the result is shown 
by the subsequent discovery of previously unknown elements, whose existence they had predicted from certain gaps existing in the genealogical table of the elements. According to Wendt ('91) and Preyer ('92), the elements have been developed in the course of the earth's history by gradual condensation from a primitive element, hydrogen, in such a way that those having a higher atomic weight have been derived from those having a lower one; finally, all have been derived from hydrogen, the element possessing the lowest atrmic weight. But here scientific theory ceases and hypothesis begins. Whether hydrogen is really the ultimate unit, and in what relation its atoms stand as ponderable or mass-atoms to the imponderable universal ether, the existence of which physics finds it necessary to assume from the phenomena of light and electricity, for the present is not known.

But if we confine ourselves to ponderable matter, to which living substance, like all other bodies, belongs, chemical analysis shows that of the sixty-eight elements of which the physical world consists, twelve only are found constantly in living substance. These twelve elements which occur in every cell are:-

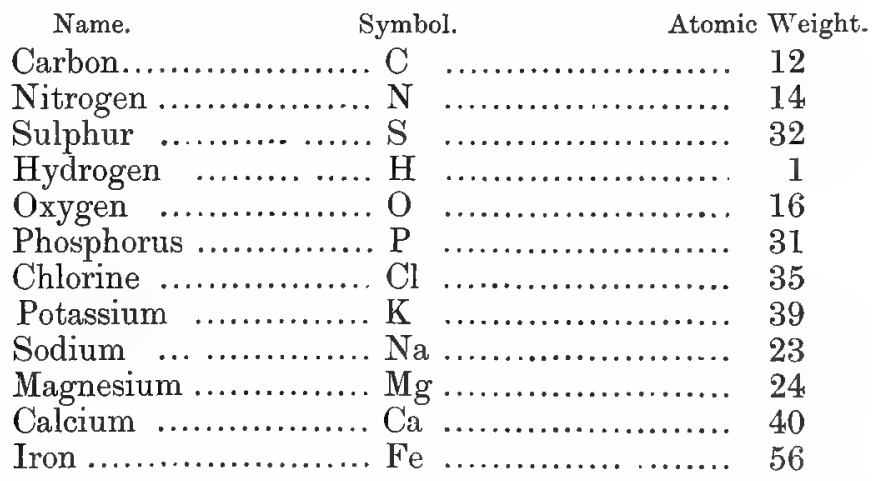

Besides these twelve general organic elements, a small number of special elements occur which are not met with in all cells, and some of which are found only very sporadically. These are:-

\begin{tabular}{|c|c|c|}
\hline Name. & Symbol. & Atomic Weight. \\
\hline Silicon... & $\ldots \mathrm{Si}$ & .. 28 \\
\hline Fluorine & $\ldots \mathrm{Fl}$ & 19 \\
\hline Bromine & $\ldots \mathrm{Br}$ & 80 \\
\hline Iodine ... & $\ldots I$ & 127 \\
\hline Aluminiu & $\ldots \mathrm{Al}$ & $\ldots \quad 27$ \\
\hline Manganes & $\ldots \mathrm{Mn}$ & $\ldots$ \\
\hline
\end{tabular}

Among these, silicon is wide-spread and fluorine is infrequent, while the others, which likewise have a very limited occurrence, 
and certain metals, such as copper, which are occasionally found in traces in living substance, perhaps possess no importance at all for the vital processes of the organisms in which they have been observed.

But no one of all these organic elements is limited exclusively to organic nature.

Carbon occurs in the air, combined with oxygen, as carbonic acid, and in large masses in the calcium carbonate of sedimentary rocks.

Hydrogen, likewise combined with oxygen, as water, covers the greater part of the earth's surface.

Oxygen occurs both free as a gas in the atmospheric air, of which it constitutes about 21 per cent., and also combined with a large number of other elements.

Nitrogen occurs likewise both in the free state in the air, comprising about 79 per cent, and also combined with hydrogen and oxygen in the compounds of ammonia, both ammonium nitrate and nitric acid.

Sulphur is wide-spread in combination with oxygen in sulphates.

Phosphorus behaves sinilarly, and is to be found everywhere in the phosphates of the alkalies and the alkaline earths.

Chlorine is very widely distributed, combined with sodium, as common salt.

Potassium occurs in combination with chlorine as potassium chloride, and with acids as nitrates, sulphates, and phosphates.

Sodium, chiefly in the form of sodium chloride or common salt, is found everywhere on the surface of the earth; it is in solution in the sea, in the earth, and forms large solid masses in salt strata.

Magnesium is a constant accompaniment of - potassium and sodium, and is similarly combined, occurring as magnesium chloride, carbonate, sulphate, and phosphate.

Calcium, in the form of calcium carbonate, silicate, sulphate, and phosphate, occurs in the vast limestone strata of the sedimentary rocks.

Iron is very wide-spread over the earth's surface in the form of sulphur compounds, oxides and their salts.

Siticon appears almost exclusively combined with oxygen in the form of silicic acid and its salts in igneous rocks.

Fluorine occurs chiefly in combination with calcium as fluor spar.

Bromine and iodine are present in many salt strata, as well as in sea-water, as sodium bromide (iodide), and potassium bromide (iodide).

Aluminium is spread over the whole earth in combination with oxygen as clay, and in the latter form, combined with silicic acid, as feldspar.

Manganese and all the other metals that are observed occasionally 
here and there in the living organism are met with everywhere in rocks in the form of their oxides, sulphur compounds, and various. salts.

This survey shows that all organic elements help at the same time to constitute the inorganic portion of the earth's surface. Since, moreover, chemical analysis of living substance has shown that no constituents but these organic elements are to be found in the organism, the important fact follows that an elementary vital substance exists no more than a specific vital force. The conceptions of a "vital ether," a "spiritus animalis," a "vital matter," etc., with which the edrlier physiology so freely dealt, have, therefore, in harmony with the advanced development which analytical chemistry has undergone at the present time, completely disappeared from the present theory of life; living substance is composed of no different chemical materials from those occurring within lifeless bodies.

Nevertheless, one fact deserves mention, viz., that the few general organic elements are not scattered irregularly here and there through the natural system of elements, but they occupy a definite position, being remarkable as elements having very low atomic weights. Hence the conclusion may with great probability be drawn that in the evolution of the elements the organic elements arose by condensation very early, and therefore existed in the very early stages of the development of our planetary system, at a time when other elements, such as the heavy metals, had not yet been formed.

\section{The Chemical Compounds of the Cell}

Living substance must be killed before its chemical composition can be learned. Paradoxical as this may sound, at present it is the only way by which a knowledge of the chemistry of living substance can be obtained. The biting sarcasm that Mephistopheles pours out before the scholar upon this practice of physiological chemistry must be quietly endured. It is not possible to apply the methods of chemistry to living substance without killing it. Every chemical reagent that comes in contact with it disturbs it and changes it, and what is left for investigation is no longer living substance, but a corpse-a substance that has wholly different properties. Hence ideas upon the chemistry of the living object can be obtained only by deductions from chemical discoveries in the dead object, deductions the correctness of which can be proved experimentally in the living object only in rare cases. This alone is responsible for the excessively slow advance of the knowledge of the chemistry of the vital process. It is evident that the greatest foresight is necessary in applying results obtained upon the dead object to conditions in the living, and it must con- 
stantly be borne in mind that the chemical relations of the latter are to be distinguished sharply from those of the former.

Although there is no fundamental difference between the elements composing living and those composing lifeless substance, in other words, although no special vital element exists in the organic world, some of the elements in living substance form unique compounds which characterise it only, and are never found in lifeless substance. Thus, there exist in the former, besides chemical compounds that occur also in the latter, specific organic complexes of atoms.

Many of these organic compounds, especially those that are of special importance to living substance, possess so complicated a constitution that thus far chemistry has not succeeded in obtaining an insight into the spatial relations of the atoms in their molecules, although the percentage composition of the molecules is known to a greater extent.

There are especially three chief groups of chemical bodies and their transformation-products, by the presence of which living substance is distinguished from lifeless substance; these are proteids, fats, and carbohydrates. Of these only the proteids and their derivatives have been demonstrated with certainty as common to all cells; hence they must be set apart among the organic constituents of living matter as the essential or general substances, in contrast to all special substances.

\section{a. Proteids}

The proteids play the most important rale in the composition of living substance, since they are absolutely indispensable to all life that exists at present upon the surface of the earth, and quantitatively they constitute the chief constituent of all the organic compounds of the cell. Without exception they consist of the elements carbon, hydrogen, sulphur, nitrogen, and oxygen; of these, nitrogen especially distinguishes proteids from the two other chief groups of organic bodies, carbohydrates and fats, so that the former, as nitrogenous bodies, are to be contrasted with the latter two as non-nitrogenous. The stereo-chemical composition of the proteid molecule is not yet known, but from analyses, in which the molecule is split up into a large number of still very complex molecules, it is known that it must have an excessively complex constitution; although it contains only the five elements $\mathrm{C}, \mathrm{H}, \mathrm{N}, \mathrm{S}$, and $\mathrm{O}$, the number of its atoms often reaches far beyond a thoulsand. In the year 1866 Preyer made the first analysis of hæmoglobin, the proteid that gives the characteristic colour to the blood, more exactly to the red corpuscles, and, as a carrier of oxygen from the lungs through the blood to the cells of the tissues, 
plays an extremely important rôle in the animal body. Preyer found the composition of hæmoglobin to be-

$$
\mathrm{C}_{600} \mathrm{H}_{960} \mathrm{~N}_{154} \mathrm{Fe}_{1} \mathrm{~S}_{3} \mathrm{O}_{179} \text {. }
$$

Although at first this formula caused surprise, a number of later analyses have since given quite similar results. ${ }^{1}$

Thus, according to Gruibler's investigations ('81), the composition of the crystallised proteid which occurs in the squash-seed may be estimated as-

$$
\mathrm{C}_{292} \mathrm{H}_{481} \mathrm{~N}_{90} \mathrm{O}_{83} \mathrm{~S}_{2} \text {. }
$$

Zinoffsky ('85) found the formula of hæmoglobin from horse's blood even still larger than Preyer-

$$
\mathrm{C}_{712} \mathrm{H}_{1130} \mathrm{~N}_{214} \mathrm{O}_{245} \mathrm{FeS}_{2} \text {. }
$$

Similarly complex formulas have been derived for the proteid that constitutes the white of the hen's egg. From all these analyses it follows that because of the mass of its constituent atoms the proteid molecule must be enormous.

The great size of the molecule explains an important characteristic of proteids, viz., that, in contrast to other bodies, they do not diffuse from solutions through animal membranes or artificial

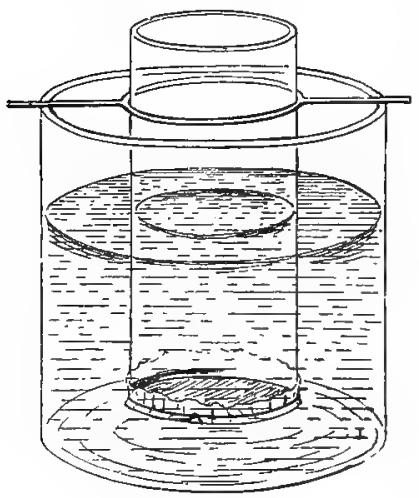

F10. 37.-Dialyzer. parchment. If an aqueous solution of common salt or any other soluble salt be placed in a wide glass tube, the lower end of which is closed by a membrane, preferably artificial parchment (Fig. 37), and the tube be suspended in a vessel of pure water, it is found after a short time that the concentration of the salt solution in the inner tube has decreased considerably, while the water in the outer vessel has come to have an equal percentage quantity of salt. Hence salt has diffused from the tube through the membrane into the outer water until its percentage composition has become equal in the two liquids. But if instead of the solution of salt a solution of egg albumin be employed, which can be obtained by rubbing up thoroughly the white of a hen's egg with about 100 cubic centimetres of water and filtering, the solution can be allowed to stand in the dialyzer (as the apparatus is called) for hours and days without a trace of albumin diffusing from the inner tube into the outer water. This phenomenon may be explained very simply from 
the size of the albumin molecule; the latter is too large to pass through the excessively fine pores of the membrane, while no obstruction stands in the way of the small molecules of salt. This property is of practical importance in the chemical investigation of proteids, for by dialysis the proteids can always easily be separated from the salts that may be present with them in solution.

The fact that proteids and a host of other substances which behave similarly do not diffuse through membranes, has led to the idea that these bodies, in contrast to diffusible substances, dissolve in water only apparently, and form no real solutions; their apparent solubility may be only a largely developed power of swelling. Proteids in a dry state are, in fact, capable of taking up very large quantities of water, and thereby gradually swelling. In 1861 Graham contrasted these bodies as colloid substances from crystalloid substances; and this distinction has been handed down and been generally accepted. The colloids are said to be capable of swelling only, not of crystallising; the crystalloids, on

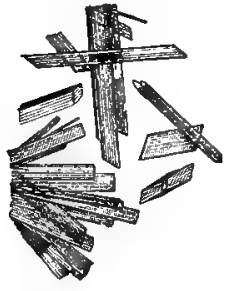

$I$

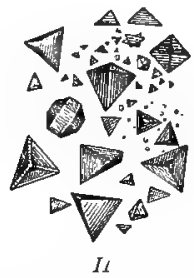

FIc. 38.-Crystals of hæmoglobin, I, From man, $I I$, from the guinea.pig, III, from the squirrel. (After Kirkes.)

the other hand, to be really soluble and capable of crystallisation. But such a sharp distinction is scarcely admissible; for in the first place, proteids are known that can form genuine crystals, like the above-mentioned proteids in squash-seeds, which occur widespread in plant seeds as aleurone-grains, and like the hæmoglobin of the red blood-corpuscles. If, e.g., whipped blood from the guineapig be shaken for a time with ether, by which the hæmoglobin is extracted from the substance of the red corpuscles and driven out into the blood-serum, and a drop of this liquid be allowed slowly to evaporate upon a glass slide, very delicate tetrahedral crystals gradually separate (Fig. 38, II), which consist of pure hæmoglobin. In the second place, under the influence of certain reagents, proteids can pass over into modifications that diffuse through membranes, without losing in the process the chemical characteristics of proteids. These modifications, which, e.g., proteids undergo in the body under the influence of the digestive juices of the stomach and the pancreas, are termed peptones; it is known 
that they arise by the hydrolytic cleavage of the original proteid molecule, so that the peptones represent the hydrates of the original proteids. Important conclusions follow from this fact. Since the proteid molecule, which was originally not diffusible on account of its enormous size, is split up in the peptonising process into the peptone molecules, which are much smaller and therefore diffusible, but which have the chemical characteristics of proteids, it follows that the proteid molecule is not simple but polymeric, i.e., it consists of a chain-like combination of many similar groups of atoms. In the transition to the peptone condition the proteid molecule is broken up with hydration into these single, similar atomic groups, all of which, however, have the chemical characteristics of proteids, but represent much smaller molecules. The inability of proteids to diffuse through membranes depends, therefore, solely upon their polymerism. Wholly analogous cases occur in inorganic nature; e.g., certain forms of silicic acid are unable to diffuse through membranes because of their polymerism. Hence it is evident that no fundamental difference exists between solutions of simple molecules, as found in peptones, and those of polymeric molecules, as in ordinary albumin.

A further physical property, which is perhaps connected with the polymerism of the ordinary proteid molecule, and which belongs to almost all proteids with the exception of their hydrates, the peptones, is their capacity of clotting, or coagulating. Coagulation consists in the passing of the substance from the dissolved to the solid condition within the solvent medium. Boiling is a method that causes coagulation in almost all proteids. In the fresh hen's egg the proteid is present in a thick clear viscous solution. In the boiled egg it has become a solid white opaque mass; it is coagulated. By boiling, proteid can be separated out of thin solutions in the form of fine curdled flakes. Other methods, such as the use of inorganic acids and alcohol, also cause proteid in solution to be coagulated and precipitated, the result being indicated by a clouding of the liquid. That the power of coagulation is in some way connected with polymerism is indicated by the fact that inorganic polymeric molecules, such as the above-mentioned silicic acid, in aqueous solution can likewise be coagulated into a jelly. If, e.g., hydrochloric acid be added to a solution of sodium silicate, free silicic acid and sodium chloride are produced; the silicic acid may then be separated from the salt by dialysis, since, in contrast to the salt, the former, like a polymeric body possessing very large molecules, does not diffuse through membranes. By the addition of a few bubbles of carbonic acid this solution of silicic acid may be changed at once into a coagulated jelly-like mass. Since our knowledge of the chemical composition of proteids is at present very incomplete, it is not easy to produce definite chemical reactions with them. Nevertheless, a number of tests have been empirically 
determined, which are characteristic for proteids and in doubtful cases make known their presence. What chemical transformation the proteid molecule undergoes in these tests is of course practically unknown. The best-known tests are the following, any one of which alone is not sufficient to prove with absolute certainty the presence of albumin :-

1. The santhoproteic test: a solution of proteid is coloured yellow by boiling with nitric acid; by the addition of ammonia the colour changes to orange.

2. The biuret test : if a solution of proteid be made alkaline by causic potash or soda, it takes on in the cold, by the addition of a drop of cupric sulphate solution, a clear violet colour.

3. Millon's test: coagulated proteid, boiled for a time with a solution of mercuric nitrate, and a little nitrous acid, becomes rose-red.

4. The hydrochloric acid test: boiling with concentrated hydrochloric acid dissolves coagulated proteids and colours the clear liquid violet.

5. The potassium ferrocyanide test: a solution of proteid to which acetic acid has been added shows by the addition of a solution of potassium ferrocyanide a white cloudiness.

6. The iodine test : the addition of tincture of iodine, or a solution of iodine in potassium iodide, serves as a good microscopic method for recognising proteids; the clot becomes yellowish-brown.

Besides these tests, a great number of others have been suggested by different investigators, but they fail in individual cases.

According to their different solubilities in water, three groups may be distinguished among the simple proteids-viz., the albumins, the globulins, and the vitellins. ${ }^{1}$

The albumins are directly soluble in pure water. To them belong egg-albumin, which forms the great mass of the white of eggs; serum-albumin, an albuminous body contained in blood-serum; muscle-albumin, the proteid of muscle-cells, soluble in water; and plant-albumin, which is dissolved in the sap of plant-cells.

The globulins are soluble in water only when it contains neutral salts, but in less quantity than in saturation. If a solution of globulin be saturated with salts, the globulin is precipitated in a flocculent mass-a phenomenon that is termed "salting out." The globulin is likewise precipitated if the solution be wholly freed from salts by diffusion in a dialyzer. To the globulins belong serum-globulin, which is dissolved in the blood-serum; fibrinogen, also a proteid of blood, which coagulates spontaneously into flakes and threads of fibrin when the blood is allowed to stand outside the blood-vessels; myosin, the globulin of muscle, which likewise

\footnotetext{
1 Neumeister' ('93).
} 
coagulates spontaneously upon standing-a phenomenon that appears in dying muscle in rigor mortis; and, finally, plantglobulin, which gives to kernels of grain their glutinous quality, and hence has been termed glutin.

The vitellins are likewise soluble in neutral salt solutions only, but, in contrast to the globulins, they are not precipitated by saturation of the solution with salts. Among them are the so-called yolkplates of the yolk of eggs, and the already-mentioned aleurone grains of plant seeds, both of which are proteids capable of crystallization.

The above-mentioned proteids occur in a free state in living substance. A very large number of proteids, however, are not free, but are chemically combined with other substances. In these compounds, which have been termed combined proteids in distinction from the simple proteids, the proteid molecule behaves in general like a feeble acid, and by the addition of stronger acids it can frequently be forced out of its compounds, the stronger acid taking its place. The proteid then becomes free. We have already become acquainted with one of these compounds, hæmoglobin, which plays in blood so important a rôle and is a compound of proteid and iron. But the most important compounds, in which proteids appear without exception in every cell, are the nucleins. The nucleins, as Altmann ('89) has shown, are compounds of proteid with nucleic acid, an acid which is itself a compound of phosphoric acid with peculiar basic bodies, the so-called nuclein bases-guanin, adenin, xanthin and hypoxanthin. The nucleins are capable of entering into further combinations with a second proteid molecule, and these extremely complex compounds are termed nucleo-proteids or nucleoalbumins. Casein, a body which for a long time has presented difficulties to the physiological chemists, is such a nucleo-proteid, combined with calcium. Casein is the calcareous nucleo-proteid of milk that is manufactured into cheese ; it has the peculiarity of not coagulating when the milk is boiled, while it is immediately precipitated when separated, as by acetic acid, from the calcium. A fourth group of combined proteids is that of the glyco-proteids, in which proteid is combined with a carbohydrate; prominent among these is mucin, which is contained in the cells of mucous glands.

Besides the genuine proteids which we have just described, there exist a number of bodies which behave in many ways similar to proteids and, therefore, have been termed albuminoids. The group of albuminoids is a true unnium gatherum; it contains a very large variety of bodies. These are partly compounds of proteids and partly bodies of similar constitution to the proteids, but which show in their chemical behaviour much less similarity and are much less known than the proteids themselves. Especially prominent among albuminoids are many of those substances that are produced by cells to serve as skeletal substances for the support 
of the more delicate parts of the organism. A detailed examination of the known reactions which the numerous albuminoid bodies present would lead too far and be superfluous for our purpose. ${ }^{1}$ It is sufficient here to cite some of the most important members of the group, all of which occur in the solid undissolved state. Such are keratin, which is contained in most horny structures produced by the epidermis-cells of the skin (horns, hoofs, hairs, feathers, and nails); elastin, which composes the elastic fibres of the cells of connective tissue and the strong yellow ligamentum nuchoe; collagen, which composes the organic ground-substance of bones and cartilage, and in boiling passes over by hydrolysis into gelatine; spongin, the skeletal substance of bath-sponges; conchiolin, the organic substance of the shells of mussels and snails; cornein, that of the skeletons of corals; and many other substances that form skeletons, especially in invertebrates.

With the albuminoids also is classed a series of highly complex nitrogenous bodies which at least are derivatives of proteids and possess the greatest importance in the life of the organism, especially in digestion. These are the unformed ferments or enzymes, such as pepsin, produced by the gland-cells of the stomach; ptyalin, by the cells of the pancreas and the salivary glands; trypsin, produced likewise by the pancreatic cells; and many others. The properties of these bodies and their róles in the life of the cell will be considered more fully elsewhere.

There appear in living substance, as constant accompaniments of proteids, certain decomposition-products of them which can be divided into two groups-the nitrogenous and the non-nitrogenous cleavage-products. The former constitute a series of substances whose chemical constitution is more exactly known. They are the products of retrogressive proteid-metamorphosis. Among them belong especially the substances excreted in considerable quantity by the higher animals in the urine. Among them urea, $\left(\mathrm{NH}_{2}\right)_{2} \mathrm{CO}$, holds the first rank; it is the richest in nitrogen of all the nitrogenous end-products of proteid-decomposition, and its artificial synthesis was accomplished by Wöhler in the year 1828 . Next to urea, uric acid, $\mathrm{C}_{5} \mathrm{H}_{4} \mathrm{~N}_{4} \mathrm{O}_{3}$, contains the most nitrogen; next to uric acid come in order hippuric acid, creatin, which originates in the muscles by the decomposition of proteid, and creatinin. Further, the nuclein bases, xanthin, hypoxanthin or sarkin, adenin and guanin are met with as end-products of the decomposition of nucleins in the living organism. Of these, especially the last in combination with calcium occurs very frequently in the skin-cells of Amphibia and of fishes, in the latter of which its crystals produce the well-known silvery sheen. Finally, there is one more group of nitrogenous bodies, the lecithins,

1 A review of the subject and the bibliography of it may be found in Neumeister: Lehrluch der physiologischen Chemie. 2nd edition, Jena, 1897. 
which stand near the fats, but contain phosphorus; they are probably present in every living cell and, according to Hoppe-Seyler, are to be regarded as cleavage-products of proteids, especially of nucleins, with which they occur.

Among the non-nitrogenous end-products of proteid-decomposition carbonic acid, which is produced by every cell, comes first in importance. Lactic acid, oxalic acid; and sulphuric acid are important. The cholesterins also are to be regarded at least as derivatives of proteids; they seem to occur in all living substance, but appear in great quantity only under certain circumstances in the form of iridescent scales, as upon the surface of the skin and the beak of birds, and in pathological conditions as gall-stones in the bile. Chemically the cholesterins are univalent alcohols, which with fatty acids can form fat-like compounds. Finally, there appear as decomposition-products of proteids certain carbohydrates, particularly grape-sugar and glycogen, and fats, which must be considered somewhat in detail in connection with allied substances.

\section{b. Carbohydrates}

In contrast to its presence in the proteids, nitrogen is wanting in the carbohydrates. The latter contain only the three elements, carbon, hydrogen and oxygen; in the natural carbohydrates the number of carbon atoms within the molecule is always six or a multiple of six, while the number of hydrogen atoms is always double that of the atoms of oxygen; hence hydrogen and oxygen are present in the same relative proportions as in water-a fact which led to the designation "carbohydrates." The carbohydrates are very wide-spread and are of great importance, especially in the manufacture of living substance in plant-cells; but there are varieties of living substance in which they cannot be demonstrated ; in other words, they are not general constituents of such substance. They present far simpler chemical relations than the proteids, and a brief glance will show their most essential features.

The natural carbohydrates may be divided into monosaccharids, disaccharids and polysaccharids, of which the two latter groups are different anhydride forms of the first group.

The monosaccharids all have the formula $\mathrm{C}_{6} \mathrm{H}_{12} \mathrm{O}_{6}$, and are, therefore, isomeric; but they are not all stereo-isomeric, that is, their individual atoms are not grouped alike in all. To the monosaccharids belong chiefly grape-sugar (dextrose or glucose) and fruitsugar (lævulose), both of which are wide-spread in plant juices, the former in great quantity also in animal tissues. One of the most remarkable characteristics of the monosaccharids is that they readily take up oxygen from their surroundings and thus reduce bodies that are rich in oxygen, a peculiarity upon which depend the most important tests for their recognition. The most re- 
liable of these reduction-tests are Trommer's test and Böttger's test. They may be performed very simply in a test-tube. The former consists in the reduction of cupric hydroxide to cuprous oxide by an alkaline solution of grape-sugar. If a few drops of a very dilute solution of cupric sulphate be added to a sugar solution, made alkaline by caustic potash or soda, until a blue flocculent precipitate of cupric hydroxide appears, on boiling the latter is reduced to red cuprous oxide or yellow cuprous hydroxide. In Böttger's test a few drops of a solution of basic nitrate of bismuth is added to the alkaline solution of grape-sugar; the former is then reduced to black metallic bismuth. A further very characteristic property of the monosaccharids is their power of ferinentation. They become decomposed by the action of yeast-cells (Saccharomyces) into alcohol and carbonic acid-

$$
\mathrm{C}_{6} \mathrm{H}_{12} \mathrm{O}_{6}=2 \mathrm{C}_{2} \mathrm{H}_{5} \mathrm{OH}+2 \mathrm{CO}_{2} \text {. }
$$

Such an experiment can be carried on best in a fermentationglass (Fig. 39), by introducing into it a solution of grape-sugar

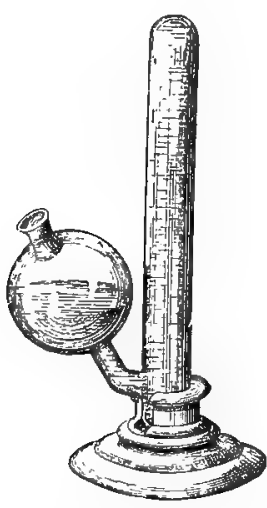

c

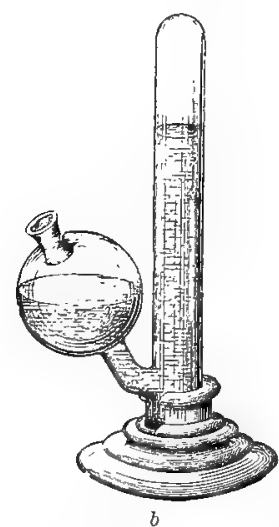

$b$

Fra, 39.-Fermentation-tube- $a$, newly filled; $b$, with carbonic acid developing. At the top of the straight limb a quantity of carbonic acid has already accumulated.

mixed with fresh yeast, so that the liquid fills completely the long closed limb of the glass. At a temperature of $c .30^{\circ}-40^{\circ} \mathrm{C}$. there appears a fairly energetic cleavage of the grape-sugar, small bubbles of carbonic acid rising continually as in a glass of champagne, and accumulating at the upper end. The more carbonic acid accumulates above, the more the liquid is forced out of the long limb into the spherical part of the vessel, until finally the former may be entirely filled with the gas. The presence of alcohol may be recognized at once by the odoux of the liquid. One more characteristic of the monosaccharids may be mentioned, 
which they share with all soluble carbohydrates - viz., the power of rotating the plane of polarised light. As their names indicate, dextrose rotates it to the right; lævulose, to the left.

The discccharids may be regarded as having arisen from the monosaccharids by the combination of two molecules of the latter and the loss of a molecule of water; this would yield the formula-

$$
\mathrm{C}_{12} \mathrm{H}_{\Omega 2} \mathrm{O}_{11} \text {. }
$$

Among the disaccharids are to be noted especially cane-sugar (saccharose), which is contained in large quantities in the cell-sap of the sugar-cane; and milk-sugar (lactose), the carbohydrate of milk. By certain methods, as by boiling with dilute inorganic acids or by the action of certain bacteria, the disaccharids can be made to undergo hydrolytic cleavage, so that they pass over into the monosaccharids. This change is termed inversion. In contact with certain fermentation-agents, especially Bacterium lacticum, the disaccharids are induced not to ferment directly, but to. pass over into monosaccharids, which are themselves subject to the fermentative action of these organisms. If Bacterium lacticum beemployed as the fermentation-agent, lactic acid results-

$$
\mathrm{C}_{6} \mathrm{H}_{12} \mathrm{O}_{6}=2 \mathrm{C}_{3} \mathrm{H}_{6} \mathrm{O}_{3}
$$

-a process which, in contrast to alcoholic fermentation by the yeast. plant, is termed lactic acid fermentation; to it is due the souring of milk exposed to the air. Finally, under the influence of another fermentation agent, Bacillus butyricus, lactic acid can be still further decomposed into butyric acid, carbonic acid and hydrogen-

$$
2 \mathrm{C}_{3} \mathrm{H}_{6} \mathrm{O}_{3}=\mathrm{C}_{4} \mathrm{H}_{8} \mathrm{O}_{2}+2 \mathrm{CO}_{2}+4 \mathrm{H} \text {; }
$$

thus a butyric acid fermentation is recognised.

The polysaccharids are anhydride stages of the monosaccharids still further removed; in them several monosaccharid molecules combine with the loss of a molecule of water, so that their formula is a multiple of $\mathrm{C}_{6} \mathrm{H}_{10} \mathrm{O}_{5}$. Among the polysaccharids occurs a series of bodies that play an important role and are wide-spread, some in the life of the plant-cell, others in many animal-cells. They are, first, starch, which occurs in all green cells of plants in the form of granules, in which the layers are arranged concentrically (Fig. 40); secondly, glycogen, which occurs as flakes. and irregular particles, especially in the cells of the liver, but in smaller quantities in many other tissue-cells; thirdly, cellulose, which constitutes the cell-membranes of all plant-cells, and has been demonstrated also in the leathery mantle of the Tunicates. These members of the group of polysaccharids may be distinguished from one another in a very characteristic manner by their behaviour towards solutions of iodine: by iodine starch is coloured an intense blue, glycogen a mahogany brown, and cellulose 
not at all; the latter, however, becomes blue in the presence of iodine and sulphuric acid.

In addition to the free carbohydrates, combinations of carbohydrat's exist in living substance-e.g., combinations with proteids, as an example of which mucin has already been mentioned.

The most important decomposition-prorlucts of carbohydrates have also been mentioned, such as lactic acid, butyric acid, carbonic acid, etc., all of which are met with in living substance.

\section{c. Fats}

The fats likewise do not belong to the general constituents of living substance, but they are wide-sprearl, chiefly in animal cells. Like the carbohydrates, the fats are non-nitrogenous, and contain only the elements carbon, hydrogen and oxygen. But chemically they differ fundamentally from the carbohydrates. For example, they represent the so-called compound ethers, or esters-i.e.compounds in which an acid has combined with alcohol with the loss of water. The alcohol that is the basis of all fats is glycerine, $\mathrm{C}_{3} \mathrm{H}_{5}(\mathrm{OH})_{3}$, and the acids that are combined with glycerine belong to the series of fatty acids, whose general formula is $\mathrm{C}_{n} \mathrm{H}_{21} \mathrm{O}_{2}$. Since the glycerine represents a trivalent alcohol,

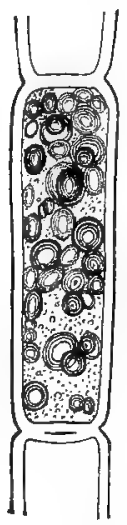

1

FIG. 40.-1. Plant-cill cuntaining starch-grains. II. Starch-grains isolated-a, from the potato; $b$, from the corn ; $c$, from the pea. in the neutral fats three atoms of fatty acid are always combined with one atum of glyc erine into tri-glycerides. The general formula of the fats is, therefore-

$$
\mathrm{C}_{3} \mathrm{H}_{5}(\mathrm{OH})_{3}+3 \mathrm{C}_{11} \mathrm{H}_{21} \mathrm{O}_{22}-3 \mathrm{H}_{2} \mathrm{O} \text {. }
$$

As examples of the fatty acids there may here be mentioned palmitic acid, stearic acid, butyric acid, valuric acid and capronic acid. In addition to these, oleic acid, which does not belong to the normal serics of fatty acids, occurs in the various oils combined with glycerine.

In correspondence with their composition, the neutral fats may by certain methorls be split up by hydrolysis into theirconstituentsi.e., into glycerine and free fatty acids: this process takes place in the organism is the result of the action of the digestive juices. It 
occurs also when neutral fats are boiled with alkaline liquids, such as caustic potash or soda. The fatty acids thus set free combine with the alkali to form the so-called soaps, which may be distinguished as potash soaps, sodium soaps, calcium soaps, etc.

The fats are all lighter than water and do not dissolve in water, but they are easily soluble in ether. A characteristic property, which is important for the microscopic recognition of the fat-droplets in cells, is their power of reducing perosmic acid to metallic osmium, the latter forming a black coating to the fat-droplet. This osmic acid reaction is not to be employed alone as a sure test in the diagnosis of fat; for doubtless other reducing substances exist, which, under certain circumstances, can be blackened by osmium; hence it should be used only in conjunction with other tests, solubility in ether, strong refracting power, etc.

The fact that fats, like carbohydrates, can appear as cleavageproducts of proteids has already been mentioned.

\section{d. The Inorganic Constituents of Living Substance}

In the case of the organic compounds of the cell the general constituents (proteids) and the special constituents (carbohydrates and fats) can be contrasted; the same distinction can be made with the inorganic compounds.

Here, also, the greater interest is associated with the general inorganic constituents, among which there are distinguished water, salts, and gases.

Water is that constituent of living substance that gives to it its liquid nature and thus renders possible the easy shifting of its particles, which is so necessary for the occurrence of vital phenomena. It is contained in the cell, in part chemically combined as water of constitution, and in part free, as the solvent medium of all sorts of substances. Accordingly, water is present in abundant quantity, constituting upon the average more than 50 per cent. by weight of living substance. If, c.g., the whole water contents of the human body be investigated, which with the great variety of the forms of tissue affords a good average, approximately 59 per cent. of water is found; this is shown especially by the detailed investigations of Bezold. The different tissues vary very greatly in this respect. Thus, bones contain only about 22 per cent. of water, the liver 69 per cent., muscles 75 per cent, and the kidneys 82 per cent. Hence it is not strange that the water contents of living substance varies much more in different species of animals, and that all intermediatestages in percentage composition are met with between the slight traces of water contained in a rotifer when dried but still capable of life, and the water-contents, amounting to more than 99 per cent., of certain pelagic Ctenophore. 
Many salts occur dissolved in water, and they are present in all living substance. The compounds of chlorine appear to be especially important, as well as the carbonates, sulphates, and phosphates of the alkalies and alkaline earths, particularly sodium chloride (common salt), potassium chloride, ammonium chloride, and sodium, potassium, magnesium, ammonium, and calcium carbonates, sulphates and phosphates.

Finally, as regards gases, there occur in all living substance oxygen and carbonic acid. When not in chemical combination, they are usually absorbed in water, and rarely, as in many unicellular organisms, e.g., Rhizopoda, in the form of bubbles of gas.

The special inorganic constituents of cells comprise a great variety of substances, but for present purposes it is unnecessary to discuss them. It is remarkable that in certain cells even free mineral acids appear, such as hydrochloric acid, which is produced by certain cells of the gastric glands in vertebrates, and sulphuric acid, which in many marine snails is secreted by the cells of the salivary glands.

\section{e. The Distribution of Substances in Protoplasm and Nucleus}

Although during the last few years our knowledge of cellmorphology has increased greatly, and microscopic investigation of the cell has revealed its finest structural relations, comparatively little is known of the chemical nature of its individual morphological constituents. Here is the point where physiological microchemistry must institute its work. The combination of microscopic observation and chemical reaction alone is able to bridge the gap between that which has become known morphologically as groundsubstance and solid constituents in the protoplasm and the nucleus, and that which gross chemical analysis has shown to be the constituents of living substance. The building of the bridge between the morphology and the chemistry of the cell is a difficult undertaking, since the majority of reactions that can be employed conveniently and easily in the test-tube, under the microscope on account of the minuteness of the objects either give very indistinct results, or are entire failures. Hence, first of all, delicate and reliable micro-chemical methods need to be devised. The first steps in this direction have already been taken, and we have begun to obtain here and there an insight into the distribution of the chemically known substances within the cell-contents.

It has been shown that the bodies that have been found as morphological differentiations in the cell-contents, differ also chemically. Especially the investigations of Miescher, Schwarz, Zacharias, Altmann, Kossel, Löwitt, Malfatti and others have proved that characteristic chemical differences exist between the 
constituents of the two essential cell-elements, the protoplasm and the nucleus.

The proteids, which are the sole general chemical constituents of the cell, occur in both the protoplasm and the nucleus, but a very remarkable difference between them has been discovered. It has been found that the compounds of proteids, containing phosphoric acid, the so-called nucleins, preponderate greatly in the nucleus, ${ }^{1}$ while in the protoplasm they seem to be wanting entirely, or at least to appear only in combination with other proteids as nucleo-albumins; the protoplasm, on the other hand, is constructed chiefly of simple proteids and proteid compounds that lack phosphorus. The employment of a simple chemical method confirms this fact. As Miescher ('74) has shown, the nucleins, in contrast to all other proteids, resist the digestive action of the gastric juice. If, therefore, cells of very different kinds be brought under the influence of artificial gastric juice, all other proteids are digested, while the nucleins remain. It is then found that the whole protoplasmic body is digested, while the nuclei are left with an inconsiderable decrease in volume and a somewhat ragged contour. If, now, the remaining substance of the nuclei be tested with the known nuclear stains, it is shown that what is wanting is the nuclear sap, ${ }^{2}$ and perhaps the achromatic substance also, for the whole remaining mass takes up the nuclear stain more or less strongly. It follows, therefore, that the chromatic substance and the nucleoli consist of nucleins, while the protoplasm of the cell is composed of other proteids. Lilienfeld and Monti ('93), in Kossel's laboratory, have endeavoured to prove by means of a micro-chemical reaction that phosphorus is localised especially in the nucleus. If ammonium molybdate be added to a substance containing phosphoric acid, a compound is formed, phospho-molybdic acid, which with pyrogallol takes on a dark brownish-black colour. Lilienfeld and Monti were able to show that in a great variety of cells the nuclei stain black because of this reaction, while the protoplasm is left unstained; but it should be mentioned that soon after the publication of their results Raciborski, Gilson and Heine raised the objection against the reaction that there was simply an accumulation of ammonium molybdate in the nucleus, which is analogous to the accumulation there of nuclear stains. Hence caution is still necessary in drawing conclusions from this reaction.

The carbohydrates appear to be limited to the protoplasm; at least, thus far no carbohydrates have been found in the nucleus. In the protoplasm they appear not rarely as solid constituents, e.g., glycogen in the form of scales and irregular particles in the protoplasm of liver-cells, starch-grains in general in the protoplasm of

1 Cf. Kossel (91).

Cf. Milfatti(?), '92). 
all green plant-cells, and cellulose as a protoplasmic product upon the surface of cells.

The fats also appear to be limited to the protoplasm. Without exception they seem to be wanting in the nucleus, but are very wide-spread in the protoplasm as fat- and oil-droplets. They may always be recognised by their great refracting power, or, in dubio, by their blackening with perosmic acid and solubility in ether.

Concerning the distribution of the inorganic constituents of the cell almost nothing whatever is known. As to the potassium compounds, however, the investigations of Vahlen appear to show that they are to be found exclusively in the protoplasm, and not in the nucleus.

These are the few facts thus far known. The chemical composition of the great mass of substances in the protoplasm that are termed granules, as well as that of the substances in solution, is thus far wholly unknown. Here an unbounded field is open to the physiological chemists of the future, and in a more distant future shall we have to look to the micro-chemical investigation of living substance for the solution of the final riddle of life.

The main points of the above examination of living substance may be summarised as follows: Living substance, as it now exists upon the surface of the earth, appears solely in the form of elementary organisms, the cells, some of which live separately, while some are united together into coherent communities. Each cell is a bit of liquid substance, usually microscopic in size, in which various constituents, partly solid, partly in solution, are stored. Only the liquid ground-mass, the protoplasm, and the somewhat more solid nucleus contained within the former can be regarded as general cell-constituents. A bit of protoplasm containing a nucleus is a complete cell, and, vice versce, there are no cells that do not possess nucleus and protoplasm. Just as very different morphological constituents may be distinguished in living substance, so very different chemical bodies are present. The elements of which they consist are only such as exist in the inanimate world also, but their number is small, and it is chiefly the elements having the lowest atomic weights that compose living substance. A special vital element does not exist, but the compounds in which these elements occur are characteristic of living substance, and in great part are absent from the inorganic world. They are, first of all, proteids, the most complex of all organic compounds, which consist of the elements $\mathrm{C}, \mathrm{H}, \mathrm{O}, \mathrm{N}$, and $\mathrm{S}$, and are never wanting in living substance. Further, there occur other complex organic compounds, such as carbohydrates, fats, and simpler substances, all of which either are derived from the decomposition of proteids or are necestary to their construction; and inorganic substances, 
such as salts and water; the latter gives to living substance its requisite liquid consistency.

In its main outlines the above is the picture that the anatomical, microscopic, physical, and chemical investigation of living substance has afforded.

\section{Living and Lifeless Substance}

But the picture of living substance is still incomplete. In the above pages there have been presented the details of its composition as known at present, but the most essential point is still wanting. In what does the characteristic difference between living and lifeless substance consist? This question is weighty, for it contains nothing less than the problem of all physiology-namely, the problem of life, which since the earliest times has had an irresistible fascination for inquiring minds.

As has already been seen, the conception of life has not been always the same. Since its origin among primitive peoples, it has become changed in diverse ways. We will now inquire whether it is possible to outline the conception scientifically by considering the differences between living and lifeless substance.

Because of the sharp distinction between objects that never have lived, such as stones, and those that have lived and died, or corpses, this undertaking must be extended in two directions -first, to the differences between organisms and inorganic substances, and, secondly, to the differences between living and dead organisms.

\section{A. ORGANISMS AND INORGANIC BODIES}

\section{Structural Differences}

In comparing organisms with inorganic substances, the mistake has been made of contrasting the organism with a crystal, instead of with a substance that has a consistency, and, in general, physical relations similar to those of living substance, i.e., with a semiliquid mass. Because of this mistaken comparison, a host of differences have been set up, the incorrectness of which is erident.

Thus, it has been said that inorganic bodies have forms constructed according to simple mathematical laws and possessingperfectly definite angles and edges, while organisms have bodily shapes that cannot be represented mathematically. It is not necessary to cite in refutation the "crystallised human folk" which Mephistopheles claims to have secil in his years of travel; the untenableness of this distinction become's clear when it is recalled that, in the first place, mathematically simple body-forms do actually occur among organisms, as in the Kadiolaria, which are 
provided with extremely delicate silicious skeletons, in many tissue-cells when pressed close together into polyhedral forms, and in many spherical egg-cells; and, in the second place, in inorganic nature the mathematically fixed body-form is wanting in all fluids.

Further, it has been maintained that inorganic bodies, such as crystals, have no organs, while the presence of these distinguishes all organisms. This also is incorrect. There exist not only organisms without proper organs, such as Amobe and all other Rhizopoda, in which the whole liquid protoplasmic body is an organ for all things, but also inorganic structures with real organs, such as machines, in which the individual parts are provided with perfectly definite functions. Yet no one will seriously regard Amcebce as inorganic bodies, or steam-engines as living organisms.

Another difference has been sought in the claim that, in contrast to all inorganic bodies, organisms are composed of the characteristic structural elements of all living substance, cells. It is true that the cell is a specific element of the whole organic world. But that which characterises this elementary constituent, that which distinguishes it from the whole inorganic world, is not its morphological character. Objects that are composed of separate form-elements can easily be manufactured out of inorganic substances. Nature has manufactured such objects in great quantity in rocks which consist of innumerable separate crystals, such as granite. That which characterises the cell is rather its chemical properties. Hence the presence of cells is not a sign of absolute structural difference.

Finaily, it has been said that inorganic bodies possess a very simple uniform structure, while organisms possess a highly complex "organisation." If by "organisation" there is understood simply the more or less complex composition of organisms out of different kinds of elementary structural particles, the cells, this statement, within certain limits, is true; although, in contrast with composite rocks, the difference is merely one of degree. But the cell must be employed for comparison, for it is in itself a complete organism. If, however, the conception of complex "organisation" be applied to the cell, it signifies merely the gross morphological variety and chemical complexity of its constituents, and such a condition can be established in a test-tube in a complex chemicophysical mixture. If by "organisation" a special kind of association of the individual constituents is understood, such as would not occur in inorganic nature, then the conception carries with it more or less mysticism, which has always been a favourite aid in explaining vital phenomena. Such a process cannot be followed in science, for science and mysticism are mutually exclusive.

Thus it is seen that a comparison of the structural relations of living and of inorganic substance does not reveal essential 
differences between the two. If the former be compared with a liquid rather than with a crystal, it is found that in its structural relations it differs no more from lifeless liquid mixtures than these differ among themselves, and, indeed, not so much as they differ from a crystal.

\section{Genetic Differences}

A second series of differences which, it is believed, have been found between organisms and inorganic substances has reference to reproduction and derivation. These differences likewise are not fundamental, and it is easy to perceive that between the two groups no real contrast in this respect exists.

It is regarded as a characteristic sign of difference that organisms reproduce, while inorganic bodies cannot do so. This is not an absolute difference, for many organisms are known that live and yet can never reproduce. Thus, it is well known that the power of reproduction is wanting throughout life in the so-called workers, those individuals in communities of ants and bees which form the great majority of the community and in which the sexual organs are undeveloped; notwithstanding this latter fact they must be regarded as living organisms. Further, reproduction in organisms consists simply in a giving-off of a certain portion of the body-substance, a division of the individual body. This fact shows most distinctly, i. .., is less masked by accompanying accessory phenomena, in unicellular organisms. An Amoba, for example, constricts itself into halves, and each half continues to live as a new Amolur. But if reproduction in its essentials consists merely in the division of substance, no fundamental difference exists between the process in a living cell and that in an inorganic body. A drop of mercury that falls upon the floor is divided into a number of small globules, all of which are drops of mercury.

It has been said, further, that organisms are always derived from other organisms, while inorganic bodies can be derived from both organisms and inorganic bodies. Thus, it is impossible to manufacture even the simplest organism artificially from inorganic substances, while it is not difficult to obtain inorganic bodiese.g., water-in a varicty of ways from both organic and inorganic substances. This appears to be an absolute difference, for it is true that in spite of all endeavours no one has succeeded in demonstrating that organisms can be formed from inorganic matters either in nature or in the laboratory. Nevertheless, this difference cannot be regarded as really absolute, for it can be r'plied that organic substance is constantly being built out of inorganic substance in the plant-body, this being the "xclusive method of construction in plants. $T$ o this it has been rejoined in 
turn that this origin of organic out of inorganic substance is possible only with the help of living organisms; Preyer ('80) has said that organisms are distinguished from inorganic bodies by the fact that they always presuppose the existence of living substance. Only in this form does the distinction hold good, at most, for the present time. Virchow's dictum, "Omnis cellula e cellula", which is the generalisation that has become necessary in the course of time from the old dictum of Harvey, "Omne vivum ex ovo," holds good only for the conditions that now prevail upon the earth's surface. If we go backward in the development of the earth, we soon come to a time when the earth was an incandescent mass, upon which no cell could exist. Cells must, therefore, have arisen at wine time from masses of matter that were not cells. At this point the following alternative is presented: Either, as the theory of spontaneous generation assumes, organisms have arisen at some time out of inorganic substances, or. as the theory of the continuity of life demands, the conception of life must be applied also to those bodies from which cells have developed, although they were totally different from the living substance of present organisms. If the former be accepted, the difference in the derivation of the two groups of bodies disappears of itself, for then not only inorganic, but also organic nature is derived from nonliving substance. Preyer decides upon the second assumption; he considers as living the mass of matter out of which cells have developed, and even the whole incandescent mass of the earth itself; and he extends still further Harvey's dictum to "Omne virum c vivo," thereby expressing the idea that life has never originated, but has existed from eternity. But the difficulty of establishing a fundamental difference between organisms and inorganic bodies upon the ground of their derivation is not thus set aside. For, in harmony with his idea that the whole incandescent mass of the earth is to be considered as living; Preyer assumes that the inorganic has arisen out of the organic. Hence, not only does organic nature, but also inorganic, presuppose the existence of living substance, and it is clear that the above-mentionerl difference in the derivation of the two great groups of natter disappears. It is seen, therefore, that even by such an extension of the conception of life as Preyer's the difference in derivation cannot $1 x$ maintained for the carlier period of the earth's development.

Just as little alsolute difference exists between the organism and the inorganic body in their development as in their reproduction and deriration from like bodic. By development is understood a series of changes undergone by the new-born organism, which make it finally like it. parents. Such changes occur in innrganic nature likewise. and are there not fundamentally different from thrs in manisms. $E \cdot g$. if a piece of 
sulphur be melted in a vessel and the melted mass be poured into water, there is obtained a tough, brown, gummy substance which has not the least external resemblance to the piece of sulphur from which it came. But if it be left for a day or two, it becomes gradually harder and more solid, its brown colour fades and changes to a yellowish, and after some time the whole mass takes on again the appearance of common hard yellow sulphur. Here the sulphur has gone through a development which has made it again like the piece from which it was derived. But even on the part of organisms development is not an absolute sign of difference, for there are organisms that live without developing. The two equal parts into which Amobe constricts itself are complete Anabo without any further process, and are distinguished by their size only from the individual from which they are derived.

Finally, an endeavour has been made, but with similar slight success, to find a distinction between organisms and inorganic substances in the manner of growth. The unfortunate contrast of the organism and the crystal, again, has led to the assertion of this difference. It has been said that the crystal grows by the apposition, the organism by the intussusception of particles; i.e., the crystal grows by laying one particle after another upon its surface, the interior remaining fixed and unchanged, the organism, on the contrary, by taking particles into its interior and storing them between those already present. If a cell as a whole be contrasted with a crystal, this is not to be disputed; but it has already been seen that as regards its physical characteristics the living substance of organisms in its essentials ought to be compared with a liquid. Liquids, however, grow solely by intussusception, i.e., if a soluble body be added to a liquid, e.g., salt to water, the latter dissolves the former and stores the molecules of the soluble body by diffusion between its own molecules-that is, there is here exactly the same process as in the growth of the organism.

Hence, the comparison of the genetic relations of organisms and inorganic bodies reveals no more fundamental difference between them than the consideration of their structural relations, and it is necessary to search further.

\section{Physical Differences}

A third group of differences which have been asserted to exist between organisms and inorganic bodies comprises the phenomena of movement. Movement, the most evident of all vital phenomena, was regarded in early times as a characteristic sign of life, and primitive people, in holding consistently to this idea, regarded winds and waves as living things. But the sea is no longer 
called living, and, on the other hand, in the resting plant-seed there is seen a condition of the organism in which, while it is not dead, not the slightest movement can be recognised. Thus the significance of movement in its primitive form has now disappeared, and in place of it more special motile phenomena have been sought as distinguishing marks between organisms and inorganic bodies.

It has been thought that a difference must be recognised in the causes that produce movements, on the one hand, of organisms, and, on the other, of inorganic bodies. The former, such as musclemovements, are said to result from internal causes- those that have their seat in the organism itself; the latter, such as the movement of waves and clouds, from external causes-those that, like the wind, act upon the object from without. The mystical vital force is here more or less evident. But we have already become convinced of the non-existence of such a force, and the claim of such a difference in the causes of movement cannot be maintained. Moreover, in many cases it is difficult to draw a sharp boundary between internal and external causes. E.g., if a steam-engine, and not winds and waves, be considered, it can be said of it, with as much right as of the organism, that it works from internal causes, for the pressure of the steam which drives the piston and puts the wheels in motion is in the interior of the boiler.

But it has been said that the difference between the causes of motion in the steam-engine and those in the organism lies in the fact that the former cannot work unless it is heated from the outside, while the latter works of itself. This is wholly untrue. The organism also must be heated if it is to continue in activity, i.e., in life, exactly as the steam-engine. Its heating is by the introduction of food. The analogy between the heating of the steam-engine and the nutrition of the organism goes very far. The carbon-containing food is burned in the organism in great part as is the coal in the steam-engine-i.e., the food-stuffs are oxidised by the oxygen taken in in respiration, as the coal is oxidised-and in both cases there is obtained as the end-product carbonic acid. If the introduction of food be interrupted, the activity of the organism ceases after a time when all the ingested food is consumed, similarly as with the steam-engine; in both, movement is stopped.

The comparison of the organism with the steam-engine allows the untenableness of the claim of another difference, closely associated with the previous one, to be at once recognised. It has been said, namely, that organisms are in dynamical equilibrium-icc, the same quantity of (nergy that is introduced into the organism leaves it again in some form-while inorganic bodies are in stable equilibrium. It is true that organisms in the adult state are in dynamical equilibrium. But, when this is put forward as a real difference in comparison with 
inorganic bodies, the crystal alone is again in mind. The steamengine, however, is an inorganic system in which dynamic equilibrium exists very clearly; for by the mediation of heat the system gives off to the outside as mechanical energy exactly as much energy as is introduced by the burning of the coal.

Finally, irritability has been brought forward as a general characteristic of organisms in contrast to inorganic bodies. In reviewing the history of physiological investigation it was seen that at first very indefinite ideas were associated with the word "irritability," and, in order to guard against misunderstandings, the conception must be definitely formulated. It can be said in general that irritability is the capacity of a body to react to an external influence by some kind of change in its condition, in which the extent of the reaction stands in no definite proportion to the extent of the influence. As a matter of fact, irritability, or excitability, is a property of all living substance, whether the organism responds to the external influence by the production of definite substances, as with secreting gland-cells, or definite forms of energy, as with muscle-cells, phosphorescent cells, and electric cells, or whether it responds by depression or even standstill of its vital activities. But irritability is not the exclusive property of organisms, for lifeless substances are likewise irritable and respond to external influences by definite changes, e.g., by the production of definite substances or of energy, in which process the extent of the production by no means corresponds always to the extent of the external impulse. The clearest examples of such cases are afforded by explosive substances. By a slight shock nitroglycerine is decomposed into water, carbonic acid, oxygun and nitrogen, the process being accompanied by a powerful evolution of energy; in other words, nitroglycerine responds to an external influence by an enormous production of energy and a change of material. Hence irritability is not an absolute sign of difference between organisms and inorganic bodies, and it is seen that a fundamental contrast between the two is afforded no more by thcir dynamical than by their structural and genetic relations. Wc will, therefore, search still further.

\section{Chemical Differences}

It is by a comparison of their chemical relations that a difference is finally found to exist between organisms and inorganic bodies.

It has been seen that a specific vital element exists in the organism no more than a specific rital force. The chemical clements that compose the organism oceur without exception in inorganic nature also. Therefore, a fundamental chemical contrast buwen organic and inorganic substance is not to be 
expected, i.e., a contrast that rests upon a difference as regards chemical elements. But a difference does exist in the kind of combinations into which the elements enter. It was seen above that chemical compounds are present in living substance, that never occur in the inorganic world; such are proteids, carbohydrates and fats. Of most importance is the fact that one group of these chemical bodies, the proteids, belong to all organisms without exception. Just as there is no single organism, whether living or dead, in which proteids are wanting, so there are no inorganic bodies in nature in which even an approximately similar substance is present. The possession of the highly complex proteid molecule is, therefore, a definite mark of distinction of the organism in its relation to all inorganic bodies.

But some have gone still further and have endeavoured to find an absolute difference between the two bodies, not only in the existence of certain compounds, but also in the order of the chemical processes in the active organism. It is said that living substance is characterised by its metabolism, in which definite compounds are formed continually, are broken down, give off their decomposition-products to the outside, and are reformed at the expense of the substances taken in from the outside is: food; hence a continual streaming of matter through the living substance takes place, being conditioned by the construction and destruction of the compounds in question. Metabolism is, indeed, a characteristic process of the living organism, and it will be seen later that upon it the vital process rests; but it is solely a process that distinguishes the living organism from the dead organism and not from inorganic substance, for it is not confined to organisms, but occurs also among inorganic bodies. A simple example of this is found in the behaviour of nitric acid in the production of concentrated sulphuric acid. If nitric acid be mixed with sulphurous anhydride, which is obtained in the manufacture of sulphuric acid by roasting sulphur ore, the sulphurous acid withdraws oxygen from the nitric acid and passes over into sulphuric acid, while the nitric acid becomes nitrous acid. If the constant entrance of fresh air and water be provided, the nitric acid is constantly reformed from the nitrous acid and gives a part of its oxygen again to new quantities of sulphurous acid, so that the molecule of nitric acid is continually being alternately broken down with loss of oxygen and built up with absorption of oxygen. In this manner with the same quantity of nitric acid an unlimited quantity of sulphurous acid can be changed into sulphuric acid. Thus here in a simple form, i.t., in a simple chemical compound, is a regular metabolism, a succession of destructions and constructions of a substance along with the gain and loss of substances, which correspunds in principle, even 
to its details, to the metabolism of organisms ; nevertheless, nitric acid is an inorganic compound,

Such phenomena are relatively rare and occur in free nature, where their conditions are not artificially established by human agency, only very seldom. Nevertheless, they do not permit the presence of a metabolism to be maintained as an absolute difference between living organisms and inorganic bodies.

Thus the fact has been established that a fundamental contrast between living organisms and inorganic bodies does not exist. In contradistinction to all inorganic nature, however, organisms are characterised solely by the possession of certain highly complex chemical compounds, especially proteids.

\section{B. LIVING AND LIFELESS ORGANISMS}

\section{Life and Apparent Death.}

In India, where mystery and magic have always prevailed, the belief seems to have existed for a long time, that many men, especially the so-called fakirs, whose existences are full of privation and self-inflicted torture, and who are supposed to possess special holiness, have the remarkable power of voluntarily putting a complete stop to their lives for a time and later resuming them undisturbed and unchanged. A great number of such cases, in which the fakirs have been buried in this condition of suspended animation and after some time have been taken from their graves, have been reported by travellers from India. James Braid ("50), the wellknown discoverer of hypnotism, has collected some of the most authentic cases, and supported them by the testimony of witnesses. One of these cases, which may serve as a type, is the following:

At the palace of Runjeet Singh, in a square building which had in the middle a closed room, a fakir, who had voluntarily put himself into a lifeless condition, had been sewed up in a sack and walled in, the single door of the room having been sealed with the private seal of Runjeet Singh. (To judge from the account, the air, as in all such cases, was not absolutely excluded.) In order to exclude all fraud, Runjeet Singh, who was not himself a believer in the wonderful power of the fakirs, had established a cordon of his own body-guard around the building; in front of the latter, four sentries were stationed, who were relieved every two hours and were continually watched. Under these conditions, the fakir remained in his grave for six wecks. An Englishman, who was present during the whole event as an eye-witness, reported as follows concerning the disinterment, which took place at the end of six weeks: When the building was opened in the presence of 
Runjeet Singh, the seal and all the walls were found uninjured. In the dark room of the building, which was examined with a light, the sack containing the fakir lay in a locked box, which was provided with a seal likewise uninjured. The sack, which presented a mildewed appearance, was opened, and the crouching form of the fakir was taken out. The body was perfectly stiff. A physician who was present found that nowhere on the body was a trace of a pulse-beat evident. In the meantime the servant of the fakir poured warm water over the head, laid a hot cake upon the top of the head, removed the wax with which the ears and nostrils were stopped, with a knife forcibly opened the teeth, which were tightly pressed together, drew forward the tongue which was bent backward and which repeatedly sprang back again into its position, and rubbed the closed eyelids with butter. Soon the fakir began to open his eyes, the body began to twitch convulsively, the nostrils were dilated, the skin, heretofore stiff and wrinkled, assumed gradually its normal fulness, and a few minutes later the fakir opened his lips and in a feeble roice asked Runjeet Singh, "Do you believe me now?"

Similar cases are reported in great number by more or less reliable witnesses. An analogous instance was observed in Europe, and is cited likewise by Braid. It is the well-known case of Colonel Townsend, of whom Dr. Cheyne, a physician of Dublin, well-known in scientific circles, narrates as follows:

"He could die or expire when he pleased, and yet, by an effort or somchow, he could come to life again. He insisted so much upon us seeing the trial made that we were at last forced to comply. We all three felt his pulse first: it was distinct, though small and thready, and his heart had its usual beating. He composed himself on his back, and lay in a still posture for some time; while I held his right hand, Dr. Baynard laid his hand on his heart, and Mr. Skrine held a clear looking-glass to his mouth. I found his pulse sink gradually, till at last I could not feel any, by the most exact and nice touch. Dr. Baynard could not feel the least motion in the heart, nor Mr. Skrine perceive the least soil on the bright mirror he held to his mouth. Then, each of us, by turns, examined his arm, heart, and breath; but could not, by the nicest scrutiny, discover the least symptom of life in him. We reasoned a long time about this odd appearance as well as we could, and finding he still continued in that condition, we began to conclude that he had, indeed, carried the experiment too far; and at last we were satisfied that he was actually dead, and were just ready to leave hin. This continued about half an hour. By nine in the morning, in autumn, as we were going away, we observed some motion about the body, and upon examination found his pulse and the motion of his heart gradually returning: he began to breathe heavily and speak softly. We were all astonished to the last 
animalcules, or Rotatoria, whose bodies, extended like a telescope, have at their anterior end a locomotor organ provided with stout cilia, which on account of the apparently wherl-like motion of the cilia has been termed the wheel-organ. Besides the Rotatoria there are found chiefly the so-called bear-animalcules, or Tardigradr, clumsy mite-like animals provided with four pairs of short stumps of feet, bearing claws; like the Rotatoria they are provided with a nervous system, digestive apparatus, etc. (Fig. 41 a). So long as this latter peculiar animal is in water, it performs all its vital phenomena like other animals. But if it be isolated and allowed to dry slowly upon a slide, it is seen that the more the water evaporates, the slower become its movements, until finally they cease entirely when the drop is dried up. Then the body gradually shrinks, the skin becomes wrinkled and folded, the form becomes gradually indistinguishable, and some time after the animal has become dried up it can scarcely be distinguished from a grain of sand (Fig. 41 b). In this dried condition it can remain for many years without undergoing the slightest change. If it be moistened again with water, the return of life to the desiccated body after its sleep can be followed with the microscope. The awakening of the tardigrade, or the anabiosis, as Preyer ('80) has termed the process, takes place somewhat as follows: The body swells up and becomes extended, the folds and wrinkles slowly disappear, the extremities project, and the animal soon assumes its normal shape. At first it remains quiet; then, after a time, varying, according to the duration of the drying, from a quarter of an hour to several hours, movements, at first slow and feeble, begin and gradually become stronger and more frequent, until after some time the animal, unaided, creeps away to resume life at the point where it was interrupted.

These highly remarkable phenomena of anabiosis are not limited to the Rotatoria and the Tardigrada. They have been noticed likewise in various other organisms in the course of investigations which in great number followed Leeuwenhoek's discovery. They have been observed in the so-called paste-eels, or Anguillulido, the small eel-like worms that live in diseased wheat-grains, in Infusoria and Amoso, and in Bacteria.

In the same group of facts belongs also the long-known capacity of plant-seeds to remain dry for many years unchanged without losing their power of sprouting; indeed, it has even been believed that this power can continue for an unlimited time. The statements are well known that wheat-grains found in the graves of Egyptian mummies after a rest of many thousand years have sprouted and bloomed. It has been settled, however, that these reports rest upon a delusion, for Mariette, the well-known Egyptologist, has shown that with genuine mummy wheat these experiments always fail. since all wheat-grains taken from the graves 
have a charred appearance, and, when brought into water, disintegrate into a clayey pulp. Nevertheless, from several observations it appears certain that many plant-seeds, when completely dried, can retain their power of sprouting for more than a hundred, perhaps for more than two hundred, years.

These rare facts are of great importance in forming a conception of life, and demand exhaustive investigation. The question to be considered is whether it is allowable to regard organisms in this peculiar condition as really lifeless.

Theoretically, in its most general expression, the distinction between living and lifeless organisms meets with no great difficulties. Our conception of life has been formed from the observation of certain phenomena which appear only in living organisms, in other words, vital phenomena. Wherever we observe vital phenomena we speak of a living organism. This characterisation of the conception of life can be simplified still more. If, for example, all the varieties of vital phenomena be recalled, it is found that they arrange themselves into three great groups,-those of metabolism, or change of substance, those of change of form, and those of transformation of energy. Every living organism exhibits changes in its component materials, since it continually takes in substances from the outside and gives off others to the outside; it exhibits changes of its form, since it develops, grows, and reproduces by constricting off certain parts; and it exhibits changes of its energy, since it transforms the chemical energy received with its food into other forms of energy. But these changes are not three wholly different processes, which are independent of one another; they are, rather, different kinds of phenomena of one and the same process. No substance exists without form or energy. Substance, form, and energy are simply the three phases in which the physical world can manifest itself in phenomena, in which matter can be considered. Every change of substance necessitates a simultaneous change in the two other phases, although in a given case one phase is more evident to the senses than another. Hence it can be said that in a general sense the vital process, the outward expression of which is perceived in the various vital phenomena, consists in changes of substance, or, in brief, metabolism. Accordingly, it is metabolism in which the living organism differs from the lifeless.

Practically, i.e., in a concrete case, this distinction is not always so simple, as is evident from the case of desiccated organisms. In accordance with the above considerations, it is a question whether these organisms in their peculiar condition possess really no metabolism, or whether their metabolism is simply depressed to so slight a degree that it is not apparent to our unaided senses in the form of vital phenomena, i.e., whether the life-process is at an actual standstill, or whether only a vita minima exists. The decision of this question is possible only by means of the most 
refined and careful methods of research. The majority of investigators have always believed that in such dried organisms there is really a complete standstill of life; but the objection has always been possible that the metabolism in this condition may be so slight that with the minuteness of most of the objects it cannot be proved by the usual methods of investigation. The experiments carried on recently by Koch' ('90) are likely to refute this objection completely. Dried animals, isolated upon a clean glass slide, take in no solid or liquid food, and direct observation shows likewise that no outgo of liquid or solid matters takes place. But Kochs has demonstrated in the following way that a respiration, i.e., an in-take of oxygen and an out-put of carbonic acid, is never present. He selected for his experiments various plant seeds, completely dried, and placed a considerable quantity of them in a wide glass tube; he extracted the air as much as possible by means of the air pump and then sealed the tube by melting. If only a slight metabolism were present in the seeds, with their considerable quantity at least a trace of expired carbonic acid could have been found. But, when after several months Kochs investigated the contents of the tube by the most delicate methods, he found not the slightest trace of expired carbonic acid or any other product of metabolism. These experiments were repeated always with the same result. Nevertheless, the seeds remained capable of life and sprouted upon being sown.

From the results of these experiments it can no longer be doubted that in desiccated organisms there is a complete standstill of life. Can organisms in this peculiar condition be termed dead? In reality they are lifeless but not dead, for anabiosis is possible after the application of water, while nothing can bring dead organisms back to life. The distinction between the dried and the dead organism lies in the fact that in the former all the internal vital conditions are still fulfilled, and only the external conditions in part have disappeared, while in the latter the internal vital conditions have experienced irreparable disturbances, although the external conditions can still be fulfilled.

Preyer illustrates this distinction very happily. He compares the dried organism to a clock that has been wound but has stopped, so that it needs only a push to set it going, and the dead organism to a clock that is broken and cannot be made to go by a push. Hence a sharp distinction must be made between dried and dead organisms. But dried organisms cannot be call'd living, for they exhibit no vital phenomena, and, as has been seen, vital phenomena are the criterion of life. It is best, therefore, to apply to them the expression "apparently dead." Claude Burnard has termed the condition of apparently dead organisms " vie latente" (latent life), an expression which Preyer has replaced with "potentielles Leben" (potential lifo), in contrast to the usual or "actuelles Leben" (actual life) of the normal organism. To use a German expression, 
it may be said that such organisms exist in the condition of "Scheintod" (apparent death).

\section{Life and Death}

It has been seen that the determination of the difference between life and apparent death is beset with practical difficulties, since it is not easy to decide experimentally whether the life-process in reality is at a complete standstill in dried and apparently dead organisms. It is still more difficult to determine theoretically a sharp limit between life and death.

In daily life it is easy to distinguish the dead organism from the living; for from the human body and from the higher animals we have formed a general conception of death, and are accustomed to consider it as nccurring at the moment when the heart, hitherto never quiet, stands still, and the individual ceases to breathe. But we here follow the superficial habit of daily life and take into consideration only the gross differences that make their appearance at that time, without noticing the continuance of certain phenomena after this all-important moment.

The criterion of life is formed only by the vital phenomena, i.e., by the various phases in which the vital process, or the metabolism, becomes evident to the senses. But if this criterion be applied to the human being at the moment usually termed the moment of death, it is found that in reality he is not then dead. A careful examination shows at once the truth of this statement.

It is true that the spontaneous gross muscular movements cease, the man becomes relaxed and quiet. But the muscles frequently remain for several hours sensitive to external influences, responding to the latter with twitchings and movements of the limbs, in other words showing vital phenomena. A moment even comes when the muscles gradually contract once more spontaneously, this is the death-stiffening (rigor mortis). Not until this has passed is the life of the muscles extinguished. Nevertheless, even then the body is not entirely dead. Certain parts only, certain organs or cellcomplexes, such as the cells of the nervous system and of the muscles, no longer show vital phenomena; but other cells and cellcomplexes continue to live unchanged long after rigor mortis has passed. As is well known, the inner surface of the air-passages, the larynx, the trachea, and the bronchial tubes, is covered with a ciliated epithelium, a layer of cylindrical cells pressed tightly together and bearing upon their surface fine hair-like appendages, with which they perform a continual, rhythmic, beating motion ( $C f$. Fig. $20 \alpha$, p. 78). These ciliated cells continue their normal activity in the corpse for days after the cessation of the heart, and thus survive after the so-called death. But even after several days the whole body is not always dead. The white blood-corpuscles, or leucocytes, the amœboid cells that are not only carried about passively in the 
blood-current but also wander about actively in all the tissues of the body and play an important role in the organic household, remain in great part living, and, if kept under favourable conditions, can live still longer.

What moment then shall be designated as the moment of death? If the existence of vital phenomena be employed as the criterion, then the moment when spontaneous muscular movement, especially the activity of the heart, ceases, cannot consistently be regarded as the moment of death, for other cell-comiplexes continue to live for a long time thereafter. We see, therefore, that there is no definite point of time at which life ceases and death begins; but there is a gradual passage from normal life to complete death which frequently begins to be noticeable during the course of a disease. Death is developed out of life.

The history of death is very different in the different classes of animals. In the warm-blooded animals death develops relatively rapidly after the standstill of the blood-circulation, as a result of the great dependence of all tissue-cells upon nourishment from the blood-current. The cold-blooded organism passes from life to death as a rule much more slowly; the definitive death, i. , the state in which no further vital phenomenon can be perceived in the body, appears in many cases only months after the animal has experienced an irreparable, fatal injury. In harmony with the greater independence of the individual organs in respect to the blood-circulation and one another, in many cold-blooded animals. individual parts also, when severed from the rest of the body, can survive for a long time, - a peculiarity upon which depends the special usefulness of such animals, e.g., frogs, for many physiological investigations. It is well known that a muscle with its nerve can be removed from a frog's body, and under proper conditions can be maintained for experimentation alive and in an irritable condition for days. The fact appears here much more clearly than in the case of man, that death is not a condition that is established in a moment, but is developed very gradually.

It may be said that in all the cases mentioned multicellular animals are under consideration, and in them one kind of cell suffers death earlier, the others later; but how is it with the single cell, which in itself represents a living organism? The history of cell-death corresponds exactly with the development of death in the multicellular organisin, except that in the former the various important points appear much more clearly. We see here also that death does not occur suddenly, but that normal life is united with definitive death by a long series of transition-stages, following one another uninterruptedly, and frequently extending through several days or, not rarely, several weeks. We have already become abundantly acquainted with the fact that nonnucleated protoplasmic masses that have been cut off from a cell do not continue living. If such a separated pice of protoplasm, 
which possesses no nucleus and whose fate is therefore sealed, be observed with the microscope, it can be seen that it passes from its normal behaviour to complete standstill of all its vital phenomena only very gradually. ${ }^{1}$ Certain marine species of Rhizopoll", r.g., Orbitolites, are well fitted for this observation; they stretch out through the pores of their calcareous shell clusters of naked non-nucleated protoplasmic threads, or pseudopodia, of considerable length, and by means of them they move, seize foodorganisms and digest fond. If such a mass of pseudopodia be cut off fron an Orbitulites under the microscope, the network of threads first flows together into a roundish droplet, which thereupon immediately stretches out new pseudopodia of the same form as in the uninjured organism, and moves as if in connection with the nucleated body. The new pseudopodia also seize food-organisms, but ar' not able to digest them. This latter fact is very important, for from it follows the fact that the non-nucleated protoplasmic droplet is not able to manufacture new body-substance. The movements of these microscopic bodies continue normal for hours, and their irritability is also maintained. But the pseudopodia are very gradually drawn in, while new ones are no longer protruded, and as a result the mass draws itself more and more into a spherical lump. It cannot yet be said that the protoplasmic mass is dead, for even upon the next day, if the object be observed at intervals of several hours, extremely slow, feeble changes of form can be perceived. Only after several days does the protoplasmic droplet swell up and disintegrate into a loose mass of granules.

Thus, death does not come to the cell immediately, but is the end-result of a long series of processes which begin with an irreparable injury to the normal body, and lead by degrees to a complete cessation of all vital phenomena. Since during the course of this process rital phenomena are still noticeable, while death as a result of the injury is unavoidable, it is advantageous to characterise by a name the time from the receipt of the fatal injury up to the definitive death as a time of uninterrupted transitions. Extending a conception introduced into pathology by K. H. Schultz and Virchow (71), I shall term it necrobiosis.

It is seen, therefore, that is impossible to draw a sharp line between life and death, that life and death are only the two endresults of a long serit's of changes which run their course successively in the organism. But if, after having established this fact, the transition-stages be left out of consideration for the moment and only the two end-results be considered, on the one side, the uninjured living organism and, on the other, the same organism killed and preserved in alcohol by the mulern technical methods, a sharp distinction between these two can be recognised in the fact that in the former the life-process gues on undisturbed, as is

${ }^{1}$ Cf. Verworn ('91). 
evident from the appearance of all vital phenomena, while in the latter it is for ever at a complete standstill, as is shown by the absence of even the slightest phenomena of life.

We are now in position to add a capstone to our characterisation of living substance-in other words, to characterise in general terms the vital process itself.

It has been shown that a fundamental difference-i.e., a difference in the elementary materials and the elementary forces-between organisms and inorganic bodies does not exist. The vital phenomena of organisms must, therefore, depend upon the same general mechanical laws as the phenomena of the inorganic world. Burt a difference does exist between the two great groups of bodies in respect to the kind of chemical compounds in which the elementary materials are associated, since in organisms generally certain highly complex compounds occur, especially proteids, which are never wanting in living substance, and are never found in the inorganic world. It is evident that this difference is of the same kind as the differences that exist between the various inorganic bodies themselves as regards their chemical composition. Nevertheless, in the possession of the complex proteids organisms have something in common in contrast to all inorganic bodies.

Further, it has been shown that living differ from lifeless organisms, whether the latter be apparently or really dead, by their metabolism-i.e., by the fact that their substance continually breaks down spontaneously, is regenerated, and accordingly continually gives off substances to the outside and receives other substances from the outside. The kind of product arising from this decomposition shows that nitrogenous compounds, especially proteids, are involved in it. Since it is known that the nitrogenous proteids, with their allies, which in part are derived from the proteids and in part are necessary to their formation, are the sole organic compounds that are never wanting in living substance, that everywhere they constitute its chief mass and alone are sufficient for its formation, it can be said that all living organisms are characterised by the metabolism of proteids.

We can thus summarise our considerations so far, and at the same time give simple expression to the problem of all physiology. The life-process consists in the metabolism of pruteids. If this be true, all physiological research is an experiment in this field; it consists in following the metabolism of proteids into its details and recognising the various vital phenomena as an expression of this metabolism which must result from it with the same inevitable necessity as the phenomena of inorganic nature result from the chemical and physical changes of inorganic bodies. 


\title{
CHAPTER III
}

\author{
ELEMENTARY VITAL PHENOMENA
}

WHAT is called life is a series of vital phenomena very unequal in importance. As regards most of the activities that constitute the daily life of mankind, some are composed of elementary phenomena, and some are secondary results of elementary phenomena. Eren those that are apparently simple and direct, such as the circulation of the blood and respiration, are not elementary. The elementary phenomena are the contraction of the heart and the respiratory muscles, which secondarily accomplish the circulation of the blood and the exchange of air in the lungs; for muscle-contraction cannot be reduced to the activity of other elements, it is the direct expression of the life of those cells in which it appears. If we wish to become acquainted with the elementary rital phenomena, we must go back to the cells in which they appear.

If all complex activities and secondary phenomena be traced back to the elementary vital phenomena that lie at their foundation, three great groups of the latter are found, which in some form are peculizr to all living substance, to every cell ; these are the phenomena associated with changes of substance, of form, and of energy. All living substance without exception, so long as it lives, shows continual changes of its material, alterations of its form, and transformations of its energy; and all vital phenomena whatsoever, when resolved into their elements, may be placed in one or more of these three great groups. In this chapter we shall endeavour to obtain a comprehensive riew of vital phenomena by recording the facts, and shall leave to a later chapter the reduction of them to mechanical causes.

\section{The Phenomena of Metabollism}

\section{A. THE INGESTION OF SUMSTANCES}

"Nourishing," in the widest sense, signifies the whole process involved in the taking-in of food-stuffs from the environment. In 
the case of the compound organism, eating and drinking constitute merely an extrinsic part of the process; whatever is thus brought to a single organ, the stomach, is for the good of each one of the many millions of cells that constitute the body. If the life of the body is to be maintained, all cells must take in certain food-substances. The following consideration must, therefore, cover two points-first, the nature of the substances that every cell needs in order to maintain its life, and, second, the mode of ingestion of those substances.

\section{Food-stuffs}

All living matter is continually undergoing decomposition and, hence, must take in substances that contain all the chemical elements of which it is constructed.

While it is a vital phenomenon of erery cell to take in foodstuffs, the latter differ in kind with every form of cell. But in spite of all specific differences in the substances that each form of cell requires for its life, all organisms may be classified into a few large groups, within each of which a general agreement in the kind of nutrition prevails.

A fundamental difference in the nutrition of plants and of animals was discovered early. All green plants take up from the earth and air simple inorganic materials from which to construct their living substance; on the other hand, all animals without exception, in order to be able to maintain life, require highly complex organic compounds.

This fact is easily confirmed. In order to prove that animals cannot exist without organic food, it is only necessary to perform suitable feeding experiments. When fed with purely inorganic matters, such as water, salts, etc., even when these contain all the chemical elements of living substance in the correct proportion, animals always die after a longer or shorter time. On the other hand, it can be shown that plants live solely at the expense of inorganic substances, by allowing them to grow in so-called nutrient solutions, which possess in the form of inorganic salts the chemical elements that are necessary to the formation of living substance. Such a nutrient solution, which contains in soluble compounds the elements $\mathrm{N}, \mathrm{H}, \mathrm{O}, \mathrm{S}, \mathrm{P}, \mathrm{Cl}, \mathrm{K}, \mathrm{T}$ a. $\mathrm{Mu}$. Ca, Fe, i.e., with the exception of carbon, all organic elements, is cumposed, according to Sachs ('82), as follows:-

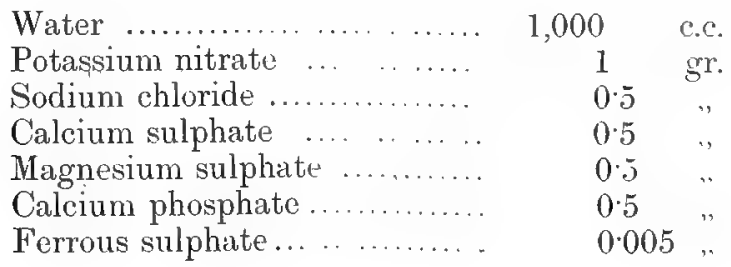


If the root of a grain of corn that has sprouted in water be placed in a cylinder containing this nutrient solution, while the upper parts project into the air (Fig. 42), the plant, when placed in the light, grows well, derelops into a large stalk, flowers and produces seed with which the experiment can be repeated. If the iron salt be wanting in the nutrient solution, the plant grows likervise for some time, but remains colourless, and microscopic examination of the leares: shows that the chlorophyll is wanting in the cells. Only after the addition of a trace of iron sulphate do the leaves become green.

As a glance at the contents shows, no carbon is present in the nutrient solution. Since, however, under all circumstances the plant requires carbon for building its organic substance, in its growth it must have taken carbon from the air; hence it is necesiary that the experiment be arranged so that the upper parts of the plant project into the air. If the air be excluded by a bell-jar, in a short time the plant dies. Carbon is contained in the air only in the form of carbonic acid; hence the plant must withdraw it from this compound, and, in fact, it appears that, when a certain quantity of carbonic acid is left under the bell-jar, after a short time all is consumed. This important fact, that the plant supplies its need of carbon solely from the carbonic acid "If the air, was discovered by Ingenhouss and de Saussure, and, after having been doubted for a lung time, now forms one of

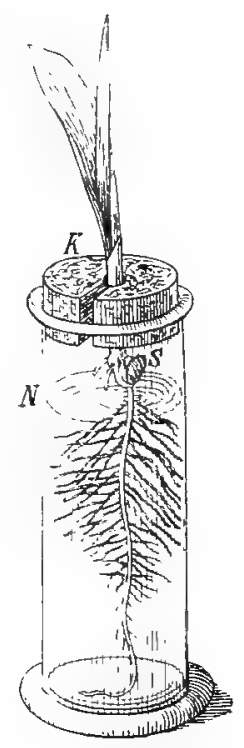

FIG. 42.-Corn-plant growing in a cylinder contrining is nutrient solution. $r$, Nutrient solution; $s$, grain of corn ; $\bar{k}$, cork. (After Sachs.)

The plant: nitrogen, however, as an experiment analogous to the above shows. cannot be extracted from the air; it is taken up sulely from the nitrogenous salts of the witer.

It follows from these experiments that plants construct their living substance out of simple inorganic compounds, from the carbonic acid in the air, which is taken up by the leares, and from the watur containing salts, which reach the plant through its roots. In contrast to this, no animal is able to build its living substance synthetically from simple inorganic compounds, even when all the chemical elements of its body are contained in them: all animals without exception require organic material already prepared.

This contrast between animals and plants is very significant, for it expresses the important fact that the animal world cannot exist without the plant world. It is true that a great number of 
animals exist, such as carnivora, which require only animal foodstuffs, especially flesh; but, if the source of their food be sought, it is always found ultimately in herbivora, and the latter cannot live without plant-food. Thus, the carnivora depend ultimately upon the existence of plants. Without plants all animals would die, for plants alone are able to manufacture from inorganic substances the carbohydrate, the fat and the proteid that animals require for their existence. The old philosophy of nature, prevalent at the beginning of the present century, was, hence, not entirely incorrect when in this sense it termed the whole animal world parasites of the plants.

For a long time it was believed that this difference in the nutrition of animals and plants is an absolute one, that all living cells, as regards their metabolism, can be divided simply into animal-and plant-cells. But it has been found that the difference txists only within certain limits, viz., only so far as animal-cells and green, i.e., chlorophyll-containing, plant-cells are concerned, for those constituents of the plant-cell in which carbonic acid is received and elaborated are exclusively the green chlorophyllbodies. There are plants without chlorophyll-e.g., the fungi-which in their metabolism form to some extent a transition between animals and green plants.

The fungi do not have the power of the chlorophyll-containing plants to extract carbon from the carbonic acid of the atmospheric air; in order to satisfy their need of carbon they require, like animals, organic substances, such as proteid, carbohydrate, etc. On the other hand, the fungi behave like plants in so far as they satisfy their need of nitrogen from the inorganic salts of the earth, while animals obtain their requisite nitrogen only from proteids and their derivatives. These facts follow from experiments with nutrient solutions, in which fungi do not grow when no organic material is at their disposal ; if, however, besides nitrogenous salts, sugar be added to such a solution, they grow vigorously. Thus, the fungi constitute a group of organisms which, as regards their metabolism, combine half animal and half plant characters. But still other relations occur in nature; for among micro-organisms numerous entirely similar transition-forms occur, and the more the very peculiar life-relations of these microscopic beings, especially the Bacteria, are investigated, the more it appears that in this group of lowest organisms the metabolic relations in general are not so sharply differentiated as in the higher organised animals and plants. Thus, very recently the clever investigator, Winogradsky ('90), has discovered Bacterio that live in the earth and construct their living substance entirely from inorganic material, chiefly ammonium carbonate and certain mineral substances. These remarkable nitrogen-bacteria (Nitromonas), therefore, although they possess no chlorophyll, behave exactly like 
green plants. Other forms of Bacteria cannot exist without organic food.

To glance at the more special nutrition of animals, as regards the organic food-stuffs a considerable difference prevail: between individual species. There are remarkable adaptations to single food-stuffs. Thus, the caterpillar of the fur-moth lives exclusively upon the hairs of fur, which consist of pure keratin. Keratin, which is closely allied to proteid, is, therefore, capable of furnishing all the elements for the formation of the living substance of the fur-caterpillar. In other cases, e.g., in carnivora, proteid alone suffices to supply all the elements necessary to the formation of the body; and lately Pflüger ('92) has shown by detailed experiments that even dogs, when forced to perform hard labour daily, can live continually upon pure proteid food. In such experiments, after a short time the dogs lose almost all their body-fat, but remain abundantly capable of work, strong and healthy. On the other hand, it is impossible to maintain an animal's life with carbohydrates or fats solely, or even with the two together. In spite of an abundance of such food, the animals consume their own body-proteid, as shown by the continual excretion of nitrogen in the urine, and finally grow weaker and die. The reason for this is evident, for, since the living substance is constantly breaking down of itself in a definite quantity, it must constantly be reconstructed if the animal is to live. But this cannot happen if no nitrogen, which is lacking in carbohydrates and fats, be given to the animal. Since, however, as has been seen, animals cannot take up nitrogen from inorganic compounds, it follows that proteids, which alone represent the nitrogenous foodstuffs, are absolutely necessary for the maintenance of animal life. Hence we arrive at the important fact that of all organic substances proteids alone are indispensable to the nutrition of animals, and in certain cases also they alone suffice to maintain the animal's life. Pfliger, therefore, distinguishes proteid as the primitive food from the carbohydrates, fats, etc., which act only as substitute foods.

In addition to food proper in the narrow sense, all organisms take in oxygen - a process that is termed respiration. Of course all organisms do not receive oxygen in the same form and from the same source. Terrestrial organisms take it in the form of gas from the air; aquatic organisms use the oxygen dissolved in the water; and the tissue-cells of animals that are provided with a blood-circulation, as well as many parasitic organisms, withdraw it from chemical compounds-the tissue-cells from the hæmoglobin of the blood, with which it is loosely combined, and certain parasites from relatively fixed combinations. All organisms take only a certain quantity of oxygen, even when more is offered; their consumption of it is not essentially increased in a medium of pure 
oxygen. Hence within certain limits living substance is fairly independent of the quantity of oxygen that is at its disposal. But all organisms withont exception absolutely require for their life a certain quantity of oxygen. If separated from it they invariably die after a shorter or longer time. Without respiration no life exists.

Finally, all organisms without exception take in water, and with it certain sults, which, in so far as they are not contained in the other food, are likewise essential to the maintenance of life, although wide differences prevail among the different organisms as regards the kind of salts required. Salts of sodium, potassium, magnesium, calcium, and iron, containing phosphorus, sulphur, carbon, and chlorine, appear to be essential to all organisms.

We have thus reviewed the food-stuffs of organisms; we will now consider how the individual cell takes in this food.

\section{The Mode of Food-Ingestion by the Cell}

Food-stuffs exist partly in the gaseous, partly in the liquid, i.e., dissolved, and partly in the solid condition; but by no means all living colls are able to take in solid food. The great majority of all cells, almost all animal tissue-cells, a great number of plantcolls, and many unicellular organisms take in dissolved food only, the latter either primarily consisting exclusively of dissolved substinces, or being transformed from the solid to the dissolved state by the agency of certain secretions outside the cell-body. Only relatively few kinds of cells are fitted for the ingestion of solid food.

The process of ingestion of gaseous and dissolved food-stuffs, which is termed resorption, is essentially different, according as the cells in question do or do not possess a cell-membrane. In cells that do not possess a membrane all dissolved food-substances of whatever kind pass directly into chemical relations with the materials of the living substance at the surface of the protoplasm. Where a membrane is present, it is necessary that the food-stuffs have the power of diffusing through membranes. The substances that cannot do this must, therefore, first be transformed into diffusible substances in order to reach the interior of the cell.

Every cell, however, is capable of ingesting gaseous and dissolved food.

In plants the carbonic acid and oxygen of the air come into rirect contact with the cells of the leares. A similar arrangement is found in the lungs of vertebrates. The finest branches of the bronchial tubes end in small blind sacs, the so-called pulmonary alveoli, which are formed by an extremely thin layer of epithelium-cells and are surrounded by a cluse network of likewise very thin-walled blood-capillarien. The axygen of the air inspired 
into the lungs passes readily through the thin walls, to be eagerly sucked up by the red blood-corpuscles and transported throughout the whole body.

Dissolved -ubstances also always bathe the surface of the cells. In the plant they ascend along with the water in fine tube-like canals and thus are brought directly to the cells. In the compound animal body some of the cells, such as those of the intestinal epithelium, are in immediate contact with the dissolved food-stuffs of the intestinal tract, while all the other tissue-cells are bathed by the blood-current, which brings to them the dissolved food in a definitely elaborated form. In such invertebrate animals also as possesis no proper blood-circulatory system, the cells either stand in immediate contact with the surrounding water or are supplied with juices that bathe the cells in fine intercellular spaces. The simplest relations, finally, exist in unicellular organisms, such as Algce, Bacteria, and others, which live constantly in a nutrient solution, either in water containing salts or in organic liquids.

The ingestion of solid food occurs in only a few cell-forms. Among unicellular organisms all Rhizopoda, most ciliate Infusoria, and some flagellate Infusorir, take in solid food. In the complex cell-community this power is possessed by the leucocytes or white blood-corpuscles, which, therefore, have been termed by Metschnikoff phagocytes (eating cells), by amoboid wandering cells, which play in the lower animals the rôle of leucocytes, by amœboid egg-cells, such as occur in sponges, and by the intestinal epitheliumcells. Among these forms of cells two types may be distinguished, according to the manner of ingestion of solid food. The one type is able to take the food-masses into its living substance at any desired point upon its surface-such are all amœboid cells, to which belong Rhizopoda, leucocytes and intestinal epithelium-cells; the other type possesses a special, constant mouth-opening-such are the ciliate and the flagellate Infusoria, which have a definitely fixed body-form with a denser cutaneous layer. All cells, however, that take in solid food are able to do it only by means of active movements of the body.

The ingestion of food by Amoza may serve as an example of the first type. The process, which has been observed in full only relatively seldom, takes place somewhat as follows. An $A m o b a$, which is being observed in a drop of water under the microscope, creeps about the glass slide by letting the living substance of its formless protoplasmic body flow here and there into broad, lobate projections (Fig. 43). Suddenly it turns toward a small alga-cell lying in the vicinity, and creeps on until it touches the cell. Its protoplasm immediately begins to flow around the latter in the form of the usual lobate pseudopodia; but the cell is shoved away by the encroaching protoplasm and the amoeba is obliged to make a new attempt to surround the cell. After several fruitless attempts it 
frequently succeeds in bringing the eell into such a yosition and so holding it fast by a delicate viscous secretion that its pseudopodia are able to grasp the alga completely. Then, by flowing more and more about the cell, the protoplasm encloses it gradually on all sides, and the alga finds itself surrounded by a thin covrring of water, forming the so-callerl food-vacuole, in the interior sf the amoba, which then crouss on unhindered. Amoba, therefore, takes in solid food by causing its protoplasm simply to surround the food-mass. But the act does not always go on so smoothly. The difficulties that arise before the food-mass, which yields continually to the pressure of the encroaching protoplasm, is so fixed that the protoplasm can enclose it upon all sides, are frequently so great that not rarely the amoba, with its pseudopodia flowing on continually in

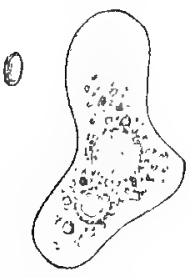

a

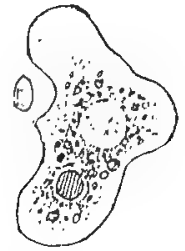

b

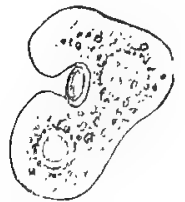

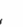

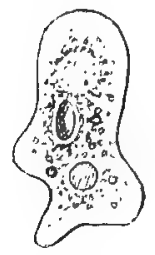

a

Fic. 43.-Anowba devouring an alga-cell. Four sinceessive stagos of the prucess of fuod-ingestion.

other directions, is taken away from its victim, and nust creep toward it anew in order to seize it, if it has not been taken entirely out of the sphere of influence of the food-mass.

The ingestion of food by other Rhizopude takes place exactly an in the case of Amoba, whether they have pseudopodia that arc thick and broad, tine and thread-like, or branched and tree-like. If the food-bodies are motile organisms, c.g., Infusoria, they usually cause the excretion of a viscous substance by stimulation resulting from their swimming against the rhizopod body; this is increased by stimulation arising from their attempts to escape; hence they stick firmly and can be drawn into the protoplasm. The amoboid wandering-cells and lencocytes also, like Amatia, ingest solid substances which exist in the blood or in the interstitial spaccs between the cells. As the admirable work of Metschnikoff ('83, ' 84 ) has lately shown, they possess very great importance in the protection of the body from infectious diseases by devouring the bacteria that have entered a wound; the $y$ thus prevent the increase of the bacteria and protect the body from further infection (Fig. 44). Finally, the ingestion of microscopic fat-droplets on the part of the intestina] epithelium-cells represents the same inode of food-ingestion. In lower animals-e.g., in worms-these colls are really amoboid cells, and by means of their pseudoporlia flow around the fat-globules of 
the digested food (Fig. 45, $A$ ). In the higher animals, such as man and other mammals, however'. the intestinal cells are somewhat. modified. They are cylindrical cells that jussess upon their free surface, turned toward the lumen of the intestine, a striated border. As Thanhoffer ('74) has shown, this striated border represents really nothing more or less than fine, pseudoporliumlike, protoplasmic processes, which can be extended and retracted, and with which the cells, exactly like Amerece, flow around the fat-droplet and draw it into its body (Fig. $45, E$ ).

The phenomena are wholly different in the seconrl type of foodingestion, where the cell has a firmer superficial layer of a fixed form, and only a small opening, the cell-mouth, which leads directly into the liquid endoplasm. Here the movement of the cilia and flagella of the cell exclusively mediates the ingestion of solid substances. The delicate $V o r-$
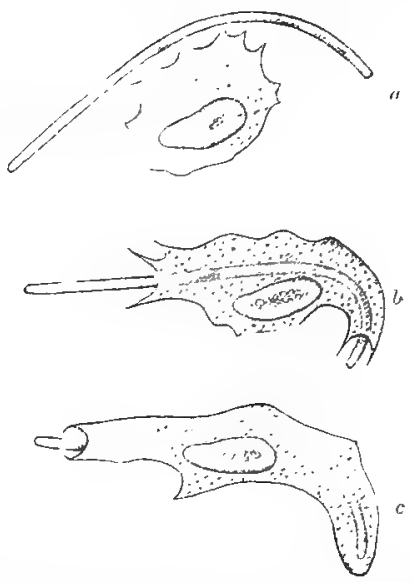

Fic. 44.-Leucocyte from the frog devoming a bacterium. Three suc. .ersive stages in the ingestion of fiml. (After Metschnikoff.)

ticella may serve as an example, a ciliate infusorian whose bell-shaped cell-body sits upon a contractile stalk and bears at its broad end a spiral-like circlet of cilia (Fig. 46).
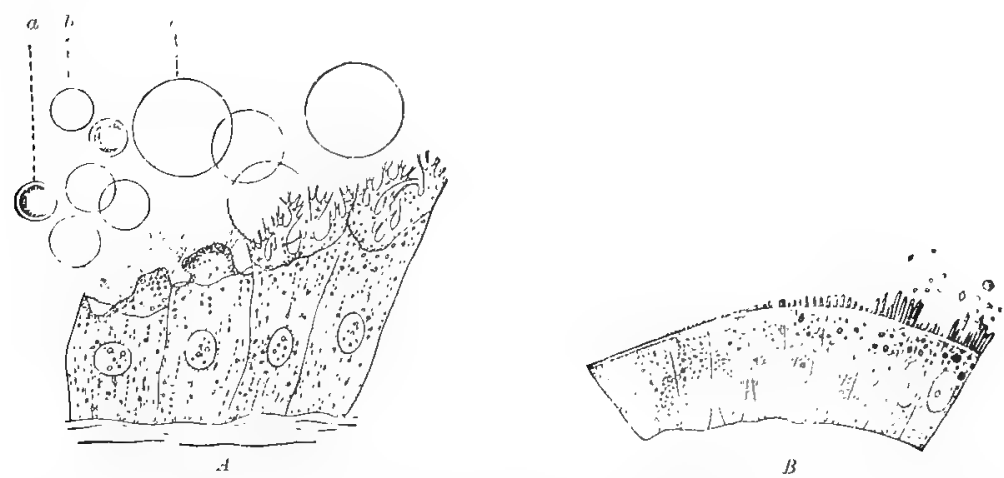

$B$

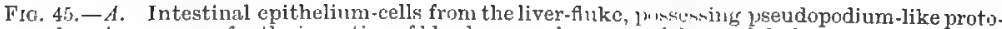
plasmaic processes for the ingestion of blood-corpuscles, $a, b, i n d$ drops of ehyle, $c$. (After Sommer.) $B$. Intestinal epitholium-cells from the vertebrate, ingesting filt. In the interior of the cells single microseopic fat-droplets are fuund. (After Thanhoffer.)

At the bottom of this spiral-like ciliater funnel is a cell-mouth, which is prolonged a short distance into the protoplasm as the cellpharynx, and then gradually disappears into the liquid endoplasm. 
The cilia of the ciliary wreath of the peristome contract continually and rhythmically, and in this way produce in the water a whirlpool, which is so directed that it sucks small particles, such as detritus, mud, bacteria, algæ, etc., which are suspended in the water, into the cell-mouth; from there, surrounded by a layer of water, they are shoved by contractions of the body into the cell-pharynx, and thence into the endoplasm (Fig. 46). The phenomena may be very easily observed, if, according to Ehrenberg's method ('38), granules of carmine or indigo be mixed with the water. It is seen at once how the Vorticella engulfs the red or blue granules and forms them in its protoplasm into balls which are surrounded by a covering of water and constitute food-vacuoles.

The mode of ingestion of food by other Infusoria is entirely similar to that of Vorticella. The free-swimming forms frequently seek fixed

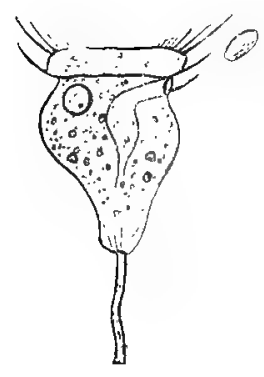

$a$

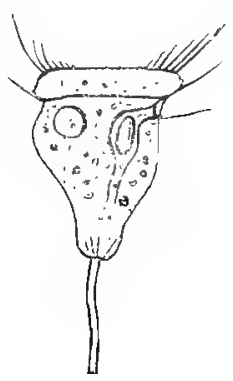

b)

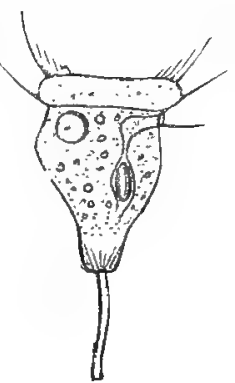

c

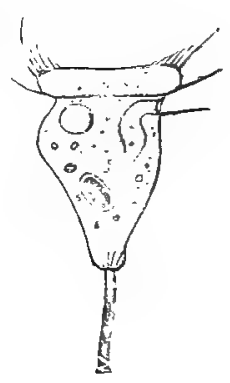

d

FIG. 46. - Vorticella in four successive stages of the process of food-ingestion. An alga-cell is being engulfed into the ccll-mouth and taken through the pharynx into the endoplasm.

food-masses and engulf them. Many Infusoria even, such as Coleps, a small, egg-shaped, ciliate form having a delicate latticelike surface, take in large balls of food which are broader than their mouth-opening by pressing the latter upon the ball by the force of the ciliary contraction so that the mouth-opening, as in a snake, is gradually enlarged. Thus they really suck the food-balls into their bodies (Fig. 47).

The ingestion of solid food on the part of the cell is, therefore, in every case brought about by active movements of the cellprotoplasm or its motile organoids.

In the ingestion of substances by the living cell, one phenomenon deserves special mention-namely, the fact of the selection of food. Of the various cells living in the same medium, each takes to itself different materials, and such as are necessary for the formation of its characteristic substance. This is clear in the tissuecells of highly organised animals, such as the human body. Here the blood-plasma is the common nutrient material for all tissue- 
cells. But from this common nutrient liquid each kind of cell removes the substances necessary for its life; the mucous cell takes substances different from those taken by the ganglion-cell, the muscle-cell substances different from those taken by the cartilage-cell, the liver-cell substances different from those taken by the sense-cell, and so on. The different cells choose entirely different materials, each one according to its need.

This phenomenon of food-selection is, perhaps, more remarkable in certain free-living cells that take in solid food. Cienkowski

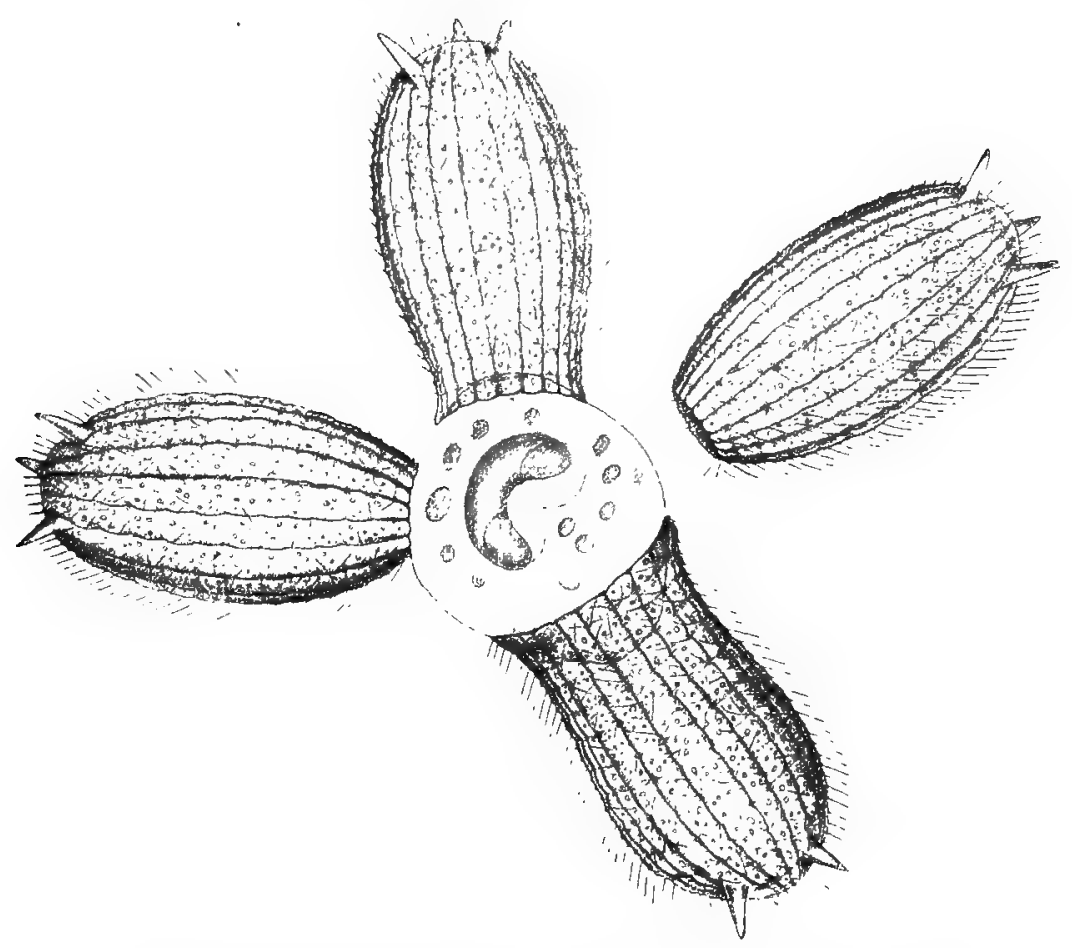

Fro. 47.-Four individuals of Coleps hivtus swarming about and ingesting a ball of food.

('65), who has studied in detail the life of the lowest Rhizopoda, the naked monads, gives an interesting description of how Colpodella and Vampyrella, two simple, naked rhizopod-cells, procure their food, which consists of living alga-cells. Cienkowski relates as follows: "Although the zoospore- and amoba-conditions of the monads are only naked protoplasmic bodies, their behaviour in seeking and ingesting food is so remarkable that it seems to be the work of conscious beings. Thus, Colpodella pugnax pierces the Chlamydomonas, sucks up the chlorophyll that flows out, and 
runs away. A second rare case of this kind is afforded by Vampyrella Spirogyree. The amoba of this species applies itself to a healthy Spirogyra, bores through the cell-wall and devours the slowly escaping primordial utricle together with the chlorophyllbands. It seems to be able to satisfy its hunger upon Spirogyra only." (Fig. 48.)

But we need not search so far. In the human body there are cells that behave similarly. As Metschnikoff ('92) has shown by his researches extending over many years, the leucocytes or white blood-corpuscles, the amoboid wandering-cells, devour and digest certain forms of bacteria present in the body, while they scorn and even directly avoid other bacteria; likewise, intestinal

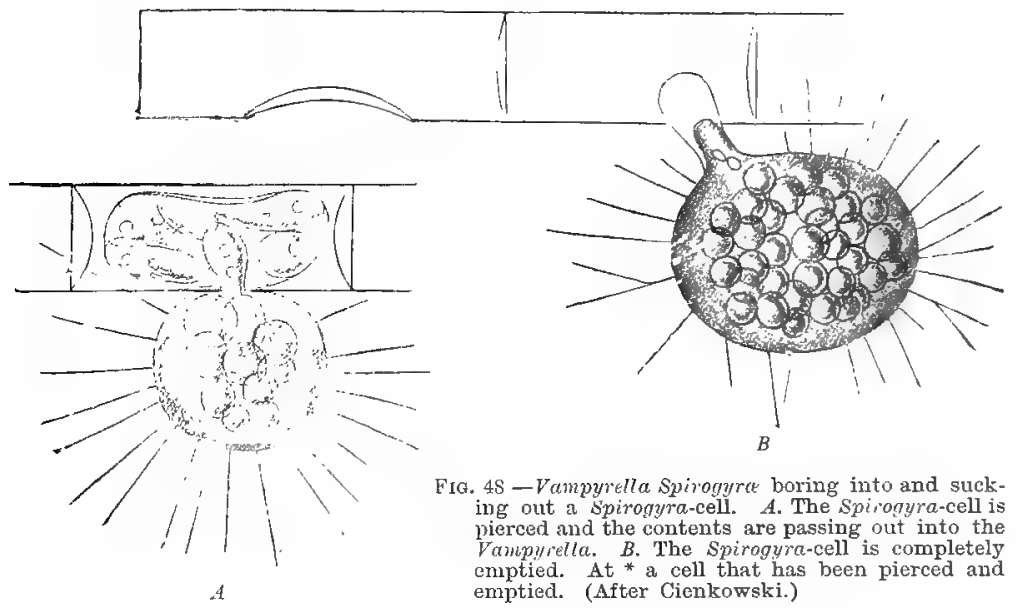

epithelium-cells, as has been seen, devour only fat-droplets, while they behave wholly passively toward other small particles that are brought into the intestine, such as granules of carmine.

Finally, another very interesting phenomenon, which has to do with the ingestion, not of food, but of substances that likewise play a role in the life of the organisms in question, has also frequently been referred to, although incorrectly, as a power of selection on the part of the cell. This is the ingestion of material for shells and capsules on the part of certain shell-bearing rhizopods. The Diffugioe, which are unicellular fresh-water Rhizopoda whose naked protoplasmic bodies are fixed in a very delicate urn-shaped or flask-shaped capsule, take up the material for their tiny dwellings with their finger-like pseudopodia out of the mud of the pools and lakes at the bottom of which they live. ${ }^{1}$ The structural material of their shells is very varied, but in many 
cases the shells are composed of one definite material (Fig. 49). Thus, forms occur that employ only cases of the silicious Algce or diatoms, while others employ only sand-grains of certain sizes, and still others particles of mud. It has been thought that the Diffugice select their material from substances at their command. But it can be proved, at least in some cases, that no real selection exists here in the same sense as in food-ingestion by the above-mentioned cells. The fact that forms from one and the same locality employ only a certain material depends rather upon the circum-

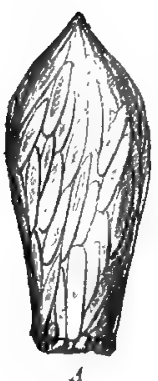

1

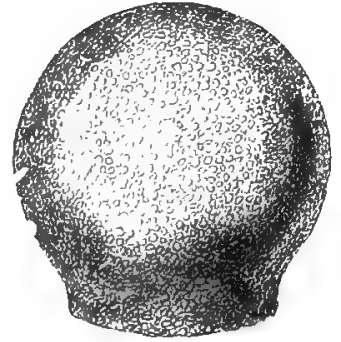

$B$

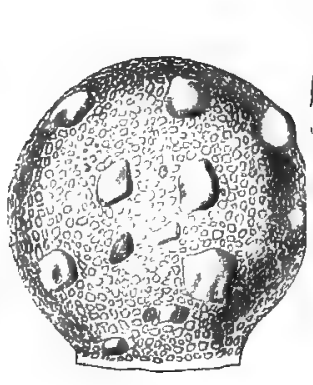

$c$

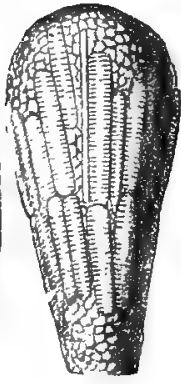

$D$
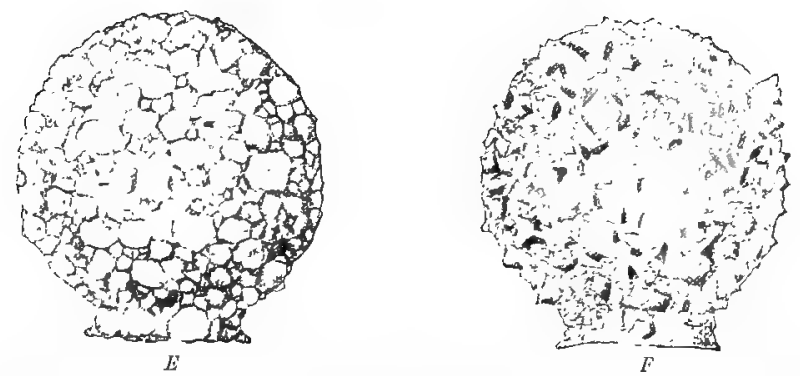

Fu. 49.-Virious Diflugia-shells, constructed of : $A$, diatom-cases; $B$, fine sand-grains; $C$, fine and coarse sand-grains; $D$, datom cases and sand-grains; $E$, coarse sand-grains ; $F$, the same form as $E$, but made of splinters of blue glass.

stance that in the given locality only this one material is at hand. If, e.g., the dwelling-place of the form that constructs its shell out of mud or substance excreted from its body be examined, it is found that here other materials, perhaps diatom-cases or sand. grains, are wholly wanting. If, however, such forms be given the possibility of getting other material, by the introduction of very finely pulverised sand or, still better, very finely ground, coloured glass into the culture-vessel in which they live, it is found that the individuals arising by reproduction surround themselves with a delicate shell of sand or splinters of coloured glass. ${ }^{1}$ The circum- 
stance that some shells possess small sand-grains, and others considerably larger ones is likewise to be referred in part to the character of the material at their disposal, in part, however, to other external conditions, such as the narrowness of the opening of the capsule, which does not allow the protoplasmic body to draw through large sand-grains. It accordingly appears that in most cases the construction of the capsule by Diffugice involves no real selection of material, and thus far no case has become known where such a selection has really been established with certainty. There is, therefore, no justification in drawing a parallel, as is often done, between the ingestion of structural material in the building of the Diffugia-capsule and the act of food-selection by the living cell.

\section{B. THE TRANSFORMATION OF INGESTED SUBSTANCES}

The process of construction of living substance out of the ingested food-stuffs can be designated best by generalising, as is frequently done, a conception of the botanists and employing the word assimilation. By assimilation in the narrow sense has been understood for a long time in botany the synthetic formation in plants of the first visible organic material, starch, out of the ingested inorganic compounds. But it is advantageous to extend the conception and employ it also for the construction of higher organic compounds, especially the proteids, and, indeed, not only in plants, but also in animals. By assimilation, therefore, is understood the sum of the processes that lead to the construction of living substance to the maximum of its most complex constitution, the synthesis of proteids. Construction, or assimilation, can then be contrasted with destruction, or dissimilation.

\section{Extracellular and Intracellular Digestion}

"Corpora non agunt nisi soluta." This old dictum plays in the life of the cell a very great rôle. In order that the ingested foodstuffs may work chemically and be of use for the construction of living substance, they must be in a dissolved condition; since, however, the food taken in by the organism is in part solid food, it must first be transformed into soluble form, and this process is termed digestion. It has been seen that only a few cells have the power of taking in solid food; in these there occurs so-called intracellular digestion, the transformation of the solid food into soluble compounds taking place in the interior of the cell. The great majority of cells, however, cannot take in solid food; in them, therefore, the transformation of the solid into the soluble form must take place outside of the cell, in order that ingestion may be possible; 
this transformation is, therefore, termed extracellular digestion, and the ingestion of the dissolved food, resorption.

The change of solid food, such as coagulated proteids, starches and fats, into soluble compounds takes place through the action of definite secretions which the cell-body gives off to the outside. These characteristic secretions are called enzymes or unorganised ferments. The result of their action can be demonstrated outside

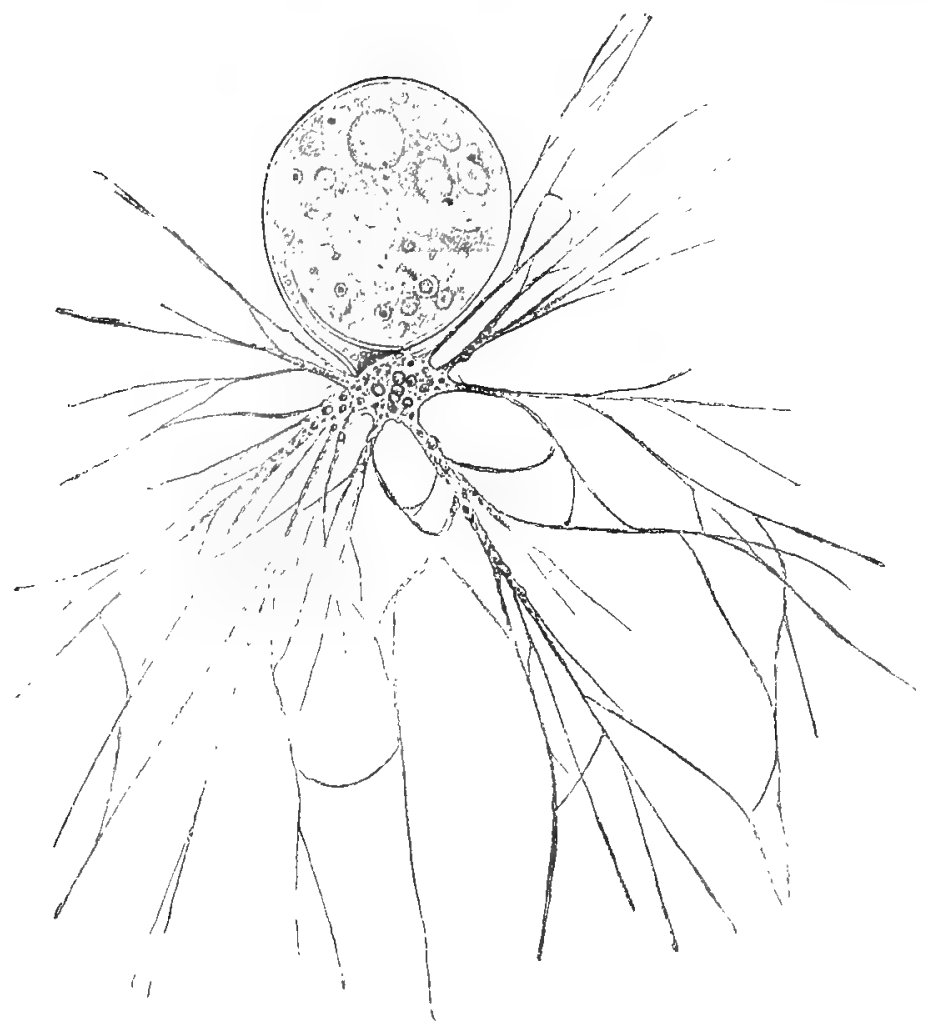

FIG. 50.-Licberkithnia, a fresh-water rhizopod, from the cgg-shaped shell of which branched pseudopodial filaments protride.

the organism by allowing an enzyme, e.g., pepsin, which is produced by the cells of the gastric glands, to act upon a bit of coagulated proteid. If, c.g., there be placed in a beaker a solution of pepsin in water to which has been added an equal volume of 0.4 per cent. hydrochloric acid, there is obtained an artificial gastric juice. If there be put into this digestive solution a flake of fibrin, i.e, the proteid the spontaneous coagulation of which causes the clotting of 
the blood outside the blood-vessels, and the beaker be warmed in a digestion-chamber to the body-temperature, it is found after some time that the willid flake of fibrin begins to swell, to become transparent upon the outside, and gradually to become dissolved in the liquid. Finally, the whole flake, as such, disappears, and in its place there is found dissolved in the liquid peptone, that modification of proteid which, as has already been seen, arises by a hydrolytic cleavage of the polymeric proteid molecule, is soluble in water, and diffuses through organic membraner. Besides the peptone there are found also certain transition-stages between the native albumin and the peptone, which are likerwise soluble in water and are termed albumoses. We shall presently discuss more in detail the peculiar manner of working of the ferments.

That which happens, in extracellular digestion, outside the cellbody, and which can be imitated even in the test-tube, takes place in intracellular digestion within the protoplasm. Likewise here the process can be followed best in the naked protoplasmic body of Rhizopoda. Lieberkiünia is a large fresh-water rhizopod, from the egg-shaped, membranous shell of which thick, branching pseudopodial filaments protrude through an opening at the pointed pole (Fig. 50). When the Lieberkïinna seizes and digests ${ }^{1}$ an infusorian that carelessly swims against its pseudopodia, it can be seen with the inicroscope that the prey first becomes attached to the pseudopodia, entangles itself more and more firmly by its strong efforts to escape, and gradually becomes surrounded either wholly or partially by the pseudopodial protoplasm (Fig. 51). For some time the movements of the infusorian continue; then they become feebler, and at the same time its body-form begins to change. It decreases in size constantly, while the liquid and granular parts of its protoplasmic body pass over into the pseudopodial protoplasm, mix with it, and are no longer seen to stream to the central body of the Lieberkiihnia. Thus, gradually, the whole body of the infusorian becomes dissolved and its liquefied contents mix with the protoplasm of its captor, until none of it is longer distinguishable. In other cases of intracellular digestion the food-body, e.g., in Amoebc and Infusoria, becomes surrounded by a food-vacuole within the endoplasm, and is dissolved in the same manner as in the exoplasm of the Licberkiitnia. Further, the observations that have been made upon the Infusoria by Greenwood ('94) are very interesting. Sho followed the fate of the ingested food-masses in the Vorticelline', especially in Carchcsium (Fig. 52), and found that, while thy are undergoing digestion, they take a perfectly definite path within the cell-body-viz., from the cell-pharynx (Cf. Vorticella. p. 146, Fig. 46) to the bottom of the cell and back to the mouth-opening, where the undigested masses are cast out. It is very noteworthy that the food-masses 
remain for a long time in the concavity which the sausage-shaped nucleus turns toward the interior of the cell, there chiefly to
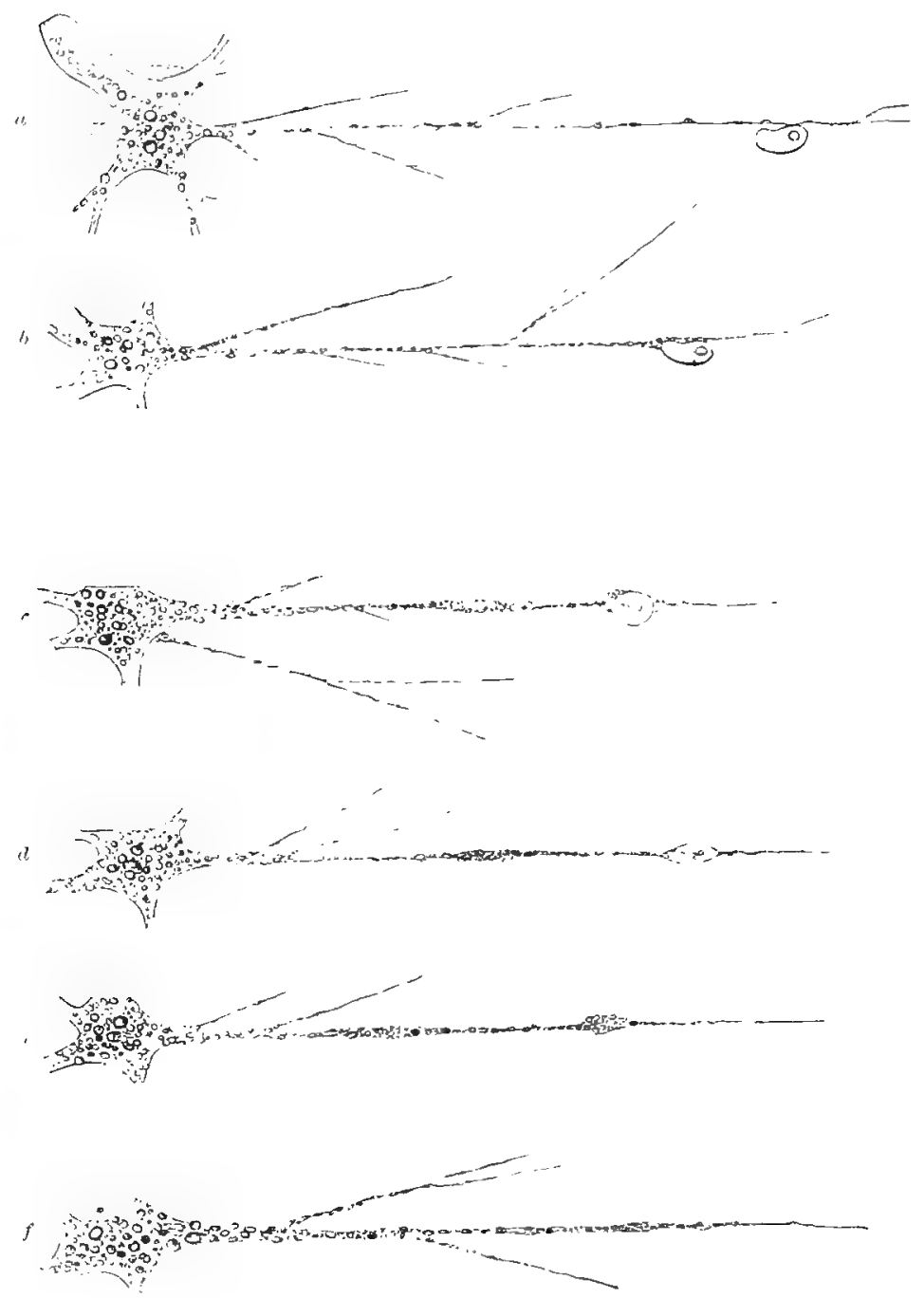

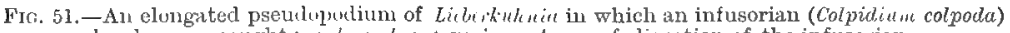
has become cuught : $", b, c, d, e, t$, various stages of digestion of the infusorian.

undergo destruction. This indicates that very probably the nucleus takes an important share in the digestion of the food-masses.

Just as proteids are transformed by pepsin in an acid and by 
trypsin in an alkaline solution, so also the insoluble carbohydrates, such as starch, are changed into soluble forms both in intracellular and extracellular digestion by the action of certain enzymes. As has been seen, starch is a polysaccharid, which represents a combination of several sugar molecules in the anhydride form. By the action of the enzyme, e.g., the ptyalin of the saliva and the pancre-

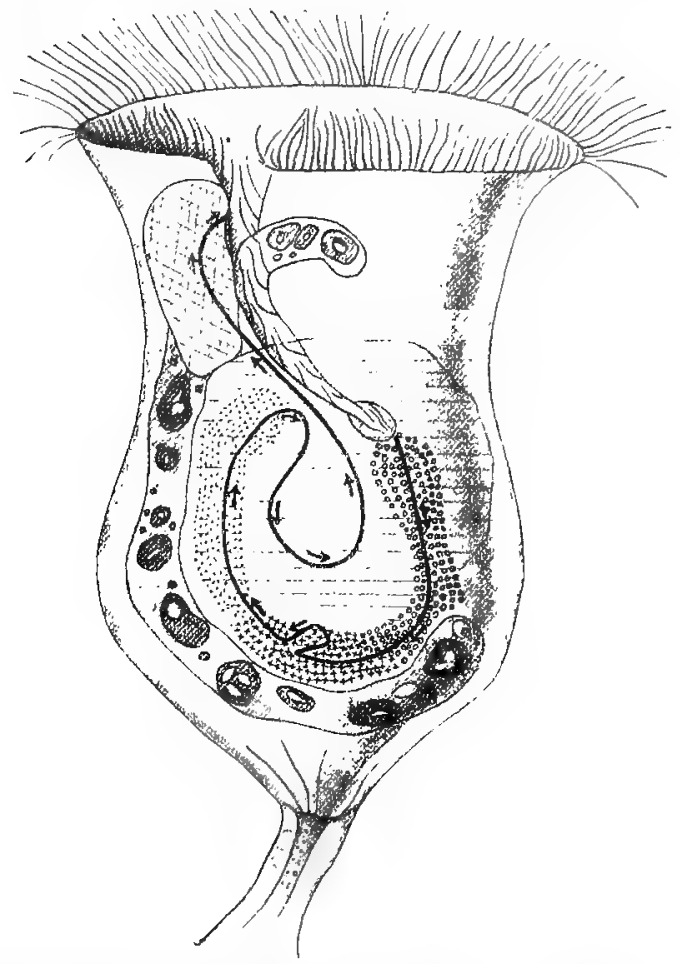

F10. 52.-Caschesium polypinum, scheme of the path taken by the ingested food in digestion and expulsion of the excreta. The food enters through the pharynx and is trausported downward (small circles), where it is stored in the concavity of the sausage-shaped nucleus (the latter is recognised by its containing darkcr hodies). It remains here for some time at rest (small crosses). Then it passes npward upon the other side (dots) and returns to the middle of the cell, where it undergoes dissolution. The excreta are removed to the outside, through the opening of the cell-mouth. The black line with arrows indicates the directiou of the path. (After Greenwood.)

atic juice in animals or the diastase in plants, the polymeric starch molecule is split up through hydrolysis into simple sugar molecules, maltose and dextrose, which are soluble in water. In the intracellular digestion of Infusorio, as M. Meissner ('88) has shown, starch grains are slowly digested from the outside, so that they appear as if gnawed (Fig. 53), and finally are completely dissolved. Yet from the striking researches of Greenwood ('86, '87) 
and Meissner (loc. cit.) it appears that Rhizopoda such as Amcebca, although occasionally taking in starch, are nevertheless unable to digest it.

Finally, fats in extracellular digestion are split up, likewise with hydration, by the fat-ferment stecupsin into glycerine and fatty acids, the latter uniting with alkalies to form soaps. Glycerine and soaps are soluble and can be resorbed. In the intracellular ingestion of the neutral fat-droplets as such, however, a direct digestion does not always take place. As Meissner has observed, Amceba

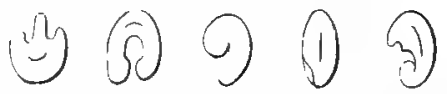

FIG, 53.-Starch-grains, which have been devoured and digested by an infusorian. (After M. Meissner.) and Infusoria retain ingested fatdroplets within their protoplasm for days unchanged, and Greenwood has found that Amoeba and Actinosphorinum do not digest ingested fat at all.

\section{Ferments and their Mode of Action}

The ferments are physiologically such an extremely interesting group of bodies that it is worth while to examine them somewhat in detail, and especially to become acquainted with their peculiar. mode of working. By ferments there is understood a series of highly complex organic bodies belonging to animals and plants, which have the remarkable peculiarity of bringing about certain chemical transformations apparently without undergoing changes themselves.

When two substances act upon each other in an ordinary chemical reaction, both undergo a chemical transformation. With the ferment this appears not to be the case, for, when a large quantity of a chemical compound has been split up by a certain quantity of an enzyme, the original quantity of enzyme is found unchanged in the liquid. Theoretically, an unlimited quantity of material can be decomposed by a small quantity of a ferment. Practically, however, this is usually not possible, because the effectiveness of the ferment gradually becomes diminished by the accumulation of substances resulting from the cleavage.

It is a question, however, whether the ferment, when acting upon other substances, really undergoes no decomposition or is itself destroyed and constantly re-formed, so that in the end the same quantity of ferment is found as at first. In inorganic chemistry there are cases analogous to each possibility.

By the terms catalytic action and contact-action in the original sense, chemists understand the property possessed by many substances of decomposing chemical compounds by simple contact. Thus, Sainte-Claire Deville and Debray have found that formic acid can be split up into carbonic acid and hydrogen, not only by certain forments, but also by finely divided iridium, rhodium and ruth- 
enium, the molecules of the metals undergoing no change. These facts are explained as follows: It is known that according to the mechanical theory of heat the atoms in every molecule are in constant vibratory motion-a phenomenon that is termed intramolecular heat. Upon contact of the molecule of the metals in question with the complex molecule of formic acid this intramolecular vibration of the atoms of the former is transferred to the latter, and combines with the latter's vibration in such a way that another arrangement of atorns results-i.e., a decomposition of the formic acid molecule. According to a different idea, it is the chemical affinity between the atoms of the molecule of the metal and certain atoms of the formic acid molecule that disturbs the intramulecular vibrations of the formic acid atoms in such a way that a rearrangement, i.c., a decomposition, takes place, without, however, the occurrence of a real combination of the atoms of the metal with the corresponding atoms of formic acid. However it be, in every case the intramolecular motion of the atoms in the molecules that are to be broken up becomes disturbed, while the catalytic molecule of the metal remains intact. Such contact-actions are widely known in chemistry. Thus, hydrogen peroxide upon contact with finely divided platinum is changed into water and oxygen without the platinum itself being altered.

In contrast to these pure contact-effects, chemistry recognises other cases in which the effective body remains unchanged only apparently. While bringing about transformations, it is continually altered chemically, but is immediately re-formed again. The end-results in the two cases must be the same, for even in the latter case at the conclusion the body in question is found in its original form. We have already become acquainted elsewhere with such a case. In the manufacture of concentrated sulphuric acid the nitric acid is continually reduced by sulphurous anhydride into nitrous acid, to be re-formed again into nitric acid with the aid of the oxygen of the air.

Which of the two cases doc's the action of ferments resemble? Thus far this question has not been decided with certainty. It is very probable, however, that among so-called ferment-actions both cases are present.

In the large group of ferments two kinds are distinguished--dissolved unorganised ferments, or 'n:ymes, and solid organised fermicnts, or ferment-organisms; the former comprise secretions which are given off to the outside by the living cell and remain constantly effective, the latter consist of the living substance of the cell itself, with the life of which the fermont-action is associated. While in ferment-organisms the ferment-action is extinguished with the life of the cell, the enzymu can be preserved as long as desired as chemical bodies, without lusing their power. The cells of youst (Gocharomyces), which "ause the alcoholic fermentation 
of beer ( $\mathrm{F}$ g. 54), are ferment-organisms, decomposing grape-sugar into alcohol and carbonic acid. 1 They produce, however, in iddition an enzyme, invertin, which is able to convert cane-sugar into grape-sugar. The two actions can be separated from one another. If the yeast-cells be killed by chloroform or ether, it is no longer possible for them to decompose grape-sugar into alcohol and carbonic acid; but the power of the inverting enzyme continues undiminished, so that the change of cane-sugar into grape-sugar goes on as well as before. In ferment-organisms the living substance exercises the ferment-action only so long as it lives, i.e., its ferment-action is associated with metabolism. This evidently indicates that in ferment-organisms there is realised the second case mentioned above, that which is analogous to the action of nitric acid in the manufacture of sulphuric acid; while the peculiar. fact that the action of the enzymes may be replaced by other substances, e.g., metals, suggests
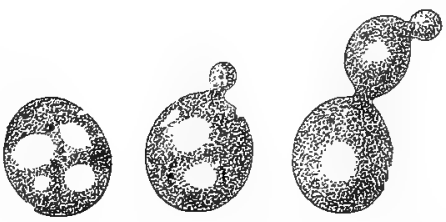

FIG. 54.-Saccharomyces, yeast-cells. (After Reinke.) the probability that they work also like finely divided metals by pure contact. At present, naturally, this question cannot be decided with absolute certainty. Like the organised ferments the enzymes are highly complex compounds, all of which probably contain nitrogen and are derived from the metabolism of proteids; they are made ineffective by substances that enter into combination with proteids, as well as by boiling; within certain limits, however, an increase of temperature is favourable to ferment-action, because thereby the intramolecular vibrations of the atoms are increased.

If the action of ferment-organisms depends actually upon a continual destruction and rebuilding of their wwn substance, then all living organisms may be regarded as ferment-organisms; for all living substance transforms food-stuffs in its metabolism while not disappearing itself. Hence the metabolism of living substance can be compared with the metabolism of nitric acid in the above case.

\section{Assimilation and Dissimilation}

\section{a. Assimilation}

The digestion of food-stuffs by the action of ferments is only a preparation for the process of assimilation. Only after the foodstuffs have been brought into the condition in which they can do chemical work, i.e., after they have become rlissolved, can their

$$
{ }^{1} C f . \text { p. } 111 .
$$


function in the construction of living substance begin to be exercised.

The process of assimilation naturally differs much according to the condition of the ingested food. Differences must be recognised also in assimilation by the two main groups of organisms, plants and animals, corresponding to the differences that have been recognised in their food. It is

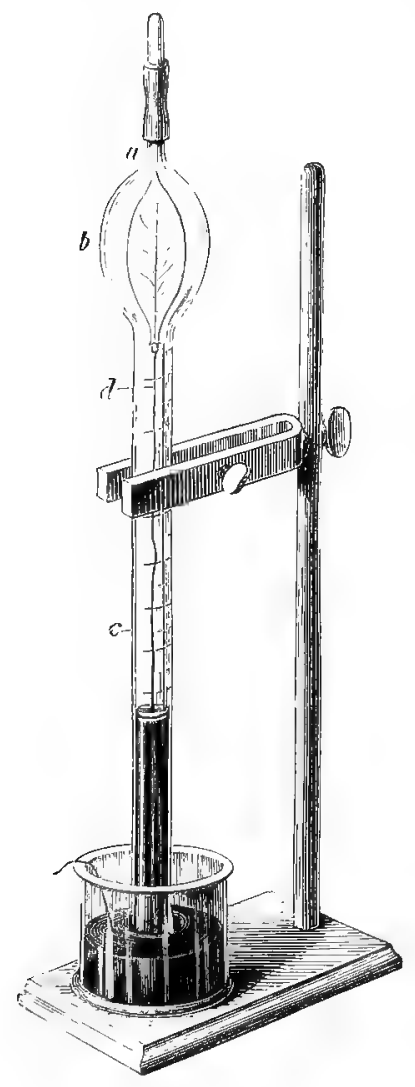

F1G. 55.-Apparatus for the investiga. tion of the cleavage of carbonic acid in the green parts of plants. (After Detmer.) evident that the processes that lead to the formation of living substance in the plant-cell must, constitute a much longer series than in the animal-cell, for the plant must construct the highly complex proteid molecule out of the simplest inorganic compounds, carbonic acid, water, salts and oxygen, while the animal obtains, already formed, the proteid food without which it cannot live, and only needs to use this in its specific manner. We will follow the processes that lead to the assimilation of proteids somewhat in detail in the two series, so far as in general they are known. The lack of our knowledge is realised here as elsewhere.

To consider first the plants, a simple experiment shows the first step which the plant takes in the series of processes that lead to assimilation. In a cylindrical tube, provided with a bulb closed above (Fig. 55) and graduated, a green leaf is placed by means of a wire, and a certain measured quantity of carbonic acid is allowed to flow in. The lower end of the tube is closed by means of mercury, and the whole is allowed to stand for some hours in the sunlight. If then the contents of the tube be tested gasometrically, it is found that the carbonic acid has disappeared, and in place of it an equal volume of oxygen is in the tube. Since the volume of the carbonic acid is equal to the volume of the oxygen contained in it, the experiment proves not only that the plant has taken up carbonic acid and given off oxygen, but also that it has given off as much oxygen as was contained in the carbonic acid. The first stage toward assimilation in the plant is, therefore, a 
cleavage of carbonic acid; this takes place in the green plant-cell under the influence of sunlight. The plant gives off oxygen to the outside. As to the fate of the retained,carbon, microscopic observation gives us information. It shows, namely, that in proportion to the destruction of the carbonic acid starch is formed in the chlorophyll-grains themselves, and is laid down in the form of small, highly refractive granules (Fig. 23, p. 81, and Fig. 56). Moreover, by a series of experiments Sachs has shown that as soon as the breaking-up of the carbonic acid ceases in darkness the formation of starch also ceases, immediately to begin again in the light along with the destruction of carbonic acid. Since starch contains, in addition to carbon, only hydrogen and wxygen in the same relative proportion as in water, it can be rerived only by synthesis from the curbon that is set free and the water that i- received through the roots. Starch is, therefore, the first assimilation-product to appear.

"If," says Sach-, ( 82 ), "starch is the first and sole vi-ible product of assimilation, it follows directly that all other organic compounds of the plant must originate by chemical metamorphosis from it." It will be remembered that no carbon was present in the artificial nutrient solution in which

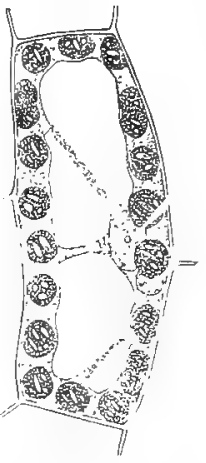

A

FIa. 56.-Starch appearing as transparent scales in chlorophyll-bodies, $A$, Chlorophyllbodies lying in the cell. $B$, Chlorophyll-bodies undergoing division. (After Sachs.) plants were allowed to grow. ${ }^{1}$ If, therefore, later the plant manufactures other carbohydrates, fats, and finally proteids, all of which contain carbon, it can employ only starch as the starting-point. Of course almost nothing is known concerning the special chemical transformations which starch undergoes further. But an idea can be furmed, at least in gross outline, of the further processes of assimilation. The fact that from the starch soluble varieties of sugar can be derived very easily by cleavage with hydration, is at once understood when it is borne in mind that starch is a polymeric molecule of the anhydride of sugar. Hence it can pass into the condition of the soluble carbohydrates, and this is necessary in order to make possible further chemical syntheses. The formation of fatty oils out of starch can also be directly observed. If unripe seeds of certain plants, e.g., Poconia, which contain carbohydrates and no fats, be allowed to lie in moist air, it is found after some time that all starch has disappeared, but fatty oil has appeared in its place. But much more complicated is the origin of proteid from carbohydrates. Since in addition to the atoms of carbohydrate proteid contains nitrogen and sulphur, 
which the plant receives through its ruots from nitrates and sulphates only, complicaterl transformations of these salts and then syntheses with the carbohydrate atoms must take place, the details of which are thus far wholly unknown. As to how, finally, the proteid molecule, synthetically formed, is employed further in the living substance for purposes of construction, at present, on account of our extremely scanty knowledge of the chemical constitution of proteids, we can say absolutely nothing. Here an enormous field is offered for future physiological investigation.

In animals, the path from the ingested food to the living proteid molecule is of course essentially shorter, for all animals without exception need for their nutrition proteids already prepared. But what happens further to the proteids that have been peptonised by digestion is not fully known. After the investigations of Salvioli ('80), Hofmeister ('82), Neumeister ('90), and others, no doubt can be entertained that the peptones as such disappear in the cells of the wall of the intestine, in other words, they are transformed in the cell itself. If pieces of the intestinal mucous membrane of a rabbit be placed in a liquid that contains peptone, in which the cells of the intestinal wall exist during life, after some time it is found that all peptone has disappeared. If, moreover, a solution of peptone be injected into the blood of an animal, in a short time the whole quantity of peptone is excreted unchanged in the urine; and in normal life the blood is always free from peptones. These two experiments prove undoubtedly that the peptones become changed on their way through the cells: of the intestinal wall. But little is thus far known as to the kind of change within the cells. Perhaps some of the peptones are broken down immediately into simpler substances by a retrogressive proteid metamorphosis. It is certain that many are changed back into proteid and pass into the juices of the body along with the proteid resorbed directly without peptonisation. This clissolved proteid circulates throughout the body with the bloodcurrent, bathes the cells of all tissues, and is withdrawn by the cells from the blood, to be bruken down within them. Hence it happens that in a remarkably short time all the proteid taken into the body, beyond a certain quantity, appears as urea, uric acid, etc., in the urine. Voit ('81) thought that this proteid that is broken down ought to be distinguished as "circulating proteid" from the "tissue proteid," which is "mployed for the formation of tissues, since he assumed that the destruction of the circulating proteid took place in the blool, in the liquids, of the body. But the reason for such a distinction has disappeared, since PHiuger ('93) and Schöndorff ('93) have shown recently by very careful investigations that the breaking-duwn of the proteid dissolved in the blood does not take place in the blond itself, but in the tissue- 
cells. Under certain circumstances, however, the cells also retain a small part of the proteid dissolved in the blood, either employing it for the increase of its living substance, as in growth, or storing it up in the protoplasm, as in fattening, in the form of reserve, i.e., passive, proteid which is not ordinarily consumed in metabolism. Under certain conditions, as in fasting or during the development of eggs, such passive, indifferent, reserve-proteid can again be drawn into the metabolism. The vitellin in egg-cells is such a substance.

Regarding the fate of the ingested fats and carbohydrates as few details are known as regarding the finer transformations of proteids. The fat which is taken as such into the cells frequently remains for a long time as reserve-material. Likewise the fat that is split up into glycerine and fatty acids and resorbed can be changed back into neutral fat in the cell; this is proved by the striking experiments of J. Munk ('84), who, by feeding fat-free soaps or free fatty acids, was able to cause a storing-up of tissue-fat in dogs that had fasted and become extremely lean. In a similar manner the grape-sugar that is split off from the carbohydrates can be transformed synthetically into glycogen in the tissue-cells, especially in the cells of the liver and the muscles, and can be stored up as such. Regarding the further fate of this stored fat and glycogen, however, it is known only that they can be consumed during fasting and during excessive muscle-work, that, therefore, they represent a reserve-material which acts in cases of need as "compensation-food" in Pfliger's sense.

\section{b. Dissimilation}

Our knowledge of the processes of dissimilation of living substances is much more meagre than that of assimilation. We really know only that living substance is continually undergoing decomposition, for this is apparent from the output of decomposition-products. But as to the path from the complex proteid compounds to the end-products, as to the special chemical transformations that take place, our knowledge is very incomplete, since as yet the composition of proteids is known very slightly.

But one fact at least is certain, namely, that the most of all those substances that result from the decomposition of the proteid molecule are not groups of atoms that were preformed as such in the molecule and are now simply split off, but they are derived from certain cleavage-products by successive syntheses; this takes place either at the moment of decomposition by rearrangement of the atoms in the proteid molecule itself, as in the case of carbonic acid, or later outside of the proteid molecule by combination with other cleavage-products and a simultaneous rearrangement of atoms, as is the case, e.g., in the form'tion of uric acid. Thus far it is not known 
that any product of proteid-decomposition originates by the simple cleavage of preformed groups of atoms.

It is important to become acquainted with the most essential derivatives of the disintegrating proteid molecule. As has been found by the investigation of the substances that are contained in living substance, ${ }^{1}$ there can be distinguished among these products of proteid transformation two groups-those containing nitrogen, and those not containing nitrogen. Representatives of each group appear in every cell, but their special composition differs in individual cases according to the characteristic metabolism of the cell.

Among the substances that contain nitrogen the most wide-spread are urea, uric acid, hippuric acid, creatin, and the nuclein basesxanthin, hypoxanthin or sarkin, guanin, and adenin. Regarding the majority of these substances, thus far it is not known how they originate from the decomposition of proteids, but for some at least hypotheses concerning their immediate forerunners have been formed. Thus, from the fact, which Schröder discovered, that ammonium carbonate introduced into the fresh, excised, still-living liver of a dog leaves the liver as urea, it has been supposed that ammonium carbonate is the forerunner of urea, that from it the liver-cells prepare urea by a transformation of the atoms and the giving-off of two molecules of water:-

$$
\left(\mathrm{NH}_{4}\right)_{2} \mathrm{CO}_{3}-2 \mathrm{H}_{2} \mathrm{O}=\left(\mathrm{NH}_{2}\right)_{2} \mathrm{CO} \text {. }
$$

But this is not conclusive, it is only a provisional hypothesis, for the possibility is not to be excluded summarily that within the organism itself still other substances are employed for the synthesis of urea. With somewhat more certainty we know the forerunner of uric acid, which is that substance in which, in reptiles and birds, the greater part of the nitrogen that is derived from the decomposition of proteids leaves the body. Its forerunner is ammonium lactate. From experiments which Gaglio ('86) carried out upon dogs it follows that the lactic acid of the blood is derived from the decomposition of proteid, for the quantity of lactic acid in the blood increases and decreases together with the quantity of proteid food, and is wholly independent of the quantity of ingested carbohydrate. While lactic acid is always found in the blood, under normal conditions no trace of it occurs in the urine; it must, therefore, undergo transformation before it is excreted. Minkowski('86) made these relations clear by an experiment, in which he showed that geese after the extirpation of the liver excrete very small quantities of uric acid but large quantities of lactic acid and ammonia, both of the latter in the quantitative relations of ammonium lactate. From this important fact Minkowski rightlyconcluded that ammonium lactate is a preliminary stage in the formation of uric acid, from which uric acid arises by rearrangement. We can also

$$
1 \text { cf. p. } 109 .
$$


conjecture with great probability as to the synthesis of hippuric acid, which arises from the decomposition of proteids, especially in the metabolism of herbivora. By boiling with mineral acids or alkalies hippuric acid is split into benzoic acid and glycocoll by hydrolysis, and by heating under a high pressure these two substances can again be united into hippuric acid with the loss of water. It is, therefore, supposed that in the body of the herbivore, where the possibility exists of the derivation of benzoic acid from proteid or the aromatic compounds of the food, and of glycocoll from gelatine-yielding substances derived from proteid, hippuric acid is formed synthetically from these two substances. As a matter of fact, not only in the body of the herbivore, but even in the carnivore, the formation of hippuric acid may be brought about artificially by introducing benzoic acid into the stomach, this acid then uniting with glycocoll into hippuric acid in an unknown manner in the tissues. In contrast with this, nothing whatever is known concerning the origin of creatin. Creatin, trigether with creatinin, which is derived from it with loss of water, is the substance in which muscle-cells give off chiefly the nitrogen that comes from the decomposition of their proteid. Just as little is known concerning its fate as concerning its origin; for, although it is found in muscles in considerable quantity, only small quantities of it appear in the urine; hence it appears to undergo in some manner transformations in the body itself. Finally, regarding the nuclein bases, it is known only that they are derived from the decomposition of nucleins and their derivatives; the details of the process are unknown.

Among the non-nitrogenous transformation-products of proteids, the most important are fats, carbohydrates, lactic acid, and carbonic acid. These also are derived from the proteid molecule, not by a simple cleavage but by rearrangement and synthetic processes. The theory that fat can arise from proteid by transformation has been much disputed. The pathological process of the so-called fatmetamorphosis of cells, in which fat appears in the place of proteid, so that at the end of the process the cells are dead and filled with fat, necessarily led to the idea that here proteid is transformed into fat. But the objection was possible that in the course of the disease the proteid of the cell is forced out by the fat coming in from the outside. Notwithstanding this possibility, this important question has been decided experimentally in favour of the former view. Leo ('85) made experimental use of the fact that phosphorus poisoning causes an extremely rapid fat-metamorphosis, especially of the liver-cells. From a number of frogs he selected six individuals of equal size and weight, killed them and determined their fat-contents. He then took six other individuals, poisoned them with phosphorus and killed them after three days. The determination of fat revealed a considerably greater fat- 
contents in the latter than in the former. This experiment proves that fat must actually have arisen in phosphorus poisoning. Franz Hofmann (72), however, performed an experiment which showed directly the origin of fat from proteid. He took a quantity of eggs from the blucbottle fly (Musca vomitoria) and divided them by weight into two equal portions. One of these portions he employed for the determination of the fat-contents, the other he laid upon blood, the small quantity of fat contained in which was likewise determined. The larve of the flies creeping out of the eggs fed upon the blood and grew. After they were grown, Hofmann determined the quantity of fat in them, and found that they contained ten times as much fat as the eggs and the blood together. On account of its minute quantity, the blood-sugar need not be considered in the fat-formation. Hence the fat could have come only from the proteid of the blood. After these experiments it is no longer doubtful that fat can originate from proteid. Neither can doubt exist concerning the origin of carbohydrates (grape-sugar and glycogen) from proteid. It has been known for a long time that in severe forms of diabetes mellitus, even with complete lack of carbohydrates in the food, the quantity of grapesugar excreted in the urine is considerably increased by the consumption of an increased quantity of proteid. Likewise, Claude Bernard has observed that in dogs in which the glycogen had been used up by fasting, glycogen is stored in greater quantity when they are fed abundantly upon pure proteid food; and in a dog that had been fed for four days with pure fibrin after fasting twenty-one days, Mering ('77) found more than sixteen grams of glycogen in the liver. Numerous similar observations have been made, and the origin of carbohydrates from proteids is now assured. The origin of lactic acid from proteid has been proved by the investigations of Gaglio ('86), which show that the lactic acid of the blood depends only upon the quantity of ingested proteid, not upon that of the carbohydrates. Finally, that cartonic acid also, which all living substance without exception expires throughout its life, is derived from the decomposition of proteid and not from that of non-nitrogenous substances, is at once evident from the fact that in carnivora life can be maintained continually with proteid food alone. This important fact proves in general that from proteid all those substances can be formed that are continually excreted by the organism, as well as all the substances that are necessary to maintain life.

Formerly a sharp distinction was drawn between animal- and plant-cells as regards the kind of chemical transformations that take place in them. It was said that in the plants synthetic processes take place almost exclusively, in the animals analytic processes only; and this idea has persisted until recent times. But that such a fundamental difference exists was energetically dis- 
puted more than twenty years ago by Pfliger ('75, 1). As a matter of fact, as the above consideration has shown, the difference consists only in that the plant-proteid of the chlorophyll-bodies has retained from early times the property of assimilating inorganic material, while animals require for the construction of their living substance organic food-material already prepared. Nevertheless, synthetic and analytic processes take place in both the plant and the animal body. In the plant the decomposition of carbonic acid must precede the synthesis of starch; in order that the starch may be further elaborated, it must first be decomposed into simple kinds of sugar, and so on. Finally, in the plant also there occurs the whole series of cleavages that are associated with the decomposition of the proteid molecule, with dissimilation, exactly as in the animal body. But syntheses take place in the animal body to a great extent. The further elaboration of the digested proteids, fats, and carbohydrates towards the construction of living substance involves extended synthetic processes, and it has been seen that the majority of the products of retrogressive proteidmetamorphosis are formed synthetically out of the cleavageproducts of the proteids. Hence analytic and synthetic processes go hand in hand in the animal- as in the plant-cell, and the old distinction into analytic and synthetic organisms is merely the expression of an earlier stage of our knowledge of the chemical processes in living substance.

\section{THE OUTPUT OF SUBSTANCES}

Living substance excretes transformation-products in the same proportion in which it receives substances from the outside and transforms them; the substances given out are as varied as those taken in. But with our slight knowledge of the transformations and with the overwhelming number of substances excreted by the various forms of cells, we can say in a very few cases only by what processes the substances are derived. As regards most of them, it is not known whether they are derived from assimilatory or dissimilatory transformations; evidently a large quantity of byproducts are formed in both the ascending and the descending portions of the metabolic series, whether by simple cleavage, or by synthesis from the cleavage-products or other substances which are excreted by the organism either for some further use or as useless products. This last point, as to whether the excreted substances are of still further use in the life of the organism, or are removed as useless products, as slag, has caused a distinction to be recognised among the substances given off. Although it is difficult to make this distinction sharp, because of the extraordinary variety of different products, the use of it is advisable 
from practical considerations. The substances given off from the cell, among which occur gaseous, liquid, and solid substances in all grades of consistency, are distinguished as secretions when they play a still further useful rôle in the life of the organism, and as excretions when they are removed to the outside as useless residue. Accordingly, secretions are contrasted with excretions. We will look for a moment somewhat in detail at the two groups of substances and at the mode of their output.

\section{The Mode of Output of Substanies by the Cell.}

Like the taking-in of food, so also the manner of output of substances varies, according as the latter are gaseous, dissolved or solid.

The output of gaseous or dissolved substances evidently takes place under the same conditions and in the same manner as such substances are taken in, for here there is the same process reversed. In many cells, e.g., in many unicellular organisms, it is very probable that the so-called contractile vacuole (Fig. 57), a drop of liquid within the cell which is alternately emptied and filled by rhythmical contractions of the protoplasm of its wall, attends to the expulsion of dissolved substances. It is supposed that the latter, together with the water that during the diastole of the vacuole streams in from all sides out of the protoplasm, accumulate in the vacuole and at its systole are given off to the outside.

It is clear that every cell excretes primarily substances that are derived from its own metabolism. But in the compound cellcommunity, especially of the animal organism, there exist also cells which in addition have undertaken for the whole body the excretion of certain other materials. Thus, the cells in the convoluted uriniferous tubules of the kidney excrete the urea that is prepared by the liver-cells and passed into the blood, by receiving it from the blood and giving it off to the outside. Other cells of the kidney, those of the so-called glomeruli, the microscopic capsules in which the blood-capillaries are twisted into knots, greedily suck up the water from the blood to excrete it as the water of urine into the pelvis of the kidney.

In the mode of output of solid substances two types again are distinguished. They are essentially different according as the excreted substances either occur in the cell itself in a dissolved condition, and become solid only at the moment of excretion, or lie within the living substance as solid masses, which are to be given off as such to the outside.

In the former case, which is realised in the excretion of most skelctal substances, such as chondrin, chitin, and lime, the same conditions are present as in the excretion of dissolved sub- 
stances in general, except that sooner or later after their exit from the living cell the substances assume a solid form. The solidifying of the excretions at the surface does not prevent the repetition of the process, and thus eventually all substances of the kind become excreted and solidified upon the outside. Thus originate the cell-membranes of tissue-cells, the cellulose coats of plant-cells, the chitinuus coats of insects, and the calcareous shells of Forcminiferc.

This process and at the same time the mode of growth of these superficial structures can be illustrated by an experiment which
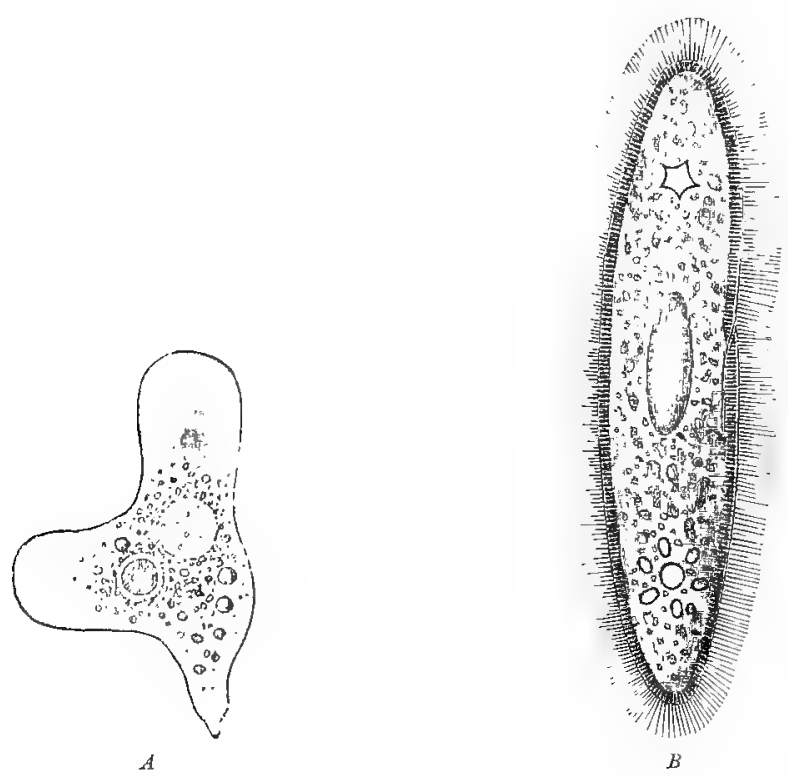

Ia. 5. $-A$, Anobla. A pale contractile vacuole lies in the cndoplasm beside the dark nucleus $B$, Priminteitut. At each pole is a star-shaped contractile vacuole; the upper is in the act of contracting, while the lower is beginning to fill itself from several small drops of liquid that are flowing together.

was suggested by Traube, and was much discussed in his time. If a drop of a thick solution of gelatine be allowed carefully to fall into a solution of tannin, there appears about the drop a so-called precipitation-membrane of gelatine tannate, since at the surfaces of contact of the gelatine and the tannin the two substances undergo a chemical combination.' This precipitation-membrane shows the peculiar phenomena of growth both in surface and in thickness, and on account of its similarity to a living cell Traube's drop of gelatine in the tannin solution has been termed an "artificial cell." Since the gelatine solution attracts water to itself, tannin in solution comes constantly through the membrane to the 
drop. At the surface of the latter the tannin is united with the gelatine, and thus the continual apposition of n'w layers leads to the thick ning of the mombrane. The water, however, presses into the interior of the drop, so that this constantly swells and increases in size. By this process there alpurar continually in the precipitation-membranc extrencly fine hol's and cracks; these, however, become closenl by new precipitate at the moment of their appurance. Thus, the artificial cell grows continually and uniformly larger until atl the gelatine is in combination. The formation and growth of the nembrane, which in the large drop take place relatively iapidly, proceed

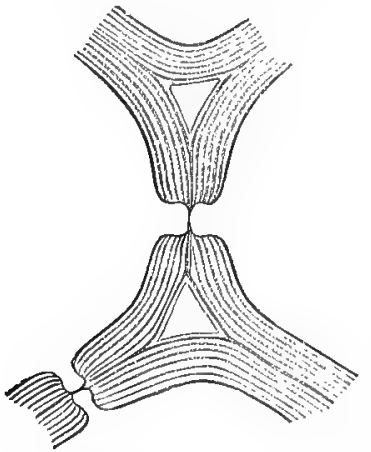

Fra. 58.-Cell-wall of a pith-cell of Clematis, showing stratified growth in thickness. (Aftor strasburger.) very gradually in the small living cell.

In botany a fruitless discussion has been going on for a long time over the question whether the cellulose-me'mbrane of the plant-cell is formed by intussusception, i.e., by the deposition of new particles betwern the old ones, or by apposition, i.e., by the deposition of particles upon the outside. ${ }^{1}$ This discussion arose in connection with Nägeli's unhaply comparison of, or rather distinction leitween, growth in crystals and growth in organisms. Lately the view has be'n gradually accepted that both morles lead to the growth of the membrane-the one to growth in surfice, the other to growth in thickness. If the protoplasmic body of the cell itself is enlarged, the membrane is extended. In the process, as a rulc, no actual cracks alpenar, as in the artificial cell, but as a result of the "xtension the spaces between the single particles of the membrane become wider and larger, so that new particles of protoplasm can enter in. But, on the other hand, the stratification of the membrane parallel to the surface, which is visible under high magnifying fowers and with increasing thickness becomes constantly more distinet, shows that incraiss in thickness by apposition is also present (Fig. 58).

If the cells in their metabolism produce substances and "xcrete them to the outside continually, "xtensive solid masses are gradually formed, which in multicellular tissurs, where the proflucts of the individual cells blend together, form the so-called intercellular substances, such as in cartilage and bune (Figs. 59) and 60). But the substances are not always "xireterl at once to the outsirle; in many cascis they are stored up as a solid miss in a vacuole in the cell itself, particle after particle lwing added to them as in a crystal. Thus, stareh-grains in plant-cells, and calcarous neerlles 
and stars in echinoderms and -ponges, are formed within the cell itsolf, and only after they have reached a certain size are they given off to the rutside by the customary node of excretion of solid bodien (Fig. 61).

Amolor shows bont the morle of excretion of substances that lie in the interior of the cell as solid masses. It has been seen that

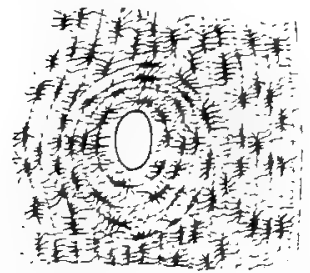

Fir, is.-Cruss-section ot bone. The compact ground-fitl-atince lie. between the star-shuped formecells. In the micluly of the section is a crosson.t. tion of a bonecanal. (After $H a t-c, h_{t}+k_{\text {. }}$ )
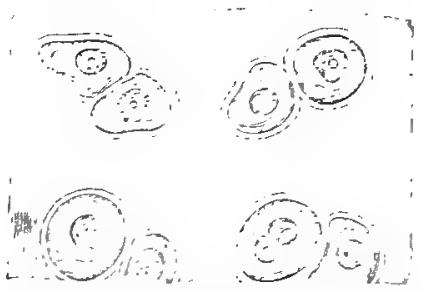

Firi, fol-Hraline cartilage. Between the individual cells a solid, byaline ground-substance has been excreted. (After Hatschek.)

in the ingestion of ford by Amola the ford-ball enclosed in a food-varnole lies finally within the protrplasm. In this racuole, which mat be termed a digestive vacuole, all digestible substance becomes rliswolverl, and passes into the protoplasm; but the indigestible residue, such a- shells of algæ and of diatroms and the chitinous masses of rotifers, remain in the vacuole, and become excreted in the following manner: By the creejing of the Amolso the digestive vacuole in the streaming protoplam comes to lie very near the surface, so that its contents are separated from the medium $n$ rel $y$ by a thin delicate wall of protoplasm. In such a case the wall break rery "asily by the protroplasm flowing in all
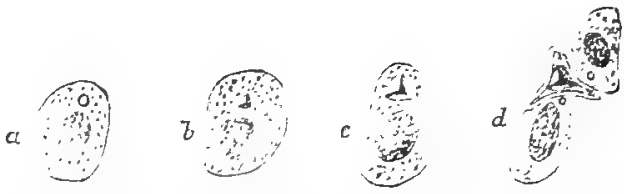

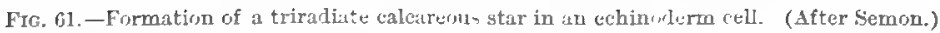

directions awily from the thinnest place, and the contents of the vacuole togetlus with the solid mass are olnptind to the outside (Fig. 62). This now of remoral of solid com-tituents from the protopla-m in formd exclurively in cell- that do not possess a membrane, and hence chiefly in ancebrid crell of all kinds.

A transition betwern the method of output of liquids and that "f solids is repunted by the sieretion of mucus. The mucous 
cells, which in the compound organism play so very important a rilc in protecting the internal surfaces and keeping them smooth and moist by their secretion of mucus, are always cylindrical. The nucleus, surrounded by somewhat more solid protoplasm, lies at the bottom of the cell-body, while the upper end of the cell, which borders the free surface of the membrane, is formed by a
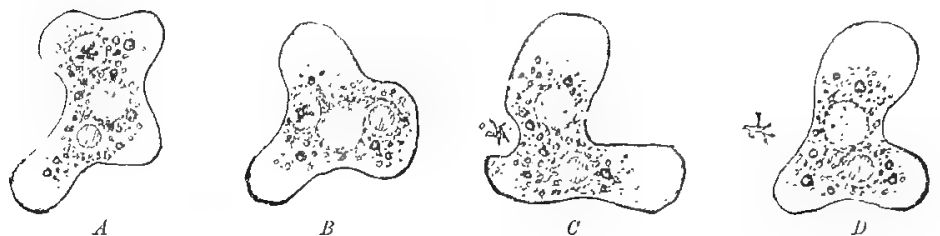

FIG. 62,-An Ameba in four successive stages of excretion of the undigested residue of food.

substance, mucigen, that is continually being transformed into mucus. During the quiet activity of the cell a little of the secretion passes constantly to the thin liquid layer that covers the surface of the tissue. But during energetic, sudden secretion the whole mass that forms the upper part of the cell is shoved out (Fig. 63) and blends with the drops cast out of the neighbouring cells into a thick, gummy covering of mucus. The
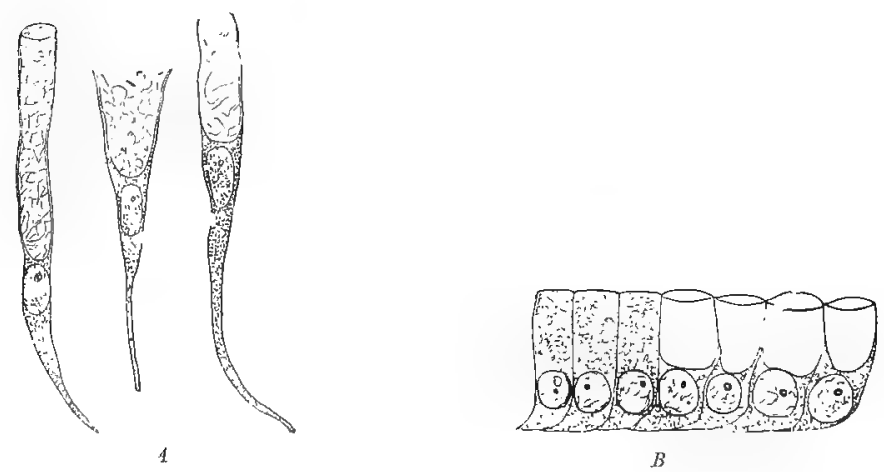

FIG. 63.-Mucous cells. $A$, 'lhree isolated cells. $B$, Seven adjacent cells, of which the three at the left are full, the four at the right aro orapty. (After Schiefferdecker.)

peculiarity of many holothurians, those cucumber-shaped forms of cchinoderms, of transforming their thick, solid skins upon stimulation in a short time into a glistening, viscous slime, is very remarkable and not yet explained. In general, the cell-physiological investigation of the process of secretion promises to afford many very interesting general physiological facts. 


\section{Secretions and Excretions}

It is neither necessary nor possible to examine here in detail the whole series of secretions and excretions which plant and animal cells afford in their metabolism; our consideration shall therefore, be limited to the most important of these.

\section{a. Secretions}

Since it is characteristic of secretions to be of use to the organism, it is easy to understand that many secretions remain continually within the organism and are not given off to the outside. Hence two groups of secretions can be distinguished, according as after their formation they are at once given off or are retained continually in the organism, whether in the cell or upon its surface ; in neither case in the cell-community of the compound organism is it always necessary that the secretion be of use to that particular cell that affords it.

Among the secretions that after their production leave the organism there are, in the first place, the ferments, which have to do with digestion and appear in both animals and plants. Thus, in animals the cells of the salivary glands produce ptyatin, which transforms starch into grape-sugar; the cells of the gastric glands, pepsin, which peptonises proteids, and rennet-ferment or chymosin, which mediates the coagulation of casein; and the cells of the pancreas ptyalin for the digestion of starch, trypsin for the peptonising of proteids, and steapsin for the splitting of fats. Ferments occur likewise in plants, such as the so-called carnivorous plants, which catch insects, hold them and digest them by the secretion of peptonising ferments. An example of such a plant is Drosera, which grows in the swamps. Whether the very effective ferments that are produced in the milky juice of some plants, such as Carica papaya, and are not cast out upon the surface, are to be regarded really as secretions in the present sense or only as excretions (by-products of metabolism) is thus far not decided, since the significance of these in the life of the plant has not yet been discovered. In unicellular organisms, further, the ferments are of great importance for the nutrition of the cell when these organisms, as is the case with the bacteria, come into contact with organic food and are obliged first to liquefy solid food-stuffs in order to be able to absorb them.

Other secretions, such as the wide-spread mucin, of which mucus consists, are of great importance. Mucin protects the cell itself from external intuences that can harm it, such as direct contact with objects; with strong stimulation the mucous cell produces a thick layer of mucus separating the former from the body that touches it; this is the case with the mucous cells of the trachea 
when a foreign body comes into the throat. Further, the mucus of the saliva serves to make masticated food smooth, so that the masses of food can glide more easily through the narrow gullet. In this lies the chief importance of the saliva in man; here, on account of its too brief action, the ptyalin, which works only in an alkaline liquid, and hence in the acid gastric juice is made immediately ineffective, can hardly exercise its amylolytic power. Finally, mucus serves for attachment, especially in the lower animals and unicellular organisms. Rhizopoda secrete upon the surface of their protoplasmic bodies a delicate mucous covering with which they stick themselves to the bottom in order to creep about, and with which also they hold fast food-organisms that swim against them, in order to draw the latter into their own bodies and digest them. A similar importance as protective media is possessed by the fats which, such as the sebum, are produced by the sebaceous glands of the skin; they protect the skin from too great evaporation and render it supple.

Further, as Stahl ('88) has shown by a series of experiments, many secretions act in a different manner solely as protective media in animals and especially plants; such are ill-smelling or ill-tasting acids and ethereal oils. The organisms are protected by them from being devoured. Most of these cases present interesting phenomena of adaptation to definite conditions, which have arisen through natural selection and constitute contrivances advantageous to the organism. The same is true also of other cases in which plants, by means of good-smelling and good-tasting secretions, such as ethereal oils and honey, attract insects whose coming and going are useful, perhaps indispensable, to the plants; the animals bear away pollen upon their legs and deposit it upon the female flowers so that the latter are fertilised. Such adaptations, often astonishingly fitting, are especially common among plants, and the physiology of secretion touches here most closely the interesting field of the mutual relations of plants and animals.

Finally, as secretions in the widest sense there may be recognised also substances produced in the cell, such as starch, aleuronc-grains, fat-d,roplets, etc., which are stored in the cell for a time as reservematerial and later are used in metabolism.

Among the secretions that after their production remain in the organism, there belong almost exclusively pigments and substances that form skeletons. The former appear mostly in the form of fine granules, remain continually in the cell-body, and possess a special importance in the colour-changes of the animal, which is not yet entirely explained. The great majority of skeletonforming substances are excreted to the outside. Sometimes they are laid down within the cell itself and later extruded, as are the calcureous needles and plates of the Holothuria; sometimes they 
are secreted at once upon the surface of the cell in the form of membranes, shells, and coatings, such as cell-membranes, the cellulose-membrane of plant-cells, the chitinous coats of insects, the silicions cases of diatoms, the delicate latticed skeletons of Radiolaria (Fig. 64), and the calcareous shells of Foraminifera;
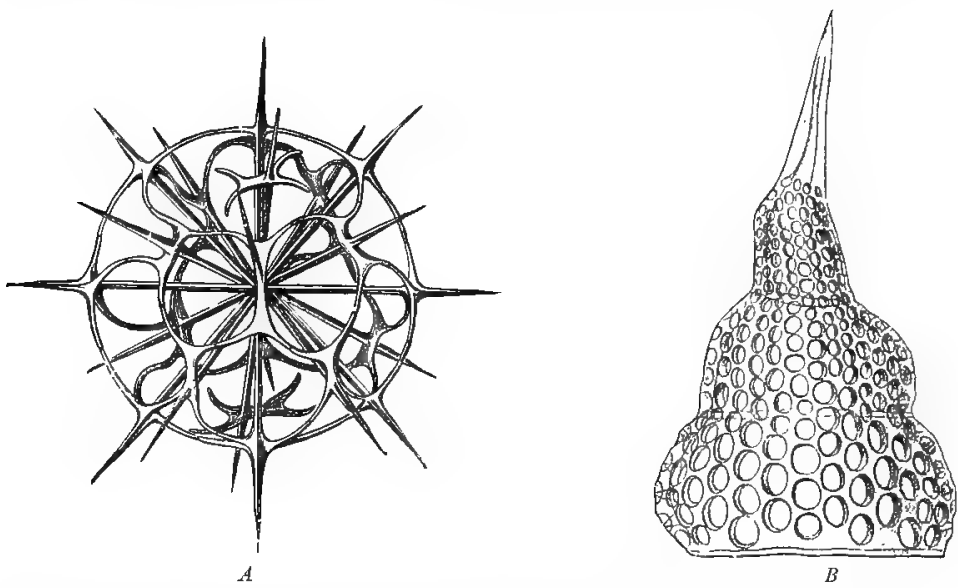

FIG. 64.-Silicious skeletons of Raliolaria. (After Haeckel.) A, Dorataspis, B. Theoconzs.

and sometimes they are stored in the tissues between the individual cells as the so-called connective substances, such as chondrin in cartilage, glutin in bone, calcium phosphate in bone, and the great number of supporting or skeletal substances which belong to the albuminoids and in the different groups of animals have compositions very different and as yet little known.

\section{U. Excretions}

The excretions are much fewer in number than the secretions. Chief among them are the products of retrogressive proteidmetamorphosis which are excreted by all living substance.

Among gaseous excretions the most important one, whose production is associated with the life of every cell without exception, is carbonic acid, the end-product of respiration; it is produced chiefly by the oxidation and the decomposition of proteid, but under certain circumstances by the fermentation of carbohydrates. As has already been seen, in addition to carbonic acid, plants excrete oxygen, which is derived from the splitting-up of the carbonic acid received from their green parts. It has, therefore, been thought that the supposed contrast in the metabolism of plants and of animals, already spoken of, is to be found 
in the fact that plants take in carbonic acid and give out oxygen, while animals, vice ver's $\alpha$, take in oxygen and give out carbonic acid. But later experiments have shown that, in reality, this contrast does not exist. It is true that animals inspire oxygen, employ it for the combustion of living substance, and expire carbonic acil as the product of such combustion. But plants do the same. In them this fundamental vital phenomenon of respiration is merely concealed by the consumption and the splitting-up of carbonic acid; the latter, however, has nothing to do with respiration itself, but is preliminary to the construction by the plant of the first organic substance out of inorganic materials. If the metabolism of plants be examined at a time when no starch-formation is going on, when no carbonic acid is being split up, but when the life of the plant is being expressed in other ways, as in the night or in darkness, it is found, by gasometric experiments analogous to those above described, that the plant consumes oxygen and expires carbonic acid like the animal. In the plant, therefore, the process of respiration is not to be confounded with the process of assimilation of starch: the latter requires carbonic acid to be taken in and split up and oxygen to be given out, and thus conceals the respiration which is constantly taking place beside it. Respiration, i.e., the taking-in of oxygen and the giving-out of carbonic acid, is a general metabolic phenomenon.

Among liquid cxcretions water occurs everywhere, and substances dissolved in water. Because of the small quantity of these various excretions, in the present state of micro-chemical reactions it is usually not possible to demonstrate them for the individual cell; hence they must be studied in the compound cellcommunity. In the plant, water is excreted and evaporated during transpiration through the so-called stomata of the leaves. By the action of special guard-cells the stomata can be closed and opened, and thus the output of water by the plant can be regulated very delicately. In animals there are special glands, the kidneys and sweat-glands, the cells of which excrete the water, together with the products of retrogressive proteid-metamorphosis, out of the body-liquids, and pass them to the outside.

Most of the non-nitrogenous products of proteid-decomposition are oxidised completely to carbonic acid and water, so that the latter leave the body as the almost exclusive end-products. But intermediate products also arise, which, excreted by certain cells, have a different fate within the body. This is true especially of lactze acid, which, among other things, is excreted by the musclecells into the blood and can be found there, but does not leave the body as such in the urine. That sarco-lactic acid or para-lactic acid is derived from the decomposition of proteids, and not from the ingested carbohydrates, is proved by the experiments of 
Gaglio ('86), already mentioned. But the sarco-lactic acid is still further transformed in the body, for, as has been seen, the experiments of Minkowski ('86) upon geese in which the liver was extirpated have shown that lactic acid, presumably combined with ammonia, is consumed in the synthesis of uric acid.

The nitrogenous products of proteid-decomposition are the wellknown substances which have already been met with frequently, especially urea, uric acid, hippuric acid, creatin, and the nuclein bases, xanthin, hypoxanthin or sarkin, adenin, and guanin. These are excreted chiefly in the urine and represent the compounds in which all the nitrogen taken in in the food leaves the body, apart from an inconsiderable quantity in the sweat and the fæeces.

The last fact, that, with the exception of the minute quantity in the sweat and the fæces, all the nitrogen is excreted in the urine, has assumed great importance in the physiology of animal organisms in connection with the circumstance that proteids and their derivatives are the sole nitrogenous substances in organisms. But, unfortunately, it has led to a false conclusion, which in itself would, perhaps, have had no immediate influence upon the development of fundamental physiological ideas, had not far-reaching and weighty deductions been drawn from it. It follows necessarily from the above-mentioned fact that all nitrogen excreted in the urine must be derived from the decomposition of proteid; but the further conclusion which, it has been thought, must be drawn from it, does not follow, namely, that the nitrogen excreted in the urine is a measure of the proteid-transformation in the body. The latter conclusion would be justified only if it were known that all nitrogenous cleavage-products of the proteid molecule, without exception, leave the body. But there is no ground for such a belief; on the contrary, no fact whatever is known which contradicts the idea that nitrogenous cleavageproducts of the proteid molecule can rebuild themselves synthetically again into proteid with the aid of new non-nitrogenous groups of atoms. This latter possibility has been overlooked, and in consequence views have arisen, especially in relation to metabolism in muscle, which, a priori, bear in themselves the stamp of improbability, but which have been accepted and handed down. Recently they have been attacked and criticised by Pfluiger ('91).

To the excretory substances resulting from retrogressive proteid metamorphosis one more group can be added, the members of which likewise are derived from the transformation of proteids, chiefly in the mutabolism of bacteria. These are the so-called ptomaines, some of which, on account of their very poisonous action, have lately been termed toxines. Upon their poisonous action chiefly depends the serious illness in the infectious diseases produced by bacteria, such as cholera, dysentery, diphtheria, and 
typhoid fever. The chemical composition of these substances has become somewhat better known recently, especially through the comprehensive and exhaustive labours of Brieger ('85-'86). Some of them, the ptomaines that were first found, which are produced by the putrefaction of proteid substances through the metabolism of the putrefactive bacteria, as in dead bodies, are nitrogenous bases that are related to the so-called allicaloids or vegetable bases, which arise in the plant-body and likewise represent very poisonous excretory substances.

Finally, we may refer here briefly to a very interesting series of substances which are produced, likewise, by the metabolism of bacteria chiefly, but also of very many other cells, and very recently have attracted the attention of investigators. These are the toxalbumins, poisonous proteids, which are produced in the metabolism of the cells by transformation from other bodies, and in the pathology of infectious diseases play an important rôle. Most of these toxalbumins are globulins and albumoses. Thus, the active constituent of tuberculin, which was obtained some time ago by Koch from the metabolic products of tubercle bacilli, is a toxalbumose, which in small doses is extremely poisonous. By the production of another toxalbumose the bacilli of diphtheria cause very characteristic phenomena of poisoning in the bodies of persons ill with diphtheria, the phenomena disappearing very slowly. The toxalbumose of the bacteria of diphtheria was the first toxalbumin which was recognised as such: it was so recognised by Löffler ('90), and was obtained pure by Brieger and Fränkel (90). No little astonishment was caused when the first poisonous proteids were recognised, since the proteids had been known so long as harmless substances, and even as absolutely necessary food-substances. And the surprise was no less when later it was found that the poisonous effects of suake-bites, which are so greatly feared, and of the blood of many fishes, such as the lamprey, are to be traced, likewise, to the poison of such toxalbumins, which are produced by the metabolism of the tissuecells and are excreted.

Solid excrctions are found almost exclusively in cells that take in solid food. In them the indigestible residue of the food is given off to the outside in the form of solid excretions in the manner already described. In a few cases the excretory substances which occur dissolved in the cell-contents are formed into solid concretions within the cell and are then cast out; this is the case. in the ciliate Infusoria, according to the investigations of Rhumbler ('88). At present it is not yet decicled whether the concretions of guanin and the crystals of calcium guanin which accumulate in many cells and are stored permanently in the protoplasm, such as in the beautifully iridescent crystalline plates and needles in the epidernis-cells of amphibians and fishes, are to be regarded as 
excretions or as substances that possess still further importance in the life of the organisms in question.

If, now, the facts of metabolism be co-ordinated, it is found that from the entrance of substances into the living cell to their exit from it, metabolism consists of a long series of complicated chemical processes which can be represented in the form of a curve with an ascending and a descending limb. The ascending limb comprises all processes that lead to the construction of living substance; the apex is formed by the synthesis of the most complex organic compounds, the proteids; the descending limb comprises the processes of the destruction of living substance into its simplest compounds. The beginning and end of the curve, i.e., the substances that enter into and go out from the organism, are best known; the components that lie at the apex of the curve are known least, and in large part not at all.

The green plant-cell, even the simple, unicellular, green alga, such as Protococcus, is a chemical laboratory in which, out of the simplest inorganic materials-carbonic acid, water, and saltsorganic substance is manufactured, analytic processes and syntheses going on hand in hand in the process. First, starch appears. Starch with the help of nitrogenous salts serves to construct proteids, in which process very various kinds of by-products arise. But the green plant-cell does not complete this gradual construction of proteids for itself alone, it does it at the same time for all animal-cells, which in the course of evolution have lost the power of manufacturing organic material out of inorganic. The organic substances produced by plants serve as food for herbivora, the flesh of herbivora as food for carnivora. Carnivora can live upon proteid food alone. Hence it is seen that of the substances that appear in metabolism some, as in plants, lead to the construction of proteid, and some, as in carnivora, are derived from the transformation of proteid. But in plants as well as in animals a constant decomposition of proteid finally takes place, and there result again, as definitive end-products of metabolism, simple inorganic compounds, essentially the same materials with which the construction of living substance was carried on, namely, carbonic acid, water, and nitrogenous salts. All metabolism, therefore, is merely a series of processes which are related to the construction and destruction of proteids and their compounds. This is true as well of the plant as of the animal.

\section{The Phenomena of Form-changes}

The form of organisms is not unchangeable. Apart from the changes that ale associated with motion, and which will be considered elsewhere, organisms show profound changes of form that 
are termed their development. Two great series of form-changes are recognised in living substance-phylogenetic or racial development, which comprises the form-changes of living substance in their totality during the earth's development; and ontogenetic or germinal development, which comprises the form-changes that a single individual goes through during his life. Haeckel ('66), who has done pioneer work of fundamental importance for the modern theory of evolution, has shown that the two series stand in intimate connection with one another; in general, germinal development is an abbreviated recapitulation of racial development.

\section{A. PHTLOGENETIC DEVELOPMENT}

The forms of living substance that inhabit the earth's surface have not always been the same. Modern palæontology, the science of fossil organisms, has revealed an overwhelming number of forms which differ from those now living the more the older the strata from which they are derived. Critical research during the last decade has relegated to the realm of fable a large number of remarkable beings with which the earlier geology peopled the earth, and has shown them to be fanciful pictures which stand upon the same plane as the rare animal forms contrived by the curious creative fancies of the Indians, the Assyrians, and the Incas; nevertheless, the discovery of well-authenticated fossil forms during recent decades has proved conclusively how utterly different from its present state was the organic world upon the earth's surface during the earlier periods of the earth's development. An overwhelming number of organisms have become known which inhabited the water and the land before man. The theory of descent has introduced a causal connection into this wealth of forms by showing that fossil organisms are not to be regarded as unique curiosities, lusus naturce, and the unsuccessful experiments of a Creator, as the previous century believed them to be. Rather are they the dead twigs and branches of a mighty, wide-spread trunk, of which the youngest and last shoots are the present living organisms; the oldest branches have sprung from a common root, the Protista, whose direct descendants, little changed, now appear in the interesting groups of unicellular beings, Rhizopoda, Bacteriu, Infusoria, and Alga. Modern morphology has succeeded by critical research in drawing in gross outline a picture of the genealogical tree of organisms, and the conception of natural relationship, which was presaged by the use of the word by the earlier systematic morphology in a figurative sense, has obtained through phylogenetic research a very real significance. The present organic world is the product of an historic development stretching back orer in enormously long space of time, in which some forms, such as the 
vertebrates, are the result of manifold and profound transformations, while others, such as the Protista, have persisted from the (arliest times in a form changed relatively little. The last fact, that in the unicellular Protiste there is recognised a group of organisms that possess in almost absolute purity the characters of the ancient ancestors of all organisms, makes these micro-organisms appear particularly valuable physiologically. But let us go somewhat more fully into the phenomena of the development of form in general.

No substance exists without form. All substance has a definite form which is the expression of chemico-physical laws that pertain partly to the nature of the substance in question and partly to the influences that it receives from the outside. Living substance is only a portion of the matter that composes the earth, and is not different in its elementary nature trom other substances. In assuming form, therefore, living substance must obey the mechanical laws of matter, as all other bodies do. If an organism has a definite form, however, there are two factors, the mutual working of which determines its further forn-clerelument-a conservative factor, which acts to maintain the form, and a mutative factor, which acts to change it. The factor that maintains form is the inheritance of present characteristics, the factor that causes change is adaptation to changed external conditions.

\section{Heredity}

Heredity is one of the most familiar phenomena, so familiar that in daily life we scarcely notice it and become conscious of it only in special cases. By heredity is meant simply the fact that in reproduction characteristics of the parents are traminitted to the offspring, so that the descendants resemble in general the ancestors. The offspring of a beetle become bectles of the same form, and from the eggs of a fowl fowls rlevelop; a dog can produce only a dog, a humin being only a human being and never other species. This transmission of the characteristics of the parents to the offspring pertains to the minutest details: not only is the external form of the body transmitted, but suecial peculiarities of motion, attitudes, habits, ete. This is seen must clearly in human beings, since by practice in distinguishing them our gaze is sharpened eren for minutice. But, as a rule, the fact of heredity strikes us only when it has to do with specially characteristic signs, when wo see transmitted from parents to children peculiar features, abnormalities of the borly, such as supernumerary fingers, hair wer the whole body or unon unusuil parts, and physical defects.

But not all peculiarities are alwars inherited. Many special characteristics are not inherited at all, others are transmitted 
from the parents, not to the next generation, but to the second or the third. This transmission of characteristics to the second or third generation, with omission of the first, is known as reversion, or atavism. Thus, in man it is frequently observed that children have peculiarities of their grandparents which are wanting in their parents throughout life. Indeed, many peculiarities, after having remained latent for many generations, can suddenly appoar again. This is frequently observed in domestic animals and cultivated plants which have been artificially bred from the wild forms and been gradually improved. When these are allowed to run wild, as a rule they go back again to the wild state; every breeder of animals and every gardener is acquainted with many such examples. It would lead too far to discuss these facts in cletail, and it would be superfluous, since a great variety of examples have become known through the immortal work of Darwin and the morphological studies that have been carried out in connection with the theory of descent.

One interesting question in the problem of heredity has recently come into the fore-ground and has been discussed very actively, namely, the question of the inheritance of acquired characteristics in multicellular organisms. Are characteristics that have arisen during the individual life through the action of external influences, e.g., mutilations and diseases, inherited, or does inheritance deal with innate characteristics alone, i.e., characteristics that have become established during the germinal development of the organism? While Darwin ('59), Haeckel ('66), Eimer ('88) and others have defended the view that acquired characteristics are heritable, Weismann $(' 92,1)$ has endeavoured to show in a long series of studies that only those characteristics are inherited the rudiments of which were already present in the germ-cells of the organism. At the first glance it seems surprising that such a question, which apparently is so easy to answer, can be the subject of such opposite views; for nothing seems simpler than to decide by experiment whether mutilations, performed upon an adult animal, are transmitted to its offspring. In fact, such experiments have been made by Weismann and others. Weismann removed the tails of twelve white mice, of which seven were females and five males, and bred five generations of descendants, a total of 849 mice, from these tailless parents, but not a single one was born without a tail; and in all the adult animals the tails had their normal length. Many such experiments have been performed, but they prove only that in the cases in question the mutilations are not inherited, and not that no acquired characteristics at all are heritable. Upon the other side a number of examples have been brought forward, from which it would appear that certain acquired peculiarities have been transmitted. But W (ismann has subjecterl all these cases to very careful criticism and has sought 
to show that for various reasons they ought not to be regarded as clemonstrative. Hence, thus far, the question is not decided. A decision can be reached only by experiment, but not by such experiments as those performed upon mice. It is a priari inuprobable in the highest degree that injuries of the tail, the fingur, or similar parts of the borly are inherited, for it is hardly to he imaginerl that the organs in yurstion stand in such a relation to the sexual cells, through which alone reproduction and inheritance occur, that their mutilation shall exercise a marked influence upon thuse colls, which is the tirst requisite of inheritince. In future experiments, therefore, mutilations must be performed upon such organs as stand demonstrably in correlation with the sexual organs, for only then would there be the pusibility of hereditary transmission. "Few such correlations, howerer, are known. In man, as is known, the derelopment of the larynx is correlated with that of the sexual organs. Men who in their youth have lost the testes by castration retain throughout life a larron retarded in its development and a high childish roice. The splendid supranos in st. Petur's at Rome, whose artistic singing is su attractive, have often afforded examples of this. Similar correlations ought first of all to be fully investigated and then to be employed for experiment, unless experimentation is to be a mere groping-about without plan, a process that leares the decision to chance. That influences which affect the germ-cells, the ovum and the spermatozoon, influence the further development in a high degree, is " priori clear, and, moreurer, has recently been shown, especially by the brothers Hertwig ( $\$ 7$ ), in a large number of striking experiments. If, now, mutilations that alter the germi-cells could be performed upon highly developed animals or upon plants, it would be pusible to decide experimentally whether mutilations as such are transmitted by means of a definite action upon the germ-cells, or whether they intluence the latter only in so far that offspring coming from those cells have other defects and abnormalities that are not like the mutilations. In the first case, there would be a real transmission of acquired characteristics, in the second not. Hence the question of the inheritance of acquired characteristies remains to be decided experimentally. W'haterer has thus far appeared upon either the affirmative or the negative side is nothing but more or less probable supposition.

Special characteristics are not necessarily inherited. But the general charilcter's of every organism which for generations have been reproduced constantly, whether they are exclusively innate or are really acquired at some time $b_{r}$ some predecessor, are constantly transmitted in their essentiali. A chinge takes place so slowly that it can sarcely be perceited within the few generations that come under ubservation during the life uf one man or of 
several, or even within many generations; this is evident from the identity of the animal world found in the Egyptian graves with that of the present.

Heredity, therefore, represents an agency upon which depends in phylogenetic development the preservation of peculiarities of form that have once been present.

\section{Adaptation}

Adaptation, which changes form, is not so immediately apparent as heredity, which maintains form. This is especially due to the fact that the phenomena of adaptation usually require long spaces of time for their observation, while heredity appears in every generation of organisms. But the results of adaptation are seen daily, usually without this fact being recognised. The fact of purposefulness in living nature, which was so marvellous to men of science in early times, even down to the middle of the present century, forced them constantly to embrace teleology, i.e., the hypothesis of a fore-ordained plan of creation, such as dogmatic theology, preserving faithfully the ancient venerated ideas, accepts to-day. This purposefulness in nature is the simple expression-or, better, the result-of the adaptation of organisms to their vital conditions in the widest sense.

Aquatic animals are adapted very perfectly to life in water, terrestrial animals to life upon dry land, flying animals to life in the air. Fishes have limbs in the form of fins, which function very perfectly as rowing-organs; terrestrial vertebrates have in place of fins legs for walking and creeping upon dry land; birds have wings constructed most fittingly, with which their light bodies, supported by bones containing air, soar through the air so perfectly that up to the present all inventors of artificial flying machines have tried in vain to imitate them. But only in single cases in the derelopment of the individual can an adaptation to other conditions be traced. Thus, the larvæ of frogs, so long as they live in the water as tailed tadpoles, breathe like fishes by means of gills, which are constructed very simply and suitably for obtaining from the water the air dissolved in it. As soon as the small frogs come to the land, the tails shrink, the gills degenerate, and the lungs develop, by means of which, like all terrestrial animals, they take air directly into their bodies. If the tadpoles be prevented artificially from creeping upon dry land, they retain their tail and gills, and the lungs do not develop even though the animals reach a considerable size. Such examples prove that all organisms are adapted rery fittingly to their vital conditions; and the later zoological and botanical investigations have shown that these adaptations cxtend frequently to the minutest details, of which an untrained obscrver would never think. 
Since the conditions upon the earth's surface have slowly and constantly changed from the time of the incandescent nebula down to the present, since fairly rapid changes of the external conditions of life continually appear in locally restricted regions, and, finally, since all organisms are constructed even to the smallest minutiæ in a manner corresponding perfectly to both general and special conditions, organisms must become adapted to their external conditions constantly and in proportion as the conditions themselves change. If this ratio between the change of external conditions and the change of the form of organisms had not existed in the past, there would have appeared within a conceivable time an extraordinary lack of fitness in the structure of organisms. But the cases in which an organ seems to be superfluous are relatively rare, and injurious mechanisms perhaps do not exist at all.

The mode of adaptation of organisms is a double one : an individual, or personal, and a phyletic, or racial, adaptation may be distinguished. The two occur very differently.

Individual adaptation acts only within very narrow limits, and in the phylugenetic changes of form has, perhaps, only a subordinate importance; it has, indeed, no importance whatever in phylogeny, if the inheritance of acquired characteristics does not take place, for it consists in the fact that changes in the external environment cause direct changes in the organism itself according to the different factors of the environment. Individual adaptation usually exprusses itself much more clearly in habits, manner of life, etc., than in form. A man, put under other conditions than his customary ones, in another land and among other people, adapts himself to his surroundings gradually in the course of years, and gradually adopts the customs, usages, activities and mode of life of the new people. Much more seldom is there observed in organisms a change in body-form through individual adaptation to vital conditions, especially because much more profound changes in the conditions are necessary to cause it, and these are not so easily endured as the relatively slight changes that lead to adaptation in manner of life. A relatively slight change in the composition of the water in which aquatic animals live, leads in most cases to death. Marine animals placed in fresh water and freshwater animals placed in sea water usually die; only a few forms have adapted themselves to both, especially such as live at the mouths of rivers, like certain fishes. A crustacean, Artemice salima, is rery interesting in this connection. Schmankewitsch ('77) established the very interesting fact that this small animal living in salt water can change itself, by slowly becoming accustomed to a higher or lower percentage of salt, into a different form of crustacean-in water of greater concentration into Artemia Millucusenii, in fresh water into Branchipus stugnalis, two forms having wholly different characteristics (Fig. 65). Similar 
cases are known in single cells. Thus A. Schneider, Brass, and O. Zacharias ('85) have produced considerable changes of form in spermatozoa, intestinal epithelium-cells, and Ancoa, by the addition of various solutions to the medium. Unicellular organisms in general, especially Infusoriu and Rhizopode, afford many favourable objects for the study of the changes that the body-form experiences as the result of changes in the surrounding medium. The following example ${ }^{1}$ is very interesting; it show that the various forms of Amela, which are usually characterised by the shape of the pseudopodia, ought not to be regarded as distinct species in the systematic scmse. Innumerable quantities of small amobæe are frequently found in the bacterial scum upon the surface of decomposing hay-infusions. When placed upon the slide, these have an essentially spherical form (Fig. 66, a). Broad, lobate pseudopodia begin gradually to be extended in various clirections, so that the form of

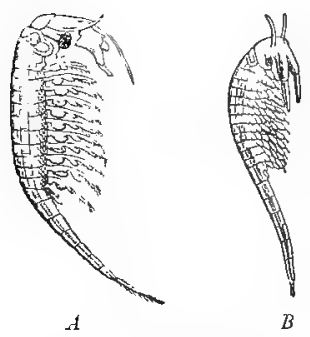

FIQ. 65.-A. Branchipus stag. nalis, fresh-water form; B. Artemia salina, saltwater form of the same crustacean. (From Semper.) Amcebce proteus (princeps) (Fig. 66, 6 ) is assumed. The creeping soon takes on one principal direction, the whole cell in a certain sense representing a single, long pseudopodium and assuming the form of Amaba limax (Fig. 66, c). In this form the. amœbæ creep about constantly, so long as they are not disturbed. If the composition of the medium be changed by making the water very feebly alkaline by the addition of potash solution, the following is observed. The amoba first contract into balls, but soon fine-pointed pseudopodia appear upon their surface (Fig. 66, d). These become longer and longer, and finally assume the appearance of long, pointed thorns. In the course of about 15 or 20 minutes the cells assume the very characteristic shape of Amcba radiosa (Fig. 66,e,f), which is known by the systematists as a very well-defined species; they remain in this condition and show the very sluggish movements of this species so long as the alkalinity of the medium continues. If they are put again into their accustomed water, their shape changes gradually to the usual limar-form. Many moulds, which can be accustomed to concentrated salt-solutions when these contain sufficient food-stuffs for MIucor, behave similarly. The hyphæ, as a rule, become considerably finer and slenderer than in the customary water. In many cases, however, changes in the vital conditions affect, not directly the form of the individual, but in a hidden manner the germ-plasm of the sexual cells, so that the uffspring assume forms different from those associated with earlier conditions; this, however, is rather to be considered under phyletic adaptation.

${ }^{3}$ Cf Verworn $(96,4)$. 
Phyletic adaptation, i.e., the gradual adaptation of the series of forms to existing vital conditions, has a disproportionately great, perhaps a determinative, significance in the form-changes of phylogenetic development. It takes place in a manner wholly different from that of individual adaptation. Darwin's immortal work ('59) consists in explaining naturally the surprising purposefulness in the organic world by revealing the mode of phyletic adaptation. According to Darwin's theory of selection the adaptation of organisms to external conditions takes place, not by the immediate change of the single individual, but by natural

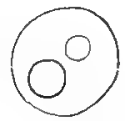

ce

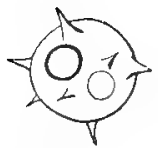

d
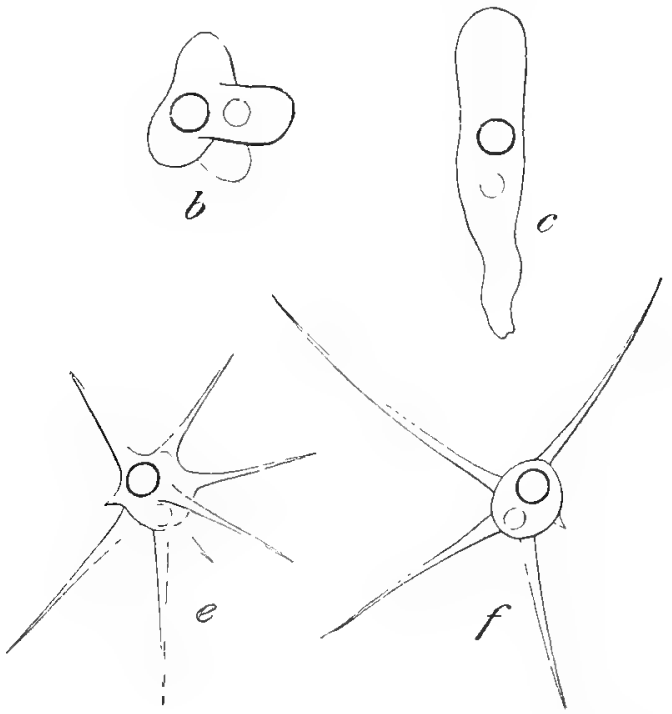

FIa. 66.-Amaba limer. $a$, Contracted; $l$, at the beginning of the formation of pseudopudia, (proteus-form) ; $c$, common lima, form: $d, e, j$, forms assumed after the addition of potash solution; $d$, at the beginning of the actiun; $t, t$, radiosa-forms.

selection among many individuals in the same manner as in the improvement of the race by artificial selection on the part of the breeder.

Starting from the fact of individual variability, i.e., the phenomenon that in every generation of uffspring from the same parents no single individual is wholly like another, although to ordinary observation the differences frequently appear very small, Darwin finds as a necessary consequence of the struggle for existence a choice, a selection, among the different individuals of every generation according to the measure of their vital power. It is known that in all organisms without exception more offspring 
wre produced in germ than as adults would find sufficient vital conditions. To cite a striking example, it has been computed that, if of the several million eggs that a sturgeon lays only one million should develop into females and reproduce to an equal extent, the third generation would find no room upon the surface of the earth, while the fourth generation could produce a quantity of eggs greater than the volume of the earth! But this remarkable condition is illusory, for only a very limited number of individuals can find the proper conditions for their existence, all others perish. But in this partly passive, partly active struggle for the means of existence it is not the chance individuals that perish, but almost exclusively those that can maintain the struggle less long, that are less adapted to the given conditions. On the other hand, those that are strongest, most powerful, most capable of life under the given conditions, will overcome in the competition and alone survive. Thus there takes place a selection of individuals most fitted for the given conditions of life; and since this selection, as in breeding, continues for many and finally innumerable generations, while the selected individuals reproduce their characteristics by hereditary transmission, a gradual adaptation of individuals to their external conditions comes about, the result or expression of which is the purposefulness, reaching to the minutest details, of organisms in relation to the conditions under which they live. If the external conditions remain for a time unchanged, adaptation acts in a conservative sense; if they change, whether locally and suddenly, or generally and gradually, as in the development of the whole earth's surface, there wccurs by selective adaptation in the struggle for existence a proportionate variation of form. The test of the correctness of this theory lies in the experiments of animal breeders, which have gone so far, especially in England, that by artificial selection toward definite aims in the course of a few years new varicties of domestic animals, especially pigeons, can be supplied to order, having these or those desired qualities. Here the artificial selection of the breeder plays the rôle of natural selection which in free nature consummates the struggle for existence.

Darwin's theory affords a comprehensive and consistent picture of the origin of form-changes in living substance from the simplest species that previously inhabited the surface of the earth down to present organisms. If the effects of the few agents that determine form are recognised, it is easy to understand naturally the phylogenetic development of plants and animals from the unicellular protists, on the one side through cryptogams and munocotyledons to the highly developed flowering-plants, and on the other side through the colenterates and worms to the highly developed arthropods and vertebrates. 
All living substance, like every physical body, must have some form, which is determined by its relations to the chemico-physical conditions of its environment. If the relations between organisms and the external world remained constantly the same, no change in the forms of organisms in the phylogenetic series would take place; and, since living substance has the property of reproduction, by heredity the descendants would always be exactly like the ancestors. Since, however, the conditions upon the earth's surface, as upon every physical body, are continually changing, and since the form of living substance, like every physical body, is under the influence of its surroundings, it must likewise continually change by adapting itself to the new conditions. Thus, there are the two opposing factors of heredity and adaptation, and the result of the action of these is expressed in the phylogenetic changes of form.

\section{B. ONTOGENETIC DEVELOPMENT}

The old myth of the metamorphoses of the multiform Proteus never found a more beautiful realisation than in the developmental history of the individual. Just as the organic world as a whole has undergone an unbroken change of form in the course of innumerable centuries, so the single individual, especially the multicellular animal, during its development into the adult organism passes through in the briefest time a long series of manifold forms until it becomes like or approximately like its parents. It does not:belong to the task of general physiology to follow the cycle of development of individual groups of organisms; by the great growth of the fundamental ideas of Darwin and Haeckel our knowledge of individual or ontogenetic development has expanded into an independent science, embryology, the great importance of which for the understanding of the present organic world has been demonstrated during the last few decades. To-day no biologist or physician, who has not became a blind specialist, is unequipped with embryological knowledge. But, although the study of the more special facts of the ontogenetic development of form must be lueft to the embryologist as his well-earned right, physiology has to deal with certain general and elementary vital phenomena, upon which the development of the individual rests. These are the phenomena of reproduction.

As should be the case with all vital processes, these phenomena should be studierl in the cell. The success of this method of treatment has already be'tn demonstrated with reproductive phenomena; morphology has laboured here intelligently and has illumined the whole field solely by means of cellular methods. As a result, we are now oriented as to the minute details of the visible events. 


\section{Grouth and Reproduction}

Reproduction cannot be separated from growth, for in the widest sense it is only a special case of growth; the earlier embryology was prompted to regard reproduction as growth beyond the measure of the individual. The general process that constitutes growth is an increise of living substance, and the essence of reproduction likewise consists merely in an increase of living substance. The difference between that which is usually termed growth in the narrow sense and the phenomenon of reproduction consists only in the fact that in the former rase the newly formed
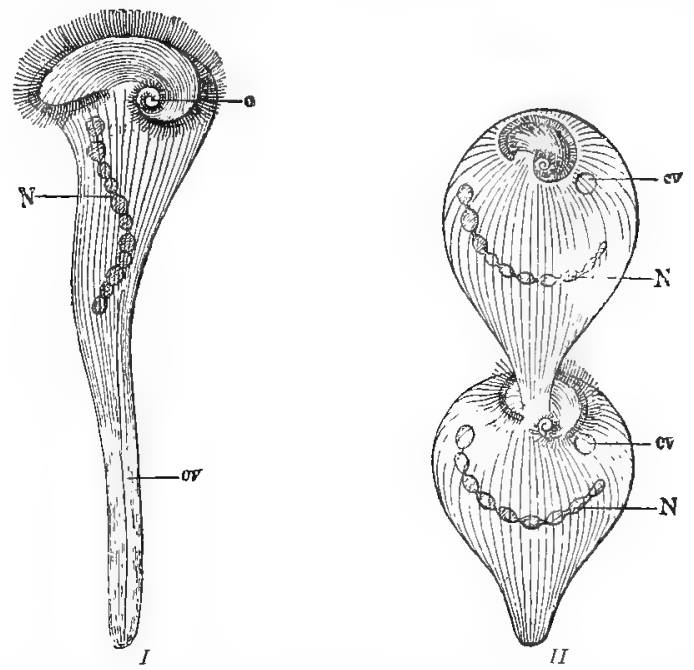

Fig. 67.-Stentor polymorphus. $N$, Mouiliform nucleus ; $o$, mouth-opening, $c c^{\prime}$, contractile vacuole. I. Young individual extended. II. Older individual in the process of division, contracted. (After Stein.)

living substance remains in constant connection with the original organism and helps to increase its volume; while in the latter case a part of the substance separates itself from the original organism, either, as in most cases, being set entirely free, or, as in the increase of tissue-cells, being separated merely by a partition-wall and remaining in place. Correspondingly, there is a large number of transitions between the growth, in the narrow sense, and the reproduction of the cell. Examples of such are afforded especially by many multinucleated cells, as, e.g., Opaline, the infusorian living in the intestine of the frog, which at tirst is uninucleated and in growth becomes multinucleated hy the repeated division of its nucleur. There vecurs here ar roproduction of the nuclei, while the 
protoplasm belonging to them remains in one mass; the final result is a very large but multinucleate cell.

Every cell exhibits, if not continually, at least at a certain time of its life, phenomena of growth; the mass of its living substance increases. This can occur only by taking in material from the outside, or, in other words, by metabolism; and the conception of growth can be rendered precise by bearing in mind that in meta-
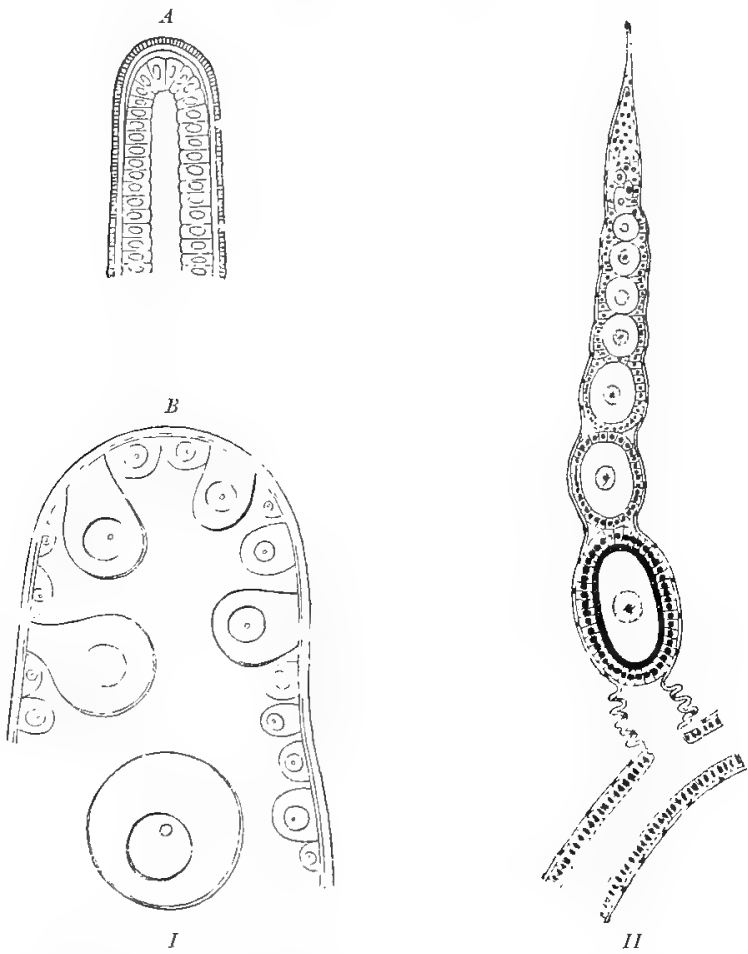

FIC. 68.-I. Formation of eggs in the sea-urchin. $A$, Piece of a young ovary with the germinal epithelium within; $B$, piece of an older ovary, in which the cells of the germinal epithelium ire develuping into eggs which are being constricted off. (After Ludwig.) II. Egg-tubes of the ovary of an insect. In the tubes lie eggs in different stages of formation. (After Hatschek.)

bolisn more living substance is built up than is broken down. But, as has been seen, the size of every cell is limited and does not surpass a certain measure. Particularly the size of every definite cell-form has a limit assigned for that particular form, which varies little. Hence, if the puantity of the living substance increases further by growth, this must lead to a "growth beyond the measure of the individual," the cell-mass must divide, i.e., it reproduces. The cell, therefore, multiplies by division; and every one of the 
parts that arise, every daughter-cell, is correspondingly smaller; it can then grow in turn until it has reached the limit of its individual measure. But in the reproduction of the cell by division, parts must pass over into the daughter-cells from both the essential cell-constituents, the nucleus and the protoplasm, otherwise the daughter-cells would not represent complete cells, and hence could not continue to live.

In another chapter in which we shall consider the mechanical explanation of vital phenomena we shall have to enquire after the deeper-lying causes of growth and of limitation in the size of cells. In this place it is necessary merely to obtain an outlook over the field of vital phenomena. If it be accepted provisionally that reproduction is merely further growth, while the size of the cell is

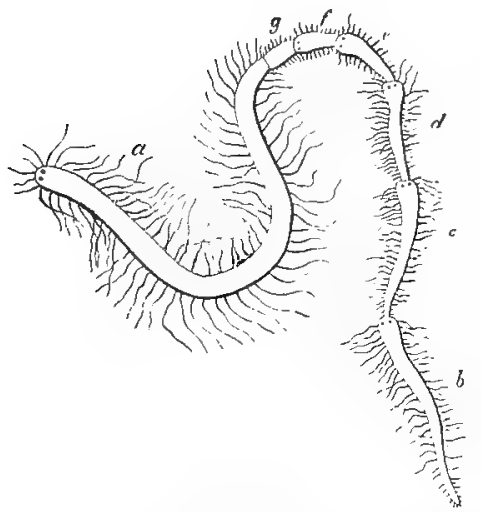

FIG. 69.-Myriunida, a worm in the process of fission. The single individuals are still hanging together like the links of $n$ chain. $a$, The original animal; $b, c, t, e, f, g$, the buds from the oldest (b) to the youngest (g). (After Milne-Edwards.) limited, it follows that all reproduction depends upon a division of the living substance of the cell. The widely different varieties of reproduction are nothing but cell-division; and Virchow has rightly extended the old dictum of Harvey, "omne vivum ex ovo, " into that which forms the basis of all inodern ideas of reproduction, "omnis cellula e celluzla."

This is at once evident in unicellular organisms. They reproduce simply by the division of their cell-body, each daughtercell assuming during the division the shape and form of the mothercell; and if, as in the Infusoria, the cells possess various kinds of appendages or organoids, the elements that are lacking become regenerated after the division of the body (Fig. 67). But in multicellular organisms, both animals and plants, special reproductive organs are developed, the cells of which become constricted off and as eggs develop by repeated cell-division into similar organisms (Fig. 68). In organisins that have separate sexes the sexual cells of the reproductive organs are different in the male and the female individuals. The male sexual cells are the sperm-cells, or spermatozoa, the female the egg-cells, or or . For the production of a new individual a union of the two sexual cells, called fertilisation, must take place, except in certain cases where parthenogenesis is present, i.e., where individuals capable of life can develop from unfertilised eggs, as with many crustacea and insects. Finally, in the lower multicellular animals, in addition to sexual repro- 
duction, there occurs asexual increase, by fission and gemmation. In both cases whole complexes of cells are separated off. In fission, e.g., in certain worms (Fig. 69), the whole body, after having reached a certain size by cell-division, is constricted into two or more parts which regenerate themselves again into complete individuals. In gemmation, e.g., in many coelenterates (Fig. 70), there is formed in one part of the body by rapid cell-multiplication a bud, which contains cells from the essential bodylayers and likewise becomes constricted off to regenerate into a new individual.

In all cases, therefore, reproduction, whether asexual or sexual, takes place by cell-division alone, and this depends upon growth. We will now follow the different kinds of cell-division

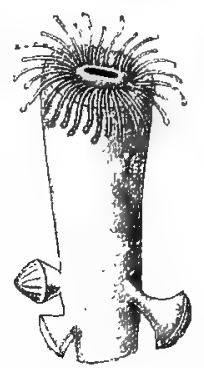

Fig. 70.-Gerumation in a polyp. (After Claus.) somewhat more in detail and consider the remarkable phenomena that take place in the cell.

\section{The Forms of Cell-division}

In order that the daughter-cells of a cell-division may be capable of life, both the nucleus and protoplasm, as already remarked, must divide. But while the division of the protoplasm is very simple, the cell-body simply becoming constricted deeper and deeper by a groove until the protoplasm is separated into two halves, in most cases there appear in the nucleus extremely complicated changes, which in most cells, both animal and plant, agree remarkably in essentials. Regarding the more minute phenomena of cell-division a literature so large as to be almost beyond mastery has appeared during the last two decades, since investigators, misled by the very peculiar behaviour of the nucleus in cell-division, adopted the erroneous view that the nucleus is the sole essential cell-constituent and must be studied as exhaustively as possible in its "active" condition. The fundamental investigations of the phenomena of cell-division comprise the admirable ones of Butschli ('76), Flemming ('82), Strasburger, ('80, '88), O. Hertwig ('76, '77, '78, '92), van Beneden ('87), Boveri ('87, '88, '90), and others, who have found objects best fitted for this purpose in the cells of young larvæ of salamanders, in the pollen-cells of lilies, and in the transparent eggs of the sea-urchin and the round-worm of the horse.

\section{a. Direct Cell-division}

The simplest form of cell-division is the direct or anitotic celldivision, which, however, is comparatively rare and, beyond certain 
unicellular organisms and leucocytes, has been met with only in very f(w forms of cells. The division of Amoba can serve as a type (Fig. 71). While the Amobo is creeping, the original spherical nucleus becomes gradually lengthened, then biscuitshaped, then constricted through the middle; the connectingpiece becomes constantly slenderer and finally breaks; and thus two new nuclei result, which immediately assume the spherical form. Then the division of the protoplasm begins; the Amoba

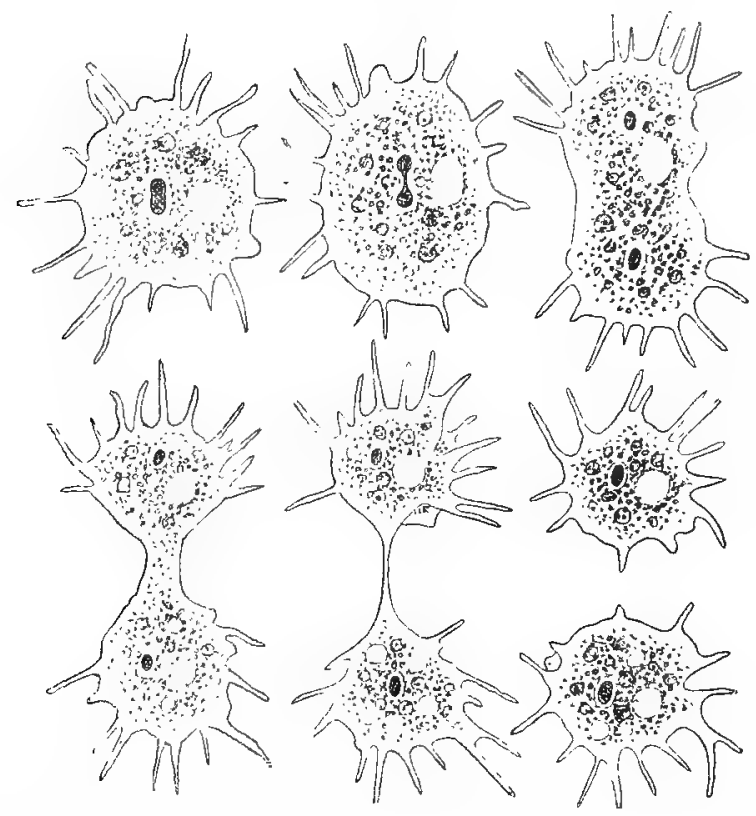

F1G. 71.-Amoba polypodia in six successive stages of division The dark body surrounded by a clear area in the interior is the nucleus, the pale body the contractile vacuole. (After F. E. Schulze.)

becomes constricted in a similar manner between the two nuclei like a dumb-bell and creeps towards the two sides, until only a thin thread of protoplasm unites the two halves, this finally breaks so that two new Amobce, each with one nucleus, result from the division. The process requires a long time, usually several hours, and does not always proceed smoothly: the protoplasm often flows together into one mass after a considerable constriction has taken place, and then flows apart again, until, finally, the uniting bridge is torn through. 


\section{b. Indirect Cell-division}

By far the great majority of all animal-and plant-cells follow the mode of the so-called indirect or mitotic cell-division, in which the protoplasm is simply constricted, while the nucleus undergoes very remarkable and typical changes of great regularity. Different authors have distinguished different stages and have designated them by different names. Two phases in nuclear division can be

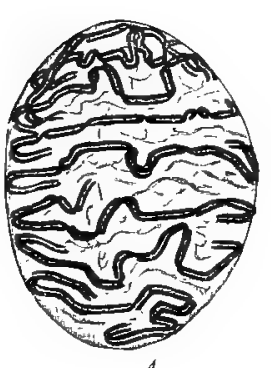

A
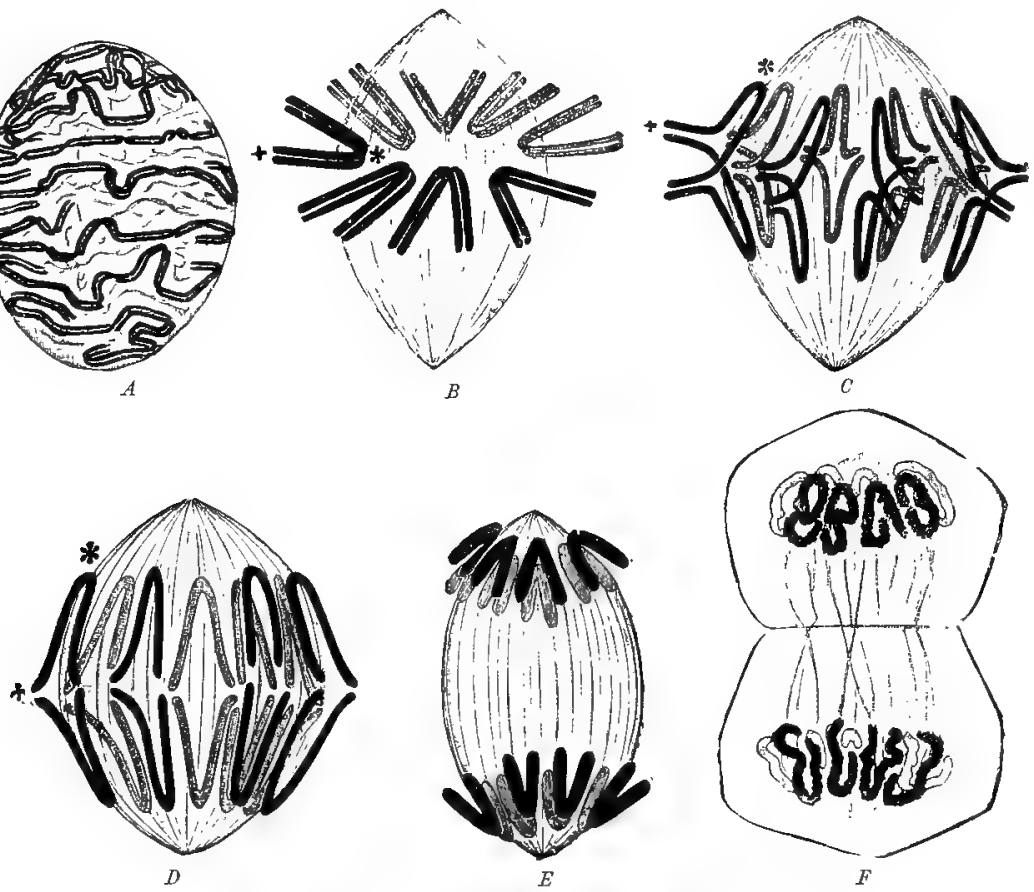

FIG. ì,-Scheme of mitatic cell.division. (After Flemming.)

very generally recognised-a progressive one, in which the changes reach their height, and a retrogressive one, in which the two nuclear halves that arise from the division go back to the "resting-stage" of the nucleus, which latter term designates the condition in which the nucleus shows no phenonena of division. A picture will put before our eyes the important phenomena of nuclear division better than all classifications and descriptions (Fig. 72).

To begin with the resting nucleus about to undergo division, it is seen that the chromatic substance, which, as is well known, 
consists of nucleins, arranges itself into threads which appear loosely rolled up into a coil (Fig. 72, A). The threads, which have given to this form of nuclear division the name of mitotic division and have approximately equal lengths, split lengthwise so that from each a double thread results. At the same time the nuclear membrane becomes dissolved, and at the two opposite poles of the nuclear mass the centrosomes, or central bodies (p. 69), surrounded by their protoplasmic radiations, now become visible, the two being united to one another by a fibrous, spindle-shaped figure which is derived from the achromatic substance mixed with the protoplasm. The double threads form loops, and group themselves in the equator of the achromatic nuclear spindle in such a way that their angles are directed towards the centre (Fig. 72, B). Presently the spindle-fibres, streaming out from the centrosomes, by their own contraction divide the double threads in such a way that one half of each is turned toward one pole, the other half toward the other

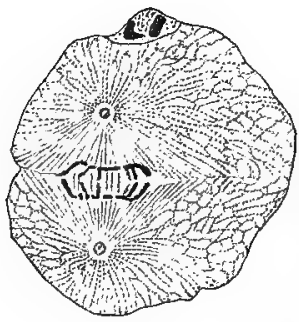

FIG. 73.-Centrosomes with protoplasmic radiation in the division of the 'egg-cell. (After Boveri.) (Fig. 72, C). Thus two groups of threads separate from each other and from the equator of the spindle (Fig. 72, D). With this the progressive phase of nuclear division is ended and the retrogressive phase begins. The two groups of chromatic threads proceed further and further toward the two poles, so that the whole equatorial part of the spindle becomes free (Fig. 72, $E$ ). Presently the spindle-fibres between the two groups begin to become indistinct, and the threads become twisted again into a coil at each pole (Fig. 72, F). During this process the whole cell-body has become constricted by a circular groove, the plane of which stands at right angles to the axis of the two nuclear poles: The groove becomes deeper and deeper, until finally the whole cell divides into two equal halves, each of which possesses a nucleus; the latter surrounds itself with a new nuclear membrane, the spindle-fibres completely disappearing, and thus returns to its resting-stage. Thus by the division of the mother-cell two daughter-cells have arisen, and these continue the growth on their own behalf (Fig. 72, F). But during the division a phenomenon has appeared in the protoplasm. Simultaneously with the appearance of the spindle, the poles of which are formed by the centrosomes, two starshaped figures begin to appear in the protoplasm, by the latter arranging itself at each pole like rays around the centrosome as a centre; the centrosomes thus become surrounded exactly like two suns by a closed circle of rays (Fig. 73). As the spindlefibres become indistinct the protoplasmic rays also disappear. 
This mode of mitotic nuclear division is the same in the different forms of cells, almost without exception and even to the finest details. But the division of the cell as a whole does not always proceed in exactly the same manner. Deviations from the type occur in various cases, especially in the division of egg-cells that contain much nutrient material (yolk). With O. Hertwig ('92)
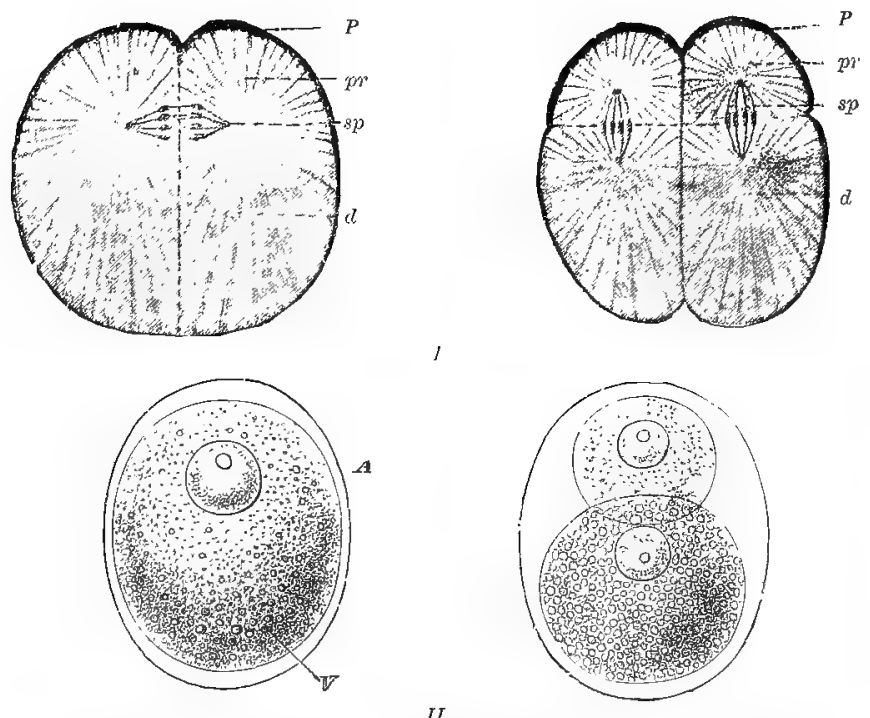

FIG. 74., 1. Division of the frog's'egg. $\quad P$, Pigmented surface of the egg; $p r$, protoplasmic pole ; $a$, pole rich in yolk; $s p$, nuclear spindle. (After Hertwig.) II. Unequal division of the egg of a worm (Fabricia). A, Protoplasmic pole; $V$, pole rich in yolk. (After Haeckel.)

all known forms of cell-division can be conveniently classified under four types--

I. Total division.

a. Equal division.

b. Unequal division.

c. Gemmation.

II. Partial division.

III. Multiple division.

IV. Reducing division.

In total division, the protoplasm of the daughter-cells is completely divided by a partition, so that complete cells always result from the division. But certain differences are here noticeable. In one case, that of equal division, the daughter-cells are entirely equal, as in the type described above (Fig. $\left.72, F^{\prime}\right)$. In another case, that of unequal division (Fig. 74), the two daughter-cells are 
unequal in size and their contents differ; the larger one contains the chief mass of the passive yolk, while the smaller one consists principally of active protoplasm. In this way differences arise which have an important bearing upon the subsequent divisions, and become constantly greater. In the third case, that of gemmation, only a very small portion of the egg-cell becomes divided off; this
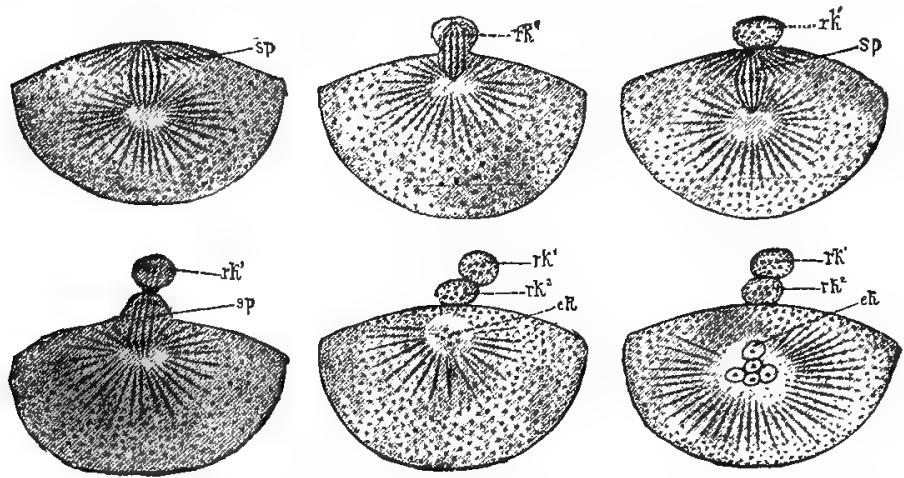

FIG. 75.-Formation of the polar bodies in the starfish; $s p$, nuclear spindle; $r k 1$, first polar body; $r k^{2}$, second polar body; $t k$, egg-nucleus.

occurs especially during the maturation of the egg in the formation of the so-called polar bodies or direction-corpuscles, where the process occurs twice in succession (Fig. 75).

In partial division the groove that separates the two daughterhalves extends not through the whole cell, but through a part
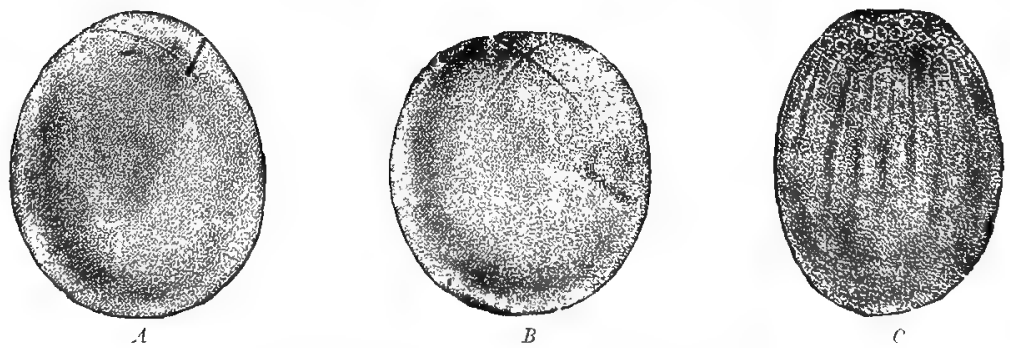

Fig. 76.-Discoidal cleavage of the egg of a cephalopod. (After Watase.)

only, so that in subsequent divisions the daughter-halves remain united on their under side by a common protoplasmic mass (Fig. 76). This form is termed discoidal cleavage.

In multiple division, no division whatever of the protoplasm appears at first, but the nuclei alone multiply in the egg-cell; later, however, they wander to the surface and there surround 
themselves with a separate protoplasmic covering. Thus there exists upon the whole surface an indifferent yolk-mass surrounded by a single layer of separate cells (Figs. 77 and 78) - a phenomenon that has been termed superficial cleavage.

A special kind of multiple division is spore-formation, which is: especially common in the Protista. The characteristic of this
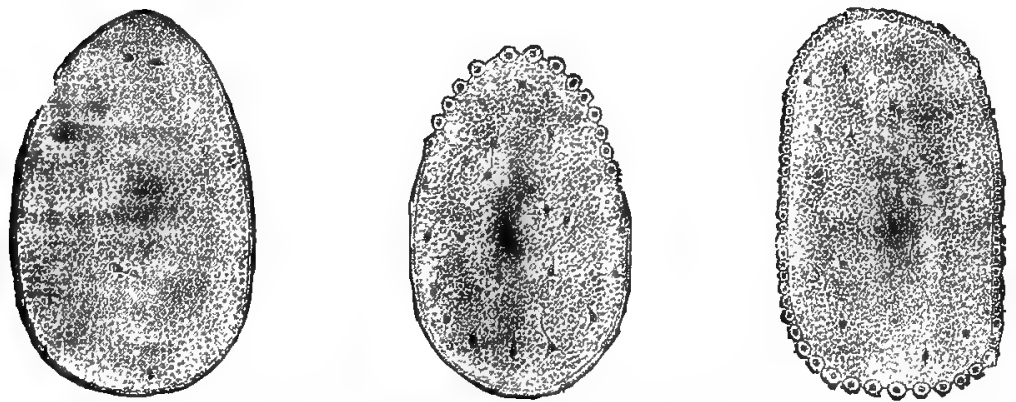

Fia. 77.-Superficial cleavage of the egg of an insect in three successive stages. (After Bobretzky.)

form of cell-multiplication is that the nucleus breaks up into a very large number of tiny granules. Each of these small nuclei surrounds itself with a certain quantity of protoplasm, so that tiny cell-territories appear, which become free as amobæ or flagellated cells, while the rest of the protoplasmic body perishes. The swarm-spore, set free, represents a very small cell containing a nucleus, and slowly develops into the form of the protistan cell from which it was derived.

Finally, in reducing division, as Weismann has termed certain processes that lead to the formation of the ova and the sperm-cells in the ovary and the testis, a slight deviation in the behaviour of the chromatic fibres of the nucleus appears during division. The sperm-cells arise by repeated division of other cells, the sperm mother-cells. The first
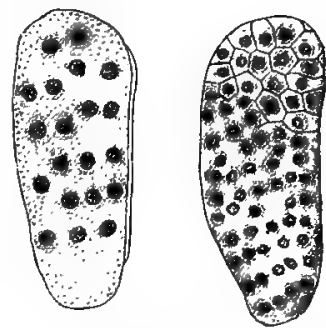

FIG. Ts.-Multiple division in the cleavage of the egg of an insect in two successive stages. (After Balbiani.)

\section{division of the sperm mother-cells} proceeds according to the type described above, but before the nuclei have returned to the resting-stage a second division takes places, each centrosome dividing into two halves which diverge from one another and attract to themselves on both sides the chromatic fibres that arise from the first division, without the latter being able to split, lengthwise as in the normal division. Thus, one half of the chromatin-loops wander toward one pole, 
the other half toward the other pole, so that by this second division each nucleus obtains only one-half as many chromatin-fibres as in a normal division (Fig. 79).
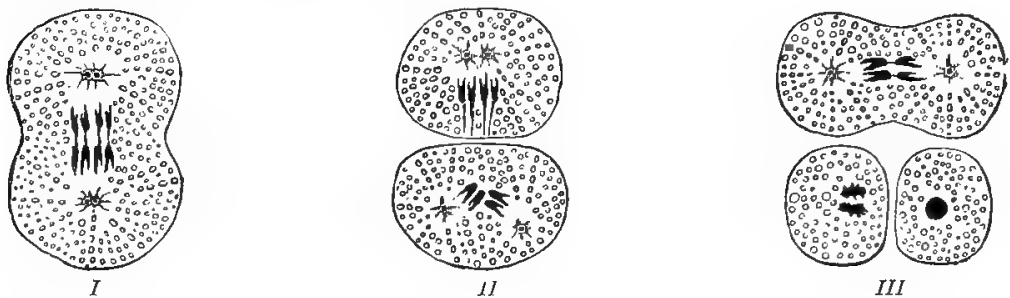

Fua. 79.-Reducing division in the origin of the sperm-cell from the sperm mother-cell of the thread-worm of the horse. (After O. Hertwig.)

These comprise the various forms of cell-division which have become known thus far. The only element common to them all is the transfer of both nuclear substance and protoplasm to the daughter-cells.

\section{Fertilisation}

The act of fertilisation is intimately associated with that profound mystery with which mankind is wont to invest its most sacred feelings. The biologist recognises that fact that the unconscious aim of normal sexual love, one of the most powerful factors that control organic life, is the microscopic act of fertilisation of the female egg-cell by the male sperm-cell. At first sight it might seem strange that so powerful motives, as are those of love in human life, culminate in so tiny a phenomenon, which cannot be perceived by the naked eye; but when it is borne in mind what the result of this act is, what an endless chain of complex processes and changes associated with the development of the new organism from the egg is caused by fertilisation, and what is the end-result of this long series of developmental processesnamely, the highly complex animal, man, with the immeasurable richness of his life--then this fact loses its strangeness, and we come to attribute to the tiny act of fertilisation an extraordinary significance, which it contains in potentia. It is no wonder, therefore, that since early times physicians and men of science have made sexual reproduction the subject of deep research. Yet it was not till after Leeuwenhoek had constructed the microscope that his pupil, Ludwig van Hammen, discovered the sperm-cells, which because of their active intrinsic movements were called "spermanimalcules" or "spermatozoa." And only the unlooked-for prrfection of the microscope in the present time has made possible the brilliant work of Bitschli, Fol, Hertwig, van Beneden, Boveri, 
and others, who have thrown light upon the minute details of the phenomena of fertilisation.

In the human being and the higher animals the process of fertilisation cannot be observed, because it is concealed in the interior of the female body, and it is not possible to keep the egg-cells alive outside of the body and there fertilise them with sperm. This latter method, however, succeeds with certain lower animals, and hence in eggs that are particularly large and transparent, such as those of the sea-urchin and the round-worm of the horse, the whole course of this interesting process has been carefully studied.

As has already been seen, the male and the female germ-cells are differentiated very differently. While the ova usually are large, spherical or amoboid cells consisting of a vesicular nucleus and much protoplasm, the latter containing the building-materials for the future development (Fig. 80), the spermatozoa are ex-
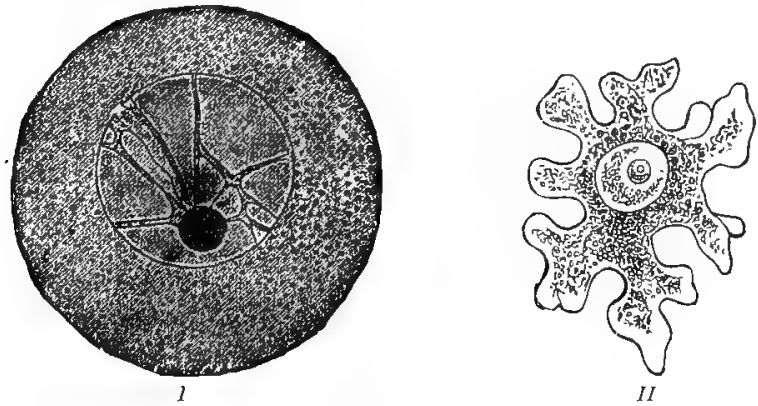

FIG. 80.-Ova. I. Spherical ovum of a sea-urchin. (After Hertwig.) 11. Amceboid ovum of a calcareous sponge. (After Haeckel.)

tremely tiny in comparison with them. The spermatozoa consist chiefly of nuclear substance, and have only a thin protoplasmic covering; in most cases the latter is extended into a motile flagellum, the tail, which is distinguished from the rest of the body, the head, and serves for the movement of the spermatozoon in seeking the ovum. The finer structure of the sperm-cell, as the detailed investigations of Ballowitz ('90) have recently shown, is very complicated, and very various differentiations occur among different animals. The accompanying illustrations present some examples of this (Fig. 81). But both the spermatozoa and the ova are always complete cells, and contain both the essential cell-constituents, protoplasm and nucleus-a fact upon which special emphasis should be laid.

Before fertilisation takes place, in some cases also during the beginning of fertilisation, there occurs the maturation of the ovum, which consists in the formation, by means of two successive divisions of the nucleus, of two buds, the polar bodies or direction- 
corpuscles, and their subsequent extrusion (Fig. 75, p. 196). Fertilisation, therefore, consists in the union of a mature egg-cell with a sperm-cell, in which process the latter seeks the former by its own locomotion. We shall become acquainted with the mode of locomotion later in considering the phenomena of movement.

The process of the union of two cells is a phenomenon that occurs not only in sexual reproduction but is constantly met with among unicellular organisms, where sexual differentiation cannot be said to exist. There, in the Protista, it is known by the name of conjugation. Conjugation occurs even among the unicellular shell-bearing Rhizopoda, e.g., in Diffugia, which is provided with a delicate capsule. In this genus two, and sometimes three, four,
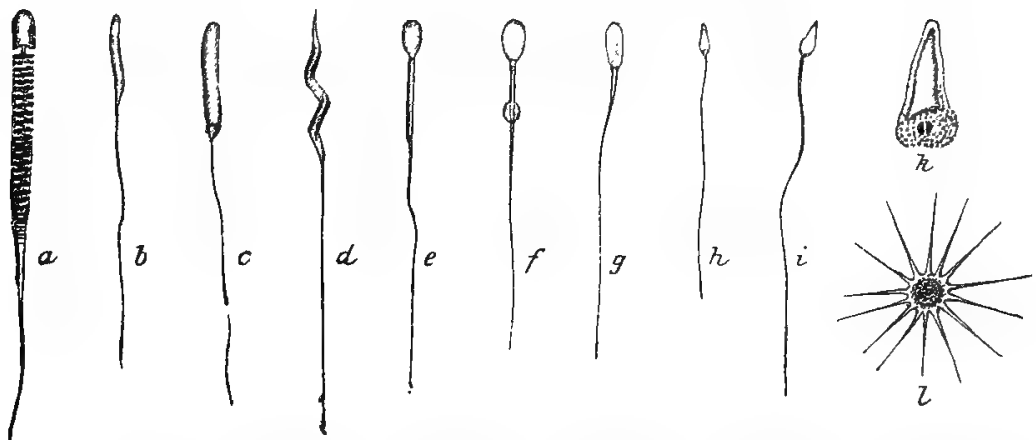

Fig. 81.-Various forms of spermatozoa, a, From a bat (T esperugo nocturna) (after Ballowitz) $; b$ and $c$, from the frog; $d$, from the finch; $e$, from the sheep; $f$ and $g$ from the pig. (After Sohweigger-Seidel.) $h$, From a medusa; $i$, from a monkey (Cercopithecus); $l$, from a crustacean. (After Claus.) $k$, From the round-worm (after Boveri).

or even more, of the sluggish protoplasmic forms creep closely together; their protoplasmic bodies lie in contact with one another, then coalesce into a common mass, and finally separate after the protoplasm of the various bodies has mixed and certain changes in the nuclei have taken place. ${ }^{1}$ The phenomena of conjugation in ciliate Infusoria have been studied very thoroughly by Buitschli ('76), Balbiani ('61), Maupas ('88), A. Gruber ('86, 2), and R. Hertwig ('88-'89). Paramecium is an oblong infusorian, completely ciliated upon the outside, and constitutes an extraordinarily favourable object for cell-physiological investigations of the greatest variety. Paramacia, visible to the naked eye, may be cultivated in great quantity in decomposing hayinfusions and may be kept in stock. It is frequently observed that an epidemic of conjugation suddenly appears throughout the whole culture, so that almost none but conjugating individuals are found. The phenomena of conjugation are as follows:-Two indi- 
viduals apply themselves parallel to one another at their mouthopenings (Fig. 82, I., o), their masses of protoplasm join together to form a bridge, and very characteristic changes in the nuclei begin. As above remarked, ciliate Infusoria have two forms of nucleusa macronucleus, or chief nucleus, and one or more micronuclei, or

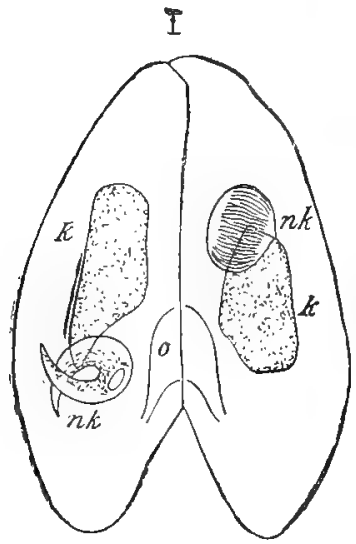

II

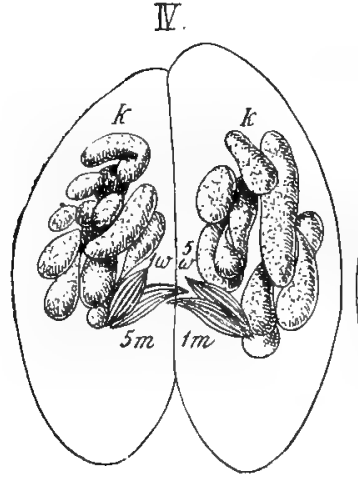

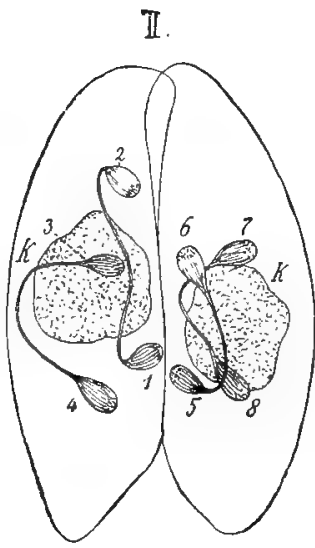

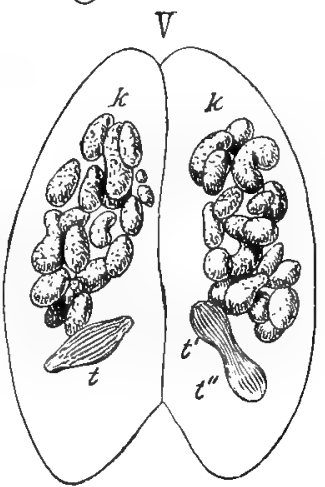

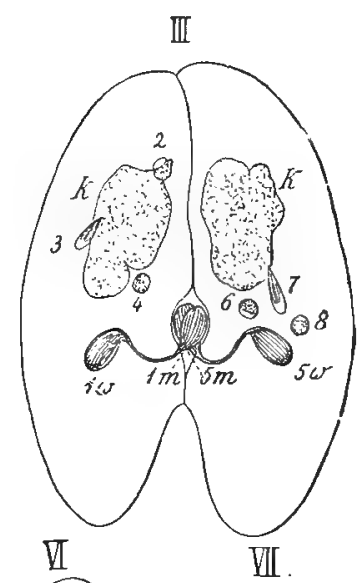

Frg, 52,-Conjugation of $P_{4}$ romcecin $m$ in the various successive stages; $K$, macronucleus: $n k$, micronucleus. I. Beginning of conjugation. II. The micronucleus has divided twice in succession. III. Three of the four portions of the micronucleus perish, the fourth divides once more into a male $m$ and a female 20 nucleus. IV. While the macronucleus is disintegrating, the two male nuclei, $l m$ and $5 \mathrm{~m}$, become exchanged and unite with the two female nuclei into a nucleus, $V$. $t$, which divides in turn into $t^{\prime}$ and $t^{\prime \prime}$. VI $t^{\prime}$ and $t^{\prime \prime}$ divide. VII. From this division arise the rudiments of the new macronucleus $p t$ and the new micronucleus $\pi k^{\prime}$. The old macronucleus perishes. (After R. Hertwig.)

accessory nuclei. During conjugation the macronucleus perishes, disintegrating and dissolving in the protoplasm. If the Paramocium be a form possessing one micronncleus, such as Paramecium ccudatum, where the relations are simplest, the micronucleus in each individual divides twice in succession, so that four partial nuclei arise. Three of these likewise dissolve in the protoplasm, 
but the fourth divides once more in each individual, and one half (the "male" nucleus) passes over the protoplasmic bridge into the other individual, so that each one of the pair now contains a "female" nucleus of its own, and a "male" nucleus from the other. These two nuclei immediately fuse together and then divide, one half becoming a new macronucleus, and the other half a new micronucleus. After such a mutual exchange of half-nuclei, the pair separate again and the conjugation is ended.

The phenomena of fertilisation in sexual reproduction are derived phylogenetically from the conjugation of asexual unicellular

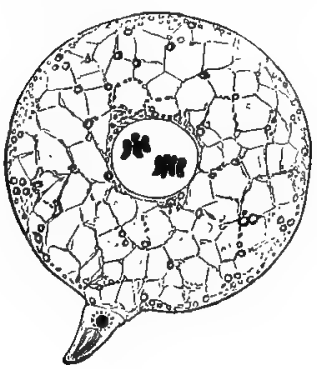

1

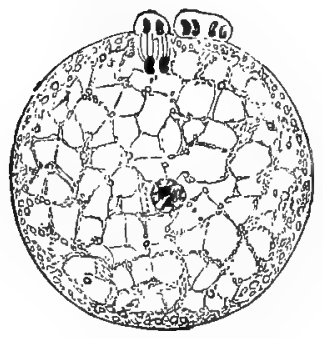

$1 V$
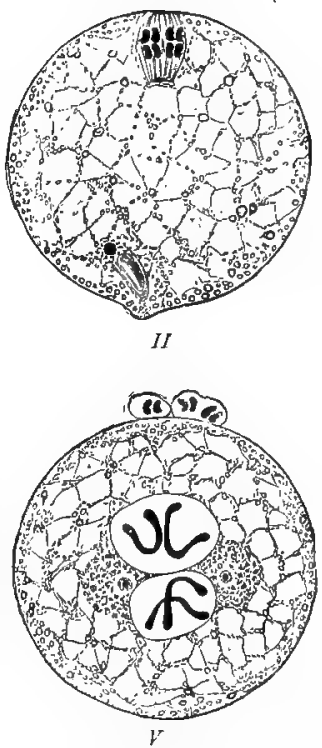
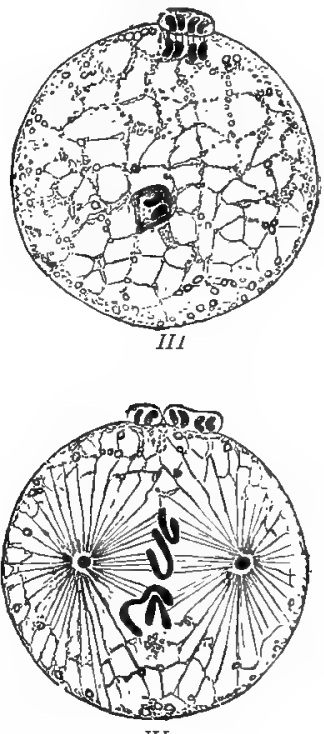

VI

FIG. 33.-Fertilisation of the ovum of the thread.worm (Ascaris megalocephala) in six successive stages. The maturation of the ovun, $i_{0}(.$, the extrusion of the polar bodies, takes place simultaneousig. (After O. Hertwig.)

organisms; essentially the same facts are found in the former as in the latter. The process of fertilisation is not entirely the same in different species; at least in the two species that thus far have been most fully investigated, the egg of the sea-urchin and that of the thread-worm of the horse, some slight differences have been observed, although the essential factors agree throughout.

We shall consider, first, the fortilisation of the ovum of the threadworm. The maturation of the ovum, i.e., the extrusion of the polar bodies, takes place while the sperm-cell is entering the egg. While the latter process is taking place (Fig. 83, I), the eggnucleus, which up to this time has lain in the middle of the egg, 
wanders to the surface (Fig, 83, II), where it divides twice in succession and gives off the polar bodies (Fig. 83, $I I I$ and $I V$ ). In the meantime, the protoplasm of the sperm-cell has fused with the protoplasm of the egg-cell and withdrawn from further observation. The sperm-nucleus, however, has wandered into the middle of the egg; to which place also the egg-nucleus, after giving off the polar bodies, returns from the periphery. The two nuclei now apply thenselves to one another, surround themselves with a transparent envelope, and show distinctly two large chromatic loops in each. At the same time, two centrosomes become visible and begin to surround themselves at opposite sides of the nuclei with a circle of rays (Fig. 83, $\mathrm{V}$ ). In the thread-worm the nuclear substances do not fuse, but the well-known spindle of nuclear division develops, beginning at the two centrosomes, and the spindle-fibres on either side draw to their respective poles one chromatic loop from the egg-nucleus and one from the spermnucleus, so that each half of the egg-cell obtains one nuclear component from the egg and one from the spermatozoon (Fig. 83, VI). The fertilisation is thus ended, and at the same time the first division of the ovum is prepared for; the latter now proceeds in the usual manner, the egg being constricted through the equator of the spindle, while the nuclei in the two halves assume their resting-form.

As regards individual points, the fertilisation of the egg of the sea-urchin proceeds somewhat differently. The maturation of the ovum is completely ended when the spermatozoon enters. Further, the egg- and the sperm-nuclei fuse completely into a single nucleus before the division into the first two cleavage-cells of the ovum takes place. Fol ('91) supposed that he had made in the further course of the fertilisation-process an observation of special interest, because it appeared to shed some light upon the behaviour of the centrosome. What he saw was the following: With the sperm-cell, a sperm-centrosome enters the ovum, which still possesses, in addition, its own centrosome. After the union of egg-nucleus and sperm-nucleus the two centrosomes come to lie at the two opposite poles of the common nucleus, which is surrounded by a simple protoplasmic radiation. Each of the two centrosomes thereupon divides, constricting itself like a dumb-bell into two, each of which wanders across to the other of the opposite side, a phenomenon that was termed by Fol the "quadrille of the centrosomes." Thus, each half of the original egg-centrosome comes into union with one half of the sperm-centrosome and finally fuses with it, so that only two centrosomes are present again at the opposite poles of the nucleus; each of these two, however, consists in half of the substance of the egg-centrosome and in half of that of the sperm-centrosome. These two centrosomes now form the poles for the following division of the nucleus 
and become surrounded each with its own protoplasmic radiation. Thus the fertilisation is ended and the division of the fertilised ovum into the first two cleavage-cells is introduced. But, unfortunately, this account by Fol concerning the course of fertilisation and the much-quoted "quadrille of the centrosomes" appears to rest upon incorrect observation. At least Boveri ('95) and, in harmony with him, Wilson and Mathews ('95) in sea-urchin eggs, and Mead ('95) in the eggs of tube-worms (Chotopterus pergamentaceus), have found that such a quadrille of the centrosomes does not exist, that rather the centrosome of the egg-cell perishes and disappears (Mead) without playing any roble, while that of the sperm-cell after fertilisation divides alone in the egg-cell into two centrosomes, each of which becomes a centre for the protoplasmic radiation and the succeeding division of the fertilised ovum.

A résumé of the essential factors of the phenomena of fertilisation leads to the following statement: Fertilisation consists in the union of two cells, the egg-cell and the sperm-cell, in which protoplasm fuses with protoplasm and nucleus with nucleus; thus, in the succeeding division of the fertilised egg-cell cach half obtains material from both the fused cells, and from both the protoplasm and the nucleus.

\section{The Development of the Multicellular Organism}

Development may be defined in a general sense as a continuous series of changes. If we leave out of consideration the reproduction of the multicellular organism by the constriction of entire parts of the body, as in gemmation and fission, where the essential cell-groups of the individual systems of organs are transferred directly from the parent organism to the buds or products of fission, the formation of the multicellular organism consists only in its development from the egg-cell. The multicellular organism develops gradually from a single cell, whether the egg develops without fertilisation, as in the interesting phenomenon of parthenogenesis (which occurs in certain lower animals and affords a real background for the ancient legend of the immaculate conception), or whether the egg has previously been fertilised, as is the general rule in the development of animals and plants.

Development is present in unicellular organisms, but here the whole cycle proceeds in a single cell. The development of the Protista forms an interesting analogy to that of multicellular organisms, both animals and plants. In the lowest forms, such as $A$ mocoba, development is identical with simple growth. An Amoebc changes simply by increasing in mass and then dividing. The halves then grow again until they become so large that they again divide. The whole developmental cycle of Amaba consists in growth up to cell-division. We see, therefore, that growth and 
cell-division ar' the simplest elements that development demands; in firet, in the whole living world there is no development without growth and cell-division. In all Protiste that reproduce by sporeformation, there occurs a development expressing itself in complex changes of form. In this case the spores, which are totally unlike the mother-cell, must pass through a series of changes of form until they become like it. The development of the Protisto has been little studied. Nevertheless, Rhumbler ('88) has followed completely and with great care that of the infusorian genus Colpoda. Colpoda is a small bean-shaped infusorian, the surface of whose whole body is ciliated (Fig. 84, $A$ ). In spore-
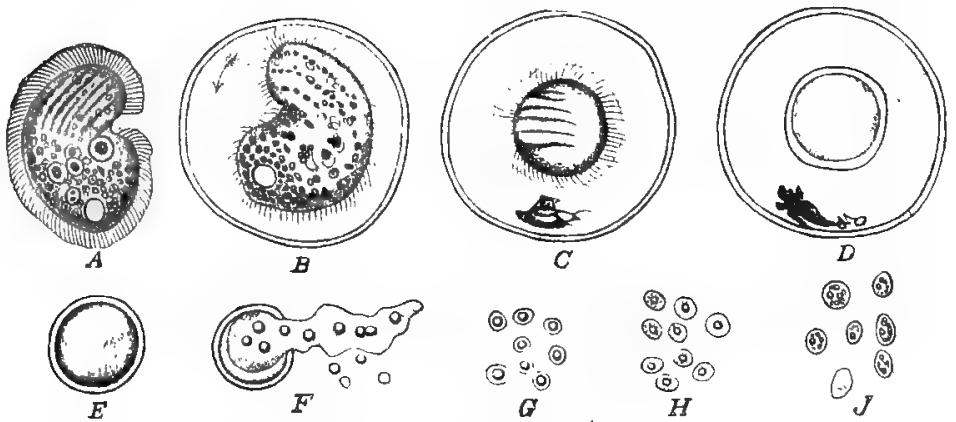

(9) (9)
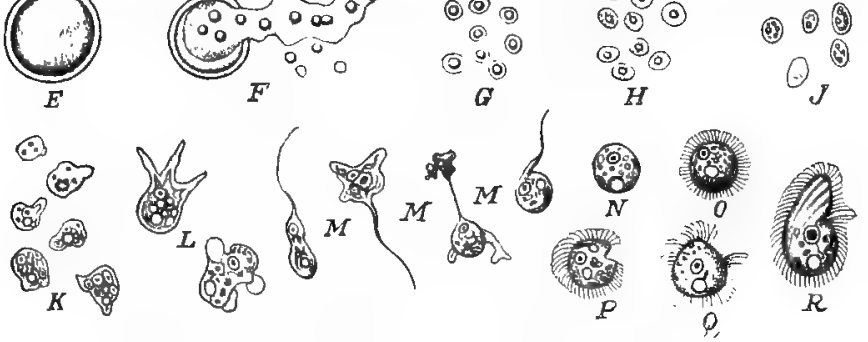

Fig. S4,-Depelopment of Colpona cucullu. (After Rhumbler.)

formation the body surrounds itself with a thick envelope or cyst $(B)$, within which by giving off water the body constantly diminishes its volume. Finally it extrudes all undigested foodparticles and draws itself together into a ball $(C)$, which loses its cilia and surrounds itself by a second smaller envelope $(D)$. The contents of this second envelope $(E)$ break up into single spores, which together with it remnant consisting of useless material burst the capsule and freely wander out $\left(F^{\prime}\right)$. From each spore $(G)$ a new individual develops by the spore transforming itself into a small amoba-like being which creeps about, takes food, grows $(H, J, K, L)$, develops a long flagellum with which it swims $(M)$, and finally contracts into a small spherical cell $(N)$, which covers its surface with cilia $(O)$, and by further growth gradually assumes the form of a Colpoda $(P, Q, R)$. Thus the developmental cycle is completed. 
That which comes to pass among the Protista in a single cell, takes place in an aggregate of cells in the development of the multicellular organism. In accordance with the above considerations concerning reproduction, the development of the multicellular organism from the unicellular egg can take place by continued celldivision only. But in this process two factors play important róles: first, the products of the division of the egg-cell do not separate as in most Protista, but remain in connection with one another; and, second, the products of division are not always alike, but by unequal division two forms of cell, wholly different from each other and from the mother-cell, can arise. In this manner is
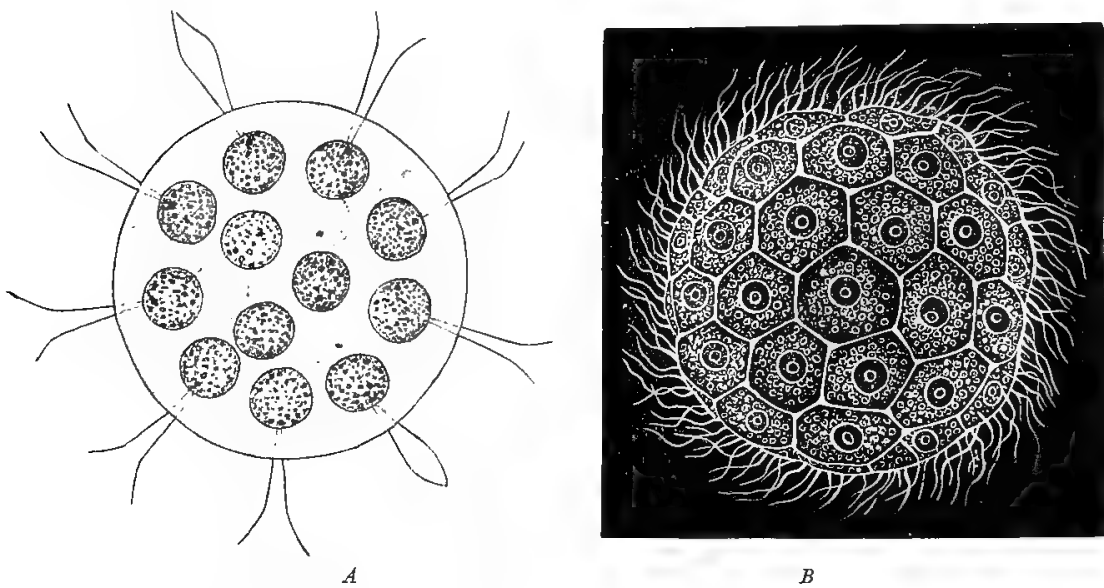

FIG. 85.-A. Eudorina elegans, B. Magosphcera planula, two multicellular organisms consisting of similar cells. (After Haeckel.)

rendered possible the origin, not only of a multicellular organism, but of such an organism with differentiation of various kinds of tissues and organs. If the first factor alone were present, there would result a cell-community consisting ' of many cells, all of which, however, would be alike. Such organisms exist among Protista (Fig. 85), and are regarded as cell-colonies that have a republican constitution, i.e., in which every cell is exactly like every other. These forms are the intermediate links between the really unicellular organisms and the animals or plants. In the bodies of animals and plants, even the lowest, the cells are not all alike, and this differentiation, through which alone the development of a complex cell-community becomes possible, depends upon the efficiency of the second factor, unequal celldivision. Hence, cell-division, both equal and unequal, and cohesion of the cells are the factors that bring about the development of a differentiated cell-community. 
We cannot go further into the special phenomena of the individual development of animals and plants, and must refer the reader to the detailed works of Haeckel ('91), O. Hertwig ('90), and Korschelt and Heider ('90), who treat embryology as an independent science. We must, nevertheless, glance at that important law which, as has already been seen, prescribes a definite path to individual development, namely, the fundamental law of biogenesis.

Karl Ernst von Baer, the founder of embryology, discovered that in the embryonic development of widely different forms of animals, stages occur that appear strikingly similar; and after Darwin's epoch-making labour Fritz Müller ('64) expressed clearly the fact that the developmental history of the individual is a short repetition of the whole course of development which the corresponding species has undergone during the development of the earth. It was Haeckel's service to formulate more exactly the fundamental law of biogenesis and emphasise the existence of a causal relation between ontogeny and phylogeny. Haeckel ('66) showed that individual development, or ontogeny, is only in gross outline a repetition or palingeny of the racial development or phylogeny, but that this repetition is frequently blurred or falsified by the appearance of phenomena that are not present in the phylogeny of the corresponding form and which, therefore, he termed the phenomena of falsified development or cenogeny. Hence, in the individual development of every organism, two elements may be distinguished : tirst, the palingenetic phenomena, which recapitulate in brief the racial development of the form in question, and, second, the cenogenetic phenomena which have arisen supplementarily by adaptation and have altered and blurred the course of the palingenetic phenomena.

The causal explanation of these facts lies in the two factors which, as has been seen, control the whole development of organic life, namely, heredity, which maintains form, and adaptation, which changes it.

The characteristics of an organism comprise more than those which it shows at any single moment of its development or as an adult animal. To them belong the whole sum of peculiarities and changes which it has shown from its simplest beginnings; for the later characteristics do not represent anything new and spontaneous, but proceed immediately and continuously from the earlier ones. If, therefore, heredity conveys the characteristics of the parents to the offspring, it must convey to the latter, not only the characteristics possessed by the parents at the moment of the production of the offspring, but the whole sum of parental characteristics, and among them those that the parents have shown during their development. Hence the peculiar course of development that the parents have gone through must be transmitted to the children, and the latter must 
go through the same development. Since this is true of every generation of parents and children, it must be true also of all the ancestors of the race, even the earliest, i.e., the children are the historic product of the whole racial development and in their developmental history must pass through the whole history of the race.

But this is true only on the condition that heredity is the sole factor that determines form. In such a case every minute peculiarity that was once present in the ancestral series of the organism would repeat itself with painful exactness in the development of the latter. Since individual development demands a relatively short time and racial development shows an inconceivable variety of changes in form, the remarkable spectacle would be presented of the ontogeny of a higher animal appearing like the picture in a constantly turning kaleidoscope, which never remains the same but presents to the eyes at every moment a different form. It is well known that this is not the case, but that the racial development is recapitulated only in bare outlines and undergoes manifold changes; these latter are the cenogenetic phenomena, which are caused by the second factor that determines form, namely, adaptation. It has been seen that the form of every organism is determined in a certain degree by external conditions. Any form that lived at a certain geological period in the racial series of an animal is, therefore, determined among other things by the conditions that prevailed upon the earth's surface at that period. The conditions now are entirely different. But not only have the conditions upon the earth become different, but the animal in its development is under wholly different conditions from the completed animal, especially if the first developmental stages are passed through within the mother's body. Since, however, these external conditions must effect an adaptation of the organism in question, it is explained why in the ontogenetic recapitulation of the phylogenetic series there appears not only a simplification but also an alteration of certain phenomena. Simplification comes about because developmental stages which at the time of their appearance represented special adaptations to certain conditions become bred out as useless and disturbing factors now when those conditions are wanting; alteration occurs by the adaptation of certain developmental stages themselves to the new conditions. It is clear that here also selection controls the change of form, and that characteristics arising cenogenetically become transmitted like original ones.

Accordingly, with Haeckel ('75), the fundamental law of biogenesis may be formulated in brief as follows: "Germinal derclopment is an epitome of racial development; the more complete, the mow the abridged dccrlopment is maintuined by heredity; the less romplete, the more a falsified development is introduced by adaptation. 


\section{The Phenomena of Transformation of Energy}

\section{A. THE FORMS OF ENERGY}

For a long time natural science has distinguished different forces which bring about the phenomena of motion in nature. In the scientific sense force is nothing but an expression for the cause of motion, for we know nothing concerning it except that it causes motion. Sense-perception is not force, it is merely motion. Accordingly, since early times, wherever different kinds of motion have been seen, different kinds of force have been assumed. It thus came about in time that a large number of forces were distinguished, which could not in any way be compared with one another, because some kinds were only special cases of others, some were combinations of several kinds, and some were not forces at all. The force of gravity, muscular force, and the force of will were all spoken of. This condition of things has not yet wholly disappeared. The forces that physics still recognises are not equivalent things, and little light has been thrown, even yet, upon the relations of certain ones to others.

In recent times, in accordance with the usage of Th. Young and Thomson, the old and easily misunderstood name "force" has been replaced by the term "energy," and what earlier wcre termed different forces are termed now different forms of energy. Thus, physics now recognises in general the following forms of energy:

1. Chemical energy (chemical affinity, attraction of atoms).

2. Molecular energy (cohesion, adhesion, attraction of molecules).

3. Mechanical energy (pressure, traction, thrust).

4. Energy of gravitation (gravity, attraction of masses).

5. Thermal energy (heat).

6. Photic energy (light).

7. Electrical energy (electricity, galvanism).

8. Magnetic energy (magnetism).

We will glance at these individual forms.

Modern natural science, as is well known, conceives the physical world to be composed of extremely small particles; it terms the particles that cannot be divided further without losing their properties, molecules, and those that compose the molecule and are indivisible, atoms. Chenical energy is that form of energy by which atoms attract one another in order to form a molecule; molecular energy that form by which molecules attract one another in order to form masses. If a mass is in motion and strikes against another movable body, it puts this likewise into motion if the impact be strong enough. The form of energy that puts in motion the body that is struck is mechanical energy. Further, masses attract one another, 
like the atoms in the molecule and the molecules in the mass; since Newton's immortal discovery it has been known that the paths of the heavenly bodies result from the mutual attractions of their powerful masses. This mass attraction, which binds the earth to the sun, and the moon to the earth, and compels a stone thrown upward to return again to the earth, is gravity or the energy of gravitation. Finally, thermal, photic, electrical, and magnetic energy are the forms of energy that put the atoms of the hypothetical ether, which fills universal space and penetrates all bodies, into those forms of motion termed heat, light, electricity and magnetism; for in accordance with the researches of modern physics the phenomena of heat, light, electricity and magnetism result merely from the vibrations of very minute particles.

But simple reflection shows that these forms of energy are not equivalent and separate. If all matter, including the hypothetical ether, is composed of atoms as its smallest physical particles, and if nothing corporeal exists beyond matter, all forms of energy, since they are associated with matter, must have their seat in atoms. In other words, atoms are the smallest particles endowed with energy, and it is evident that the forms of energy that are assumed for the motions of masses, such as gravity, must have their seat in atoms. Now, a priori, it is in the highest degree improbable that every atom is provided with eight different forms of energy. Scientific experience, which shows that everywhere in nature apparent multiplicity can be traced to unity, suggests that all these different forms of energy may be traced to a single form. As a matter of fact, molecular and mechanical energy and energy of gravitation, upon the one side, have been put into close relations with one another, as well as thermal, photic, electrical and magnetic energy upon the other side; and very recently electro-chemical researches have made it appear that a very close relation exists between chemical and electrical energy. Hence we have a wellfounded hope that before very long physics will succeed in demonstrating all forms of energy to be merely the expression of one and the same form, which appears different under different conditions; just as chemistry hopes to be able sometime to reduce the multiplicity of the chemical elements to the properties of a single original element, perhaps the universal ether.

The probability that the different forms of energy are only different modes of appearance of one and the same energy, amounts almost to a certainty in the light of the fact that one form of energy may be changed into another form, and in nature is continually so changed. As is well known, this all-important fact finds expression in the law of the conservation of energy, which was discovered and founded by Robert Mayer and Helmholtz, and which has become the foundation of our whole modern view of nature. This fact is explicable only in accordance with the idea that energy 
itself is always the same, and that the different forms of its appearance are merely cloaks, which may be exchanged according to the conditions at the moment.

Just as we speak of different forms of energy, wo can distinguish in the single form two different modifications, according as the energy itctually produces motion or only has potentially the capacity of putting into action under proper conditions. Physicists term these two modifications kinetic energy (also actual energy, or energy of motion) and potentiul energy (energy of position). The energy of gravitation, c.g., is kinetic when it draws a stone to the earth at the moment when the stone is set free; it is potential so long is the stone is fixed above the earth's surface. Likewise, chemical "ner'y is kinetic when it brings two atoms to ench other'; but it is potential when an atom has no other one in its ricinity that it can attract. Kinetic energy passes over constantly into potential energy and rice erese.

The law of the consermation of ene'ry, therefore, controls all that hippens in nature: it is the fundamental law of energetics. According to it, is has already been seen, energy in the world nurer originates or disappears; the sum of energy in the world is constant, just as the law of the conservation of matter expresses the same constancy in the quantity of matter. Where a certain quantity of energy seems to originate or disappear, in reality it simply goes wer into another form or modification. If, e.g., an electric current be passed throngh a ressel containing water, the clectrical energy seems to be lost. But in reality it does not go out of existence, for it has been seen that the molecules of the water are decomposed into their hydrogen and oxygen atoms, and these accumulate in a gaseous state upon the two poles of the electrical conductors. Hence the electric current has performed work and has separated the atoms of the molecules of witter from one another. But the atoms of hydrogen and oxygen set free have a chemical affinity for one another; hence in the experiment the kinetic energy of the electric current has simply been transformed into the potential energy of chemical affinity. If, therefore, the separite atoms of hydrogen and oxygen be brought again into union under proper conditions, the chemical potential pisses over again into kinetic energy, and a certain quantity of heat is liberated thereby. This heat can be transformed again into electricity in a thermo-electric apparatus, and, if the technical difficulties would allow the whole experiment to be carried out with sufficient exactness, it would be found that the same quantity of electricity has again been obtained as was consumed previously in the splitting-up of the water. During all transformations the original quantity of energy remains the same. In order to have a unit for the measurement of any quantity of energy, physicists have chosen, in accordance with Joule's 
researches upon the relation of heat to mechanical energy, a certain quantity of heat as the unit of heat or calorie. A calorie is that quantity of heat that is necessary to warm one kilogram of water from $0^{\circ}$ to $1^{\circ} \mathrm{C}$. Heat was chosen with good reason as that form of energy which may serve as a unit of measure for all others, for it holds a peculiar position in relation to all others; it is the sole form into which all others can be transformed completely. When, therefore, it is desired to express in numbers a quantity of any desired form of energy, e.g., mechanical or chemical energy, the latter is expressed in measures of heat, that is, in the number of equivalent calories. Thus, one calorie computed in the form of mechanical work corresponds to the quantity of energy that is needed to raise a weight of 424 kilograms one metre high; in other words, the mechanical equivalent of one calorie is 424 kilogrammetres and, vice versa, one calorie is the heat-equivalent of 424 kilogrammetres. In the same way the quantity of all other forms of energy can be expressed in heat-equivalents. The calorie is the unit of measure for all energy.

\section{B. THE INTRODUCTION OF ENERGY INTO THE ORGANISM}

Life has often been compared with fire, an idea which plays a rôle in the oldest mythological folk-views of nature and, as is well known, first assumed a fixed form in the philosophy of Heraclitus. In many points the comparison is fitting. To extend it somewhat further, the organism is the burning coal which is being constantly consumed, the breath is the smoke, and the food is the freshly added fuel which constantly replaces the old. Just as the burning mass of coal represents a physical system in which a continual transformation of energy is taking place, potential energy being introduced with the fuel and transformed into two forms of kinetic energy manifest outside, namely, heat and, by proper arrangement, as in the steam engine, mechanical work, so an organism is a physical system in which a similar transformation of energy continually takes place. Just as by heaping new coal upon the fire, energy is added in the potential form, so also, at least in the animal organism, by far the greater part of all the energy introduced is potential energy. The introduction of energy is considerably less evident to the eye than the production of energy; the latter results from the transformation of the introduced potential and is expressed in movements and other visible work.

\section{The Introduction of Chemical Energy}

Since confused ideas concerning the transformation of energy in chemical processes are wide-spread, it will be advantageous first to glance at the general facts. 
By chemical energy is understood, as is well known, the capacity of atoms to attract other atoms; this property has also been termed chemical affinity. Every atom, regarded as isolated, represents accordingly a small magazine of energy. The chemical energy in it is potential so long as the atom has no opportunity to unite by means of its affinity with another atom. But, as soon as two atoms combine, a part of the potential, corresponding to the strength of their aifinities, passes over into kinetic energy and is set free in the form of heat, light, mechanical energy, etc. Since, further, chemical affinity is quantitatively very different in different kinds of atoms, the stronger the combining affinities, the more energy is set free. A chemical compound, must, therefore, contain less potential energy, the stronger the affinities are that have brought together its atoms. Vice ver's, if two combined atoms become separated, a certain quantity of kinetic energy is absorbed in the process, and after the separation the same quantity appears again in the potential form as the free affinities of the atoms. Thus there is a complete cycle.

An example will make this relation more evident. Suppose a strong glass cylinder to be inverted over a mercury trough and to contain in a small space free from mercury a gaseous mixture consisting of two-thirds hydrogen and one-third oxygen; such a mixture consists of molecules whose atoms contain large quantities of potential energy in the form of chemical affinity for one another. If, now, the conditions be made such that the atoms of oxygen and hydrogen can combine, the atoms rush eagerly toward one another, unite and give off to the outside all their stored potential in the form of heat, light, and mechanical energy. A spark appears, the cylinder becomes heated, and the mercury is forcibly driven down. The latter soon rises again, for the vapour that results from the union of the atoms of oxygen and hydrogen becomes condensed with the increasing cooling into water, which finally occupies only a minute space within the cylinder. Thus, in the synthesis of water from hydrogen and oxygen the potential energy of chemical affinity is transformed into kinetic energy and is set free as heat, light, etc. Hence the molecule of water has lost to its environment this quantity of energy, and this can be exactly determined. Vice vers $\alpha$, the atoms of water can be separated again into atoms of hydrogen and oxygen by introducing from outside the same quantity of energy. Electrical energy serves best for this purpose. If an electric current be passed through water, atoms of hydrogen and oxygen are set free at the poles in the same degree as the electrical energy disappears. Hence energy is absorbed in separating the atoms of the watermolecule; but this encrgy appears again as the potential of chemical affinity in the free atoms, for, when the free hydrogen and oxygen are brought into combination, kinetic energy is obtained anew, and so on. 
This consideration is very important, for from it there follows a principle of far-reaching significance which usually is not formulated with sufficient clearness, viz.: In the combination of atoms kinetic energy is liberated; in the separation of atoms kinetic energy is absorbed.

This principle, which is a necessary sequence of the law of the conservation of energy, must be considered as a fundamental one for all chemical transformations, and forms the starting-point for an understanding of all the phenomena connected with the transformation of energy within the living organism. That as a rule it has not been established and applied with sufficient clearness, is to be ascribed chiefly to the fact that in certain cases at first sight it suffers apparently an exception. To make the relations clear, this must be considered, at least briefly.

To express in terms of heat the energy that is transformed in a chemical process, there are recognised processes in which heat is evolved and processes in which heat is absorbed. In accordance with the nomenclature of thermo-chemistry, the heat that is evolved in a chemical process is termed the positive thermo-chemical equivalent, the heat that is absorbed, on the other hand, the negative thermo-chemical equivalent. From the above considerations, it would be expected that all synthetic processes, i.e., all processes in which bodies unite, would be accompanied by an evolution of heat, for in every synthesis atoms become united, and in every union of atoms energy is liberated. Vice versa, it would be expected that all decomposition-processes, i.e., all processes in which united atoms become separated, would be accompanied by an absorption of heat. If the conceptions of synthesis and decomposition are employed in their pure significance, this is always the case. Nevertheless, at first sight there appear certain exceptions to the rule. For example, some syntheses are known in chemistry, such as that of hydrogen iodide, which are accompanied by an absorption of heat; on the other hand, there are many decompositions, especially of the more complex compounds, such as nitroglycerine and otherexplosives, in which a powerful evolution of energy takes place. These are undeniable facts, but, if the details of these processes be analysed somewhat fully, the apparent paradox becomes at once clear and in reality confirms the law. Since no free atoms are known, but since the similar atoms of every chemical element are united always into molecules, or groups of atoms, it is evident that unless whole molecules enter into combination without rearrangement of their atoms or are split off from a combination as preformed groups, then a decomposition of the active molecules into their atoms must precede every synthesis, and a synthesis of the free atoms into new molecules must follow every decomposition. Hence, no synthesis occurs without previous decomposition, and no decomposition without subsequent synthesis. Accordingly, it is clear that under certain circumstances heat can be absorbed in a synthesis: for example, 
when, as in the iodine molecule, the atoms of iodine or, as in the hydrogen molecule, the atoms of hydrogen have a greater affinity for one another than the iodine atoms have for the hydrogen atoms. In these cases more energy becomesabsorbed, in order to separate from one another the atoms of the iodine molecule and the atoms of the hydrogen molecule than becomes free when the atoms of iodine and hydrogen unite into a molecule of hydrogen iodide, and, since in every calorimetric experiment the end-result and not the intermediate processes come under observation, it is explained why at the end of the reaction there must be an absorption of heat. The reverse is the case in the decomposition-processes accompanied by an evolution of heat. It is well known that nitroglycerine (glyceryl tri-nitrate), upon being shaken, explodes with an enormous evolution of energy, being decomposed into water, carbonic acid, oxygen and nitrogen. These products of decomposition are not preformed stereochemically in the molecule of nitroglycerine, but they arise from a synthetic rearrangement of the atoms set free by the decomposition. Since the atoms of water, carbonic acid, oxygen and nitrogen, have greater affinities for each other in this arrangement than in their position in the nitroglycerine molecule, a small quantity of energy suffices to cause the decomposition of the latter, while from the resulting syntheses an extraordinary quantity of energy becomes free. Hence as the end-result there is an evolution of heat. Therefore, just as in the synthesis of hydrogen iodide, strictly speaking, the absorption of heat is not to be credited to the synthesis, so in the dynamite explosion the evolution of energy does not come in reality from the decomposition of the nitroglycerine molecule. This fact should be clearly understood. But, since, when a synthesis is spoken of, the preceding decomposition is left out of account, and when a decomposition is spoken of, the subsequent synthesis is similarly treated, it is more exact to express the fundamental law of the transformation of energy in chemical processes in the following form: If in a chemical process affinities become united rather than separated, energy is liberated; if affinities become separated rather than united, energy is absorbed.

To return from our excursus, it is clear from the discussion that chemical energy can be introduced into the organism only when the food-stuffs contain affinities for the satisfying of which an opportunity is afforded within the organism. Hence substances must be introduced into the body, which undergo among themselves chemical transformations with the evolution of heat. This takes place in two ways, which we have just become acquainted with, viz., first, by the introduction of simple substances possessing strong affinities, and, second, by the introduction into or synthesis within the body of complex compounds which are easily decomposed and, like explosive bodies, furnish decomposition-products that combine synthetically into new 
substances with a rearrangement of their atoms. Free affinities come into the body with oxygen especially; and it is well known that in the combination of oxygen with other substances, or, in other words, in combustion, a great quantity of energy is liberated. Hence the process of oxidation plays an extremely important rôle in all life; and, as has already been seen, the comparison of life with fire is a very happy one. Complex compounds come into the organism, especially in the case of animals, with the organic food; there they undergo a long series of transformations, which thus far have not been followed, in which decompositions and syntheses proceed together to the construction of the living proteid molecule. Living proteids may be classed with explosive bodies. They tend toward decomposition; and out of the complexes of atoms set free there arise synthetically by rearrangement, partly immediately after the decomposition and partly later in combination with substances newly introduced, chemical compounds the origin of which under certain circumstances is again associated with the evolution of energy.

In the present condition of our knowledge it is not possible to follow in detail the intricate series of chemical processes, the decompositions and syntheses and the transformations of energy associated with them, from the first cleavage of carbonic acid and the synthesis of the first product of assimilation in the plant to the decomposition of the living proteid in the plant and the animal. It is known, however, that the final products of metabolism, such as carbonic acid, water, urea, etc., are extremely poor in chemical energy. The larger quantity of chemical energy introduced into the body with the food must, therefore, have been transformed into other forms of energy upon its way through metabolism, and thus results the work of the organism.

\section{The Introduction of Light and Heat}

It has been said that the main quantity of all the energy that is introduced comes into the body as chemical energy. For the animal organism this statement holds good without limitation; for the plant, however, it needs a correction. It is true that in the plant the energy at the expense of which its work goes on is likewise pre-eminently chemical; but a part of this potential is not introduced into the body as free, available energy, i.e., in the form of free affinities, such as oxygen possesses; another form of energy must first be introduced in order to create free affinities in the former. It is well known that carbonic acid and water are necessary for the synthesis of the first product of assimilation. ${ }^{1}$ But carbonic acid and water as such are poor in chemical energy

${ }^{1} C f$. p. 158. 
because their atoms are coupled together by very strong affinities. Hence, in order to make them free and serviceable for new labours, they must first be split up, and for this an introduction of energy is necessary. The energy that performs this cleavage is light in combination with the chemical energy of the living plant-substance. Without light no plant-life is possible, and since without plant-life no animal-life can exist, it may be said that without light no life whatever would exist. Hence, although light plays an essential rôle as a direct source of energy only in the plant, it is as indispensable for the maintenance of life upon the earth's surface as the chemical energy of food.

The places in the plant where light effects the cleavage of carbonic acid are the green parts of the plant-body, and hence especially the leaves. This can best be demonstrated by the experiment on assimilation already described. ${ }^{1}$ This experiment shows that in the part played by the rays of light in the cleavage of carbonic acid in the green plant-cell, two factors are present, the intensity and the wave-length of the rays. The efficiency of the light increases with the intensity, so that in a brighter light, more carbonic acid is split up than in a feebler one. Moreover, with the same intensity the rays of red light (not those of yellow, as botanists formerly supposed) are the most effective. Engelmann (' 81,1 ; '94) in a series of researches placed this beyond all doubt by a microscopic method that depends upon the action on bacteria of the oxygen set free in the cleavage of carbonic acid. At the same time these researches confirmed the view that the cleavage of carbonic acid in the green plant-cell takes place in the chlorophyll-bodies only, and established the fact that the cleavage begins at once upon the admission of light and ceases immediately upon darkening. Hence the dependence of this property of the chlorophyll-body upon light is extremely close.

The heat that comes into the living organism from the outside, partly by radiation and partly by conduction, plays, like light, a rôle in the chemical transformations within living substance. Since with increasing temperature the power of decomposition increases in all chemical compounds, the heat that is introduced takes part especially in the processes of decomposition in the living substance. The rôle of heat as a source of energy may be recognised especially clearly in the so-called cold-blooded animals. These are better termed animals possessing a changeable temperature (poikilothermal), since in contrast to the so-called warmblooded animals, or animals possessing a uniform temperature (homothermal), the temperature of their bodies changes continually with that of their environment: with a high external temperature they may have a body-temperature equal to that of the warmblooded animals. When the temperature of the medium in which

${ }^{1} C f$. p. 158. 
they live is high, these animals, such as insects and reptiles, are extremely lively, move about much, and show in general an intense activity. With decreasing temperature the liveliness of their movements decreases, and at $0^{\circ}$ in many cases vital activity is hardly to be observed in them, the transformation of energy has almost ceased. "Wherever we look into the realm of living organisms," says Pfluger ('75, 1), "we see how the intensity of vital processes, and hence decomposition, varies proportionately with the temperature. When I observe the lively, moving, nimble lizard in summer, and then see how the same animal, exposed to a temperature below $0^{\circ}$, becomes gradually quiet and sinks into a death-like torpor, and inquire what is the reason why the animal becomes again so active in warmth, appearance tells me that it is because heat has been introduced into the organs; heat puts the atoms into vibration and promotes dissociation." The heat that is introduced serves in this way directly as a source of energy for the work of the organism.

This completes the enumeration of the sources from which the organism receives energy. The other forms of energy have almost no importance in this respect.

\section{THE PRODUCTION OF ENERGY BY THE ORGANISM}

At present it is wholly impossible to follow the tortuous paths taken by the energy that is introduced in its changes through the living body. Scarcely a beginning has been made in investigating the transformations that this energy undergoes under the various conditions found by it in living substance. There is here needed a long series of exhaustive special researches and especially a detailed knowledge of metabolic processes, before an intelligible conception can be formed of the mechanism of these transformations. The field of physiological energetics offers rich problems full of reward for the future, which thus far have been scarcely noticed. Only the final links of the chain of metamorphoses, the outward achievements of the living organism, are now known with certainty.

The evolution of energy outward, especially that of mechanical energy, which expresses itself in the movements of the living body, is undoubtedly the most evident of all vital phenomena; it is more or less the first criterion of life for the untrained observer, and perhaps this is the reason why physiology from early times has made the phenomena of movement a favourite object of research. Less evident, because either uncommon or difficult to observe, is the production, on the part of living substance, of other forms of energy, such as light, heat and electricity. 


\section{The Production of Mechanical Energy}

All living substance moves, i.e., the single points of its material system change their positions in space. There results, according to the special conditions, a shifting of the single particles, the external form remaining the same, a change in the external form, a change of place of the whole (locomotion), or several of these changes at the same time. But although motion in itself is a general phenomenon of life, all forms of living substance do not show the same kind of motion. The variety of modes of motion that may be observed in different organisms is very great. Nevertheless, all may be classified in accordance with the manner of their occurrence into a few large groups, of which only certain ones, on account of their wide distribution, possess any considerable importance. Since the motion of living substance is the most evident vital phenomenon, and special interest is therefore lent to it, we are justified in considering it somewhat in detail.

It is useful first to classify the various modes of motion into:

(a) Passive movements.

(b) Movements by swelling of the cell-walls.

(c) Movements by change of the cell-turgor.

(d) Movements by change of the specific gravity of the cell.

(e) Movements by secretion on the part of the cell.

(f) Movements by growth of the cell.

(g) Movements by contraction and expansion of the cellbody :

Amœboid movement.

Muscular movement.

Ciliary movement.

\section{a. Passive Movements}

In passive movements the cause lies outside the part that is moved. Passive movements in living substance are, therefore, not a vital phenomenon of the elements that are moved, but the expression of vital phenomena in the environment. The movement of the red blood-corpuscles, the streaming of the blood-plasma in the blood-vessels of the human body, are passive movements; for the blood-corpuscles and the plasma possess no intrinsic power of movement; they are only passively driven by the activity of the heart, which works like a suction- and force-pump in the system of branching tubes filled with blood. This streaming of the blood in the fine capillary vessels can be observed very beautifully under the microscope, if a frog, paralysed by the South American arrow poison, curare, be placed upon a cork plate and the web between the toes of the hind leg be stretched out by needles over an opening 
in the plate. A picture full of interest for every observer will then be presented (Fig. 86). The much-branched network of the capillary system is seen, and in it the blood flows with its apparently yellow corpuscles so slowly that one can easily follow every individual corpuscle as it winds its way in the clear plasma through the fine canals and sinuosities.

Even in the single cell such passive movements are found. The fine granules that lie embedded in the protoplasm of the naked cells of rhizopods show a streaming movement, especially in the long, thread-like pseudopodia of marine species; this so-called granular streaming presents a spectacle as fascinating as the streaming of the blood in the capillaries, although going on much more slowly. Like pedestrians in the street, or like ants, the

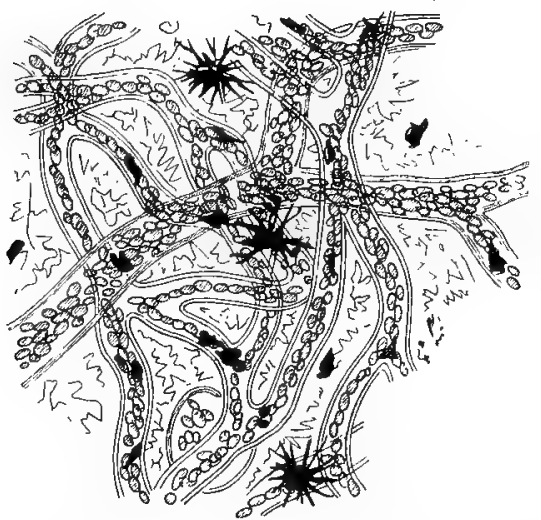

FIG. 86.-Capillary circulation in the web of the frog's foot. (After Ranke.) granules take their selfestablished paths, now in a centrifugal, now in a centripetal direction, now standing still, now turning about, and now again proceeding. This granular streaming does not come about by the active progressive movement of the granules themselves; but by their being passively dragged along by the liquid protoplasmic ground-substance in which they lie embedded, and which has constantly an active flowing motion.

Another interesting form of passive movements that occur in the living cell is the so-called Brownian molecular movement. There lives in fresh water a small, unicellular, green alga, Closterium, of a delicate crescent-shape (Fig. 87, I). In its protoplasm at each end of its body is a vacuole of liquid, in which as a rule lie fine granules which show Brownian motion. By strong magnification it may be seen that the granules are continually dancing about each other with a delicate trembling motion, but without moving to any considerable distances. The dancing continues tirelessly and unceasingly. Here the object in which this peculiar motion is seen is living. More frequently, however, it can be observed in dead cells, and it has long been known in the so-called salivary corpuscles in the saliva, which are dead leucocytes (white blood-corpuscles). These leucocytes are swollen into a spherical form by the absorption of water, and possess a nucleus surrounded by granular protoplasm (Fig. 87, II). 
Upon strong magnification the granules of this swollen protoplasm show clearly molecular motion. That the strange Brownian molecular movement does not occur in living organisms exclusively, follows from the fact that all light, microscopic granules of whatever kind, when suspended in water or any other easily moving liquid, show it. Among the most beautiful lifeless objects adapted for this purpose and occurring in the organism are the fine crystals (Fig. $87, I I I$ ) in the calcareous sacs that lie in the body-cavity of the frog on each side of the spinal column between the transverse processes of adjacent vertebræ. If some of the white substance be placed in a drop of water and examined under a cover-glass with a high power of the microscope, the wonderful picture of this restless, trembling dance of lifeless crystals is pre-
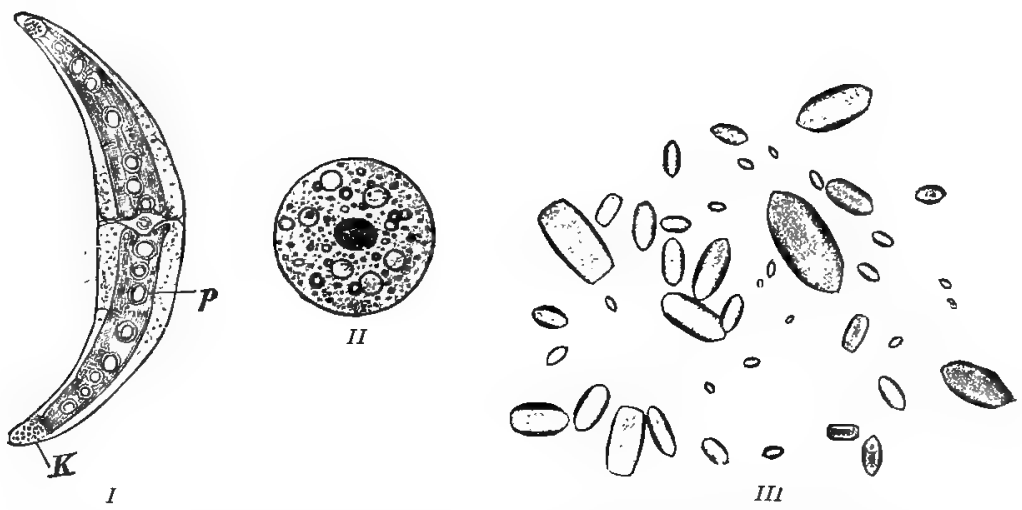

Fig. 87.-Brownian molecular movement. I. Closterium (after Strasburger). In the vacuoles, $k$, at the two ends of the crescent-shaped body there are numerous granules in active molecular. motion. II. A so-called salivary corpuscle, a dead and spherically contracted leucocyte from the human saliva, in the swollen contents of which the granules are in dancing motion. III. Crystals from the calcareous sacs of the frog; when put into water they show a restless, dancing motion.

sented in its most graceful form, especially in the smaller crystals. ${ }^{\mathrm{L}}$ When the English botanist Brown in the year 1827 discovered such peculiar motions in plant-cells, it was believed that the motion of the fine granules was an active one, resulting from the vibrations of their molecules, and it was accordingly termed " molecular motion." In accordance with more modern ideas this view became untenable, and for a long time the significance of the puzzling phenomenon was not understood. But in the year 1863 Wiener, and soon afterwards Exner, studied very carefully the physical conditions of the motion, and found an explanation that is in entire accord with our present ideas of the molecular condition of liquids. In fact, the behaviour of the molecules of a liquid even requires such phenomena of motion of small light par- 
ticles suspended in it. As is well known, the molecules in a liquid are conceived to be in constant motion, crowding together, bounding against one another, pushing away, moving off and again colliding. This motion of the molecules cannot be seen even with the strongest magnifying powers, for liquids appear homogeneous because their molecules are too small to be perceived even microscopically. But the result of the motion can be observed in small, light granules suspended in the liquid; if the molecules possess the given kind of motion, they must strike the particles continually, so that with their delicate mobility the latter are put into a trembling, dancing motion. Hence, the so-called Brownian molecular movement of small granules is a purely passive movement caused by the constant slight impulses given to the granules by the dancing molecules of the liquid. An excellent proof of the correctness of this view is afforded by the fact that the Brownian movement gains in intensity with increasing temperature of the liquid. This might have been predicted from the fact that the motion of the molecules of the liquid is greater the higher the temperature; it finally becomes so great that the individual molecules are driven violently apart, that is, the liquid evaporates.

\section{b. Movements by Swelling of the Cell-valls}

Movements that are caused by swelling of the cell-walls constitute a variety intermediate between passive movements and all those mentioned below, which latter depend upon the activity of living substance. The phenomenon of swelling, as is well known, is due to the fact that between the molecules of a dry, expansible body, brought into a moist environment, molecules of water become stored, being attracted so strongly by the molecules of the body that they force the latter powerfully apart; during the process the volume of the body becomes markedly increased. If the swollen body comes again into an environment free from water, e.g., dry warm air, it gradually gives off its water, diminishes its volume proportionately and shrinks; upon being again moistened, it swells again. The organic products of the metabolism of plants, especially the cellulose walls of plant-cells, are peculiarly prone to swell. This is not associated in any way with the life of the plant-cell, but goes on for an indefinite time in the cellulose of dead cells, in the same manner as in that of living cells. In order that a movement in one direction may be brought about by the increase in volume caused by the swelling or by the decrease in volume caused by the drying of an expansible object, such as the stem of a leaf or a membrane, the different sides of the object must be capable of swelling differently, one side strongly, the other feebly or not at all. Were all parts equally capable, there would result a uniform enlargement toward all sides. If, however, 
one side of an elongated structure swells more than the opposite, the former becomes lengthened more than the latter, and the result is a bending of the whole structure, which takes place suddenly or gradually as the swelling is rapid or slow.

The well-known resurrection-plants (Selaginella lepidophylla), which of late have frequently come to Europe from the American deserts, are characteristic objects for the observation of swellingmovements. During a drought their leaf-stalks are brought together like the fingers in a closed fist, but when moistened they bend out as in the open hand, the leaf-stalks strongly swelling
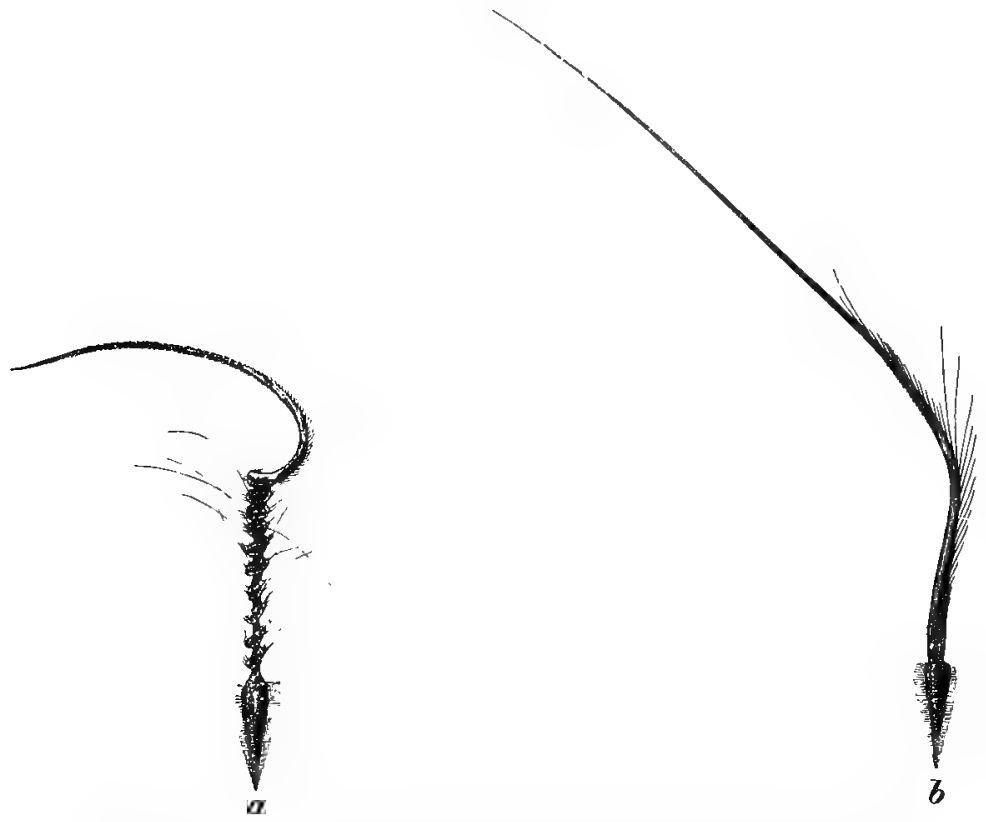

FIa. 88. - Seed of the crane's bill (Erodium ciratarium), $a$, in the dry, $b$, in the swollen state.

upon their inner side. The well-known rose of Jericho, which is simply the dry, dead branch of a crucifer (Anastatica) growing in the Arabian deserts, behaves similarly. Its spreading when placed in water has led to the common belief that the rose of Jericho is resurrected to a new life, while in reality the phenomenon depends merely upon the swelling-movements of the dead branch. Selaginclla, however, is a real resurrection-plant in so far as it can remain for years completely dry without losing its capacity of life. The seeds of many species of crane's bill likewise show very clearly the phenomena of swelling-movements. Erodium cicutarium has seeds that are provided with a long stalk 
beset with hairs; in a drought this stalk is rolled up like a corkscrew into a beautiful spiral (Fig. 88, a), but when moistened it. becomes straightened, one turn after another unrolling itself by swelling and extension of the inner side (Fig. 88, $b$ ).

The movements of the so-called elaters on the spores of the horse-tail are very interesting and striking because of their rapidity. The ripe spores of the horse-tail are spherical cells surrounded by a cellulose-wall. This wall is split into two bands the elaters (Fig. 89), which run in a spiral from above downward around the whole ball, being fastened to each other and to the spore itself at a certain spot in the equator. If the spores, slightly moistened, be brought under the microscope, the two bands are seen to lie in two parallel spirals and form a closed capsule about the spore (Fig. 89, a). If they be allowed to dry, the two spirals become extended into straight bands (Fig. 89, $\breve{b}$ )

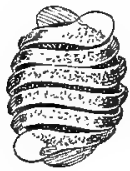

a

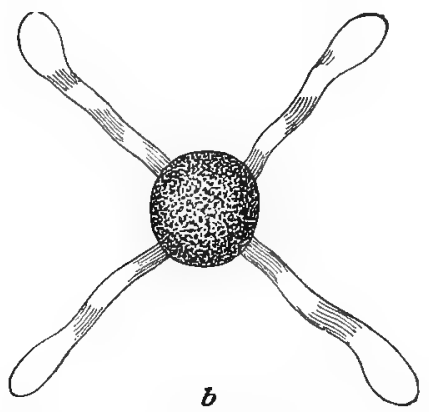

FIa. 89.-Spore of a horse-tail. a. The elaters in the moist state are curled around the cell $b$. The elaters in the dry state are rapidly spread apart.

through the drying and shortening of their outer sides. If, while observing with the microscope, one breathes upon them in this extended state, they are seen to coil themselves in spirals about the spore with excessive rapidity, their outer surfaces extending by swelling. At the moment when the moisture of the breath disappears, the bands extend again with equal rapidity; and the experiment can be repeated, like all experiments on swelling, as often as one wishes.

Swelling-movements are very common among plants, and some of them play an important rôle in plant life. The great power that can be developed by swelling can be realised from the fact that huge rocks can be split with wedges of swelling wood. 


\section{c. Movements by Change of the Cell-turgor}

With movements caused by a change of the cell-turgor, we begin the consideration of those phenomena of motion that presuppose normal life in the object in which they appear. With the death of their substratum they are extinguished. Turgescence-movements are chiefly found among plants; and it is necessary, therefore, that certain peculiarities of the plant-cell be recalled.

The plant-cell, as is well known, is a cylindrical capsule, the alls of which are formed by an elastic membrane of cellulose.

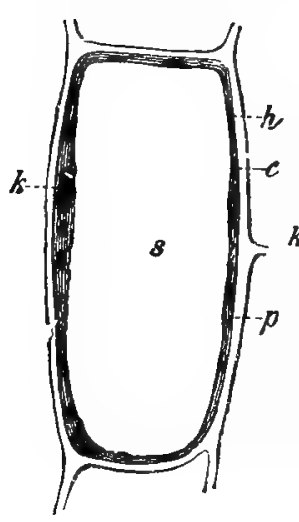

$A$

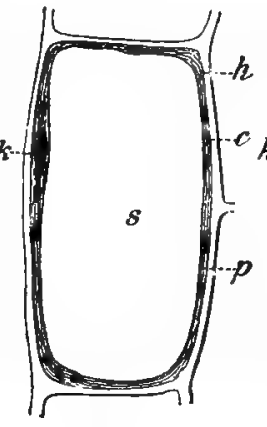

$B$

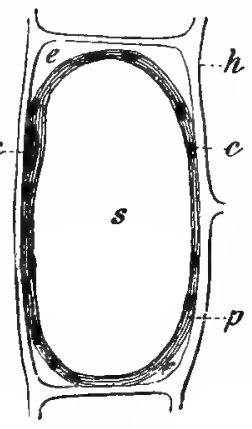

$c$

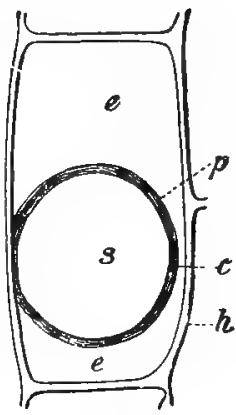

$D$

F1G. 90.-Scheme of cell-turgor of a plant-cell ; $h$, cell-membrane $; p$, primordial utricle ; $k$, nucleus ; $r$, chlorophyll-bodies; $s$, cell-sap; $e$, infiltrating salt solution. In $A$, the cell is in complete turgescence, the primordial utricle lies close to the cell-membrane. In $B$ the turgor has decreased as a result of the action of a salt solution, the cell has become smaller, but the primordial utricle still lies in contact with the cell-membrane. In $C$ the turgor has become still less, the primordial utricle is beginning to be pulled away from the cell-menbrane, which latter has reached its minimum. In $D$ the primordial utricle has contracted completely, because the osmotic effect of the salt solution acting from the outside has reached a very high degree. (After de Vries.)

The inner surface of the capsule is covered by a thin but continuous protoplasmic layer, the so-called primordial utricle, which encloses like a sac or bladder a liquid, the cell-sap, and as a rule sends through the large vacuole strands of protoplasm which branch lengthwise and crosswise (Fig. 90; in this figure the strands are wanting). Various chemical substances, which have been produced by the vital activity of the cell, are dissolved in the sap. In its nsual uninjured condition the protoplasm is impermeable to these substances, hence they cannot diffuse from the interior to the outside through the primordial utricle. But the protoplasm is likewise impermeable to many substances that are dissolved in the water outside the cell, and which, therefore, 
cannot diffuse into the cell. Now it is known that the molecules of such soluble substances as salts, sugar, etc., attract water, every molecule taking to itself a number of molecules of water. The molecules of the former are said to act "osmotically." As Van t'Hoff has recently shown by his important researches, the osmotic pressure is proportional to the number of molecules dissolved in the unit of volume. If, therefore, there are stored within the cell-sap strongly osmotic substances, and outside the cell in the water substances that are less osmotic, and if the wall of the primordial utricle is impermeable to these dissolved substances, an equalisation by diffusion cannot take place; but, since the primordial utricle allows pure water to pass through it unhindered, water must be drawn by the osmotic substances of the sap into the interior and held there permanently. The result of this process is that the pressure in the primordial utricle becomes constantly greater the more osmotic substances are dissolved in the sap, i.e., the more the concentration of the sap increases. The primordial utricle of the cell, therefore, must be extended from within outward; and this tension, stretching the elastic cellulose wall, is the turgor of the cell. It is evident that the turgor will become greater, that the cell must be put more upon the stretch, the more osmotic substances accumulate in the sap and the less in the surrounding medium.

From this brief consideration it is clear that the turgor of the cell can be changed in different ways. First, the quantitative relations of the osmotic substances within and without the cell can change, by the concentration outside or inside becoming increased or decreascd. If, e.g., substances in solution be added gradually to the surrounding medium, water will be drawn out constantly from the interior, and the turgor will decrease. This phenomenon has been termed, with little appropriateness, plasmolysis. Further, the turgor can likewise be changed by the wall of the primordial utricle from some cause becoming permeable to the substances in solution in the cell-sap. Then an equalisation by diffusion must take place, and the tension of the cell-wall must disappear. Finally, a change in turgor will take place when the tension of the primordial utricle increases or decreases because of active changes in its protoplasm. If, e.g., the protoplasm contracts, the contraction will partially or wholly overcome the osmotic pressure opposing it, and the result will be that a corresponding quantity of water minus the osmotic substances will be pressed out from the sap through the primordial utricle. When the contraction of the primordial utricle ceases, the osmotic substances of the sap will attract more molecules of water, and the turgor will again increase.

The result of diminishing the turgor must in all cases be the same. The primordial utricle, which previously was stretched from within outward by the tension, will shrink together, and its 
circumference will become smaller (Fig. 90). But what is more important for the present purpose is the diminution in size of the whole cell with decrease of the turgor, for the tension of the elastic cellulose coat will be decreased to the same extent as that of the primordial utricle, and, as a result of its elasticity, the wall will assume finally a circumference corresponding to its decreased tension (Fig. 90, $B, C, D$ ).

In the movements of plants now under consideration a change of turgor takes place solely by the contraction of the primordial utricle of certain cells for some cause, either spontaneously or as the result of stimulation, in such a manner that water is squeezed out of the cells; the phenomenon passes away after some time, and the turgor again appears pari passu with the disappearance of the contraction. There thus appears under certain circumstances a sudden diminution of the turgor and with it a diminution in the size of the cell, and only gradually does the previous condition return.

In order that upon this principle a microscopic movement may take place in a plant, the cells that undergo the change of turgescence must have a definite arrangement. If in one of two parallel rows of cells the turgor is suddenly diminished, so that the cells become smaller, while in the other it remains unchanged, the first row must shorten. Hence, according to simple mechanical principles, a bending will occur with the concavity upon the shortened side. At the same time the other side will be extended passively. If, later, a gradual increase of turgor and a lengthening of the cells upon the shortened side takes place, the elasticity of the other side will assist the extension.

Such a diminution of turgescence appears in many plants, often very suddenly, both spontaneously and after mechanical stimulation, and the result is a sudden movement of certain parts. In most cases both the arrangement and the shape of the cells that cause the movement are very complicated. As a rule, at the base of the motile leaves, or petioles, small enlargements, called pulvini, are developed, the cells of which can diminish their turgor very rapidly. One of the best-known examples of this kind is the movement of the petioles in the sensitive II imosc pudica, which in the "waking" state, i.e., during the day, are upright with the leaflets extended (Fig. 91, I, A, and $I I, A)$, while in the "sleeping" state, i.e., at night, they are depressed and the leaflets are folded upward together (Fig. 91, $I, B$, and $I I, B)$. If a Mimosa. in the waking state be vigorously shaken, the night position is suddenly assumed in the daytime.

Upon the same principle depend numerous other movements of the sensitive plants, such as those of the leaves of clover, the stamens of barberry, the insect-catching organs of carnivorous plants, and many others. 

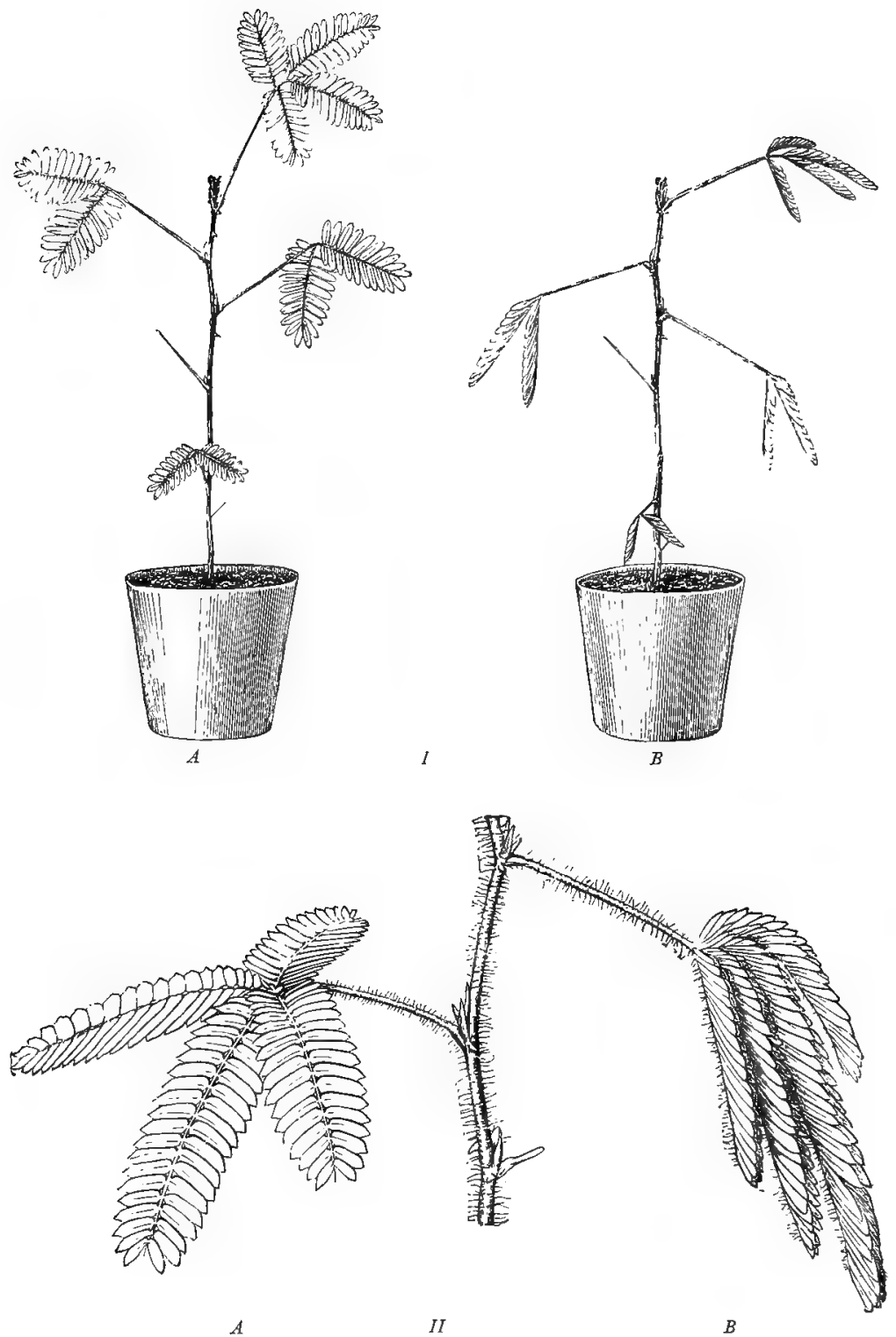

Fia. 01.-Wimosa pudica. I. Whole plant; $A$. in the dity position; $B$. in the night position. $l 1 . A$. A branch undisturbed (day position); $B$. a branch of the same stalk stimulated (night position). (II. after Detmer.) 


\section{d. Movements by Change of the Specific Gravity of the Cell}

Among the wonderful forms of animals, mostly of glassy transparency, that lead a pelagic life in the upper strata of the sea and lately as plankton have become the object of detailed investigation, there are many that are endowed with the remarkable capacity of slowly rising or sinking in the water without the use of any locomotor organs. These are especially the Radiolaria, Ctenophora, and Siphonophora. Some unicellular, fresh-water organisms, such as Actinosphcerium, also possess this power. Since all external causes for this mysterious suspension, such as currents of water, may be excluded, and since the movement of special organs of the body does not share in it, it can depend only upon changes in specific gravity, and this has been demonstrated. As has already been seen, ${ }^{1}$ protoplasm is heavier than water. Hence a cell that lies upon the bottom can raise itself only when substances that are lighter than water appear and accumulate in the protoplasm.

It is well known that certain fresh-water Rhizopoda, especially Arcella and Diffugia, which are provided with delicate capsules, are heavier than water, and usually creep about upon the bottoms of ponds and puddles between particles of mud and decaying leaves, can actively raise themselves by developing a bubble of carbonic acid in their protoplasmic bodies; when it has become sufficiently large, they rise to the surface like a small balloon. Engelmann ('69) first carefully investigated this fact. At times in a culture-vessel containing Difflugia, when conditions favour the development of carbonic acid in the protoplasm, the movement of individuals from the bottom to the surface becomes epidemic. If the carbonic acid is then given off, the individuals sink again to the bottom. In this manner there may arise in nature a very considerable change of habitation, which under certain circumstances, as when the Protista have come under unfavourable conditions, can be of great usefulness to the species.

In an analogous manner take place the rising and sinking of the Radiolaria and, in all probability, those of the Ctenophora and many other pelagic animals. Thalassicolla nucleata, e.g., is a large globular radiolarian of $3-4 \mathrm{~mm}$. in size, which represents a single cell, the nucleus of which, surrounded by protoplasm, lies in a spherical central capsule (Fig. 92). The whole extracapsular protoplasm is filled with innumerable vacuoles, so that it appears like a mass of foam, and it is bordered externally on the side of the sea-water by a solid layer of jelly. This vacuole-layer is the portion of the cell that is lighter than the sea-water, and

$$
{ }^{1} C f . p 97 \text {. }
$$


maintains the undisturbed Thalassicolla suspended at the surface of the sea. ${ }^{1}$ 'This can be made out readily by remoring from the living animal single constituents of the cell, by cutting off the layer of jelly, isolating the vacuole-layer and extirpating the central capsule with its contents. All constituents, when isolated, sink to the bottom of the water, except the vacuole-mass; this remains at the surface, and, if submerged, continually returns to it. ${ }^{2}$ Correspondingly, the whole Thalassicolla begins to sink as soon as the vacuole-layer collapses by the bursting of the vacuoles, which takes place as a result of stimulation, in nature especially from the impact of violent waves. Then the cell falls into more quiet depths, and thus is protected from entire destruction; the

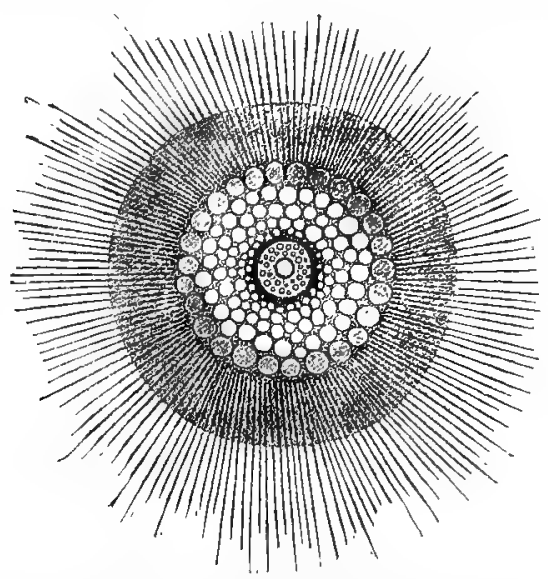

FIG. 92.-Thalassicolla nuclecta, a spherical radiolarian cell in section. In the middle of the central capsule, which is surrounded by black pigment, lies the vesicular wucleus. The central capsule is surrounded by the vacuole-layer, which is enveloped by a zone of jelly and sends through the latter radiating, thread-like pseudopodia.

vacuole-layer can regenerate itself, and the Thalassicolla, increasing in volume, in quiet weather rises again from the depths to the sunny surface. The great importance of this manner of movement for the life of pelagic organisms is evident.

It is a question how the contents of the vacuoles can become lighter than the surrounding sea-water. The cause of the appearance of vacuoles, the formation of which can easily be observed in any isolated central capsule, consists in the accumulation throughout the protoplasm of osmotic substances, which cause the water to come in from the outside to them through the protoplasm. The size of the vacuole increases in proportion as the formation and concentration of osmotic substances in the protoplasm increase, for an equalisation of the osmotic pressure in the liquid of the vacuole

${ }^{1}$ Cf. Branclt ('85).

"Cf. Verworn ('93). 
and in the surrounding water must always take place, i.e., the liquid of the vacuole must always contain in solution the same number of molecules as the water. But it must be assumed that these are molecules of substances different from those in the water. If, therefore, we imagine some of the substances dissolved in the liquid of the vacuole to possess low specific gravity, we can understand how, upon the whole, the contents of the vacuole can be lighter than the water. $\mathrm{K}$. Brandt ('95) has recently made it very probable that it is the carbonic acid produced by the protoplasm that, dissolved in the liquid of the vacuole, lowers the specific gravity of the protoplasm below that of the sea-water. If the vacuole-layer is developed to a sufficient extent, the specific gravity of the whole cell will be less than that of the sea-water, i.e., the cell will float at the surface. If by the bursting of the vacuoles the volume of the layer becomes diminished, or if in the cold, when the metabolism sinks to a minimum, the production of carbonic acid becomes greatly decreased, the radiolarians will sink again.

\section{e. Hovements by Secretion}

Movements that come about through secretion by the cell are limited to a few groups of organisms, particularly the Algce, Desmediacece, and Oscillarice. The principle of this mode of motion is extremely simple. It consists simply in the cell lying upon the bottom and pressing out at a definite place upon its surface and in a definite direction a mass of secretion, usually of a slimy nature; this sticks to the bottom, and the motile cell-body thereby thrusts

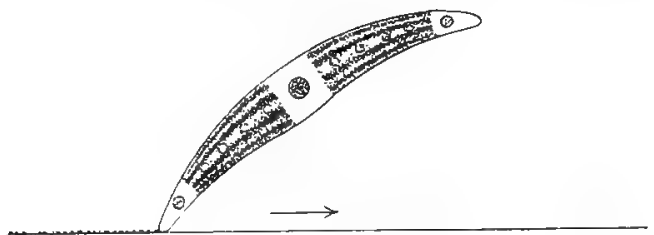

Fra. 93._Closterium, a desmid, shoving itself along the bottom by a secretion of slime. The non. secreting end swings freely in the water.

itself forward in a definite direction, just as a fisherman pushes his boat off the shore with a pole. If the secretion continues, the cell glides slowly along.

In this manner the Desmidiacece move themselves. The crescentshaped Closterium (Fig. 93), which we have already become acquainted with in considering the Brownian molecular movement, secretes a slimy substance at each end of its unicellular body. While it thus clings to the bottom with one end, the other end floats freely in the water, so that the whole body is directed 
upward obliquely at a certain angle. The Closterium shoves itself slowly forward, as Klebs ('85) and Aderhold ('88) have shown, by the attached end expelling a mass of slimy secretion (Fig. 93), the cell maintaining approximately its angle of inclination to the bottom. But in gliding forward it alternates its two poles, the swinging pole sinking, adhering and secreting, while the previously attached pole rises and swings freely. Thus the alga gradually moves forward upon its support.

As regards the movement of the Diatomres. the small, brown, boa -shaped or rod-shaped Algo, provicled with an extremely

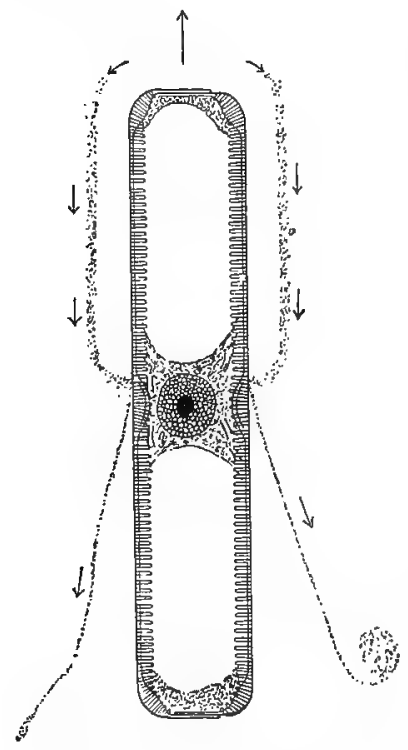

FIG. 94.-Diatom with threads of slime extruded. (After Butschli.) delicate silicious shell, which are found in enormous variety in both fresh and salt water, a literature almost too vast for review has appeared. When these unicellular forms are observed in a drop of water upon a slide, they are seen gliding forward upon the bottom in the direction of their long axis in a peculiar hesitating manner, sometimes slowly, sometimes rapidly, and often going backward with the poles reversed in direction. It seems impossible to discover any sort of locomotor organs in the body. The numerous investigators who, like Max Schultze, Engelmann, and others, earlier studied this graceful form of motion, adopted widely different views as to its origin. Afterward, from the researches of Butschli $(92,2)$ and Lauterborn ('94), it appeared as if it depended upon the above principle of the extrusion of a slimy secretion. Biitschli and Lauterborn succeeded in showing that certain forms of

Diatomece are enveloped in a covering of jelly and extrude peculiar threads of secretion, which can be made visible by adherent granules of india ink (Fig. 94). But recently the very detailed investigations of 0 . Miiller ('93, '94, '96, '97) have shown that these threads have a subordinate significance in the progression of the Diatomece, and that the mode of motion of these small cells is much more complicated, and perhaps more allied to movement by protoplasmic streaming.

As to the long, blue-green, thread-like Oscillarix, which consist of many cells arranged one after another in a row and creep slowly through the water like the Diatomea, it is highly probable that they really shove themselves along the bottom by the expulsion of 
a secretion. Recently, Schewiakoff has shown the same also for the Gregarince (Fig. 22, b, p. 80), which are parasitic unicellular organisms that likewise perform very slow, gliding movements without special locomotor organs.

\section{f. Movements by Growth}

Movements that are associated with the growth of cells need only be mentioned briefly; their principle needs no elucidation. All growth is accompanied by movement, for, as a cell increases in volume, it becomes expanded. Hence growth-movements are common to all living substance, but they take place so slowly that they can scarcely be followed with the eye. If, however, growing objects be compared with their earlier stages after considerable spaces of time, if the sprouting seed be first considered and then the plant that has developed from it with all its branches, leaves and Howers, it is evident that extensive movements have taken place, by which the building material has been transported to the places where it is laid down. Growth-movements are recognised also especially clearly in long plant-stalks or tendrils, when the cells grow or multiply more rapidly upon one side than upon the other, so that the part becomes curved. But the most apparent movements caused by growth are in those cases in which the mechanical energy developed by growth is not continually set free, but is accumulated in the form of tension, and finally by some stimulus is suddenly transformed into kinetic energy; this appears most beautifully in the seeds and fruits of certain plants, $e . g$., Imputiens, which, upon being touched, suddenly burst with a jerking motion and throw out their contents. It is not necessary to go further into the mode of growth-movements, since their principle is plain and they are met with at every step in living nature. That the phenomena of growth are powerful sources of energy is clear when it is recalled that trees growing between rocks are able to force apart huge masses of stone by their roots.

\section{Movements by Contraction and Expansion}

Finally, movements that are produced by the contraction and expansion of the cell-body, and which are usually termed, in brief, contraction-phenomena, are distinguished from all other organic modes of motion by the fact that they consist of changes in the form of the surface of the living substance itself, which changes are associated with an alternate shifting of position of its particles. All contraction-phenomena comprise two phases of movement, that of contraction and that of expansion. The particles of living substance arrange themselves with reference to one another in contraction, so that the mass presents a smaller surface, 
in expansion, so that the same mass presents a larger surface. Transition from one phase to the other alone renders possible phenomena of motion.

It is evident that only bodies of more or less liquid consistency can show such movement; only a liquid can diminish or increase its surface by rearrangement of its particles, becoming spherical or spread out, according as its surface-tension is equal in all directions, or becomes greater in some places and less in others. A solid, stiff body, even if it is elastic, cannot manifest contraction-phenomena of this kind, because its particles cannot change their mutual positions. Hence, it is of fundamental importance for the occurrence of contraction-phenomena that living substance possess a liquid consistency. As a matter of fact, all living substance, as has already been found, is more or less liquid, a condition that is imposed upon it by the high percentage of water in its contents, and, therefore, the common view is well founded that all living substance possesses contractility, although many cells are known, such as certain Algce and Bacteria, which in spite of their leading an active life can perform no contractionphenomena, because they are surrounded by a stiff membrane. Contractility, i.e., the property of executing contraction-movements, is, however, a general property of living substance, and hence demands detailed consideration.

Among the phenomena of movement brought about by contraction and expansion in accordance with the above principle there can be distinguished, according to the peculiar differentiation of the substratum in which they are observed, three groups, which are termed:

Amoboid movement (protoplasmic streaming);

Muscular movement (movement of smooth and cross-striated muscle-fibres);

Citiary movement (movement of flagella and cilia).

Amceboid movement, the original form of contraction-phenomena, is found wherever there exist naked protoplasmic masses, that is, cells the protoplasmic bodies of which are not surrounded by a cell-membrane, or wherever, as in plant-cells, there is within the membrane a free space for morement. As examples there may be mentioned especially the manifold representatives of the great protistan group $R$ hizopule (Figs. 95 and 98); further, in the animal cell-community, leucocytes and amœboid wandering-cells of various kinds (Fig. 96), amceboid egg-cells of certain animals, such as sponges (Fig. 17, a), pigment-cells of widely different organs ${ }^{1}$ (Fig. 97),

1 The view often expressed in recent times, that in the movements of pigmentcells there is a change of place of the granules of pigment without a simultaneous. change of form of the protoplasmic body, appears to me wholly untenable. 
intestinal epithelium-cells (Fig. 45); and, finally, various kinds of plant-cells (Fig. 24, $a$, and Fig. 35). The movement of Amoba can serve as a type (Fig. 95). This organism is the lowest of all living things, and its formless body holds within itself the whole secret of life. Taken with a pipette in a drop of water from the bottom of a pond and brought under the microscope upon a slide, the amœba-cell appears as a small grey semitransparent droplet of a more or less pronounced spherical form; in the central portion lie the nucleus and usually a contractile vacuole, surrounded by a more or less granular endoplasm, while the peripheral layer consists of a more hyaline exoplasm. If this drop of living substance be observed for some time, it is
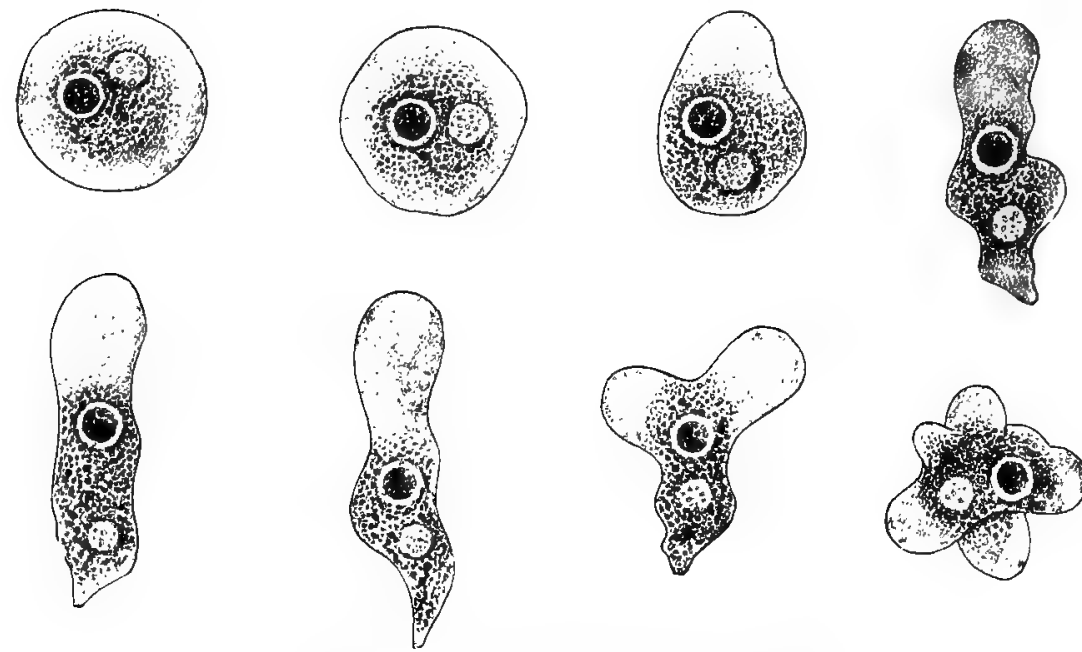

FIG. 95.-A the bu in eight successive stages of movement.

seen that at some point of its surface the spherical mass bulges out in the form of a lobate projection; this becomes constantly larger and extends itself farther and farther, more protoplasm flowing into it constantly; the phenomenon spreads from the peripheral parts toward the centre, so that a continual streaming takes place from the centre toward the periphery in this so-called pseudopodium (Fig. 95). Frequently the whole protoplasmic mass of the amoba flows over into this one lobate projection, so that the body forms a single extended mass, as can be observed especially in Amaba limax. Frequently, however, the centrifugal protoplasmic streaming of the pseudopodium becomes interrupted, while at the same time at another point of the surface a second pseudopodium is formed in the same manner by a centrifugal 
flowing of the protoplasm into the medium, and a third may follow this, so that the amoba protrudes it: substance in various directions, and thus considerably increases its surface. This extension of pseudopodia, this flowing of substance into the medium, represents the phase of expansion. While a new pseudopodium is being extended, protoplasm usually flows out of another one, from the periphery back to the centre to afford material for the new one, that is, the old pseudopodium is drawn in. This retraction of pseudopodia, this centripetal back-flow of the protoplasm and diminution of the surface associated with it, represents the phase of contraction. If all pseudopodia are drawn
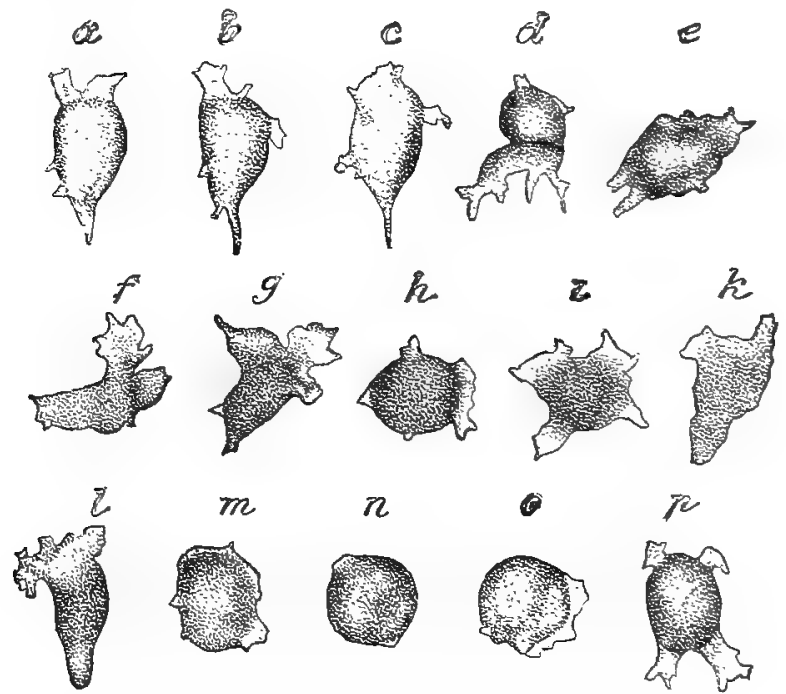

Fra. 96. - A leucocyte (white blood-corpusele) of the frog, in rarious stages of morenent. (After Engelmann.)

in, the amœba-cell again assumes a spherical form. The spherical form is, therefore, the expression of most complete contraction in naked protoplasmic masses. Then undisturbed, howerer, simultaneous contractions and expansions usually take place in the sime amoba at different points on its surface. Hence the pseudopodia are not preformed. Substance flows out, now here, now there, is mixed continually and flows back again, and this changeable play is the amœboid movement.

In the various amœboid protoplasmic masses the form of the pseudopodia varies greatly, according to the special consistency and composition of the living substance. As has already been seen,${ }^{1}$ there occur among the numerous forms of rhizopod-cells

$$
{ }^{1} C f . \text { p. } 76 .
$$


pseudopodia that are short and blunt, incised, thick and fingershaped, slender and thorn-like, straight and radiating, long and thread-like, dichotomously branched, or reticulate. But all these varieties, which are united with one another by innumerable transitions, are produced in the same manner, namely, by protoplasm streaming out into the medium centrifugally from the central cellbody. In organisms possessing long, flose pseudopodia, such as Foraminifera (e.g., Orbitolites, Fig. 98), the protoplasm must travel a long way from the centre to the tip of the constantly
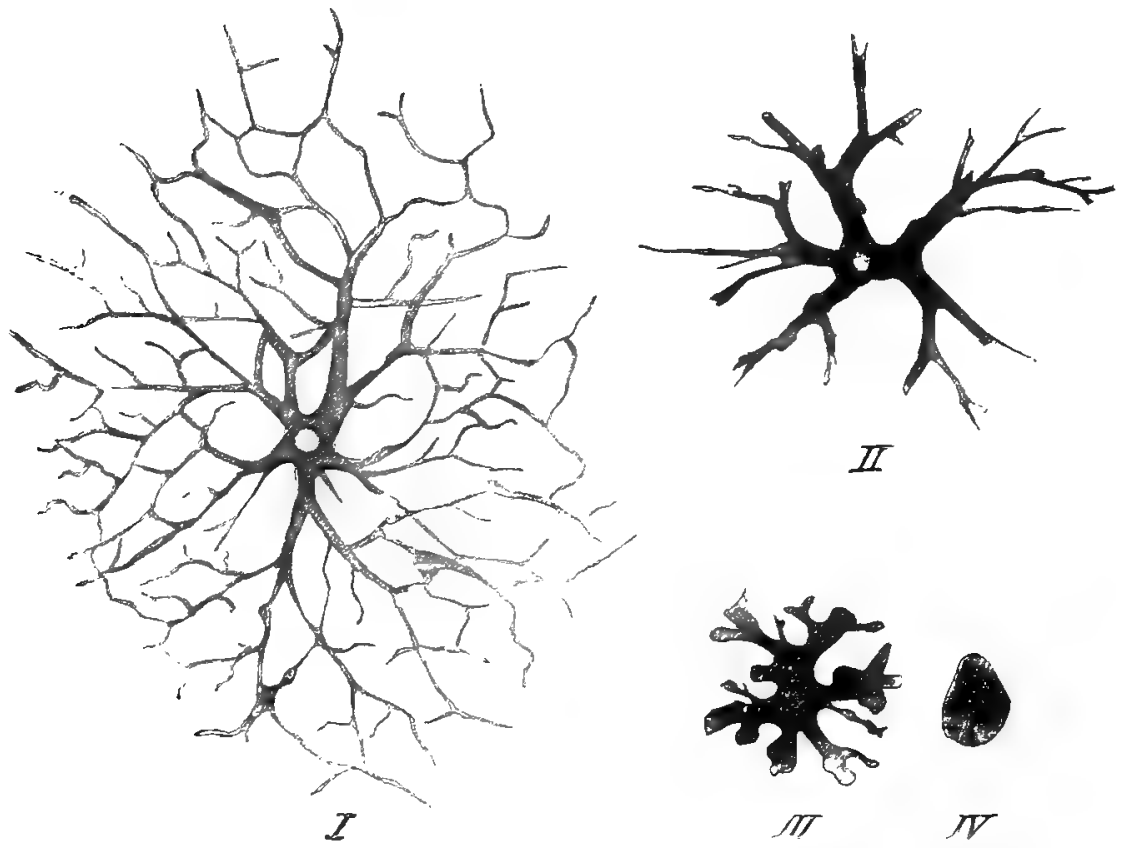

F1G. 97.-Pigment-cells from the skin of the frog; $I$, extended ; $I I$, slightly contracted; III, strongly contracted; $I V$, wholly contracted; the clear spot in the centre of the cell-body is the nucleus.

lengthening process; in these fine threads the microscope shows the protoplasm with its granules flowing like the water of a slow stream. This extremely fascinating phenomenon constantly charms the observer and has been vividly described by Dujardin ('41), Max Schultze ('54), and Haeckel ('62), as granular or protoplasmic streaming. In the retraction of such pseudopodia the protoplasmic particles must again travel over the same path in the reverse or centripetal direction. In pseudopodia that are extended to a considerable distance and remain extended for a considerable time, two currents, a centrifugal and a centripetal, are always 


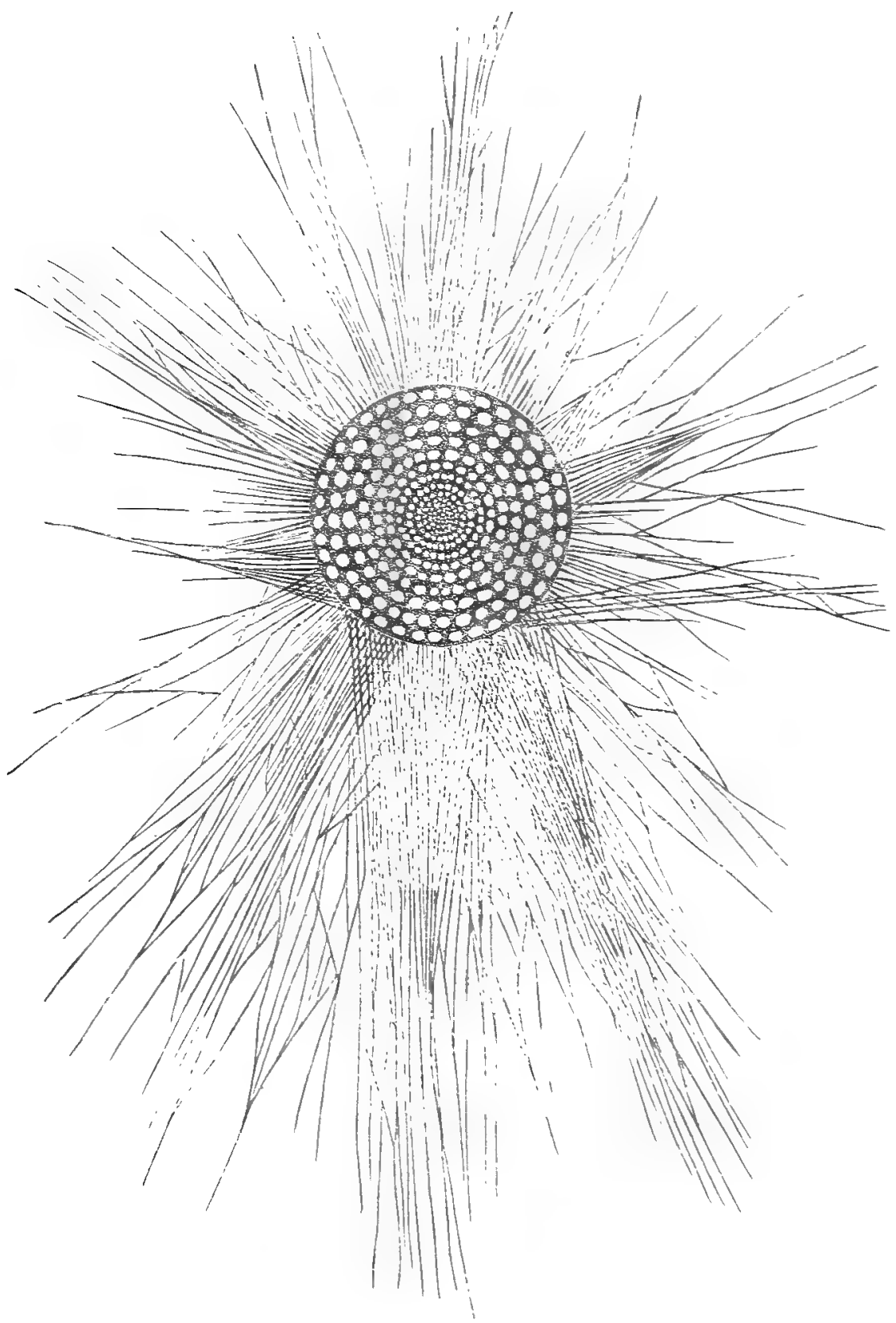

FIG. 9s,-Orbitolites complanatus, a rhizopod-cell from the Red sea (small specimen magnified about forty times; natural size of adults upon an arerge $5 \mathrm{~mm}$.). The central protoplasmic body is enclosed in a round, disc-shaped, ealcareous shell, which consists of innumerable chambers arranged essentiall in concentrie rings. The protoplasm of each chamber contains one or more nuclei. At the periphers of the shell numerous the, straight, filose pseudopodia protrude, often resching in large specimens a length of almost $20 \mathrm{~mm}$. ; these branch greatls and analgmate with one another. Vers benutiful protoplasmie and granular stresming mag be seen upon them. 
noticerl, in thick pseudopodia the former plainly upon the preriphery, the latter in the axis of the strand. According as the former or the latter preponderates, the penrlopodium gradually extends or shortens itself. If the two are erpually strong, it remains extended, its length not changing. The phenomena of contraction and 'xpansion may be studied very aisily and fully in the long, filose Miculoportia of Forminifor, such as Orbitolites (Fig. 98). The phasi of expansion, i.e., extensim, ("onsists always in a centrifugal flow of the living substance into the surrounding medium, the phase of contriction, i.c, retraction, in a centripetal fluw from the puriphery to the central cell-body. Expansion is characterised by an increvise of surface, contraction by an effort toward a spherical form.

Protuplasmic streaming in plant-cells follows the same plan. A coll from a stamen-hair of Trenderentia virpinime is a cylindrical, ('tisenl cellulosi-capsule (Fig. 99, A), in which the protoplasmic cellbuly with its nucleus is anclosed. The prutiplasm forms upon the iuncr wall a continuous, extremely delicate layer, the so-called yrimorlial utride, from which there extend in various directions through the lumen of the capsule, filled with cell-sap, protoplasmic strands which anastomose with "ne another and at one point lodge the nucleus. Both in these long, protoplasmic strands and in the primordial utricle a continual, protoplasmic streaming is visible, which (arresponds perfectly to the protoplasmic streaming in the pseudopodia of Rhizopoda. When the protoplasm in the various strands flows in an inco-ordinated, irregular direction, the movement is termed by the botenists circulation; when it follows continually one definite direction, rotation. This phenomenon would correspond, therefore, to the protoplismic movement of a rhizopod-cell, such as (Jintelites, in an undisturbed state, in which the protoplasm in clongatul pseudopodia streams continually both in a centrifugal and a contripetal direction, i.e., in which the phases of contraction and of expansion are equally developed. In the plant-cell such a complex system of curronts has arisen by division of the mass of protoplasm into single, anastomosing strands, so that the distinction betwe'n centrifugal and centripetal currents no longer holds; the silme is true, also, of large Rhizopoda, such as the plasmodia of Myromyectes, the whole body of which resolves itself into a richlybranched, pseudopodial network. The phase of contraction may, how'ver, be brought out here very clearly and easily by stimuli. As in the Rhivolodi, it is characterised by the protoplasm becoming contracted into globules (Fig. 99, $B$ ), which flow together and under certain circumstances finally form a large lumpy mass about the nucleus. This is the complete analogue of the phase of contraction in the $R$ hizmpule, where the pscudopodia retract themsives so that the boty assumes a more or less spherical form. Hence, the phenomena of protoplasmic streaming are, in principle, 
exactly the same in plant-celis as in Riricipola: Mas schultze ('63) has discussed rery fully the analogt of protoplasmic morement in the two cases.

The amount of work that can be performed in amœboid morement has thus far not been ascertained, but the derelopment of energy does not appear tu be considerable.

Muscular morement is the specifi: form of morement of the animal organism, by which apparently it is distinguished from all plants. All the gross and rapid niass-morements of the whole animal budy or of single Trstems of organs. all thuse remarkable morements which of all rital phenomena produce most the impression of liring. depend upon the contraction of muscle-fibres. Such morements mislead ordinary observers into ascribing to animals a higher stage of life than to the plants, the latter being considered to stand mnch nearer to lifeless nature than to the animals.

In contrast to amoboid protoplasmic morement, muscular morement is especially characterised by the fact that its factors are co-ordinated in space, in so far as the particles of a muscle-fibre shift themselves in one definite direction. Of course it can be said that in a long straight. filose psendopodium the particlesflow likewise in a definite lirection: but this direction is not continual, for, in retraction the particles mingle
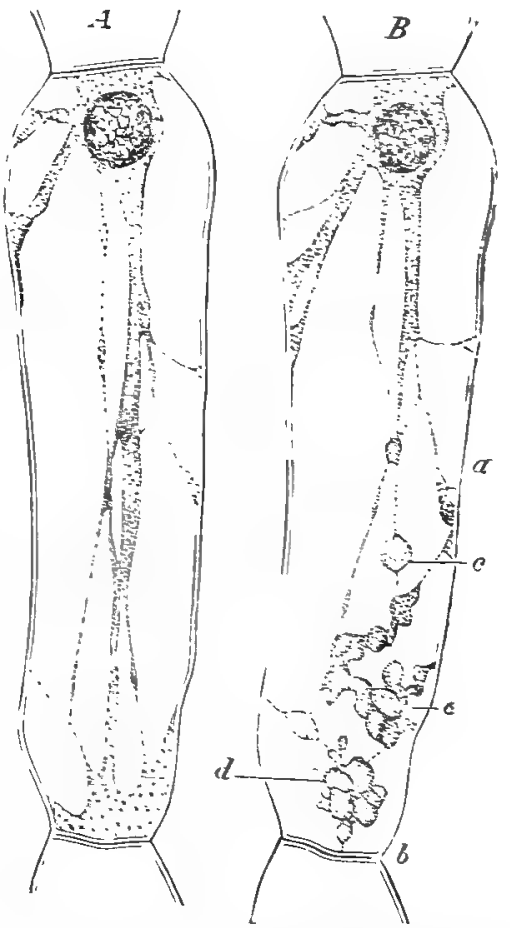

FIo. 90.-Cell from in stamen-hair of Tradecentin sincer. A, Quiet protoplasmie streaning in the strumds of protophasn : $B$, the pnotoplasm his contrincted into lumps and globules at $a, b$. c. d. (After Kiibne.) again with others and separate from one another in all possible directions. In contrist to this. the particles that in a muscle-fibre are the seat of contractionphenomena are constantly present as special structures in the lest of the cell-protoplasm and cannot directly mix with it. It is customary to term the whole muscle-cell a mustiible, and these specially differentiated contractile strips in it 
muscle-fibrilla; the fibrillæ lie embedded in the protoplasm of the fibre, which can be termerl with Rollett, in brief, sarcrplasm, arranged in various ways but all in the same direction. They represent specially differentiated organoids of the cellprotoplasm.

In accordance with the rarying structure of the contractile inuscle-fibrillæ, two groups of muscle-fibres or muscle-cells are distinguished, the smooth and the cross-striated. In smooth muscle-fibres the fibrillæ, which lie embedded in the sarcoplasm parallel to one another, are completely homogeneous threads in

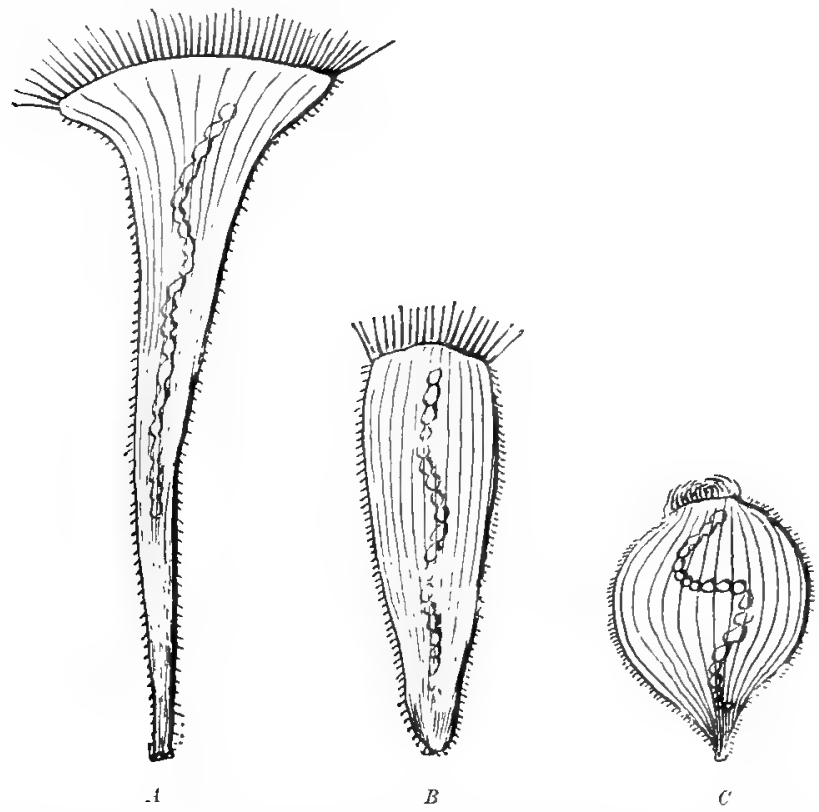

FIG, 100.-Stentor rosul, w, a ciliate infusorian containing numerous parallel muscle-fibrillp (myoid-fibres) in the exoplasw. $A$, Extended ; $B$, half-contracted (free-swimming); $C$, wholly contracted.

which every cross-section is like every other one. Cross-striated muscle-fibres, on the other hand, contain fibrillæ that from one end to the other are divided into many segments, all of which possess a corresponding but complicated structure.

The simplest forms of smooth muscle-cells are found among Infusmia. In many ciliate Infusoria, such as Stentor, the cell-body represents such a muscle-cell of the simplest kind; it contains, "mbedded in the external layer of its protoplasm, smooth muscle-fibrillæ, the so-called myoids, arranged approximately parallel to one another (Fig. 100). Other Infusorir, especially 
the delicate Vorticella, possess a single, smooth muscle-fibre, composed of several fibrillæ cemented together; this extends outside the body as a thick strand and, surrounded by an elastic sheath to the inner wall of which it is fixed in an elongated spiral, serves the cell-body as a stalk for attachment (Fig. 101). In smooth muscle-cells that are united in the cell-community to form tissues, the protoplasmic body is reduced very much in quantity in comparison with the contractile fibrillæ. It either forms merely a small sarcoplasmic mass containing the nucleus, which is enclosed by a long, spindle-shaped covering of contractile, fibrillar substance,

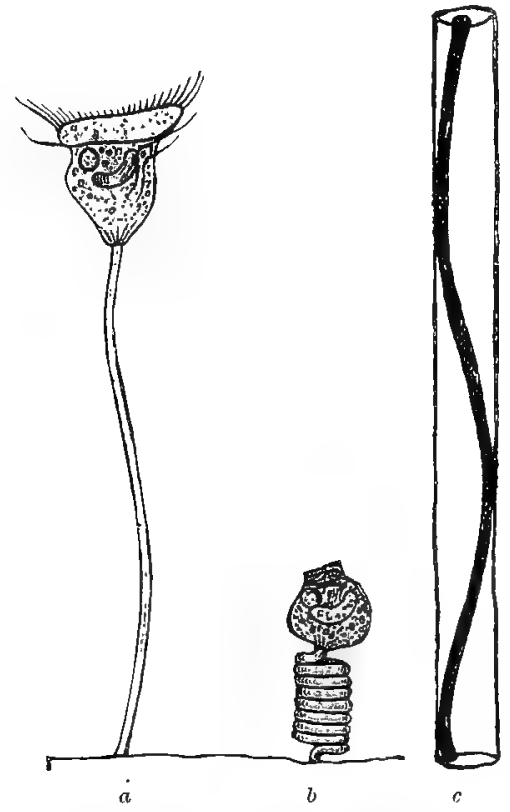

FIG. 101.-Vorticella. $a$, Extended; $b$, contracted (the stalk-muscle is not seen in $a$ and $b) ; c$, stalk-sheath containing muscle-fibre, strongly magnified.

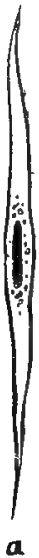

Frg. 102.-Smooth muscle-cells $-a$, from the bladder of the frog; $b$, from the retractor muscles of fresh-water Bryo$20 a$.

as in the smooth muscle-cells from the bladder of the frog (Fig. 102,a), or it lies as a small cell-body in the middle, lateral to the contractile bundle of fibrilla, as in the retractor muscles of fresh-water Bryozoa (Fig. 102,b).

The structure of cross-striated muscle-fibres is far more complex. As a type of these, which, like the smooth muscle, appear in manifold modifications, the insect muscle-fibre may serve, the structure of which has become known in minute detail, especially through the striking and extended investigations of Engelmann and recently Rollett. The cross-striated muscle-fibre 
of insects is a long thin cylindrical cell, consisting of sarcoplasm, which is bounded externally by a somewhat denser layer, the sarcolemma, and contains numerous nuclei elongated longitudinally (Fig. 103). Embedded in this sarcoplasm, and extending parallel from one end of the fibre to the other, lie the regularly segmented muscle-fibrillæ (Fig. 104, A). If the muscle-segments of a fibrilla be examined with very high powers, it is found that they all possess the same structure, the same arrangement of their constituents being repeated in every segment. Each segment is separated from the two adjacent segments by the so-called Dobie's line [Zwischenscheibe] (Fig. 104, z), and contains
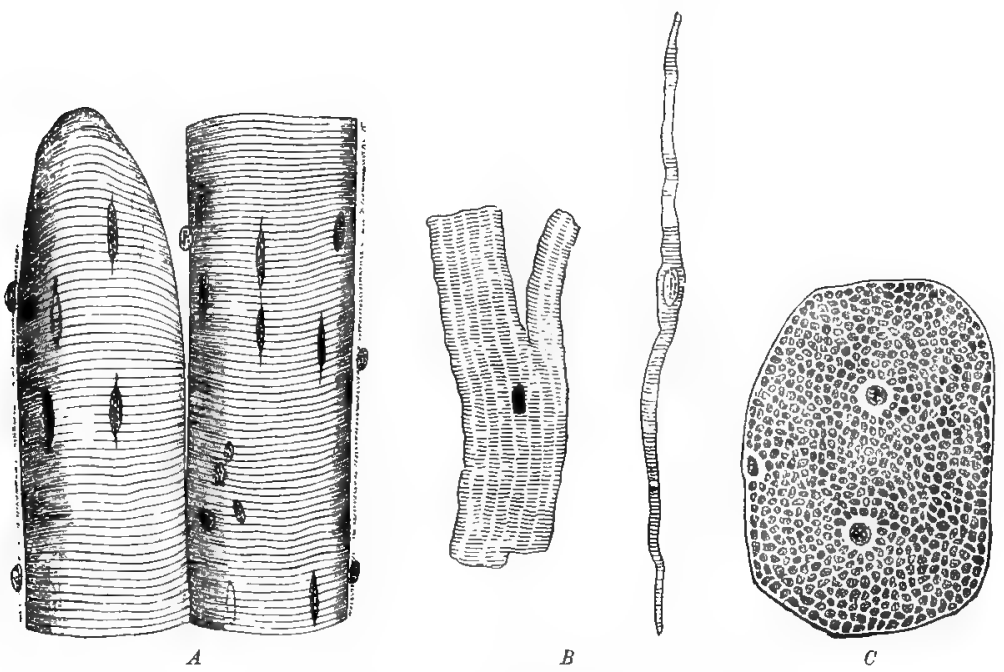

TIr. 103.-Cross-striated muscle-fibres. $A$, Two excised pieces of nuscle-fibre (at the left above, the end of a fibre); the cross-striation is clearly to be seen, likewise many spindle-shaped muscle-nuclei. (After Schiefferdecker.) $B$, Two uninucleated cross-striated muscle-cells fron the heart, at the left from man, at the right from the frog. (After Disse.) C, Crosssection of a muscle-fibre of an insect; three nuclei are to he seen and, embedded in the sarcoplasm, the cross-sections of innumerable fibrillæ. (After Rollett.)

two different substances, of which the one is doubly light-refracting, or anisotropic, and lies in the middle of the segment (Fig. 104, $q$, or $q+m+q$ ), while the other is singly refracting or isotropic, and in two portions borders the anisotropic substance (Fig. 104, i). In the middle of the anisotropic layer there appears more or less distinctly a clearer zone, which is termed Hensen's disc or Hensen's line [Mittelscheibe] (Fig. 104, m). Finally, there occur in many muscle-fibres, but not as a constant constituent of all, one or two accessory discs [Nebenscheibe] (Fig. 104, n) lodged in the isotropic substance. The general constituents of the muscle-segment are the anisotropic layer and the two isotropic: layers bordering it; of these the anisotropic substance is darker, 
denser and more strongly refracting, while the isotropic substance appears richer in water, brighter, less dense, and less refracting. In every muscle-fibre similar discs of the individual fibrillæ lie in the same transverse plane, so that the whole fibre appears regularly banded or cross-striated (Fig. 103, A). The cross-striated musclefibres of vertebrates often reach a, very considerable length, although they represent only a single, multinucleate cell-e.g., the fibres from the long skeletal muscles of man are more than a decimetre in length, and each fibrilla in them extends from one end to the other.

In the movement of both smooth and cross-striated musclefibres, two phases can be distinguished, as in amœboid movement-

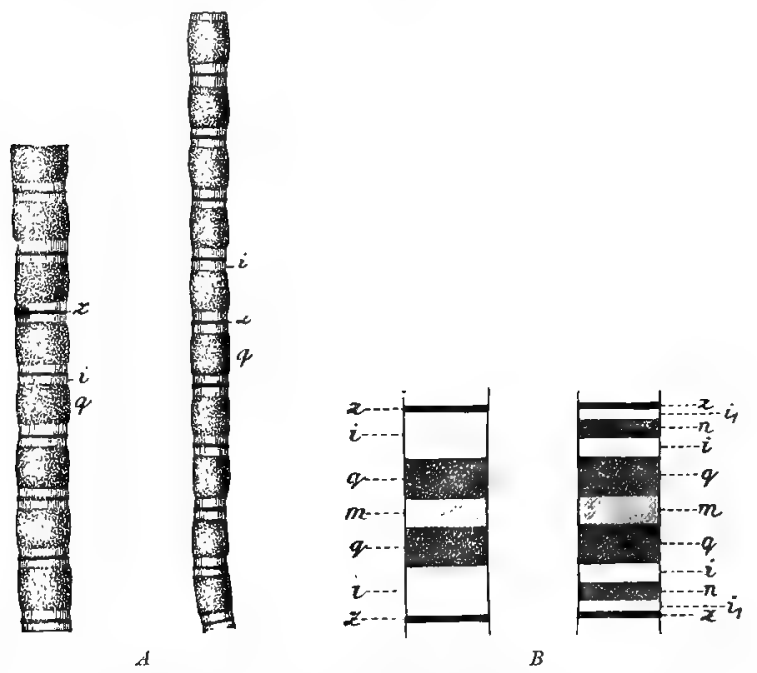

F1a. 104.-A, Two isolated muscle-fibrillse; $z$, Dobie's line; $i$, isotropic substance; $q$, anisotropic aubstance. (After Ranvier.) $B$, Two single muscle-segments; $z$, Dobie's line ; $i$, isotropic substance; $q$, anisotropic substance containing Hensen's disc, $n$." The segment at the right possesses an accessory disc, $n$, in the isotropic substance.

that of contraction and that of expansion. Contraction consists of a shortening and thickening of the fibrillæ. This process passes from the place of its origin in the form of a contraction-wave over the whole fibrilla. The particles, therefore, shift themselves in the longitudinal direction in such a manner that they come to lie beside one another in a larger cross-section. In this way the whole surface of the fibrilla becomes diminished, although not to its minimum, the spherical form, as is the case in naked protoplasmic masses. The simultaneous contraction of the single fibrillæ in either a smooth or a cross-striated musclecell evidently causes a shortening and thickening of the whole 
fibre. If the contraction proceeds very rapidly, as in the fibrilla of infusorian cells and cross-striated muscle-fibres, the fibre gives il very quick twitch, the single events of which cannot be followed by the eye. Thus, the stalk of Vorticella contracts suddenly, assuming a screw-shape as a result of the spiral winding of the muscle-fibre and drawing the head of the animal tightly down to the foot of the stalk (Fig. 101, b). The smooth muscle-fibres of the tissues, on the contrary, generally contract extremely slowly and never show sudden twitches like the infusorian myoids and cross-striated muscle-fibres. But, while in the smooth musclefibrilla no further events, apart from the change of form, are to be noted microscopically, the cross-striated fibrilla, in correspondence with its complex structure, shows in the phase of contraction highly characteristic changes of its striation (Fig. 105). During the contraction of a single muscle-segment the following phenomena, which Engelmann ('73, '75, '78) first carefully' analysed, may be observed. The segment becomes shorter and

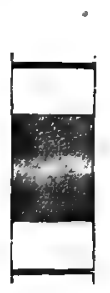

1

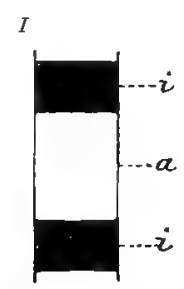

2

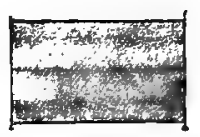

1

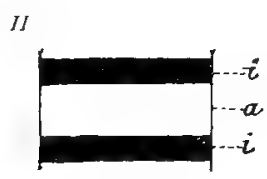

2

FIG. 105.-Single muscle-segment; $I$, extended; 11 , contracted ; 1 , in ordinary light; 2 , in polarised light; $a$, the anisotropic disc ; $i, i$, the isotropic discs.

thicker, which is to be expected from the shortening and thickening of the whole fibrilla. Meanwhile, remarkable changes occur in the relation of the isotropic to the anisotropic substance. The latter increases in volume and the former decreases, while the volume of the whole segment remains unchanged. At the same time the anisotropic substance, which before was denser and darker, becomes less dense and lighter, i.e., less refracting, while the isotropic substance undergoes the reverse changes, becoming denser and darker, i.e., more refracting than it was before. These changes are extremely important, for they show that contraction consists in " passage of substance from the isotropic discs into the anisotropic, and, moreover, of substance that is of less consistency than that of the anisotropic disc. Recently, by means of photography Schäfer ('91, 2, 3) has studied more carefully the microscopic changes in this process, and has discovered the interesting fact that in the anisotropic disc extremely fine tubes run parallel to one another and in correspondence with the direction of the fibres almost up to Hensen's disc (Fig. 106); in contraction the isotropic 
substance flows into these tubes, so that the lumen of each is enlarged and the whole segment becomes broader and shorter. All these complex phenomena of contraction proceed with excessive rapidity from one muscle-segment to the following, so that one contraction-wave after another passes metachronically over all the elements of the whole muscle-fibre until the latter is completely contracted. The expansion of smooth and cross-striated muscle-fibres. shows exactly the reverse of all these events observed during the contraction. The fibrillæ extend, becoming gradually longer and thinner from the point where the contraction-wave previously began, so that now a wave of expansion proceeds from here over the whole fibrilla, until the latter is completely extended. In the single segment of the cross-striated fibre, also, the changes are exactly the reverse

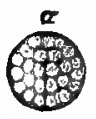

6

IIIIIII)

भiIIIII

F'I 106.-Muscle-segments from the wasp, containing tubes of anisotropic sub. stance; $a$, anisotropic substance seen from above; $b$, from the side; $c$, three muscle-segments. (After Schäfer.) of those that appear in contraction. The segment becomes longer and thinner, the anisotropic substance decreases in volume, and becomes darker, denser, and more highly refracting, while the isotropic substance gains in volume and becomes lighter, less dense and less refracting, until the resting state is again reached. In the expansion of the cross-striated musclc-fibre, therefore, substance that possesses slight consistency passes from the anisotropic to the isotropic discs.

Both smooth and cross-striated musclefibres are united in the cell-community into tissues, the muscles. Wherever rapid and powerful muscular effects are to be brought about, as in the skeletal muscles and the heart, the muscles are composed of cross-striated fibres, while the slow, sluggish movements of the involuntary organs, such as the stomach, the intestine, and the bladder, depend upon the activity of smooth muscle-cells. The contraction of muscle reaches its highest, and indeed, an astonishing rate in the wing-muscles of many insects, e.g., gnats, where, as Marey has shown, 300-400 contractions in a second can be carried out. It is evident that the effect of the contractions will be very considerable where very many fibres compose a muscle. In fact, even in relatively small muscles an enormous transformation of energy takes place. Thus, such a small muscle as the calf-muscle (gastrocnemius) of the frog, which measures scarcely a centimetre in crosssection in its thickest place, is capable, according to Rosenthal's observations, of raising a weight of more than one kilogram. The work that the heart-muscle performs is enormous. Zuntz ('92) has (alculated that the heart of a man beating normally performs in one day a work of about 20,000 kilogram-metres-a labour that would be sufficient to raise a weight of 20,000 kilograms one metre 
high. It is easy from this to compute the enormous labour performed by this organ during the whole life of a man. The muscle is the most perfect dynamic machine known.

Ciliary movement, finally, is no less wide-spread than the two sther forms of contraction. The infusorian that bustles about actively in the water of a puddle moves by the strokes of flagella or cilia. The spermatozoon that in fertilisation strives after union with the egg-cell is driven forward by the vibrations of its flagellum. The cells of ciliated epithelium that line air-passages keep the mucous membrane clean by their activity, and by the rhythmic beating of their cilia shove to the outside foreign bodies that have come into the passages in swallowing. The host of Infusoria is numberless, flagellated spermatozoa are wide-spread among both plants and animals, and there is scarcely a group of animals whose bodies do not possess in some spot ciliated epithelium.

Like muscular movement, ciliary movement is co-ordinated -i.e., the motile particles are shifted in a definite direction. This is rendered possible by the fact that the contractile elements, as in the muscle-cell, are developed as constant differentiations of the cell-protoplasm in the form of short, hair-like appendages of the cell-body. According as the cell possesses one cilium or a few long ones, or many short ones, the term flagellated cell (Fig. 107, $C, D, E)$, or ciliated cell (Fig. 107, $A, B$ ), is employed. The phenomena of ciliary motion result from the performance of vibratory movements by the flagella or cilia.

The following are the chief characteristics of ciliary motion. In contrast to most forms of muscular motion, which with few exceptions (Infusoria, heart-muscle) come about only as the result of external impulses from the nervous system, ciliary motion is automatic, i.e., the impulses that lead to it arise in the cilia themselves; there is no known case in which the motion is at all under the influence of the nervous system. It has been determined by vivisection experiments ${ }^{1}$ that the cause of the motion is seated in the protoplasm of the cell-body, for, if the isolated cilia possess absolutely no protoplasm at their bases, they are wholly motionless. Further, most cases of ciliary motion are distinguished by their rhythm, for except in certain flagellate and ciliate Infusoria the cilia contract always at regular intervals, at least during pronounced activity. The vibrations become irregular only during the transition to the resting-stage or under the influence of external factors. Finally, a third characteristic, which belongs only to multiciliated cells, is the metachronism of the motion of the individual cilia. The individual cilia of a row, beginning at one end, contract in exactly the same rhythm and succession, so that every beat of the first cilium is followed by a beat of the second, then of the third,

$$
{ }^{3} C f \text {. Verworn }(90,2) \text {. }
$$


the fourth, and so on. A cilium never contracts spontaneously out of order, it never makes a movement before the preceding cilium in the row has moved; it always begins its movement
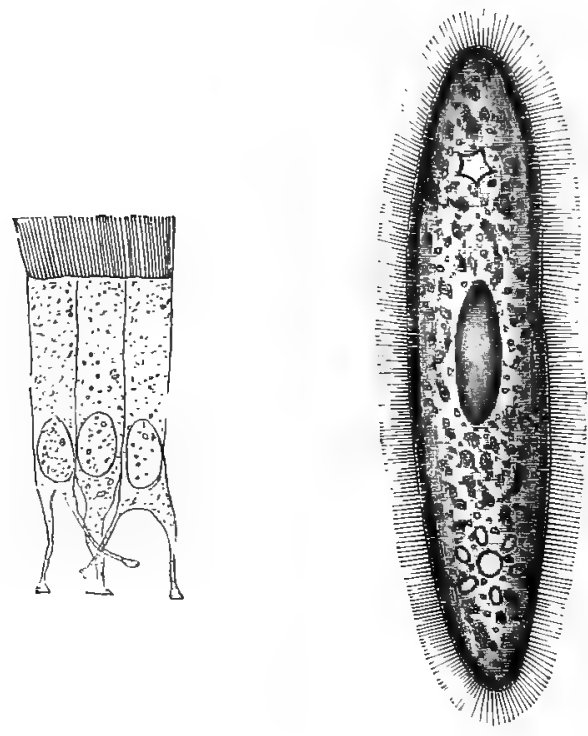

$A$

$B$

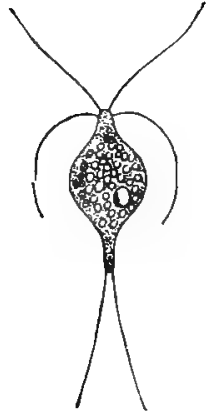

$C^{\prime}$

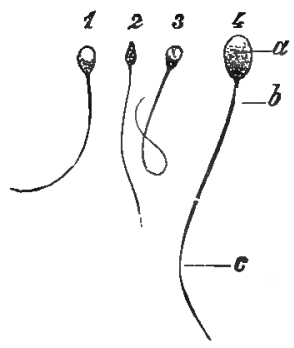

$D$

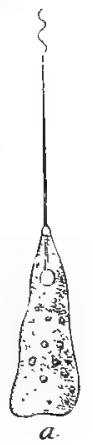

$B$

FIG. 107.-A, Three ciliated epithelium-cells from the humitn epididynis. (After Schiefferdecker.) $B, P a r a n a c i u m$ andia, a ciliate iufusorian ; $C$, Hexcmitus infatus, a flagellate infusorian possessing six fligella (After Stein.) $D$, Human spermatozon; $a$, head, $b$, $c$, flagellum. (After Stöhr.) E, Peranema, a flagellate infusorian, with its flagellum contracting, $a$, feebly, $b$, more strongly.

immediately after the movement of the preceding cilium has begun and before it is ended. It thus happens that, considering the row from above downward, the movement of each upper cilium slightly 
precedes that of each lower one (Fig. 108). In other words, the uppermost cilium gives the sign to the others; if the uppermost one is at rest, the others rest; if it contracts, they also contract in order; and this is true not only of the cilia of the single cell, but,

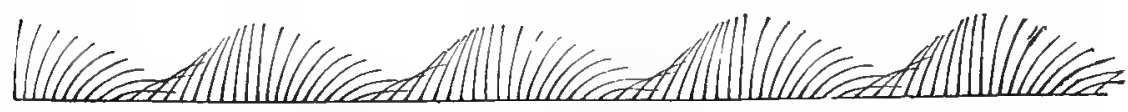

FIG. 108.-Motion of a row of cilia, in profile.

in ciliated epithelium, of the cilia of all the cells in a row. In this manner there occurs an extremely delicate and regular play of the cilia, which has fascinated many observers and gives the impression of regular waves passing over the ciliated row, somewhat as the wind sweeps over a field of grain. When several parallel rows of cilia are present, the cilia standing beside one another in adjacent rows beat synchronously, just as the fibrillæ lying beside one another in a muscle-fibre contract at the same time.

The phases of movement of the individual cilium can best be studied in the swimming-plates of the Ctenophora. ${ }^{1}$ The body of these remarkable animals consists of a delicate transparent jelly, and possesses eight stripes or ribs (Fig. 109) extending from one

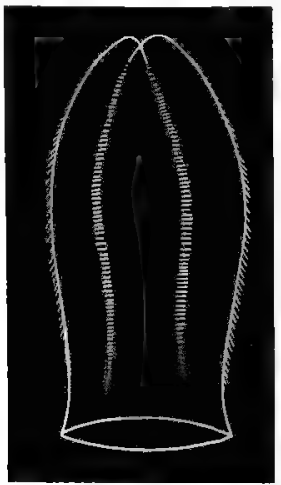

Frg. 10\%-Beroe ovata, a ctenophore, natural size. Of the eight rils or rows of swimming-plates extending from the upper (seuse-)pole to the lower (mouth-) pole, only the four rows of one side are to be seen, two from the front, and two from the side.

pole to the other; each rib consists of a row of plates, the swimming-plates, that lie upon; one another like tiles upon a roof. Each swimming-plate is about $2 \mathrm{~mm}$. long, and consists of a considerable number of cilia, cemented together, which belong to the cell-bodies lying beneath. On account of their extraordinary

${ }^{1} C f$. Verworn $(90,2)$. 
size, the unusual simplicity of their arrangement in rows, and the fact of the rhythm of their beat being frequently very slow, these swimming-plates serve as no other object does for experimentation and observation. As was said above, the plates are formed of many cilia cemented together, but each cilium evidently makes exactly the same movement as the whole plate, so that observations made upon the whole plate may be transferred to the conditions in a single cilium. On account of the size of the object observations can be made with the naked eye or with a weak lens. If a single swimming-plate be observed in profile, it is seen that in the resting-position it lies flat against the body, so that it shows two curves, a greater one of smaller radius immediately above the base, and a smaller one of greater radius and in the opposite direction in the upper half (Fig. 110). This

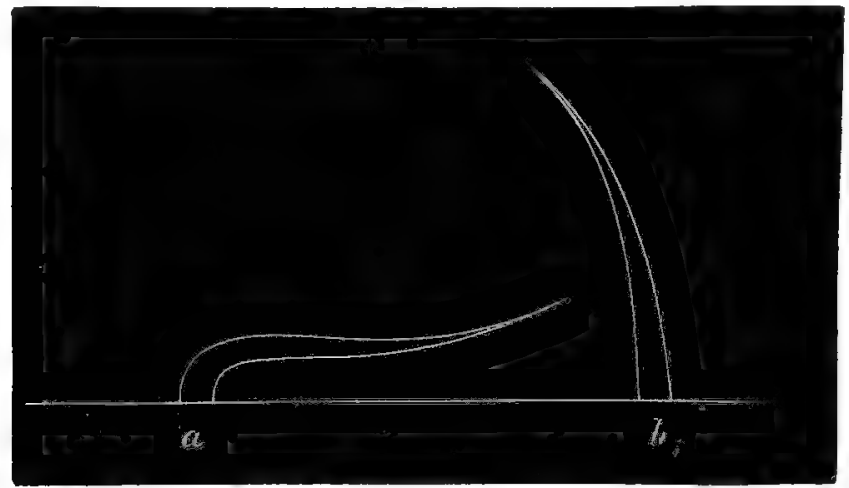

FIG. 110.-Swimming-plates of Besoe in profile. $a$, In the resting-position; $b$, in the position of extreme contraction.

is the position of rest. If now the plate performs a stroke, the lower curve beginning from the base of the cilium extends itself completely, even giving place to a slight curve in the opposite direction. Hence, in the position of extreme swing the plate stands erect with a slight curve toward the opposite side. The progressive phase of the stroke is thereby completed. Now follows the retrogressive phase, in which the plate falls back again into its position of rest, the original curve at the base gradually coming back until the plate again lies against the body. The retrogressive phase proceeds more slowly than the progressive. Because of this fact and by means of the upper curve-into the special significance of which we shall go no further-it is rendered possible that the motor effect of the progressive phase is not balanced by the retrogressive phase; otherwise the animal would remain continually in the same place in the water. The movement of 
individual cilia can be followed in Infusoria under the microscope, if the stroke be slowed by placing the objects in a thickish medium, such as a solution of gelatine. It is then found that the resting-position, from which the cilium performs its movements, is changeable. At one time the cilium lies more against the body, at another time it stands more vertical ; hence the amplitude of the swing, and thus the amount of the motor effect can be very finely graded (Fig. 111).

It follows from the change of form of the individual cilium in carrying out the stroke, that in the progressive phase a contraction, starting from the base of the cilium, takes place on the side toward which the stroke is carried out, for a simple measurement shows that this side is shortened when it passes into the position of extreme swing. At the same time the opposite side is drawn
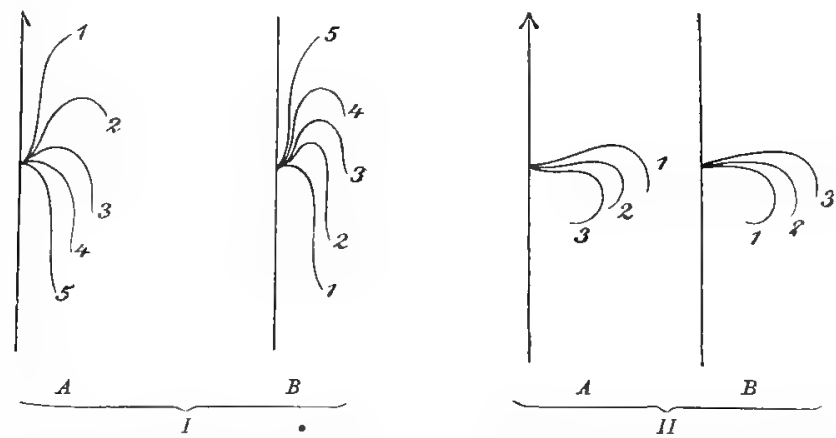

FIo. 111.-Morement of a single cilium of a ciliate infusorian (Urostyla grandis, border-cilium) from two different resting-positions, $I$ and $I I$. A. Progressive, $B$, retrogressive phase of the movement in several successive stages. The arrows indicate the direction toward which the body is driven.

over passively, being extended necessarily, according to simple mechanical principles. In the retrogressive phase the contracted side relaxes, and to the same extent the cilium, as a result of the elasticity of the extended side, bends back into the position of rest. The progressive phase, therefore, is the phase of contraction, the retrogressive phase that of expansion of the single stroke of the cilium. The play of the ciliary movement comes about by the rhythmic alternation of the two.

But all cilia do not contract in one plane like those of the swimming-plates of the ctenophores. Many, especially certain flagella, describe more complicated paths, funnel-shaped, screwshaped, and like the path of a whip-lash, and accordingly the earlier physiologists distinguished several forms of ciliary movement. But whatever the path of vibration of the different cilia may be, the same principle lies at the basis of all, viz. : that a 
contractile side contracts from the cell-body outward, and thereby the opposite side is extended; in the phase of expansion the latter, by its elasticity, brings the cilium back into the position of rest. According to the relative positions of the contractile and the passively extended substances there results a movement in a plane or a more complicated form.

The work performed in ciliary movement is much less than that of muscular movement. Engelmann, Bowditch and others have calculated the work of ciliated epithelia, and recently Jensen $(' 93,2)$ has measured the force of a single ciliate-infusorian cell, Parcemecium, which is well fitted for a great variety of investigations. Jensen determined that a Paramocium, which possesses a length of about $0.25 \mathrm{~mm}$., is able to raise a weight of $0.00158 \mathrm{mgr}$., i.e., about nine times the weight of its own body.

The view is sometimes expressed that amœboid movement has nothing in common with muscular movement, and the latter nothing in common with ciliary movement, that the three are utterly different in kind. The above brief examination is sufficient to show, however, that these three forms of contraction constitute a single group in contrast to all other modes of motion. It is true that they show among themselves certain differences, and that at first sight they appear quite unlike one another, but it has been seen that they all rest upon the same principle, namely, that of alternating diminution of surface (contraction) and increase of surface (expansion) by means of a rearrangement of the particles of the living substance. That in amoboid movement this shifting of the particles is wholly without rule, while in muscular and ciliary movements it is orderly, proves only that the two latter represent a higher stage of differentiation than the former. That, however, they stand in the closest genetic connection with amoeboid movement, that they have become cvolved from it phylogenetically, is proved by numerous cases of transition, on the one hand between amoeboid and muscular movement, and on the other between amoeboid and ciliary movement. Engelmann ('81, 2) has found rhizopods (Acanthocystis) possessing straight, filose, unbranched pseudopodia, which are capable of contracting longitudinally with excessive rapidity, and from which a small muscle-fibre is distinguishable only by its constant differentiation: Engelmann has fittingly termed these pseudopodia myopodia. Moreover, many cases have been observed where filose pseudopodia of amoboid cells carry out pendular vibrations, at first irregularly and slowly, later rhythmically, until they have developed into genuine, constant cilia. In view of such facts no proof is needed to place beyond doubt the genetic connection of the three forms of contraction, even if careful observation of their single factors had not proved sufficiently clearly the identity of the principles upon 
which they are based and their relationship in comparison with all other modes of motion.

The contraction of living substance follows the same principle "verywhere, whether the living substance creeps about as an Amaba upon decaying leaves in a pool of water, whether as a white blood-corpuscle it forces its way through lymph spaces in the tissues of the animal body, whether as a protoplasmic network it circulates in the cellulose-capsule of a plant-cell, whether as a muscle-fibre it performs the contractions of the untiring human heart, or, finally, as a cilium on the oviduct of woman it transports the unfertilised egg-cell to the uterus to undergo fertilisation,-everywhere there is the same phenomenon of alternating contraction and expansion of the living substance by means of the reciprocal rearrangement of its particles.

\section{The Production of Light}

In the movements of living substance, especially in the phenomena of contraction, the transformation into kinetic energy of the potential energy introduced into the body as food, comes out very clearly. This is much less evident in the production of other forms of kinetic energy, such as light, heat, and electricity, for the demonstration of which very complicated methods and sensitive instruments are often required.

Next to the mechanical energy of movement, the production of light is most evident to the senses, and has always had a mysterious fascination for the observer. It has a curious charm, when at night the water of a quiet sea breaks into a bright, yellow glow at every stroke of an oar, or when in southern climates in the spring, the mild night air is filled by innumerable sparks, which silently flash up and circle about, and then disappear.

The emission of light by living substance is wide-spread. It is an especially significant fact that, of the wonderful pelagic animals whose delicate transparent bodies occupy the upper strata of the sea and float about as plankton, almost all possess luminous power. Associated with this fact is the presumption that the luminous capacity of living substance is possibly much widerspread than is realised, that we do not see the light because the organisms are not transparent, or because the production is too feeble to allow the light to be seen through thick body-layers; indeed, it is not impossible that in our own bodies certain cells may be photogenic. In most cases, as in luminous insects, the power of emitting light is a peculiarity specially perfected by selection and possesses its own significance for the life of the animals in question. In pelagic marine animals also such a significance is certainly present; as a rule, these animals emit light 
suddenly and only upon stimulation, and hence it may be supposed that the light serves as a means of frightening enemies (Fig. 112).

The spontaneous emission of light is much less common. It appears especially in certain putrefactive bacteria that live upon decaying sea-fish and flesh (Bacterium phosphorescens), as well as in mushrooms (Agaricus), and certain insects (Elater, Lampyris).

Numerous researches have been carried on respecting the nature of the light, e.g., those of Panceri and Secchi on Salpoe (Pyrosoma), those of Moseley on deep-sea colenterates (Alcyonaria), and more recently, especially those of Langley and Very ('90) upon the lightning-bug (Pyrophorus noctilucus). To obtain a comparison

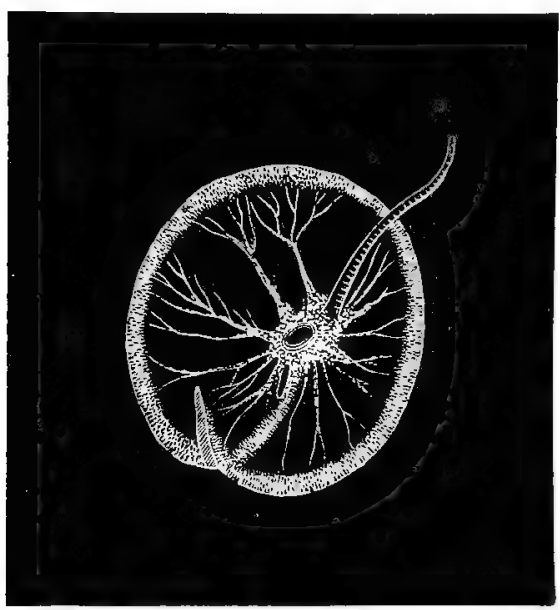

FIa. 112.-Noctiluca milianis, a pelagic flagellated cell which becomes luminous upon stimulation. that an enormous literature upon the subject has appeared. Pfliiger ('75, 1, 2) has collected a series of physiologically interesting accounts. It appears therefrom that very different views have been put forward upon the origin of the light in organisms. The idea early met with great approval, especially among non-specialists, that organic light depends upon the presence of phosphorus, to the mild light of which it has a certain external similarity. But exact investigations have shown that it has nothing whatever to do with phosphorus. This follows from the fact, among others, that the emission of light presupposes life in the cell. It can be observed in the single cell, a free-living bacterium from decaying fish, an infusorian or radiolarian from sea-water, or a tissue-cell of a composite animal- or plant-body ; but in every case the photogenic substance is produced only in 
the cell-metabolism, although R. Dubois ('92) has shown that in certain animals, e.g., the boring mussel Pholcs, the substance can be extruded from the body as a cell-product without immediately losing its luminous power. Phosphorus is an active poison for all living substance; hence, in the free state, in which it becomes luminous, it is wholly incompatible with the life of the cell. A trace of free phosphorus or luminous compounds of phosphorus has never been found in luminous animals. Nevertheless, it can be stated with certainty that the luminosity of living substance is associated, as in phosphorus, with very slow oxidation-processes. This follows especially from the fact that the light continues only in the presence of oxygen. Moreover, Fabre (55) has found that the luminous mushroom, Agaricus, produces much more carbonic acid, when emitting light, than at other times. Finally, there belongs here a fact that was observed by

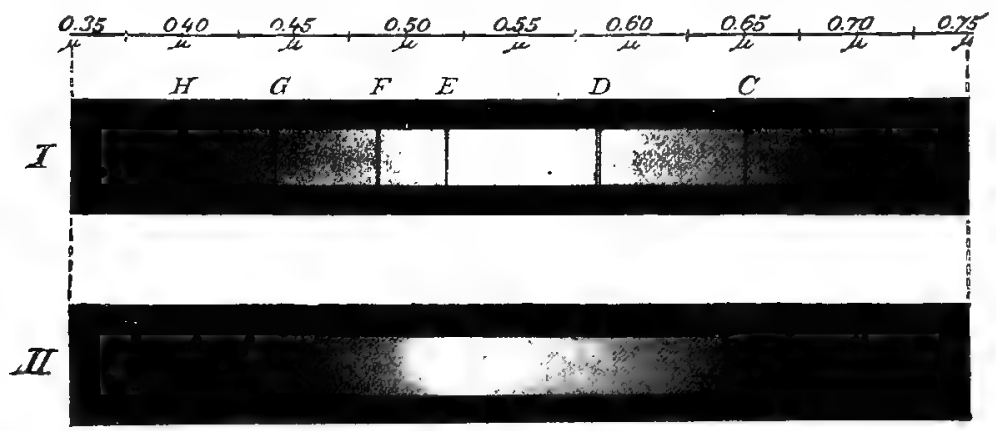

FIa. 113. $-I$, Solar spectrum; $I I$, spectrum. Pyrophorus noctilucus. (After Langley and Very.)

Max Schultze ('65) in the cells of the photogenic organs of lightning-bugs, namely, that these photogenic cells stand always in the closest connection with the tracheæ, which serve as breathingtubes; and, if they be placed under the microscope with perosmic icid, they withdraw oxygen from the latter, a fact which may be recognised by the appearance of a black precipitate. The photogenic cells, therefore, absorb oxygen actively. Pfliger appropriately says concerning it: "Here, in the wonderful spectacle of animal phosphorescence nature has given us an example that shows where the taper burns that we call life." "It is certainly no rare exception, but only the special expression of the general law that all cells are burning continually, although with our corporeal eyes we do not see the light."

As regards the special processes of oxidation with which the luminosity of living organisms is associated, at present, with our very scanty knowledge of metabolism, almost nothing can be said with certainty. The beautiful researches of Radziszewski ('80) 
more than any others have elucidated this subject. Radziszewski studied in detail the conditions under which chemical substances. exhibit phenomena of phosphorescence, and found that a whole series of organic bodies emit light when they are slowly combined with active oxygen in an alkaline solution. Such bodies comprise especially many fats, etherual oils, hydrocarbons and alcohols. In many the light appears at ordinary temperatures, in others only upon warming. If, $\epsilon . g$, oleic acid be added to an alcoholic solution of potassium hydrate in a test-tube, a light lasting for a short time may be observed in the dark while the acid is being dissolved. If, after the light has ceased, a drop of a solution of peroxide of hydrogen be added to the liquid, a clear strip of light is seen to pass through the test-tube along with the drop of peroxide of hydrogen as it falls to the bottom. This is due to the fact that the peroxide of hydrogen gives off active oxygen to the oleic acid. The same phenomenon of light is shown still more clearly when oleic acid is dissolved in pure toluol, which likewise is capable of phosphorescence, and the solution is poured over a. piece of potassium or sodium hydrate. The intensity of the light can always be increased by shaking, because the free atoms of oxygen are thus brought more into contact with the molecules of the phosphorescent body. If, e.g., into a glass bulb containing a mixture consisting of equal parts of pure toluol and cod-liver oil (which latter always contains in addition to oleic acid free atoms of oxygen), there be thrown a few pieces of potassium or sodium hydrate, and the whole be gently warmed and placed in the dark, no light is seen at first. But, if the contents of the bulb be gently shaken, there is seen " at once a beautiful light streaming through the whole mass like a flash of lightning."

It is in the highest degree probable that the luminosity of living substance depends upon analogous processes. Fats, oils, etc., are wide-spread in living substance, and Panceri believes of certain luminous marine fishes that the liquid fat is the luminous body. Substances that give an alkaline reaction are likewise found everywhere in living substance, and the luminosity of organisms is associated with processes of oxidation. Thus the same conditions are present in living substance as in the experiment of Radriszewski.

\section{The Production of Heat}

The production of heat is much less apparent to the senses than that of light. While we can observe the latter readily in the single cell, the amount of heat produced by the single cell, because of the small size of the object, cannot be measured with cur crude instruments for the measurement of temperature. Nevertheless, it must be assumed that in the interior of every living cell 
heat is produced, for chemical processes are there present that are accompanied by the production of kinetic energy, and heat is the form of kinetic energy that is evolved in all such processes without exception, either alone or in addition to other forms of energy. In fact, there is even good ground for supposing with Pfliger that in single molecules of living substance temperatures of several thousand degrees Centigrade become developed suddenly. This may be the case in the production of a molecule of carbonic acid, since the heat yielded by the combustion of carbon amounts to 8,000 calories. But the molecule of carbonic acid is excessively small, and it is surrounded in the cell by an enormous number of other molecules which possess a very low temperature. Hence, the heat that suddenly flashes up is counterbalanced as rapidly as it appears; and, since all heat-forming molecules are not produced simultaneously, but appear now here and now there between large masses of other molecules, it is evident that the total temperature of the cell resulting from the equalisation of all the various individual temperatures cannot reach a remarkable height. Further, with our crude methods of heat-measurement, we cannot yet measure the actual heat given off to the outside by a single cell, since the greater part is lost in the process by conduction and radiation. It is, therefore, necessary to employ for the determination of the heat-production, not a single cell, but large cellcomplexes, such as considerable masses of tissue or whole organisms.

The production of heat is most evident in the bodies of homothermal, or so-called warm-blooded, animals. It has already been seen that the earlier division of animals into warm-blooded and cold-blooded has been rery fittingly replaced by that into homothermal and poikilothermal animals, i.e., those that maintain under all external conditions the same body-temperature and those whose body-temperature rises and falls with the temperature of the environment. Homothermal animals show most clearly the production of body-heat because they havecontrivances for storing up heat in themselves to a certain definite degree and maintaining it at this degree by an extremely delicate regulating mechanism. Hence, with an external temperature not too high the body of the homothermal animal is always warmer than the surrounding medium. This may be determined readily by the method of thermometric measurement. Thus, the body of man possesses in its interior a constant temperature of $37^{\circ}-39^{\circ} \mathrm{C}$, upon its surface a temperature somewhat less, corresponding to the external cooling, in the mouth-cavity about $37^{\circ} \mathrm{C}$., and in the axilla about $36.5^{\circ} \mathrm{C}$. Birds with their active metabolism have the highest body-temperature, e.g., the swallow more than $44^{\circ} \mathrm{C}$. But that poikilothermal animals can attain consiclerable temperatures when under conditions in which the heat produced by them is stored and 
not given off to the medium by conduction or radiation, is proved by the fact that bees in their hives can produce temperatures of from $30^{\circ}$ to $40^{\circ} \mathrm{C}$. Even plants can raise their temperature above the temperature of the surroundings, as can be determined thermometrically, especially in sprouting and in vigorous growth where the metabolic processes are particularly active. Sachs was able to determine with a thermometer a rise of temperature of $1.5^{\circ} \mathrm{C}$. in peas which were allowed to sprout in a funnel under a bell-jar (Fig. 114). Very remarkable temperatures have been observed in the spadices of the peculiar Aroïdeue during

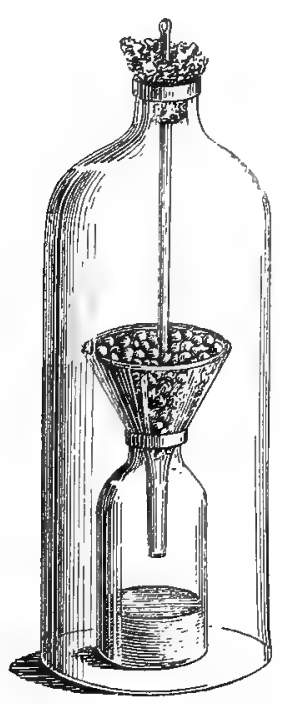

FIG. 114.-Apparatus for demonstrating the rise of temperature in the sprouting of peas. Under a bell-jar is a funnel containing sprouting peas, into which projects a thermometer. (After Sachs.) their development: here not rarely a rise of $15^{\circ} \mathrm{C}$. is found. A rise which under favourable conditions can amount to more than $14^{\circ} \mathrm{C}$. is produced also by yeast-cells in the fermentation of sugar solutions.

For the determination of delicate changes of temperature, especially in the tissues of poikilothermal animals, the rough method of measurement of temperature by the thermometer is not sufficient, and hence the finer method of thermo-electric measurement has been employed. As is well known, in a thermo-electric element, which consists of two pieces of different metals soldered together at one end (the best metals are German silver and iron, or antimony and bismuth), an electric tension is produced by slight warming of the soldered place. If the two free ends of the metals be joined by a wire so that a closed circuit exists, an electric current can be led off from them, the presence of which is shown by the deviation of a magnetic needle in the vicinity. For the demonstration of very feeble currents especially sensitive apparatus is needed, such as the multiplier and the galvanometer, the magnets of which are moved by very delicate currents. The multiplier consists of a suspended and easily moved astatic system, i.e., two horizontal magnetic needles which are fastened together parallel one above the other, so that the north pole of the one lies above the south pole of the other. In the region of the lower needle the wire of the circuit is wound into a coil consisting of an exceedingly large number of turns, so that when the current goes through it, all the individual turns tend to deviate the needle in the same direction. The upper needle hangs above a disc divided into degrees, so that here the deviation of the needle can be measured (Fig. 115). In the 
galvanometer (Fig. 116) the magnet has the form of a ring which is surpenderl by a silk fibre in the space within the coil ; a small mirror is connected with the ring and accompanies all the movements of the latter (Fig. $116 \beta \gamma$ ). At some ristance from the apparatus stands a telescope bearing a scale, the image of which by careful adjustment can be observed through the telescope in tho mirror of the galvanometr (Fig. $116 \gamma$ ). The slightest deviation of the ring-magnet is shown in the telescope by a shifting of tho image of the scalc. Accorling to the extent of 1.his shifting the strength of the elestric current can be computed,
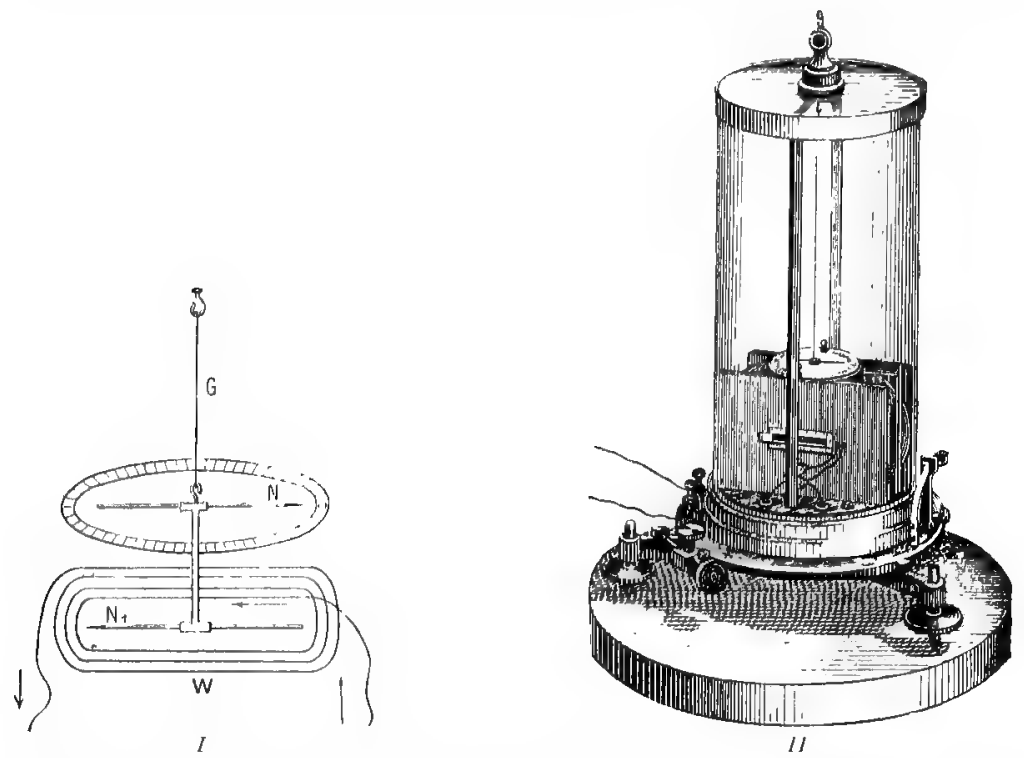

Iira. 115.-Multiplior. I, Plan. An astatie Hyston, with tho north polos $\mathbf{N}$ and $\mathrm{N}^{\prime}$, is susponded upon a bllk fibro $G$. Around the lower noodlo is $n$ coll of wiru $W$; tho upper needlo moves abovo a graduatod disc. (Aftor Landols.) II, Multiplior comploto. (Aftor Cyon.)

and hence empirically the amount of heating of the thermo(lectric element, or, better, i whole series of thermo-electric clements. Thus the most delicate changes of temperature that a living tissue undergoes can be determined. By investigations of this kind it has been established that a high' ' $\mathrm{temperature} \mathrm{is} \mathrm{pro-}$ duced by greater activity of the cells of a tissue, e.g., a gland or a muscle, than by less activity or during rest. This result is in close accord with our ideas concerning the production of heat, for the grcater activity of the cells depends upon a greater metabolism in them, and heat results from chemical transformations in the cell. It is an old experience that one can warm himself by vigorous muscular activity. 
All measurements of temperature, whether by the thermometer or the thermo-electric method, serve only to determine the temperature that prevails in some one place in the organism at some one time. They give no particulars regarding the quantity of heat that the organism or the individual tissue produces. But it is possible to determine the quantity of heat by investing the number of heat-units, or calsries, that the living body gives off to the outside in a certain time. Thus calorimetry has developed by the side of thermometry. As is well known, a calorie is that

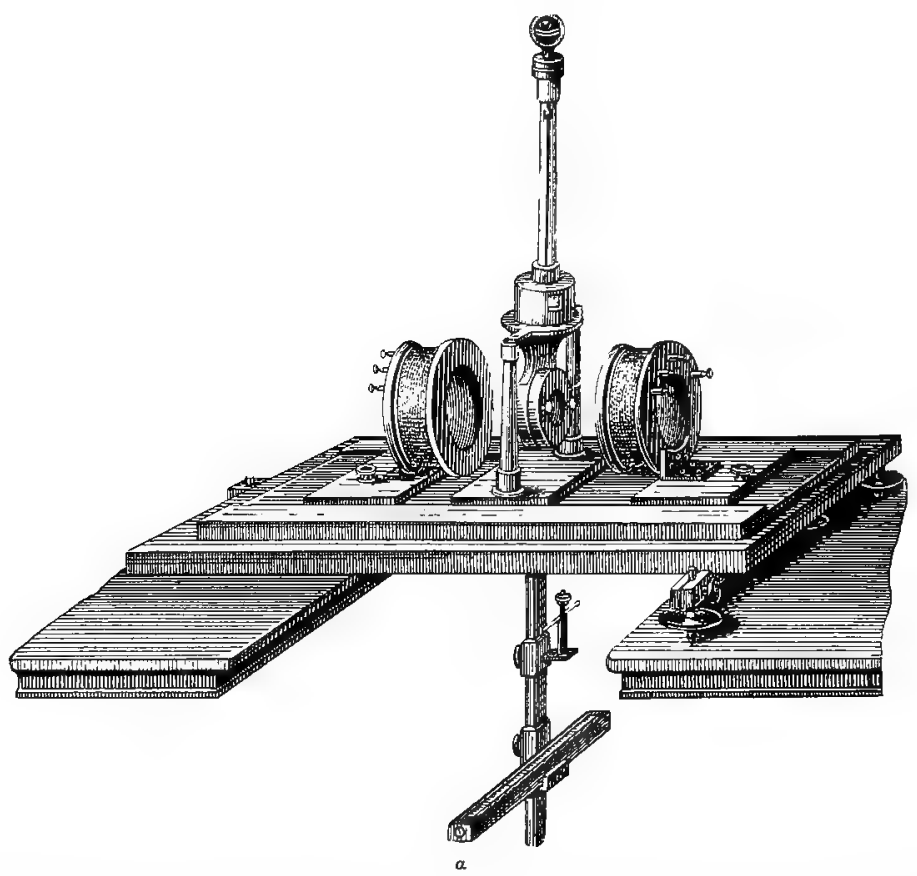

Fig. 116 a.-Mirror galvanometer. Upon a board is an upright, supported by two columus; the upper portion consists of a glass tube in which hangs a silk fibre suspending a ring-magnet in the lower portion. At the two sides are two coils of wire. (After Cyon.)

quantity of heat that is necessary to warm one kilogram of water from $0^{\circ} \mathrm{C}$. to $1^{\circ} \mathrm{C}$. In order to measure the number of calories that a living body, for example an animal, produces in a definite time, the water-calorimeter has been constructed (Fig. 117). This consists of a box having double walls that may be closed upon all sides. The space between the two walls is filled with water, the animal is placed in the box, and the whole is protected from cooling or warming from the outside by a non-conducting covering. The heat produced by the animal is communicated to the water 
and raises its temperature; the latter can be read off upon a thermometer projecting into the water. Various contrivances serve to reduce the sources of error that depend upon possible loss of heat. From the quantity of water and the warming of it in a

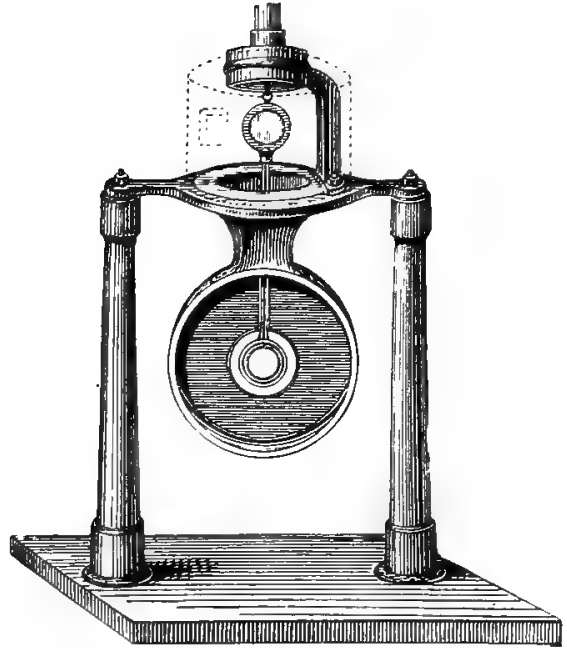

$\beta$

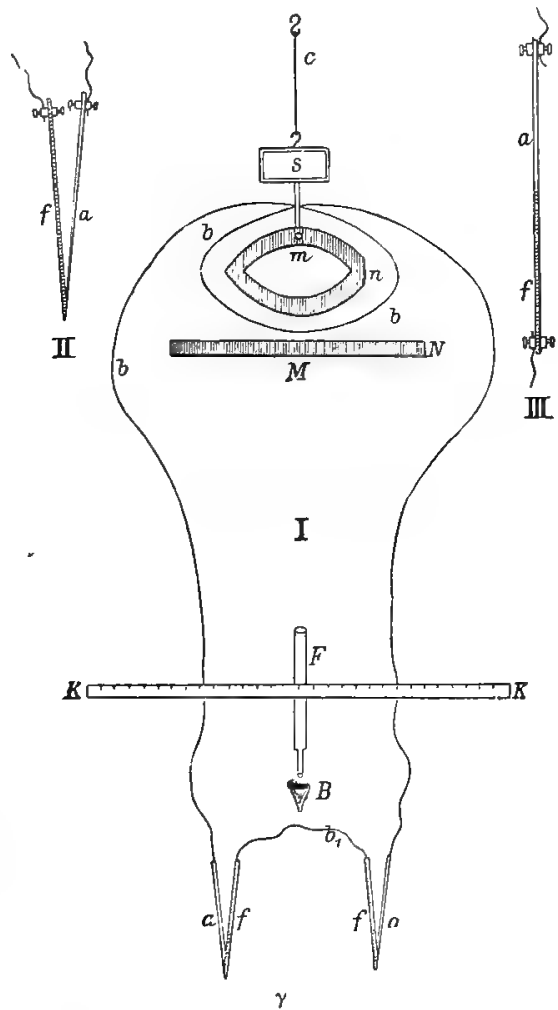

FIG, 1 lf 8. - A portion of the galvanometer enlarged. The two columns sustain a compartment within which is the ring-magnet in connection above with a small mirror; the latter is suspended in a case (outlined in dotted lines) by the silk fibre, and accompanies all the movements of the magnet. (After Cyon.)

Fro. $116 \gamma$-I. Arrangement of apparatus for thermo-electric measurement ; $a$, $f$, thermo-electric needles, which are joined together on one side by the wire $b$, and on the other by the wire $b_{1} ; b$ is coiled about the ring-magnet $m$ having the north pole $n$. The magnet $m$ is suspended by a silk fibre $c$ and fastened to a mirror 8 . In front of the ring-magnet is a straight magnet $M$, having the north pole $N$, at such a distance that the ring-magnet can still point toward the north. An extremely slight current suffices to cause it to deviate from its position. In front of the galvanometer is a telescope $F$ with a scale $K K$, the image of which the observer $B$ can see in the mirror $s$ of the galvanometer; thus he pereeives every movement of the mirror or of the ring-magnet by the shifting of the image of the scale. II and III. Different forms of thermo-electric needles, $a$, German silver ; $t$, iron. (After Landois.)

definite time, the heat-production of the animal can be determined with approximate exactness. In recent times the watercalorimeter has been replaced by the air-calorimeter, in which the cage containing the animal is surrounded by a closed air-chamber; 
the air of the chamber is expanded by the heat given off by the animal, and from the amount of the expansion the quantity of heat produced may readily be computed. Partly by one method and partly by another, Dulong, Desprez, Helmholtz, Rosenthal, and Rubner, have determined the quantity of heat produced by the animal body. Since all such heat is derived from the chemical energy of the food introduced into the body, and since all the energy of the body, in case the latter performs no work, is

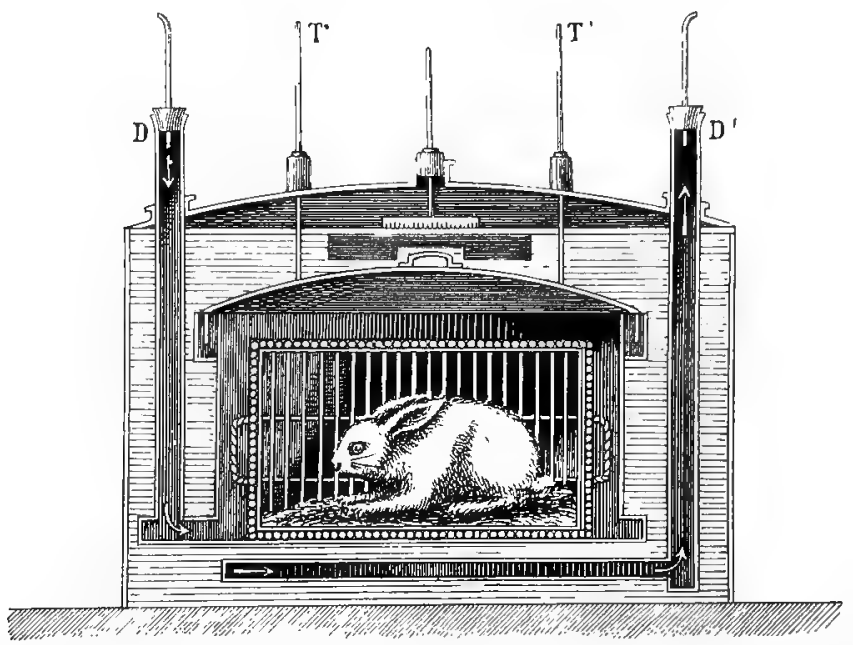

FIG. 117.-Dulong's water-calorimeter. A box with double walls; the wide space between the two walls contains water, through which a tube runs in spiral coils to the interior of the box for the admission of air from the outside at $\mathbf{D}$ to the animal, and for the removal of the used air through $\mathrm{D}^{\prime}$. At $\mathrm{T}$ and $\mathrm{T}^{\prime}$ are thermometers. (After Rosenthal.)

transformed finally into heat, the quantity of chemical energy that is introduced into the body with the food, expressed in calories, must according to the law of the conservation of energy be equal to the quantity of heat given off from the body to the outside. As a matter of fact, in the experiments this result has been attained with all desired exactness, and thus the validity of the law of the conservation of energy for the living body has been experimentally confirmed.

\section{The Production of Electricity}

As with heat, so thus far the production of electricity cannot be proved upon the single cell, because even our most delicate arparatus is too gross. Here also masses of cells are required. But the production of electricity can be perceived without special means of aid in far fewer cases than the production of heat, since all homo- 
thermal animals show the latter. The former may be observed without further aid only where it occurs in large proportions, i.e., only in the electric fishes, whose powerful shocks were known even to the ancients. The history of the science of animal electricity is associated closely with the discovery of galvanism and with the names of Galvani and Volta. It is certainly a noteworthy fact that the discovery of the physical fact of galvanism required for its starting-point physiological phenomena.

In September, 1786, Aloisio Galvani was making investigations upon the terrace of his house in the ancient university city of Bologna on the influence of atmospheric electricity upon a frog's leg from which the skin had been removed. Several years before he had carried on similar researches with the aid of his wife, Lucia, since early deceased. In the course of his experiments he stuck a copper hook through the frog's spinal column, which was still in connection with the nerves. When he laid this preparation upon the iron railing of the terrace he noticed to his astonishment that whenever the hook touched the railing, the frog's leg attached to it executed violent contractions. This simple observation is said to have been the starting-point of the discovery of contact electricity, the inconceivable range of which in relation to civilisation is only now appreciated. Alessandro Volta discovered the explanation of this phenomenon by establishing the fact that in the contact of two different metals with a moist conductor an electric tension arises, which is equalised in the form of an electric current as soon as the metals are joined with one another. In Galvani's experiment the nerves and muscles of the frog constituted such a moist conductor between the copper hook and the iron railing; the current went through the muscles and stimulated them so that they contracted. This correct interpretation of Volta was opposed by Galvani, who imagined that the twitch of the frog's leg might be caused by electricity originating within the leg itself; but this error is said to have led him fortunately to a new discovery. In labouring to prove to Volta that the contact of metals was not necessary for the production of the twitch, he endeavoured to bring out the twitch without metals; and he succeeded in this by placing the free end of a freshly prepared nerve of a frog's leg in contact with the flesh. In this experiment, as is now known, the nerve is stimulated by the electric current produced in the muscle itself; and so Galvani became the discoverer of animal electricity, as previously, although unwittingly, he had discovered contact electricity.

Pfaff, Humboldt, Ritter, Nobili, Matleucei and others laboured in the further development of the science of animal electricity, but it was reserved for the classic investigations of du BoisReymond ('48-'84) to place this field of physiology, which was then half-mystical and constituted one of the chief supports of the doctrine 
of vital force, upon a clear, exact foundation by creating for the first time sure and comprehensive methods of research. In the beginning, for evident reasons, only the muscles and nerves of the frog served as objects of experiment; but soon du Bois-Reymond brought into the range of his studies the interesting phenomena of the electrical fishes. And numerous inquirers, such as H. Munk, Hermann, Engelmann, Bernstein, and most recently Biedermann ('95), investigated the electrical phenomena of plants and various animal tissues. We are indebted to the researches of Hermann for the key to an understanding of the electrical
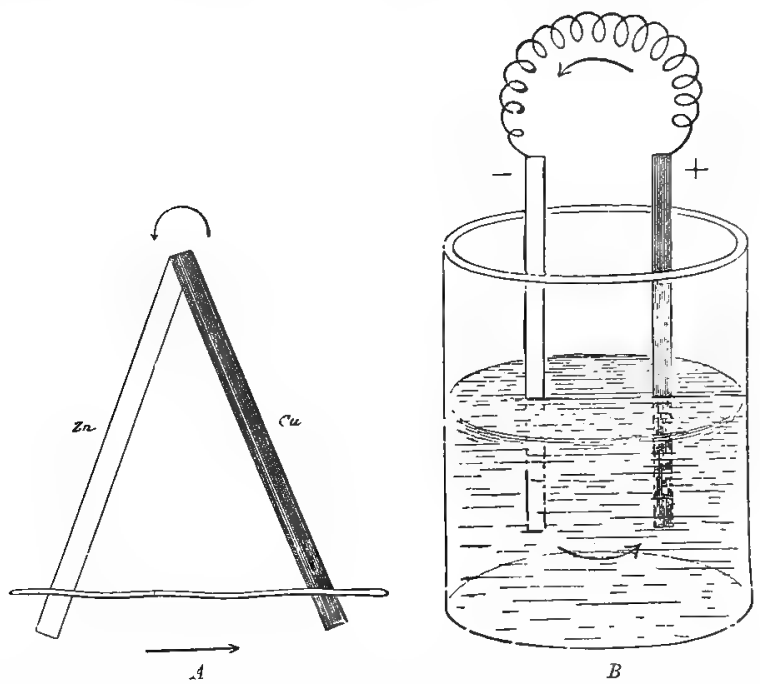

FIG. 118. $-A$, Simple arrangement for the production of a galvanic current. $Z$ n, Zinc ; $C_{2}$, copper; the two joined below by a moist thread. The arrows indicate the direction of the current. $B$, Simplest form of a galvanic element. Two metal strips (copper aud zinc) dip into a liquid and are joined together by a metal at their free ends. The current goes in the direction of the arrow.

phenomena of living substance. But it is due indisputably to the fundamental labours of du Bois-Reymond that the science of animal electricity has become one of the best-known branches of physiology.

The simplest method of obtaining a galvanic current, as is well known, is that of soldering together at one end two strips of different metals, e.g., copper and zinc, and bringing their free ends into contact with a moist conductor, e.g., a moist thread (Fig. 118, A). At the moment when the free ends of the metals are joined by the conductor, an electric current begins to flow in the closed circuit, passing from the zinc through the conductor to the copper and from the copper through the 
soldered place back to the zinc and circulating as long as the circuit is closed. This arrangement corresponds to Galvani's original experiment in which the nerve represented the moist conductor between the two metals, copper and iron. This principle for the production of a galvanic current has been employed in somewhat more perfect form in galvanic elements (Fig. 118, B), in which a liquid is employed as the moist conductor, while the two metals, the lower ends of which dip into the vessel containing the liquid, are in contact with one another at their upper ends by a copper wire in place of the soldering; this has the advantage of allowing the current to be conducted by means of the flexible wire wherever it is needed.

Following the views of Clausius upon the phenomena of electricity in liquids, Sohncke ('88) has presented a very clear idea of the origin of the galvanic current. According to Clausius ('57) the molecules in a liquid are in constant motion and constantly crowd upon one another, the result being that some split into their constituent atoms while other atoms unite into molecules. Hence simultaneously and at all times free atoms and whole molecules are present in the liquid. But while the closed molecule as a whole is electrically indifferent (e.g., water, $\mathrm{H}_{2} \mathrm{O}$ ), its various kinds of constituent atoms, when free, have different kinds of electricity (e.g., hydrogen, $\mathrm{H}$, positive, oxygen, $\mathrm{O}$, negative). Within the liquid the free atoms retain their charge of electricity. If they come in contact with atoms charged similarly they break away from them; if they meet those charged dissimilarly, they remain no longer free but unite with the latter chemically into a molecule which is electrically indifferent. But the situation is changed when there is introduced into the liquid a metal plate that exercises upon one kind of the free atoms a chemical attraction. These atoms then accumulate on the surface of the metal, which is non-electric and a conductor, and give off their electric tension to it by conduction.

If, therefore, into a vessel containing acidified water a zinc plate be dipped, free atoms of oxygen accumulate upon its surface and give off their negative electricity to it; in other words, it becomes negatively charged. If at the same time a copper plate be dipped into the liquid, atoms of hydrogen collect upon it and give to it their positive charge. There arises, therefore, an electric tension between the two metals; if now the free ends of the copper and the zinc plates be joined by a metallic conductor, this tension is able to equalise itself. During this process, however, new atoms become attracted to the place of contact of the metals with the liquid and become chemically united; thus the tension becomes continually re-established, and in this way a continual galvanic current is produced.

As is known from the researches of electro-chemistry, especially 
since the brilliant work of Arrhenius led to the great development of this science, in all chemical processes a disturbance of electric equilibrium takes place. In every chemical decomposition positively and negatively electric atoms or groups of atoms appear. If similar chemical processes take place at all points of a physical system and to the same extent, no current can be led off from it, for no tension exists between the leadingoff points, because both positive and negative groups of atoms arise in equal quantity (Fig. 119, l). But if in the system, such as a liquid mass, different kinds of chemical transformations, spatially separated, go on, so that there appear at one point a larger number of groups of atoms positively charged, and at another point a larger number negatively charged, an electric tension develops between these two points; and, so long as the processes continue, a galvanic current can be led off from the
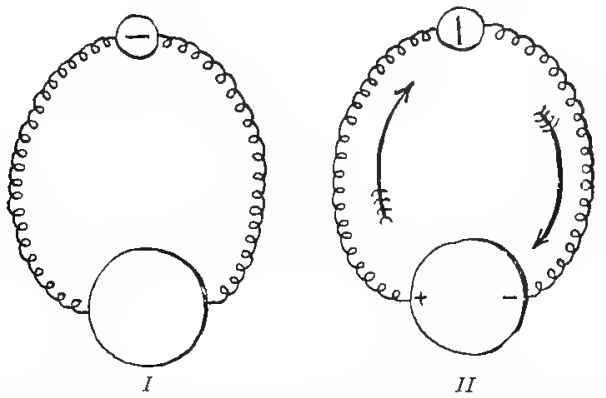

FIG. 119.-Schematic, I. A drop of liquid in which the chemical processes are alike at all points is without a current. $I I$. A drop of liquid in which at two different points chemical processes of different kinds occur gives a current. The large circle is the drop of liquid, the small one the multiplier with the raagnetic needle; the two are united by wires.

points to the outside (Fig. 119, II). The conditions under which a galvanic current can appear may be expressed, therefore, as follows: A current can be led off to the outside from a physical system when chemical processes take place in it that produce differences in the electric charge at the two leading-off points.

This proposition is valid for living as well as for lifeless substance. The living substance of a cell is a drop of liquid in which complex chemical transformations continually take place. If these be alike at all points of the cell, no current can be led off (Fig. 120,I); if, however, they be qualitatively or quantitatively different at two different poles, so that differences in the electric charge appear, a tension between the two poles results; and if these could be joined together by a conductor, a current would be obtained in the closed circuit. Naturally, this experiment cannot be performed upon a single cell on account of the 
minuteness of the latter, but the rule must hold good as well for the cell-complex, the tissue. As a matter of fact it may be demonstrated in the latter, and Herman's "differential theory" ('67-'68), according to which a current may be led off from a tissue (muscle, nerve, mucous membrane, etc.) only when different processes are taking place at the leading-off points, is merely the expression of the actual relations. In a resting uninjured muscle, e.g., the sartorius of the frog, which is the best object for demonstrating the truth of this, no current is present, because the same internal processes are taking place at every point (Fig. 121). If, however, at two points in the muscle a difference be produced artificially by warming one point, by cutting the muscle across, which is associated with a local decomposition of living substance, or by making a contraction-wave pass over the muscle, an electric current is obtained; the warmed, dying or contracting part becomes negative to all other parts. Tissues
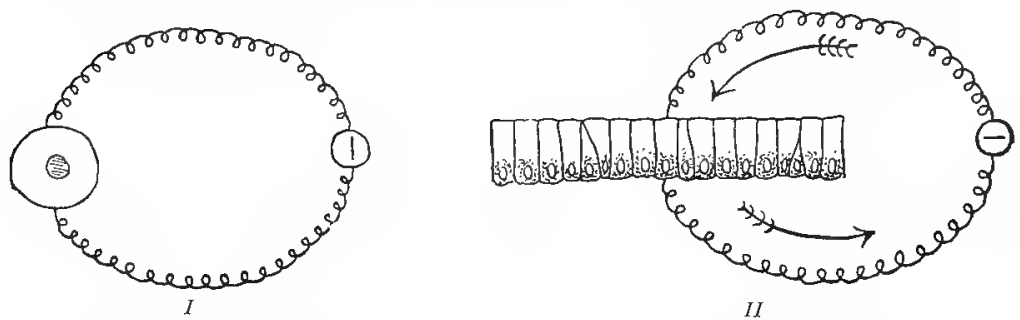

FIG. 120.-Schematic. I. A cell in which at all points of the living substance similar chemical processes are taking place is without a current. II. Polarised cells $\left(e_{0} g\right.$, , cells of mucous membrane) in which at one pole chemical processes are present that differ from those at the other give a current.

whose cells do not possess polar differentiation never show a current in the undisturbed condition, but relatively strong currents can be led off always from glands and mucous membranes, even when undisturbed; here the cells are polarised in such a. manner that the lower part of the cylindrical cell-body contains different substances and transformations of substances from the upper part (Fig. 120,II): The fact discovered by Mendelssohn is interesting, that an excised nerve, when led off from both crosssections, shows an axial current which runs in a direction contrary to the nerve-conduction, i.e., in motor nerves centripetally, in sensory nerves centrifugally.

All such currents may be demonstrated, like those arising thermometrically, by means of the multiplier or the galvanometer (Figs. 115 and 116, p. 259). But a special arrangement of the leading-off electrodes is necessary to avoid false results. If a current be allowed to pass for a time through a wire, the ends of which dip into a moist conductor, electrolytic decomposition- 
products of the moist conductor appear at the two ends of the wire, the electrodes, and accumulate there. The precipitation of these products at the two poles produces an electric tension that leads to a current, the so-called polarisation-current, flowing in a direction contrary to the original one. It is evident that the stronger the polarisation-current becomes, the more must the intensity of the original current be thereby diminished. If, therefore, a current be led off from a living tissue by means of metallic electrodes, after a short time a polarisation-current appears that completely obliterates the tissue-current. In order
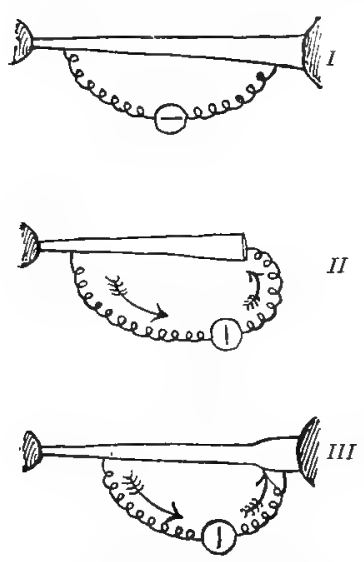

FIg. 121.-Bchematic. Sartorius muscle of the frog. At the two ends are the bony attachments. $I$. When urinjured and at rest, it is without a current. $I I$. When injured (cut across), it shows a current, the injured place being negative. III. When active (a contraction wave is passing from the right through the muscle), it shows a current; the active place is negative. to avoid this inconvenience, so-called non-polarisable electrodes have been constructed, which consist of non-metallic conductors. The most convenient of these non-polarisable electrodes are the brush electrodes suggested by Fleischl, which consist of a glass tube closed at one end by a stopper of plastic clay, and filled with a concentrated solution of sulphate of zinc. A short, soft, pointed camel's-hair brush is stuck into the stopper, and into the solution of zinc sulphate there is dipped an amalgamated zinc rod, to which the wire is fastened (Fig. 122). The brushes of two such electrodes, each of which is attached to a movable stand, are laid upon the living tissue. Experience has shown that in this manner the disturbing phenoinena of polarisation are avoided.

In the electrical phenomena of most animal- and all plant-tissues the currents are always so feeble that especially sensitive apparatus is necessary for their demonstration; but in the interesting electrical fishes there are currents of extraordinary strength, although the well-known tale of Alexander von Humboldt, that the South American electric eel is able to stun horses by its shocks must rest upon an error. In contrast to the currents of other tissnes, those of the electric fishes are characterised chiefly by their short duration and great intensity; they appear as brief, strong electric shocks, which can be given off by the animal several times in succession, either spontaneously or upon stimulation. This is comprehensible when it is considered that the production of electricity in these animals serves as a means of defence, which has become differentiated to this great efficiency during the evolution of the race. In accordance with this fact special 
organs are developed in the electric fishes for the production of electricity alone. It is most interesting that these electric
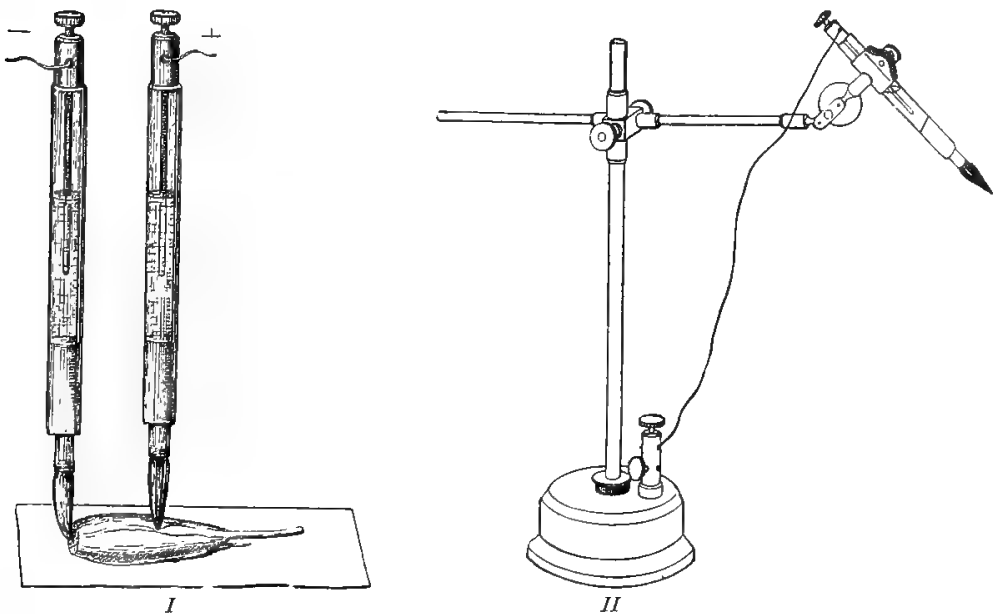

FIo. 122.-Non-polarisable electrodes. 1. Two non-polarisable electrodes laid upon an excised gastrocnemius muscle of the frog. II. A non-polarisable electrode in its stand.

organs have the same embryonic origin as cross-striated muscles, to which also in their adult state they possess great similarity.
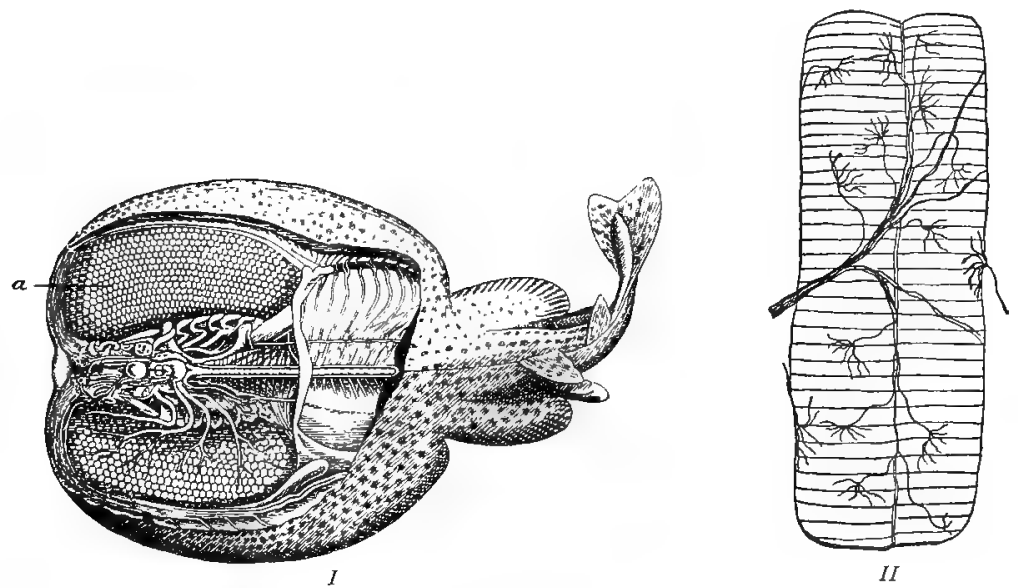

FIa. 123.-I. Torpedo marmoratus; the skin is partially cut away so that the electric organ, $a$, is visible; it consists of numerous polygonal columns, which are here seen in cross-section. (After Ranvier.) II. Two electric columns from the torpedo seen en face with the electric nerves branching over them. (After R. Wagner.)

The electric organ of the torpedo is composed of numerous long columns, hexagonal in cross-section, which correspond to 
muscle-fibres (Fig. 123). Each of these columns is composed of transverse discs lying symmetrically upon one another (Fig. 124, A); these are exactly homologous with the cross-striation of the musclefibre, but do not possess doubly refracting elements, and do not undergo changes of form during activity. Still greater is the correspondence in structure of the electric columns and crossstriated muscle in the half-electric or pseudo-electric fishes, e.g., Raja clavata (Fig. 124, B). A very interesting and obvious change
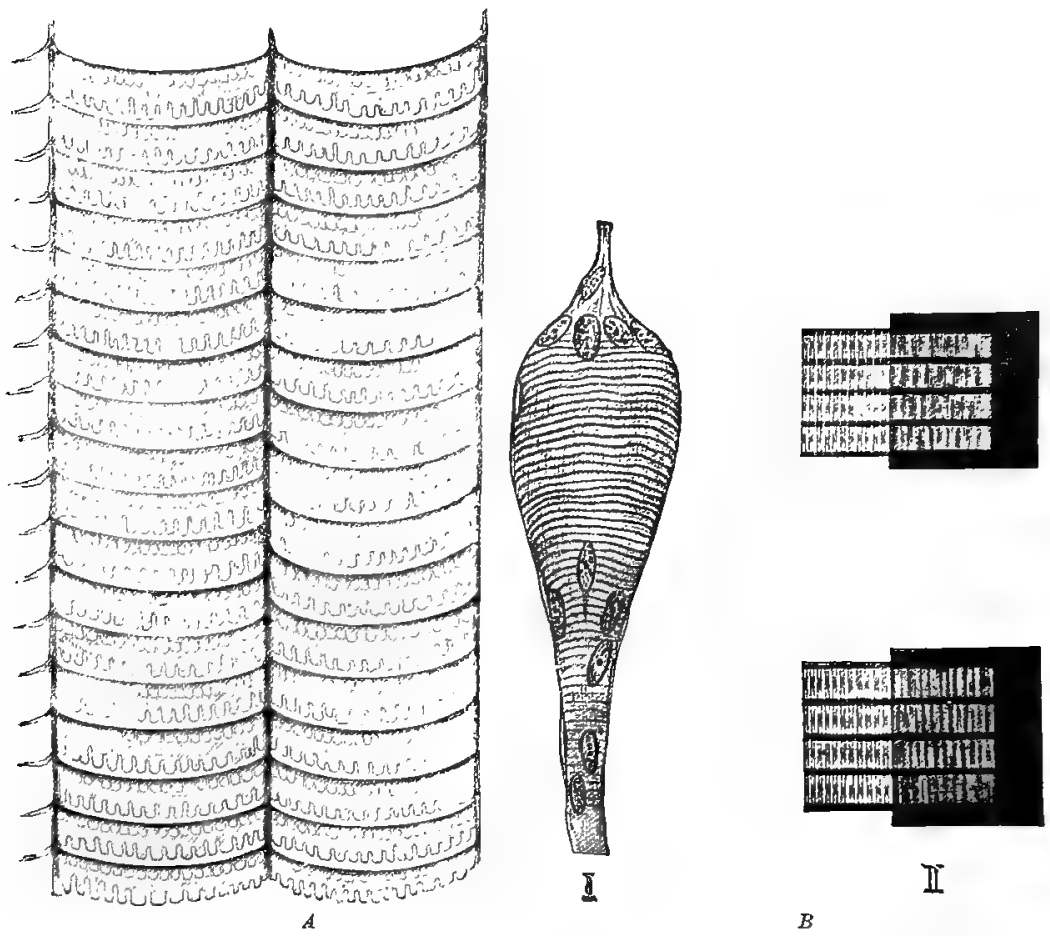

FIG. I24.-A. Two electric columns of Gymnotus electricus. (After Schultze.) B. I. Columans from the pseudo-electric organs of Raja clavata. II. $a$ and $b$. Single segments of I, more strongly magnified; the left half in ordinary light, the right half in polarised light. (After Engelmann.)

of function is here presented, for the electric organs develop out of genuine, contractile, cross-striated muscle-fibres ; and, as contractility is lost, the electric properties come into greater prominence. The similarity with the muscle is also evident during the activity of the completely-developed organ; for just as the muscle in a single twitch gives only a brief current, so in the electric organ the current is momentary, although of incomparably greater strength. 
The above picture of the transformation of energy in living: substance is as incomplete as was that of metabolism. As in the latter, so in the former, only the beginning- and the endcomponents of the series are known. Energy enters the living body as chemical energy, light and heat. Jight and heat are consumed in providing more chemical energy-light in splitting up in plants carbonic acid, which has in itself very slight value in respect to energy, into atoms of carbon and oxygen possessing free affinities, heat in causing a re-arrangement in the labile compounds of living substance by an increase in intensity of the intramolecular vibrations. Chemical energy is, therefore, the source of all other forms of energy in the organism; by its transformation there are derived mechanical energy, light, heat, and electricity. In the same proportion in which these are given out by the organism, chemical energy disappears. Hence the end-products of life, carbonic acid, water, ammonia, etc., possess extremely little chemical energy; into such the introduction of new energy from the outside, in both light and chemical substances, is necessary, in order to make available in the plant new potential energy in the form of free affinities. These are the beginning and the end of the series. But what in detail are the complex transformations in the living body, what forms of energy in each special case proceed directly from the introduced energy, what intermediate .and retrograde changes the chemical energy passes through, until it leaves the body again in the form of mechanical movement, light, heat and electricity - these are subjects that in great part are still obscure. More light may be expected here with the advance of knowledge regarding the more special metabolic processes, for the transformation of energy cannot be separated from metabolism.

For convenience, in this chapter, the phenomena of changes of :substance, of form, and of energy have been considered separately. In reality, these three groups cannot be separated from one another, for the possession of form and energy belongs to the essence of substance. Every change of substance is at the same time a change of form and energy. This is inherent in the nature of our conception of matter, and applies to living as well as lifeless matter. What has been treated separately under these three heads is one and the same event merely looked at from different points of view. In brief: All vital phenomena of a body are the expression of a continual change of the substance of which it consists. 


\section{CHAPTER IV}

\section{THE GENERAL CONDITIONS OF LIFE}

THE living substance of organisms forms a part of the mass of matter that composes the earth. As has been seen, the differences between it and lifeless substances are not fundamental in nature, for the elements that constitute the former constitute also the latter. The differences between organic and inorganic substance are no greater than the differences between many inorganic substances, and consist merely in the mode of union of the elements. It is important to familiarise ourselves with the thought of living substance, not as something mystical, which has no connection with and stands in contrast to all other substance, but as a part of the matter that constitutes the earth's crust. It is evident that life is conditioned wholly by the character of the environment, that. the evolution of living substance must be inseparably connected with the evolution of the earth. Accordingly, the composition and the form of the living substance that now covers the earth's surface are to be considered from exactly the same point of view as, for example, the composition of the present sea, i.e., as something that has gradually become, and exists in its present state only because the conditions are such as they are at the moment. Just as the sea with its salt could not have existed as it does now before the water had appeared upon the earth in a liquid state, so also living substance could not then exist with its present composition, for it contains upon an average more than fifty per cent. of water. But just as water was obliged to assume its present form when certain conditions in the earth's evolution were fulfilled, so living substance vos obliged gradually to take on its present character to the extent to which the present conditions of the earth's surface were perfected. The separation of living substance out of the mixture of materials of the earth's crust is only one result of the earth's evolution, like the separation of rocks, salts, or water.

The same idea is arrived at from another starting-point, when, not the elementary composition, but the vital phenomena of living substance are considered. It is an error easily conceived and due 
to superficial impressions to consider the organism as a closed system, independent of its environment. The fact of metabolism shows this at once; for, if the organism lives only so long as it takes in matter from the outside and gives off matter to the outside, it stands in the closest dependence upon the external world; the latter conditions its life.

Thus arises the conception of conditions of life, i.e., conditions that must be fulfilled in order that the life of the organism can exist. It is evident that every change of such conditions must exercise an influence upon the life of the organism. Hence, in order to complete a picture of the mutual relations of the organic world and its conditions, it is necessary not only to investigate the latter as they are now, but, so far as possible, as they were in the earlier periods of the earth's evolution. A few fixed points may thus be obtained for the consideration of the question of the origin, the descent and the evolution of life upon the earth.

\section{The Present Conditions of Life upon the Earth's SURFACE}

All the conditions of life are not equally necessary for all organisms living at the present time. What is absolutely necessary for the existence of one organism may even endanger the life of another. Marine animals when brought into fresh water soon die, and fresh-water animals placed in sea-water experience the same fate. This principle holds good not only for large groups of organisms but for every individual form as well. Every individual organism requires for its existence definite special conditions, without the fulfilment of which it cannot continue to live. These special conditions of life are as manifold as the innumerable forms of organisms themselves. To describe them is to describe the natural history of every organism, and their investigation belongs to the field of special plysiology. But in contrast to them there are other requirements that must be fulfilled for all organisms if the latter are to live, and these must, therefore, be termed general conditions of life. General physiology deals with the latter. In the following pages we shall be able to glance at the special conditions only momentarily, when they are of particular interest and present peculiar adaptations of living substance to peculiar circumstances.

It is usual to consider under conditions of life only external factors, such as food, water, oxygen, temperature, etc. But in contrast to these crternal conditions there are internal conditions, which are inherent in the composition of the organism, and the absence of which, like that of the external factors, is followed by death. 


\section{A. THE GENERAL EXTERNAL CONDITIONS OF LIFE}

\section{Food}

The presence of food is required by the fact of metabolism. If living substance is continually undergoing spontaneous destruction, then, in order that it may continue to live, a stream of substances must come into it from the outside, which comprise all those chemical elements that are necessary to its construction. Such chemical substances constitute food. Accordingly, water and oxygen belong to the general conception of food; it is not customary, however, to include them therein. Following the usage, we shall consider them separately, and shall take up, first, food in the more special sense.

The twelve organic elements of which all living substance is composed (p. 100) must come into the body of the organism in some form as food. In this lies the general significance of food. But the chemical compounds in which these elements are introduced into the body are as manifold for the various forms of organisms as the organisms themselves. A general food for all organisms does not exist; and it has already been seen ${ }^{1}$ that according to the kind of food-stuffs and the manner in which living substance is constructed from them, organisms may be divided into several large groups, such as green plants, fungi and animals. While the green plants are able to construct their living substance out of inorganic material only, carbonic acid and solutions of various salts, animals without exception require organic food, and cannot live without complex organic compounds, such as proteid, carbohydrate, fat, etc. The fungi stand in a certain measure between these two groups, since they can supply their need of nitrogen from inorganic salts, although they require organic compounds for their carbon. An exception to this condition is shown by the interesting nitrogen-bacteria only, which derive both their nitrogen and carbon from anmonium carbonate, and thus like the green plants live exclusively upon inorganic food-stuffs. But, however in individual cases food may be procured, without food of some kind no living body can continue to live.

Regarding quantitative conditions of food, the maximum and the minimum of food that the living body requires, which is different for every form of organism, only a few special cases have been investigated thus far, and these are among the higher vertebrates exclusively. These are questions that still require detailed answer, and, if treated from the cell-physiological standpint, are capable of yielding results equally important theoretically and practically. Thus far individual values for the whole

$$
{ }^{1} \text { Cf. p. } 138 .
$$


organism have been obtained in the case of men only. Voit ('81) has shown that an adult man performing active work can subsist upon a daily food comprising 118 grs. of proteid, 56 grs. of fat and 500 grs. of carbohydrate. With such a diet the man is in metcubolic equilibrium, i.e., the quantities of elements excreted in the urine, the sweat, the expired air, and the fæces are equal to those that are introduced with the food. But, more specifically, these values for the individual elements, such as nitrogen, carbon, etc., introduced with the food must be determined separately, since the body when, e.g., in carbon equilibrium is not necessarily always in nitrogen equilibrium. It is thus found that nitrogen equilibrium can be obtained with a quantity of proteid of only 50 grs. (which corresponds to 7.5 grs. of nitrogen), provided only that the quantity of the non-nitrogenous food-stuffs, carbohydrates and fats, is correspondingly increased. $7 \cdot 5$ grs., therefore, would correspond to the daily minimum of nitrogen with which a man can continue to exist.

The minimum of food necessary to the maintenance of metabolic equilibrium and life is of great importance. If the income of food rises above the minimum, metabolic equilibrium is disturbed only in a very slight degree, slightly smaller quantities of elements appearing in the excreta than are taken in with the food. These very small quantities remain in the bady and serve for the increase of living substance and the storing up of reservesubstances, a phenomenon that in husbandry is termed fattening. But this depends upon many factors, which are as yet known exactly only in part. If, on the other hand, the quantity of food falls below the minimum or becomes zero, the condition of hunger or inanition appears, in which the metabolic equilibrium becomes more and more disturbed. This condition has been investigated more fully.

It is worth while to follow somewhat fully the changes experienced by the living organism in the condition of inanition. Every living cell under normal conditions possesses within itself in greater or less quantity substances at whose expense the vital process continues for a time if the food-supply be cut off. These are its reserve-substances. It is a general fact that during inanition the reserve-substances disappear first. Plant-cells that are filled with starch grains consume these when they are brought into the dark, i.e., when they are forced to hunger, for in the dark no assimilation of starch from carbonic acid and water, in other words, no nutrition, takes place. Infusoria, whose cell-bodies in their infusions, where they revel in a superfluity of food, contain all sorts of particles, and hence appear opaque and granular, become clear, transparent and free from granules, when placed in water containing little food-stuff; their cell-bodies become gradually smaller (Fig. 125). The cell, therefore, does not die immediately 
at the moment of the withdrawal of food, but lives for some time at the expense of the materials of its own cell-body. If these be consumed, it gradually perishes, just as a clock that is not wound up gradually runs down and then stops. The phenomena of inanition have been studied most carefully in compound multicellular organisms, especially vertebrates, and an important task in this field is left for cell-physiological investigation.

Since it is a characteristic peculiarity of living substance that it is continually undergoing spontaneous decomposition, it is clear that in fasting animals metabolic equilibrium must be disturbed. In the decomposition-products of living substance, nitrogen, carbon, hydrogen, oxygen, etc., are continually being excreted, while there is no new incorne. The result is that, as in the individual cell so in the multiceliular organism, the living substance is gradually consumed and the
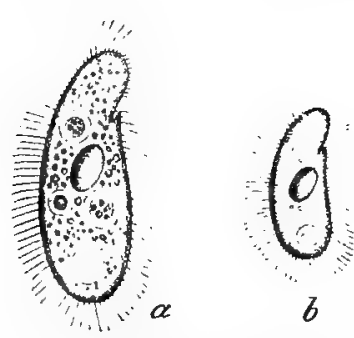

FIa. 125.-colpulum colpoda, a ciliate-infusorian cell. $a$, In the normal condition; $b$, in the condition of inanition. The cellbody has become smaller and more transparent, and the granules in the interior have disappeared. Magnification in botl cases 260. (After observations and drawings by Jensen.) organism decreases in weight. The animal lives for some time upon its own tissues. It is, therefore, conceivable that as regards their excreta fasting herbivora are like carnivora. The urine of herbivora, which during normal nutrition is alkaline and turbid, becomes during inanition acid and clear like that of carnivora; for during inanition herbivora live upon their own, that is, upon animal tissue, and hence in a certain degree become carnivorous. The living substance gradually consumes itself, until the body-weight has undergone so great a loss that the animal dies. By many experiments Chossat ('43) established this limit of decrease of weight, and found

that with widely different animals death appears when the loss of weight has reached approximately 0.4 of the whole body-weight. This limit is reached by different animals at very different times. Frogs live longer than a year, and Proteus anguineus, a peculiar amphibian of the Adelsberg grotto, lives several years without food. Man dies in a relatively short time. In earlier times opportunities for investigating human beings who were fasting for a long time were rare, and the early results are to be accepted with caution. Thus, in the year 1831 in Toulouse a convict, who would take only water, is said to have died only after sixty-three days. In later times, with the appearance of the Irofessional faster physiologists have had more frequent opportunity. for making exact investigations on fasting men. Luciani ('90) has produced a striking monograph upon fasting, based upon investigations of the well-known siucci, who undertook a thirty 
days' fast under his charge. By this case it is proved beyond doubt that under favourable conditions a normal man can exist at least thirty days without food.

The different tissues partake in the loss of weight of the body in very different degrees. While the cells of many tissues become affected greatly and very rapidly, those of others experience only slight changes. This is shown by the following experiment of Chossat. Two pigeons of the same brood, and of like size, sex, and weight, are employed. One is killed at once, and its individual tissues are weighed. The other is allowed to fast until it dies, and then its tissues are likewise weighed. In this manner whatever changes of weight that the individual tissues have experienced during inanition are determined. It is thus found that fat-tissue has lost approximately 93 per cent. of its weight, the tissue of the spleen, the pancreas and the liver $71-62$ per cent, that of the muscles $45-34$ per cent, that of the skin, the kidneys and the lungs $33-22$ per cent., that of the bones 17 per cent., and that of the nervous system only about 2 per cent. Fat-tissue is, therefore, the most affected, the nervous system the least. Of course this difference in the increase in weight of the individual kinds of tissues or cells is not to be regarded as depending solely upon a different rate of decrease on the part of each kind of cell by the cessation of the income of food-stuffs. Luciani, rather, holds rightly the view that another factor in addition plays a rôle, viz., that among the different tissue-cells a contest over the food takes place, such that some cells seize upon the reserve-substances present in the body more greedily than others, and, after their consumption, appropriate also the material of the other cells in order to maintain their metabolism. This is indicated at least by an interesting observation of Miescher-Rusch ('80). When salmon migrate from the sea up the Rhine they are strong, muscular animals in good nutritive condition. During their stay of six to nine months in the river they fast. Their muscles, especially those of the back, decrease enormously in volume, while the sexual organs develop extraordinarily. Here, therefore, a struggle for existence between the tissue-elements of the sexual organs and those of the muscles takes place, in which the former prove superior and appropriate the substance of the latter for their own needs. Likewise between other tissue-elements in other animals in the condition of inanition, a struggle for existence takes place, although not in so remarkable a manner as in the salmon. The final result of all fasting is always death. The clock finally runs down if it is not wound up.

The assertion that death is the ultimate outcome of fasting requires a certain correction. It is true of organisms only so long as they continue in the condition of actual life. Organisms in the state of latent life, such as dried Rotifere, Tardigrada, spores of 
bacteria, and seed-grains, require no food; for, as has been seen, ${ }^{1}$ no metabolism can be found in them even with the most delicate means of investigation. Hence, when food is wanting in their environment, they do not die. Here the clock has merely stopped, it has not run down.

In order, finally, to obtain an idea of the far-reaching adaptations of individual organisms to special vital conditions of a very unusual kind, so far as they have to do with food, it is necessary only to glance at the peculiar vital

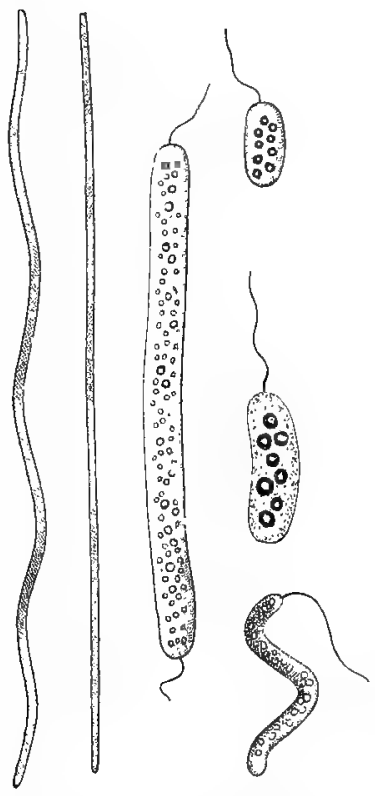

TIG. 126. - Various forms of sulphurbacteria. The granules in the in terior are particles of sulphur. (After Schenk and Warming.) relations of certain forms of Bacteria, which have become known recently, especially through the striking work of Winogradsky ('88).

The sulphur-bacteria (Beggiatoa) constitute a family of microbes that live in decaying pools and puddles of both fresh and salt water. These remarkable beings, which swarm about in the water in the form of short rods or long threads (Fig. 126), can exist only when considerable quantities of sulphuretted hydrogen are available. Their metabolism requires this gas, since they manufacture from it, by oxidation, free sulphur, which they store up in their tiny cell-bodies in the form of fine, strongly refracting granules (Fig. 126); by continued oxidation they transform the sulphur further into sulphuric acid, and in this form excrete it to the outside. If the sulphur-bacteria be brought into spring-water that contains no sulphuretted hydrogen, they perish after they have oxidised and excreted the sulphur present in their bodies.

Sulphuretted hydrogen, a gas that is poisonous to most organisms, belongs, therefore, among their essential conditions of life. Without it they cannot continue to exist.

Winogradsky ('88) has pointed ont a similar special adaptation to peculiar vital conditions in the iron-bactcria. Bog.ironore moors are very generally known, occurring wide-spread in marshy regions, with an vily, iridesent scum upon the surface of their water and thick, reddish-yellow mud below. These are the abode of the iron-bacteria, and the production of bog-ore is in 1 Cf. p. 132. 
part their life-work. They require for their metabolism ferrous carbonate, which is dissolved in the water. They absorb this and oxidise it into ferric carbonate, which they give off to the outside. The excreted ferric carbonate in time passes over into simple ferric oxide, which is insoluble, and forms a yellowishbrown precipitate upon the gelatinous covering excreted by the bacteria, in which their bodies lie. If the iron-bacteria be cultivated without ferrous carbonate, their vital phenomena become gradually feebler and finally come to a complete standstill. Hence the presence of this substance belongs among the conditions of life of these remarkable microbes.

These examples suffice to show how peculiar may be the special conditions of life among different organisms as regards food. This is not the place for their further consideration; they belong to the province of special physiology.

\section{Water}

Living substance is liquid. It is necessary to remember this fundamental physical property. The liquid jelly-like condition of living substance is due to the water that it contains, which fact can be proved easily by evaporating the water. Only liquid, not solid masses, only substances that contain water can be living, for only with the liquid state is metabolism compatible. Hence in the organism all substances that are solid and hard, such as the connective tissues of the teeth and the bones, are not living. Similarly, vital activity is lessened along with the withdrawal of water. In dried Rotifera and Tardigrada, and in dried seeds, no vital phenomena can be perceived. Life begins to manifest itself only when the seeds are made to swell by the addition of water, only when the substance of their cells becomes again liquid. Water, therefore, belongs to the general conditions of life. This conclusion is very simple and clear. But there are cases where, even in places of the greatest drought, organic life continually exists. In spite of their dryness the waste, burning deserts of Arabia and Africa, which present to the traveller most powerfully impressive pictures of eternal lifelessness, and whose sands are moistened scarcely once in a year by showers of rain, harbour manifold varieties of animals and plants. This apparent exception depends upon the fact that all desert-organisms are peculiarly adapted to life in long drought, and they manage extremely frugally and economically with the little water that comes to hand at long intervals of time. One is astonished in the driest desert to come upon green plants that contain abundant juices, plants (Mescmbryanthemum crystallinum) beset over and over with cells, which harbour such quantitics of clear water that the latter 
appear like small crystal droplets (Fig. 127). These desert-plants have a remarkable power of retaining water, either by storing up in their cell-sap soluble substances that possess great attraction for water, or by being covered over their whole surface with a fine layer of wax, so that with the stomata closed scarcely a trace of water can pass by evaporation out of the plant-body. Moreover, they possess usually much-branched roots extending very far and superficially in the soil, and these greedily suck up every trace of water that moistens the earth. The desert-animals also, such as the snails,

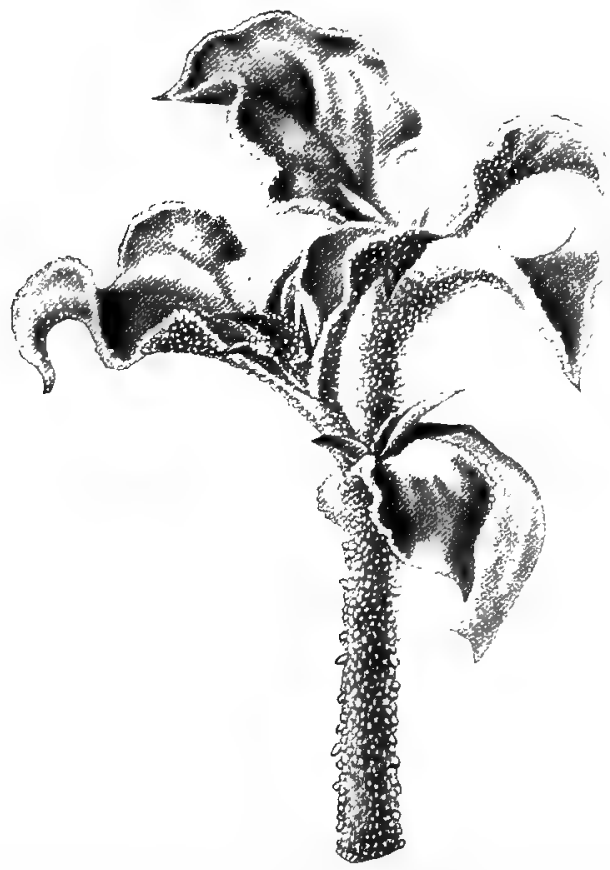

FIG. 127.-Mesembryanthemum crystallinum, a desert-plant from Southern Africa. The whole stem and the under side of the leaves are beset with clear crystal-like water-cells.

which are confined to their dry home because of their slight powers of locomotion, protect themselves by limiting their excretion of water to a minimum. The snails close the opening of their shells with a thick, double cover, so that scarcely a trace of water can be lost from the body by evaporation. Hence, in all these cases the lryness of the environment does not extend to the living substance of the organisms. On the contrary, here, as everywhere, the living substance is liquid, and in fact all desert-organisms have an actual, not a latent, life, although their life is depressed to a minimum. They show directly how the intensity of life increases 
and decreases with the rise and fall of the water-contents. If a slight shower of rain comes, activity immediately begins, the plants grow and bloom, and the sluggish animals awake from their summer sleep.

In a manner somewhat different from that of the desert-plants and animals, other organisins which at times are obliged to undergo a lack of water are adapted to life in drought, since at such times they assume a quiescent phase and are protected against drying. Such quiescent phases occur especially among unicellular organisms, as in the spores of Bacteria (Fig. 128) or the cysts of Rhizopoda and Infusorice (Fig. 84, p. 205), which enclose the living cell-substance in a thick, completely impervious skin.

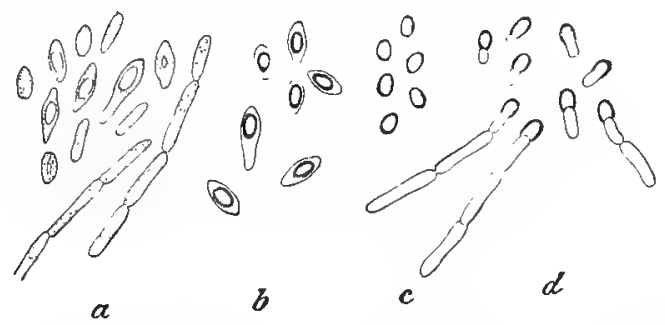

Fia. 128.-Bacillus butyricus, forming spores. $a$, Beginning of the process: $b$, ripe wwres still within the bacilli; $c$, spores after the dissolution of the membrane of the mother-cells; $d$, spores beginning to germinate and to allow the bacilli to come forth. (After Migula.)

The seeds of plants likewise belong to these permanent conditions of organisms. But in all these cases life is latent; no trace of vital phenomena can be demonstrated in them by means of the most delicate methods. It would appear that in all such cases life stands still, like a wound-up clock that has been suddenly stopped.

From these facts the importance of water for the maintenance of life is evident. Without water life cannot exist. With the increase and decrease of the water-contents of living substance within certain limits the intensity of life rises, falls, and becomes zero.

\section{Oxygen}

It was Priestley, the discoverer of oxygen, who recognised the fundamental importance of this gas for life upon the earth; by his epoch-making discovery of the gas and its properties he gave a real background to Mayow's ingenious comparison of respiration with combustion. In respiration free oxygen is taken up by the living substance, and in return carbonic acid is given off; hence a combustion, an oxidation of carbon, must take place in the 
living substance. If, therefore, as has been seen, ${ }^{1}$ all organisms without exception respire as long as they live, $i . e$, if the processes of oxidation are an integral link in the chain of metabolic processes, it necessarily follows that the presence of oxygen belongs to the general vital conditions of living substance.

As is well known, the composition of the atmosphere, as regards its essential constituents, is as follows: Nitrogen and argon, 79.02, oxygen 20.95 , carbonic acid 0.03 volumes. This composition is essentially the same at all times and all places upon the earth's surface. If, therefore, land organisms be considered-and upon them have been made the greater number of the investigations regarding the dependence of living things upon oxygen-it may be said that they live continually in an atmosphere in which in round numbers 21 per cent. of oxygen is present. The striking investigations of W. Miuller and Paul Bert have, however, shown that organisms are not bound exclusively to this percentage and the pressure of one atmosphere, but within certain limits are independent of the partial pressure of oxygen. W. Müller ('58) found, for example, that mammals can continue to exist with 14 per cent. of oxygen, they begin to be disturbed at 7 per cent., while at 3 per cent. death by asphyxia takes place; on the other hand, they thrive in pure oxygen at a pressure of one atmosphere. In like manner a series of experiments published by Paul Bert ('73) shows in animals a far-reaching independence of the partial pressure of oxygen. In atmospheric air animals can still exist with a minimal pressure of about $250 \mathrm{~mm}$. mercury and with a maximal pressure of fifteen atmospheres; in pure oxygen the minimum of pressure is consiclerably lower, but a pressure of two atmospheres for plants and of three atmospheres for animals is fatal. In general, it follows from the experiments of Paul Bert that the effects of a too small percentage of oxygen can within certain limits be compensated for by a rise of pressure, and the effects of a too high pressure by a fall of the percentage. The remarkable fact that organisms in pure oxygen with too high partial pressure die, and, as Paul Bert has shown, die of asphyxia, has been made clear by Pfliger ('75, 1), by means of an analogy between living substance and active phosphorus. As is well known, in atmospheric air phosphorus becomes oxidised actively, gives out light, and evolves fumes of phosphorous acid, while in pure oxygen it is not oxidised at all. So living substance in pure oxygen with a high pressure ceases to oxidise, and hence appears the paradoxical phenomenon of death by asphyxia in pure oxygen.

The minima and maxima of the percentage and the partial pressure of oxygen are very different for different organisms, and thus far are known only in a few cases. These details are of little 
interest here. It is, however, interesting to glance at the results of complete removal of uxygen.

The final results of complete removal of oxygen are evident. If oxygen be a general condition of life, all living substance must perish after its complete withdrawal. This has been shown by experiments that have been performed partly upon single cells, partly upon tissues, and partly upon multicellular organisms. But different kinds of cells perish after different intervals of time, some very rapidly, some gradually, just as do different organisms upon withdrawal of food. The cells of the nervous system are
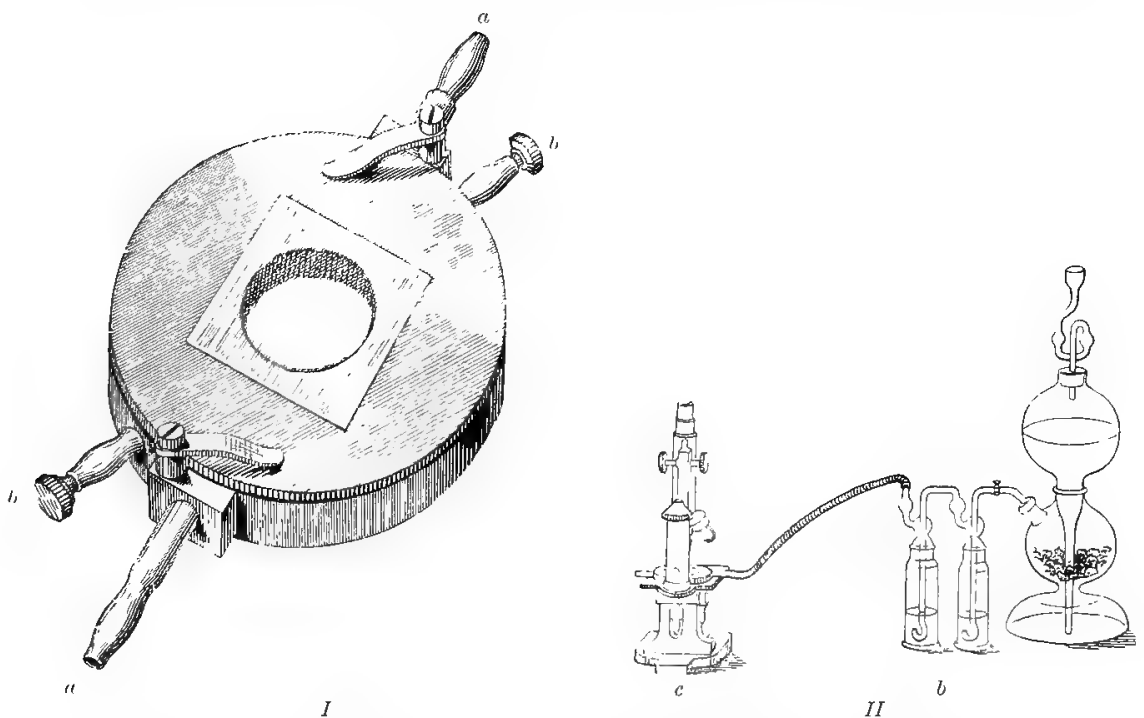

Fig. 129.-I. Engelmann's gas-chamber. An annular space is closed below by a glass plate and above by a metal cover, the latter having in its middle a cover-glass for the examination of a hanging drop; $a, a^{\prime}$, are tubes that open into the cavity of the ring and serve to heat the latter by conveying through it warm water; $l, b^{\prime}$ are tubes that open into the glass-covered chamber and serve for the passage of the gas ; the drop hanging upon the cover-glass with its living contents is bathed by the gas in the chamber. II. Arrangement of the experinent for investigation in pure hydrogen. $a$, Kipp's apparatus for the preparation of hydrugen; $b$, two wash-bottles for purifying the hydrogen; $c$, microscope, upon which is the gas-chamber con-" taining the hanging drop.

the most sensitive to absence of oxygen. Hence without oxygen the higher vertebrates, in which the movements of respiration, the activity of the heart, etc., are dependent upon the cells of the nerve-centres, perish very soon with violent phenomena of stimulation. Other kinds of cells, however, continue to live for a considerable time even in a medium wholly free from oxygen.

By the use of hydrogen, a gas absolutely indifferent to the organism, oxygen may be readily and completely excluded without introducing into the experiment other harmful factors. Since in a closed space atmospheric air, in which oxygen is the sole 
effective constituent, at least for animal-cells, can be very easily removed and replaced by hydrogen, it is only necessary to prepare chemically pure hydrogen by means of Kipp's apparatus and conduct it through a closed gas-chamber. The most convenient gaschamber fur microscopical investigations is that devised by Engelmann (Fig. 129, I). The cells to be investigated are placed in such a chamber and observed in a hanging drop of the liquid in which they live. By a series of experiments' Kiihne ('64) has shown that after replacing the air by hydrogen Amolse gradually suspends its movements after about 24 minutes. From this condition it can be brought back to life by a renewal of atmospheric air. But, if it remains for some time longer in the absence of oxygen, it dies. The movements of large plasmodia of Myxomycetes in a medium free from oxygen often cease only after three hours, and later the plasmodia die.

For the study of the question how the two phases of contractionmovements, namely, expansion and contraction, are influenced by the withdrawal of oxygen, the most favourable objects are marine Rhizopoda, possessing long pseudopodia, over which the movement of each particle of protoplasm is extended for a very considerable distance. Such a one is Rhizoplasma Kaiseri, a naked rhizopod possessing a nuninucleated, orange-red cell-body, from which radiate out in all directions fine, anastomosing pseudopodia, in which the protoplasmic streaming is uncommonly active (Fig. 130, I). If a Rhizoplasma ${ }^{1}$ be placed in the Engelmann gas-chamber and a current of oxygen be passed through, after one and a half to three hours the effects of the withdrawal of oxygen become noticeable. The centrifugal current in the protoplasm, which before was very active, so that the pseudopodia were extended, becomes feebler and feebler and finally ceases. But the centripetal current continues for a while longer, so that the pseudopodia slowly shorten; gradually, however, the centripetal current also diminishes and soon is scarcely noticeable. The protoplasm has accumulated, at the places where the pseudopodia branch, into tiny masses, which are not spherical and spindle-shaped, as when contracted because of strong stimulation, but are more pointed, angular and toothed. In this form the Rhizoplasma is finally completely motionless (Fig. $130, I I)$. Specimens possessing shorter pseudopodia finally draw them completely in. Hence, by the withdrawal of oxygen, the phase of expansion (the centrifugal protoplasmic strearning) first comes to a standstill, and then gradually the phase of contraction (the centripetal protoplasmic streaming). If now atmospheric air be introcluced, after about five minutes tips of new pseudopodia begin to project from the central cell-body. After about ten minutes, active streaming is again apparent upon the old psendumalia. A new current from the centre appears upon them, 


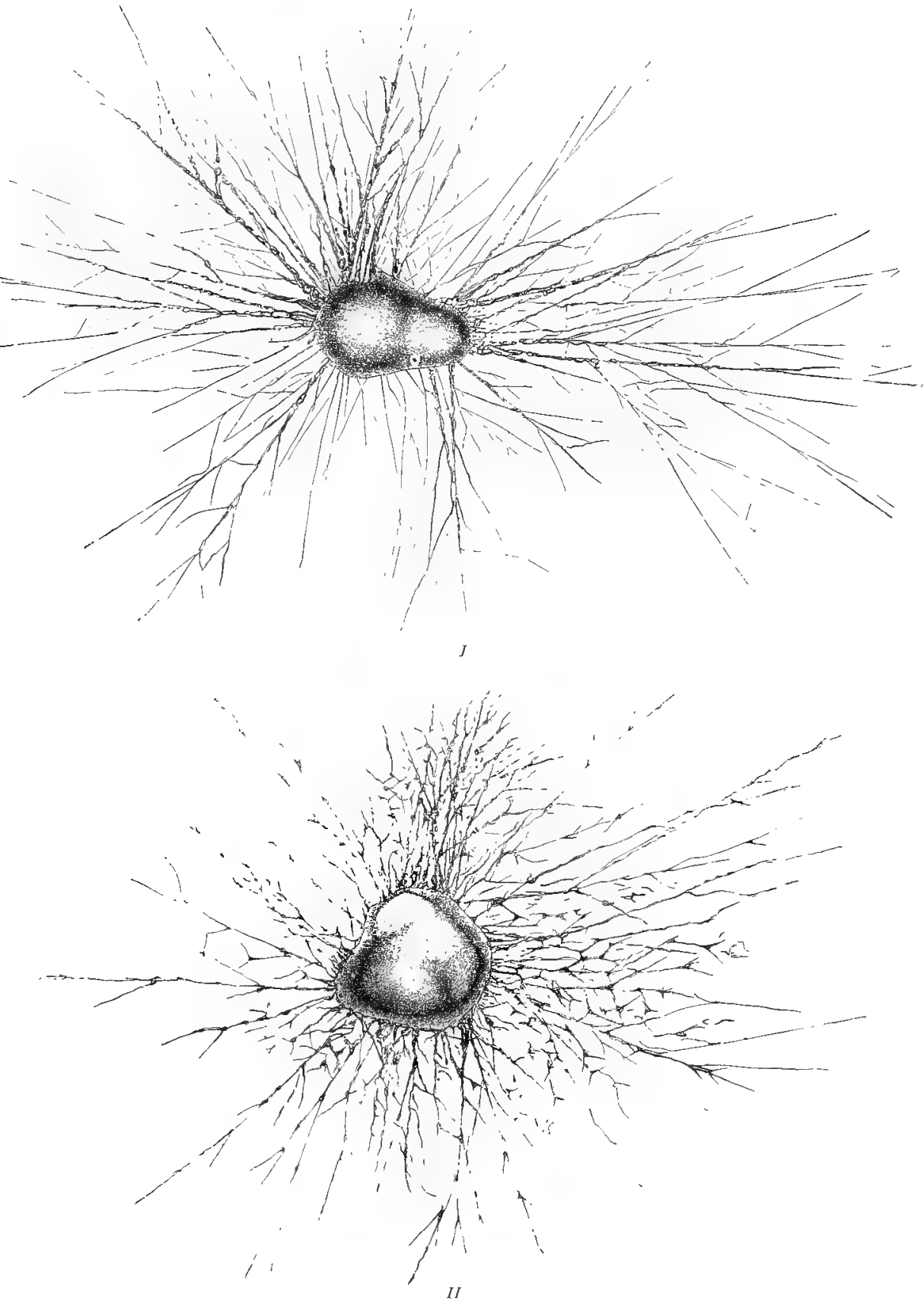

FIG. 130.-Rhizoplasuc Fuiseri: I. Normal individual, with extended pseudopodia and active protoplasmic streaning. II. Standstill of the protoplasmic movement after the withdrawal of oxygen ; the protoplasm forms small, angular accumulations at the places where the pseudopodia branch. 
and the small accumulations of protoplasm break up, their substance flowing partly centrifugally, partly centripetally. In this manner the pseudopodia again become smooth, their streaming becomes more active, and after a half-hour the same appearance is present as at the beginning of the experiment.

Engelman was able also to determine that ciliated cells are capable of maintaining life for several hours without oxygen. Hermann ('67-'68) has shown the same for muscle by placing one of two exactly similar gastrocnemius muscles of the frog in a cylinder containing pure hydrogen, the other in a cylinder filled with air containing oxygen, and testing their irritability by means of electric stimuli, which both muscles received at the same time. The muscle in pure hydrogen lived several hours before becoming inexcitable, while the muscle in oxygen continued to live unchanged. From all these experiments it follows that certain cells and tissues can maintain life for a considerable time in a medium free of oxygen.

This fact, especially in regard to muscle, has been variously employed as the basis of an unjustified conclusion. Since Hermann has shown that no free oxygen can be extracted by means of the gas-pump from an excised bloodless muscle, the inference has been drawn that muscle, while able to perform movements for a long time without external oxygen, works solely by means of cleavage-processes. This conclusion is unjustified, since, from the fact that no free oxygen can be pumped out of a muscle, it ought not to be inferred that no oxygen whatever capable of being used for oxidation is longer present in the muscle. On the contrary, it is very probable that in the muscle, perhaps in the sarcoplasm, there exists in combination oxygen that during activity is continually being consumed by the contractile particles for their oxidisation. As a matter of fact, hæmoglobin has been found in the muscles of some invertebrates that possess in their blood no hæmoglobin whatever. It must hence be supposed that in cells that continue to live for a long time in the absence of oxygen, oxidation-processes still take place, ccrtain complexes of atoms of the living substance withdrawing the oxygen for their own oxidation from others that contain it in loose combination, until finally all the oxygen is consumed and combined into the cleavage-products. However this may be, in the absence of oxygen all living organisms perish after a shorter or longer time. Without oxygen no life can exist permanently.

There are some apparent exceptions to this principle; there are organisms that apparently can continue to live without oxygen.

At first sight the green plants appear to form such an exception, and at one time they were really believed to do so. In one respect 
these plants are the exact reverse of animals: they take up carbonic acid and give off oxygen. So long as the sunlight acts upon their green leaves, they need no oxygen. A green plant, therefore, can be kept alive in a space free from oxygen, if it be allowed to stand in the light and receive carbonic acid. But this taking-in of carbonic acid and giving-out of oxygen is not the plant's respiration. In reality, as has already been seen, ${ }^{1}$ the plant like the animal inspires oxygen and expires carbonic acid. This fact is simply disguised by the process of assimilation. During the night, however, when assimilation ceases in the darkness, the plant inspires oxygen and expires carbonic acid; and, if it be cultivated in a closed space, it lives during the night upon the oxygen that it has set free during the day by the cleavage of the carbonic acid that it has taken in. The process of assimilation of carbonic acid is, therefore, to be sharply separated from that of respiration. The two phenomena are entirely distinct from one another.

But in a peculiar kind of organisms, the so-called Anaërobia, the relations are even much less clear than in the plants. The Anaërobia are organisms, belonging chiefly to the Bacteria, that can continue to live with complete absence of oxygen. Many of them even perish when they come in contact with free oxygen. Since Pasteur, the father of Bacteriology, first asserted the reality of such rare beings, their actual existence has frequently been doubted, but there is no longer any
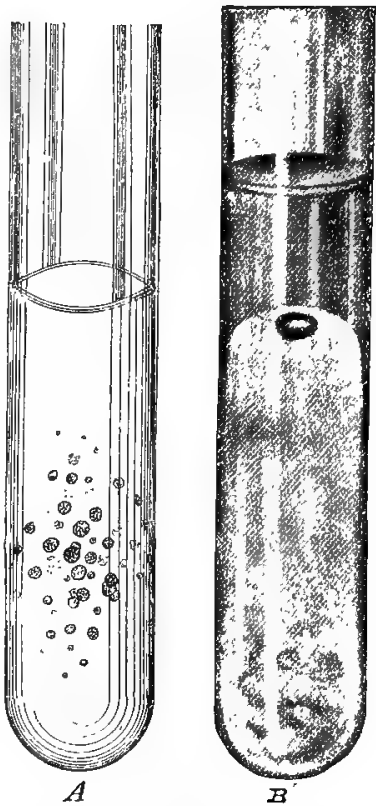

Fia. 131.-A. Culture of the bacteria of symptomatic anthrax. (After Migula.) The spherical colonies lie in the interior of nutrient gelatine excluded from the air. $B$, Culture of the bacteria of tetanus. The bacteria have liquefied the lower part of the nutrient gelatine in the test-tube and have formed a bubble of gas, which lies at the upper end of the liquefied mass. They have grown only in the lower parts of the test-tube, separated from the air by a thick layer of gelatine. question of the correctness of this claim. Thus, e.g., the bacteria of symptomatic anthrax and of tetanus grow anaërobically (Fig. 131). So, also, the vibrios of cholera are able to live admirably in alkaline nutrient media with absence of air; under these conditions thoy increase rapidly in the intestine, where scarcely a trace of pure oxygen exists. This fact is the more remarkable since when brought into contact with air 
they show themselves to be unusually greedy for oxygen. Since it cannot be supposed that without oxygen they are capable of increasing so remarkably as they do in the intestine, and since their greed for free oxygen is acknowledged, it must be assumed that they as well as other Anaërobia, such as the bacteria of tetanus and the bacilli of symptomatic anthrax, are capable in the absence of free oxygen of withdrawing oxygen from the salts of the alkalies that occur in their media-in other words, they are able to take oxygen from fixed chemical compounds. This assumption requires experimental proof, and the same may be said also of the other anaërobic parasites of the intestine, which, as, e.g., the thread-worms, according to Bunge's researches ('83), are capable of living in active movement for $4-5$ days in a medium completely free from oxygen.

Finally, organisms in the condition of latent life occupy an exceptional position in respect to oxygen, as to all other vital conditions that bear directly upon metabolism. They require no oxygen, just as they require no food and no water and yet are capable of life. The fact is not unaccountable, for where metabolism cannot be demonstrated, no oxidation-processes are found.

\section{Temperature}

Besides the conditions characterised by the introduction of matter (food, water and oxygen), upon which metabolism directly depends, certain dynamic requirements must be fulfillad, if life is to be maintained. Among them, before all others, is a temperature within certain limits.

It is well known that chemical compounds are influenced in a marked degree by temperature. In general, high temperatures lead to the dissociation of compounds that at low temperatures can readily exist unchanged. Living substance is a mixture of numerous chemical substances, among which occur highly complex compounds in an extremely labile condition. It is evident, therefore, that living substance also must be dependent in a marked degree upon temperature, that life can exist only within definite temperature-limits. These limits, the minimum and maximum of temperature, are of course very different for different forms of living substance. Temperatures in which some organisms thrive are fatal for others. It is not necessary here to determine for individual species the higher and the lower limits, but it is important to find out what are the minimum and the maximum at which life in general can exist upon the earth's surface.

The observation has frequently been made that poikilothermal animals and plants can be frozen without losing their vital capacity. Thus, in his polar expedition in the year 1820 John Franklin saw 
carps, which, after having been frozen solid, revived and moved about actively upon being warmed before a fire, although in specimens that were killed the intestines were so solid that they could be removed as a single piece. Likewise, by careful warming Dumeril revived frogs that had been frozen solid in water of $4^{\circ}$ to $12^{\circ}$; and Preyer ('80), who has collected considerable testimony upon this subject, made the observation that frogs frozen solid could be revived if their internal temperature had not reached $2.5^{\circ} \mathrm{C}$. Romanes made similar observations upon Medusce (Aurelia aurita), whose delicate jelly-like bodies were pierced by abundant, fine ice-crystals. But all these statements are to be accepted with some criticism. The fact is not to be doubted that these animals can be actually frozen solid in ice and yet be revived by careful thawing, but in all the observations it is not certain whether the living substance of the cells themselves possesses a temperature below $0^{\circ} \mathrm{C}$. As is well known, all cells produce a certain quantity of heat in their metabolism, and as a result of this when they are frozen their internal temperature is always slightly higher than that of the surrounding ice. It is, therefore, possible that in all the observations the living substance of the cells itself was not cooled to $0^{\circ}$ or below $0^{\circ}$. Hence, more exact investigations were needed in order to decide the question whether the living cell itself undergoes withont harm cooling of its substance to or below $0^{\circ} \mathrm{C}$. Such experiments have been performed by Kühne, and more recently and in great detail by Kochs.

Kiihne ('64) placed upon ice in a watch glass a drop of water containing many amœbae, and found that gradually, in proportion to the cooling, the movements became slower and slower, until finally they ceased altogether and the amœbae lay completely motionless. If the drop were again brought to the usual roomtemperature, the movements would begin again; the amobae, therefore, were still alive. But the result was different when the drop was frozen. Then, even after warming, the amœbae remained motionless and could not again be revived.

More recently, Kochs (90) performed very detailed experiments upon frogs and water-beetles. He froze these animals in glasses containing water. If the temperature was not very low, there remained around the animals, surrounded by ice, a liquid mass of water, the temperature of which was $2^{\circ}$ below the zero-point, as was shown by boring through the mass of ice. If, after boring, this last layer of water was frozen, the animals could still be revived by warming, provided that they had not been frozen longer than five to six hours. By sawing through such a preparation it was shown that the animals were not frozen solid internally. But, if the experiment was extended so that the animals were thus frozen, which was the case when they wore brought into cold air of $4 \mathrm{C}$., all attempts at resuscitation were in rain. 
In the light of these experiments the assumption that organisms always perish when the living substance of the tissue-cells itself is frozen solid, appeared very highly probable. But in opposition to them recently Raoul Pictet ('93) has established facts in accordance with which our ideas must apparently be wholly changed.

This well-known investigator, who has made a number of surprising and extraordinarily valuable discoveries concerning the chemical effects of very low temperatures, recently carried out in his laboratory experiments upon the physiological effects of such temperatures. The objects of his experiments were protected by wood from contact with the metal walls of the cold vessel in which they were placed, so that they were exposed to the low temperature of the air only. It was thus shown that different animals behave very differently. Fishes that were cooled down to $-15^{\circ} \mathrm{C}$. in a block of ice remained living after careful warming, although others in the same experiment could be ground to powder like ice. But upon cooling to $-20^{\circ} \mathrm{C}$. the fishes died. Frogs endured without dying a temperature of $-28^{\circ} \mathrm{C}$., myriopods $-50^{\circ} \mathrm{C}$., snails $-120^{\circ} \mathrm{C}$., and bacteria even less than $-200^{\circ} \mathrm{C}$. In view of these surprising experiments it can hardly be doubted that in individual cases the living substance of cells can be frozen to ice without losing its capacity of life.

These phenomena suggest the question whether in frozen organisms there is really a complete standstill of the vital processes-a question that Preyer believes must be answered in the affirmative. Theoretically, there is nothing opposed to this idea; for, when it is seen how with falling temperature the energy of the vital processes constantly decreases, it must be believed that in time a point may be reached where they cease altogether. The possibility that the cell-liquid itself can freeze without abolishing the vital capacity of the cell, would support this view; for, as has been seen, life cannot exist without water in the liquid state. It would be expected therefore, that, as soon as the water in the living substance has passed over into the solid state, the chemical transformations in the cell would be at a standstill. But conclusive experiments for the decision of this question are thus far wanting. If it should be established that living substance in the frozen condition can be maintained for years capable of life, just as certain dried organisms can be so maintained for years, decades, and even centuries, then the probability would approximate to certainty that life in frozen organisms is really at a standstill. At present this is not settled. One fact that is opposed to this iclea is the observation made by Pictet, that frozen organisms cannot endure a farther fall of temperature beyond a certain point. Upon thawing they cannot be revived. If life were really at a complete standstill, it would be difficult to understand why 
a farther sinking of the temperature should still be of influence. For the present, therefore, we inust forgo a definitive solution of this question.

The establishment of the maximum of external temperature meets with difficulties similar to those surrounding that of the minimum. In every case the maximum is represented by the point where the proteids in the living substance of the cell coagulate. The proteids play in the life of the cell the most essential rôle, and it is conceivable that, when the dissolved albumin passes over into the solid state, metabolism, in other words life, must cease. Accordingly, it might appear very simple to determine the maximum of temperature at which life can still exist. But the temperature of coagulation is very different for different proteids, and, moreover, there are kinds of organisms that still live even at temperatures at which all proteids must long since have coagulated.

In a similar manner as with the minimum, Kuihne ('64) performed experiments upon Ameba regarding the maximum of temperature, and found that, when creeping actively at ordinary temperatures, it contracted at $35^{\circ} \mathrm{C}$., but still remained capable of life; after being heated to $40^{\circ}-45^{\circ} \mathrm{C}$. it could not be revived by cooling. Thus, Kuihne was able to establish that one proteid of the amoba-cell, which he regarded as contractile substance, coagulated at $40^{\circ} \mathrm{C}$, another at $45^{\circ} \mathrm{C}$. For plant-cells Max Schultze ('63) found the death-point to be at $47^{\circ} \mathrm{C}$. In contrast to these, various other authors have given accounts of remarkable cases in which organisms exist at much higher temperatures. The most remarkable testimony was the observation of Ehrenberg ('58), who found living ciliate Infusoria and Rotiferc between the threads of Oscillaria in the hot springs of Ischia at a temperature of $81^{\circ}-85^{\circ} \mathrm{C}$. Hoppe-Seyler ( 77 ), who tested this statement of Ehrenberg at Casamicciola, Ischia, found considerably lower temperatures. Alyar, when exposed to hot vapours, were living at $64 \cdot 7^{\circ} \mathrm{C}$, but, when in water, the highest temperature in which they existed was only $53^{\circ} \mathrm{C}$. Hence it is certain that organisms are still able to live in water of $53^{\circ} \mathrm{C}$.

Some time ago very detailed investigations were undertaken in the hot springs of the Yellowstone Park in North America, and living algæ were found at much higher temperatures. The older statement of Ehrenberg does not appear therefore, to have been incorrect.

Although these statements are surprising, a well-authenticated and easily observed fact is known that is much more remarkable. This is the behaviour of the spores of certain bacteria to high temperatures. Koch, Brefeld and others, have shown that the spores of the bacillus of splenic fever (Bacillus anthracis) and the hay-bacillus (Bacillus subtilis) can enrlure 
temperatures of more than $100^{\circ} \mathrm{C}$. without losing their capacity of life.

For the present an explanation of these puzzling facts is wanting. It can only be assumed that the proteids in these organisms occur in a condition in which they cannot be made to coagulate by high temperatures, even, as in the case of the spores of the hay-bacillus, by a boiling temperature. The two assumptions, that, in spite of the temperature of the surrounding medium, the living substance is not heated to the coagulation-point of the proteid, and that the vital capacity is maintained in spite of the coagulation of the proteids in them, are equally improbable. It is not yet known upon what molecular changes the process of coagulation is based, and by what conditions apart from the known factors its appearance is influenced. When more is known upon these questions, some light will be thrown also upon the puzzling phenomena mentioned above.

\section{Pressure}

Like temperature, the pressure surrounding bodies has an influence upon their chemical constitution. This is especially noticeable in cases where the chemical body exists in a medium with the constituents of which it is in chemical relation. If this condition is fulfilled, if a chemical body exists in a gaseous or liquid medium containing substances that have a chemical affinity for it, then, by an increase of the pressure, a chemical combination between the body and the substances in the medium can take place, and by a subsequent decrease of the pressure a decomposition into the previous constituents can occur. This phenomenon depends upon an antagonism between the vibrations of the atoms and the pressure. With a greater pressure the atoms become crowded together, hence more atoms of the medium are able to come into contact with atoms of the body; with a less pressure the vibrations become again so great that the atoms are disengaged from the loose combination.

Living substance exists in such a condition. It lives in a medium, either air or water, with which it can undergo chemical exchange. It is clear, therefore, that the pressure, either of the air or of the water, will have a great significance for life, and that a pressure within definite limits must belong to the general vital conditions.

Unfortunately this condition has been very little investigated thus far, and at present it is possible to state only in part under what pressure of air or water life in general is still possible, and botween what limits of pressure it is confined in its present form upon the earth's surface. The (xperimental investigation of this 
problem will require specialised methods, and the values for the individual constituents of the air and the water, such as oxygen, carbonic acid, etc., must be separately determined manometrically.

In discussing oxygen as a general condition of life, we became acquainted with the importance of the partial pressure of this gas, ${ }^{1}$ and learned that pure oxygen at a pressure of more than three atmospheres is fatal to homothermal animals, while with ordinary air the same result appears at a pressure of $15-20$ atmospheres. Death likewise follows when the partial pressure of the oxygen falls too low.

The venturesome method of balloon-travel has been einployed to collect facts regarding the height at which the pressure of the air becomes so small that danger to human life results. The balloon trip that was made out of Paris in the year 1875, by Spinelli, Sivel and Tissandier, has become famous. They rose with considerable rapidity, and without any disturbance reached a height of 7,000 metres. At about 7,500 metres, Tissandier relates, they felt constantly increasing weakness and apathy, which soon increased to complete absence of the power of motion, although their minds still remained clear. They could no longer perform voluntary movements, nor could they even use their tongues for speaking. After Tissandier had made the observation that the balloon had passed a height of 8,000 metres, and after vain efforts to communicate this fact to his two companions, he lost consciousness. When he awoke, they had descended to 7,059 metres. Then Spinelli, who also had awaked, threw out sand in order that they should not fall too rapidly. As a result of this the balloon again rose, and the aeronauts again lost consciousness. When Tissandier awoke a second time they had sunk to 6,000 metres, and the barometer showerl that the balloon had reached a height of about $\$, 500$ metres. Spinelli and Sivel never regained consciousness.

The minimum of air-pressure under which plants and animals can still remain alive can be determined by the air-pump. In such an experiment the most important thing for animals is the partial pressure of oxygen, for plants that of carbonic acirl.

As regards the water-pressure under which life can exist, far fewer facts are known than as regards the pressure of the air. The interesting deep-sea investigations of the last ten years have shown, in opposition to earlier ideas, that living organisms exist even in the greatest depths of the se'a, where darkness always prevails and bodies are subject to a pressure of several hundred atmospheres. This pressure is so great that upon its sudden withdrawal, as when the animals are drawn to the surface, they burst. Fishes come up swollen, with their scales standing out and their intestines protruding from their mouths (Fig. 132); this is observed

$$
{ }^{1} \text { Cf. p. } 282 .
$$


even in the fishes that live in the depths of the Lake of Constance. The height to which the pressure can rise before all life ceases has thus far not been investigated. The diminution of the waterpressure to the pressure of the atmosphere resting upon the water, by means of an air-pump, appears to be without influence upon organisms living in the water. But a great diminution of the

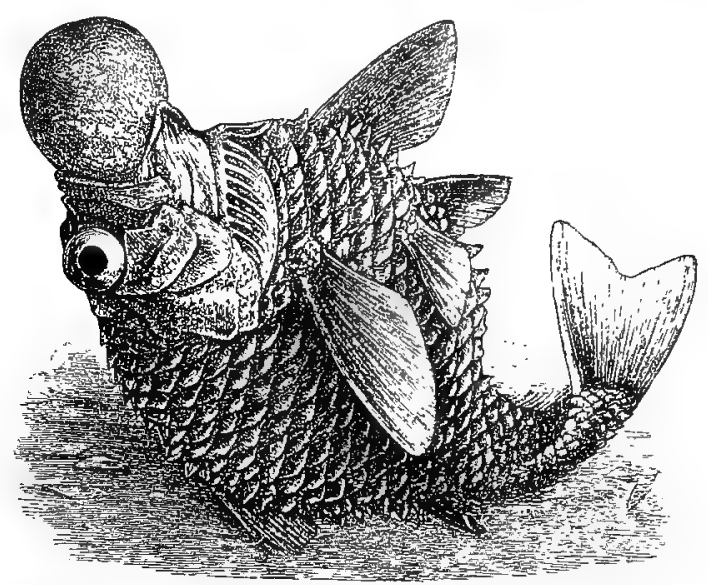

Fig. 132.-Neoscopelus macrolepidotus, brought to the surface from a depth of $1500 \mathrm{~m}$. The eye and the intestines are swollen out and the scales are falling off, owing to the great tension of the skin over the body. (After Keller.)

water-pressure is not possible without altering the liquid state of the water. Here the question of the minimum of water-pressure passes over into that of the minimum of air-pressure, and the partial pressure of the contained gases, water-vapour, oxygen, etc., and becomes connected with the questions of the importance of moisture in the atmosphere, oxygen, etc., as general conditions of life.

\section{B. THE GENERAL INTERNAL CONDITIONS OF LIFE}

The conditions, thus far spoken of, namely, a supply of food and other substances, a definite degree of temperature, and a certain pressure, comprise all the general conditions of life that must be afforded by the medium. Others, such as light, are likewise external but not general conditions, and pertain only to certain organisms or groups of organisms.

But along with the general external conditions there are associated others that must be firlfilled also in order that life can continue. These lie within the organism itself, and constitute the general internal conditions of life.

Obviously the chicf requisite for the existence of life through 
the fulfilment of all external conditions is the presence of a substance, capable of life, in which vital phenomena can take place. Hence, if a tiny drop of living substance be imagined in a medium in which all the external conditions of life are fulfilled, it must be assumed that it will remain living so long as disturbing influences do not enter from without. But experiments contradict this.

A small mass of living substance can be easily obtained by cutting off with a fine scalpel under the microscope a piece of hyaline protoplasm from a living cell, e.g., Anıcba. The piece cut off is living ; this is recognised from the fact that after the opera-
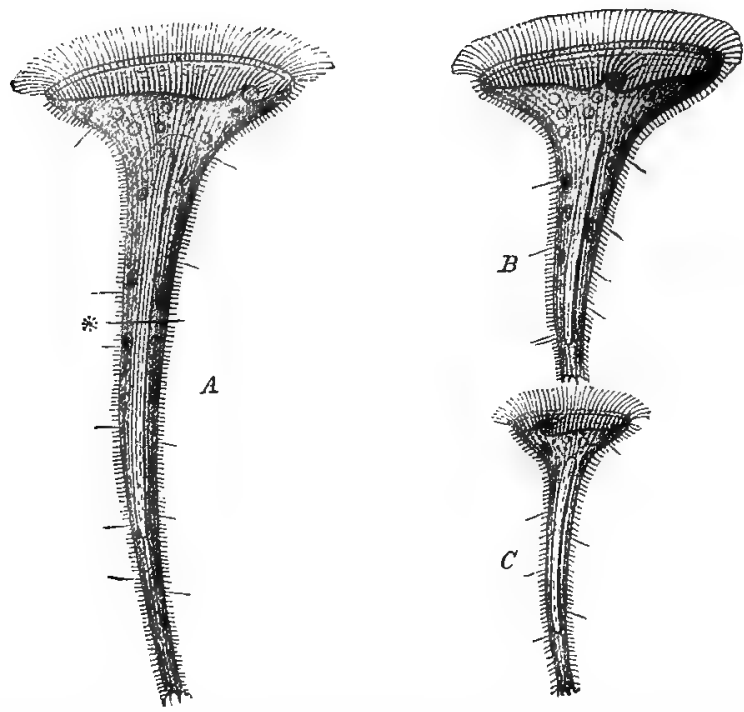

Fro. 133. - Stentor roesclit, a ciliate-infusorian cell. The clear, extended, rod-shaped mass in the interior is the nucleus. $A$, Cut into two nucleated pieces at *; at $B$ and $C^{\prime}$ the nucleated pieces have become regenerated into whole Stentors and continue to live.

tion it still performs such movements as the whole Amoba, performs. The external vital conditions, moreover, are all fulfilled, for the part exists in the same medium and has the same external relations as the whole $A$ moba. Nevertheless, it lasts for a short time only, it soon dies and cannot be restored to life by any agency. Every like experiment without exception upon any other cell yields the same result (Fig. 133). In all such cases a certain mass of living substance exists in a medium in which all external vital conditions are fulfilled, and yet the mass cannot continue living. Hence some factor among the general conditions of life is wanting. 
Inasmuch as there exists upon the earth at present no living substance that is homogeneous throughout, the absent factor, as shown by the experiment, is the natural coherence and correlation of the essential parts of the organism.

This is true equally of the cell-community and the individual cell. But the objection may be raised that in many cases parts and even whole organs can be separated from an organism without endangering its existence. This is true, but in all such cases the parts are such as are not absolutely necessary to the maintenance of the individual, whether it be because they are present in abundance and can be replaced in function by others, or because they are not closely related to the other parts, and, therefore, when separated, represent complete individuals. A polyp can be cut into two parts, both of which continue to live, and from a polypstalk a single polyp can be cut off without dying. In the above experiment upon $A$ moeba the nucleated cell-body continues living even after the separation of a portion of the protoplasm, because it still possesses a quantity of protoplasmic particles of the same kind as were removed. But the piece of protoplasm that is cut off perishes, because its connection and correlation with the nuclear mass have ceased.

The living substance that now exists upon the earth's surface is recognised only in the form of cells, either alone or bound together into cell-communities. The cell contains as its essential constituents two different substances, the protoplasm and the nucleus. ${ }^{1}$ Wherever a little protoplasm and a little nuclear substance exist in union, there is a cell ; and only such is capable of life when the external vital conditions are fulfilled. A large cell can be divided into many pieces capable of life, so long as the requirements are complied with that every piece shall possess some protoplasm and a little nuclear substance, and that the disproportion between the two masses shall not exceed a certain limit. ${ }^{2}$ With some skill it is not difficult to perform the experiment upon large unicellular organisms. But, if a cell be so divided that the nucleus is separated from the protoplasm, both parts invariably perish.

Since the cell is the general elementary constituent of organisms, the individual of the lowest order, the association of nucleus and protoplasm in the cell may be established as a general internal condition of life. Only where these two are united can life continue to exist.

A physical phenomenon takes place when, on the one hand, a material substratum is present in which it can take place, and, on the other, certain external conditions are fulfilled. The same holds good of vital phenomena. Vital phonomena appear with

$$
{ }^{1} \text { Cf. p. } 7 \text { 1. }
$$$$
\text { ' } C f \text {. Lillie ('96). }
$$ 
the same necessity that characterises the appearance of physical phenomena, when matter capable of life is present, and when the external general and special conditions of life are fulfilled. In other words vital phenomena are an expression of the correlation of living substance and the surrounding medium, or, as Claude Bernard ('79) says: "Vital manifestations result from a conflict between two factors-the organiser living substance and the medium."

In considering this correlation the question comes up: How was it with life at a time when conditions wholly different from present ones prevailed upon the globe? Was life then able to exist? When and how did it arise?

\section{The Origin of Life tron the Earth}

As is well known, the earth was once in a fiery condition, like the sun from which it came. The hard rocks and solid metals that now compose its solidified crust were then in a molten state; its liquid nucleus was surrounded by an atmosphere of incandescent gases; its particles were in violent motion, and its temperature measured thousands of degrees.

The idea that the earth in its evolution once passed through such a condition is now an accepted generality of all branches of natural science. Astronomy, physics, geology, geogony, mineralogy and chemistry, all agree in this. Moreover, modern science, with the help of the telescope and the rpectroscope, has brought the fact directly before us, that even now, everywhere in the universe, the same process of evolution that the earth once passed through is being repeated, and that there exist upon other heavenly bodies conditions analogous to each stage of the earth's evolution. There now exist in space gaseous nebulac, molten spheres, and solid, ice-cold masses, the last representing the present condition of the moon and the future fate of the earth.

The fact that the earth was once' in a condition in which its temperature was enormous and not a drop of water existed upon it, in short, a condition in which the vital conditions that are now regarded as indispensable to the existence of organisms were wanting-this fact will always be an important factor with which all speculations upon the origin of life upon the earth must deal. In the light of this we will consider the rarious views upon the origin of life that have been founded upon a scientific basis by various men of science, and will endearour to form some idea respecting it, even though the idea be only a general one. 


\section{A. THEORIES CONCERNING THE ORIGIN OF LIFE UPON THE EARTH}

\section{The Doctrine of Spontaneous Generation}

The modern doctrine of spontaneous generation (archegony, abiogenesis, generatio spontanea or aquivoca, etc.) in its general form is as follows. Since there was a time in the evolution of the earth when the existence of the living substance that now inhabits the cool surface of the latter was absolutely impossible, living substance must have arisen from lifeless substance at some later period.

The question accordingly arises, how and under what conditions were the first organisms created?

To the ancients, even to a mind having so comprehensive a knowledge of nature as that of Aristotle, the idea presented no especial difficulties that animals, such as worms, insects and even fishes, could come into existence out of mud. Only at a relatively late time and particularly in connection with the researches of Redi and Swammerdamm upon the development of insects, were these crude ideas laid aside as incompatible with established scientific knowledge.

But the doctrine of spontaneous generation obtained a new point of support, when the invention of the microscope led to the discovery of a world hitherto wholly unknown and excessively rich in forms, when it was found that whenever an aqueous infusion of dead organic substance was prepared, after a short time an abundance of minute living beings developed in it, which even yet are termed Infusoria. It was fully believed that in Infusoria organisms had been found that were produced by spontaneous generation out of the dead substances in the infusion. This view necessarily seemed all the more probable because the Infusoria were the lowest and simplest beings that had been known up to that time. But in this case also it was established later that the organisms did not originate spontaneously, but were developed from germs that were previously contained in the substances or came into the vessel through the air. Milne Edwards, Schwann, Max Schultze, Helmholtz and others showed that if the substances had previously been freed from germs by boiling, and if germs were prevented from entering through the air, the development of Infusoria never took place, however long the infusion was allowed to stand.

When, later, the smallest of all micro-organisms, the Bacteria, began to attract strongly the attention of the scientific world, and when it was found by refined methods of investigation that these minute beings or their germs are present everywhere in the air, the earth and the water, the doctrine of spontaneous generation 
seized upon them and claimed that they as the lowest organisms are continually arising at the present time from lifeless matter. But modern bacteriology, with its admirable and delicate methods, for which it is indebted to its founders, especially Pasteur and Robert Koch, has refuted this doctrine again. It has shown that by the exclusion of all germs that can come to the preparation from the outside, even the richest nutrient medium, containing all the substances required for the nutrition of bacteria in the most favourable mixture, remains free from micro-organisms; and, on the other hand, that a whole world of diverse forms develop in the medium as soon as it is left standing, for a brief time, open to the air.

Along with this continual strife over the doctrine of spontaneous generation, attempts have been made, even down to very recent times, to manufacture living organisms artificially in the laboratory. The latest of these attempts is associated especially with the name of Pouchet, who was the last active adherent of the view that it is possible to produce artificially from lifeless matter unicellular organisms, such as bacteria, yeast, and similar microbes, simply by mixing the necessary constituents and putting them under favourable external conditions. Even when at times these experiments have seemed to lead to positive results, the bacteriologists have always appeared with their critical methods, and have shown that in every case there was a development of germs that had come in from the outside or were already present in the vessels used for the experiment. These attempts are really not different from the undertaking of the famulus Wagner to compound man himself from chemical mixtures in a retort. How can one hope to produce chemically even the simplest organism when the chemical composition of living proteids, the most important substances of which all living substance consists, is at present completely unknown?

To Haeckel ('66) belongs the credit of having removed from the early absurd ideas of spontaneous generation their sound kernel and of having transferred it to a purcly scientific soil. For him the question is indifferent, whether at the present day living substance arises anywhere spontaneously or not. To-day, more than thirty years after Haeckel wrote, and after our knowledge of the lowest organisms and their reproduction has made so enormous a development, the great majority of investigators are inclined to answer this question negatively. Nevertheless, Haeckel was the first to draw sharply the conclusion that because there was a time when the earth was in a condition that excluded all organic life, living substance must have originated at some time in the earth's development from lifeless substances. According to him this time cannot be dated earlier than when the watervapour, suspended throughout the atmosphere, had been precipi- 
tated in the form of liquid. Further, he justly lays the greatest value upon the principle that the organisms that arose by spontaneous gencration must have been, not cells but the lowest and simplest organisms that can be imagined, "completely homogeneous, structureless, formless lumps of proteid." It is conceivable that these living proteid lumps arose from the mutual action of substances dissolved in the primitive sea. But Haeckel expressly refuses to discuss in detail the "how" of their origin: "Every detailed portrayal of autogony is for the present inadmissible, for the reason that we can form absolutely no satisfactory idea of the peculiar condition presented by the earth's surface at the time of the first appearance of organisms." From the very simple and low organisms that arose spontaneously, which on account of their simplicity Haeckel termed Monera, there have been derived by continuous descent the cells and all forms of organisms that to-day inhabit the earth's surface.

This in its essentials is the doctrine of spontaneous generation in its present form. Notwithstanding the fact that its conclusion is so simple and obvious, it has been contradicted on many sides and has led to the establishment of other theories upon the origin of life upon the earth.

\section{The Theory of Cosmozod}

The theory of germs of lower organisms capable of life moving about in space, or, as Preyer has termed it in brief, the theory of cosmozoc, was the first to appear in recent times in opposition to the doctrine of spontaneous generation. Its founder was H. E. Richter ('65, "70, and '71). Starting from the idea that small solid particles are moving about everywhere in space and in the rapid flight of the heavenly bodies are continually being stripped off from them, Richter assumes that, at the same time and attached to these solid particles, germs of microorganisms capable of life are also continually being thrown off from such heavenly bodics as are inhabited and carried to others. If such germs come to other heavenly bodies whose state of development presents favourable vital conditions, especially moderate heat and moisture, they begin there to develop and become the startingpoint of a host of organisms. Somewhere in space, Richter thinks, there have always been heavenly bodies upon which life exists in the form of cells. The existence of living cells in the universe is eternal. "Omne vivum ab ceternitate e cellula," says Richter, modifying anew, according to the precedent of Virchow, Harvey's old dictum. Organic life, therefore, has never originated but has always been transferred from one world to another. Thus, according to Richter, the problem of the origin of life upon the carth is not: How has life arisen upon the earth? but: How has 
it come to the earth from other worlds? and this question he answers by the theory of cosmozoa.

For the possibility that germs capable of life came from space through the atmosphere to the earth's surface, without perishing from the incandescent heat arising from the enormous friction, Richter believes that he finds a support in the assertion of observers that in many meteoric stones traces of coal and even humus and petroleum-like substances oceur. If these can come to the earth without undergoing combustion it is possible that germs capable of life also pass through the atmosphere without losing their vital capacity.

That organic germs can endure a long journey through space from one heavenly body to another without water and food cannot be doubted, if in apparently dead organisms, such as the spores of micro-organisms, there be recognised substance actually capable of life that can continue in its apparently dead condition for a long time without water and food and yet revive as soon as it comes under the required vital conditions.

Independently of Richter, some years later Helmholtz and Sir William Thompson discussed the question whether life may not, perhaps, have been transferred from other heavenly bodies to the earth; and both termed this view not unscientific. Helmholtz ('84) says: "Meteoric stones sometimes contain hydrocarbon compounds; the intrinsic light of the heads of comets shows a spectrum that is very similar to that of the incandescent electric light in gases containing hydrocarbons. But carbon is the characteristic element of the organic compounds of which living bodies are composed. Who can say whether these bodies that swarm everywhere through space do not spread also the germs of life wherever a new world has become capable of affording a dwelling-place to organic creatures? And this life we might, perhaps, have reason to regard as even allied to our own in germ, however various may be the forms in which it night adapt itself to the conditions of its new dwelling-place." That meteors can be the bearers of such germs Helmboltz holds to be entirely possible, since large meteoric stones in passing through the atmosphere of the earth are greatly heated upon their surface only, while in their interior they remain cool. Helmholtz says, further, regarding the theory of cosmozoa: "I cannot contend against one who would regard this hypothesis as highly or wholly improbable. But it appears to me to be a wholly correct scientific procedure, when all our endeavours to produce organisms out of lifeless substance are thwarted, to question whether after all life has ever arisen, whether it may not be even as old as matter, and whether its germs, passed from one world to another, may not have developed where thuy found favourable will." "The true alternative is evident: organic life has either begun to exist at some one time, or has existed from eternity.' 


\section{Preyer's Theory of the Continuity of Life}

By considerations of another kind, Preyer ('80) has arrived at a theory regarding the derivation of life which is opposed to the theories both of spontaneous generation and of cosmozoa.

Preyer cannot accept the idea of spontaneous generation for the following reasons. If it be assumed that at some one time in the earth's development living substance has arisen spontaneously from lifeless substance, it must be claimed that this is possible even now. But the failure of the innumerable experiments directed toward this problem has made it in the highest degree improbable. On the other hand, the supposition that spontaneous generation was possible only once in the primæval past, but now no longer occurs, is likewise improbable; "for the same conditions that are essential for the maintenance of life and are now realised, must necessarily have been realised also at the time of the supposed origin of living from inorganic bodies; otherwise, the product of spontaneous generation would not have been able to continue living." In other words, if spontaneous generation were once possible, it is difficult to see why it is not possible now.

Preyer is likewise not able to accept the theory of cosmozoa, because he sees in it not a solution, but only a postponement of the problem, i.e., a shifting of it from the earth to some other world, the problem itself, however, always remaining.

Proceeding from the inductive fact that organisms are always derived from other organisms similar to them, that thus far observation has never been able to establish the origin of any organism without a parent, Preyer raises the question whether the problem of spontaneous generation may not rest upon a false conception, when it demands that living substance shall at some time have originated from lifeless substance. Must it not rather be formulated in the reverse order: has lifeless originated from living substance? Organisms are always derived from other living organisms; but inorganic, lifeless substance is continually being derived from both lifeless substance and living organisms, either being excreted from the latter as dead matter or remaining after their death. In contrast, therefore, to the doctrine of spontaneous generation, Preyer puts forward the theory that living substance is the primary thing, and that lifeless substance is derived from it secondarily by excretion. ${ }^{1}$ He thus demands that continuity in the derivation of living substance has never been broken. "Whoever interrupts the series of successive generations of orginisms by the introduction of a generation without previous parentage; in other words, whoever denies the continuity of life is arbitrary." 
Omne vivum e vivo-this proposition has never experienced an exception.

The consequences following from this idea are rery interesting. If life upon the earth has never been derived from lifeless, but always from living substance, life nust have existed when the earth was still an incandescent body. In fact, Preyer so concludes. He is, therefore, obliged to give to the conception of life a considerably wider scope than usual; and to regard as living not only present living substance, but also the incandescent liquid masses as they existed at one time in place of protoplasmic organisms. "If, however, we free ourselves," says Preyer, "from the wholly voluntary and really improbable thought that only protoplasm of the quality existing at present can live, and from the old prejudice, which is sustained simply by convenience, that at first only the inorganic existed, then we will not shrink from the one great step further, we will lay aside spontaneous generation and recognise that rital motion has had no beginning. Omne rivum. viro!"

In accordance with these considerations, Preyer sketches somewhat as follows the picture of the derivation of life upon the earth. Originally the whole molten mass of the earth's body was a single giant organism. The powerful morement that its substance possessed was its life. When the earth's body began to cool, the substances that could no longer remain in the liquid state at that temperature, as, e.g., the heavy metals, were separated out as solid masses, and, since they no longer had a share in the vital movements of the whole, formed dead, inorganic substance. Thus arose the first inorganic masses. This process continued. It is remembered that at first hot, molten masses represented the life of the earth. "When in the course of time these compounds became solidified upon the surface of the globe, or, in other words, died, compounds of the elements that thus far had remained still gaseous and liquid appeared, and these became gradually more and more like protoplasm, the basis of the living substance of the present day. With the decrease of temperature and the lessened dissociations there must constantly have appeared more complex compounds, chemical substitutions, denser bodies, and more involved and correlated movements of the parts which were being massed closer together. Thus, the first forms of plants and animals, resembling one another and made possible by advancing differentiation, were able to exist."

"We do not say, therefore, that protoplasm as such existed from the beginning of the earth's formation; or that without beginning it wandered as such from elsewhere out of space to the cooled earth; or, still less, that without life it became compounded upon the planets out of inorganic bodies, as spontaneous generation would have it; but we maintain that the movement that exists in 
the universe without beginning is life, and that, after the bodies now termed inorganic had been separated out upon the cooling surface of the incandescent planet by iis intense vital activity, and were not able to return again into the hot liquids which gradually decreased in quantity because of the progressive decrease in temperature of the carth's crust-we maintain that, after this had occurred, protoplasm must necessarily have remained over. The heavy metals, once organic elements, no longer melted, and did not return into the circulation from which they had been cast out. They are the signs of the rigor mortis of the gigantic, cooling, primæval organisms, whose breath perchance was Iuminous iron-vapour, whose blood was liquid metal, and whose food was meteorites."

\section{Pftüger's Idea}

In one of the most suggestive works in physiological literature Pfluiger ('75,1) has discussed very fully the question of the origin of life upon the earth, and has defended the idea of spontaneous generation, that living substance originated upon the earth itself out of lifeless substances. Pfliiger's ideas are especially valuable in that in a strictly scientific manner he discusses the problem in intimate connection with the facts of physiological chemistry, and follows it out far into detail.

The essential point of Pfliger's investigation is constituted by the chemical characteristics of proteid as that substance with which life in its essentials is inseparably united. There exists a fundamental difference between dead proteid, as it occurs, e.g., in egg-albumin, and living proteid, as it constitutes living substance; this difference is the self-decomposition of the latter. All living substance is continually being decomposed, in some degree spontaneously and more through outside infiuences, while dead proteid under favourable conditions remains intact for an unlimited time. The chief condition of this decomposition is intramolecular oxygen, i.e., the oxygen that occurs in the living proteid molecule, and is continually being received by it from the outside through respiration. That this oxygen is the essential condition follows from the facts that during the decomposition carbonic acid is contimually being formed, and that carbonic acid does not arise from living proteid by direct oxidation of the carbon and a simple splitting-off of the carbonic acid molecule, but by dissociation, i.e., by an internal rearrangement of the atoms and the separation of new atomic groups from one another. Living substance must contain oxygen already in combination in the living molecule, and in the decombosition a rearrangement must take place, otherwise it cannot be conceived how, as PHiger has shown upon frogs, animalis can exist longer than a liuy without free oxygen in an 
atmosphere of nitrogen and yet constantly expire carbonic acid. Why the addition of oxygen will transform a more stable molecule into a more labile condition becomes clear when it is borne in mind that, as Kekiile has shown, in all organic chemistry there is no single molecule that contains enough oxygen to oxidise all the hydrogen-atoms of the molecule to water and all the carbon-atoms to carbonic acid. For this reason molecules are more or less stable and not inclined to dissociation, unless other chemical causes bring in some lability. If, however, sufficient oxygen be introduced to ensure the possibility of the oxidation of the atoms of carbon and of hydrogen into the stable molecules, carbonic acid and water, by intramolecular rearrangement, the power of decomposition must become increased, for the affinity of carbon and hydrogen for oxygen is very great. Thus, the great inclination of living substance to decomposition is conditioned essentially by the intramolecular oxygen.

A comparison of the decomposition-products of living proteid and those obtained by the artificial oxidation of dead proteid is of great importance. The significant fact here appears, that the non-nitrogenous products of the latter agree essentially with those of the former, but that "the great majority of the nitrogenous products [of the latter] have not even a remote similarity to the majority of those arising in the living budy." It follows that, as regards its non-nitrogenous groups of atoms, its hydrocarbon radicals, living proteid cannot be essentially different from dead proteid, but that a fundamental difference must exist as regards the nitrogenous radicals. Here a starting-point for further consideration is afforded by the fact that of the nitrogenous decomposition-products of living proteid, such as uric acid, creatin, and, moreover, the nuclein bases, guanin, xanthin, hypoxanthin, and adenin, a part contain cyanogen, $\mathrm{CN}$, as a radical, and a part, like urea, the most important of all the nitrogenous decompositionproducts of living proteid, can be produced artificially from cyanogen compounds by a rearrangement of the atoms. This points strongly to the probability that living proteid contains the radical cyanogen, and thus differs fundamentally from dead or food-proteid. Pfliger thereupon says: "In the formation of cell-substance, i.c., of living proteid, out of food-proteid, a change of the latter takes place, the atoms of nitrogen going into a cyanogen-like relation with the atoms of carbon, probably with the absorption of considerable heat." That considerable heat is absorbed in the formation of cyanogen follows from the fact that, as calorimetric investigations show, cyanogen is a radical possessing a great quantity of internal energy. By the addition of cyanogen to the living molecule, therefore, there is " introduced into the living matter energetic internal motion."

Accordingly, the great property of decomposition possessed by 
living proteid is explained as the result of the absorption of oxygen; for, since the atoms of cyanogen are in active vibration, the carbon-atom of the cyanogen at the approach of two oxygenatoms will pass out of the sphere of influence of the nitrogenatom into that of the oxygen, and will. unite with the latter into carbonic acid. Thus the cause of the formation of carbonic acid, i.e., of the decomposition of living substance, lies in the cyanogen, and the condition is the intramolecular introduction of oxygen.

The idea that it is the cyanogen especially that confers upon the living proteid molecule its characteristic properties is supported especially by many analogies that exist between living proteid and the compounds of cyanogen. In the first place, a product of the oxidation of cyanogen, cyanic acid, HCNO, possesses great similarity to living proteid. Pfliger calls attention to the following interesting points of comparison. Both bodies grow by polymerisation, by chemically combining similar molecules like chains into masses; the growth of living substance takes place thus, and in this way also the polymeric cyamelid, $\mathrm{H}_{n} \mathrm{C}_{n} \mathrm{~N}_{n} \mathrm{O}_{n}$, comes from cyanic acid, $\mathrm{HCNO}$. Further, both bodies in the presence of water are spontaneously decomposed into carbonic acid and ammonia. Both afford urea by dissociation, i.e., by intramolecular rearrangement, not by direct oxidation. Finally, both are liquid and transparent at low temperatures, and coagulate at higher ones, cyanic acid earlier, living proteid later. "This similarity," says Pflüger, "is so great that I might term cyanic acid a half-living molecule."

These points of view yield most important suggestions concerning the question how life may have arisen upon the earth. "When we think of the beginning of organic life, we must not think primarily of carbonic acid and ammonia; for they are the end of life, not the beginning." "The beginning lies rather in cyanogen."

Hence the problem of the origin of living substance culminates in the question: How does cyanogen arise? Here, organic chemistry presents the highly significant fact, that cyanogen and its compounds, such as potassium cyanide, ammonium cyanide, hydrocyanic acid, cyanic acid, etc., arise only in an incandescent heat, e.g., when the necessary nitrogenous compounds are brought in contact with burning coal, or when the mass is heated to a white heat. "Accordingly, nothing is clearer than the possibility of the formation of cyanogen-compounds when the earth was wholly or partially in a fiery or heated state." Moreover, chemistry shows how the other essential constituents of proteid, such as the hydrocarbons, the alcohol radicals, etc., can likewise arise synthetically in heat.

"It is seen how strongly and remarkably all facts of chemistry point to fire as the force that has produced by synthesis the 
constituents of proteid. In other words, life is derived from fire, and its fundamental conditions were laid down at a time when the earth was still an incandescent ball."

"If now we consider the immeasurably long time during which the cooling of the earth's surface dragged itself slowly along, cyanogen and the compounds that contain cyanogen- and hydrocarbon-substances had time and opportunity to indulge extensively their great tendency toward transformation and polymerisation and to pass over with the aid of oxygen, and later of water and salts, into that self-destructive proteid, living matter."

Pfluger thereupon summarises his ideas in the following sentences: "Accordingly, I would say that the first proteid to arise was living matter, endowed in all its radicals with the property of vigorously attracting similar constituents, adding them chemically to its molecule, and thus growing ad infinitum. According to this idea, living proteid does not need to have a constant molecular weight; it is a huge molecule undergoing constant, never-ending formation and constant decomposition, and probably behaves toward the usual chemical molecules as the sun behaves toward small meteors."

"In the plant, living proteid simply continues to do what it has always done since its origin, i.e., regenerate or grow; wherefore I believe that all proteid existing in the world to-day was derived directly from the first proteid. Therefore, I am doubtful about the occurrence of spontaneous generation at the present lime. Comparative biology also points unmistakably to the idea that all living substance has taken its origin from a single root only."

\section{B. CRITICAL}

\section{Eternity or Beginning of Living Substance}

Among the ideas regarding the derivation of life upon the earth that are contained in the theories just presented, two notions stand in sharp contrast to one another. This contrast finds expression in the alternative already set forth by Helmholtz ${ }^{1}$ : "Organic life either has begun to erist at some one time or has existed from eternity." The former notion lies at the foundation of the doctrine of spontaneous generation, the latter at that of the theory of cosmozoa, and in a certain sense at the basis of Preyer's theory also. Evidently the two notions are mutually exclusive. If one is accepted, the other must be rejected. To which of the two shall we adhere?

We will test first the theory of cosmozoa. According to it life has never originated, but has existed in the universe from eternity, and a Loc. cit. 
has simply been transferred from one world to another. In the present condition of our knowledge it is scarcely possible to obtain a direct contradiction of this doctrine and conclusive proof of its impossibility. This will be true so long as experience does not suffice to enable us to recognise as wholly impossible the transfer of protoplasmic germs capable of life from one world to another. But, although direct contradiction of the doctrine is at present impossible, the thought that living substance has existed from eternity and has never originated from inorganic substance appears in the highest degree improbable.

As a comparison of organisms and inorganic bodies has shown, ${ }^{1}$ organisms originate only from those chemical elements that occur in inorganic matter also, and differ from the latter only in the chemical compounds of which they are composed. The essential compounds of living substance, proteids, do not stand, therefore, in fundamental contrast to inorganic compounds, and differ from the latter no more than these differ from one another. Hence any general consideration that is formulated regarding the derivation of living substance, especially of proteid, must be equally applicable in its fundamental points to inorganic compounds, such as minerals, feldspar, quartz, etc. But it is shown more clearly in non-living than in living substance to what untenable consequences the idea that lies at the foundation of the doctrine of cosmozoa leads; for, if it be assumed that the complex compounds of living substance, especially proteids, have never originated, but have existed from eternity somewhere in space and have come thence to the earth, with the same logic and the same degree of probability it must be assumed that inorganic compounds also, quartz and feldspar, have always been present as such somewhere in space, and have come to the earth through space from another world. And if this line of thought be carried out to all chemical compounds composing the earth,-and it is as probable of them as of the compounds of living substance-it would lead to the absurd conclusion that all of the earth's compounds have wandered already complete as such from outside into our planetary system. Scarcely any man of science would be willing to accept this conclusion; every geologist is acquainted with examples of minerals that demonstrably have originated as such chemically upon the earth, and every chemist manufactures daily in his laboratory chemical compounds out of simpler substances. No thinking chemist, indeed, now doubts that even the so-called chemical elements did not exist originally as such, but that those elements possessing high atomic weight have been derived by condensation from those having less atomic weight. If the final conclusion be deduced from the cosmozoan ideas, all evolution, not only of living substance but of the whole earth, must be denied; for, if all compounds have existed as such from eternity 1 Cf. p. 118 it folg. 
and have never originated from simple substances, evolution has not taken place. This is the inexorable consequence of a full acceptance of the cosmozoan doctrine. We repeat that one has no right to assume for feldspar a principle of derivation different from that assumed for albumin. Both are compounds of chemical elements.

One fundamental fact in plant physiology practically contradicts the assumption that life has never originated from inorganic substances; namely, at the present time living substance is continually being formed in the plant-cell from simple inorganic compounds, carbonic acid, water, sulphates, nitrates, ete. Between the small seed put into the earth in the spring and the huge plant that grows from it during the summer, an enormous quantity of living substance has been formed out of the purely inorganic substances of the environment, and when winter comes, almost the whole quantity of this living substance returns again to simpler inorganic compounds. It is here seen how inseparably related are inorganic and organic nature, how living substance is originating continually from lifeless substance, and is continually being decomposed again into lifeless substance. Nägeli ('84), one of the most talented botanists, says rightly: "One fact-that in organisms inorganic substance becomes organic substance, and that the organic returns completely to the inorganic-is sufficient to enable us to deduce by means of the law of causation the spontaneous origin of organic nature from inorganic." "If in the physical world all things stand in causal connection with one another, if all phenomena proceed along natural paths, then organisms, which build themselves up from and finally disintegrate into the substances of which inorganic nature consists, must have originated primitively from inorganic compounds. To deny spontaneous generation is to proclaim a miracle."

In a sense entirely different from that of the doctrine of cosmozoa, which has met with little acceptance, Preyer, in his theory, interprets life as without beginning and eternal. $\mathrm{He}$ says: The living substance now inhabiting the earth's surface is derived by continuous descent from the substances that once in a melted condition constituted the earth's mass. Not to term the latter substances living would be arbitrary, since no sharp limit can be established. Since, however, these substances are derived from the sun's mass, and the latter forms simply a portion of the matter of the universe, which is in eternal motion, so life, which itself is only a complex process of motion, is as old as matter.

It is evident that the essential difference between Preyer's theory and the doctrine of spontaneous generation consists in a different understanding of the conception of life. Following the usage of language, the doctrine of spontaneous generation terms living only living substance as it is now recognised, in contra- 
distinction from lifeless substance; while Preyer extends the conception much farther, even to incandescent mixtures, which have not the slightest similarity to present living substance except that they are in energetic motion. If this wide extension of the conception of life be accepted, no objection can be raised against the other consequences of Preyer's theory. It is, however, questionable whether it is judicious or allowable to carry the vital conception so far.

The conception of living substance, as scientifically established at the present time, has arisen from an exact comparison of existing living organisms and existing inorganic bodies. As has been seen, ${ }^{1}$ there is but one absolute difference between these two, and this consists in the metabolism of proteids. No inorganic body possesses proteid. On the other hand, proteid is not wanting in any organism; and that which constitutes the life of an organism, wherein the living differs from the dead organism, is the metabolism of the proteid. This difference between living organisms and dead inorganic bodies, although not fundamental or elementary, is, nevertheless, profound and affords the sole means of sharply characterising living substance. If it be nullified by terming "living" bodies that cannot contain proteid, such as the incandescent masses of the once fiery globe, the advantage afforded by a sharp definition is wholly lost, and the conception of living substance is dissipated.

But here, from the standpoint of Preyer's theory, the question may be raised: If the living substance of to-day is derived in uninterrupted descent from molten mixtures, where is the limit beyond which the substance may be termed living? This question assumes a postulate that is wholly unsupported, viz., that there was a gradual, uninterrupted transition between the molten mixtures and the proteids. Hitherto we have laid great stress upon the idea that no fundamental difference exists between lifeless substances and organisms; but it cannot be proved that an uninterrupted transition between molten substances and organisms existed. It is known, moreover, that when two chemical compounds act upon one another, the resulting substances are not necessarily joined with the original substances by transitionstages, however different they may be from them. Regarding the relations that may have prevailed upon the earth's surface when water was precipitated as liquid, we cannot form even an approximate idea. The idea that living proteid originated without transition by the action of bodies wholly different from it chemically, when the proper conditions existed, would be at least as probable as the idea of a gradual descent associated with uninterrupted transitions.

Further, Preyer implies that the incandescent masses to which ${ }^{1}$ Cf. p. 136. 
he extends the enception of life have had a metabolism. This camnot be substantiated. It camnot be doubted, indeed, that these misses possessed an extremely nurgetic internal motion; and, liti is nothing but a complex motion, to which every other molecular motion is allied in principle. Nevertheless, vital motion, metabolism, is a complex motion very sharply characterising the living organism; it consists in the continual self-decomposition of living substance, the giving-off to the outside of the decompusition-products, and, in return, the taking-in from the outside of 'crtain substunces, which give to the organism the material with which tw regenerate itself and grow by the formation of similar groups of atoms, i.e., by polymerisation. This is characteristic of all living substance. But that this peculiar complex motion cxisted in the incandescent mixtures of the earth and has suffered no interruption from that timo down to the present living substance, is in a high degree doubtful. Mixtures of this kind, which, as lava, can be observed at the present day in volcanoes, and there are still at so high a temperature that in flowing from a cleft of the crater over a precipic the present the wonderfully fascinating sprectivele of an incindescent waterfall--these extremely liquid mixtures, howercr mobile they may be, show no metabolism in the real sense, and hence should not be termed living. Nor can the original incandesent mass of the earth bo so termed deliberately, howerer impressive and suggestive Preyer's theory is. There then remains as the sole difference between Preyer's doctrine and that of spontineous generation the point involved in the very unessential question, whether living substance has come from lifeless substance gradually and by imperceptible transitions: or whether it has been formed more directly, as is the product of two bodies in a chemical reaction in a test-tube, and has taken on its charineteristic properties. In neither case will the conclusion be avoided that living substance once came from substances that are ('ustomarily termed lifeless.

\section{The Desent of Living Suldsture}

Upon the basis of the idlas dereleped by Pthigere we are now in a position to form in gross outline an approximate iclea of the origin of life upon the earth. The beginnings of living substance reach down into the time when the "arth's surface wis still incandesent. 'The compounds of 'ranugen then present constitute the essential miterial from which living substance took its urigin. With their property of realy decomprosition they wre forced into correlation with various kinds of compounds of carbon, whose origin was due likewise to the wreat heat. When water was precipitated in the form of liquid upun the "arth's surfice, these compounds entered 
into chemical relations with the water and its dissolved salts and gases, and thus originated living proteids, i.e., extremely labile compounds, which like other compounds containing the cyanogenradical are distinguished by their tendency toward decompositionand polymerisation, and which form the essential constituents of living substance. This first living substance, which was formed spontaneously out of lifeless substance, was very simple and showed no differentiations. It is very probable that it did not have the morphological value of cells, i.e., that its mass was not yet separated into different substances, such as nucleus and protoplasm, but rather was homogeneous in all its parts, as Haeckel assumes for his Monerc.

Such an idea of the origin of living substance has at present some degree of probability in its favour. It is quite possible that in the future it will be considerably modified in its details. Yet further speculation at present regarding the details is of little value, since the stage upon which living substance made its first appearance and the conditions then prevailing are known so indefinitely. But with living substance already present upon the earth we are upon firmer ground; for here is the point where the doctrine of descent, founded by Lamarck and Darwin, and developed especially by Haeckel, Weismann and their pupils, comes in and elucidates the farther history of this substance down to the present day.

It would lie outside the purpose of these pages to speak of the whole enormous complex of ideas that led to the founding of the doctrine of descent. It is sufficient to point to the chief factors, the correctness of which no thinking man of science at present doubts.

As is well known, the throry of descent teaches that all the multifarious organisms that live to-day and have lived at any time upon the earth's surface are derived in unbroken descent from the first and simplest living substance that originated from lifeless substances, and that, therefore, all organisms stand in true genetic relationship to one another. The continuity of the organic series during historic time needs no special proof; for simple observation shows that every organism is derived from another organism similar to it, that the continuity of descent is never broken. But for the long geological periods elapsing between the appearance of the first organisms and historic time, direct observation is naturally wanting. Here nature has preserved certain records in which are found entered, although more or less incompletely, the history of the evolution of the organic race.

The first record is deciphered by Palcontology, or the science of fossils. Fossils are the testimony that nature has laid down in the strata of the earth's crust regarding the existence and character of earlier organism.. By the study of fossils palæon- 
tology reconstructs to a certain degree the organic world that inhabited the earth's surface at the times when the strata were formed. Thus the ancestry of existing animals and plants is learned. It is seen that existing forms are very similar to those that occur in the latest strata; that the forms become more dissimilar the farther we go toward the earliest strata; and that large groups of organisms, which are now considered to be widely separated from one another, have in the older strata common ancestors, which combine in themselves the characteristics of several groups. In the very earliest strata are found lower animals and plants only, no vertebrates and no flowering-plants occur. For every one who is not wedded to a blind, supernatural faith concerning creation, and who does not prefer, in accordance with the biblical account, to think of every form of organism as proceeding byitself from the hand of a personal Creator, there is only a single natural explanation of all palæontological facts; namely, that the whole world of organisms, living to-day and living in the past, forms a single, great genealogical tree, the germ of which was the first living substance that appeared upon the earth. This germ developed into a mighty growth with innumerable branches and twigs and leaves; its last shoots are seen in the organisms of to-day, its older branches lie buried in the earth. Unfortunately, the palæontological record is very imperfect; for, on the one hand, only a very small fraction of the earth's strata is accessible to investigation - the greater portion of the crust is covered by the sea; and, on the other hand, the preservation of organisms is very incomplete, since they can be imbedded only under very definite conditions without becoming destroyed by the impact of the waves, by decomposition, etc. In fact, organisms that did not possess protecting skeletal parts have been preserved hardly at all, because their delicate bodies disintegrated immediately after death. It thus comes about that in the investigation of the oldest and simplest organisms, which possessed no protecting skeletal parts, the palæontological record fails.

Comparative anatomy deals with the second record, which is presented in the homologies of the individual organs of existing organisms. By the dissection of organisms into their smallest parts and by the comparison of individual organs and systems of organs belonging to different groups of organisms, comparative anatomy establishes the fact that as regards their essential organic systems certain groups of organisms agree with others to a certain extent. This fact can be interpreted rationally only by the assumption of a natural relationship between such organisms; in general such a relationship is closer, the more homologies occur, and the more remote, the more differences are present; for the homologies can be due only to the fact that at some time in the early past the organisms had common 
ancestors which possessed the features in question. Of course the record of comparative anatomy is also very incomplete, for existing organisms are only the surviving tips of the various twigs of the genealogical tree, between which the other twigs and branches have perished. But here the palæontological record supplements the facts of comparative anatomy up to a certain degree very satisfactorily,

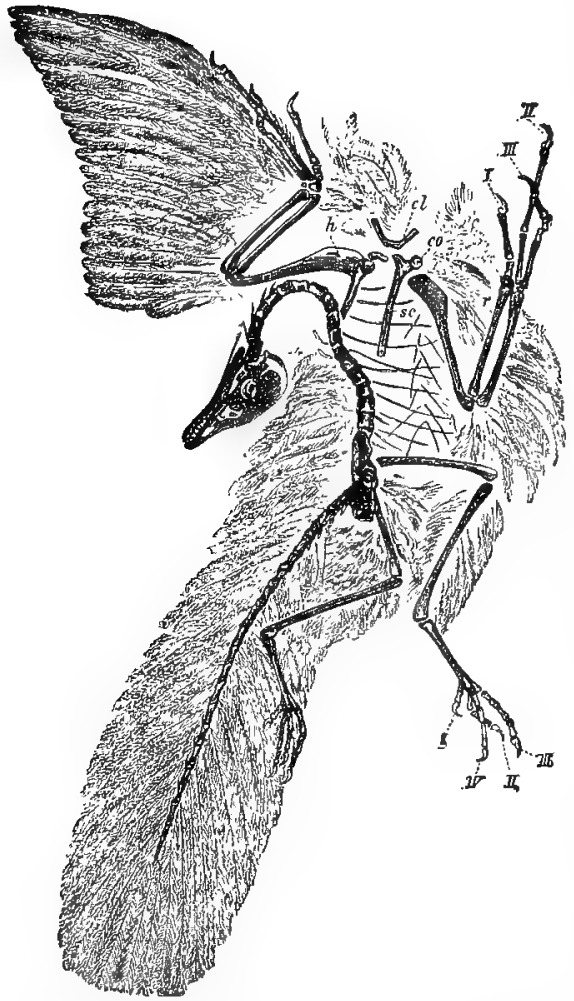

FIo. 134.-Anchaopteryx machume, s. lithographicus. cl, Clavicle ; $c o$, coracoid ; $h$, humerus; $r$, radius; $\imath$, ulna; $c$, carpus; $s c$, scapula; $I .-I V$. digits. (After Zittel.) by making the dead branches accessible to comparison with the still living ones. An example will illustrate this. Upon comparative - anatomical grounds the conviction was formed that birds stand in very close relationship to reptiles, but forms that might be considered as common ancestors of the two or were close to their ancestors were not known. There was then discovered in the quarries of the lithographic slates of Solenhofen a fossil animal about the size of a pigeon, the now wellknown Archceopteryx macrum characteristics of both bird and reptile; it had the jaws of a lizard with tecth, the spinal column of a lizard, and a long lizard-like tail; but its whole body was covered with bird's feathers, which were inpressed upon the

rock most delicately (Fig. 134). By this and similar palæontolugical discoveries the kinship of the birds and the reptiles, which was inferred from comparative anatomy, was very brilliantly confirmed. Similar examples may be cited in great number.

Finally, embryology, or individual germinal development (ontogeny), deals with the third important record of descent. As is well known, the germs of plants and of animals from their simplest condition, the egg-cell, pass through al long series of develop- 
mental stages before they come to resemble the mother from which they are derived.

Since ancestors transmit their characteristics to their descendants, these developmental stages become of extraordinary importance in gaining a knowledge of the ancestral series; for, since they represent, in gross, forms inherited from ancestors, they indicate, although in rude outline only, the developmental forms that have once appeared in succession in the ancestral series. In other words, the forms that appear in the germinal development or ontogeny of an individual recapitulate in gross the series of forms of the ancestors of the organism in question. This fundamental law of biogenesis, which was founded by Haeckel, and which has been discussed in detail elsewhere, ${ }^{1}$ enables us, by means of a critical examination of the ontogenetic development of an organism, to reconstruct to a. certain degree its phylogenetic descent.

From all these facts of palæontology, comparative anatomy, and embryology-for the full appreciation of which reference must be had to the works of Darwin, Gegenbaur, Haeckel and their pupils, which have laid the foundation for an understanding of them-the conclusion must necessarily be drawn that existing organisms are derived in uninterrupted descent from the first living substance that originated from lifeless substance. Moreover, at the same time the path is indicated that has been taken by living substance in its development upon the earth. The phylogenetic research of modern morphology has succeeded in discovering this path in general, and thus reconstructing in its gross outlines the genealogical tree of organisms. Although much opposition was expressed at first to the provisional scheme of genealogy that Haeckel presented thirty years ago as an induction from the facts then known, there are few morphologists now who do not accept Haeckel's idea in its essential points. There now prevails essential agreement regarding the phylogenetic relations of the large groups of organisms, although, as to the smaller groups and the special relations, many far-reaching differences of opinion still exist; the latter will be set aside only gradually and by new discoveries. In accordance with Haeckel's ideas and upon the basis of the present condition of its knowledge, modern morphology has pictured somewhat as follows the genealogical tree of organisms: 
Metaphytu

(Plants)
Metazoo

(Animals)
Angiospermas

(Plants having

covered seeds,

common flower.

ing-plants)

Gymnosperma

(Plants having

naked seeds, conifers)

Filicinece.

(Ferns)

Muscinea

(Mosses)
Echinodermata Arthropoda Tunicata Mollused, (Starfishes, sea. (Crabs, in- (Tunicates) (Clams, \begin{tabular}{l|l} 
urchins, etc.) sects, etc.) & snails, etc.)
\end{tabular}
Vertebrata

(Back-boned animals)

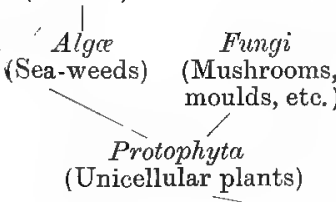 \\ $\underbrace{\begin{array}{c}\text { Fungi } \\ \text { (Mushrooms } \\ \text { moulds, etc. }\end{array}}_{\begin{array}{c}\text { Algce } \\ \text { (Unicellophytar plants) }\end{array}}$}

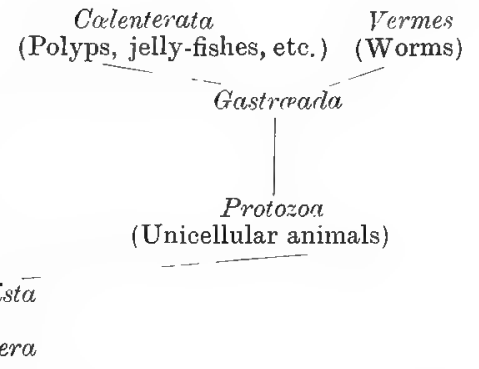

Scheme of the Genealogical Tree of Organisms.

From the first living masses, which Haeckel terms Monera, there were developed, by differentiation of the homogeneous substance into nucleus and protoplasm, the first unicellular organisms, Protista. The Protista constitute the group from which, on the one side, plants, and, on the other side, animals have been developed; they comprise the lowest organisms now living. Even among the Protista a differentiation as to metabolism took place, and they were divided into the Protophyta, i.e., those having plant-metabolism, and the Protozoo, i.e., those having animal-metabolism. The former continued to construct their living substance out of inorganic substances, while the latter simplified their metabolism by employing the organic substance prepared by the former. From the Protophyta are derived all plants (DIet $\alpha$ phyta), from the Protozoa all animals (Metrizoa), as follows:-From the Protophyta two branches went off, the sea-weeds (Algce) and the moulds, etc. (Fungi). Of these two the former group developed, and from it arose in direct descent the mosses (Muscinece), from them the ferns (Filicinece), from the ferns the plants that have naked seeds (Gymnospermoe), and from the latter, finally, the plants that have covered seeds (Angiosperma). The last group shows the 
highest differentiation of the plants. From the Protozoa, on the other hand, arose the Gastroacala, very simple animals consisting of only two layers of cells (entoderm and ectoderm). Probably no representative of this group is now living, but their presence in the genealogical series must necessarily be inferred from the very general appearance of the gastrula-stage in the development of all animals. From the Costrceade developed on the one side polyps, jelly-fishes, etc. (Colenterata), and upon the other side worms (Vermes). The latter gave origin to four groups comprising respectively star-fishes, sea-urchins, etc. (Echinodermata), crabs, insects, etc. (Arthropode), tunicates (Tunicuta), and clams, snails, etc. (Mollusca). Of these the tunicates became the progenitors of the back-boned animals ( Vistcbrita), the most widely differentiated representatives of the inimal kingdom. The present living organisms form merely the last shoots of all the branches of this great genealogical tree.

A glance over the racial development of organisms from their first appearance down to the present time shows that living substance has undergone in the course of the earth's development a remarkable change in form and organisation; in these respects. existing organisms are widely differentiated in very different directions.

Darwin's theory of selection has afforded a natural explanation of this phenomenon. This theory proceeds from the fact that all individuals of the same species, even descendants from the same pair of parents, differ from one another more or less markedly. This phenomenon is known as individualvariability, and is the result partly of sexual intermixture (Weismann's amphimixis) and partly of the action of various external influences upon the germ-plasm of the individual embryos, whether within or without the maternal organism. Of these more or less different individuals of the same generation Darwin shows that in the stm mggle for existence only those continue to live that are best fitted to the external conditions of life, while those that are less fitted perish as a result of the competition with the former. Thus, only those that are best adapted to the existing external conditions can reproduce and transmit their characteristics to their descendants. In this survival, in this selection of the fitter individuals, lies the natural selection of Darwin; and it is evident that with the continuance of the process organisms nust become adapted to existing vital conditions very perfectly. Hence the form, the organisation, and, in general, all the characteristics of living substance are in the closest correlation with the external conditions upon the eirth's surface; if these change, the characteristics of organisms must correspondingly change.

But it is a question whether, in the course of time, natural selection is the sole factor that causes organisms to change. 
Adaptation to external conditions as a result of selection presupposes a continual inheritance of innate characteristics, and Weismann $(92,1)$ holds the view that the inheritance of innate characteristics alone comes into the question of change in the organic world. Since Darwin believed that acquired characteristics also are transmitted, Weismann, as the defender of the onesided theory of selection, is, in a certain sense, more Darwinian than Darwin himself. Others, such as Haeckel ('66), Eimer ('88), and Herbert Spencer ('93), are also of the opinion that the inheritance of such characteristics as are acquired during the individual life is of great importance in the transformation of organisms. Naturally there always arises here the question whether these characteristics are properly adapted to external conditions or not. If not, they likewise are soon set aside by selection in the struggle for existence. But at the present time the question whether only innate or also acquired characteristics are inherited, constitutes the point of chief interest for those who theorise upon heredity; and, in spite of much discussion, it still waits for a definitive answer. ${ }^{1}$

If, finally, a brief examination be made of the nature of the changes that living substance has undergone from its origin down to the present, the fact appears that it has developed from simple to constantly more complex forms and organisation. The result is that the most complex organisms occur at the present time, being represented by the flowering-plants and the vertebrates, in which special parts have become widely differentiated for the exercise of very special occupations. It has frequently been said that in the developmental series of organisms from the earliest beginnings down to the present there may be seen a continual advance-a progressive perfecting. This idea embraces an error which it was the whole endeavour of the Darwinian theory to avoid, viz., that of teleology. The conception of advance, of perfecting, involves a goal toward which the advance is directed. Without this it is an empty conception. In reality, however, there does not exist in the development of organisms a predestined goal toward which the development is striving any more than in any chemical reaction. Organisms can only follow, and must follow in a definite direction, when the proper external conditions are present. Changes in them are dependent solely upon changes in their environment. The employment, therefore, of the idea of advance or perfecting is evidence merely of an anthropocentric standpoint; we introduce ourselves into the development as the goal. For whatever reason this is done, the goal is an artificial thing which does not exist in nature; the assumption that mankind is more perfect than an amoba is not justified by reality. It is simply a conventionality to call development a perfecting. ${ }^{1} C f$. p. 180. 
Nature itself has no goal to strive for, its method is eternal development, i.e., change without cnd.

To draw, now, the final conclusions from the above discussion, the fact stands out clearly and distinctly that life from its beginning on has been dependent upon the external conditions of the earth's surface. In a mathematical sense, life is a function of the earth's development. Living substance could not exist while the earth was a molten sphere without a solid, cool crust; it was obliged to appear with the same inevitable necessity as a chemical combination, when the necessary conditions were given; and it was obliged to change its form and its composition in the same measure as the external conditions of life changed in the course of the earth's development. It is only a portion of the earth's matter. The combination of this matter into living substance was as much the necessary product of the earth's development as was the origin of water. It was an inevitable result of the progressive cooling of the masses that formed the earth's crust. Likewise, the chemical, physical and morphological characteristics of existing living substance are the necessary result of the influence of the external conditions of life upon the internal relations of past living substance. Internal and external vital conditions are inseparably correlated, and the expression of this correlation is life.

\section{The History of DeATH}

Our consideration of vital conditions culminated in the fact that vital phenomena not only can exist, but must appear with the same inevitable necessity as every other natural phenomenon, when a certain complex of conditions is fulfilled. If these conditions are wanting, life is wanting.

The appearance of life upon the earth was one consequence of this fact. Another consequence, which is now to be considered, was the development of death.

\section{A. THE PHENOMENA OF NECROBIOSIS}

If one or more of the special vital conditions under which an organism exists fail, vital phenomena cease, life comes to a standstill. Excepting the few cases of apparent death, this standstill is always real death. But, as has already been seen, ${ }^{1}$ death never appears instantaneously. There is no sharp limit separating Jife and death, there is rather a gradual transition between them; in other words, death undergoes development. Normal life upon the one 
hand, and death upon the other, are merely the remote end-stages in this development, and are united to one another by an uninterrupted series of intermediate stages. The two end-stages may be easily and sharply distinguished, but it is impossible to draw a sharp line at the place where life ceases and death begins. Hence this transition from life to death is termed necrobiosis, a word that was introduced into pathology by K. H. Schultz and Virchow. Virchow ('71) distinguishes between necrobiosis and necrosis by means of external characters, speaking of necrobiosis when the original form of the part in question is completely destroyed and done away with, and of necrosis when it is still retained in death. But, however practicable this external difference may be in the judgment of gross relations, of whole organs or tissues, it has little importance theoretically, for whether the end-result assumes this or that form frequently depends upon wholly accessory matters. If, e.g., a cell has a solid wall, its form long remains, although the protoplasmic body may long since have perished; but if its protoplasm is naked, the cell usually disintegrates into a formless mass of granules; nevertheless, the essence of the process that leads to death may be the same in the two cases. Hence it seems advantageous to lay aside this distinction and so to extend the conception of necrobiosis that it may include also the so-called necrotic processes. There is then understood by necrobiosis those processes that, beginning with an incurable lesion of the normal life, leads slowly or rapidly to nnavoidable death. The frequent synonymous conception of degeneration has the disadvantage that it has more than one significance and is employed for many very different phenomena.

The phenomena of necrobiosis introduce a subject which, on account of its enormous practical importance, has been developed as an independent science and has assumed large proportions; this is pathology, the science of diseases. The following considerations will, therefore, largely pertain to this subject, and an endeavour will be made to analyse the death-process.

Since the cell is the proper seat of life, it must be the object. of study in the investigation of necrobiosis as in that of vital phenomena. The death of compound organisms with their widely differentiated organs and tissues depends simply upon the death of the individual cells composing the cell-community. But the phenomena that lead to death are very different in the individual forms of cells. This depends partly upon the condition of the living substance that characterises each form, and partly upon the nature of the causes that lead to the death of the cell. It is, therefore, evident that necrobiotic phenomena must be very manifold. Nevertheless, they can be brought into two great groups, which differ fundamentally from one another. In one group the normal vital processes drop out gradually without under- 
going an essential change; these phenomena may be termed histolytic processes. In the other group the normal vital processes are turned into a perverse course by the fatal lesion, and degenerate before they come to a complete standstill. These are termed metamorphic processes.

\section{Histolytuc Processes}

The simplest forms of the histolytic processes are the atrophies. They are mostly chronic processes, and consist in the gradual constant decrease in extent and final complete cessation of the ascending phase of the metabolism of the cell in question, that is, of the processes that lead to the construction and regeneration of living substance. The result is that the living substance, continually undergoing decomposition in a certain measure, loses in volume constantly; the cell becomes constantly smaller, until finally the remnant, having come to an extreme, disintegrates -technically speaking, the cell or the tissue "atrophies."

Cases of atrophy of an organ or tissue are wide-spread in the organic world, and play a great rôle both in the normal development of animals and in pathological conditions.

Among those that appear in the development of the normal organism and are especially well known are the phenomena of histolysis or degeneration of embryonic organs, which are particularly characteristic of animals that have a pronounced metamorphosis or larval development. These histolytic processés have been carefully followed recently in the atrophying tail of the tadpole of the frog by Looss ('89). In its essentials histolysis follows a corresponding course in different forms of cells. There is noticeable first a loosening of the cement-substance that unites the cells together into the tissue, so that the cells adhere to one another less closely. During this a visible change begins in the protoplasm. "The cell-substance gives up its normal characteristic structure. The spongioplasm, present originally in the form of a more or less pronounced spongy framework and usually capable of staining intensely, draws itself together, the individual strands become thicker, and, finally, the whole disintegrates into a larger or smaller number of spherical droplets, which lie within the hyaloplasm. The latter stains less or not at all, and has likewise come together into a homogeneous mass." The ground-substance in which the globules lie first begins to dissolve, and later the globules themselves become liquefied. Thus, finally, of the whole protoplasm there remain only a few insoluble granules, and these are devoured by the leucocytes which creep about as phagocytes in all tissues. The nucleus of the cell usually resists destruction considerably longer, but finally becomes the victim of a similar process. Its ground-substance disappears very 
soon; the chromatic substance and the nuclear membrane gradually shrink together and disintegrate into single fragments, which likewise are finally dissolved. The muscle-fibres, though in other respects very different, behave similarly. The individual fibrillæ swell and become cemented to one another. At the same time

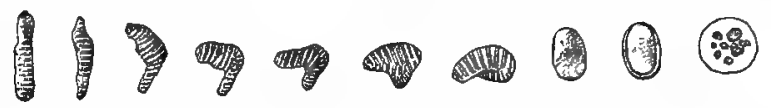

FIG. 135.-Histolysis of muscle-fibres in the tail of the larva of the frog. (After Looss.)

the isotropic and the anisotropic substances begin to mingle together, so that the cross-striation gradually disappears. The double refraction of the anisotropic disks also fades away. At the same time the fibres disintegrate into small round fragments, which finally undergo solution (Fig. 135). The processes of histolysis go on in a wholly analogous manner in most other cases, e.g., in the degeneration of the larval organs of insects, the muscles of the salmon, and the thymus-glands of human beings. But from the investigations of Metschnikoff ('83), Kowalevsky ('85, '87), and others, it appears that in many insects, especially in the fly-larva, where the degeneration of the larval tissue proceeds uncommonly
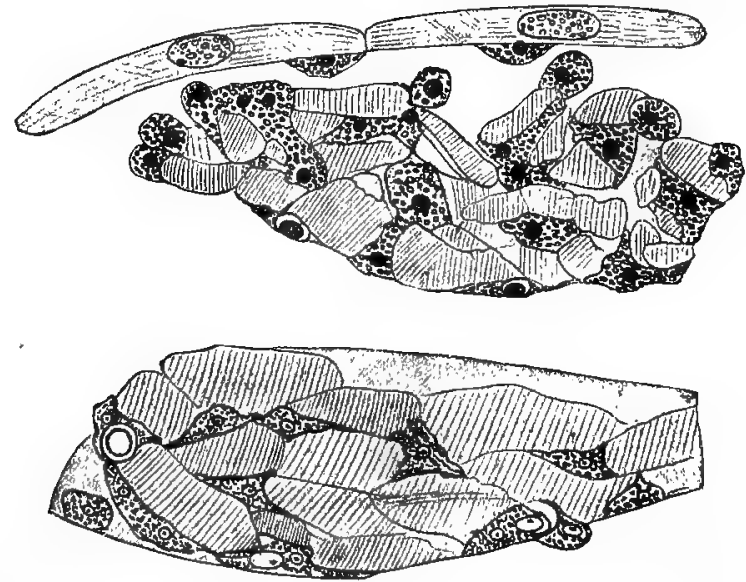

Fic. 136.-Fragments of muscle-fibres in the metamorphosis of the fly-larva, destroyed by leucocytes. The darker, granular cells are the leucocytes. (After Kowalcrsky.)

rapidly, the histolysis is performed chiefly by the leucocytes, which as phagocytes devour the tissue-cells that have not yet disintegrated (Fig. 136). It must be supposed that here also the inauguration of histolysis proceeds from the tissue-cells themselves, and that the leucocytes devour the cells that are already beginning to atrophy. 
The whole difference lies in the fact, as has been set forth by Korotneff ('92), that where a very rapid disappearance of the tissue is concerned, the leucocytes exercise greater activity and begin their work earlier. Among the atrophies in normal life belong, further, the phenomena of senile atrophy, which consists in a very slow and constantly progressive degeneration of the various tissues, and is never wanting in extreme old age.

Next to the normal atrophies are the pathological ones, which appear in the organism when diseases have created the proper conditions for them. Thus, e.g., in human beings the muscles of the leg atrophy when, as a result of disease, the knee-joint has become ossified and immovable. Such atrophies, which occur as a result of disuse of the organ, are termed, simply, atrophies from disuse. In these pathological atrophies the processes are, in
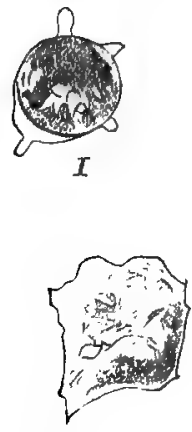

$\checkmark$
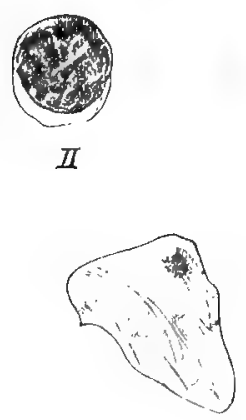

V
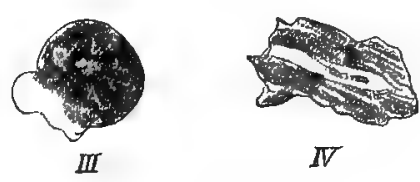

IV

FIa. 137.-Degeneration of leucocytes in acute leukremia. $I$ and $I I$, Normal leucocytes; the dark mass is the cell-nucleus, the clear border, the protoplasm. III-VII, Stages of the dissolution. (After Gumprecht.)

general, the same as in normal ones; nevertheless, at times remarkable phenomena appear. Thus, in muscles that have atrophied because of disease, a very great increase of nuclei is frequently found, while Looss was able to determine with certainty that in the muscle-atrophy of the histolytic tail of the tadpole the nuclei were neither increased nor diminished. Further, the tissues atrophying because of disease are at first, as a rule, much more solid and compact than those that undergo normal histolysis -a circumstance that is perhaps based upon the considerably longer duration of the pathological atrophy, during which the dissolved masses have more time to be discharged. But these are all special, accessory factors.

The degeneration of leucocytes has recently been followed in detail especially by Gumprecht, ('96) in acute leukæmia. It is interesting, since the dissolution of the nucleus takes place in a 
very simple manner. The nuclear membrane disappears, the contents of the nucleus mix with the protoplasm, the chromatic substance becomes gradually paler, until the whole leucocyte becomes a homogeneous mass, which disintegrates with swelling and formation of vacuoles (Fig. 137).

To the atrophies may be added a series of death-processes, which, although they have little similarity to one another, are grouped in pathology under the common name of necroses. ${ }^{1}$ In general they have a more acute course than atrophies.

Among the various necrotic processes several important forms can be distinguished, which are characterised by definite peculiarities. One of these is mummification or dry gangrene. In this

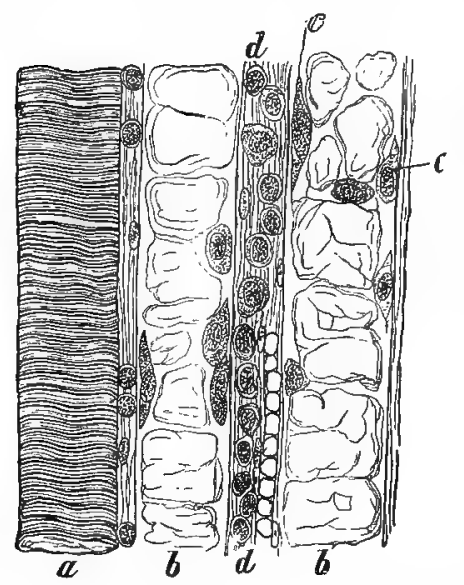

F1G. 138. - Waxy degeneration of muscle in typhoid fever. a, Normal cross-striated muscle-fibre; $b$, fibres disintegrated into waxy flakes; $c$, muscle-nuclei; $d$, connective tissue. (After Ziegler.) the tissue-cells shrink into solid, leather-like masses on account of a loss of liquid, so that when the process has reached its end the tissues appear dry, hard and friable. Mummification occurs normally in the drying-up of the remnant of the umbilical cord of the new-born child; in fathological conditions, as after burning or freezing the ends of the fingers and of the toes; particularly in old age; and also in the dryingup of embryos that develop in the abdominal cavity of the animal or the human being instead of in the uterus and, being incapable of birth, die within the body of the mother. Such embryos assume gradually a hard, mummylike consistency, because the liquid contained in them is absorbed by the mother's body. A second important form of necrosis is coagulation-necrosis, first investigated in detail by Weigert ('75, '77, '78, '80), which consists in the coagulation of the proteids of the tissue-cells in question. With the coagulation-necroses may be classed the usual rigor of dying muscles, which along with gradual contraction transforms the muscles into stiff organs and causes the rigidity of corpses. Weigert himself does not allow this classification, regarding the co-operation of lymph as essential to the occurrence of the coagulation-necrosis. But the process in rigor mortis, although transitory, is the same in principle: for the myosin, the proteid that is characteristic of and contained in solution in the living muscle, coagulates in dying and thus produces the stiffen-

\footnotetext{
' ( $f$. Cohnhein ('77-'si) and Ziegler (95).
} 
ing; as a result of other transformations in the muscle the rigor passes away, this process being accompanied by muscular relaxation. A typical coagulation-necrosis in Weigert's sense occurs in muscle under pathological conditions, especially in connection with fevers, such as typhoid; this is the so-called maxy degeneration, which consists in a coagulation of the muscle-substance with loss of its cross-striation and a separation into waxy-appearing flakes (Fig. 138). Similar coagulation-processes occur in other tissuecells, especially in active inflammations of the mucous membranes, as in pharyngeal diphtheria. Finally, among the coagulationnecroses in the wider sense there can be classed the phenomena of cell-death that appear when, for the purpose of

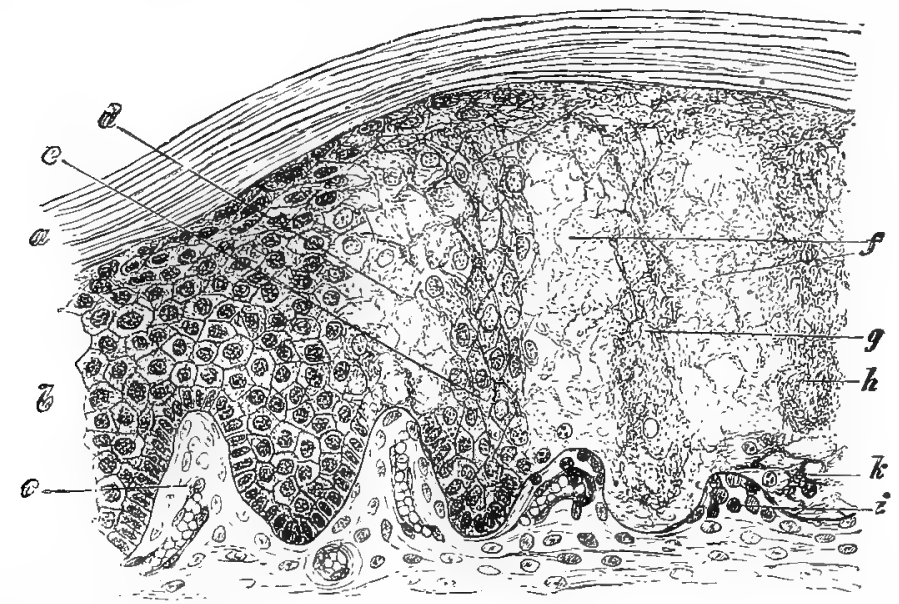

T'IG. 139.-Liquefaction at the edge of a blister caused by burning. ft, Horny layer of the epidermis ; $b$, rete Malpighit of the epidermis ; $c$, normal papilla of the dermis; $d$, cells swollen and already partly liquefied ; $e$, partly normal cells ; $f$, liquefied mass ; $/ /$ and $h$, swollen cells with nuclei destroyed; $i$, sunken papille; $k$, coagulated exudation. (After Ziegler.)

anatomical or histological preservation, living tissue is placed in liquids that cause coagulation, such as mineral acids, alcohol, sublimate, etc. These are the most acute cases of cell-dcath, and for this reason these liquids are especially well-fitted for killing and preserving. By their application the living cell is killed suddenly; it thus has not time to undergo extensive change, but in a moment is fixed in a condition very similar to that of life. In a third form of necrosis, liquefuction, the tissue-cells become completely liquefied, their protoplasm disintegrating into a granular detritus and the nuclei and cell-boundaries dissolving until the tissue is changed into a thickish liquid. Such softenings occur especially in the formation of blisters after burning (Fig. 139), and frequently combine with coagulation-pheno- 
mena. Not rarely, different forms of necrosis occur combined, and they become complicated especially by secondary factors, such as putrefaction. The latter is the case with moist gangrene, decay, etc., all of which are produced by the action of putrefactive bacteria upon necrobiotic tissue, and some of which represent postmortem phenomena. Further, certain other forms of necrosis have been more or less identified by pathology, but these pathological classes are distinguished much more by the macroscopic phenomena of the end-result than by the microscopic events in the cell itself. The former naturally depend upon various kinds of accessory circumstances that are not immediately conditioned by the pure phenomena of cell-death.

Finally, one more series of phenomena may be added to the atrophies and necroses; these accompany the death of cells living in aqueous media and are wide-spread among organisms; they are the phenomena of granular disintegration. ${ }^{2}$ The one thing held in common by all kinds of granular disintegration is that at the end of the process the cell in question forms a more or less loosely coherent mass of individual granules.

Granular disintegration can be observed most easily in many Infusoria, when their protoplasm is especially rich in water. This is the case in the large, cylindrical Spirostomum ambigurm which has a soft, superficial layer of exoplasm. If such Infusoria be wounded by being cut into two pieces under the microscope, it happens very frequently that the pieces disintegrate away regularly from the surface of the wound. Death can be followed by the eye, and its course resembles that of a spark that passes over a fuse and leaves behind it merely a loose mass of ashes. It creeps over the whole body, seizing upon particle after particle, surprising cilium after cilium in normal activity, and forcing them directly from active life into a standstill, until that which a moment before was in active motion is changed into a dead mass of granules (Fig. 140).

These very acute cases in infusorian cells, which interest every observer who sees them for the first time, are not well-adapted to a study of the more delicate protoplasmic processes, since, with the protoplasm already very granular, it is difficult to decide how far the granular material of the disintegrating masses consists of the preformed granules, and how far it is formed directly as such by the process of death. In this respect many Rhizopoda, such as the marine Hyalopus Dujardinii (Fig. 141, I), which are completely hyaline and absolutely free from granules, are extraordinarily suitable. If one of the smooth, clear pseudopodia be cut off by a knife under the microscope, it begins gradually to undergo granular disintegration from the place where it was cut (Fig. 14i, $I I$ and $I I I$ ). Then, either very soon or in the ${ }^{1} C f$. Verworn $(96,1)$. 
course of a few hours, the time varying according to the thickness and size of the piece, there is seen in place of the transparent protoplasmic mass, a collection of small granules and globules, between which lie isolated, larger, round droplets of hyaline protoplasm (Fig. 141, III, D, b), and sometimes one or more faint, round, transparent bubbles (Fig. 141, III, D, a), all being loosely held together by a very delicate viscous mass. There is no doubt that this collection of granules and globules has arisen by the transformation of a mass of living substance that originally was wholly clear. In the study of this process with stronger magnifying powers an interesting fact appears. In the normal life of the cell a characteristic difference in the behaviour of the protoplasm of the pseudopodia of the Hyalopus during the phase of expansion and that of contraction may be recognised. While during the former, i.e., extension, the protoplasm appears completely homogeneous, during the latter it assumes the typical alveolar structure of Buitschli, ${ }^{1}$ and, if the contraction becomes very strong, as after. stimulation, the protoplasm becomes uneven and knobbed upon the surface (Fig. 141, $V$ and $V I$ ). Exactly the same phenomenon appears in the development of granular disintegration. The protoplasm begins to assume the alveolar structure; then the alveolar walls are gradually drawn together in uneven and lumpy masses; they burst here and

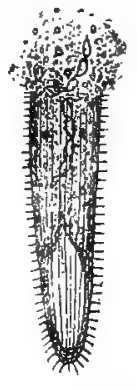

I

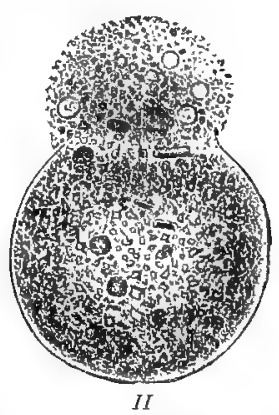

FIG. 140.-Grumular disintegration. l, Piece of a spirostomam disintegrating from the wounded place. II, Pelomysu disintegrating as the result of overstimulation upon one side. there, and become rounded off into small globules and droplets; these are held together in a loose, granular heap merely by the viscous liquid of the burst vacuoles, which frequently flows together into a large, viscous drop (Fig. 141, IV). Thus, granular disintegration depends upon a supramaximal contraction.

This fact is of great interest, for, if the histolytic processes be followed comparatively in different cells, it is found to be a common law that all elements, the contractility of which can be clearly expressed, and hence especially all naked protoplasmic masses, such as Rhizopoda, protoplasmic drops from tissue-cells, contractile fibrillx, muscle-fibres, etc., without exception die in the phase of contraction. Amoba and leucocytes (Fig. 142) in necrobiosis, as in every contraction, assume a more or less conpletely spherical form (Fig. 142, B). Rhizopoda possessing long 

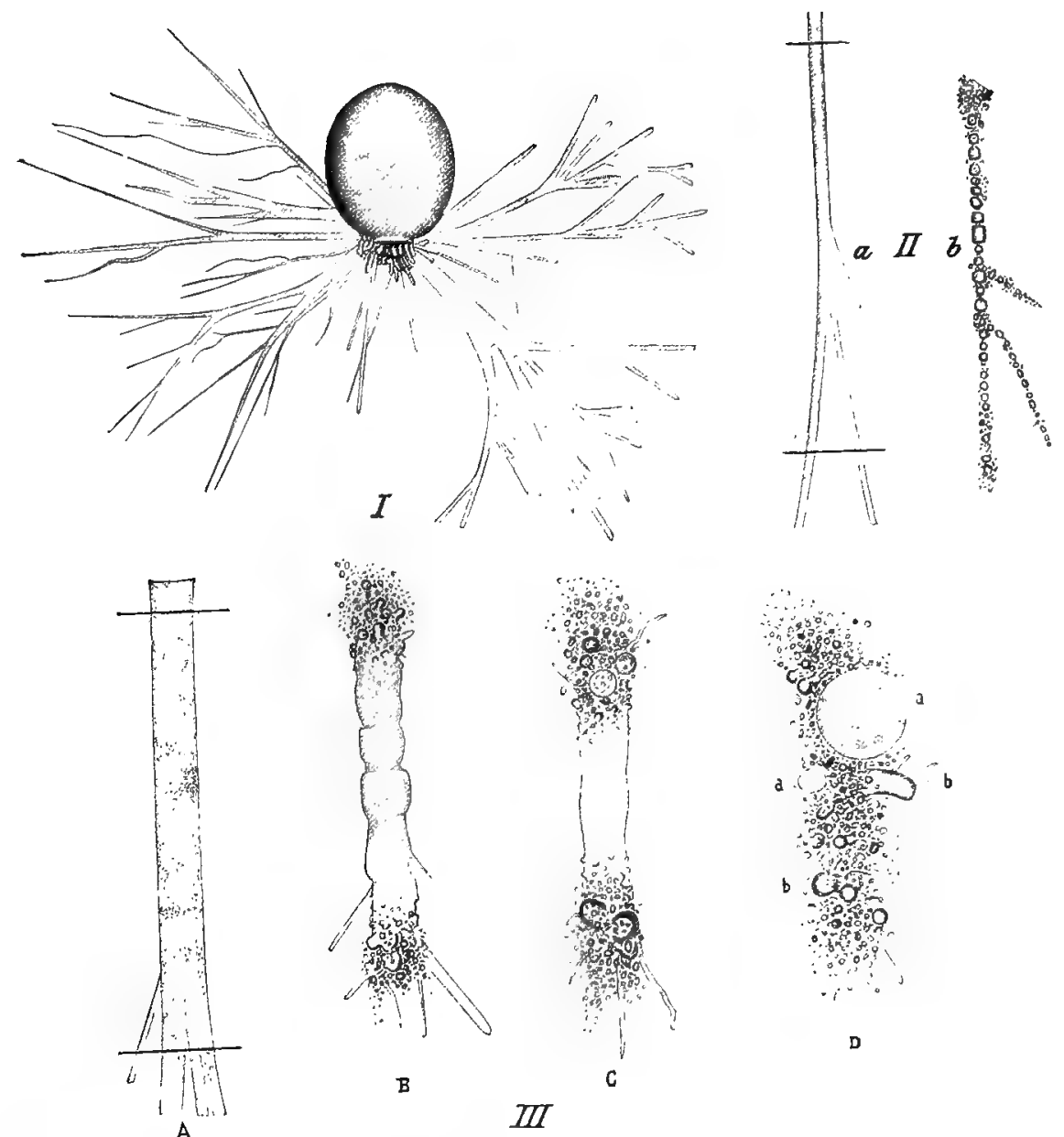

D
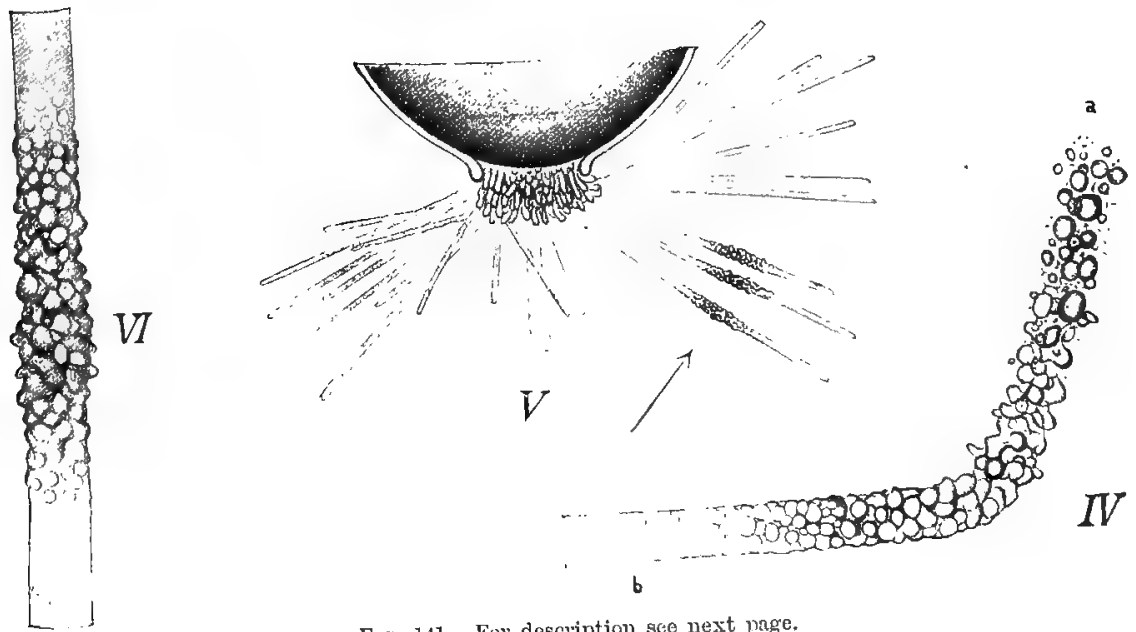

Fia. 141.-For description see next page. 
Frr, 141.- Hyalopus (G,omia) Dn, (molinii, granular disintegration. 1, Whole individual ; numerous pseudopodia are extended from the ogg-shaped, membranous shell; at the left they are being drawn in. $I I$ and $I I I$, "Pseudopodia cut off; granular disintegration is developing; the globules and droplets of protoplasm are held together simply by a loose, viscous connectingmass; between them lie scattered larger droplets of hyaline protoplasm $(I I I, D, b)$, and viscous globules $(I I I, D, a)$. IV, Pseudopodium which has been cut off at $a$, and from that point on is undergoing granular disintegration, highly magnified; at a the granular disintegration is completed, the globules are soparated ; at $b$ disintegration is beginning, beings ushered in by the formation of vicuoles; between these two points occur all transition-stages. $\Gamma^{r}$, Opening of the shell of Hyalopus, with extended pseudopodia; three have been stimulated at the place indicated by the arrow and have assumed an irregular contour. $V I$, Place of stimulation of a pseudopodium strongly magnified; vacuoles are shown, the protoplasm of whose walls is înegularly contracted. Comparison with $I V$ shows the agreement of the two.

Jiseudopodia draw in the latter and become lumpy, or the threadlike pseudopodia become varicose and disintegrate into small globules (Fig. 143). Bits of protoplasm from the interior of cells that have a constant form, e.g., plant-cells or tissue-cells, or even from free-living cells, always become rounded into spherical drops (Fig. 34, a, p. 94). Contractile fibrillæ and muscle-fibres pass into rigor mortis, i.e., they contract for the last time (p. 133), and only when the rigor has passed away, when death is completed, do
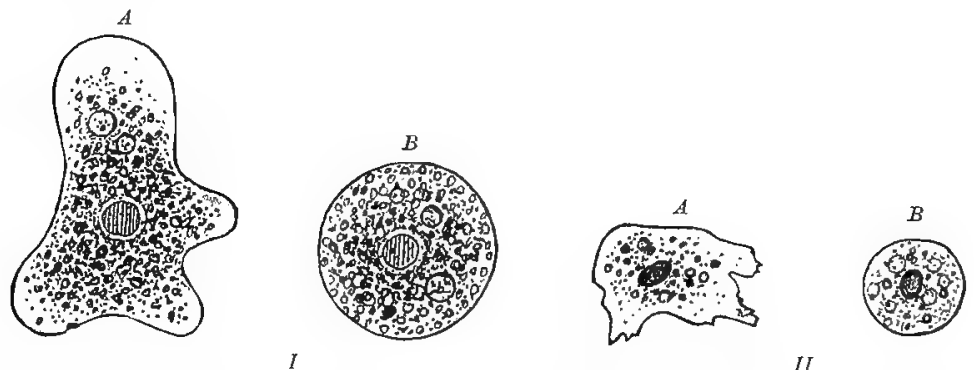

II

Fra. 142. $-I$, Amoeba ; $A$, normal ; $B$, in necrobiosis. $I I$, Leucocyte; $A$, normal; $B$, in necrobiosis.

they become again passively extended by the action of elastic elements. In brief, it is found everywhere that protoplasm whose contractility can in any way be expressed dies in the condition of contraction.

It would be of value to determine, by a comparative investigation of necrobiotic phenomena, still other peculiarities common to histolytic processes. As Israel ('97, 1, 2) has rightly emphasised very recently in his researches upon the death of the cell, especially the kind of death and the duration of necrobiosis should be studied. Only through the comparative history of death can an understanding of necrobiotic phenomena, which is now largely wanting, be hoped for in time, and with it will come an advance in our knowledge of the vital process itself. 


\section{Metamorphic Processes}

In contrast to simple histolytic phenomena, metamorphic processes are very clearly characterised by the fact that the metabolism of the cell does not merely come gradually to a standstill, but is previously turned into a perverse course, in such a way
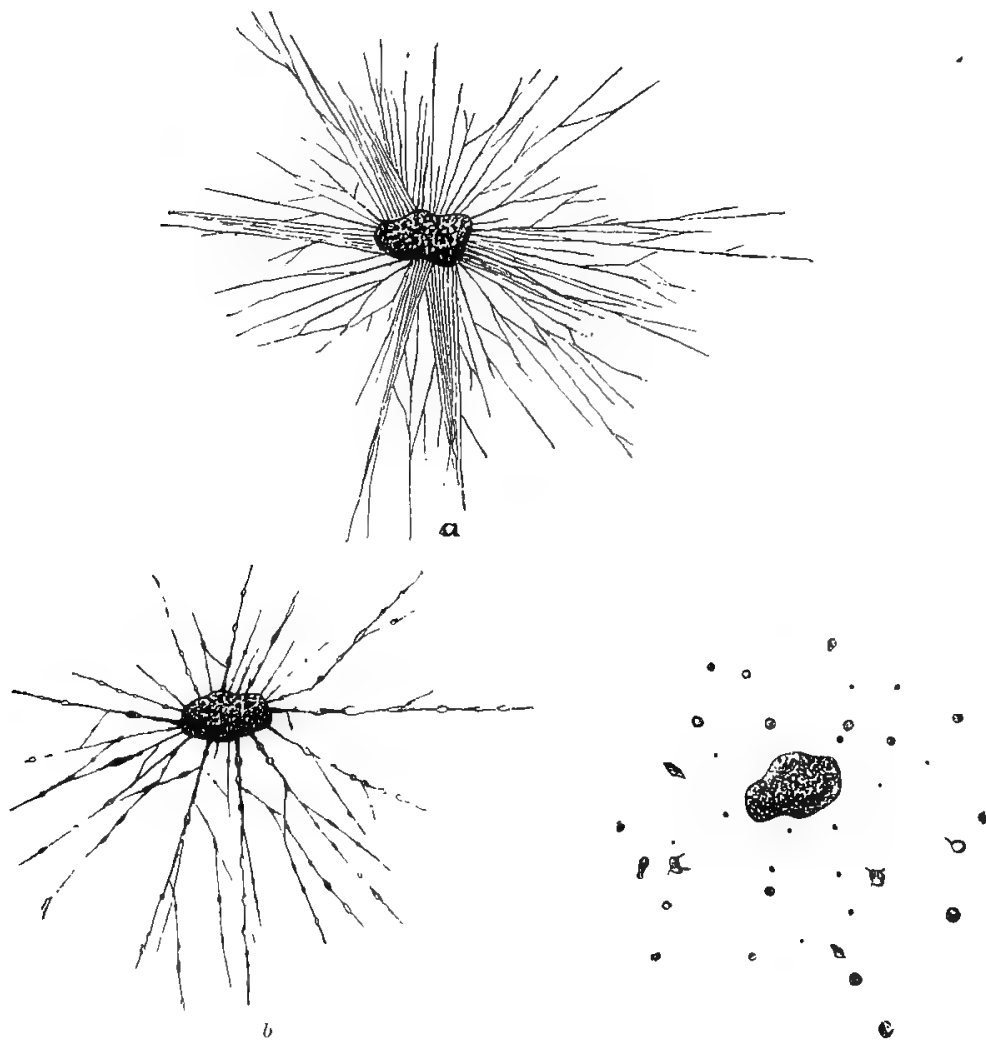

FIG. 143.-Necrobiosis of a non-nucleated protoplasmic mass of Orbitolits ; $a$, the protoplasmic mass has put out still normal pseudopodia; $b$, the pseudopodia are beconing varicose and partly drawn in; $c$, the protoplasm of the pseudopodia that are not drawn in has disintegrated into drops and globules.

that substances which in the normal cell are either not manufactured at all or appear only as intermediate stages, are produced in quantity as a result of the disturbed metabolism, and necumulate within the cell until the latter perishes. The forms of metamorphic processes that are most frequent, best known, and for physiology most important, are fatty degeneration, or fat- 
metamorphosis, mucous degeneration, amyloid degeneration, and calcification.

To consider first the phenomena of fat-metcmonphosis, we must avoid confounding these with apparently similar processes, viz., the deposition of fat or fatty infiltration in fattening, obesity, etc. In these latter also there is a great accumulation of fat in the cells. in question, but this fat has not arisen by a disturbance of the inetabolism of the cells themselves; on the contrary, it or its constituents has entered into the cells from the outside and has there been deposited. If much fat or materials from which fat can be formed be introduced into the body in the food, such fat becomes deposited by preference in certain parts within the cells, as in the cells of the subcutaneous connective tissue, and thus arises corpulency, the panniculus adiposus. Of course it is not impossible that in many cases of corpulency fat arising pathologically within the body also enters into the cells of the subcutaneous connective tissue and is there deposited. But even here there is always a fatty infiltration of the cells from the outside. In contrast to this, in fat-metamorphosis fat is formed within the cell itself at the expense of its living substance, and there accumulates until the cell is permeated with innumerable, large or small proplets and dies. Such fat-metamorphosis, which ends with the death and disintegration of the cell, occurs in certain places in the healthy body as a normal phenomenon; thus, it is present in the cells of the lacteal glands at a time when they are secreting milk, when a woman is nursing. It is found that at this time in the lobes of the mammary glands microscopic fat-droplets appear in the protoplasm of the older cells (Fig. 144); these gradually increase in number, while the protoplasm gradually dies, and the cell tinally becomes a round droplet, full of small milk-globules. The dying protoplasm gradually disintegrates, the fat-globules become free, and the whole mass, i.e., the fat-globules in their liquid, becomes secreted as milk, milk being nothing more than an emulsion of the fat of butter in a solution of salts, proteids, sugar, etc. The younger gland-cells succeed the older, fattyrlegenerated and disintegrated cells, and pass through the same (hanges, and thus the process of milk-formation continues longand uninterruptedly. What occurs as a normal process in the rells of the lacteal glands occurs under pathological conditions in much greater extent in very various tissues, and leads almost always to incurable and fatal losses, since as a rule no reparation is made by the younger cells. "The production of milk," says Virchow ('71), "in the brain instead of in the lacteal glands, constitutes a form of brain-softening. The same process that in one place affords the happiest and sweetest results, in another induces a painful and bitter wound." Such fatty degenerations appear especially in long-continuing, chronic 
diseases, such as tuberculosis, heart-diseases, kidney-diseases, etc., in the kidney, hcart, liver, blood-vessels, etc. (Fig. 145); and their causes always lie in disorders of nutrition, especially in a disturbance of the process of taking in oxygen through the blood. If, e.g., insufficient oxygen is carried to the cell, or if for other reasons its capacity of receiving oxygen is diminished, the fat, which probably in most cells appears in traces, is not burned, i.e., oxidised, as happens normally, but is stored up and accumulates in quantity. For this reason also in habitual drinkers and after phosphorus-poisoning, where as a result of the ingested alcohol or phosphorus the income of oxygen is diminished, a considerable fat-metamorphosis of the tissues, especially of the liver-cells, always takes place: and pathology recognises a whole series of cases where fat-metamorphosis can be traced to the same causes. It is highly probable that in all processes of fatmetamorphosis the fat originates from the decomposition of

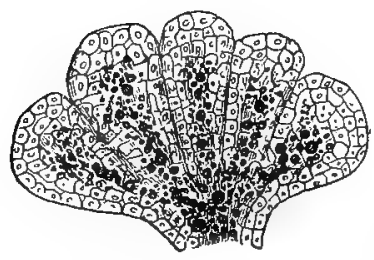

FIG 144,-Fat-metamorphosis in the formation of milk in the lobes of the lacteal glands. (After Virchow.)

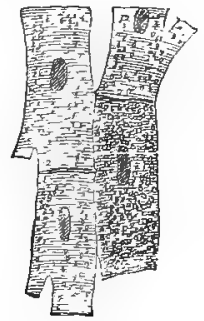

Fra. 145.-Fat-metamorphosis of cardiac muscle-cells; the granules in the cells consist of fat. (After Ziegler.)

proteid. It is known that in the decomposition of the proteidmolecule both nitrogenous and non-nitrogenous complexes of atoms appear. Moreover, it has been seen, ${ }^{1}$ that fat can be formed from proteid; and Leo ('85) has shown in the case of fat-metamorphosis after phosphorus-poisoning that the fat originates within the body. Since now, thirdly, it has been found that the excretion of urea is considerably increased after phosphorus-poisoning, the conclusion is justified that after phosphorus-poisoning proteid is decomposed in greater degree, and that the non-nitrogenous complex of atoms that arises during the decomposition is the fat deposited in the cells, while the nitrogenous portion is transformed into urea and given off to the outside. The origin of fat, at least in all fat-metamorphoses, must be regarded as wholly analogous.

The phenomena of mucous metamorphosis form a complete counterpart to those of fat-metamorphosis. As in the latter fat, so in the former mucus, is formed from the living substance of the

$$
1 \text { Cf. p. } 163 .
$$


cell. In many cases the mucus that appears contains genuine mucin, in others it consists of mucinoid substances, but it is always a compound of proteid with some kind of carbohydrate. ${ }^{1}$ It is seen, therefore, that in mucous metamorphosis the origin of the mucus lies in the proteid. Mucous metamorphosis occurs also normally in the healthy body, especially in the cells of the mucous membranes of the respiratory and intestinal tracts, as well as of the urogenital system. In the formation of mucus by these mucous cells under normal conditions the whole cell never perishes, but a part only of its protoplasm is transformed into mucus. Almost always mucous cells are cylindrical cells; their basal part contains the nucleus, and their upper end bounds the free surface of the mucous membrane. It is always the upper, free end of the cell-

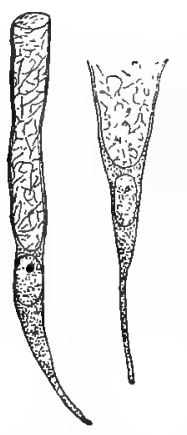

$-1$

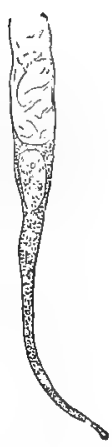

FIG. 146.-Mucous cells. $A$, Three isolated mucous cells ; $B$, seven mucous cells united. The three. at the left are full, the four at the right are empty. (After Schiefferdecker.)

body, the protoplasm of which is transformed into mucus, swelling up into a transparent mass containing separate protoplasmic granules; each mass, having no boundary, unites with the mucous masses of the neighbouring cells into a coherent mucous covering. The process is a continual one and is increased by certain external influences. The lower part of the cell-body containing the nucleus continues to live (Fig. 146), and constantly shoves upward new masses of mucus-forming substance, or mucigen, which become transformed into mucus in proportion as they move along. A complete transformation of the whole cell-body into mucus, accompanied by the death of the cell, occurs in many lower animals upon strong external stimulation; the phenomena of this process are very remarkable. They are most remarkable in certain forms of sea-cucumbers, or holothurians, belonging to the Echinodermata, plump animals, whose bodies are covered by a tough, brown, leather-like skin and resemble a cucumber. If Holothuria Poli,e.g., 
which lives in the Mediterranean Sea, be brought into the air, the thick, hard skin begins gradually to liquefy into a viscous mucus, and after a few hours becomes completely softened. If small pinholes be bored through an excised piece of the skin this mucous liquefaction, as Semper ('68) showed, can be rapidly induced; around each hole the cells begin at once to swell up and disintegrate, and the whole piece is changed finally into a thick liquid mass, which, when touched, can be drawn out into glistening threads. Many species of the holothurian genus Stichopus are said to transform their skin in a very short time into a thick mucus. It would be extremely interesting to investigate both chemically and microscopically this wholly unique case of a sudden mucous metamorphosis of so solid and tough a structure as is the holothurian skin. Krukenberg ('82) alone has made a partial study of it. The mucous metamorphosis of epithelium-cells, leucocytes, etc., which occurs in the human body, especially in intense catarrhs, is well known: in these cases the cells in

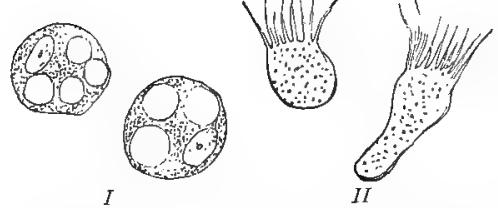

Fia. 147.-Mucous-metamorphosed cells. I, Leucocytes; II, ciliated cells. (After Ziegler.) question die with a swelling and transformation of their living substance into mucus (Fig. 147).

In the phenomena of amyloid metammphosis, in contrast to the processes hitherto considered, a substance is formed which, so far as is known, does not occur at all in the normal body. This substance, which glistens like wax or lard-which probably has conferred upon the disease in question the name of waxy or lardaceous degeneration-was first termed by Virchow amyloid substance, because with iodine staining it behaves like plant-amylum and cellulose, under certain conditions being coloured blue by the iodine. Later it was recognised as a proteid-like body, for it contains nitrogen and gives certain proteid reactions; hence, for the present it is classed in the comprehensive group of albuminoids. Its behaviour with the aniline colour, methyl violet, is very characteristic; with it it takes on a beautiful ruby-red colour, while healthy tissues are coloured blue. By its character as an albuminoid, amyloid substance points plainly to its origin. It can be derived only from the proteids of the cell, and, although thus far nothing is known in detail concerning its origin, it may safely be considered as a metamorphosed proteid, which is excreted to the outside by the cell and stored up. It never seems to be stored within the cell itself, it is always found rather in the connective substances cementing the cells, especially in the walls of the small blood-vessels (Fig. 148). But, in proportion as the cells secrete it, they die, whether as the result of perverse metabolism, the 
product of which is the amyloid substance, or because passively they are torn apart, pressed upon, asphyxiated and killed by the substance accumulating in masses. Amyloid metamorphosis is a secondary phenomenon of disease, appearing especially in connection with long-existing, chronic diseases, such as tuberculosis, longcontinued suppurations, etc., in the abdominal organs, especially the spleen, liver, kidneys and lymphatic glands. This indicates that nutritional disturbances of the tissues, very gradually developed and profound, cause the cells to be put into the condition where their proteid changes gradually into amyloid substance. Beyond what has been stated, amyloid metamorphosis remains still one of the most enigmatical among the metamorphic processes, although it is wide-spread and possesses great importance in pathology.

Finally, calcification is in a certain sense a counterpart to amyloid metamorphosis; for, as in the latter amyloid substance, so in the former lime-salts are formed by the cells, and are either excreted to the outside or deposited in the dying cell-substance itself. The formation of bone in the normal body is analogous to the former. Large skeletal bones develop from a cartilaginous basis; the cartilage-cells excrete into the ground-substance calcareous salts, especially calcareous phosphate and carbonate; particles of these press gradually upon one another, blend together, and thus form the solid bony substance, in which the bone-cells continue to live as so-called bone-

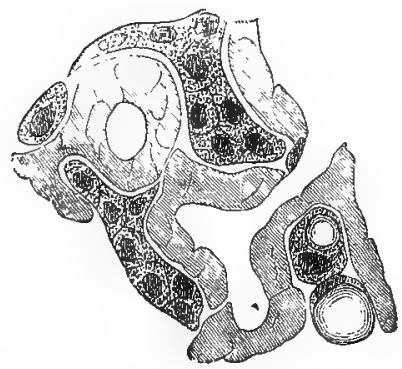

Fre. 148.-Amyloid degeneration of the capillaries of the liver; the cells are forced apart by the amyloid masses stored up between them. (After Ziegler.) corpuscles. This process, which appears absolutely necessary in the development of the vertebrate organism, occurs also under pathological conditions, especially when in old age or after certain diseases the cartilaginous discs in the joints ossify. In these cases the same phenomena are present, excepting that, as a rule, the cells by which the lime-salts are excreted later die. Besides this ossification, there occurs also under pathological conditions a true calcification of the cells themselves, in which the lime-salts become stored within the dying cell, until finally the living substance has wholly disappeared and its place is taken by a cemented calcareous mass. This happens in the walls of the arteries (Fig. 149, $A$ ), so that they become brittle and afford an opportunity for hæmorrhages; if the latter take place in the brain they constitute apoplexies, or so-called paralytic strokes. Further, in certain brain-diseases the ganglion-cells of the brain become calcified, and there are 
found, e.g., in the brains of idiots, "petrified" ganglion-cells in the true sense of the word (Fig. 149, B).

Besides the forms of metamorphic processes here presented, pathology recognises others, such as pigment-atrophy, hyaline degeneration, colloid metamorphosis, etc., at the basis of which there is always the same principle, namely, that the metabolism of the cells takes a perverse course, and forms substances that normally are formed either not at all or only in slight quantity, the final result being the death of the cell. But in the cases

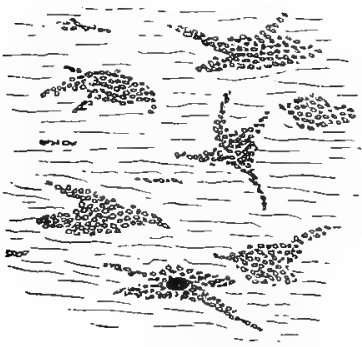

A.

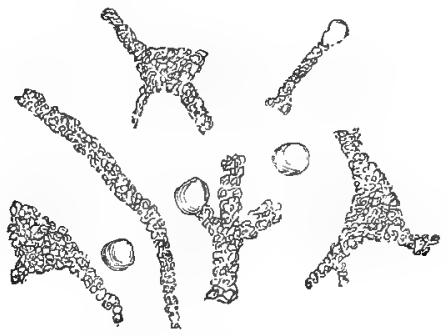

$B$

FIG. 149.-Calcification of cells. A, Calcified cells in the wall of a blood-vessel. $B$, Calcified ganglion-cells from the brain of an idiot. (After Ziegler.)

mentioned these substances and their genesis are much less known than in the metamorphic processes that have been discussed; hence it does not appear necessary in this place to go into them more fully.

In general, metamorphic processes, especially the genesis of the substances that arise in them and the disturbances of normal metabolism upon which they rest, need greater elucidation; naturally this will come in proportion as the knowledge of metabolism in general becomes extended.

\section{B. 'THE CAUSES OF DEATH}

The causes that lead to death are as manifold as are its phenomena. We have already touched here and there upon some of the special causes, but it is impossible to treat these in every individual case. It is necessary, however, to go somewhat more fully into the general causes, because with them is joined the interesting question whether death is for all living organisms the lira nccessitas that it is for mankind-in other words, whether there are organisms whose bodies are immortal. 


\section{External and Internal Causes of Death}

If we start from the fact that life can only arise, and, moreover, must arise, as soon as a certain complex of conditions is fulfilled, the causes of death in their general form are evident; for death must then take place so soon as the general conditions of life disappear. In accordance with the distinction between external and internal conditions of life, a distinction must also be made between external and internal causes of death, according as death is due to the removal of the external or of the internal vital conditions.

To examine, first, the external carses of death, the fact does not require detailed consideration that withdrawal of oxygen, water and food-stuffs, and, further, exceeding the necessary limits of temperature and pressure, lead to death, except in the case of organisms that under certain conditions pass into the state of apparent death. But these do not include all the external causes of death. All these conditions may be fulfilled and yet death be brought about by the action of external causes. Hence we must reckon among the external conditions of life the absence of such influences as are destructive to living substance, especially chemical and electrical influences.

The chemical influences that produce fatal effects are the poisons, and they are innumerable. All chemical substances that come into chemical relation with any of the essential constituents of living substance so that the mechanism of metabolism thereby suffers disturbance, cause death, sometimes after very brief, sometimes after long-continued action, death following very rapidly or constituting the end of long, necrobiotic changes. If, e.g., mineral acids or metallic salts act upon the living substance of a cell, the cell inevitably dies, because all proteid is precipitated or chemically combined by these substances so that metabolism must cease. Other substances that are poisonous to all living substance are the anæsthetics (chloroform, ether, alcohol), the vapours of which by continued action finally bring all vital phenomena to a standstill, whether in plants, animals or unicellular forms. ${ }^{1}$ To what change of the living substance this peculiar effect of anæsthetics is due is for the present wholly unknown; and the same must be said of the great majority of poisons that act, some upon all living substance, and some upon certain cells only.

Like poisons, electricity in great intensity also acts harmfully to living substance by producing chemical changes in it. It is well known that chemical compounds in solution can be decomposed by a galvanic current. The componnds of living substance are

$$
\text { l c } f \text {. Bernard ('78). }
$$


likewise decomposed by strong galvanic currents, so that the living substance is killed and disintegrates.

Thus, external causes of death are superficially clear and distinct, although the details of their actions are still largely unknown.

It is entirely different, however, with the internal causes of death. They are still very obscure. Many investigators believe that there are no internal causes of death that are based upon the properties of living substance, and they explain the appearance of death in old age in people who have never been ill by the gradual accumulation of small, imperceptible disturbances during the whole life. This is the most frequent explanation of the phenomenon. But it appears very insufficient. Johannes Müller ('44) was not satisfied with it. In the chapter upon the Mortality of Organic Bodies in his handbook, he says: "The question why organic bodies perish, and why organic force passes from the parts producing it into the young, living products of the organic body while the old parts die, is one of the most difficult in all general physiology. We are unable to answer this question, but can merely present the associated phenomena. It is insufficient to answer that inorganic influences gradually wear away life, for then the organic force would be obliged to begin its diminution at the beginning of the individual. Yet it is well known that at the time of puberty organic force is still so complete that it multiplies itself in the formation of germs. There must, hence, be a very different and deeper-lying cause that conditions the death of individuals, while assuring the transmission of organic force from one individual to another and in this way its immortality." Many such objections may be made. Were the view correct that death is brought about by the summa. tion of the actions of external injuries, it would be expected that a man who lives very regularly and avoids as much as possible all harmful things would necessarily live much longer than one who lives irregularly and exposes himself to many hardships. But, even if such a difference in the duration of life should occur, in many cases it would always be minute, for the oldest men have not lived much beyond 120 years, and not all of these persons have followed an especially regular course of life. Another circumstance comes in. In all men, without exception, whether during their life they have been exposed to the greatest or the least dangers, whether they have been often or never ill, or whether they have had this or that disease, the same phenomena of old age finally appear, consisting of atrophic processes of almost all organs. With special reference to the last circumstance Cohnheim ('77-'80) rightly indicates another explanation in saying: "The constancy with which, no matter whether many or few, and especially what pathological phenomena have occurred in the life of an individual, a more or less pronounced atrophy appears in all organs of his 
body in old age, in my opinion speaks very evidently for the idea that the conditions of senile atrophy, so to speak, are physiological." Minot ('90, '91) also adopts the same standpoint in his researches upon growth and the phenomena of old age. In fact, when man is considered not as something completed and unchangeable; when, rather, his whole development is observed, and it is seen how, although living always under the same external conditions, he changes gradually after birth; how even in childhood many organs, such as the thymus glands, normally atrophy, although not the slightest injuries from the outside act upon them; and now later in all women even in the prime of life the sexual organs degenerate, etc., etc.,-it can no longer be doubted that senile atruphy, which leads finally to death from the feebleness of old age, is simply the end of the long developmental series that man, like every animal, must pass through during his individual life. In reality there is no standstill in the life of the organism. As the adult organism develops gradually from the small egg-cell without the slightest change of its external vital conditions, as is the case in many animals living in the water, so it develops also, although at a different rate, gradually farther to a senile, and finally to a dead, organism. The egg-cell is the beginning, death in old age the natural end of an unbroken development, the cause of which lies in the peculiar composition of the living substance of the egg-cell. It would, hence, be more correct, in place of the current view that death is conditioned by the continual summation of external causes, to believe that the causes of so-called natural death exist in the living organism itself.

This view is justified at once if the history of death be considered, not simply with reference to mankind, but comparatively. The fact that the idea of death as an end-result of the developmental series appears so late in the history of science is closely associated with the prevalent view, that man, when grown, has finished his development and exists for years and decades in a stationary condition. This view is thoroughly false, and is due simply to the fact that man's development takes place much more slowly during his adult life than during his embryonic and youthful stages. In reality, development never ceases. Changes are seen clearly enough when the conditions of the adult are compared at long intervals of time. Although no new organs are formed in the meantime, the man of thirty years is a different being from the man of forty years, the man of forty from the man of fifty and sixty. A stationary condition is never present; celldivision, upon which from the egg-cell on all development depends, takes place in adults and even in old men, although it becomes constantly slower and slower. What is difficult to recognise in man is shown at once by a glance at the relations that prevail 
among insects. While in man adult life is extremely long in comparison with that of the embryo, in most insects the reverse prevails. Many insects die very soon after copulating or depositing the eggs; the only individuals to live longer are those that do not copulate. The best example is afforded by the day-tlies. The completely-developed adult individuals frequently live but a few hours, dying immediately after depositing the eggs. These facts prove most strikingly that the causes of death are not to be found in the summation of many external injuries, but are already established within the organism itself, and death is simply the natural end of development. In other words, the problems of development and death belong inseparably together, the latter is merely a part of the former.

We will now summarise the results of these considerations once more and in somewhat different words. The idea expressed regarding the causes of natural death is based upon the important fact that the organism undergoes uninterrupted change from its individual origin to its death. The various parts of the organism, however, take part in this change in very different degrees and at very different rates. In this manner there comes gradually in the life of every organism a time when the action of its mechanism has experienced such a disturbance through the changes that the individual parts have undergone in its development, that it passes into death. For the multicellular organism this means that from internal causes the various cells and cellgroups of its organs become gradually so changed in their development, that with the close dependent relation among all cells, tissues, and organs, the disturbance of their co-operation becomes constantly greater until the organism dies. The immediate causes of death may be very different for the different cells of the multicellular organism. Many of the cells and tissues invariably die from causes lying outside of them but within the organism, because the parts upon which they are dependent, which belong to their external conditions of life, as, c.g., the nerve-centres, have undergone disturbances and have died. If the ganglion-cells whose activity controls the movements of respiration have perished, respiration ceases, the heart stands still, blood circulates in the tissues no longer, the tissue-cells are no longer nourished, and all the tissues alike perish sooner or later, because their external conditions of life are withdrawn. But, if the individual tissue-cell does not die from external causes, exactly the same is true of it as of the cell-community - the condition of its living substance undergoes uninterrupted change from internal causes, and there gradually develops a point of time when the disturbances in the co-operation of its constituents have become so great that life ceases. These statements do not, indecd, disclose the special evonts in living substance, the result of which is death, no more 
than they reveal the mechanism of development and life in general; but they afford a simplification and a sharper formulation of the problem, and bring us somewhat nearer to an understanding of it.

The problem of development and the problem of death contain the same question, namely : Why does living substance continually change during its individual life? Deeper penetration into the chemism of the living cell will alone be able to reveal the special causes of this phenomenon.

\section{The Question of Physical Immortality}

If natural death be considered from the standpoint just presented, a question which during the last decade has been actively discussed upon the scientific side constantly obtrudes itself, viz., Are there not organisms for which death is not a necessity?

Evidently an organism can be imagined the development of which is such that a disturbance that makes impossible the cooperation of the individual parts never appears. This would be the case if the uninterrupted changes that appear during the development of the organism in question form a series composed of members recurring periodically. Such a development could perhaps be represented schematically in the form of the solution of a periodic continued fraction, which, transformed into a decimal fraction, would give a periodic series, while the development of an organism that is destined to die might be compared to the solution of a definite fraction. Theoretically, such a hypothetical organism would necessarily be immortal under external conditions that always remained exactly the same. It is, however, a question whether such organisms really exist.

Weismann ('82, '84) believes that this question can be answered in the affirmative, and it is interesting to follow his discussion. He finds a fundamental difference between multicellular organisms and unicellular Protista. Starting from the thought that the term death can be employed only where a corpse exists afterwards, he considers all multicellular organisms as mortal, and all unicellular organisms as immortal. In multicellular organisms no case is known where sooner or later the body does not die. In unicellular forms, however, this is not true. A unicellular infusorian, e.g., never becomes a corpse unless it is the victim of an external catastrophe. It grows and divides into two halves when it has reached a certain size, but each half likewise grows and later divides and so on, and Weismann believes that this continues without end. But since the two halves are wholly alike, and since the species can be maintained only by continued division, a corpse is never found, and a half never dies without external 
causes. Hence, according to Weismann's idea, unicellular organisms are immortal. Weismann, therefore, disputes the view that death is a phenomenon grounded in the nature of living substance, and does not believe that it depends upon "purely internal causes inherent in the nature of life itself." He holds death, rather, to be a phenomenon of adaptation which has been evolved in the course of the organic development of the earth as advantageous, and he represents its appearance in the organic series somewhat as follows: In the unicellular Protista all the functions of the body including that of reproduction are localised in a single cell. If, therefore, natural death were a necessity for the unicellular organism, reproduction would terminate with death; and, since with the equality of the parts resulting from division the same holds good for all, after a short time the species in question would become extinct. Hence in unicellular forms death is impossible, Weismann maintains, because otherwise the species would become extinct. In multicellular organisms, on the other hand, the higher we go in the series, the more a contrast develops between the sexual cells, which serve for reproduction only and hence for the maintenance of the species, and the cells of the rest of the body, which in the higher animals have completely lost the power of reproducing the species. Here, therefore, there is the possibility of death without the maintenance of the species thereby being endangered; for, if only one reproductive cell really reproduces, if only one egg develops, all the rest of the body can die without the species becoming extinct. Since now, as Weismann says, "the unlimited duration of the individual would be a luxury without any advantage," according to the well-known principles of selection immortality has been lost as disadvantageous and death has been evolved. "In unicellular animals it was impossible to establish normal death because the individual and the reproductive cell were one and the same; in multicellular organisms, however, somatic and reproductive cells were separate, death became possible, and we see that it was established."

It cannot be denied that these deductions of Weismann sound very plausible; nevertheless, they are not invulnerable, and have already called forth much active contradiction.

Especially has the claim always been contested that unicellular organisms should be considered immortal for the single reason that their body never becomes a corpse. In defining the conception of death, emphasis has been laid by Weismann's opponents largely upon the cessation of the individual life, and it has been said: If the unicellular organism divides into halves, its individual existence is therewith ended; but where the individual existence ceases, the term immortality cannot be used, since in reality the individual has perished; death and reproduction here coincide. It is evident that here there is simply a contest over ideas, which 
leaves untouched the phenomena themselves, for in the end it is a matter of taste, whether the appearance of a corpse, or, what is more general, the end of the individual existence, is regarded as the essential factor of death.

The fundamental distinction which Weismann makes between unicellular and multicellular organisms respecting immortality may be attacked from another side. As has been seen, Weismann's theory of the immortality of unicellular organisms rests upon the supposition that the reproduction of these forms by division can go on without end, without any remnant, any corpse, being left over. It is a question whether this supposition is correct.

A few years ago Maupas ("88) carried out upon Infusoria a series of striking researches, from which it appears that in that group this is not the case. He bred Infusoria in cultures for many generations, and found that after a large number of successive divisions the individuals gradually showed changes that led inevitably to death, unless after a long period of dividing, leading often to hundreds of generations, the opportunity was given them to conjugate, i.e., to enter into a correlation that corresponds in unicellular organisms to the process of fertilisation in higher animals. ${ }^{1}$ Only when a series of divisions was followed by a period of conjugation were the individuals separating after conjugation in condition to divide again unchanged without passing gradually into death. If, however, the individuals were isolated after every division, after some time they inevitably died. There is here presented, therefore, a real phenomenon of old age, which corresponds completely to the senile atrophy of tissue-cells in man and the higher animals, and Maupas himself was forced to reject Weismann's doctrine of immortality. But at this point, to save the doctrine, Gruber ('89) speaks a word for Weismann and says: "It is true that those individuals that by chance do not conjugate, perish, but the material of the others lives on for ever." Since now, in nature conjugation is the custom-for, otherwise, the Infusoria would long since have become extinct-the members of this group, Gruber thinks, are really immortal. Although the justice of this argument is to be recognised, another fact should be noticed. R. Hertwig ('88-'89), who studied very carefully the events of conjugation, found that a part of every cell dies during the process, viz., the macro-nucleus and a part of the daughternuclei, derived by continuous division of the micro-nuclei. These constituents of the cell break up into small fragments, which finally become completely dissolved in the protoplasm. ${ }^{2}$ In other words, portions of the individual actually die. That the materia] derived from their disintegration is finally consumed again by the cell, like the ingested food, does not banish the fact that these parts really die. The cells that disintegrate in the histolysis of a

$$
1 \text { Cf. p. } 200 .
$$$$
{ }^{2} C f . \text { p. } 201 .
$$ 
tadpole's tail and the death of which no one will deny, are likewise employed again as material for the construction of other organs. But, if in the conjugation of the Infusoria there are really dying parts, really partial corpses, the fundamental contrast between unicellular and multicellular organisms, maintained by Weismann, disappears, and the whole difference consists simply in the quantitative relation of the surviving and the dying substance; in multicellular organisms only the body-cells die, while the reproductive cells continue to live. In general, it would be wholly incorrect to say that in multicellular organisms an exceedingly large mass, namely, the whole body, dies, and only tiny masses, the ova or spermatozoa, remain living, while in Infusoria the greater part remains living and the smaller part dies. There are examples among animals where the relation does not differ at all from that in Infusoria. A female frog, e.g., produces in the course of her life a mass of eggs that in relation to her body is even considerably greater than the mass of cell-substance that in the infusorian body in conjugation remains living in contrast to that which dies. If, therefore, the frog and, in general, the multicellular organism are mortal, the unicellular Infusoria are mortal also; in both cases it is only a part of the living substance of the individual that is transmitted to the descendants.

Not only in the life of the Infusoria, but also in that of other unicellular organisms there are periodically recurring events, in which parts of their body perish. Many Protista reproduce by the formation of spores. If this process be followed in a large radiolarian, c.g., Thalassicolla, which has been studied in detail by R. Hertwig and Brandt, it is found that the nucleus in the central capsule breaks up into many small nuclei, which surround themselves each with a protoplasmic mass, and develop into many small swarmspores; the large, extracapsular, protoplasmic body and also a part of the intracapsular protoplasm, which is not consumed in the formation of spores, perish completely. Here, likewise and perhaps still more evidently than in the Infusorin, there are really partial corpses. We see, therefore, that with the great majority of unicellular organisms, with all whose course of development has thus far been studied in detail, Weismann's idea does not agree.

Finally, the possibility is not to be dismissed that there may be, or may once have been in the course of the phylogeny of living substance, Protista, whose cycle of development is so simple that their living substance simply grows constantly without conjugation and without spore-formation, and, when they have reached a certain volume, divides without any remnant, and continues to grow and divide as long as the external conditions allow. According to Weismann's idea, such Protista would be really immortal beings. But at this point the weakness of the doctrine of immortality appears perhaps most distinctly. If Weismann's standpoint be accepted, that 
not the cessation of the existence of the individual, but the transformation of living substance into a corpse, i.e., into lifeless substance, is the criterion for the conception of death, then the question of the existence of immortal organisms coincides with that of the immortality of living substance in general. But the conception of living substance as immortal will be accepted by scarcely any one who bears in mind the characteristic peculiarity of living substance, viz., that it continually decomposes, or, in other words, dies. There is no living substance that, so long as it is living at all, is not continually decomposing in some parts, while being regenerated in others. No living molecule is spared this decomposition; the latter, however, does not seize upon all molecules at the same time; while one is decomposing, another is being constructed, and so on. One living particle affords the conditions for the origin of another or several others, but itself dies. The particles newly formed in turn give origin to others and, likewise, die. In this manner living substance is continually dying, without life itself ever becoming extinct. Hence, there is no immortality of living substance itself, but merely a continuity in its descent. Life as a complex motion has never become extinct from the time of its first appearance upon the earth down to the present, but living substance in the form of bodies is dying continually. Life as a complex motion does not possess true immortality any more than it has existed from eternity. Just as the earth in its development has passed through a time when no life could yet exist, so it will again pass through a time when all life must become extinct. The moon now shows us the fate that hangs over the earth. From the liquid drop which once was cast off from the great, glowing mass of the earth, it has in a briefer time passed through essentially the same development as the earth which gave it its origin. The intense cold that now prevails upon it will sometime take possession of the earth, and annihilate all life upon the latter. So far as the physical world is concerned, immortality and eternity are the properties not of any special material system, such as living substance, or of any special complex motion, such as life, but only of elementary matter and its motion.

Heraclitus compared life with firc. As has been shown above, such a comparison is a pertinent one. Our consideration of vital conditions makes this more evident. It has been shown that life like fire is a phenomenon of nature which appears as soon as the complex of its conditions is fulfilled. If these conditions are all realised, life must appear with the same necessity as fire appears when its conditions are realised ; likewise, life must cease as soon as the complex of its conditions has undergone disturbance, and with the same necessity with which fire is extinguished, when the conditions for its maintenance cease.

If, therefore, all vital conditions had been investigated in their 
minutest details, and it were possible artificially to establish them exactly, life could be produced synthetically, just as fire is produced, and the ideal that existed in the imagination of the medixval alchemists in their attempted production of the homunculus would be achieved.

But, notwithstanding the fact that this theoretical possibility cannot be denied, every attempt at the present time to produce life artificially and to imitate in the laboratory the obscure act of spontaneous generation must appear preposterous. So long as our knowledge of internal vital conditions, i.e., of the composition of living substance, is so imperfect as it is now, the attempt artificially to compound living substance will be like the undertaking of an engineer to put together a machine the most important parts of which are wanting. For the present the task of physiology can consist only in the investigation of life. When physiology shall actually have accomplished this, it may think of testing the completeness and correctness of its achievement by the artificial inauguration of life. 


\title{
CHAPTER V
}

\author{
STIMULI AND THEIR ACTIONS
}

WHEN investigating a phenomenon of nature the physicist is not satisfied with determining the conditions under which it exists; he endeavours to learn also how it is affected when the conditions are altered.

Life is a phenomenon of nature. In the preceding pages we have become acquainted with its manifestations and the conditions of its appearance, and we have seen the results of an entire removal of those conditions. It remains for us to learn how vital phenomena are affected when the conditions are altered and new ones are allowed to surround the living substance. Vital phenomena are called spontaneous, when all the external conditions of life continue unchanged, and phenomena of stimulation, when other influences act upon them. This distinction is a valid one, but it must be borne in mind that spontaneity is not absolute, that as a matter of fact spontaneous vital phenomena depend upon the interaction of living substance and the environment no less than do the phenomena of stimulation. The former represent merely the reaction of living substance to normal, constant external vital conditions; the latter, the reaction of living substance to changed external vital conditions. In many cases it is quite impossible to decide whether a given phenomenon is spontaneous or a result of stimulation, since even in nature the external conditions of an organism do not remain constant, but frequently change in a manner that eludes even the most exact methods of investigation. In order, therefore, to study undoubted phenomena of stimulation we have recourse to the experimental method, and produce the phenomena artificially by causing stimuli to act upon living substance. In so doing we secure the incalculable advantage of keeping in hand and controlling exactly the conditions under which the phenomena exist, and thus are able to experiment with vital as with simple physical phenomena. 


\section{The Nature of Stimulation}

In accordance with the foregoing statements, a stimulus may be define as every change of the external agencies that act upon an organism. If a stimulus comes in contact with a body that possesses the property of irritability, i.e., the capability of reacting to stimuli, the result is stimulation. It is necessary to examine somewhat in detail the general characteristics of the process of stimulation.

\section{A. THE RELATION OF STIMULI 'TO VITAL CONDITIONS}

\section{The Varieties of the Stimulus}

If every change of the agencies that act upon the organism from without is able to stimulate, it is evident that innumerable kinds of stimuli exist. Not only may every existing condition of life be changed, but new conditions may appear and affect the organism. Notwithstanding this possibility, stimuli may be classified according to their qualities into a few large groups. A natural classification is possible in accordance with the forms of energy which the different stimuli represent; for the operation of every external agent upon a body depends upon a transformation of energy.

In accordance with this principle all influences of a chemical nature may be grouped as chemical stimuli, including not only changes in the income of food, water, and oxygen, but other chemical changes which ordinarily do not come into contact with the organism. Among chemical stimuli belong also the processes by which in the animal cell-community the nervous system influences the tissue-cells dependent upon it; for every nerve stimulation has at its foundation a chemical transformation of nerve-substance, which is transmitted to the cells of the tissues and acts towards the latter as a chemical stimulus. In accordance with our modern ideas upon the metabolism of living substance, the old conception that nerve stimuli are merely electrical stimuli, and that nerves behave as copper wires, can find credence no longer.

All purely mechanical influences that affect the organism may be termed mechanical stimuli, including those that consist in changes of pressure, such as pushing, shaking, pressing, pulling, and sound-vibrations, those that manifest themselves by molecular attractions, such as cohosion or adhesion in the surrounding medium, and those that depend on the action of gravitation.

Thermal stimuli comprise changes of the temperature that surrounds the organism. 


\section{Photic stimuli comprise changes of light.}

Electrical stimuli comprise electrical changes.

The above classes include all forms of energy that come into relation with the organism. It is observed that in this enumeration magnetism is wanting. But it is now known with certainty that magnetism exercises no effect whatever upon living substance, and cannot properly be termed a stimulus. To it was ascribed at one time a most far-reaching and remarkable influence over the living organism; this was when the physician Mesmer popularised the so-called "animal magnetism," and when the possibility of magnetising human beings, animals and plants, by means of magnets was believed in. But later research, and especially the discoveries of the Scotch physician, James Braid, showed that the phenomena that were observed in those cases from which gross deception was excluded were phenomena of hypnosis, and had nothing whatever to do with magnetism; in their production a piece of glass, a polished button, a gas-flame, or any other visible object had the same significance as a magnet. In accordance with the mysterious attraction that all mysticism is wont to exercise over the human mind, there are found even at the present time, not only among the visionary adherents of spiritualism, but even among acute physicians, some who are convinced of the action of strong magnets upon certain individuals, especially upon hysterical women. But from all observed cases sober investigation has invariably torn away the veil of mystery, and has revealed either fraud on the part of the "mediums" or self-deception on the part of the observers. Careful experiments upon the influence of magnets upon the living organism have always yielded negative results. The recent, extended researches with very strong electromagnets by Peterson and Kannelly in America demonstrate the utter ineffectiveness of magnetism upon living matter.

Stimuli, therefore, comprise chemical, mechanical, thermal, photic, and electrical changes in the environment of the organism, and no others.

\section{The Intensity of the Stimulus}

In order to form a clearer idea of the relation of stimuli to vital conditions, we must turn our attention to the intensity of the former.

Every external vital condition can be fulfilled in different degrees: food, oxygen, etc., may be introduced in small or large quantities; the temperature may be low or high; in brief, every vital condition can vary gradually within very wide limits without life thereby being endangered. Nevertheless, limits to most vital conditions are known, both an upper and a lower limit, and these are termed respectively maimum and minimum. Continual life 
is possible only between these. If they are overstepped, death develops. But all points between the two limits are not equally favourable to life. The intensity of the life-process is less when the vital condition is near its maximum or minimum, than when it has an average value. That degree of any vital condition at which life thrives best, at which the intensity of the life-process is greatest, is termed the optimum. But the optimum is not always

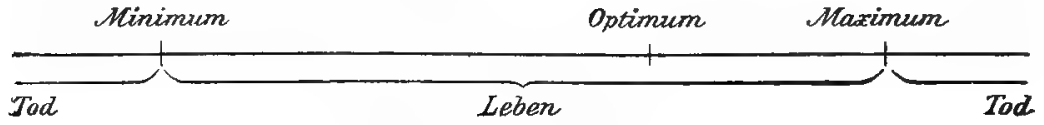

Diagram of Vital Conditions.

intermediate between the maximum and the minimum, in many cases it lies nearer the former, in others nearer the latter.

In accordance with the above diagram of vital conditions the conception of the stimulus may be at once appreciated. If an organism exists at the optimum of any vital condition, e.g., of temperature, then every deviation of the temperature, whether in the direction of the maximum or the minimum, acts as a stimulus. That degree of any vital condition to which the organism is adapted, represents its optimum, it represents the indifferent point of stimulation; here the stimulus is equal to zero. If the condition changes toward the maximum or the minimum, the intensity of the stimulus simultaneously increases until it reaches the maximum or the minimum. The stimulus, therefore, has a minimum, which coincides with the optimum of the vital condition in question, and two maxima, the one at the minimum, the other at the maximum of the condition. With supra-maximal stimulation death develops. If, therefore, a diagram of stimulation be constructed, the same points must be designated as in the diagram of vital conditions ; but other names must be given them, for the optimum of the conditions becomes the zero-point of

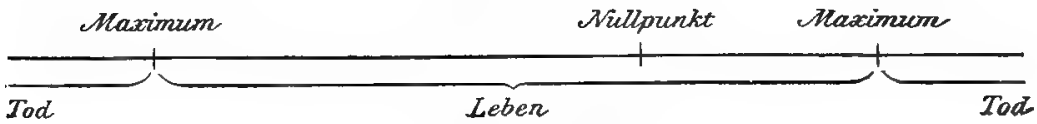

Diagkam of Stimulation.

stimulation, the minimum and the maximum both become maxima. Every change of intensity between the zero-point and either maximum acts as a stimulus.

This diagram comprises all varieties of stimulus, even those which, like certain chemical and electrical stimuli, under normal conditions do not come into relation with the organism at all. The intensity of these latter varieties considered as vital conditions 
is zero: in other words, the complete lack of them corresponds to the optimum. They can, therefore, have but one maximum, so that for them only the right-hand portion of the diagram comes into consideration. They are included in the general definition of the stimulus, namely, every change of the external agencies that act upon an organism; this definition holds good as well for those agencies which, like heat, function in a definite degree as vital conditions, as for those which, like electricity, under usual circumstances are absent from the environment of the organism, and, therefore, do not exist as conditions of life.

In considering the intensity of the stimulus, one more point requires mention. Let us imagine an organism or part of an organism, e.g., a muscle, under conditions in which no stimulus affects it, and let us bring to bear upon it a stimulus, e.g., the galvanic current, which varies in intensity from zero upward and can be graded easily and delicately. Then we should expect the muscle to exhibit phenomena of stimulation, i.e., to perform a contraction, as soon as the intensity is increased above 0 . But this does not happen. The intensity can be increased considerably before the muscle performs even the slightest twitch. Only when the intensity has reached a certain degree does the muscle respond with a contraction; from here on the contraction is never wanting, and, up to a certain degree, becomes more energetic the more the intensity is increased. The stimulus, therefore, begins to operate only at a certain intensity, and this point is termed the threshold of stimulation. Below the threshold the stimulus is ineffective; above it the effect increases with increasing intensity of stimulus. For the different forms of living substance the value of the threshold is very different. Thus, nerve-fibres are put into activity by extremely feeble galvanic stimuli, while Amoba demands very strong currents. The same is true of all other varieties of stimuli in relation to the various forms of living substance.

\section{Trophic Stimuli}

For the sake of convenience our considerations thus far have been based upon the idea that a certain contrast exists between vital condition and stimulus, in so far as the former represents a stable given state, and the latter every change of that state. This sharp distinction cannot be maintained for the reason that vital conditions are not wholly stable and continuous factors, but in nature are constantly undergoing variations. Hence, under certain circumstances certain vital conditions can be considered also as stimuli, or what is the same thing, certain stimuli function as necessary vital conditions. A few concrete cases will make this at once clear. 
With all those organisms that do not exist in a constantly uniform nutrient medium, that rather must seek their food, food is available only at irregular intervals. Periods of lack and periods of superfluity alternate with one another. If such an organism has had no food for some time, if, e.g., an Amceba, which nourishes itself upon Algce, has been deprived of food for some time and by chance comes to a place where Algo exist, these food-organisms operate as a stimulus upon it and cause it to creep toward and ingest them. Here food acts as a stimulus, although it is a necessary vital condition. Analogous cases exist in the cellcommunity. The simplest example is afforded by the green plants. Light forms one of their most important vital conditions. Without light no cleavage of carbonic acid, no formation of starch, no assimilation, takes place in the green parts of the plant; the plant dies. Yet this condition undergoes the widest variations in intensity, for light continually alternates with darkness and, therefore, acts as a stimulus. Not only can the process of assimilation be regarded as a phenomenon of stimulation, but the light-stimulus produces, in addition, a series of other, very evident reactions which express themselves in motion. In the animal cell-community, also, cases in which stimuli are a vital condition are known in great number. The stimulating impulses that are produced in the central nervous system become transmitted to the tissue-cells through the nerve-fibres. A muscle, e.g., moves only when a stimulus is conducted to it from the brain or the spinal cord through its nerve. If the nerve be cut or in any other way be made incapable of transmitting the impulse from the central nervous system, the muscle can no longer move, and after a time atrophies. In less degree a muscle becomes feeble and decreases in mass when it is used little, i.e., when few impulses are sent to it from the central nervous system. This condition is termed atrophy from disuse. This is true not only of musclecells, but of all tissues to which, through their nerves, stimulating impulses are no longer conducted. In cases where, by disease, a portion of a nerve has become temporarily impassable to stimuli, medical treatment endeavours, often with success, to hinder the atrophy of the tissue supplied by the nerve by stimulating it artificially by electrical currents, and in this action of the galvanic current lies the sole therapeutic importance of elcetricity. The strengthening of an organ by use belongs also in this categury. By continued use, as every gymnast, fencer, oarsman, and mountainclimber knows, a muscle of moderate strength can be transformed in a short time in to one of marked strength and endurance, the mass increasing very considerably. The effect of all exercise depends upon the fact that stimulating impulses are sent continually into the organ in question, putting it into activity.

From these examples it is evident that certain stimuli can be 
at the same time very important vital conditions; and these stimuli, which are necessary to the continued maintenance of life, without which the nutrition, the metabolism, of the organs in question cannot continue undisturbed, are termed trophic stimuli.

Trophic stimuli do not stand in contrast with other stimuli; the term "trophic" simply signifies a special peculiarity of the action, and very different stimuli can have a trophic effect. As regards trophic stimuli that in the animal organism are transmitted through the nerves to the tissues, it has been believed that special trophic nerve-fibres and nerve-centres must be assumed in addition to the fibres and centres of known function; such nervefibres are asserted to have nothing whatever to do with the peculiar function of the tissue supplied by them, but merely regulate its nutrition and metabolism. This idea of so-called trophic nerves has produced in physiology and medicine much mischief and confusion, and recently has misled many men of science into the most fantastic ideas and supposed discoveries. But for every critical investigator, who is wont to associate a definite idea with the conceptions with which he deals, the confused idea of trophic nerves is simply a piece of the old mysticism of the vitalists. It is seen that the assumption of special trophic nerves and peculiar trophic stimuli, existing in addition to other stimuli, is not needed in order to explain the phenomena, but that the nerves that influence the characteristic function of every tissue regulate thereby the metabolism of the cells in question; in other words, every nerve serves as a trophic nerve for the tissue that it supplies, since the impulse which it conveys represents a vital condition for the tissue.

\section{B. THE IRRITABILITY OF LIVING SUBSTANCE}

\section{The Conception of Irvitability and the Nature of Reactions}

Every process of stimulation requires two factors: a stimulus, and a body that is irritable. If the two factors come into correlation there results a phenomenon of stimulation, a reaction. We have considered stimuli; we will now consider irritability.

A definition of irritability (excitability) that shall have general application, must be formulated somewhat as follows: The irritability of living substance is its capacity of reacting to changes in its environment by changes in the equilibrium of its matter and its energy. All other factors that might be included in the definition would be applicable to special cases only. Yet, frequently, the general conception, without being exactly defined, has more or less unconsciously been made to include special factors. For example, as regards the quantitative relations of the stimulus and the reaction, that case has been regarded as the type in which an 
enormous quantity of energy, the reaction, is produced by an excessively small quantity acting as the stimulus; hence the onesided view of irritability as the capacity of responding to slight stimuli with a disproportionately great evolution of energy. This case, although representing a special condition, is very obvious and wide-spread, and it is worth while to consider its details.

If, as an irritable body, a muscle with its nerve be selected, and as a stimulus the mechanical stimulus of pressure, the following arrangement can be made (Fig. 150). The calf-muscle (gastrocnemius) of a frog, the nerve of which (sciatic) has been freed,

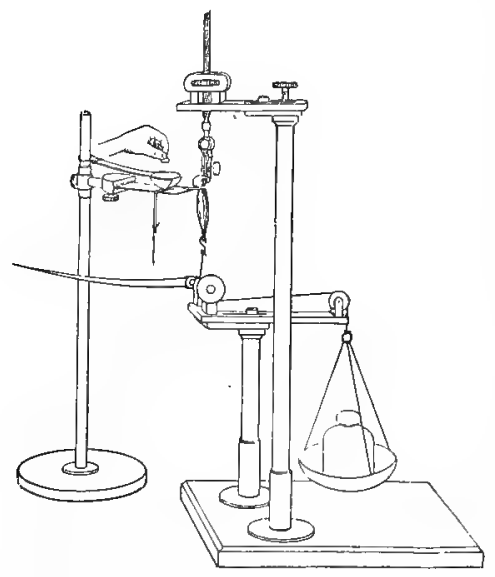

Fig. 150.-Apparatus for the demonstration of the inequality of the stimulus and the reaction. A nerve-muscle preparation is suspended upon a myograph; the muscle is loaded with a weight of $100 \mathrm{gr}$. and its nerve is laid over a glass plate supported by a stand. Upon the nerve rests a small aluminium pan having a sharp keel on the lower side, and into this a weight of $10 \mathrm{gr}$. falls from a height of about $1 \mathrm{~cm}$. At the moment of stimulation the muscle contracts and raises the $100 \mathrm{gr}$. about $1 \mathrm{~cm}$. is suspended in a muscleholder, the thigh-bone to which the muscle is attached at its upper end being fastened by a clamp. The lower end of the muscle with the tendon of Achilles is separated from the bone, and in the tendon a slit is made, into which a hook attached to a long thread is fastened. This thread is carried over two easily moving wheels, and, at its other end, is attached to a pan containing a weight of 100 gr. The nerve of the muscle-preparation lies stretched out upon a horizontal stand. Every stimulation of the nerve causes a twitch of the muscle. If, now, a weight of $10 \mathrm{gr}$. be allowed to fall upon the nerve from a height of about $1 \mathrm{~cm}$., so that the nerve is mechanically stimulated by the presssure, at the moment of stimulation a twitch of the muscle occurs, and the muscle raises the weight of $100 \mathrm{gr}$. to a height of about $1 \mathrm{~cm}$. Here the quantity of energy that corresponds to the work of the muscle is approximately ten times greater than the quantity of energy that has operated as a stimulus upon the muscle; and under favourable conditions the disproportion can be even much greater. According to the law of the conservation of energy it is clear that the considerable quantity of energy that is set free externally in the reaction cannot be derived by the transformation of the small quantity that has been introduced into the organism in the stimulus. It must, therefore, have come from the organism itself, and must have been stored pre- 
viously in the latter as potential energy. Hence in this case the irritability depends upon the fact that great quantities of potential energy are accumulated in the living substance of the muscle, so that the introduction of only a small quantity is needed to transform it into actual energy. But such irritability and such a reaction are not limited to living substance solely. Analogous conditions may be established in lifeless bodies. A spring stretched and held by a fine thread that maintains the tension in equilibrium represents a body in which a great quantity of potential energy is stored, although the body is in complete rest. If the thread that holds the spring be touched lightly with the edge of a sharp knife, the spring flies back with great force and performs external work. By a small stimulus, represented by the cutting of the thread, the potential energy of the spring has been transformed into actual energy; the cutting of the thread has, as is said, "discharged" the energy of the spring. In explosive bodies also there is such a discharge, and since there it is a discharge of chemical tension, the similarity of it with the processes of discharge in living substance is still greater, for in the latter also potential energy is stored up in the form of chemical tension. In a quantity of nitroglycerine the size of a pea there is contained such a quantity of potential energy that it needs only a slight impulse to produce a powerfully destructive effect. Like the nitroglycerine molecule, living substance is explosive, although in a manner that does not call forth so injurious effects.

But the processes of discharge, as has been said, are only special cases of reactions, and the relation between stimulus and reaction may be wholly different in other cases; for, on the one hand, there are stimuli, such as fall of temperature, withdrawil of food, and exclusion of oxygen, which consist not in the action but in the withdrawal of energy; and, on the other hand, there are reactions, such as those of narcotics, which are expressed not by an increase, but by a decrease and even a complete suppression of the production of energy. Accordingly, it is characteristic of the process of stimulation that no definite, generally valid, relation as regards the quantity of energy exists between the stimulus and the reaction. Hence, a conception of irritability that is to be generally valid must be formulated as above. As regards reactions, it must be said: The general action of all stimuli upon living substance consists in a change of spontancons vital phenomena.

With the enormous multiplicity of vital phenomena in accordance with the composition of living substance, and with the great variety of stimuli, it is a miori conceivable that the phenomena of stimulation must be very manifold. Moreover, to increase the variety of the reactions still more, not only the different varieties of the stimulus, but also the different intensities, as well as the time and place of the stimulation, cum call forth under circumstances very 
different phenomena. This great multiplicity in the phenomena of stimulation, in combination with the fact that general reactions have not yet been investigated systematically, make it at present very difficult to deduce from the facts general laws for reactions. Nevertheless, it is possible to establish empirically for groups of stimulation-phenomena common peculiarities.

The changes that spontaneous vital phenomena experience under the influence of stimuli are of various kinds. In the first place, the phenomena may continue unchanged in quality and undergo quantitative changes only. This may be expressed either in an augmentation of all, or of single phenomena-the reaction is then termed excitation [Erregung]-or in a diminution of all or single phenomena-the reaction is then termed depression [Lähmung]. ${ }^{1}$

In the second place, spontaneous vital phenomena may be wholly changed in kind, so that wholly new phenomena appear which otherwise do not occur at all in the life of the cell. Such reactions occur, e.g., in the metamorphic phenomena of necrobiotic processes, ${ }^{2}$ where under many influences not yet wholly known the cells of the body form substances, such as amyloid substance, which are completely foreign to them in normal life. These reactions have been very little investigated, and, so far as one can now judge, it appears as if they are only secondary results of quantitative changes of normal vital phenomena. Thus, it can be imagined that in metamorphic processes the appearance of foreign substances in the cell depends upon the fact that, as a result of chronic stimulation, one or more processes in the normal metabolism are gradually decreased or have entirely dropped out, so that compounds that normally are formed, but on account of immediate further transformation do not accumulate, are now stored in quantity, because the processes in the metabolism that are necessary to their transformation no longer exist. For the present, however, this must remain an hypothesis. The following consideration will have to do chiefly with the phenomena of excitation and depression. It is not superfluous sharply to emphasize our conceptions of stimulus, excitation, and depression, as well as the relations of these to one another, since not rarely in physiology because of the false idea, usually assumed, that a stimulus must always produce excitation, much confusion and difficulty in the judgment of phenomena have arisen. These can be avoided if the following definitions be accepted:

1 [The best English equivalent of the word Erregung seems to me to be "excitation." The translation of the word Lähmung has given some trouble. The customary English equivalent of the word is "paralysis," but it is easy to see that such a rendering would not convey the exact meaning of the author. After considering and rejecting various proposed terms, I have finally decided to aropt as the opposite of excitation the comparatively unobjectionable word "depression." - F. S. L.]

2 (y. 1. 330. 
1. Every change in the external ital conditions of un organism constitutes a stimulus.

2. Eve'y augmentation of "vital phenomenon, either of one or of all, constitutes excitation.

3. Every diminution of a rital phenomenon, either of one or of all, constitutes depression.

4. The action of stimuli can consist of ercitation or depression.

\section{The Duration of Reactions}

Another question, that of the duration of reactions, which naturally thus far has received much less systematic treatment, is of no less interest, for it is in the closest relation with subjects, such as those of adaptation, immunisation, etc., which are of far-reaching practical importance. It is to be expected that these subjects, which afford very promising problems for experimental cell-physiological research, will soon attract more attention. For the present only a few disconnected discoveries of a very general nature can be specified.

In general, it may be said that the duration of the reaction depends primarily upon the duration and intensity of the stimulus, and that after the cessation of the latter the reaction passes away the more rapidly, the briefer and feebler the stimulus was. A few special cases demand particular attention.

To consider first the relations under prolonged stimulation, usually during the continuance of the stimulation the reaction undergoes a change in accordance with the intensity of the stimulus. With feeble stimuli there is, after some time, an abatement and finally a cessation of the reaction: the living substance becomes accustomed, or adapted to the stimulus. Such phenomena may easily be observed in very different objects and with very different varieties of stimuli. Thus, as Engelmann $(79,1)$ and others $^{1}$ have shown, it is possible to accustom many unicellular organisms to relatively strong salt solutions which at first call out distinct phenomena of stimulation. If an Actinosphorium that has extended its pseudopodia in the customary, ray-like manner be placed in a weak solution of sodium bicarbonate, it gradually draws in its pseudopodia from all sides and becomes spherical. But soon minute projections reappear upon the surface, extend and lengthen, until the organism has assumed its original form and become completely normal. By successively increasing the concentration, the same result can be produced many times in succession. Such adaptations may be brought about to weak solutions of poisons, high temperatures, strong light, etc. If the stimuli are strong, no adaptation takes

\footnotetext{
$1(c)$. Terworn $(89,1)$.
} 
place, but the phenomena of fatigue and exhaustion develop (these will be discussed elsewhere); irritability gradually decreases, and death finally results. In contrast to these phenomena both of adaptation and fatigue, in a few cases with prolonged stimulation the reactions continue with equal intensity. An example of such cases is afforded by the muscles of the mammalian body, which exist in a certain state of excitation, or, to use the common term, possess a "tone." Such are especially the muscles that close the urinary bladder and the anus. These muscles are in a constant state of contraction, which is caused by stimuli that come from the cells of the nervous system and act uninterruptedly upon the former. The skeletal muscles also possess a constant, feeble tone, which is maintained by feeble stimuli coming mostly from the periphery and transmitted to them through the nervous system.

With brief stimulation the reactions give place, usually soon after its cessation, to the normal condition of the organism, but

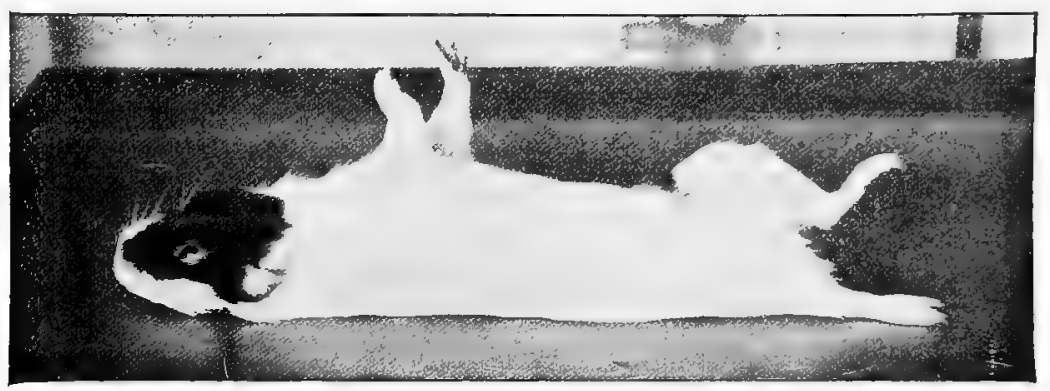

FIo. 151.-Guinea-pig, lying motionless upon his back, with the muscles of the extremities tonically contracted. The legs stand out stiflis.

there are cases in which the extinction does not begin immediately, but a long, under some circumstances a very long, after-effect exists. Thus, a single brief stimulus can put into long-continued, tonic excitation certain ganglion-cells and the muscles innervated by them. If, $\iota . g$., we seize a guinea-pig with the hands firmly but without great pressure, and turn him suddenly upon his back, he makes a few, brief, defensive movements and then lies motionless. It can be seen that the muscles of the extremities, which just before had made the defensive movements, are strongly contracted, so that the limbs stand out stiffly (Fig. 151). Whon the animal is undisturbed, this condition of tonic excitation may continue for a half-hour.

The phenomena of prolonged reflex tone after brief stimulation may be seen still more clearly in frogs that have been deprived of their cerebrum. If such a frog sitting quietly in the customary squatting attitude (Fig. 152, A) be gently stroked by two fingers along the sides of the spinal column, he raises himself upon his 
extremities by contracting their muscles, and stands, sometimes more than an hour, in this grotesque position (Fig. 152, B). By the proper operations it can be determined that by the mechanical stimulation of the skin the ganglion-cells at the base of the mid-

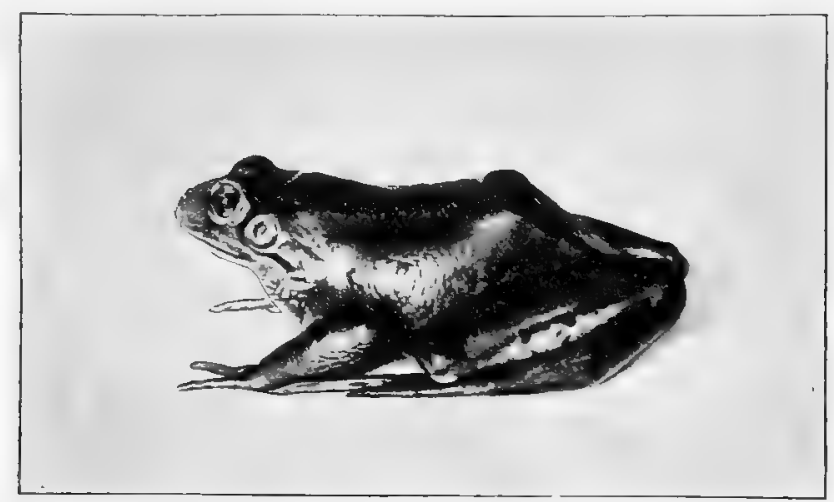

$-1$

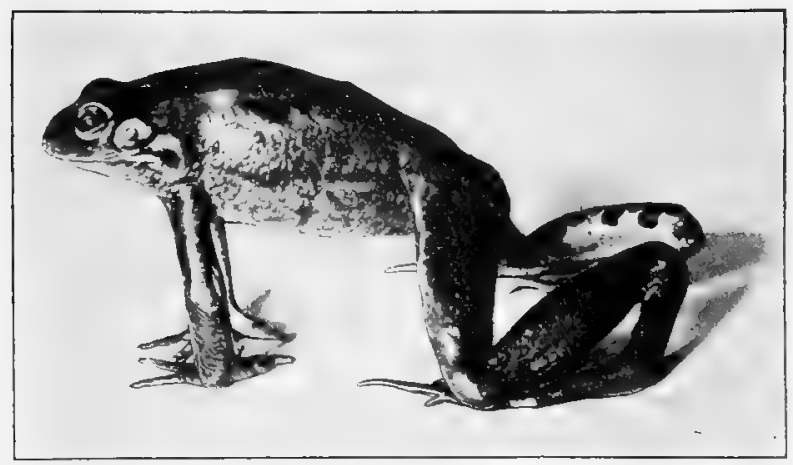

$b$

Fro. 15‥-Frog that has been deprived of his cerebrum. A, In the customary squatting attitude. $B$. In the attitude of general retlex tone; the muscles of the limbs and the back are in constant contraction, so that the frog stands immobile upon his raised legs in the attitude of a frightened cat.

brain are put into a tonic state of excitation, which is communicated to all the body-muscles that are innerrated from that point. ${ }^{1}$

The after-effects of many chemical stimuli, 'specially the bacterial poisons, are the most interesting and of most practical importance. It is an old experience that after recorery from certain infections diseases, snch as small-pox, scarlet ferer, and measles, the bodies of men and animals are immune to further infection from the same source. It is well known that the modern thera-

${ }^{1} \mathrm{Cf}$. Verworn $(96,5)$. 
peutics and prophylaxis of the infectious diseases are based upon this fact, especially the inoculation- and injection-methods of Jenner, Koch, Pasteur, Behring, Roux and others. We know how to produce immunity at will by the artificial introduction of weakened inoculation-substance, of metabolic products of the excitant of the disease in question, or of blood-serum from animals that have been exposed to the infection. In all these purely empirical methods of treatment we are totally ignorant of what goes on in the body; we can only say that the poisoning by the bacterial poisons produces in the cells an after-effect, which can continue in many cases, such as diphtheria, only a relatively short time, but in others, such as small-pox, for many years. A phenomenon is here presented, the explanation of which is as yet scarcely hinted at. But it is to be expected that cell-physiological researches, which replace with the simplest relations the complex and abstruse conditions presented by human and animal bodies, will be of the greatest service in assisting toward an understanding. In fact, investigations upon unicellular organisms with various chemical substances have shown that analogous phenomena are to be met with in these forms. Thus, by accustoming Infusuria to weak solutions of corrosive sublimate, Davenport ('96) has made them immune toward solutions of such strength as were at once fatal to non-immunised individuals. Cell-phrsiological research opens here an uncommonly wide and fruitful field. The systematic investigation of reactions in the single cell is of fundamental importance not only theoretically, but also for practical medicine. ${ }^{1}$

\section{The Conduction of the Stimulus}

Inseparably connected with irritability is another property of living substance, viz., the power of conduction of the stimulus. "If a mass of living substance be stimulated locally, as can be done very simply by touching it or pricking it with a fine needle, the reaction is not limited to the point stimulated, but spreads from that place more or less over the neighbouring parts.

The capacity of conducting the stimulus belongs to all living substance, but in very different degrees. While one kind conducts rapidly and far, another conducts slowly and only to the nearest surroundings.

The capacity of conduction is most pronounced in those forms that are developed exclusively for that purpose, riz., the animal nerve-fibres. Nerves conduct with enormous rapidity and to distances measured by meters. Helmholtz has computed that in a frog's nerve the stimulus is transmitted at a rate of $26 \mathrm{~m}$. per second. In man the rate is still greater, approximately $34 \mathrm{~m}$. in

1 Cf. Verworn ('96, 2). 
a secrond; in the lobster, as Lem Frederieg and Van de Velde have shown, it is less and amounts to about i $\mathrm{m}$. in a second. Various methods have buen devised for determining the rate of transmission in the nerve, an undertaking that with the great rapidity of the process is not easy. The principle of all these methods depends upon the determination of the difference in time between the appearance of a muscle-contraction, when the nerve belonging $t_{0}$ it is stimulated very near the muscle, and its appearance upon stimulation of the nerve at a more remote place (Fig. 153). For this purpose the spring-myograph of du BoisReymond can be employed, an apparatus that serves for the graphic representation of a muscular movement (Fig. 154). The apparatus consists of a muscle-holder in which the gastrocnemius muscle of a frog, the nerve of which is freed, is fastened by the femur; the muscle is connected with a lever, which accompanies every contraction and by means of a fine point records it upon a smoked glass plate which is shoved rapidly by. The glass plate moves in a sledge-like frame in a vertical plane in front of the writing-lever, and is put in motion by a spring. Simultaneously with the release of the spring an electrical stimulus is let loose upon the nerve; moreover, a tuning fork is made to vibrate, and traces its vibrations, likewise by means of a writing-point, upon the blackened glass plate. If the nerve be stimulated once at a distance of about $3 \mathrm{~cm}$. from the muscle, and once immediately at the muscle, the first contraction follows

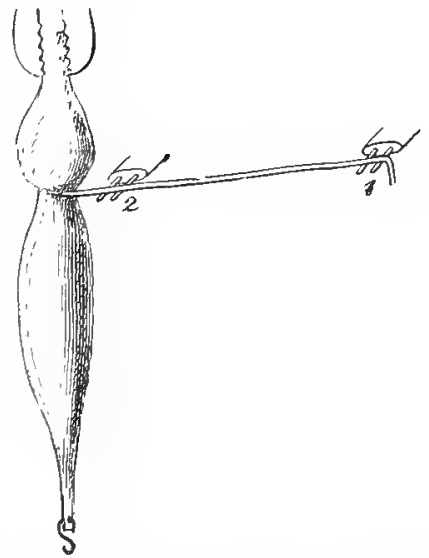

Fic, 153.-Gastrocnemius muscle of a frog with the sciatic nerve. The femur to which the nuscle is attached, is clamped in a muscle-holder, and the nerve is atimulated first at 1 , then iat $\stackrel{0 .}{*}$ a short time later than the second, because the first stimulus has a longer stretch than the second to pass over before it can act upon the muscle. This difference in time that in both cases elapses between the moment of stimulation and the appearance of the contraction, can be measured with extreme exactness upon the blackened plate, unon which the contraction is traced in the form of a curve, by the number of vibrations of the tuning fork that are traced simultaneously (Fig. 155). Since the number of vibrations of the tuning fork in one second is known, the duration of a single vibration can easily be computed, and from the number of vibrations that lie between the beginning of the second contraction and that of the first, the time can be calculated that elapsed 
while the stimulus was passing over a piece of nerve $3 \mathrm{~cm}$. in length. It is thus found that the rate of conduction of the

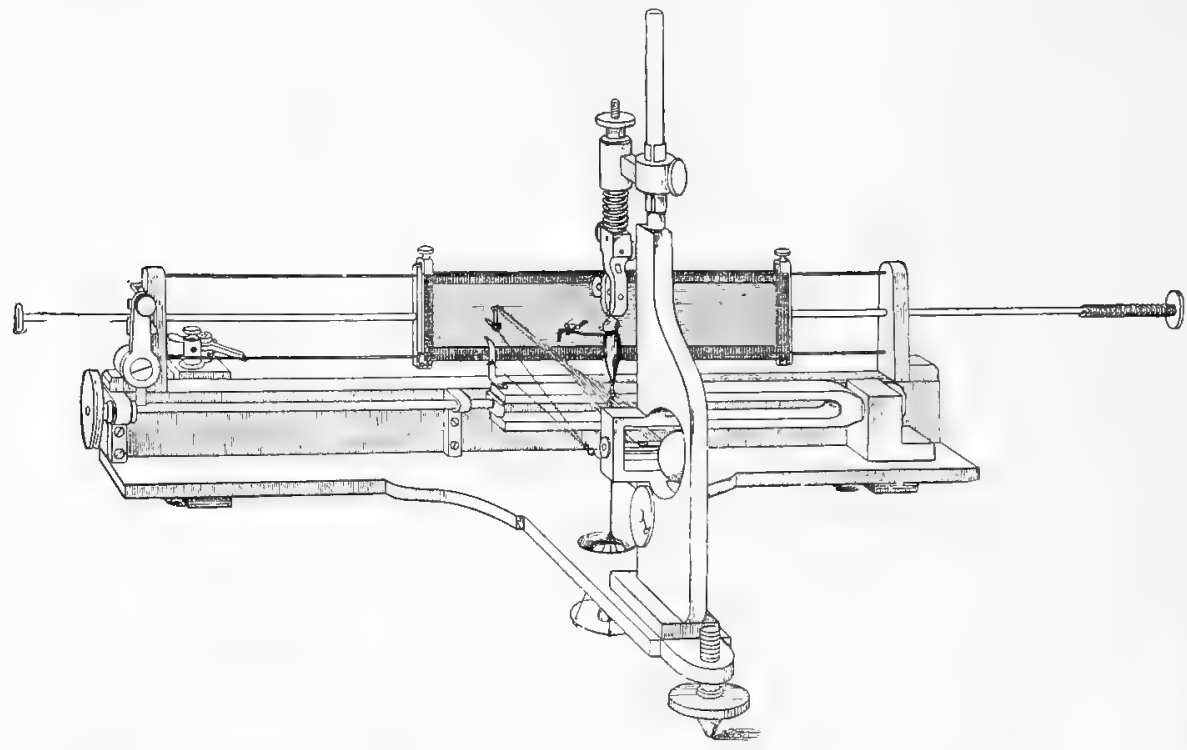

Fig. 154.-Du Bois-Reymond's spring-myogruph.

stimulus in a frog's nerve under normal conditions amounts to approximately $26 \mathrm{~m}$. in the second.

Other forms of living substance conduct the reaction considerably more slowly and some to a very short distance only, the effect being gradually extinguished with the distance. In very slowly cunducting objects the rate of conduction can be followed with the

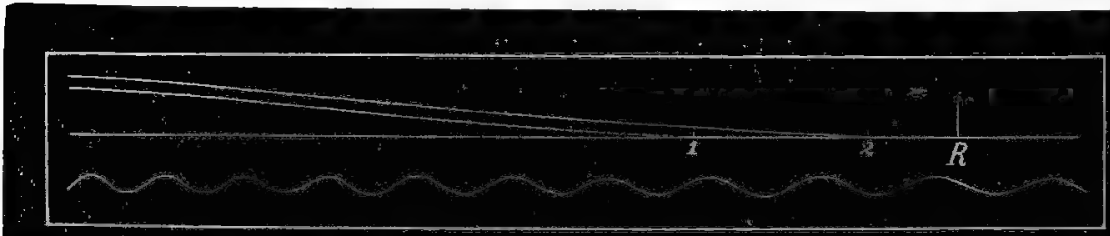

FIC. 155.-Ascending limb of the myographic curve taken with the spring-myograph, $R$, Moment of stimulation: 1 , beginning of the contraction upon stimulation of the nerve at a remote place (Fig. 153); $\approx$, beginning of the contrizetion upon stimulation immediately at the muscle. Below, tho curve of the tuning fork.

eye. Thus, in Diffugia the rate of conduction of the excitation can be very easily recognised under the microscope in the 
long finger-shaped pseudopodia by the fact that drop-like projections form on the surface of the pseudopodial plasma, beginning at the place of stimulation. If such a pseudopodium be stimulated only slightly at the tip by contact with a needle, the reaction extends a short distance only, the surface of the pseudopodium becoming slightly undulating (Fig. 156, a). But if it be stimulated more strongly, the reaction is stronger and is transmitted considerably farther (Fig. 156, b). The reaction diminishes in extent as the distance from the place of stimulation increases, and finally it is extinguished. ${ }^{1}$ Very slight conduction is found in many rhizopods that have thread-like pseudopodia, e.g., Onbitolites ( $C f$. Fig. 98 , p. 238). Here even with the strongest stimulation, such as cutting across a pseudopodium, the excitation is limited to the immediate vicinity of the place stimulated, the protoplasm there being drawn together into one or more small globules. These " globules glide centripetally for a very considerable distance along the pseudopodial thread, which thus begins to shorten, while the globules gradually dissolve and allow their substance to flow into the central body (Fig. 157).

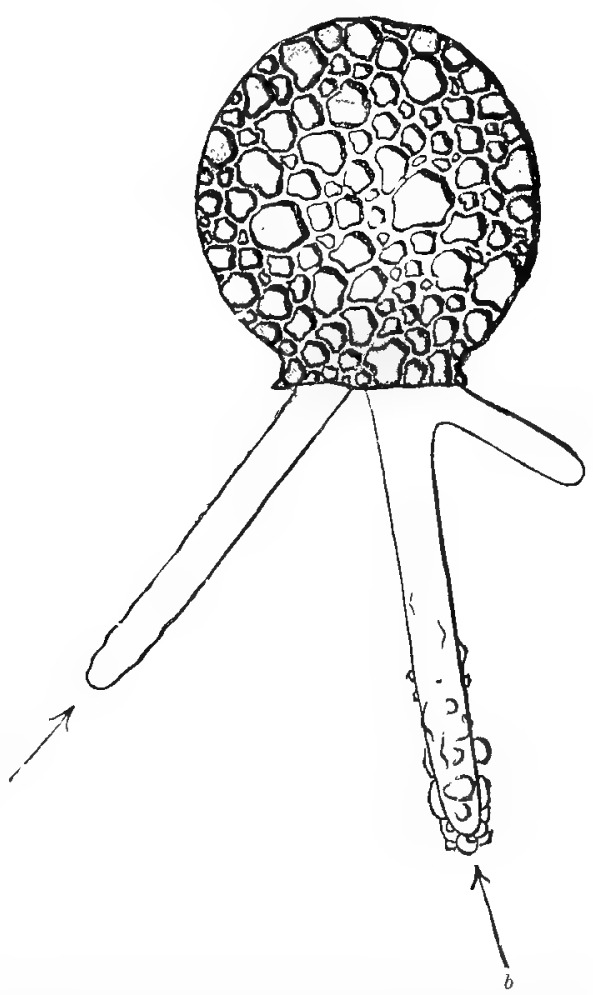

FIG.156. Diffugia urceolata. Three finger-shaped, hyaline pseudopodia are projected out of the urn-shaped shell made of sand-grains. At a feebly stimulated locally; at $b$ somewhat more strongly stimulated.

Their movement is not to be regarded as a conduction of the excitation, ${ }^{2}$ but only as the expression of the transport of substance by the stimulated protoplasmic mass to the cell-body; for the protoplasm in the vicinity of the globules exhibits no phenomena of excitation, but streams on quietly in a centrifugal direction.

] $C f$. Verworn $(' 89,1)$.

${ }^{2}$ In the first edition of this book this was so regarded; but later studies upon the Rhizopoda of the Red Sea have convinced me that conduction of excitation and transport of substance are to be separated from one another in naked protoplasmic masses. $C f$. Verworn $(' 96,3)$. 
But between the very slight power and rate of conduction possessed by Orbitolites, and the very great power and rate possessed by the nerve, there are found in the various living forms the greatest variety of transitions. The cross-striated muscle-fibre

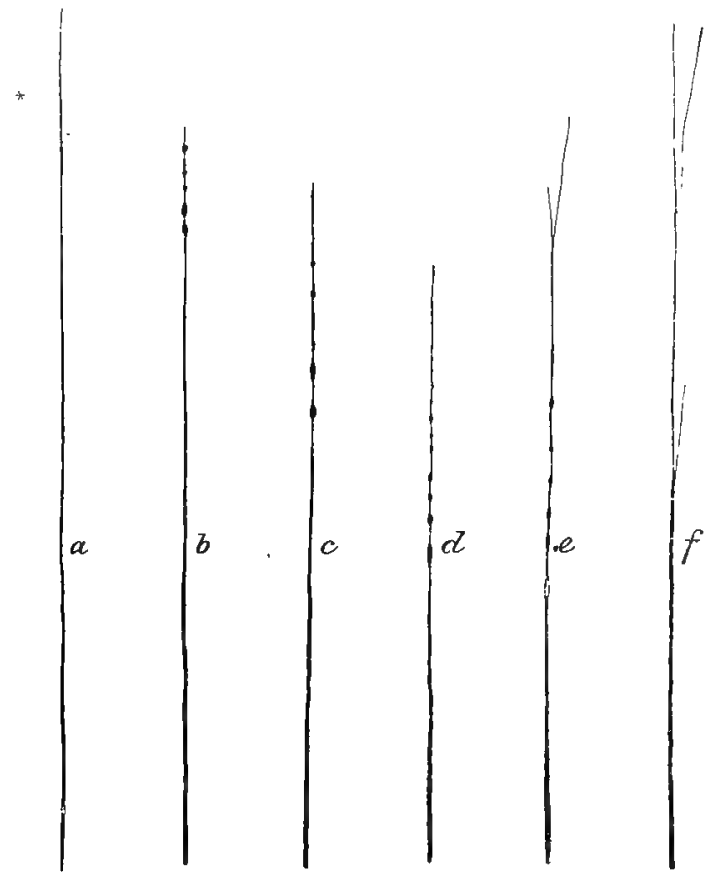

Fic. 157.-Pseudopodium of orvilolites. $\alpha$, At * cut across ; $b$, effect of stimulation (formation of protoplasmic globules) limited to the immediate vicinity of the place stimulated; $c-f$, transport of substance. The stimulated masses are transported along the pseudopodium to the central cell-body, and their substance becomes gradually spread out $(e, f)$; the unstimulated protoplasm exhibits no phenomena of excitation but continues to flow centrifugally, and the pseudopodium soon lengthens again $(e, f)$.

conducts considerably more slowly than the nerve, the smooth muscle-fibre still more slowly than the cross-striated, and so on. Thus, according to the rute of conduction, living substances can be arranged in a long series showing most delicate transitions.

\section{The Phenomena of Cell-Stinulation}

After this general discussion of the individual elements of the process of stimulation we can pass to the consideration of the phenomena of stimulation themselves.

Since the single cell does not allow the various vital phenomena to be recognised with equal readiness, but according to its specific 
work permits one phase to come more to the front, whether it be metabolism, or change of form, or transformation of energy, it is advantageous to select for the study of any vital phenomenon a specific form of cell in which the vital phenomenon in question is expressed especially clearly. By this method the phenomena of changes of substance, of form and of energy may be considered separately in different objects. But this ought never to lead us into considering these different groups of phenomena as mutually independent. They are merely different phases of one and the same process.

\section{A. THE ACTIONS OF THE VARIOUS STIMULI}

\section{The Actions of Chemical Stimuli}

The number of chemical bodies that when brought into contact with living substance enter into chemical relation with its constituents is enormous, but thus far only a few of them have been investigated as regards their stimulating effects. A comprehensive, comparative, cell-physiological investigation of chemical stimuli and their actions, undertaken from a systematic point of view, would require a very long time, but would surely yield very valuable results. For the present, our knowledge of these stimuli and their effects is so full of gaps that a systematic summary of it is not possible. We must, therefore, limit ourselves to the consideration of a few typical phenomena.

\section{a. The Phenomenc of Excitation}

In general, increase in the quantity of ingested food-stuffs acts as a chemical stimulus to augment metabolism. The best example is afforded by the cells of the various tissues of the human body, the most essential food-stuff of which is proteid. As Voit ('81) has shown, a strong man, working hard, needs 118 grs. of proteid in order to maintain his nitrogenous equilibrium intact, i.e., in order to replace the quantity of nitrogen derived from the destruction of the living substance of his cells and excreted in the urine. If this quantity of ingested proteid, which is a necessary vital condition, be increased, as is the case with most men living under good conditions, the greater quantity is not employed for the construction of new cells, for the increase of living substance, but is taken up by the tissue-cells from the blood, passed over into living proteid and split up, to leave the body again almost completely in the urine as the products of retrogressive proteidmetamorphosis (urea, uric acid, creatinin, etc.). The increase of the proteid-income beyond a certain measure (118 grs.)accomplishes, 
therefore, a corresponding increase of both the assimilatory and the dissimilatory phases of the metabolism of the tissue-cells.

A similar condition exists among plants. The carbonic acid of the air serves the plant as food and is split up in the chlorophyllbodies of the living cells. The carbon set free is then employed, together with the water received through the roots, for the synthesis of starch, or assimilation. If more carbonic acid be brought to the plant than is contained in the air as its necessary vital condition, the splitting-up of carbonic acid and the assimilation of starch are increased in equal measure up to a certain degree. The increase of the quantity of food, therefore, conditions also an increase of metabolism.

But this does not always hold good. Regarding oxygen, we know, at least, that its increase in quantity beyond the amount necessary for life is essentially without influence upon the metabolism of the tissue-cells. The tissue-cells of the human body are within wide limits independent of the percentage and the partial pressure of oxygen in the air, and experience no. augmentation of metabolism with increase of the income of oxygen. Whether the same is true of free-living cells and the cells of lower animals still needs investigation.

In many cases the increased income of food that is accompanied by an increase of metabolism causes also a clearly recognisable increase in change of form. While in the tissue-cells of the human body, as has been seen, the food that is introduced beyond the necessary quantity is under normal conditions destroyed excepting an extremely small fraction, and is not employed for the increase of living substance, in many unicellular organisms, especially in Bacteric and Infusoria, an increase of the assimilatory processes, and in unequal measure of the dissimilatory processes also, takes place with increase of food. The result of this is an increase of living substance, a "fattening," which is expressed in rapid growth and continued cell-division. If, e.g., putrefactive bacteria (Bacterium termo, Spirillum undula, etc.) be transferred from a liquid in which they are living in small numbers, into a good nutrient solution, such as an infusion of hay, they at once begin to increase enormously, until from the fow bacteria with which the nutrient solution was infected many millions may have developed. If there be placed in such a hay-infusion swarming with putrefactive bacteria a Paramecium, which nourishes itself upon such bacteria, in a few days it may be seen that from this one infusorian thousands have been produced, so that they give to the liquid a milky cloudiness. Thus the assimilatory phase of the metabolism of these micro-organisms becomes enormously increased by superfluity of food.

Under pathological conditions also similar phenomena occur in the tissue-cells of the human body, and modern pathology 
recognises a whole series of analogous cases in the various kinds of pathogenic neoplasms or tumours, to which belong also malignant cancers. These tumours (carcinoma, sarcoma, myoma, fibroma, etc.) arise by the rapid division of the cells of a normal tissue, e.g., the epidermis. There thus results in the particular place an enormous increase of cells, a growth, which leads frequently to a very extensive tumour and completely chokes the neighbouring tissues in which it grows, so that they become incapable of life and perish. Without doubt, in many cases this rapid cell-increase is due to chemical causes acting upon the cells in question. Although thus far it is an open question whether or not tumours, especially carcinoma, are a result of infection by certain micro-organisms, the majority of pathologists incline to the view that they are to be traced to a change in the nutrition of the cells.

Much more evident than the effects of chemical stimuli upon metabolism and form-changes are the effects upon the transformation of energy, especially upon movement. Regarding the effects upon the amoboid movements of naked protoplasmic masses, such as Rhizopoda, Amceba, Myxomycetes, Polythalamic, and the protoplasmic bodies of plant-cells, the classic investigations of Max Schultze ('63) and Kühne ('64); over thirty years ago, have afforded information. The most
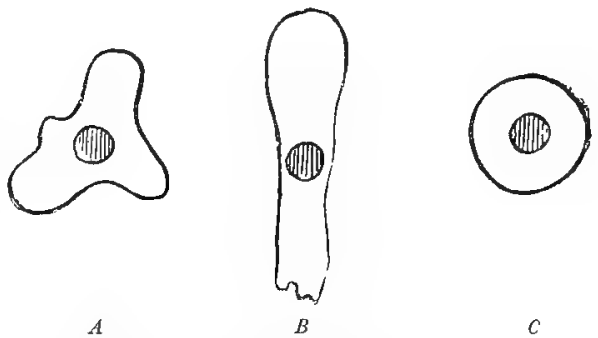

$A$

FIG. 158.-Amaba. $A$, With pseudopodia extending in different directions. $B$, Creeping, with a long pseudopodium in one direction (form of $A$ maba linnas). $C$, Contracted to a ball upon chemical stimulation. wide-spread effect here is the calling-out of a contraction, i.e., the retraction of pseudopodia, frequently after a preliminary acceleration of the protoplasmic streaming at the beginning of the action. The greatest variety of chemical substances can produce this reaction. If, e.g., to a drop of water in which many amœbae exist there be added a $1-2$ per cent. solution of common salt, or a solution of $0 \cdot 1$ per cent. hydrochloric acid, or of 1 per cent. potassium hydrate, or other acids, alkalies and salts in weak solution, the amœbae immediately draw in their pseudopodia and assume a spherical form (Fig. 158). Carbonic acid exerts the same effect, if the amobae be exposed in a gas chember ${ }^{1}$ for some time to the action of the gas. Other naked protoplasmic masses behave similarly toward these chemical stimuli. The delicate Actinosphorium Eichhonnii, which with its straight, ray-like pseudopodia appears like a minute sun, when 
brought into contact with these stimuli, likewise gradually draws in its pseudopodia, the protoplasm becoming contracted into numerous, small globules and spindles, which slowly flow centripetally into the cell-body ${ }^{1}$ (Fig. 159).

Upon the effect of chemical stimuli upon ciliary motion, Engelmann ('79, 1) and Rossbach ('71) especially have carried out detailed investigations. Here also the greatest variety of substances, such as acids, alkalies and salts, carbonic acid and various alkaloids, have like effects, which always consist in an augmentation of the activity of the cilia or flagella, the rate of their beat being considerably increased. The result is a considerable augmentation of the motor effect, which can be clearly observed in free-living ciliated cells, such as Infusoria, in a great acceleration of their

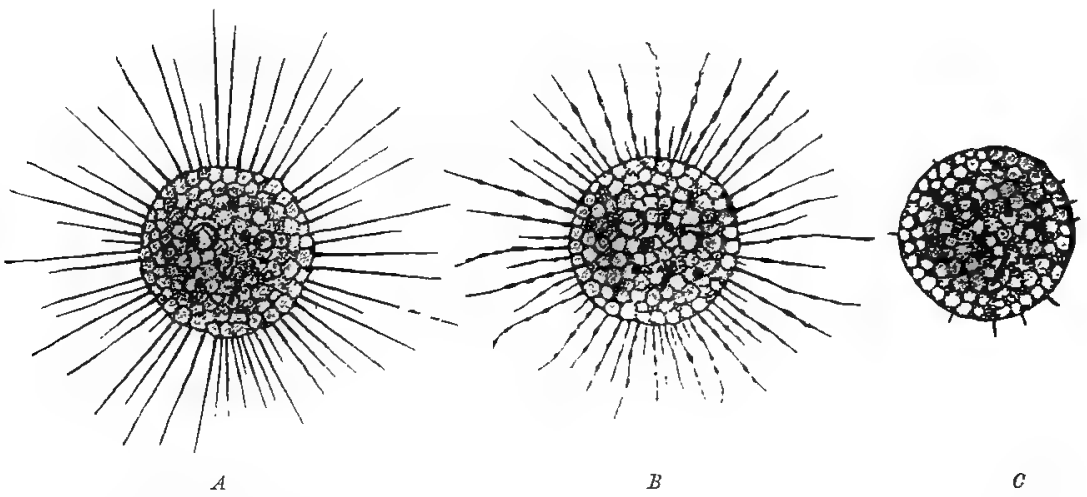

FIG. 159.-Actinospherium under chemical stimulation. $A$, Unstimulated; $B$, at the beginning of the stimulation; $C$, after the stimulation has continued for some time (the pseudopodia are almost entirely drawn in).

motion. After the addition of chemical reagents the Infusoria by the strokes of their cilia rush madly through the field of view.

Numerous chemical stimuli act upon the different forms of muscle-fibres (myoids, smooth muscle-fibres, cross-striated musclefibres) in a manner analogous to that upon naked protoplasmic inasses, by calling out contractions. If to a drop of water in which many Vorticelloe exist, waving their bodies gracefully upon their extended stalk-muscles, chemical substances of the above-mentioned kinds be added, all the Vorticello immediately draw together, their stalk-muscles suddenly contracting in their elastic sheaths, and coiling up into delicate spirals (Fig. 160, $b$ ). Cross-striated muscles likewise contract suddenly upon chemical stimulation. If, e.g., the sartorius muscle of a frog, which forms a small band of nearly parillel, cross-striated muscle-fibres, be clamped in a muscle-holder

\footnotetext{
1. Cf. Verworn ('89. 1).
} 
by means of the attached leg-bones, and a thread, passing over a wheel and attached to a small weight, be drawn through the pelvic bone, to which the muscle is also attached, every movement of the muscle can be observed in a signalling-lever, which is fastened to the wheel (Fig. 161). It, now, a dish containing ammonium carbonate be brought under the muscle, the latter is chemically stimulated by the vapours of the ammonia, and performs contractions, which can be shown clearly by the lever and can be traced upon a smoked drum. Biedermann ('80) observed a very remarkable phenomenon in the sartorius muscle when he let it hang in a temperature of $3^{\circ}-10^{\circ} \mathrm{C}$. in a solution of 5 grs. common salt, 2 grs. alkaline sodium phosphate, and 0.5 grs. sodium carbonate in one litre of water (Fig. 162). The muscle then showed rhythmic contractions, a phenomenon that otherwise is neverobserved in this muscle during life, and suggests constantly the rhythmic motion of cardiac musclefibres.

The chemical effects of stimulation in contractile substances, thus far spoken of, consist of contractions. But certain chemical stimuli produce expansion. Such, e.g., are food-stutf's, and especially oxygen. These phenomena have been discussed elsewhere. ${ }^{1}$ They consist chietly in the fact that in an atmosphere free of oxygen Amcba and marine Rhizopoda cease the formation of pseudopodia and undergo a diminution of expansory processes, developing the latter again when new oxygen is introduced. Kuihne (l. c.) has observed the same in Myxomycetes, in the reticulate plasmodia of Didymium, which lives upon decaying leaves. When he introduced a dried, and, therefore, completely motionless, piece of the plasmodium into a vessel filled with water boiled and hence free of oxygen, which was shut off by mercury from the air, it remained in complete rest. But as soon as a few bubbles of oxygen were added to the Didymium, the latter began to extend pseudopodia and to spread itself out in an arborescent

$$
{ }^{1} \text { Cf. p. } 284 .
$$


manner on the inner surface of the vessel. From these experiments it is very clear that oxygen acts as a stimulus, giving rise to the expansory phase of protoplasmic movement.

The production of other forms of energy besides that of movement is also excited by chemical stimuli. Since it would, however, lead too far to consider all the excitation-effects of such stimuli, only the facts connected with the production of light will be presented.

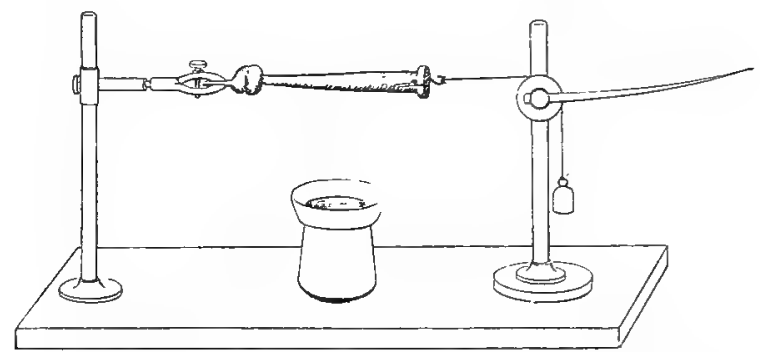

FIG. 161.-Chemical stimulation of the sartorius muscle of the frog.

For the investigation of this the unicellular organisms are best fitted, for in them all conditions are simplest and most easily observed. It is known of many unicellular organisms, Bacteria, Radiolaria, etc, that they develop light as the result of chemical, as of various other stimuli. But light-production has been investigated most frequently and in most detail in the Noctilucas,

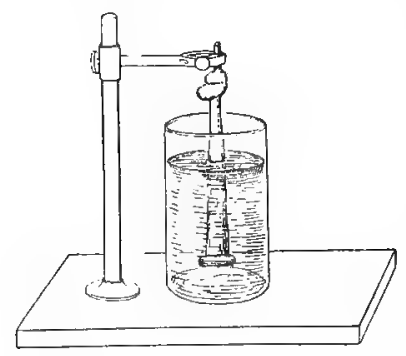

Fra. 162.-Production of rhythmic contractions in the sartorius muscle by chemical stimulation. the peculiar Flagellata which usually produce the light on the surface of the water in our northern seas (Fig. 163). Recently Massart ('93) has studied again in detail the action of chemical stimuli upon them. In a vessel containing sea-water, in which the Noctilucce rested quietly upon the surface without emitting light, he placed carefully with a pipette various substances, such as distilled water, a concentrated solution of common salt, a solution of sugar, etc., and in each case let the drop spread slowly over the surface of the sea-water. The result was that as soon as the liquids introduced came into contact with the Noctiluce, the latter became brilliantly lighted, and the pleasing spectacle was presented of a slowly widening, glowing circle, spreading over the surface of the watcr. A similar phenomenon can be observed very well in Radioleria, especially in the large Thalcssicolla, which emits light activaly upon a change in the concentration of the sea-water in 
which it exists, or upon being transferred to fresh water. The various luminous Bacteria, which produce, e.g., the luminosity of dead sea-fish, behave similarly.

Finally, the living substance of nerves and ganglion-cells can be ('xcited by chemical stimuli. The excitation in the nerve-substance itself is not visible without special methods; but a clear expression of it in motor nerves is exhibited in the contraction of muscles supplied by them. If, $c . \%$, the sciatic nerve of a frog be stimulated by its central (nd being dipped into glycerine, a concentrated solution of conmon salt, or a solution of a mineral acid, an alkali, a metallic salt or sugar, contractions of the leg-muscles of the frog take place, and prove that the nerve is excited. Excitation by chemical stimuli can be observed in the excised nerve also by means of the galvanometer through the

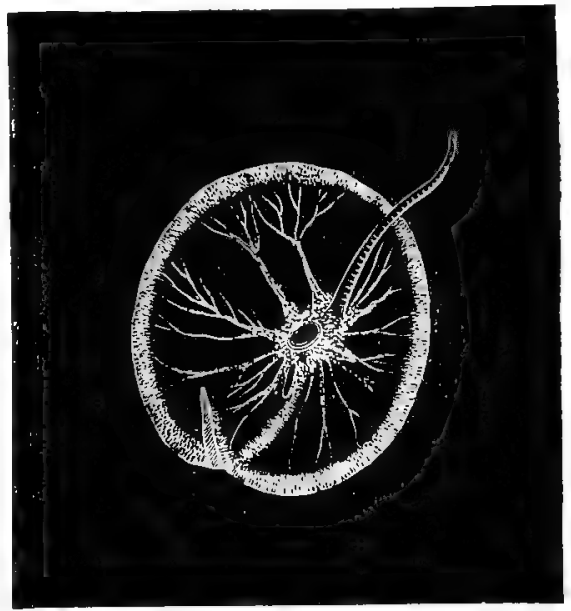

FıG. 163.-Noctiluca miliaris, a marine flagellateinfusorian cell.

development of electricity, which influences the current derived from the resting nerve.

\section{b. The Phenomena of Depression}

In contrast to the exciting effects of the chemical stimuli just mentioned are the effects of certain chemical substances, which depress or wholly suppress vital phenomena. These substances are, hence, termed nareotics or ancesthetics. Among them belong especially those that depress all forms of living substance and all vital phenomena: alcohol, ether, chloroform, and chloral hydrate. With these belong the great group of alkaloids, comprising morphine, quinine, veratrine, digitaline, strychnine, curare, etc., some of which act upon a great variety of living cells, while others affect specific cells only, especially those of the central nervous system.

The depressing effects of narcotics upon the phenomena of metabolism have been studied especially by Claude Bernard ('78). This well-known Parisian physiologist showed that metabolism is suppressed by chloroform-narcosis in very different forms of cells. If yeast-cells, which, as is well known, in the course of their 
metabolism split up grape-sugar into carbonic acid and alcohol, be placed in two fermentation-tubes (Fig. 164), one of which contains a pure solution of grape-sugar, the other some chloroform-water mixed with a similar solution of grape-sugar, there appears at once, under otherwise completely identical conditions, in the first tube a fermentation, as is evident from the carbonic acid rising and accumulating above (Fig. 164, $A$ ), but in the second tube an entire absence of fermentation (Fig. 164, $B$ ). If the contents of the second tube be left open to the air for a time, so that the chloroform evaporates, fermentation appears there later. The chloroform-water, therefore, only inhibits the metabolism of the yeast-cells without killing them.

In plant-cells also the depression of metabolism is very easily brought about, especially the cessation of the cleavage of carbonic
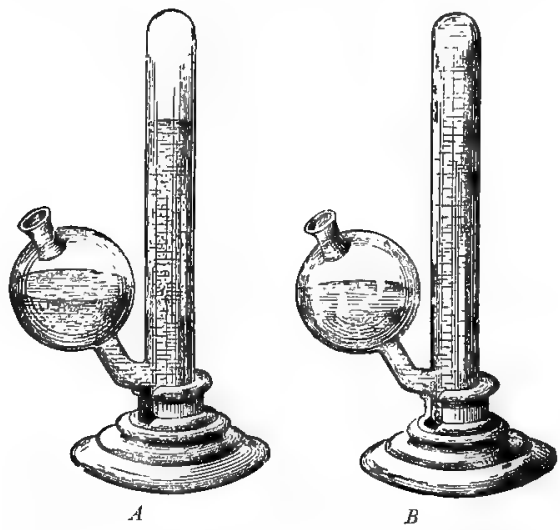

FIG. 164.-Fermentation experiment. A, Production of carbonic acid in a solution of grapesugar by means of yeast-cells. $B$, The solution of grape-sugar is not fermenting because the yeast-cells are narcotised by chloroform-water.

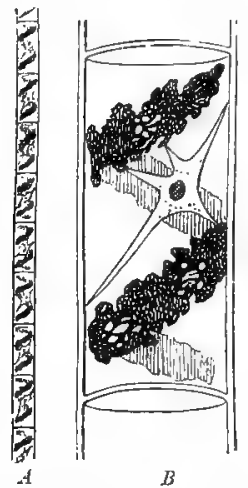

F1G. 165.-Spirogyra, a filose Alga. $A$, Piece of a thread consisting of many cells arranged in a row. $B$, Single cell with the characteristic spiral band of chlorophyll and the star-shaped protoplasmic body.

acid in chlorophyll. Claude Bernard employed for this a filose, aquatic Alga, Spirogyra, the cylindrical cells of which are arranged lengthwise one after another in fine threads and possess a delicate, spirally wound band of chlorophyll (Fig. 165). Under two bell-jars, of which one was filled with water containing carbonic acid, the other with water containing carbonic acid and chloroform, he placed a quantity of Spirogyra threads and exposed the jar to the sunlight. After some time the cells in the first jar had evolved a considerable quantity of oxygen, while in the second the cvolution of oxygen and, therefore, the dissociation of carbonic acid, were wholly absent.

Corresponding with the cessation of metabolism, the phenomeni 
of form-changes are also depressed in narcosis. Growth and celldivision cease. In order to prove the inhibition of growth, Claude Bernard arranged the following experiment (Fig. 166). Two empty, cylindrical flasks were provided, above and below, with openings that were closed by rubber stoppers, each pierced by a glass tubc. In each of these flasks there was placed, half-way up, a moist sponge, and upon the latter sprouting plant-seeds were laid. Through the lower opening one flask $\left(e^{\prime}\right)$ communicated by means of a rubber tube (V) with a glass cylinder $(t)$, which contained at its bottom a layer of ether (S); through the stopper of the cylinder, beside the tube $\mathrm{V}$, an open glass tube $(a)$ projected

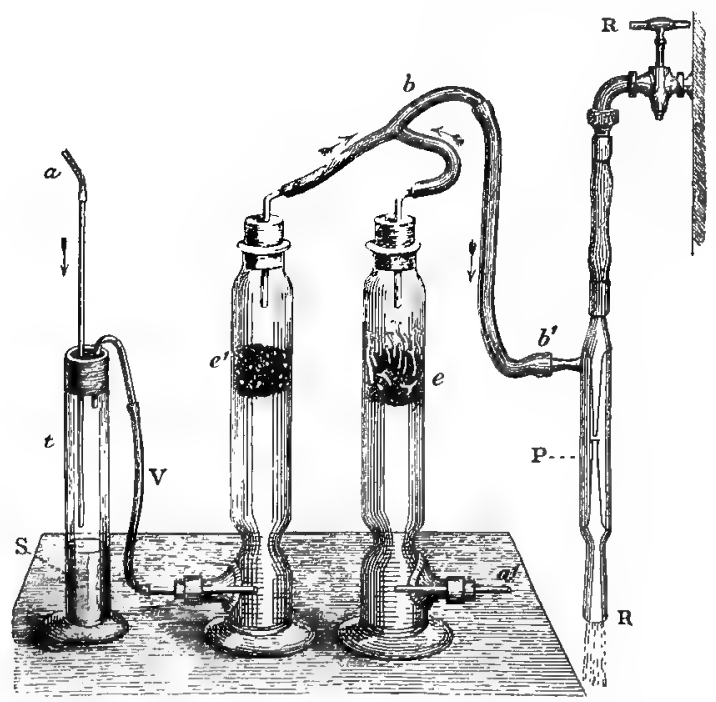

IIG. 166.-Apparatus for the comparison of germinating plant-seeds in the normal condition and in narcosis. (Aftor Claudo Bernard.)

from the outside down to onc-half the depth of the cylinder. The lower opening of the other flask (e) communicated through the glass tube $\left(a^{\prime}\right)$ directly with the outside air. To the glass tubes that led outside through the stoppers in the necks of the flasks, a forked rubber tube $(b)$ was fastened, which was in connection with an aspiration-apparatus $(\mathrm{P})$. If the water of the water-pipe $(\mathrm{R})$ was let through the aspirator, it sucked the air through the two glass flasks, of which the one received pure air directly from the outside through the tube $\left(\alpha^{\prime}\right)$, while the other took in through the glass cylinder $(t)$ air charged with ether-vapour. In this way a continuous stream of pure air passed through the germinating seeds of the one cylinder and a stream of ether-vapour 
through the seeds of the other. After some days under this arrangement the seeds that were in pure air had grown out into long seedlings (e), while those bathed by the ether-vapour showed no growth at all, without, however, having lost the capacity of germinating in pure air.

The brothers Hertwig ('87) have investigated the depressing action of solutions of chloral hydrate upon cell-division in eggs of the sea-urchin. When they let a $0.2-0.5$ per cent. solution of chloral act for some time ( 5 minutes-3 hours) upon eggs that were about to develop, cell-division did not go on. Both the nucleus and the protoplasm remained in the stage of division in which they already

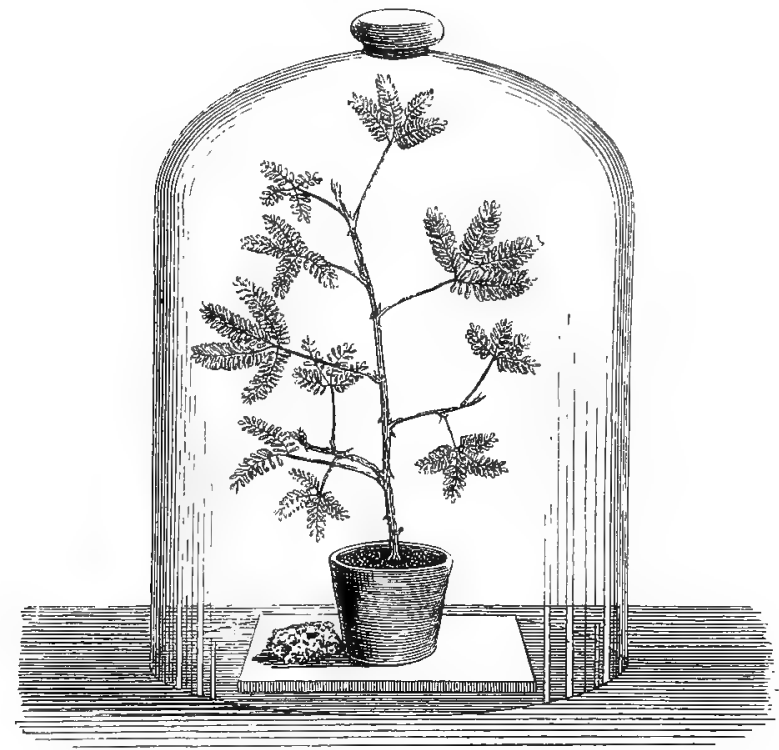

HIo. 1t7.-. Wimosa pedica in ether-narcosis. (After Claude Bernard.)

were, while the formation of rays about the centrosomes was completely absent. Only after the eggs had been washed for a considerable time with pure sea-water did the development and division of the cell proceed again.

Finally, the phenomena of transformation of cnergy are also depressed by narcosis. Both the spontaneous production of energy and the capacity of reacting to stimuli are diminished, and finally wholly cease. Among the phenomena of motion Claude Bernard has shown this for the turgescence-movements of Mimosc pudica. ${ }^{1}$ If a pot containing a Mimosa be placed under a bell-jar, under which is a sponge soaked with ether (Fig. 167), the spontaneous move-

$$
1 \text { ('f. p. } 227 .
$$


ments cease, and, moreover, after some time it is no longer possible to call forth by stimuli the well-known movements, which consist of a falling of the branches and a folding together of the leaves. The irritability is extinguished, the plant is in narcosis. "What a singular thing," says Claude Bernard, "plants can be anæsthetised like animals, and absolutely the same phenomena can be observed in the two."

Like the turgescence-movements, the growth-movements of plants also cease in narcosis, and the secretory movements of the Diatomeo, Oscillarioe, and Desmidiacees ${ }^{1}$ are wanting.

Contraction-movements are also depressed by narcotics; but, as a rule, at the beginning of the influence a short stage of excitation is noticed, in which the movements are accelerated. The protoplasmic movements of Amorba cease after the cells have contracted into a ball. As Binz ('67) found, quinine especially exerts a powerful paralysing action upon the amœboid movements of leucocytes. Engelmann ('68) carried out extensive investigations upon the depressing action of narcotics upon ciliary motion. When he let the vapour of ether or chloroform act upon the ciliated cells of the pharyngeal mucous membrane of a frog in a gas-chamber, after a rapid preliminary stage of excitation, in which the motion was accelerated, a standstill of the cilia took place. If the duration of the action was not too long, the motion appeared again after the introduction of fresh air. According to the observations of the Hertwigs ('87), similar behaviour was exhibited by the flagella of spermatozoa that had been brought to complete standstill by etherand chloroform-vapours, as well as by small doses of quinine and chloral hydrate, so that the fertilisation of the ovum was hindered by the absence of their movements. In Infusoria also by the introduction of chloroform-water, after a short stage of excitation in which the cells whirl madly through the water, ciliary motion is inhibited. In Stentor, in addition to this fact, the paralysis of the myoids by the chloroform-water can be observed at the same time. In their undisturbed condition the Stentors are extended in the form of delicate trumpets with their aboral pole attached at the bottom (Fig. 168, A). From time to time, partly spontaneously and partly as a result of stimulation, they jerk together into stalked balls (Fig. 168, C) by the contraction of their tine myoid-fibres that extend from the upper to the lower end of the cell-body in the exoplasm. In narcosis, however, after a sudden twitch at the beginning of the influence, they assume a stage of moderate contraction (Fig. 168, B), their cilia cease to beat, and their bodies do not shrink into the customary ball either spontaneously or upon stimulation, until by transference into fresh water the narcosis is ended. Like the smooth myoid-fibres, the irritability of cross-striated skeletal muscles also is completely abolished by narcosis. A frog's muscle

$$
{ }^{1} \text { Cf. p. } 231 \text {. }
$$


that is carefully and slowly bathed with air containing the vapour of ether cannot be made to contract by any kind of stimulus. Nevertheless, the vital processes in the muscle are not at a complete standstill, as is evident from the fact shown by Biedermann ('85) that the narcotised muscle produces electricity when stimulated, just like the contracting muscle in the normal state. The stimulated part, as well as the artificial cross-section, appears by galvanometric investigation electrically negative to the resting part, as in normal conditions. In narcosis, therefore, certain metabolic processes must still remain
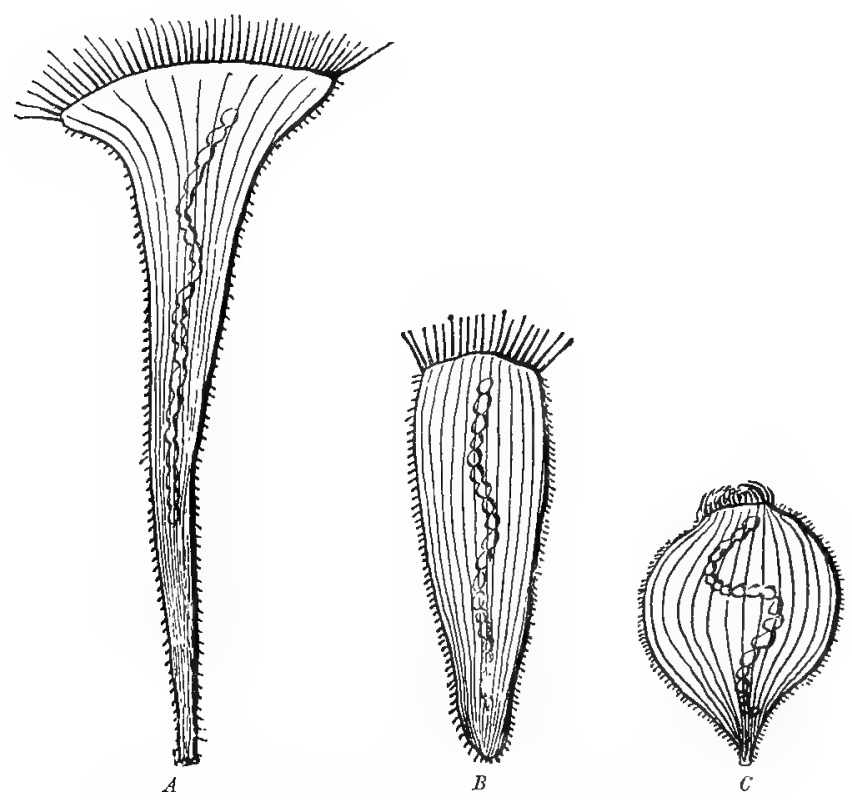

Frg. 168.-Stentor corrters. $A$, Wholly exteuded in rest; $B$, in the condition of moderate contraction as in free swinming; $C$, completely contracted.

undisturbed; and perhaps this is true not only of muscle but of the narcotic conditions of all living substance.

Recently, Massart ('93) has been able to abolish completely the development of light in Noctiluce by alcohol, by laying several sheets of filter-paper wet with alcohol over the vessel containing the sea-water in which the organisms were swimming quietly upon the surface; the vapours of the alcohol were thus forced into contact with the Noctiluce. After a short time the latter could not w. induced by any stimulus to emit light.

Finally, best known are the depressing effects of narcotics upon the activity of the ganglion-cells of the central nervous system, 
both those that produce motor impulses, and those that are the seat of the sensations, of consciousness. In their anæsthetising action upon the cells of the central nervous system lies the extraordinary practical importance of the narcotics. Through the abolition of sensations, especially of pain, they confer enormous benefits upon mankind. But their misuse, especially that of alcohol and morphine, by inflicting irreparable injuries upon the

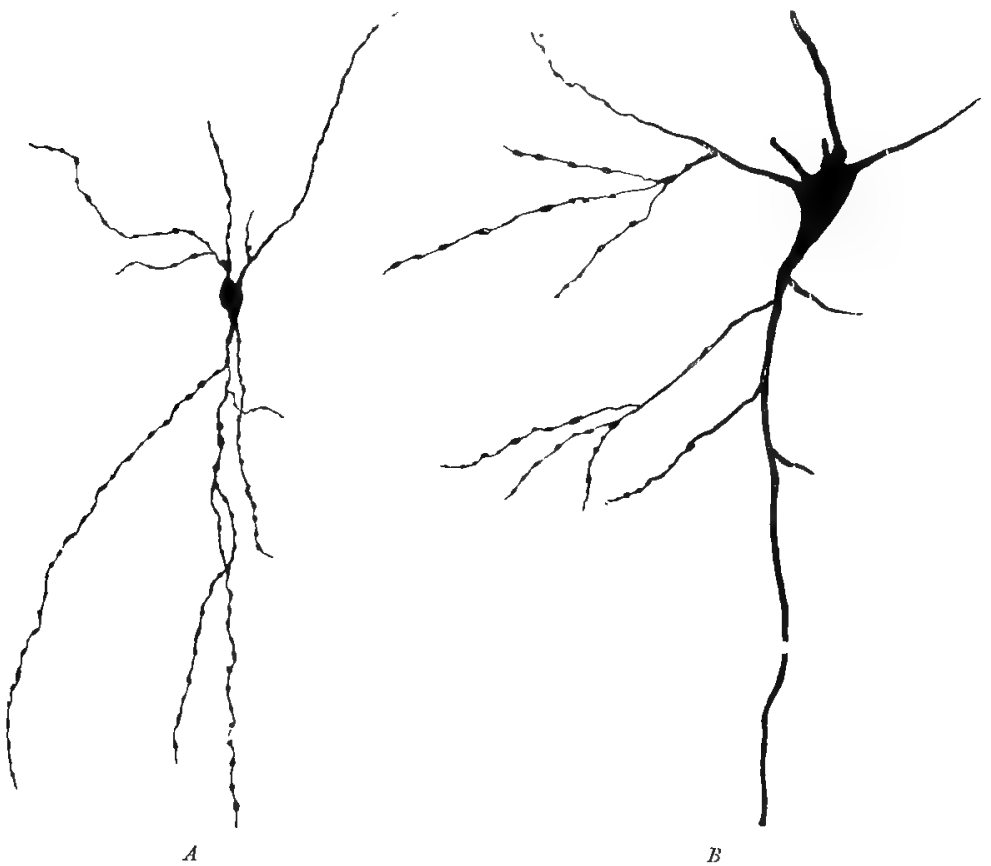

Fra. 169.-Gauglion-cells of a morphinised dog, stained by Golgi's method. In $A$ all, and in $B$ nost of the protoplismic processes have assumed a moniliform appearance. (After Demoor.)

cells, produces most destructive effects and transforms the benefit into a serious evil.

Recently a number of investigators, such as Meynert, Lepine, Duval, Solvay and others, have put forward the view that ganglion-cells possess the power of amœboid motion, their protoplasmic processes, or dendrites, being able to shorten and lengthen. Hence it is highly interesting to show, as Demoor ('96) has very recently succeeded in doing, that under the influence of morphine in narcosis, and also of other stimuli, distinct phenomena of contraction can be observed in the dendrites of the ganglion-cells, or neurons, which correspond exactly to those contractile phenomena that 
strong stimuli produce upon the branched pseudopodial filaments of Rhizopoda. The two pictures agree completely ( $C f$. Fig. 169, $A$ and $B$ ). The dendrites of the neurons, for example in the brain of a dog, like the pseudopodia of the rhizopods, assume a very characteristic moniliform appearance in morphine- or chloralnarcosis, their protoplasm accumulating in numerous small globules and spindles. Evidently this phenomenon, which can be

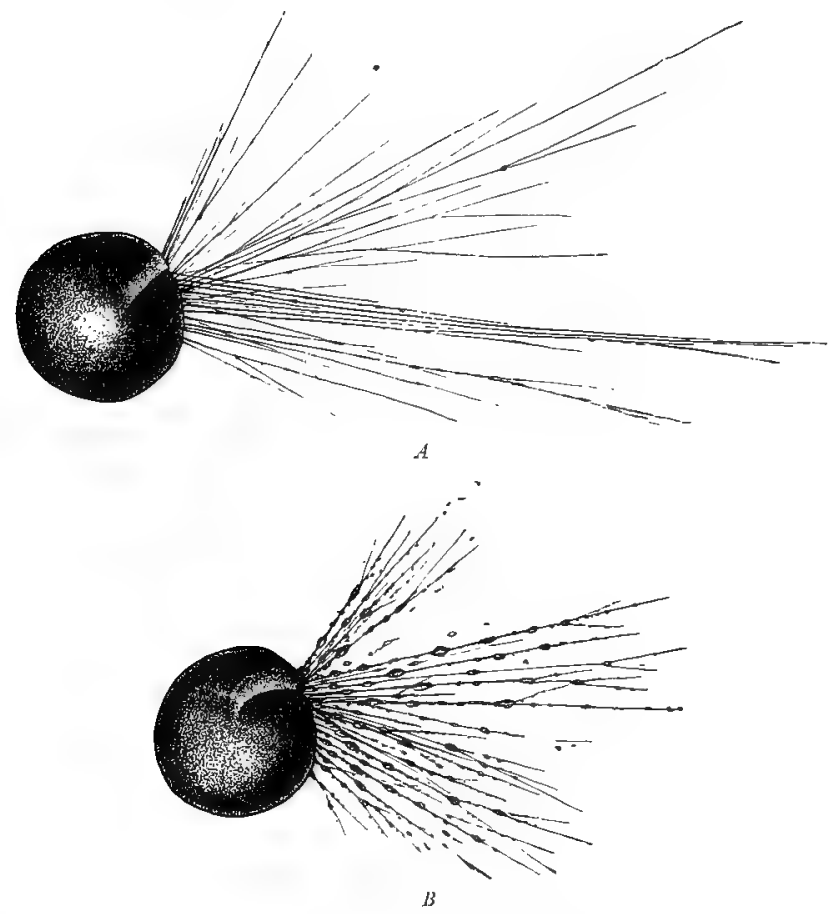

Fig. 170.-Amphistegina lessoniz. Filose pseudopodia project out through the opening of the lenticular, calcareous shell. $A$, Normal ; $B$, in chloroform-narcosis.

produced only by an excitation of contraction, is an effect of the stage of excitation which the narcotics, as we have seen, cause in other forms of living substance before paralysis begins. In this condition the ganglion-cells are gradually paralysed, and during their narcosis preserve this form of pseudopodia. The same is readily observed ${ }^{1}$ also in the narcosis of Rhizopoda (Fig. 170), e.g., Amphistegina, Orbitolites, Rhizoplasma, etc.

$$
\therefore C f \text {. Verworn }(96,3) \text {. }
$$




\section{The Actions of Mechanical Stimuli}

All changes in the pressure-relations of living substance in its environment may be termed mechanical stimuli. The effects of diminution of pressure have not thus far been investigated in detail, hence only the effects of increase of pressure are to be considered here.

Increase of pressure can take place in various ways, ranging from a light touch to a vigorous squeezing or complete crushing of the living substance, from a brief shock to a continuous and lasting pressure, or from an irregular shaking to rhythmically intermittent impacts, such as a tuning-fork produces.

\section{a. The Phenomena of Excitation}

Among the excitation-effects of mechanical stimuli upon the phenomena of metabolism the clearest is that of the production of substance, secretion, in unicellular organisms. Actinosphoerium, e.g., when completely at rest, floats in the water, with many pseudopodia extended straight in all directions and evolving no secretion. Absence of secretion is evident from the fact that ciliate Infusoric belonging to the Hypotricha, which have cilia on their ventral side only and by means of them run over objects in the water like lice, not rarely walk along quietly upon the extended pseudopodia of the Actinosphorium without sticking to them. But if one of the Hypotricha is actively swimming and bounds against a psendopodium, the mechanical stimulus is sufficient to cause at the place of contact the secretion of a viscous substance, which holds fast. the infusorian as prey. ${ }^{1} \quad$ A single strong shock likewise causes the secretion of slime upon the pseudopodia, so that small particles suspended in the water remain sticking to them. Such secretion as the effect of mechanical stimulation is wide-spread in the naked protoplasmic bodies of Rhizopoda. The slime becomes directly visible in the large marine radiolarian Thalassicolla. It is possible with little trouble to extirpate from the round body of T'halassicolla, which has the size of a pea, the central capsule, which is. pierced with extremely fine pores and contains protoplasm and nucleus. If this be done without injury to it, after a short time the capsule begins to regenerate into a complete radiolarian, i.e., to form new pseudopodia, and gelatinous and vacuolar layers ( $C f$. Fig. 171). After the pseudopodia have become extended like a circlet of rays from the yellow spherical body, there is noticed between them an extremely delicate, very liquid slime, which is excreted by the pseudopodia and represents the rudiment of the new gelatinous.

1 Cf. Verworn ('89, 1). 
layer. If in this stage the radiolarian be given a strong shock, it may be seen that the liquid mass of slime increases and becomes at the same time thicker and firmer; this is manifested more distinctly when the shock is repeated. ${ }^{x}$ The mechanical stimulation promotes visibly the secretion of slime.

No excitation-effects of mechanical stimulation upon the phenomena of form-changes, upon growth and cell-division, are thus far known.

Effects of excitation upon the phenomena of the transformation of energy have been investigated very fully, and a great
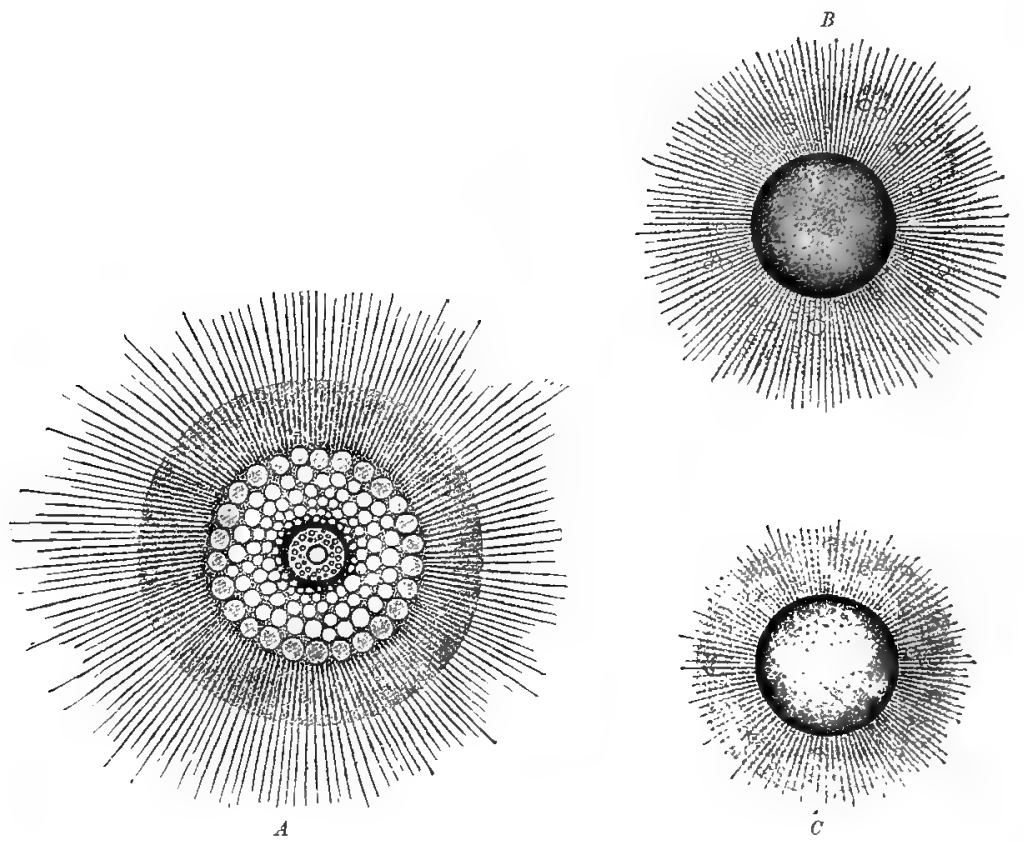

T'IG. 171.-Thalassicolla nucleata, a spherical radiolarian cell. A, Uuinjured individual in optical section. In the middle lies the central capsule, containing the nucleus, surrounded by black pigment. $B$, Central capsule removed. It has already surrounded itself with a new circlet of pseudopodia. $C$, The same central capsule after strong stimulation. The pseudopodia are somewhat drawn in, and between them a thick mass of slime has been secreted. $B$ and $C$ strongly magnified.

mass of observed facts exists. From these we will select the typical phenomena.

Those connected with motion, which are called out by mechanical stimuli, constitute here also the chief point of interest. The production of turgescence-movements in the so-called sensitive plants, such as the delicate Mimosa mudica, is generally known. Mimosa, which resembles a small Acacic tree, during the day and while

${ }^{1} \mathrm{Cf}$. Verworn ('91). 
undisturbed holds its primary petivles, which spring from the trunk, directed obliquely upward. The secondary petioles, which bear the rows of leaflets, are spread out wide apart, and the leaflets themselves stand horizontal and widely extended (Fig. 172, A). But as soon as the pot in which the plant is growing is shaken, the picture changes almost immediately. The primary petioles fall down as a result of the decrease of the turgor of the cells of their pulvini, the secondary petioles turn toward each other, while the leaflets are raised and lie with their upper surfaces together (Fig. 172, $B$ ). The plant, when left at rest, remains for some time in this position, and then very gradually returns to its original condition, the cellturgor again increasing at the corresponding portions of the pulvini. In the single leaflet the position of stimulation can be called out

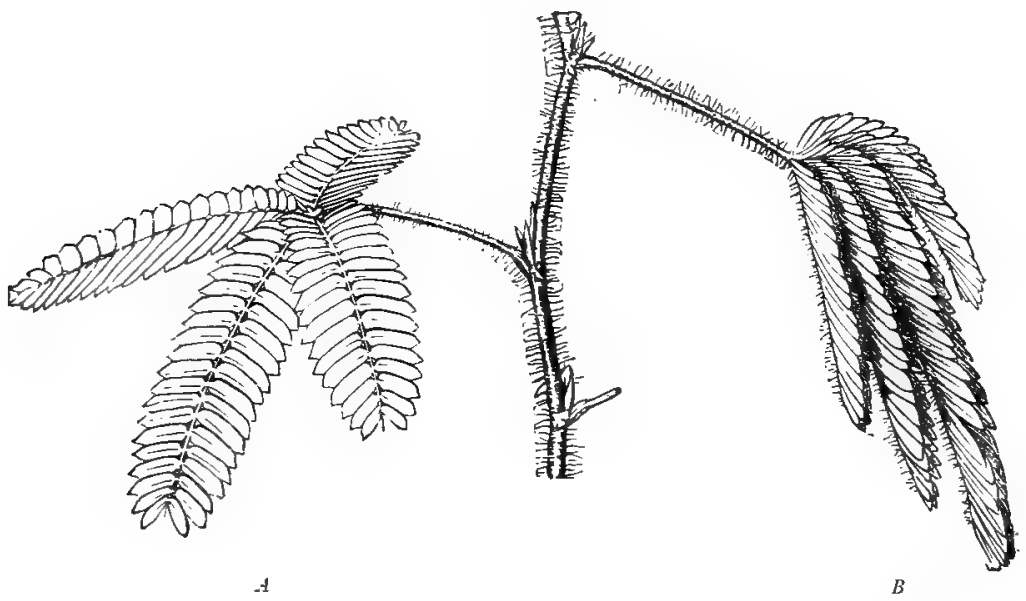

FIo. 172.-Mimosa pulicu. -t, A brauch unstimulated and extended; $B$, a branch stimulated and fallen, with its leaves folded. (After Detmer.)

also by a very gentle local touch. If the touch be stronger, the leaflets mar be seen to move in succession like a row of dominoes. thus affording a very striking demonstration of the transmission of the stimulus.

Among the contraction-movements resulting from the mechanical stimuli the contraction-phase only is thus far recognised with certainty, although it is not improbable that in many cases very delicate tactile stimuli may produce expansion. Thus, the contact of an amoboid protoplasmic mass with a smooth support might influence by cohesion the extension of the pseudopodia.

In the naked protoplasmic bodies of Rhi zopode a single shock upon the extended pseudopodia, such as can be produced by a rigorous rap of the slide under the microscope, produces more or less pro- 
nounced phenomena of contraction, according to the very various grades of irritability of the different species. ${ }^{1}$ An $A$ moeba or an Actinosphorium, stimulated in this way, ceases momentarily its centrifugal protoplasmic streaming, i.e., the extension of its jseudopodia; upon stronger stimulation there may be a partial indrawing of the pseudopodia, a transitory centripetal streaming. Other forms, such as Difflugia, react more energetically (Fig. 173). With a gentle shock the pseudopodia become slowly more or less retracted, their previously smooth contour becoming wrinkled

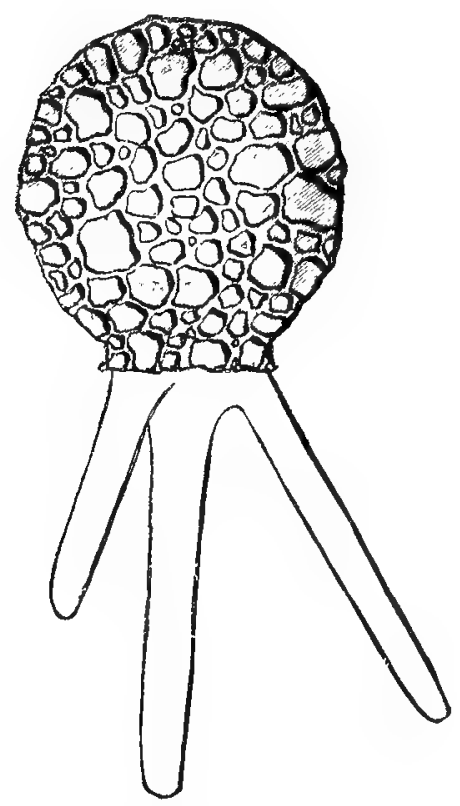

4

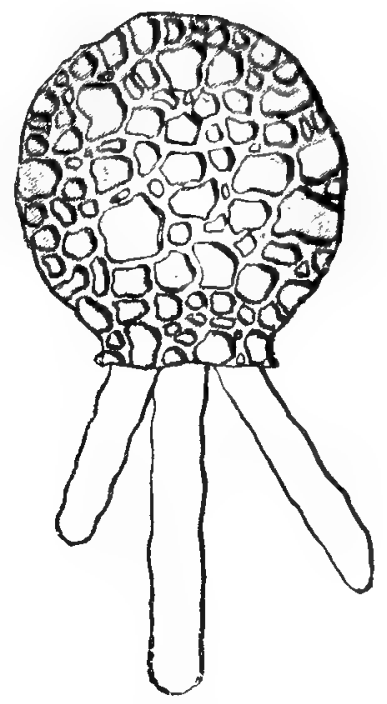

B

Fia. 173.-Diflugia. From the shell of sand-grains project three finger-like pseudopodia. $A$, Unstimulated; $B$, stimulated by a gentle shock.

(Fig. 173, $B$ ); with a stronger shock the pseudopodia are frequently drawn with such force into the protoplasmic body, that their ends, being fastened to the support by means of a sticky secretion, are torn off. With stronger stimulation the change in the pseudopodia is much more pronounced than with feebler: they become not only wrinkled, but on their whole surface small droplets swell out from the smooth contour $;^{2}$ the more the reaction llevelops, the larger become the droplets; they flow together into ، myelin-like mass, and are distinguished clearly by a strongly refractive strand visible in the axis of the pseudopodium (Fig. 174);

${ }^{x} C f$. Verworn $(' 89,1)$.

${ }^{2} C f$. Fig. 156, p. 363. 
finally, the latter is wholly drawn in, and its mass mingles with the rest of the body-protoplasm. Among the marine Polythalamia also there are many forms that possess very great irritability ; these draw
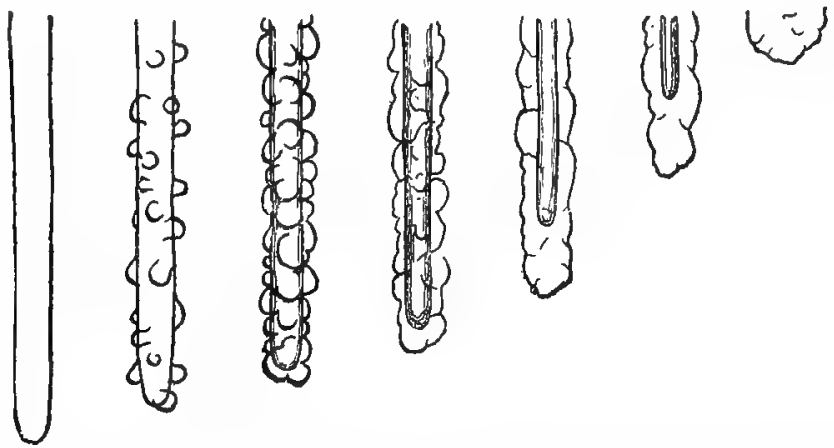

Fia, 174.-Contraction of a pseudopodium of Diffugia lobostoma after vigorous shaking Seven successive stages of retraction.

in their whole richly-branched net-work of pseudopodia upon a single shock.

In the same manner there can be observed upon the slide under the microscope the effects of a shock upon the motion of flagella and cilia. E.g., Peranema, by means of the regular lashings of the flagellum at its anterior end, moves through the water quietly and in a straight line (Fig. 175). But, if the slide be given a brief shock, there follows at once an energetic lashing of the whole flagellum, which gives the cell another direction. It then continues its way quietly as before, with only the end of its flagellum vibrating. The mechanical stimulus, therefore, has had the result of intensifying the stroke of the flagellum. The same thing can be observed in the ciliary motion of the ciliate Infusoric. If a Paramncium be observed in quiet and not too rapid locomotion, moving through the water by the play of its cilia as by innumerable small and rapidly moving oars, it is seen that upon being jarred it suddenly accelerates its motion, returning immediately, however, to its previous rate. This fact may be observed much more distinctly in Pleuronema
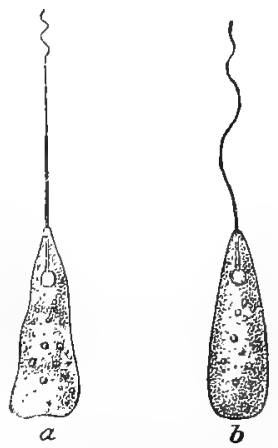

Fra. 175. - Peranema, a flagellate-infusorian cell. $a$, Swimming quietly; $b$, stimulated by shaking chrysalis, a small bean-shaped infusorian, which usually lies in the water for a long time absolutely still and keeps its long, ray-like cilia completely quiet (Fig. 176). At the moment when it is slightly shaken, it suddenly makes a few, very 
energetic strokes with its cilia, so that it springs through the water like a flea, and immediately thereafter becomes quiet in another spot. Similar cases exist in great abundance among the active Infusoria. It is found everywhere that mechanical stimuli cause energetic strokes of cilia.

Infusorian life offers innumerable opportunities to observe the effects of mechanical stimuli upon muscular motion. Smooth muscle-fibres (myoids) are wide-spread among Infusoric ; and just as everything in the life of these Protista, which are in endless. activity, takes place with great rapidity, so their contractile fibres. react upon the slightest jarring with a sudden, strong contraction. There are few sights in the microscopic world so pleasing as the
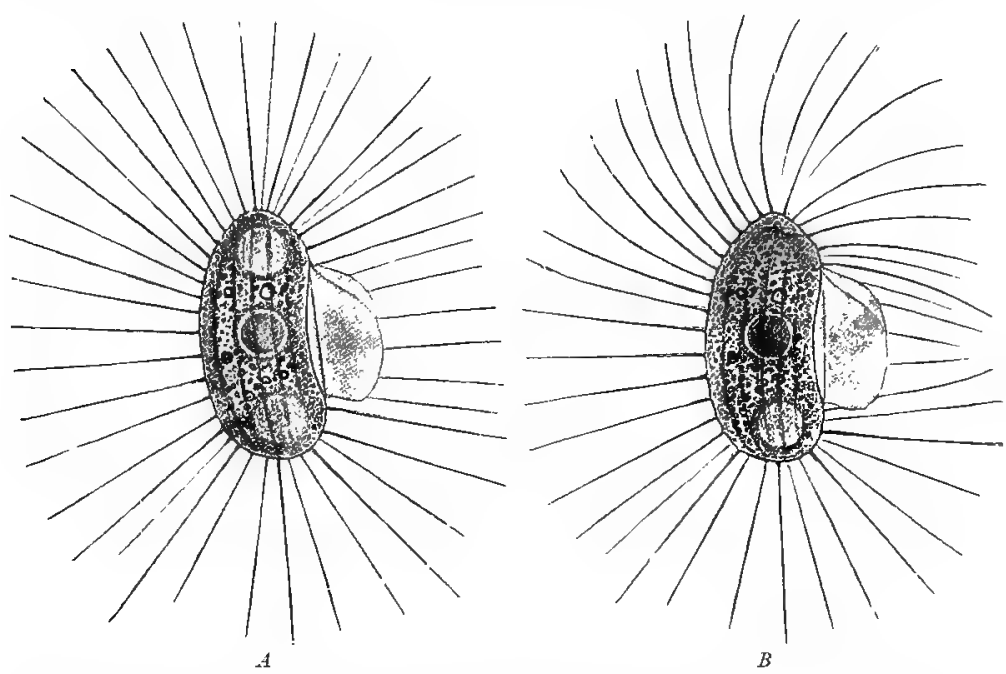

FIG. 176.-Pleuronema chrysalis. A, Lying still. $B$, In the act of springing, upon being stimulated by shaking ; the cilia are just performing a stroke.

contraction of a much-branched tree of Vorticellinoe upon very slight. jarring (Fig. 177). At the moment of the impulse all the stalkmyoids contract suddenly and simultaneously, and the stalks are coiled in delicate spirals (Fig. 177, B). Stentor also, which in rest has its beautiful, trumpet-shaped body unfolded, at every jar suddenly draws itself into a stalked ball by the contraction of the many myoid-fibres lying in the external layer of the body (Fig. 168, p. 376). The cross-striated muscles of the higher animals behave similarly, without of course possessing the same high grade of irritability. In order to cause contraction, by means of a mechanical stimulus, in a frog's muscle, for example, a stronger shock to the muscle-substance is needed than in the case of an infusorian. 
Every one who has been at the sea-shore upon a quiet summer evening has observed the production of light by mechanical stimuli. The curious, transparent animal life that in quiet weather exists at the surface of the water has the wonderful power, irrespective of the classes to which the animals severally belong, of glowing brilliantly at every movement of the boat or the oars, or every dash of the waves. Where the water contains much plankton, such as Noctilucce, Radiolaria, eggs of Ctenophora, etc., the effect of a shower of sparks in the water can be produced as well indoors. Every time sea-water is stirred in a glass the unicellular organisms, at the moment of the touch with a glass rod, flash up brightly, and then immediately sink again into
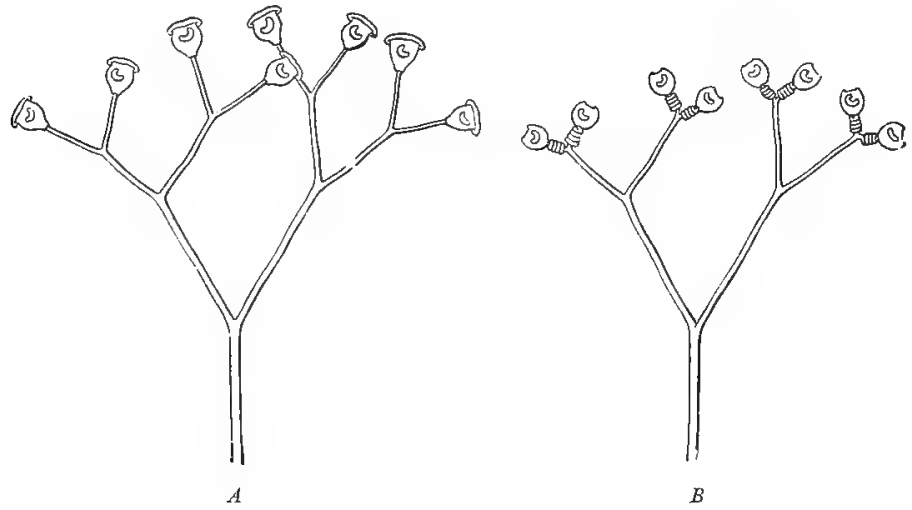

FIa. 177.-Carchesium polypinum, a branched colony of Vorticellina. A, Unstimulated; $B$, stimulated by jarring. The single individuals jerk together by the contraction of the myoids of their stalks.

darkness. The experiment can be repeated innumerable times, and the spectacle that is presented is of wondrous beauty.

Before leaving the excitation-effects of mechanical stimuli, one more group deserves attention, viz., the results of rhythmically repeated shocks. Phenomena that are produced only incompletely by single shocks are expressed much more strongly by summation, providing that each succeeding impulse follows before the stimulus of the preceding one has passed away. This fact is demonstrated most distinctly in contraction-movements, where one contraction is superimposed upon another so that there is no time for expansion to develop between them; a genuine cramp then appears, which is termed mechanicul tetanus. The peculiarity of tctanus lies in the fact that, although composed of many single contractions, on account of their rapid succession it gives the impression of a continual process. The simplest method of producing rhythmic shocks is either to shake the objects in a shallow basin by means of a rotating toothed wheel that has wide 
teeth, or to fasten them upon a thin glass slide upon one limb of a tuning fork of the proper pitch and draw a violin bow across the other limb. Observation immediately after the experiment shows that Amceba, Actinosphorinm, and other rhizopods have drawn in
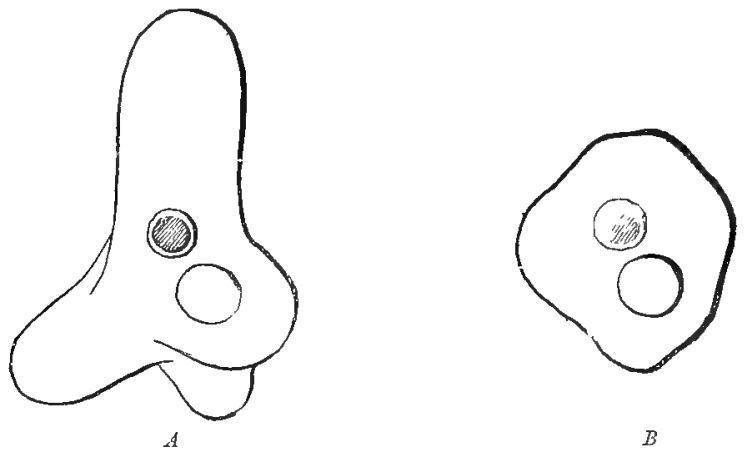

FIG. 178.-Amoob. $A$, Normal ; $B$, after tetanic stimulation upon a tuning fork.

their pseudopodia completely and are in the stage of complete contraction, i.e., more or less completely spherical (Fig. 178). If the experiment be interrupted after a brief period of shaking, according to the time of the interruption the various stages in the formation of tetanus can be observed. The pseudopodia are then

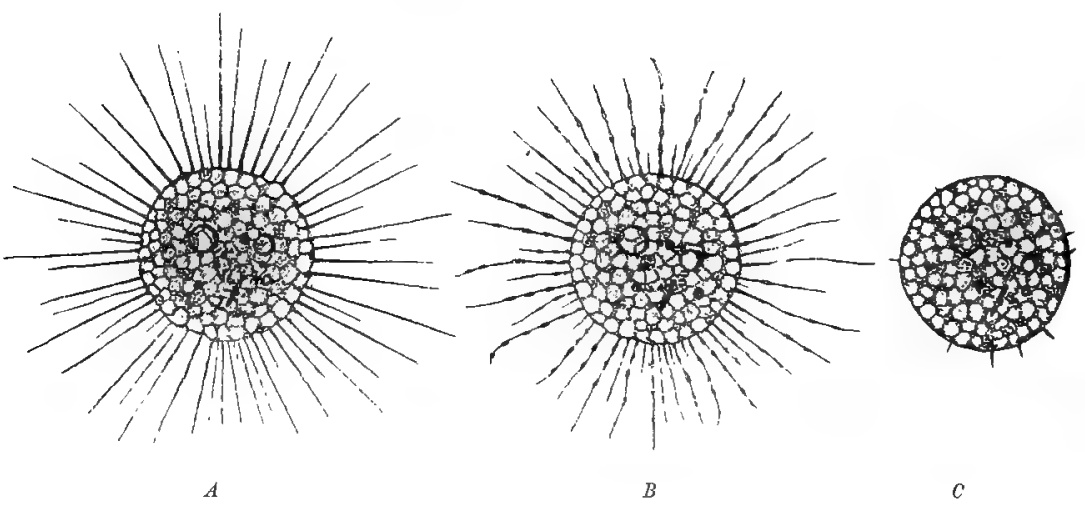

Fra. 179 -Actinospharium. A, Undisturbed; $B$, at the begimning of strong tetanic stimulation; $C$, in complete mechanical tetanus.

incompletely retracted. The phenomena in long, thread-like pseudopodia, such as in Actinospharium or Orbitolites, are characteristic (Figs, 179 and 180). With very slight shaking the pseudopodia remain smooth and straight, as they were when 
undisturbed, and their protoplasm flows slowly but constantly without exception in a centripetal direction. But, if the shaking be more intense, ther assume a varicose appearance, their centripetally streaming protoplasm collecting into many small spindles and globules, of which the smaller flow into the adjacent larger ones, while the larger ones constantly approach the central protoplasmic body"; upon long-continued action all protoplasm flows into the cell-body. ${ }^{1}$ This curious formation of droplets upon pseudopodia is a peculiarity of strongly and continually stimulated protoplasm which is general in Rhizopoda that are provided with filose pseudopodia; it is only a special case of the general law that strong stimuli cause naked protoplasmic masses

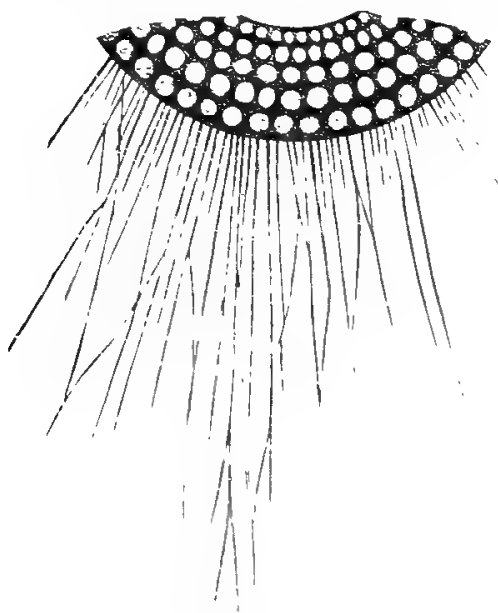

4

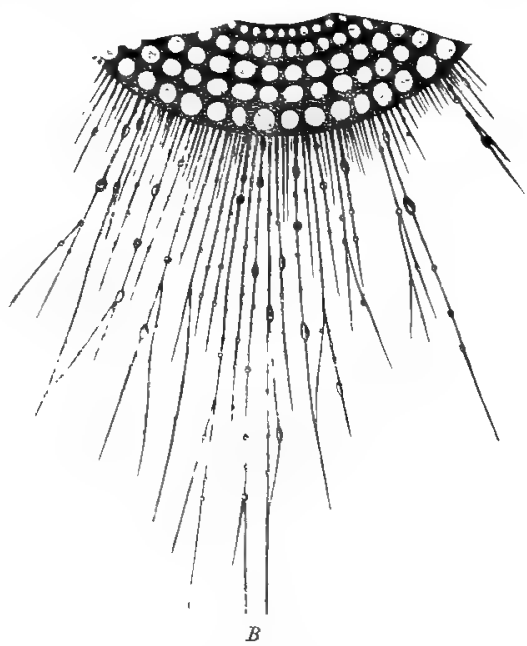

FIt. 180.-Orbitolites. A part of the surface of the shell, with extended, thread-like psendopodia. $A$, Unstimulated ; $B$, after rigorous shaking.

to assume the spherical form. The same tendency toward the formation of globules, which all stimulated protoplasw as a whole shows, is also noticeable in its individual parts. ${ }^{2}$

Ciliary motion is increased greatly by rhythmically intermittent shocks, so that Influsoria stimulated in this way rush madly through the water for a considerable time after the stimulation has ceased. A real tetanus, however, in which the cilia remain bent in the position of contraction, appears not to occur, at least thus far such has not been observed. The rhithmic motion of the cilia continues, and is changed in its rate and amplitude only.

1 ('r. Terworn $(92,1)$.

(r. herewitl the phenomena of necrobissis, p. 329, Fig. 142. 
On the other hand, in the muscle mechanical tetanus can be produced very easily. Vorticella, stimulated in the above-described manner, passes at once into tetanus. The stalk-muscle remains continually contracted. The tetanus is frequently so great that the cell-bodies are loosened from their stalks and swim away. A short time after the cessation of the stimulation the isolated stalks extend again, but rarely continue to live for any length of time. Cross-striated muscle can likewise be put into tetanus by a hammer acting rhythmically and arranged to strike carefully upon the muscle; the latter remains contracted throughout the duration of the stimulus.

One might easily be misled by external appearances into regarding the tetanus of contractile substances as a phenomenon of depression, for Anveba, Actinosphocrium, muscle, etc., during the tetanic condition are apparently in complete rest and motionless, like the same objects when a narcotic has acted upon them. But the two conditions have absolutely nothing to do with one another. The difference is fundamental, as is shown by an investigation of the metabolism. While in narcosis the metabolism undergoes a real depression, researches have shown that in tetanised muscle the metabolism is considerably increased. The decomposition-products of the living substance, such as carbonic acid, lactic acid, etc., undergo an extraordinary increase in quantity; certain substances that have accumulated in the muscle, such as glycogen, become consumed; and the production of heat becomes increased to a considerable degree. It follows from this that in the tetanic condition the vital process experiences a considerable augmentation, that tetanus is a phenomenon not of depression but really of excitation.

The production of light by Noctiluca upon intermittent stimulation appears to be a continual process analogous to the tetanus of contractile substances. After a short time this phenomenon decreases very considerably in intensity. ${ }^{1}$

\section{b. The Phenomena of Depression}

The phenomena of depression that are called out by mechanical stimuli are as rare as the exciting effects of such stimuli are manifold and wide-spread, and they have been little investigated.

Horvath (78), and later in agreement with him Reirike ('80), marle the statement that the growth of Bacteria the cultures of which are continually exposed to regular shocks, is interfered with, in other words a depression of growth takes place. Later the validity of these experiments was called in question by others; but recently Meltzer ('94), in a dotailed series of 
experiments, confirmed in essential the observations of Horvath and Reinke by showing that regular vibrations are able to produce not only an inhibition of growth, but under certain conditions even complete death and granular disintegration of the protoplasm.

Further, Engelmann ('79, 1) made the observation that the motion of Diatomece and Oscillarioe ceases upon shaking. But here the question is undecided whether the standstill is to be interpreted as a phenomenon of depression or the expression of tetanic excitation, like the cessation of protoplasmic motion in tetanised Amcebce.

Finally, in the pressure-paralysis of nerves we have, at all events, a real phenomenon of depression which is to be classed with those produced by narcotics. This pressure-paralysis, which appears when a nerve is compressed for a time but not too strongly, is generally known as the "feeling of going to sleep" of the limbs. Besides the subjective phenomena, the "going to sleep" expresses itself in a diminution or complete interruption of the power of conductivity of the compressed nerve, so that for some time the muscles supplied by the latter cannot be stimulated through it to contraction. A short time after the cessation of the pressure the power of conduction is again established.

This comprises approximately all the facts known regarding depressing effects of mechanical stimuli.

\section{The Actions of Thermal Stimuli}

The employment of thermal stimulation allows far fewer variations to be made than that of mechanical or even chemical stimulation, for only a rise or a fall of temperature can act as a stimulus upon living substance. In accordance with the nature of the thermal stimulus rapid rhythmical variations of temperature cannot be produced, since heat requires a long time to be communicated to a body or to disappear from it. Hence it is impossible to produce a thermal tetanus corresponding to mechanical tetanus. Thermal stimulation is thus very simple, and its effects are likewise simply shown.

\section{a. The Phenomena of Excitrition}

Starting from the average temperature at which a cell normally exists, which, therefore, represents the optimum of the vital condition, it is found to be a general law that up to a certain point excitation increases with increasing temperature. This holds good for very different vital phenomena and for very different forms of living substance. 
The best example of the excitation of metabolism by increasing temperature is afforded by the activity of yeast-cells, since in the quantity of carbonic acid that is derived from the decomposition of grape-sugar there is given an excellent measure of the increase of metabolism. The evolution of carbonic acid in a solution of grapesugar containing yeast is always more active with increasing temperature up to about $30^{\circ}-35^{\circ} \mathrm{C}$, when it becomes very violent. ${ }^{1}$ The bubbles of carbonic acid rise in the fermentation-tube as in sparkling champagne. Plant-life likewise affords many clear examples of how with rising temperature within certain limits the vital phenomena, such as cleavage ' of carbonic acid, formation of starch, of proteid, etc., increase in intensity; it is here found that the temperatures at which the excitation reaches its maximum are very different, not only for the different forms of living substance, but also for the various metabolic processes in the same object. It is observed also in animals that metabolism increases proportionally with the temperature; and Spallanzani showed for cold-blooded animals, especially for snails, that the consumption of oxygen is thus increased. Whatever may be the details of the metabolism, the law holds good everywhere in the living world, that the intensity of metabolism increases with increasing temperature.

It should be mentioned, however, that there is, apparently, an exception to this general law. This is shown by the behaviour of homothermal (warm-blooded) animals. It is a well-known fact that warm-blooded animals undergo a decrease of metabolism with rising temperature. Man in winter has a much more active metabolism than in summer, he consumes most food at the lowest, least at the highest degrees of teinperature. Thus far this remarkable paradox has been little explained, and Pfluger ('78), who has studied the subject in detail, arrives at a solution of the apparent contradiction only by the aid of certain hypotheses. As is well known, the peculiarity of warm-blooded animals in contrast to all others is the possession of a mechanism in their nervous system that regulates reflexly the temperature of the body and maintains it at a constant height, however great variations the external temperature may undergo. The metabolism, which is the source of heat-production in the animal organism, is, however, in the warm-blooded animals, the servant of the heat-regulating mechanism. If the external temperature is low, the metabolism and with it the production of heat are increased reflexly through the nervous system from the skin, in order to compensate for the greater loss of heat by the body; and, vice versa, if the external temperature is high, the metabolism and with it the production of heat undergo, likewise reflexly, a corresponding depression. The increase of metabolism of the cells in cold and the decrease in

${ }^{1} C f$. von Liebig ('70). 
warmth are, therefore, not called out directly by the temperature, but by stimuli that come from the central nervous system. But the paradox is not thus removed, it is merely deferred. The excitation of the central nervous system, which affords the stimuli, is brought about along the path of the temperature-nerves from the surface of the body, the skin, by cooling or warming; and thus the question remains still open, how increasing temperature can produce a depression, and falling temperature an increase of excitation in the central nervous system. In order to answer this question in harmony with the general law of temperature, and also to set aside the apparent paradox, Pfliger ('78) formed the following very plausible hypothesis. He supposes "that the central organ of the sense of temperature contains two substances as substrata of two different specific energies: the excitation of one of these substances manifests itself in consciousness as the feeling of warmth, the excitation of the other as the feeling of cold. One would then have to suppose further that the two substances are related in such a way that the excitation of one is decreased, when that of the other is increased, and vice versa." As a matter of fact, such conditions are frequently recognised in the central nervous system. Upon this supposition it is clear that with rising external temperature the heat-centre must be excited and the cold-centre be depressed, while with falling temperature the heat-centre must be depressed and the cold-centre excited. If, therefore, the cold-centre is connected with the nerve-trunks that influence metabolism, depression of it by increased external temperature must have as a result a depression of the metabolism, and vice versa. Thus the law of temperature would preserve its general validity. The idea is, however, only hypothetical.

The augmentation of vital phenomena by increase of temperature is also evident in form-changes, where in general it is clearly expressed, especially in organisms that are undergoing development and in cells whose living substance is growing and reproducing. Thus, plant seeds begin to germinate at a certain temperature; Indian corn at approximately $9^{\circ} \mathrm{C}$, seeds of the date at approximately $15^{\mathrm{c}}$ C. 1 From these points on, with increasing temperature, growth increases constantly up to about $30^{\circ}-40^{\circ} \mathrm{C}$. Numerous observations have been made upon Bacteria which have shown the same relation. The hay-bacillus, according to the investigations of Brefeld, begins to grow at $6^{\circ} \mathrm{C}$, and with rising temperature increases constantly and more rapidly up to $30^{\circ} \mathrm{C}$. The bacillus of tuberculosis, as Koch has shown, begins to grow first at $28^{\circ} \mathrm{C}$., and reproduces most rapidly at $37^{\circ}-38^{\circ} \mathrm{C}$. The fact that this bacillus begins its growth at so high a temperature is due to its parasitic manner of life in the tissues of warm-blooded animals, with whose body-temperature the optimum of its growth coincides.

1 Cf. Sachs ('82). 
De Bary ('87), in his lectures upon Bacteria, has collected a number of similar examples from the life of these organisms. Investigations of other objects, such as animal egg-cells, leucocytes, etc., would supposably give wholly analogous results.

But the excitation-effects upon the phenomena of changes of energy, especially upon motion, are most directly noticeable. Here, also, in general, an increase of motion accompanies increasing temperature. In following these phenomena in single living cells, the warm stage devised by Max Schultze for this purpose can best be employed. It consists of a horseshoe-shaped brass plate, widened out at its curved part so as to cover a greater surface (Fig. 181). Under this surface, which is pierced by a diaphragm, there is a

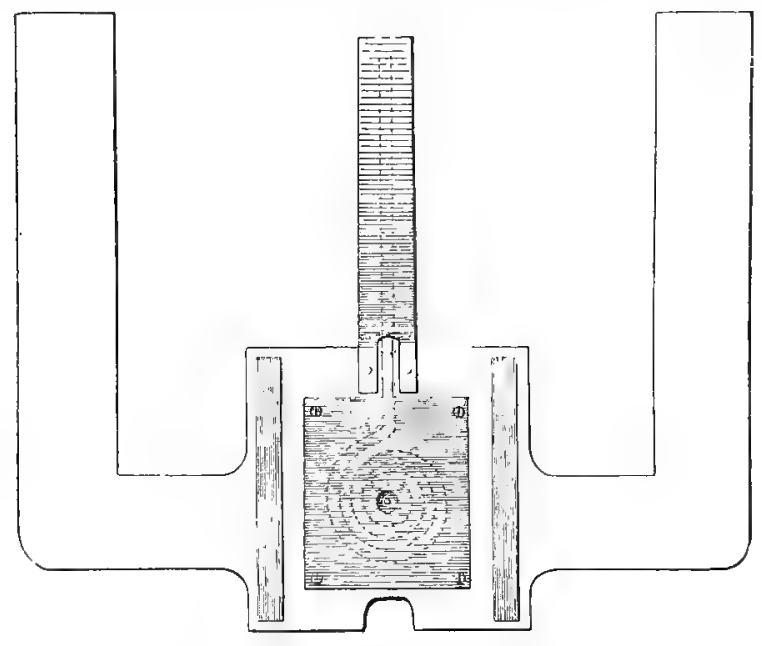

FIG. 181.-Warm stage. (After Max Schultze.

thermometer spirally wound, the upper end of which projects upon a scale between the two limbs of the horseshoe-shaped stage. The whole is fastened upon the stage of the microscope, and beneath the two ends of the horse-shoe spirit-lamps are placed, which slowly heat the stage. With the thermometer the height of the temperature prevailing in the middle of the stage can easily be controlled.

In this way it can be demonstrated that the protoplasmic movement of Amceba, as Engelmann ('79, 1) found, is always more active with increasing temperature and that, as Kiihne ('64) first established, these Protista fall into strong contractions at $35^{\circ} \mathrm{C}$., assuming a spherical form, just as after strong chemical or mechanical stimulation ( $C f$. Fig. 183, $B$, p. 395). Other rhizopods such as Actinusytherinm, Orbitolite's, etc. (Cf. Figs. 179 and 180), as 
well as leucocytes of various species of animals, behave in all respects analogously; and even the protoplasmic streaming of plant-cells shows the same phenomena. Max Schultze ('63) and Nägeli ('60) measured the rate of granular streaming in the protoplasmic threads of the cells of Tradescantia and Nitella with increasing temperature, and saw how it became constantly greater; and Kiihne (l.c.) found that the protoplasm of the cells of the stamenhairs of Tradescantia at a temperature of $45^{\circ} \mathrm{C}$. exhibits powerful contractile phenomena, becoming collected into globules in the typical manner ( $C f$. Fig. 35, p. 95).

In the exciting effect of rising temperature upon protoplasmic motion an important fact is to be noticed, which is of great importance in the explanation of many phenomena to be treated later. This is the fact that the two phases of the motion, that of expansion and that of contraction, are not equally excited. ${ }^{1}$ This can be established best in marine Rhizopoda that possess long, thread-like pseudopodia, in which the protoplasmic particles have to pass over a very long path. E.g., in the action of rising temperature upon Rhizoplasna (Fig. 130, p. 285) it is seen that up to about $31^{\circ}-32^{\circ} \mathrm{C}$. both phases are gradually excited, so that the protoplasmic motion is accelerated; but expansion outweighs contraction, so that the pseudopodia extend farther and farther and become more numerous. At about $31^{\circ}-32^{\circ} \mathrm{C}$., the two phases are equally excited. If the temperature be still more increased, contraction more and more outweighs expansion, and with a very slow increase of temperature up to about $39^{\circ}$ and $40^{\circ} \mathrm{C}$. the pseudopodia finally become completely retracted. Hence the curves of excitation of expansion and of contraction do not coincide, but have their maxima at different places. Without doubt a similar condition is to be observed in other contractile objects and with other stimuli, and it would be a very fruitful task to make further studies in this direction.

Ciliary motion is likewise gradually augmented by increasing temperature up to a certain degree, as Engelmann $(79,1)$ has observed in ciliated epithelia, and Rossbach ('71) in Infusoria. The oral mucous membrane of the frog is a convenient object for the observation of the ciliary motion of an epithelium. It is easy to loosen from the palate and cut off a piece of this ciliated membrane a centimetre square, the ciliary motion of which is directed toward the oesophagus. If we stretch such a piece with four needles upon a cork frame (Fig. 182) and cover it with a cover-glass, we can observe the ciliary motion for days, if the object be protected from drying, and study its rate, either directly under the microscope, or by the passage of bloodclots or particles of coal-dust laid upon it. In such a preparation it is easy to determine that the rate and energy of the motion ${ }^{1} C f$. Verworn ('96, 2, 3). 
increase with increasing temperature. The same phenomena can be observed as easily and perhaps more clearly in Infusoria with the warm stage. Rossbach, who first made such investigations upon various Ciliata, describes how the ciliary motion suddenly increases in rate, so that at $25^{\circ} \mathrm{C}$. the Infusoria begin "to shoot here and there like arrows," and at $30^{\circ}-35^{\circ} \mathrm{C}$. their motions become really furious.

Muscle behaves analogously. If a frog's muscle be hung in a 0.5 per cent. solution of common salt, the temperature of which

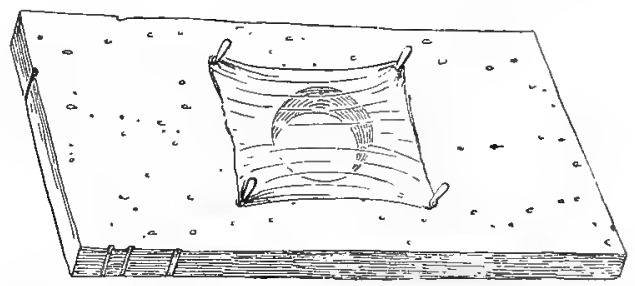

FIG. 182.-Oral mucous membrane of the frog stretched upon a cork frane.

is rapidly increased, the muscle shortens gradually with increasing temperature from about $28^{\circ} \mathrm{C}$. on, until at about $45^{\circ} \mathrm{C}$. its contraction has reached its maximum. But if the muscle be dipped suddenly into a salt solution of $45^{\circ} \mathrm{C}$., there appears at once a sudden contraction. The irritability of muscle is also increased with rising temperature.

Thus, everywhere in living nature the law is met with, that within certain limits increasing temperature acts to augment vital processes.

\section{b. The Phenomence of Depression}

Falling temperature produces effects opposite to those of rising temperature. If the temperature be constantly lowered from the average at which an organism normally exists, it is found that the vital phenomena constantly decrease in energy, and that from a certain low degree on-which point is very different for different organisms and different phenomena-they are no longer perceptible. Thus, at temperatures below $10^{\circ} \mathrm{C}$. yeast no longer decomposes grape-sugar; at $2^{\circ}-3^{\circ} \mathrm{C}$. the development of sea-urchin eggs undergoing division is at a standstill; at a little above $0^{\circ} \mathrm{C}$. Amoba ceases its motions, and when cooled rapidly is fixed in the form which it possessed (Fig. 183, $C^{\prime}$ ). At a certain lower degree protoplasm takes on cold-rigour. Warming above this point is sufficient to dissipate the rigour and allow the phenomena to appear again. But, if the temperature is reduced below this point, a point is finally reached at which the vital capacity is abolished, 
from which warming cannot restore life. This minimum of temperature lies of course with different organisms at very different heights. Thus, as has been seen, Kiihne showed that Amobu dies upon freezing, that is, upon being cooled to a little below $0^{\circ} \mathrm{C}$.; while Pictet found for Bacteric that they could endure cooling to more than $-200^{\circ} \mathrm{C}$. without losing their vital capacity. ${ }^{1}$ The question whether in any living substance a point is ever reached by cooling where the vital processes are at a complete standstill withont
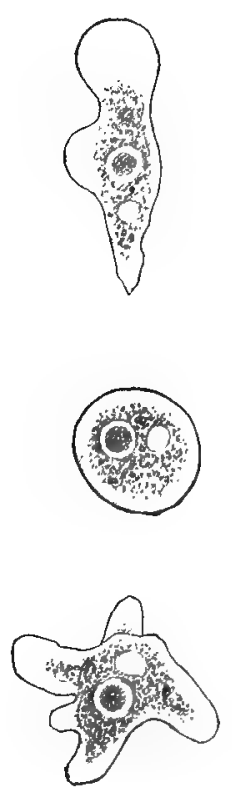

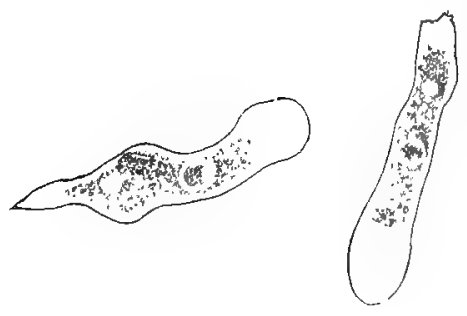

$A$
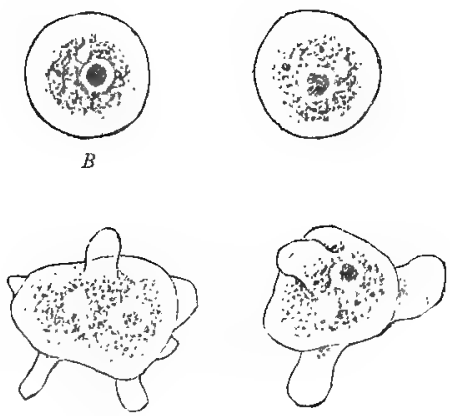

C

Fig. 183.-Forms of the body of Amaba limux at different temperatures. A, At $25^{\circ}$ C.; the amoebr have an extended wedge-shape, and show active protoplasmic streaming. $B$, At $40^{\circ} \mathrm{C}$.; the amoebæ have assumed a spherical form, and are in heat-rigour. $C$, At $2^{\circ} \mathrm{C} . ;$ the amobe show a lumpy cell-body, from which numerous small pseudopodia project; movement is noticeable only after very long-continued observation.

the vital capacity being extinguished, is at present no more decided than the same question regarding narcosis. Cold-rigour and narcosis are wholly analogous states: in both, vital processes are not perceptible, from both by restoring the normal conditions the living substance is restored to life, and from both by intensifying the unusual condition, i.e., by deeper narcosis and further cooling, it passes over into irreparable death. This latter fact, that jncreased narcosis and cooling abolish the vital capacity of paralysed organisms, ought rather to speak in favour of the view

$$
1 \text { Cf. p. } 290 .
$$


that in this depressed condition the vital processes are not wholly extinguished, that a vito minima exists. Decisive experiments upon this point are wanting. ${ }^{1}$

These phenomena of depression by cold are, however, not the only ones that are called out by changes of temperature. High degrees of heat, like low degrees of cold, depress vital phenomena. It has been seen that increase of temperature acts to stimulate, and that at a certain height the vital processes can become even violent. But, if the temperature rises beyond this point, the intensity of the processes suddenly decreases with extraordinary rapidity, and vital phenomena become imperceptible. With yeast-cells warmed above $40^{\circ} \mathrm{C}$. no evolution of carbonic acid can be observed in a solution of grape-sugar; the eggs of sea-urchins undergoing division or fertilisation, when warmed above $30^{\circ} \mathrm{C}$., are at a standstill in the stage in which they already were; amobæ warmed above $35^{\circ} \mathrm{C}$. maintain their spherical shape; and at the same temperature the cilia of ciliated cells remain greatly curved, i.e., in the state of contraction; in brief, the protoplasm falls into heatrigour (Fig. 183, B). If the objects are cooled after a brief action of these high temperatures, they recover slowly; but, if the action continues too long, or the temperature rises a little more, a return to life is impossible. The point where the vital processes act most intensely, i.e., the maximum of metabolism, is, therefore, very near the point of heat-rigour and the maximum of temperature, beyond which death results, while it is very far removed from the point of cold-rigour and the minimum of temperature. In other respects the analogy between cold-rigour and heatrigour is complete; both are phenomena of depression. It is, therefore, disadvantageous, and it leads to false ideas, to employ the expressions cold-tetanus, and heat-tetanus for cold-rigour and heat-rigour, as is sometimes done. Rigour is the direct opposite of tetanus : rigour is a phenomenon of depression, tetanus a phenomenon of excitation. Cold- or heat-tetanus cannot be produced at all, since the rhythmic intermittence of the stimulus belongs to the conception of tetanus, and in temperature this can hardly be obtained. Hence, confusing the two conceptions leads only to erroneous ideas.

Thus, life is embraced between two points of temperature, that of cold-rigour and that of heat-rigour, at which the vital processes have their minimum, or are at a complete standstill. Between these points they go on perceptibly, and the more actively the more the temperature rises from the point of cold-rigour up to near the point of heat-rigour. Shortly before the latter point is reached the vital processes have their maximum. From here on their intensity suddenly sinks with increase of temperature up to the point of heatrigour. Hence, if we had an exact measure for the intensity of every 
metabolic process, such as we possess, e.g., in the quantity of carbonic acid split off in the fermentation of yeast, it would be possible to represent the individual factors of the vital process and therewith the individual vital phenomena as a mathematical function of temperature in the form of a curve of which the abscissa would indicate the temperatures, the ordinates the

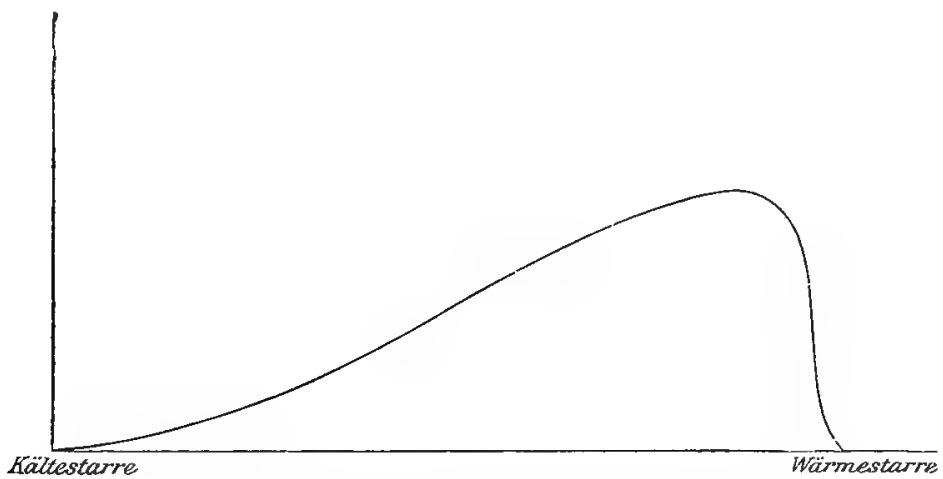

FIG. 184.-Curve of excitation with increasing temperature. The abscissa represents the temperatures, the ordinates the excitation

intensity of the vital phenomenon in question (Fig. 184). Since the individual factors of the vital process, both those belonging to assimilation and those belonging to dissimilation, are dependent upon temperature in very different degrees, in the construction of these individual curves it would be possible to express in the clearest and most graphic manner the very complex relations of metabolism with every change of temperature.

\section{The Actions of Photic Stimuli}

When light is spoken of as a stimulus, the chemical, not the thermal, activity of the light-rays is meant. In this sense, light, when compared with other varieties of stimuli, is to a certain extent peculiar, since it has been found that all varieties of living substances do not react to it, while they do react to chemical, mechanical, thermal, and galvanic stimuli.

In the higher animals it is almost exclusively the sense-cells of the visual organs that possess the capacity of reacting to light. In most tissue-cells, so far as research thus far has gone, this power is wanting. In some lower vertebrates, such as the remarkable salamander, Proteus anguineus, which lives in the streams of the Adelsberg grotto, the whole skin, as Raphæl Dubois has shown, is sensitive to light-rays; and in many inverte- 
brates, such as the earth-worm, eyes are completely wanting, and only the cells of the skin are similarly sensitive. Many unicellular forms possess the power of reacting to photic stimuli, even those that have no organs specially developed for the perception of light, and in the chlorophyllaceous Protista and plants the irritability to light is generally wide-spread.

On the other hand, there are a host of cell-forms, as, e.g., the majority of tissue-cells and ciliate Infusoria, which, according to experiments thus far, are not affected in the slightest degree by light-stimuli when the thermal effect of the latter is excluded. But recently an observation has been made, which deserves great attention in considering the question as to the irritability to light of such cells as hitherto have been regarded as insensitive.

The development of modern electrical technique has revealed methods of producing electric light of very enormous power, which surpasses sunlight in intensity, and which is not sufficiently described by the common word "dazzling." The term "destructive" or "destructively luminous" should be applied to it; for in the electric works, where labourers are exposed to such light, it has often been observed that the skin of these persons exhibits genuine phenomena of necrosis in the uncovered parts of the body. The cells of the epidermis die, the upper layers of the skin scale off, and the lower layers show signs of intense inflammation and ulcerations like burns. It is not the thermal effects of the light that are shown in these phenomena, but the chemical effects of those rays of the spectrum that have a short wave-length; this can be determined by inserting media that absorb the heat. Hence there can be no doubt that we have to do here with very strong photic effects upon cells whose living substance is affected only in very slight measure by the intensity of the light-rays that under usual circumstances come to the earth's surface.

This fact is worth consideration, for it raises the question whether cell-forms whose living substance has been regarded as wholly insensitive to light of the usual grades of intensity do not react to photic stimuli of greater intensities, and, moreover, whether all living substance, just as it reacts to heat, is not also influenced by light, its different varieties responding to different intensities. This possibility must certainly be weighed. Yet, so long as conclusive experiments upon this point are wanting - and such can be carried out with little difficulty and in a short time in a large clectrical establishment-we must hold to the facts as stated above.

Absolute darkness can best be considered as the indifferent point, i.e., that point of intensity at which light exerts no stimulating effect. Beyond this point with increasing intensity the stimulating effect begins. 


\section{a. The Phenomena of Excitation}

The whole organic world of to-day is directly dependent upon the metabolic action of light. The old philosophers of nature, as has been seen, characterised animals, in a certain sense not incorrectly, as parasites upon plants. It is true that carnivora nourish themselves upon animal substances; but this animal food is derived from herbivora, and thus the carnivora also are thrown back upon the plants. But plants cannot exist without the influence of light. The sun's rays give the stimulus that causes the chlorophyll bodies of the plant-cells to decompose the carbonic acid of the air into carbon and oxygen, and from the carbon, with the water taken in through the roots, to produce synthetically the first organic substance, the first product of assimilation, starch. Further, the sun's rays also give the impulse to the production of the green chlorophyll colouring-matter itself; this follows from the fact that plant seeds, sprouting in the dark, produce a whive or bright-yellow plant, which grows for a time at the expense of the reservesubstances stored up in the plant seed, but which becomes green only when exposed to the light. Only after it becomes green is the plant able to decompose carbonic acid and form starch. Thus, the first organic product, from which all other organic substance is derived, originates from the action of the photic stimulus of the sun's rays.

This assimilatory action of sunlight does not belong to all lightrays in equal measure. As has already been seen, with equal intensities the red rays have the strongest action.

As regards most of the objectively perceptible effects of light upon the retinal cells in the eyes of man and of animals, so far it is uncertain whether they depend upon the direct stimulation of the cells in question, or upon reflex stimulation through the central nervous system. Nevertheless, metabolic effects must be present in the retinal cells, since their results in the central nervous system, to which the excitation is transmitted through the optic nerves, we subjectively feel as colours, and objectively recognise in other men or animals in the movements that are called out by photic stimulation through the mediation of the central nervous system.

As regards the excitation-effects of light upon form-changes, thus far nothing is known.

Numtrous effects upon changes of cnergy, especially in motile phenomena, have been recognised.

In certain fresh-water ponds there is found concealed between mud and sand, in almost total darkness, an awkward, sluggish, amoba-like rhizopod, Pelomyr. The lumpy, naked protoplasmic

${ }_{1} C f . \mathrm{p} .21 \%$. 
body, sometimes almost $2 \mathrm{~mm}$. in size, contains, in addition to a large number of spherical nuclei, innumerable small grains of sand and particles of mud, and hence appears opaque. The movements of Pelomyxa are like those of a sluggish Amoeba. The lumpy, protoplasmic droplet sends out here and there beyond the dark contour of the body and usually in a jerking manner a flat, hyaline pseudopodium, into which the internal mass streams with its nuclei, sandgrains, etc. As a rule, when the protist is left to itself undisturbed, after some time, as in Amoba limax, ${ }^{1}$ a definite direction is taken, in which alone the protoplasm continues to How, and the body thus assumes an extended form (Fig. 185, A). But, if, while creeping, the Pelomyxa be stimulated mechanically by jarring, or chemically by the addition of salt solutions, or thermally by warming, it contracts immediately and like all naked protoplasmic masses assumes a spherical form (Fig. 185, B). In this peculiar organism Engelmann ('79, 2) discovered a pronounced irritability to light: when the Pelomyxa was creeping in the darkness lazily and quietly with its
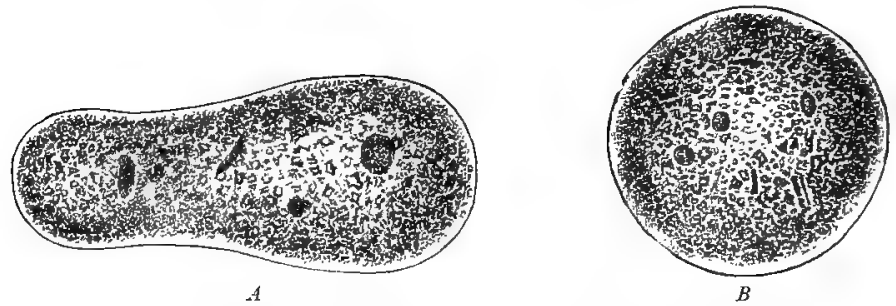

Fia. 185.-Pelomyxa palustits. $A$, Unstimulated, crceping; $B$, stimulated, contracted.

form extended, sudden illumination had the effect that has been spoken above in connection with chemical, mechanical and thermal stimulation. The protoplasmic body contracted suddenly into a ball, and all motion ceased, to reappear at once, however, upon darkening. Slow increase in the intensity of the light from darkness on had no decided influence. The protoplasmic masses of many Myxomycetes, which likewise show contraction-phenomena upon photic stimulation, behave in all respects similarly.

Engelmann, who has made many discoveries concerning the physiology of the unicellular organisms, found also a peculiar Bacterinm, which proved extraordinarily sensitive to photic stimuli. This form, which Engelmann ('83) called Bactcrium photometricum, moves about actively in a drop of water by the stroke of the flagellum which the ends of the body of every motile Bacterium bear. This motion continues only so long as the Bacterinm is exposed to the influence of light. "If it be brought into darkness, the motion "grarlually ceases, and the Bacterinm remains still. But, so soon as 
light again acts upon it, its motion begins anew, and Engelmann was able to determine by means of a spectral apparatus that it is the rays of the orange and the ultra-red which especially exert this stimulating effect upon its motion.

Isolated examples of the excitation of ciliary motion by light occur among the ciliate Infusoria, which in general thus far have shown themselves not irritable to light. In another connection ${ }^{1}$ we have become acquainted with Pleuronema chrysalis (Fig. 186), which in the undisturbed condition lies still in the water without moving its long, leaping cilia, and only from time to time makes a quick spring by a sudden stroke of the latter. If these small
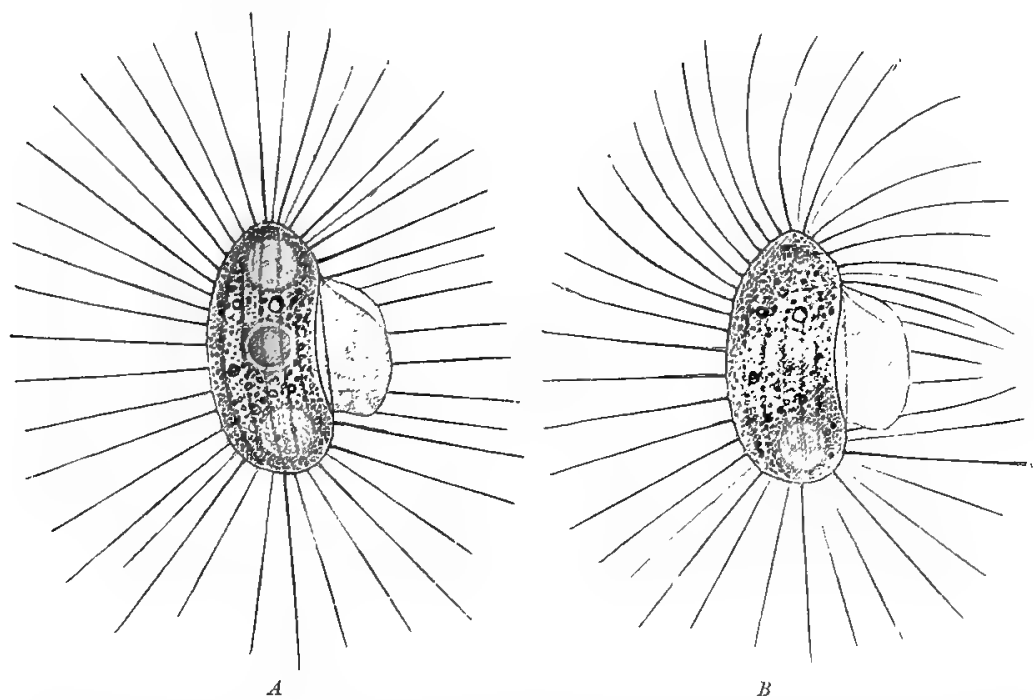

Fia. 186.-Pleuronema chrysalis. A, Unstimulated, lying quiet; $B$, stimulated, in the act of springing by the stroke of its cilia.

Infusoria, which as a rule are observed in great quantity together, are lying still upon the slide in one spot, in ordinary daylight a leaping movement can be induced in them by removing the screen over the mirror of the microscope, and the motion is repeated frequently, when the screen is not shoved in again. ${ }^{2}$ The ciliated cells jump about wildly like a crowd of excited fleas, until they are again shaded. The motion of the cilia does not begin at the exact moment at which the light strikes them but only after a latent period, which continues for about $1-2$ seconds. By the insertion between the source of light and the stage of the microscope of coloured glasses and liquids, the penetrability of which to waves of definite wave-lengths has previously been established spectroscopic-

\section{${ }^{1}$ Cf. p. 383.}

"Cf. Verworn ('89, 1; appendix). 
ally (Fig. 187), it can easily be demonstrated that this jumping movement is not a thermal effect of light, but is caused chiefly by the blue and violet rays, and, therefore, the rays that are least effective thermally. The same effect can be produced by heat-rays, but ordinary daylight is not sufficient for this ; it requires sunlight of considerable intensity, such as can be obtained by the concentration of direct sunlight by means of a concave mirror.

As regards the motion of cross-striated muscles, no instance is thus far known in which light has exerted an influence upon it with certainty. Nevertheless, some time ago Steinach ('92) showed that certain smooth muscle-fibres can be made to contract by light stimuli. In fishes and Amphibia the sphincter iridis, a muscle which in contraction narrows the pupil of the eye, is, as Steinach found, composed of smooth muscle-fibres which contain a brown pigment. These fibres are stimulated by light directly, without the

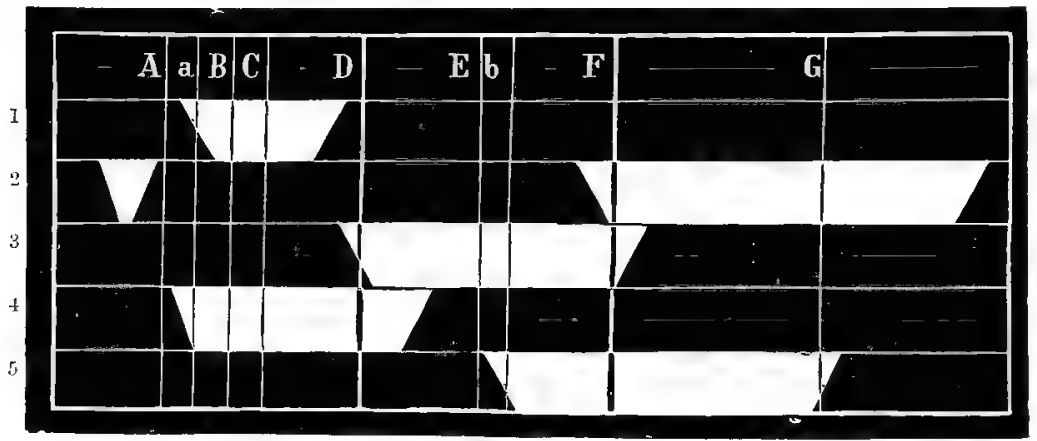

FIG. 187.-Spectra of various media ; 1 , of a red glass ; 2 , of a cobalt glass ; 3 , of a green glass ; 4 , of a solution of potassium bichromate; 5 , of an ammoniacal solution of a cupric salt.

mediation of the central nervous system; this is proved by the fact that even the excised muscle can by illumination be made to contract.

Just as in many cases contraction-movements are caused by light, the peculiar motion of the Diatomece can be influenced in a certain sense by the same stimulus. As Engelmann ('82) has found, this ceases when the organisms are put into a dark chamber and oxygen is excluded. But it immediately begins again, when light is allowed to act upon them. This phenomenon, as Engelmann showed, is due to the fact that, with the exclusion of oxygen, the oxygen necessary to the motion of the Diatomece is soon consumed. If the latter be put into darkness, their movements immediately "ease; if they be brought into the light, they split up carbonic acid into carbon and oxygen, by means of their yellow colouringmatter, which is allied to chlorophyll, and in this manner themselves produce the oxygen that is necessary to their movements. 


\section{b. The Phenomena of Depression}

While light, at least in its ordinary intensity, is, according to experiments thus far, not a general excitant of living substance, it is still less a depressant. The few depressing effects of light that have been reported must be received as such with great caution ; they have been little investigated and their suggested interpretation is extremely doubtful.

The phenomenon, e.g., that the growth of plants in the light is less than in the dark might be regarded as a phenomenon of depression; it might be imagined that light directly inhibits certain metabolic processes that are necessary to growth. But the growth of plants is a very complicated phenomenon, one in which many different factors play a rôle, and, as Sachs ${ }^{1}$ has already emphasised, at present it is impossible to judge how far light as such has a share in its occurrence.

Another depressing effect of light might be sought in the influence of the latter upon the production of light by many luminous marine animals. For example, the statement has often been made that pelagic animals, such as Ctenophore and Siphonophora, when brought from the light into the dark, do not emit light, and only after they have remained in the dark for some time can they be made by stimuli to do so, at first feebly and later more strongly. The power of producing light in these organisms appears, therefore, to be depressed by the influence of light, and since the unicellular Noctiluce are said to behave similarly, it should not be assumed that the phenomenon depends upon a secondary effect of light, mediated by the sense-organs and the central nervous system. But the matter is very uncertain, for, although the doubtful phenomenon has been observed by several persons, thus far it has never been investigated.

Little more is known of the depressing effects of light, and the question whether light is able to call out phenomena of depression at all must remain for the present undecided.

\section{The Actions of Electrical Stimuli}

In many respects the electrical stimulus stands in peculiar contrast to other stimuli. In nature it comes into contact with living organisms only in exceptional cases; this is true also of many chemical stimuli but of no others. Nevertheless, it possesses many properties that make its employment upon living substance especially easy and convenient. It can be graded in intensity more conveniently than any other and with a fineness that answers the highest requirements. Further, its employment can be limited in time in any desired manner. These great advantages, which have

${ }^{1}$ Cf. bibliography: Sachs, J., . Ueber den Einfluss, etc. 
been put to their utmost practical use in the ingenious methods of electrical stimulation, are the reason why in the special physiology of the vertebrates, wherever the effects of stimulation of definite organs are studied, the electrical stimulus is employod almost exclusively. Of the various methods of producing electricity (friction, contact, induction), we employ for stimulating purposes in physiology exclusively the galvanic current, obtained by contact or induction, because this offers the greatest advantages by reason of its constancy and certainty, its

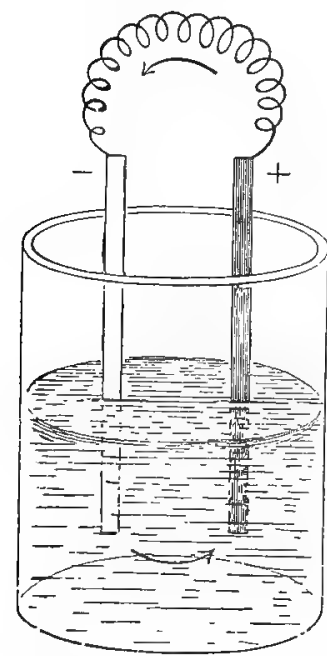

FIG. 188.-Galvanic element. The free pole of the zinc $(-$ ) is joined to the free pole of the copper $(+)$ by a wire ; a circuit is thus formed, in which the direction of the current is indicated by the arrows. convenient handling and applicability, and the capability of its being finely graduated in intensity and duration. Since the methods of galvanic stimulation have developed to very great complication and delicacy, it will be advantageous briefly to consider some of the most important apparatus.

As has been seen elsewhere, ${ }^{1}$ a galvanic tension arises when two strips of different metals or certain other bodies are dipped into a feebly acid liquid. A strip of copper and a strip of zinc, the lower ends of which dip into a vessel containing dilute sulphuric acid, while the upper ends project freely into the air, constitute the most primitive form of a galvanic element (Fig. 188); in it between the two free ends of the zinc and the copper a tension exists of such a kind that the end of the copper is electrically positive, the end of the zinc electrically negative. If the two ends be united by a metallic conductor, such as a wire, at the moment when the union is established, the electrical tension becomes equalised. Since, however, the tension is being constantly renewed at the place of contact of the metals with the liquid, there results a continuous equalisation, which is termed a constant galvanic current. The continuity of copper, wire, zinc, liquid, and copper, forms in a certain sense a closed circuit, in which the current flows. This galvanic current has always the same direction; outside the liquid it flows from the copper, the positive pole, through the wire to the zinc, the negative pole. In the liquid its direction is evidently reversed; from the zinc, through the liquid, back to the copper; but this reversal need occasion no confusion, since it is customary to term the poles outside the liquid the positive and negative poles. The copper is the positive, the zinc the negative 
pole, or, as it is also said in order to express in words the direction of the current, the copper $(+)$ is the anode, the zinc $(-)$ the kathode.

This primitive form of galvanic element, upon which, slightly modified, is based the very powerful chromic acid dip-element in which carbon and zinc dip into dilute chromic acid, has proved for many purposes unserviceable. If the circuit be kept closed for a considerable time, i.e., if the metallic union between the two ends of the metals be not interrupted, or, as is said, the current be not "broken," it is found that the current is not so strong as at the beginning. This depends upon the fact that certain substances, the so-called polarisation-products, have become formed by electrolytic decomposition, have accumulated at the two ends of the metals within the liquid, and by contact with the liquid give rise themselves to a galvanic current, which is opposed to the original current and, therefore, gradually weakens it. In order to prevent the appearance of this polarisation-current and thus to maintain the intensity of the original current as constant as possible, the expedient has been employed of dipping the two metals into different liquids, which are separated from one another by a porous partition-wall of clay, and are so constituted that they destroy the efficiency of the polarisationproducts at the very moment of their appearance. Hence a polarisation-current cannot develop, and the electromotive force of the element remains constant. Such constant elements are in use in various forms. The best-known forms and those that are employed most in physiology are that of

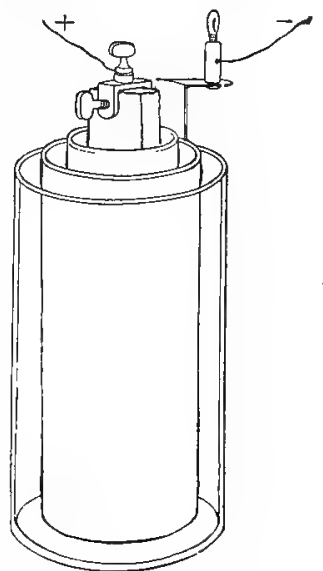

Fig. 189.-Bunsen's element. The carbon plate $(+)$ stands in a clay cylinder contain. ing concentrated nitric acid. The cylindrical zine plate (-) surrounds the clay cylinder, and stands in a vessel containing dilute sul. phuric acid. Daniell, in which zinc dips into dilute sulphuric acid, and copper into a concentrated solution of copper sulphate; that of Bunsen (Fig. 189), in which zinc dips into dilute sulphuric acid, and carbon into concentrated nitric acid; and that of Grove, in which zinc dips into dilute sulphuric acid, and platinum into concentrated nitric acid. In all, the free zinc pole is the kathode.

These galvanic elements are sources of electricity; from them at any moment a galvanic current can be led off very conveniently wherever desired. In order to stimulate galvanically a living object, e.g., a nerve-muscle preparation of a frog, it is necessary simply to cut the wire that joins the two metals of an element and insert the preparation between its ends; the current then 
flows through the preparation (Fig. 190, I). But, in order to be able at any moment conveniently to interrupt the circuit and again to close it and thus to control arbitrarily the influence of the stimulus upon the preparation, there is inserted into one wire a so-called galvanic 7cey, which consists of a cup set into a plate of insulating hard rubber and containing mercury into which one end of the wire dips, while the other is in metallic connection with a small lever; at any moment the lever can be dipped into the mercury or withdrawn, so that the metallic conduction can be established and again interrupted, or, in other words, the current can be made and broken (Fig. 190, II).

When currents are allowed to act on the preparation for a considerable time the metallic wires themselves should not be

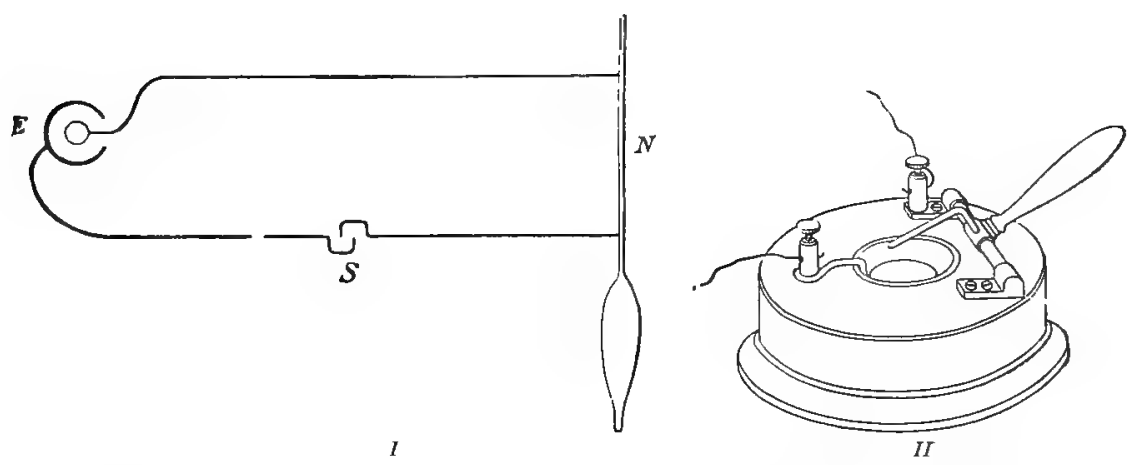

FIr. 190.-1, Circuit between the element $E$ and the nerve $N$ of a nerve-muscle preparation ; in the circuit is the key $S$. $I I$, Mercury kej.

laid as electrodes on the nerve, the muscle or other tissue, since at the place of contact of the metal with the preparation, which latter is a moist conductor, opportunity would be given for the development of polarisation-currents, which would themselves stimulate the preparation and thus disturb the experiment. In order to avoid this, so-called non-polarisable electrodes have been constructed, which allow no polarisation-current to develop at the place of contact with the preparation. ${ }^{1}$ These non-polarisable electrodes consist in their most convenient form of a short glass tube, closed below by a stopper of plastic clay, into which a short soft camel's-hair brush projects; the lumen of the tube is filled with a concentrated solution of zinc sulphate, into which dips a zinc rod connected with the conducting wire (Fig. 191). The electrodes are held in adjustable stands and can be handled with extreme ease, the pointed brushes being laid upon the preparation.

After having become acquainted with a reliable source of

$$
\text { t } C f . \text { p. } 268 .
$$


electricity in the galvanic element, it is necessary to graduate finely and in any way desired the intensity of the current. For this purpose we must consider somewhat fully the fundamental law which formulates the facts regarding the intensity of electricity. This is Ohm's law; it may be stated as follows: the strength of a current is directly proportional to the electromotive force, and inversely proportional to the resistances: $I=\frac{E}{W}$.

The electromotive force depends upon the kind and number of the elements. Many elements have only slight electromotive tension, others very high tension; and if two or more elements be coupled together so that unlike poles are joined with one another, the current is considerably stronger than that afforded by a single element. According to Ohm's law, the chief means of strengthening or weakening the intensity $I$ of a current consists in increasing or diminishing the number of the elements, for thereby the electromotive force $E$ is increased or diminished. But this graduation by change of the electromotive force is very crude and does not ullow delicate changes to be made. Hence, where finer graduations are required, the second factor upon which, according to Ohm's law, the intensity

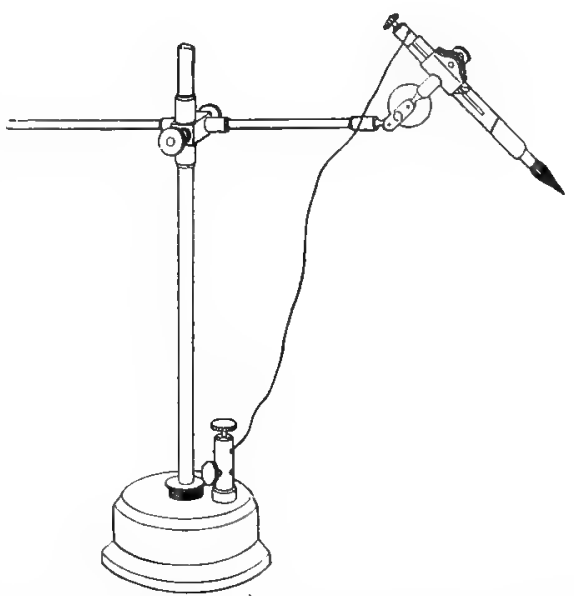

FIo. 191.-A non-polarisable electrode. A glass tube closed by a stopper of clay and filled with a concentrated solution of zinc sulphate is held in the movable stand. A moist camel's-hair brush sticks into the clay stopper, and a ainc rod, to which the wire is carried, dips into the solution. The nerve of the pre. paration is laid over the brushes of two such elec. trodes. depends, is employed, namely, the resistances $W$. The resistances are of two kinds : on the one hand, internal resistance, that which exists in the element itself, especially in the liquid, which is a moist and, therefore, a bad conductor of electricity; on the other hand, external resistance, which exists outside the element in the kind, the length, and the diameter of the conductor. The latter especially can be graduated very delicately.

Metals are good conductors, and for this reason metallic wires, and best copper wires, are always selected as conductors outside the element. Their resistance is less, the shorter the conduction and the greater their cross-section. A very ready means of increasing the 
resistance and thereby diminishing the intensity of the current by any desired amount is that of lengthening the conducting wire and using wires that have a small cross-section.

Upon these facts is based a principle which comes into general use in apparatus that is employed for graduating the intensity of the current, viz., the principle of the accessory or short circuit. If, c.g., a circuit from an element $E$ (Fig. 192, I) extend through copper wires to a preparation $N$, a galvanic current of a definite intensity, which can easily be measured, flows through the preparation, although the latter as a moist conductor affords considerable resistance. But, if into this circuit a short circuit be introduced by joining two opposite points of the metallic conductor by means of a cross-wire, a small circuit is made to.
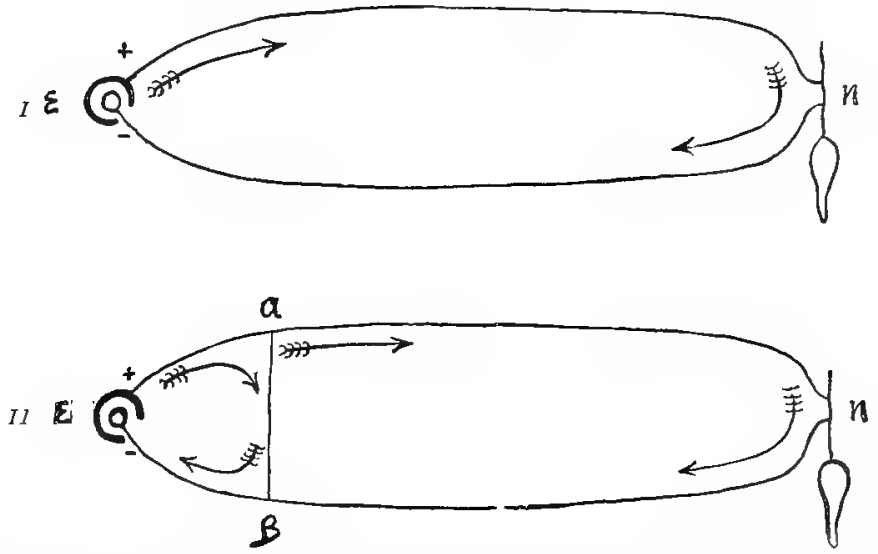

FIG. 192.-Scheme of the short circuit. $I$, A simple circuit; $I I$, long circuit with short circuit. $E$, element; $N$, nerve-muscle preparation; $A, B$, short circuit.

branch off from the large one ; in the former the resistance is considerably less than in the latter, since its conductors are metallic and shorter than those in the latter. The result is, as Ohm's law directly teaches, that in the long circuit a current of slight intensity passes, which is so feeble that under certain circumstances it has no effect whatever upon the preparation, while in the short circuit there is a current of considerable intensity. Hence in the long circuit, in which the preparation is, there are two extremes of intensity: with the short circuit broken, a considerable current, and with the short circuit closed, a very slight current. From the latter extreme to the former the intensity can be graduated very delicately by successively increasing the resistance in the accessory circuit, until it becomes so great that the circuit hardly conducts at all. Then nearly the whole current gous through the large circuit and the preparation. 
Du Bois-Reymond employed the principle of the accessory circuit in his rheochord, an apparatus that serves to increase the intensity in the circuit of a preparation as desired, by shunting definitely measured resistances into an accessory circuit. For resistances fine wires of definite length are employed, which can be introduced one by one into the accessory circuit. The apparatus (Fig. 193) consists in its essential parts of a thick bar of brass, the continuity of which is broken at definite distances so that it is really a series of separate metal blocks, which, however, can be joined into a single bar by the introduction of metallic connecting pieces. Each of these brass blocks is joined to the adjacent block by a very thin conducting wire of a definite length, and upon the wire that joins the first two blocks a metallic slide can be shoved to and fro, so that the wire can be shortened or wholly cut out by shoving up the slide. This whole apparatus is inserted as an accessory circuit into the circuit of the preparation in such a way that the two wire poles lead from the source of the current to the brass bar, and from there two other wires lead to the preparation. If, now, all the connecting plugs of the metal blocks are inserted between the blocks, so that the brass bar is continuous, the condition that is represented in Fig. 192, II is obtained. A strong current passes through the short circuit, because there is little resistance there, while through the long circuit a very feeble current Hows, because there the preparation affords considerable resistance. But

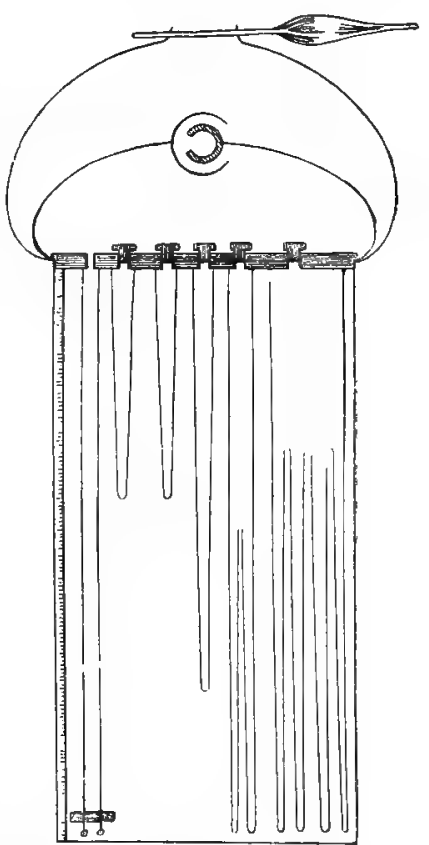

FIG. 193,-Du Bois-Reymond's rheochord the weak current going through the circuit of the preparation can be strengthened very conveniently by increasing the resistances in the shortcircuit, and this is accomplished by shoving down the slide, farther and farther, so that the current inust pass through a constantly greater stretch of the first wire of the rheochord, the distance being measured upon a scale. The resistances can be strengthened still more by removing one by one the connecting plugs between the metal blocks. The result is that finally the current in the short circuit must traverse all the wires of the rheochord, which with their fineness and length form a very considerable resistance. But the more the resistances 
increase in the short circuit, the more does the current that passes through the circuit of the preparation increase in intensity, and, since the resistances are measured exactly, the intensity of the latter current can be graduated very delicately.

Finally, we must consider the methods of allowing a current of momentary duration to act upon the preparation, and of producing currents of momentary duration in rapid rhythmic succession. These are presented by the phenomena of induction.

If two coils of wire are in the same vicinity but not in contact with one another, and if a constant current be allowed to flow through one, the so-called primary coil (Fig. 194), at the moment of the making of this primary current there appears a current in the second, the secondary coil. This induced current is of very brief duration; it exists at the moment of making the primary current, but disappears at once. So long as the primary current passes through the primary coil, not the slightest current is
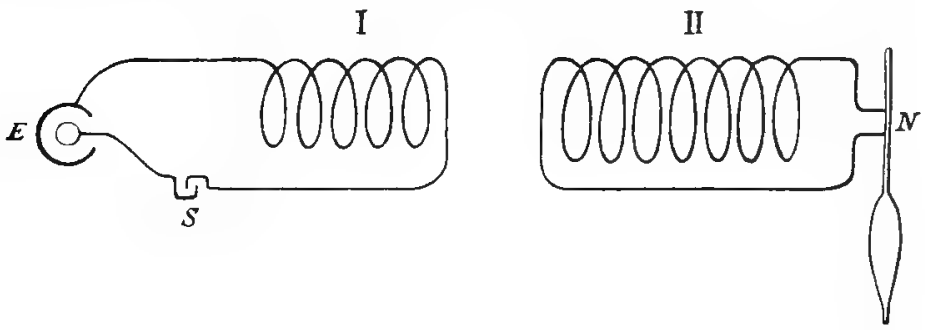

FIG. 194.-Scheme of induced current. I, Primary coil ; $B$, element; $S$, key. II, Secondary coil; $N$, preparation.

present in the secondary coil; but a brief induced current appears again in the secondary coil as soon as the primary current is broken. Thus, an induced current appears only at the moments of making and breaking the primary current. The making induced current is, however, in certain respects essentially different from the breaking induced current. While the direction of the former is opposite to that of the primary current, the breaking induced current has the same direction as the latter. This fact is important, for it explains at the same time another difference between the making and breaking shocks. If the current in the primary coil be made, it induces at the time of its appearance not only in the secondary coil, but also in the turns of its own coil, a current in the opposite direction; and this cxtra current running in opposition to it hinders the increase of the primary current, until the latter has reached its greatest intensity, when the induction-effect ceases. The case is different at the breaking of the primary current, for the extra current that then appears in the turns of the primary coil, has the same direction as the primary 
current. Hence, an essential difference between the making and the breaking induction-shocks is observed in the secondary coil, since with the former, on account of the slow increase of the primary current, the electric tension is more gradually equalised than with the breaking shock, where the equalisation takes place very suddenly. If, therefore, we wish to let a very sudden current act upon a living object, we employ exclusively the breaking induction-shock. The intensity of the induction-shocks may be graduated by the distance that is allowed between the primary and the secondary coils. The intensity is less with greater distance, greater with smaller distance, and greatest when the secondary coil, which is always made somewhat the larger, is shoved completely over the primary.

The sledge-inductorinm of du Bois-Reymond, which is one of the most essential of all pieces of physiological apparatus, is constructed, in accordance with the above principles, for the pro-

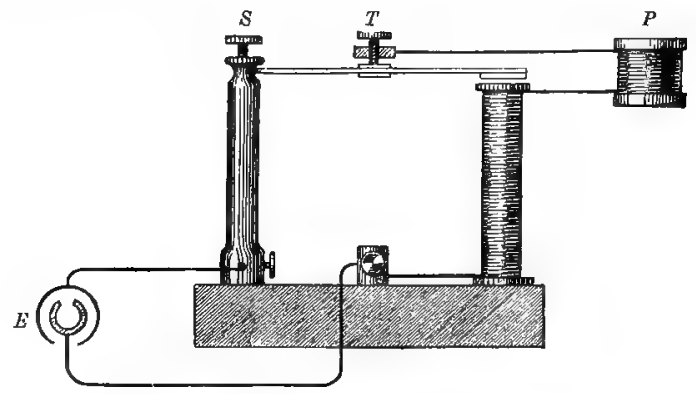

F'IG. 195.-Neef's or Wagner's hammer.

duction of induced currents ; in it the secondary coil slides upon a sledge-like track (Fig. 196). It is arranged also to produce single induction-shocks rapidly and rhythmically. The contrivance that makes this possible is the Neef or Wagner hammer (Fig. 195), which is based upon the following principle. A's is well known, the galvanic current has the peculiarity of transforming into a magnet a piece of soft iron, around which it flows, as long as the circuit remains closed. If the current be broken, the magnetism disappears. In Neef"s hammer there is a brass column $S$, which bears a straight spring. This spring, to the free end of which is fastened a small hammer of soft iron, in its resting position touches an adjustable screw $T$, which is in connection with a wire $P$; the wire forms a coil about a soft iron rod which stands upright under the hammer, and ends in a second small brass column. The two brass columns bear screws to hold the conducting wires coming from the element $E$. If the current of the element be made, the following happens: the current enters through the brass column $S$, 
passes through the spring into the screw $T$, through the coil $P$, about the iron rod, into the second smaller brass column, and then returns to the element. The result is that the soft iron rod becomes magnetic and attracts the hammer that is suspended above it. Thereby the contact of the spring with the screw $T$ is broken. By the breaking of this contact, however, the current is broken, the magnetism in the soft iron rod consequently ceases, and the hammer springs up by reason of the tension of the spring. As a result of this the spring again touches the screw $T$, and the current is made again. By this ingenious contrivance, as long as the element remains in the circuit, the current is continually made and broken in rapid rhythmic succession. In du Bois-Reymond's sledge-

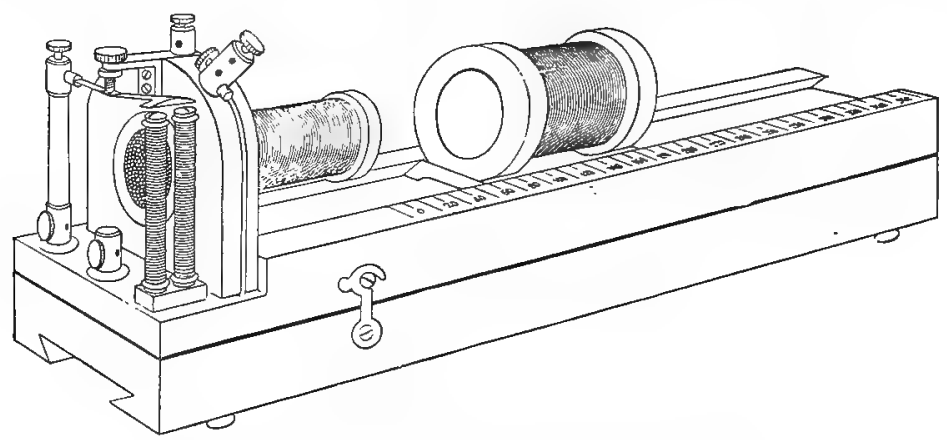

FIG. 106.-Du Bois-Reymond's sledge-inductorium.

apparatus (Fig. 196) such a hammer is inserted into the primary circuit, and by its play there occurs in the secondary circuit for every opening and every closing an induction-shock; thus a rapid succession of shocks takes place. When sent through living substance, these act as rapid intermittent stimuli and produce a tetanus.

For the construction of most of these pieces of apparatus we are indebted to the inventive genius of du Bois-Reymond alone, who has created a method that has become, and will remain, indispensable in many fields of physiology.

We will now pass from this excursus regarding the technique of galvanic stimulation to the effects that the galvanic stimulus exerts upon living substance.

\section{a. The Phenomena of Excitation}

It is a noteworthy fact that, although electrical stimulation is one of the most common methods in use in physiology, it has been employed almost exclusively upon the nerve- and the muscle-fibre, and only occasionally upon plant-cells and unicel- 
lular organisms. This fact is associated with the one-sided development of physiology as the science of the organs of vertebrates. If experiment be limited to the organs of the higher animal body, where almost all tissues depend upon the nervous system, stimulation of the tissues may be indirect, through the nerves supplying them; in most cases it is necessarily so, since the extremely finely branched nerve-fibres lying between the tissuecells can be excluded with difficulty. Methods of excluding completely the influence of the nervous system are known only in the case of muscle, by means of the very remarkable arrowpoison of the Mexican Indians, curare. The cells of gland-tissue, mucous membranes, connective tissue, etc., cannot be freed from the influence of the nerves supplying them, and hence, if an electric current is allowed to act upon them, because of the much greater irritability of the nerve-fibres there is always obtained not a direct stimulation of the tissue-cells alone, but a simultaneous stimulation of the nerve-fibres which transmit their own excitation to the gland-cell, the connective tissue-cell, etc. In order to put a tissue into activity by stimulation, it is of course sufficient, and it is very convenient, to stimulate it indirectly through its nerves; but the effect of a direct stimulation of the tissue itself cannot thus be studied. It follows that all the innumerable experiments with electrical stimulation upon the vertebrate body have to do almost exclusively with stimulation of nerve or muscle.

This fact has led to many erroneous ideas regarding the excitation-effects of galvanic stimulation. When attention was confined chiefly to the stimulation of muscle, whether directly or indirectly through the nerve, it was customary to consider contraction as the expression of excitation in the muscle. This was undoubtedly correct. But the more or less manifest thought was incorrect, that excitation exists only where contraction appears, and that no excitation is present where there is no contraction. This view has led to very many errors, many of which are not yet corrected. Thus, the idea is still maintained by many physiologists, that only variations in intensity of the galvanic current act to stimulate, and only these when they occur with a certain rapidity; i.e., that only an increase or diminution in the strength of the current, taking place at a certain rate, produces reactions, and not a current continued with a constant intensity, or one that is very gradually increased or decreased. It was believed that this conclusion could be drawn from the following facts.

If a constant current be allowed to flow through a muscle or its nerve, the muscle contracts only at the moment of making, when, therefore, the intensity of the current suddenly increases; it expands again immediately, remains extended during the whole duration of the current, and at the moment of breaking, when the 
intensity suddenly falls, performs a second contraction. Further, if a current so weak as to be ineffective be allowed to flow through the preparation and then its intensity be gradually increased, it can be made very strong without the muscle making the slightest contraction. But, if a current of the same intensity be allowed to act suddenly upon the preparation, there is an energetic contraction at the making. Likewise by induction-shocks a much stronger contraction is obtained upon breaking, when, as has been seen, the electric tension is equalised suddenly, than upon making, when the equalisation takes place more slowly. These and similar phenomena have led to the mistaken idea that only the variation of a current at a certain rate acts as a stimulus, and not the continued constant current, and there has been an inclination to transfer this idea to other varieties of stimuli. This error is not unnatural in view of the facts that for a long time muscle was the sole object employed for experiment, and contraction represented the sole obvious expression of the excitation. More careful investigation has shown that during the continuance of a constant current the muscle goes into a peculiar condition which du BoisReymond has termed electrotonus, and in which its irritability is peculiarly changed. It has also been known for a long time that in the employment of somewhat strong currents the muscle does not extend completely after the making, but continues during the whole duration of the current in a state of feeble closing tetanus. An attempt, involving great effort and trouble, has been made to interpret this latter fact otherwise, and also many other facts that favour the idea that the muscle can be in a state of excitation without showing a sudden twitch or continuous contraction. If experiments had not been limited to the muscle or the nerve, but if other objects, such as unicellular organisms, which express excitation in a greater variety of ways, had been employed, and if the question had been followed comparatively, this error, that stimulation results only from variations in the current, and not from the current itself, would evidently have been avoided.

The one-sided study of galvanic stimulation in muscle and nerve has led to another incorrect iden, viz., the general law of polar excitation of living substance by the constant current. If a constant current be allowed to flow through a living object, it is observed that the whole stretch passed through by the current is not stimulated simultaneously, but that the excitation appears primarily at the place of entrance or outgo of the current, that is, at the anode or the kathode; from here it can spread secondarily over the whole object because of the continuity of the living substance. Hence the anode and the kathode are the only places where the current stimulates directly; but under what circumstances the former is the point of excitation, and under what the: 
latter, has been reduced to a very definite principle, and this principle finds expression in the law of polar excitation.

If a constant current be sent through a motor nerve, the nerve becomes stimulated at the kathode upon making, and from here the excitation extends through the conducting nerve to the muscle, which latter then performs a contraction. Upon breaking the current the excitation of the nerve takes place at the anode and extends from here to the muscle, so that the latter contracts. In the year 1859 Pflüger confirmed this law of polar excitation of the nerve. Its correctness can be proved in various ways, best by the following experiment. A constant current is allowed to flow in different directions through the nerve of a nerve-muscle preparation, first, descending, i.e., with the anode lying nearer the central end of the nerve, the kathode nearer the muscle, and, secondly, ascending, i.e., with the anode lying nearer the muscle and the kathode nearer the central end of the nerve; and both times the contraction of the muscle is recorded upon the plate of a myograph. ${ }^{1}$ It is then found from the length of the latent period that at the making of the descending current the muscle contracts earlier than at the making of the ascending current, but that at the breaking the relation is reversed; the difference in time equals the duration of the process of transmission of the stimulus along the intrapolar stretch of nerve. It thus appears that the excitation upon making must start from the kathode, and upon breaking from the anode. This law of polar excitation was recognised by Bezold ('61) as valid for cross-striated muscle, and Engelmann ('T0) showed that it can also be applied to smooth muscle. Later investigations, especially by Biedermann ('79, '83, '84, '85), afforded a number of new proofs of its validity. It was then assumed that, like muscle and nerve, all living substance is stimulated by the galvanic current at the kathode upon making, and at the anode upon breaking. But it has here been demonstrated again how the one-sided investigation of nerve and muscle may lead to errors, which can be avoided by comparative physiological research, for the testing of other forms of living substance, especially various kinds of free-living cells, has shown that a generally applicable law of polar excitation of living substance does not exist. Since the phenomena in question in unicellular organisms. afford a striking example of the fact that excitation is caused not only by variations of current, but also by the continued current, we will here consider them somewhat in detail.

In the year 1864 Kühne made the peculiar obscrvation that Actinosphcerium Eichhornii (Fig. 198) obeys a very different law of excitation. But this discovery remained isolated and unnoticed for more than two decades. Only when certain other effects of the galvanic current, constituting galvanotaxis, were 
discovered, was Kühne's observation recalled and confirmed by means of more complete methods. The examination of a long series of free-living cells followed, all of which follow a law of polar excitation differing in various ways from that of nerve and muscle. ${ }^{1}$

The best method of employing galvanic stimulation with nonpolarisable electrodes upon a slide under the microscope, is to use a slide (Fig. 197) upon which two strips of porous clay, such as is used in the porous cups of the galvanic element, are cemented parallel to one another $\left(a, a_{1}\right)$; the ends of these strips are united by a wall of insulating cement (colophonium and wax) $\left(b, b_{1}\right)$; thus a small open box is formed, in which can be placed a drop of water containing the objects to be investigated. The brushes of ordinary non-polarisable electrodes are laid upon the two parallel strips. It is possible by means of this small contrivance to send nearly parallel currents through the microscopic objects, and, at the same

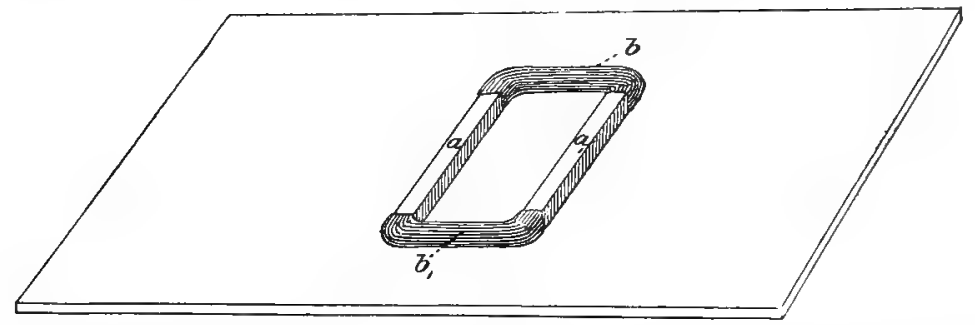

FIc. 197.--Slide bearing a small box for the galvanic stimulation of microscopic objects. $a, a_{1}$, Strips of fired clay; $b, b_{1}$, insulating walls of cement, which with the strips form a box, in which the objects are placed.

time, to observe the effects of the stimulation under the microscope. If Actinosphoerium be stimulated by a constant current in this way, when it has protruded its pseudopodia from its spherical body, like the rays of the sun, it is found that, at the moment of making, phenomena of contraction may be observed in the pseudopodia that are extended in the direction of the anode and the kathode, the protoplasm coming together into small globules and spindles and streaming toward the body (Fig. 198). The pseudopodia that are extended perpendicularly to the direction of the current remain at rest. Hence, at the moment of closing there is both an anodic and a kathodic excitation of contraction. The excitation at the anode is the stronger of the two. Throughout the duration of the constant current the following is noticeable: At the kathode after the making, the phenomena of excitation gradually disappear, and the pseudopodia there assume their former smooth appearance, while upon the side of the anode the excitation

Cf. Terworn ('89, 2, 3). 
continues as long as the current remains. This latter is expressed in a constant advance of the phenomena of contraction. The protoplasm constantly draws back from the anode toward the body, and soon the pseudopodia are wholly drawn in. Then the contraction is noticed upon the body itself: the protoplasm of the walls of the vacuoles retracts more and more toward the interior, the vacuoles collapse, and the protoplasm itself disintegrates partly into its granules. This process of amalgamation and disintegration continues as long as the current flows, but gradually decreases in intensity. Hence there can be no doubt that the constant current stimulates throughout its duration. At the moment when the

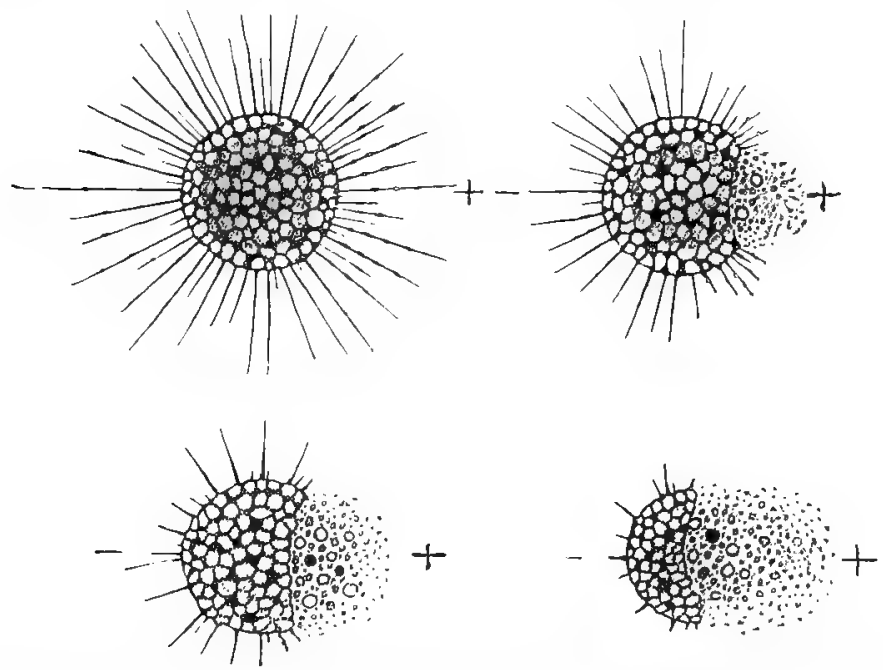

Frg. 198. - Actirosphatiun Fichhonii in four successive stages of polar excitation by the constant current. The protoplasm is disintegrating from the side of the anode.

current is broken the amalgamation-process at the anode ceases at once. A few phenomena of stimulation are noticeable at the kathode, the pseudopodia again showing contraction-phenomena and their protoplasm flowing together into globules and spindles. But this effect gradually ceases and there is no complete retraction of the kathodic pseudopodia. If the current be not broken, the body of the Actinosphoeraum disintegrates from the anode constantly, but in the course of time more slowly, until finally, if the current is feeble, the process wholly ceases. If, however, the current is stronger, the disintegration proceeds rapidly until the whole body has fallen into a lifeless mass of granules. Hence, Actinosphorium is stimulated to contraction by the mething of the constant current at both the anorle and the hathorde, ly the brealing only at the kathode. 
Exactly like Actinosphcerium, many marine rhizopods, such as Orbitolites, Amphistegina (Fig. 199), and others, are stimulated by the making of the current to contract strongly at the anode and feebly at the kathode; this phenomenon appears much more distinctly and purely in long, thread-like pseudopodia than in Actinosphorium, since in the former the globules and spindles that are so thoroughly characteristic of all strong excitation of contraction develop at the two poles especially beautifully.

In the ciliated epithelia of vertebrates Kraft ('90) saw likewise that upon passage of the constant current the ciliary motion was

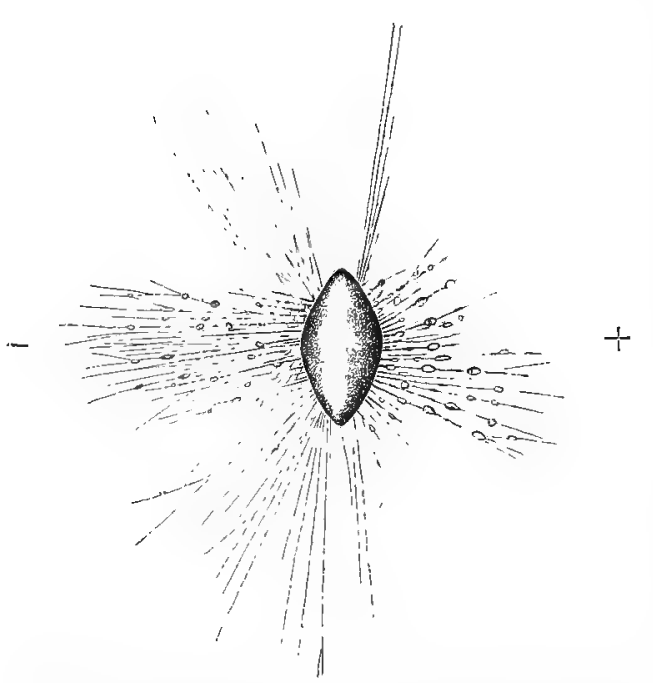

ErG. 199.-Amphistegina lessonii. (Cf. Fig. 170, p. 37s.) The lenticular calcareous shell stands upon its sharp edge, and from the opening, which is directed toward the ground, sends in all directions thread-like pseudopodia; upon these may be clearly recognised at the anode a very strong, and at the kathode a very feeble, excitation of contraction. accelerated at both poles upon making. As regards the polai effect of the breaking he could not come to any definite conclusion. Finally, Loeb $(96,4)$ has found very recently that in Amblystoma, an American urodele, the cells of the cutaneous glands are stimulated at the anode by the making of the current so that a whitish secretion is extruded at that pole, in whatever direction the current is sent through the body.

Pelomyxa ${ }^{2}$ behaves somewhat differently and also differently from muscle. If this lump of protoplasm be stimulated by a constant galvanic current, an excitation appears at the moment of making only at the anode, being expressed by a sudden, jerk-like contraction followed immediately by disintegration at the anodic side (Fig. 200). At the breaking of the current the same phenomenon occurs upon the kathodic side, while the disintegration at the anode immediately ceases. If, however, the current be kept made for a long time, the body disintegrates gradually from the anodic side into a dead mass. Hence, Pelomyxa shows likewise that the continued constant current acts as a continued stimulus. The irritability always becomes less, the longer the current remains made. If, after the action has continued for some time, the current

$$
{ }^{1} \text { Cf. Verworn ('92, 2). }
$$

${ }^{2}$ Cf. p. 400. 
be broken, the breaking frequently is no longer stimulating, and, in order to obtain a result on remaking, considerably stronger currents must be employed than before. The decrease of irritability under long action of the current is also the reason why in Actinosphcerinm, with the intensity of the current remaining equal, the amalgamation-process constantly decreases in intensity. Living substance loses in irritability under the long action of a stimulus. The law of excitation of Pelomyxd runs, therefore, as follows: Pelomyxa is stimulated to contraction at the unode upon making, and ret the kathode upon breaking.

Another form of polar excitation, which is perhaps still more interesting, is shown by Amoba proteus. ${ }^{1}$ If a constant current be sent through the body of the amoba when the latter is extending its pseudopodia in various directions, it is seen that the body assumes at once the typical form of Amcebc limar, i.e., the extended form in which the protoplasm flows in a single direction, the body in a curtain sense representing a single, large, thick pseudopodium.
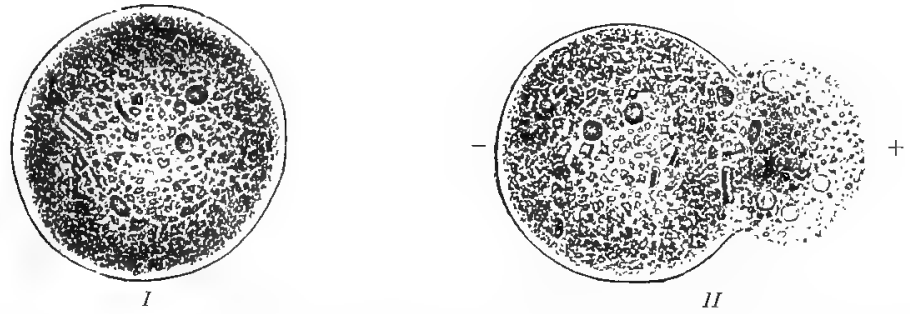

Fia. 200.-Pelomyxa palustiv. I, Normal, spherically contracted. $1 /$, At the moment of making, the protoplasm begins to disintegrate at the anode.

It is thus shown that the extended body is stimulated to contraction at the anode, for here the characteristic vacuoles of Buitschli develop in the protoplasm, and the body retracts strongly upon this side. At the kathode, on the contrary, there exists an excitation of expansion, for here the protoplasm spreads out into a broad lobe. The phenomena are seen best when the direction of the current is suddenly reversed, so that what was previously the anode now becomes the kathode, and vice verso (Fig. 201).

Wholly analogous relations have been demonstrated recently by Ludloff ('95) in Paramacium. By the making of the current phenomena of contraction are shown in the external form of the body at the anode; by strong currents that end of the body is compressed into a point, the liquid of the trichocysts is pressed out and, becoming coagulated in the form of threads, surrounds the end of the body with rays (Fig. 202, B). The polar excitation of the ciliary motion is much more characteristic. The cilia at the two

${ }^{1} C f$. Verworn ('96, 4). 
poles of the body are stimulated in opposite senses, the anodic to contract, their motion being stronger in the direction of the posterior end, and the kathodic to expand, the motion of these being stronger in the direction of the anterior end, in whatever relation to the direction of the current the body may be fixed (Fig. 202, $C^{\prime}$ ). Hence in Amoba, as in Paramacium, the making of the curront produces at the two poles opposite effects, leading to contraction at the anode and to expansion at the kathode.

But, in reality, the polar effects of the galvanic current on muscle, as the later researches of Biedermann $(90,1,2)$ on smooth and cross-striated muscles have shown, are more complex than the law of excitation of muscle, in the form in which it has thus far been expressed, declares. The conception of excitation has hitherto been limited to the augmentation of these processes that in

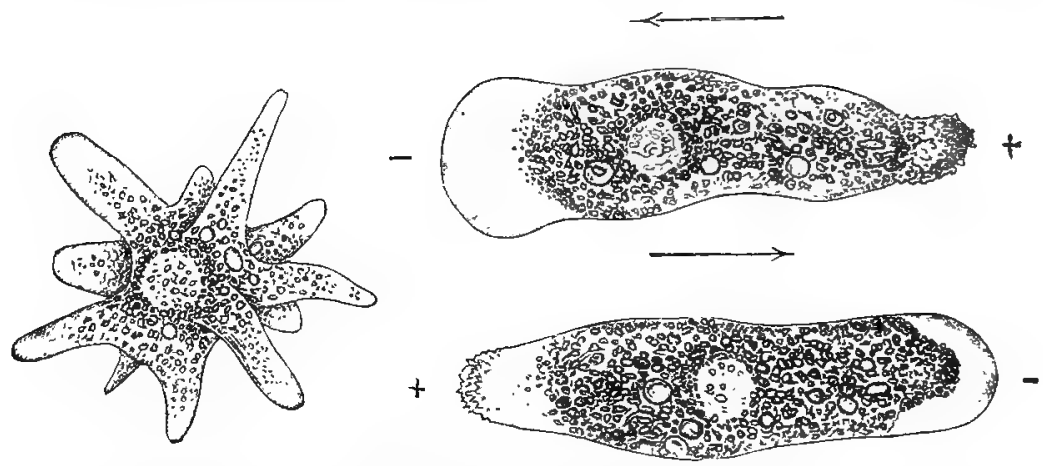

F1G. 201.-Anoba proteus. At the left an unstimulated individual possessing numerous pseudopodia; at the right two individuals stimulated by the galvanic current. At the anode a typical contraction is shown, at the kathode a strong expansion; this is noticed especially clearly upon sudden reversal of the direction of the current.

contractile substances find their expression in contraction. Expansion (relaxation) has customarily been regarded as a phenomenon of depression. This is incorrect. By depression is meant a diminution or complete cessation of the vital processes in question, as is exemplified by narcosis. Expansion, however, is based upon an augmentation of processes, just as is contraction. The confounding of expansion and depression leads to false ideas. The two conceptions should be sharply separated, and the term excitation should be extended to include the augmentation of those processes that in contractile substances find their expression in expansion. From Biedermann's researches it follows that the making of the constant current produces in the muscle not only an excitation of contraction at the kathode, but at the same time an excitation of expansion at the anode. In a muscle that is at the maximum of its extension the excitation at the anode can apparently not find 
expression, for a muscle completely extended cannot be extended further. But that the process at the anode is as is stated above is seen at once when smooth or cross-striated muscles that are partially contracted are stimulated. In such muscles at the moment of making the current a local expansion takes place at the anode. Biedermann was likewise able to establish upon heart-muscle the
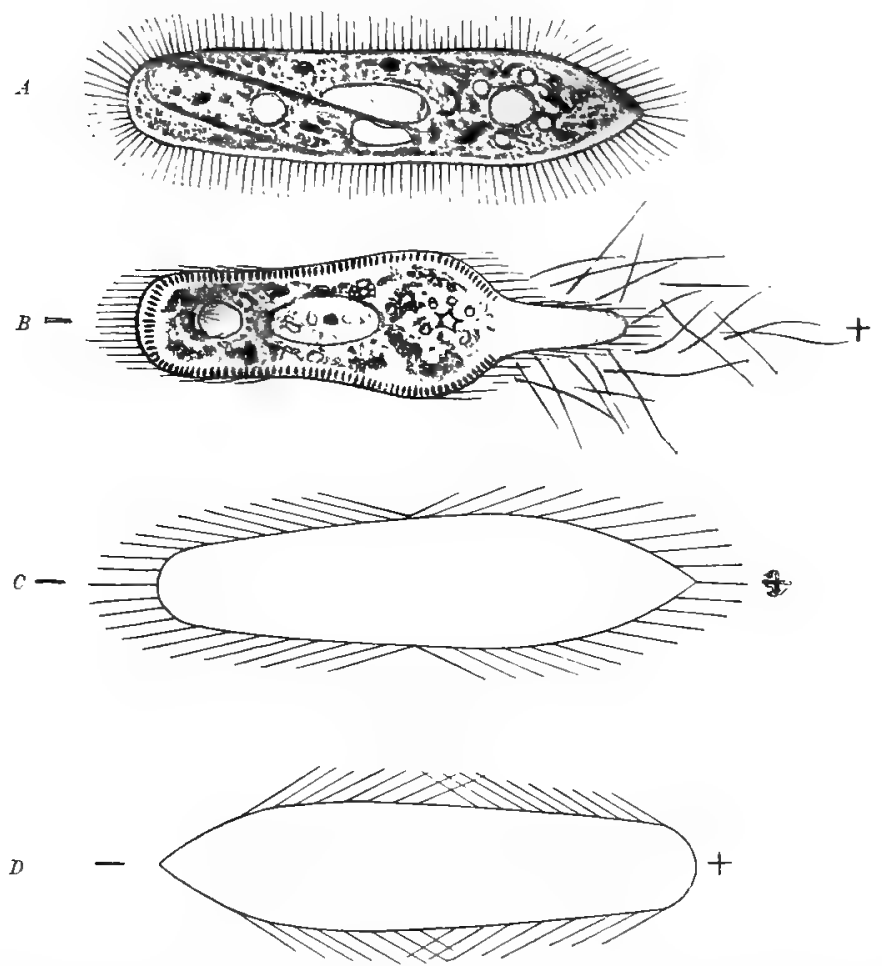

Fic. 200.-Parancecium auelia, polar excitation. A, Unstinulated individual. $B$, Action of at strong current; the anodic end has become compressed into a point and the contents of the trichocysts have been extruded. $C$, The positions taken by the cilia (only the outline of the body is sketched); at the anode the cilia are bent more strongly toward the pointed hinder end of the hody, at the kuthode more toward the blunt forward end. $D$, The same, the position of the body being reversed. (After Ludloff.)

reverse fact, that upon breaking, in addition to the excitation of contraction at the anode, an excitation of expansion takes place at the kathode. It is interesting that the effects at the two poles upon making are the opposite of those upon breaking. The phenomena in the nerve afford a complete analogr to this. In the nerve also there are opposite effects at the two poles. This is expressed in the change in irritibility that manifests itsulf at the poles when a galvanic current is passed through the nerve. For example, 
experiments on the stimulation of nerves that are in the electrotonic condition have shown that upon the making of the current the irritability rises at the kathode in comparison with the normal, but at the anode is depressed; this relation is completely reversed upon breaking, so that for a short time after breaking an increase of irritability at the anode and a decrease at the kathode are noticeable. Thus, opposite processes exist at the two poles upon
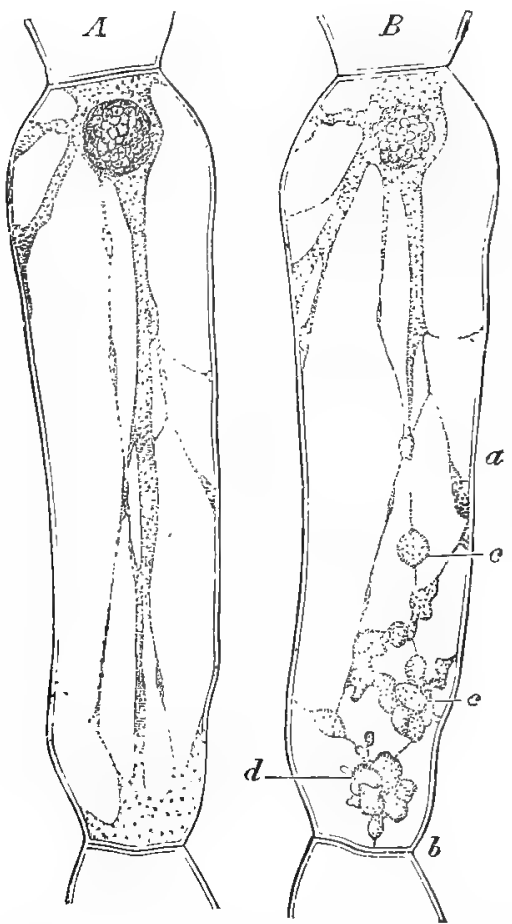

FIG. 203.-Tradescantia virginira. A cell from a stamen-hair. $A$, Unstimulated ; $B$, stimulated by an induction-current. The protoplasm has flowed together into globules and lumps at $a, b, c, d$. (After Kithne.) making, and each is reversed upon breaking. Whether similar relations between the effects of making and breaking on the one hand, and those of the two poles on the other, will be discovered in many free-living cells, later experiments must show. But that the opposition in the effects at the two poles upon making, which exist in muscle and nerve, is not to be generalised for all living substance, is shown by the simple fact that in Actinosphorizm, Orbitolites, and Amphistegina, it is not present; in these forms an excitation of contraction alone appears at both the anode and the kathode.

In summarising briefly our knowledge of the polar effects of the galvanic current, it can only be said that the primary effects of the constant current are localised at the points of entrance into (anode) and exit from (kathode) the living substance; in the different forms of living substance the kind of excitation at the kathode and at the anode upon making and upon breaking are very different; hence, no general law of polar excitation, applicable to all living substance, can be formulated.

We will here leave the polar effects of the galvanic current and take up the various kinds of excitation-phenomena caused by electric stimulation. The effects upon contractile substances have already been considered to some extent. Contractile effects that are manifested outwardly in motion will now be examined. 
Expansion-effects of galvanic stimulation are mostly inconspicuous externally, and it has already been seen that only in certain cases is it possible to observe them at all. But contractioneffects are everywhere noticeable. Typical phenomena of contraction have already been seen in Actinosphos'ium and Amphistegina in the formation of globules and spindles in the protoplasm of stimulated pseudopodia. Amoba and leucocytes, as Golubew ('68) and Engelmann ('69) have shown, when acted upon by single inductionshocks, draw in their pseudopodia and assume a spherical shape. The protoplasm of plant-cells, as Kühne ('64) demonstrated in the cells of the stamen-hairs of Tradescantia virginica, is induced likewise to form globules by repeated making and breaking of the constant current or by single induction-shocks (Fig. 203); this is also characteristic of naked protoplasm, and can be produced locally by the local application of stimuli. The activity of cilia, as Engelmann $(79,1)$ and, more recently, Kraft ('90) have observed in ciliated epithelia, is increased to greater rapidity by the galvanic current, the frequency and amplitude of the stroke, and hence the useful effect, being especially influenced. In the single flagellum of the flagellate cell also, e.g., in Peranema, the exciting effect of the electric current can be observed expressing itself with a single induction-shock by an energetic stroke in the otherwise uniformly rhythmical beat (Fig. 204). In the myoids of Infusoria, e.g., in the stalk-myoid of Vorticella, in smooth musclecells, and in cross-striated muscle-fibres, excitation by a single, brief electrical stimulus, such as a single induction-shock, is expressed

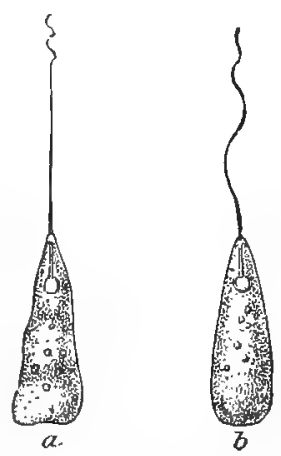

FIG. 204.-Peranema, a flagellate infusorian. $a$, Swimming quietly; $b$, stimulated by an induction-shock. by a contraction; with cross-striated skeletal muscles this can be recorded graphically by means of a myograph (Fig. 205).

But before bringing to an end the consideration of reactions in contractile substances, the effect of rapidly successive galvanic stimuli deserves attention. The best means of putting a contractile structure into tetanic contraction is afforded by the rhythmic induction-shocks of du Bois-Reymond's sledge-apparatus with the hammer in action. An amœeba or a leucocyte under the influence of rhythmically successive induction-shocks remains in contraction, i.e, preserves its spherical form, as long as the action continues. With the same kind of stimulus muscle likewise is in continual contraction. Muscle here affords a much more favourable opportunity than with mechanical stimulation to follow the origin of tetanus and to demonstrate the fact that tetanic contraction consists of discontinuous, single contractions, which follow 
one another so rapidly that between them there is no time for the muscle to extend. In order to study the details of tetanic contraction, a myograph is employed (Fig. 206), the writing-lever of which traces the movement of the muscle upon stimulation in the form of a curve upon a revolving drum. If the muscle be stimulated by means of a single, not too strong induction-shock, so that it performs only a moderate contraction, a single curve is obtained, the ascending limb of which represents the phase of contraction, the descending limb the phase of expansion (Figs. 205, $I I$, and 207, I). But, if several induction-shocks be allowed
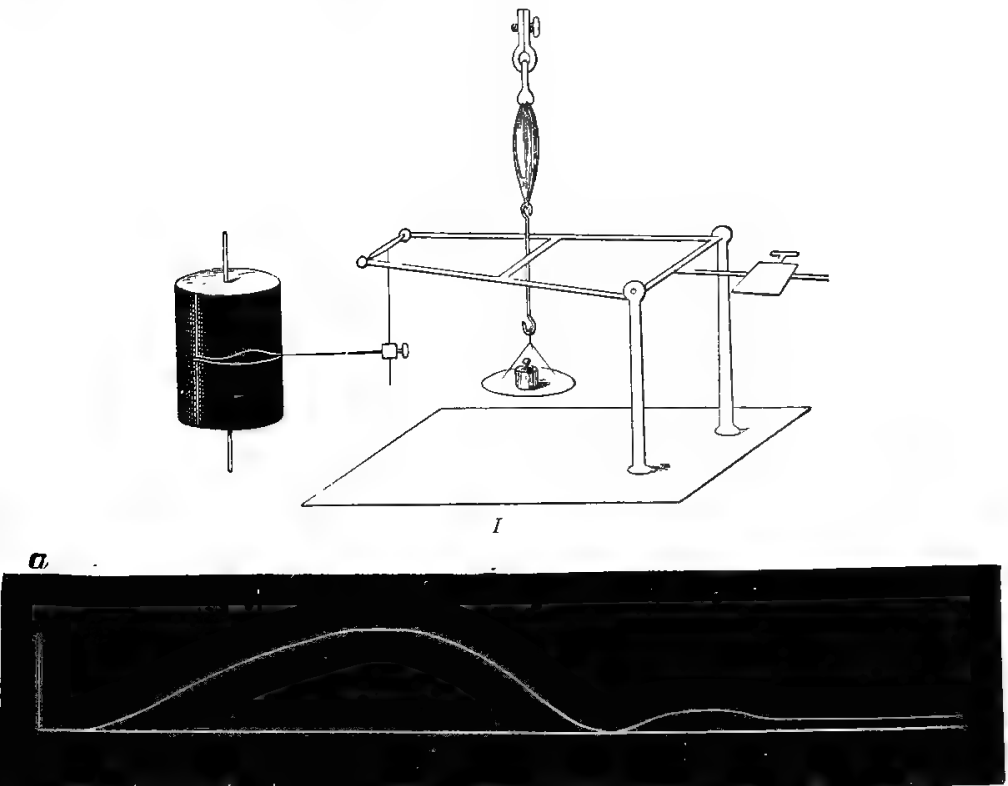

71

Fra, 205.-I, Myograph. II, Curve of contraction. (After Helmholtz.) a, Moment of stimulation by an induction-shock.

to act upon the muscle in succession and at regular intervals in such a manner that each succeeding stimulus reaches it at the moment when it is just beginning to extend, the first contractions are superposed, i.e., the shortening of the muscle is continued with every succeeding contraction; the shortening of each contraction may be regarded as representing the resting point of the muscle, and from this the shortening of the next contraction rises. Thus, with every succeeding stimulus the curve of shortening rises like steps and reaches a certain height, at which it is then maintained, still allowing the regular variations 
between the individual stimuli to be recognised clearly (Fig. 207, II). If induction-shocks be allowed to act upon the muscle in more rapid succession, as they are produced in the secondary coil by the play of Neef's hammer, the effect of each single shock is no longer to be distinguished as such, but a smooth curve results, which rises rather steeply and then, if the stimulation be not continued too long, maintains itself at a uniform height as a straight line (Fig. 207, III). Thus, by increasing the rate of succession of the stimuli, it is possible to follow the formation of complete tetanus from the completely finished single contractions through all the transition-forms of incomplete tetanus, and thereby to afford the proof that, in reality, tetanus is a discontinuous con-

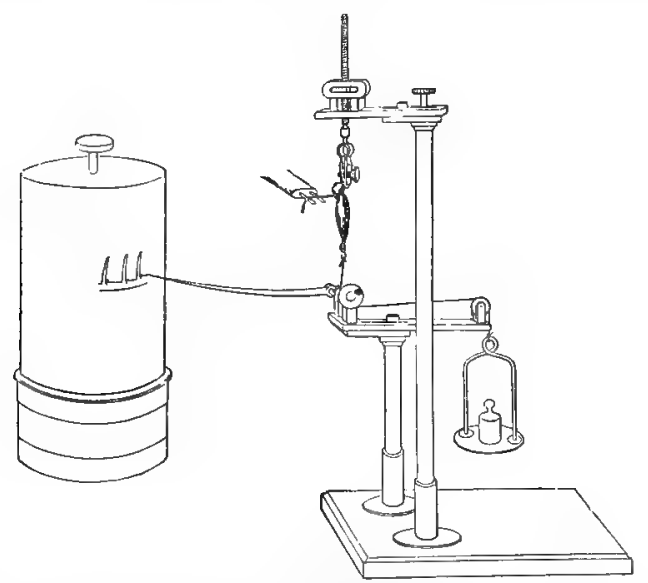

FIG. 206.-MIuscle-writer. The nerve-muscle preparation is fixed in the muscle-holder; the norve is stimulated by pointed platinum electrodes; and the muscle records its movement upon a rotating, blackened drum by means of a writing-lever.

traction. All continued contractions that are performed in the human body under nervous influence are, like tetanus artificially produced, discontinuous phenomena composed of many single contractions following one another in rapid succession.

It should be mentioned that there are forms of living substance that are not influenced at all by induction-shocks, either by single ones or by shocks succeeding one another rapidly or slowly, howerer strong they may be. Such objects are Orbitolites, Amphistegina, and other marine Rhirnoulin. Their protoplasm requires for reaction a longer duration of the stimulus than the lightning-like induction-shock possesses. ${ }^{1}$

As regards other excitation-effects of galvanic stimulation, mechanical motile effects are produced, not only in contractile

${ }^{2} C f$. Verworn $(92,2)$. 
substances, but in plants that, like Mimosa, move by , changes of turgescence. If single induction-shocks be allowed to act upon
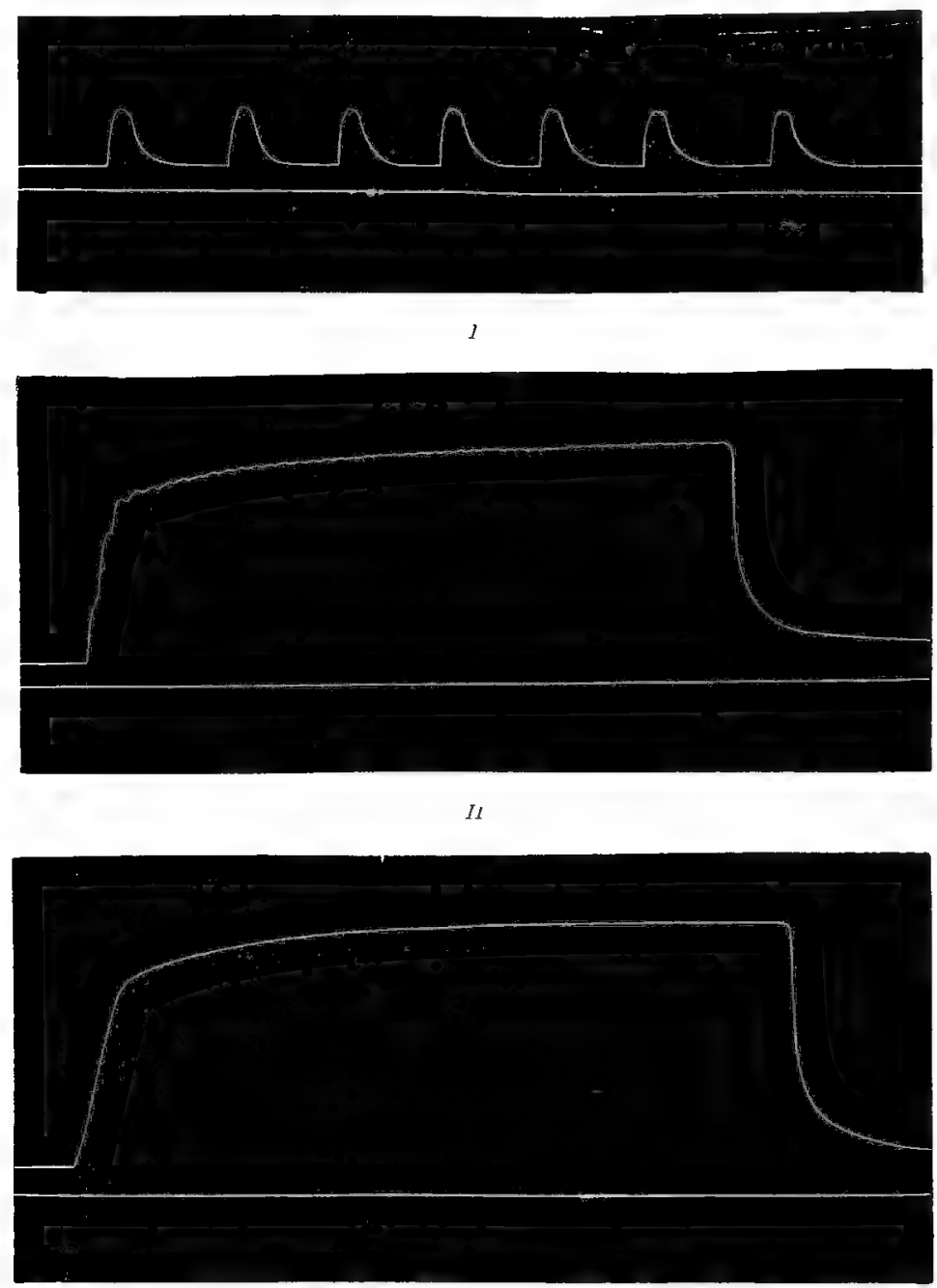

III

FIa. 207.- Myographic curves from the gastrocnemins of the frog. $J$, Single contractions produced by single opening induction-shocks. II, Incomplete tetanus, produced by opening in duction-shocks following in rapid succession. III, Complete tetmus, produced b5 inductionshocks in very rapid succession.

a IIimosa the branches and leaves of which are outspread, they have exactly the same effect as mechanical stimulation: the 
branches drop at once and the leaves flap together in the typical manner.

Other forms of energy are also made manifest by galvanic stimuli. Thus, exact thermo-electric measurements have shown that the temperature of muscle rises in activity, although very slightly, and that, in general, the production of heat stands in inverse proportion to the performance of work.

That electricity is also produced in the contraction of muscle caused by galvanic stimulation is already clear from our previous knowledge; an electric tension appears between every contracted point and every resting point, the former being negative to the latter. When, therefore, a contraction-wave runs over the muscle from one end to the other, an action-current can be led off from the two ends at the moment when the wave begins, since, while the one end is contracting, the other is at rest.

Light also can be produced by galvanic stimulation in pelagic phosphorescent animals, such as Radiolaria and Noctiluca.

It is evident from our previous considerations that all this evolution of energy in its various forms must be combined with an excitation of metabolism; it is chiefly the much-investigated muscle that has shown this. The muscle excited to constant activity by stimulation of any sort consumes more oxygen than the resting muscle, it consumes the glycogen stored in it, it produces more carbonic acid than the resting muscle, and, in place of the neutral or alkaline reaction of the latter, it shows an acid reaction. All these changes show very clearly that in muscle when put into activity by stimuli a considerable augmentation of metabolism takes place.

\section{b. The Phenomena of Depression}

In electrical stimulation also the depression-effects are wholly subordinate in comparison with the phenomena of excitation, and, while the latter have been investigated extensively and carefully, the former are little known. Nevertheless, there appear to be cases in which the galvanic current, especially through long influence or great intensity, is able to produce phenomena of depression. Whether the diminution of the irritability of the nerve that takes place at the anode upon the making of strong currents and at the kathode upon the breaking, and can lead to complete loss of the power of conduction at the place in question without any real destruction, is to be regarded as a phenomenon of depression is more than doubtful ; more extended experiments especially directed to this point are needed. But apparently genuine depressing effects of the galvanic current have been discovered with regard to ciliary motion by Engelmann ('79, 1) and Kraft ('90). 
The gill-filaments of bivalve mussels are covered by a ciliated epithelium, the cilia of which on account of their length are especially well-fitted for the observation of ciliary motion. When Engelmann stimulated these filaments by means of a single, strong induction-shock, the cilia went into rigor, exactly as the cilia of Injusoric and ciliated epithelia go into heat-rigor after strong thermal stimulation. They bent into the form of a hook in the direction of the stroke, their motion ceased and they remained in this position the longer, the stronger the induction-shock had been.

Kraft made an analogous observation during the long-continued action of the constant current upon ciliated epithelia of vertebrates. At the beginning of the action the ciliary stroke was accelerated, first at the two poles, but then, by a spreading of the excitation in the tissue, in the whole intrapolar portion; with long duration of the current the acceleration decreased gradually and gave place to a depression of the activity, amounting to complete standstill in the whole intrapolar portion. Hence it appears that the same relation is present here as in other, e.g., chemical, depressions, viz., the stimulus in question calls forth first a stage of excitation and then with stronger or longer action a depression. But all these relations have been too little investigated to permit a definitive interpretation.

\section{B. THE DIRECTIVE EFFECTS OF UNILATERAL STIMULATION}

Among the physical phenomena that are employed for the amusement of children in civilised countries, those of nagnetism usually have great attraction for the childish mind. The remarkable facts, that the magnetic needle, freely suspended, under all circumstances directs one end toward the north pole of the earth, that small boats and animals prorided with an iron pin and swimming in a basin of water follow the slightest motions of the magnetic needle with unfailing certainty as if conjured by a magician, that iron filings, strewn upon paper, arrange themselves in very characteristic curves over a magnet placed below them-all this has greatly fascinated us as children. Magnetic phenomena must have made the same deep impression upon the fervid fancy of the people of the Orient, who in many respects have retained childish qualities even to the present time. In the tales of the Arabian Nights there is a vivid expression of this, which still takes strong hold upon the childish heart, in the gloomy stories of the magnetic mountain and the fright of the helpless mariners who saw their ship, attracted by the invisible force, crash upon the smooth metallic rocks.

In the adult the sense of wonder and fascination connected with the magnet is largely lost because of our being accustomed to its 
peculiar effects. But the sensations of our childhood are again awakened when we meet with effects analogous to those that the magnet exerts upon the needle, namely, attraction and repulsion, transferred to living nature as results of a great variety of stimuli; and when we see that these stimuli are capable of exercising upon organisms an effect that forces them under certain circumstances to turn toward or away from the source of the stimulus with the same irresistible power and the same unfailing certainty as the magnetism forces the iron. The moth returns with deadly certainty to the light; although it has singed its wings innumerable times, it cannot resist the fascinating power and finally falls into the flame.

Since, in the higher animals, as a result of the co-operation of the nervous system, these phenomena possess a complexity that renders their examination more difficult and not rarely diminishes the certainty of the reaction, it is advantageous to consider them more especially from the standpoint of cell-physiology.

It is necessary to the occurrence of the phenomena in question that differences in stimulation exist in different parts of the body. If stimuli act equally upon all sides, all the effects of stimulation described in the preceding section occur, but a directive effect is necessarily absent. Only unsymmetrical stimulation can control the direction of motion.

\section{Chemotaxis. ${ }^{1}$}

The word chemotawis is applied to that property of organisms that are endowed with the capacity of active movement by which when under the influence of chemical stimuli acting unilaterally they move toward or away from the source of the stimulus. Where there is an approach to the source of the stimulus, there is positive chemotaxis, where there is a removal from the source negative chemotaxis. Unilateral stimulation with chernical stimuli is only realised when the concentration of the substance in question gradually increases from the living object in one direction.

Discovered first by Engelmann in Bacteria, observed by Stahl in Myxomycetes, studied systematically and more fully by Pfeffer, and recently investigated in leucocytes by Massart, Leber, Gabrits-

1 Although the words " chemotropism" " heliotropism," etc., have been long in use, I have decided, after considerable delay, to exchange then in this edition of the book for the words "chemotaxis," "phototaxis," etc. ; my reason is that the former not only sound heavy, but suggest objections from the philological standpoint. I come to this conclusion with some reluctance because for some time I have been endeavouring to extend the former terminology from the few, earlier known "tropisms" to the corresponding phenomena associated with other stimuli, and admit new tropisms, thus indicating at once by the term used the fact that all the phenomena belong in the same class. Now, however, when the analogy of the phenomena that result from the various kinds of stimuli is fully recognised, I believe it advantageous to replace the less fortunate terms by the newer expressions, already much employed. 
chevsky, Metschnikoff and others, chemotaxis has now become recognised as a phenomenon of wide distribution among free-living cells and of extraordinary significance, not only for unicellular organisms but also for life in the cell-community.

Among naked protoplasmic masses chemotactic phenomena were first observed by Stahl ('84) in Myxomycetes. He allowed the yellow reticulate plasmodium of Aithalium septicum, which lives upon tan, to creep upon moist strips of filter-paper, and then hung up the strips with one end in water that was deprived of oxygen and shut off from the oxygen of the air by a layer of indifferent oil, while the other end was in contact with the air. The result was that the protoplasm of the strands that dipped into the water gradually streamed completely out and accumulated above the layer of oil upon the moist filter-paper in the air. Hence it was positively chemotactic to the oxygen of the air. That it was not the water itself which the plasmodia sought to avoid, as might perhaps be supposed, follows from the fact that the plasmodia are positively chemotactic to water and always creep from dry to moist, thus manifesting a specific hydrotaxis. The strips of filter-paper in the experiment must always be kept moist, in order that the chemotaxis toward water may not interfere with that toward oxygen. The plasmodia behave chemotactically toward other substances also, especially the tan that serves them as food. Thus, in Stahl's experiments the protoplasmic masses always crept toward pieces of tan or toward little balls of paper that were soaked with an extract of tan, and collected there, a form of positive chemotaxis which Stahl termed trophotaxis because it plays an important rôle in the habit, which is wide-spread among unicellular organisms, of searching for food. Leber ('88), Massart and Bordet ('90), Metschnikoff ('92), Buchner ('90), and others have discovered chemotactic properties in the leucocytes of vertebrates, and a relation has been found here that is of the greatest importance with respect to the behaviour of organs toward infectious diseases. As has already been seen, ${ }^{1}$ Bacteria excrete certain metabolic products, such as the toxines, which recently have attracted greatly the attention of investigators. These products exercise upon leucocytes a very pronounced chemotactic effect, and cause them to creep in great swarms to any place in the organism where Bacteric have entered and multiplied. At the place of infection a dense accumulation of leucocytes takes place and in certain cases, as Matschnikoff has shown, they devour the bacteria and determine in part the further course of the infection (Cf. Fig. 210). If the bactcria are not present in too great numbers, they may succumb in the struggle with the leucocytes, which latter in a certain sense represent the police of the body in comparison with the weaker intruders, and the infection is stopped. If the bacteria prove the 1 c. p. 175. 
stronger, an extension of the infection throughout theorganism takes place, and the course of the disease is then determined by other factors.

In order to demonstrate the positively chemotactic action of bacterial products upon leucocytes, the following experiment of Massart can be performed. According to a method first devised by Pfeffer, a short capillary tube is filled with a culture of the pusforming Staphylococcus pyogenes albus, and one end is sealed, The tube is laid in the abdominal cavity or under the skin of a rabbit, and left for some 10 to 12 hours. After this time it is found by microscopic examination of the tube that through the open end a dense swarm of leucocytes has penetrated into the interior, and has closed the opening like a thick white stopper (Fig. 208). In other words, the leucocytes are induced by the bacterial substances to creep from the tissues of the animal into the capillary tube. A critic will at once raise the objection that perhaps it is the nutrient solution in which the bacteria are cultivated, which acts chemotactically upon the leucocytes. This objection can be nullified, if, as Massart has done, there be put into the animal for

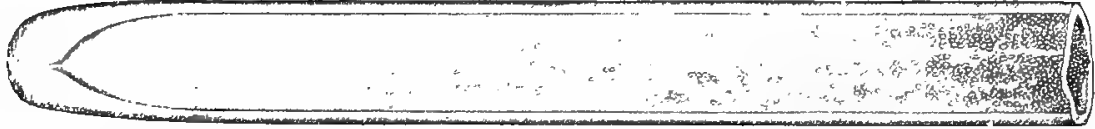

FIG. 208.-Chemotaxis of leveocytes toward pus-cocei. The leucocytes have wandered in dense crowds into a capillary tube, which contains a culture of Staphylococcus; they may be seen especially at the opening of the tube.

purposes of control a similar capillary tube containing similar nutrient liquid but without the bacterial culture. In such a case the leucocytes do not enter. That it is not simply the bodies of the bacteria themselves, but the metabolic products excreted by them, which have the chemotactic effect, may be proved by employing for the experiment a culture liquid that has been sterilised and wholly freed from the bacterial bodies, and in which, therefore, only the dissolved metabolic products of the bacteria in question exist. The result is then the same as when the culture is employed directly: after some time the tube is filled with leucocytes that have wandered in. What is true of the culture of Staphylococcus pyogenes albus has been found also in many other pathogenic Bacteria, and there is no doubt that further investigations upon the relations between leucocytes and bacteria will make clear a whole series of points which thus far in the history of infectious diseases have been very obscure.

Moreover, leucocytes appear chemotactic, not only toward the metabolic products of Bacteria, but also, as Buchner has found, toward the proteids of the bodies of the Bacteria themselves, and 
whole series of substances of non-bacterial origin. Thus, Buchner found that broth made of wheat flour and that made of pea flour possess especially strong chemotactic power. Finally, Sicherer ('96) has recently shown that under proper conditions the leucocytes of warm-blooded animals outside the body exhibit their chemotactic properties toward very varied substances for a long time, as clearly as in the living body itself.

The chemotaxis of leucocytes plays an important rôle in the development of many animals. This is made clear especially by the beautiful investigations of Kowalevsky ('87) upon insects. When the fly-larva changes into the complete fly-a metamorphosis that takes place fairly rapidly-the organs of the larval body, such as the creeping muscles, become superfluous, and

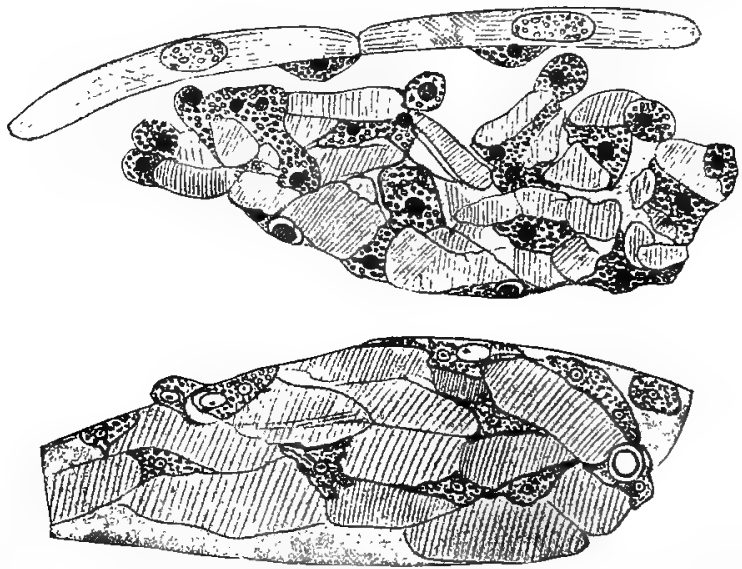

FIG. 209.-Leucocytes destroying the muscles in the metamorphosis of the larva of the fly. The granular masses are leucocytes, the striped masses bits of muscle. (After Kowalevsky.)

begin to degenerate. The substances formed at the beginning of this degeneration have a strong chemotactic action upon the leucocytes; the latter wander into the degenerating organs in great crowds, and as genuine phagocytes devour the disintegrating inasses, and thus accelerate their removal (Fig. 209). It is characteristic that the phagocytes manifest their activity only in insects in which the metamorphosis takes place very rapidly; but that in others, as in the moth, and in the degeneration of the tail of the tadpole, they have no share. Nevertheless, Metschnikoff was able to demonstrate analogous phenomena in the development of star-fishes.

Chernotaxis is wide-spread in the Hagellate Bacteria, Infusoria, and swarm-spores. In liacteria the phenomenon was first discovered by Engehmann ('81, 1 ; '94), and was at once emplored practically 
in an ingenious manner. Engelmann observed that certain forrns of Bacteria that live in decomposing infusions accumulate in great numbers in the neighbourhood of the sources of oxygen. Thus, in an exposed drop under the microscope a dense accumulation of these microbes takes place at the edges of the drop, where the oxygen of the air has the freest access. Under the cover-glass, likewise, the Bacteria congregate in the neighbourhood of the edge, and form a dense wall parallel to it. Bubbles of air, as well as plant-cells whose chlorophyll sets free oxygen in the light, act in the same way, especially when lack of oxygen is produced to a certain extent by covering the edges of the cover-glass by a layer of oil. Engelmann employed this extraordinary irritability of Bacteric toward oxygen as the basis of a method for the microscopic demonstration of very small quantities of oxygen, and this has become very important in our knowledge of the assimilatory action of various kinds of light upon the green plant-cell. ${ }^{1}$ By the external exclusion of air from a drop containing Bacteria, the place may be found where only the slightest traces of oxygen are present, and these are situated where there is a dense accumulation of the microbes. A beautiful example of such is afforded by the following observation. ${ }^{2}$ In a drop under the cover-glass a large diatom (Pinnularia) was in the field of sight, and, since in the light
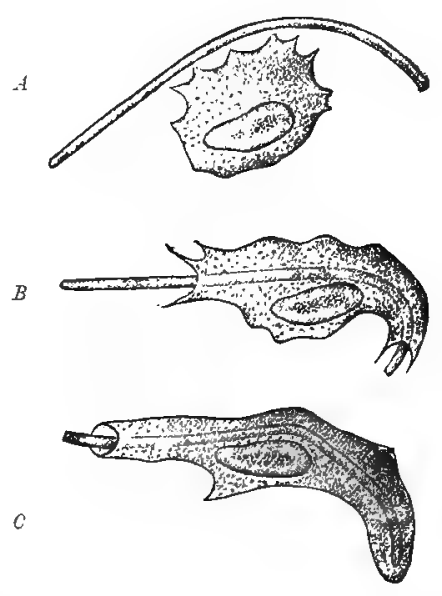

Fio. 210.-Leucocyte devouring a bacterium of splenic fever. (After Metschnikoff.) it gave off oxygen by reason of the activity of its chromophyll, it was closely surrounded by a wall of motionless Spirochatce. In the other parts of the field almost no Spirochoto were visible. The diatom suddenly moved a little distance, and then lay still. The Bacteria, thus separated from their source of oxygen, lay quiet for a few moments; but soon an active movement began among them, and they swam in dense crowds again to the diatom. After one or two minutes almost all were congregated again about it in a dense mass, and motionless as before (Fig. 211, I). Engelmann has recently figured similar observations (Fig. 211, II and $I I I$ ).

The striking and systematic investigations of Pfeffer ('84,'88) upon chemotaxis had their starting-point in observations on the spermatozoids of ferns, in which chemotactic relations to the egg-cell were found. It is now known that analogies to this exist in almost

$$
1 \text { Cf. p. } 217 .
$$$$
\text { 'Cf. Verworn ('89, 1). }
$$ 
all organisms, and function as the indispensable condition of the fertilisation of the egg-cell by the spermatozoon in animals as in
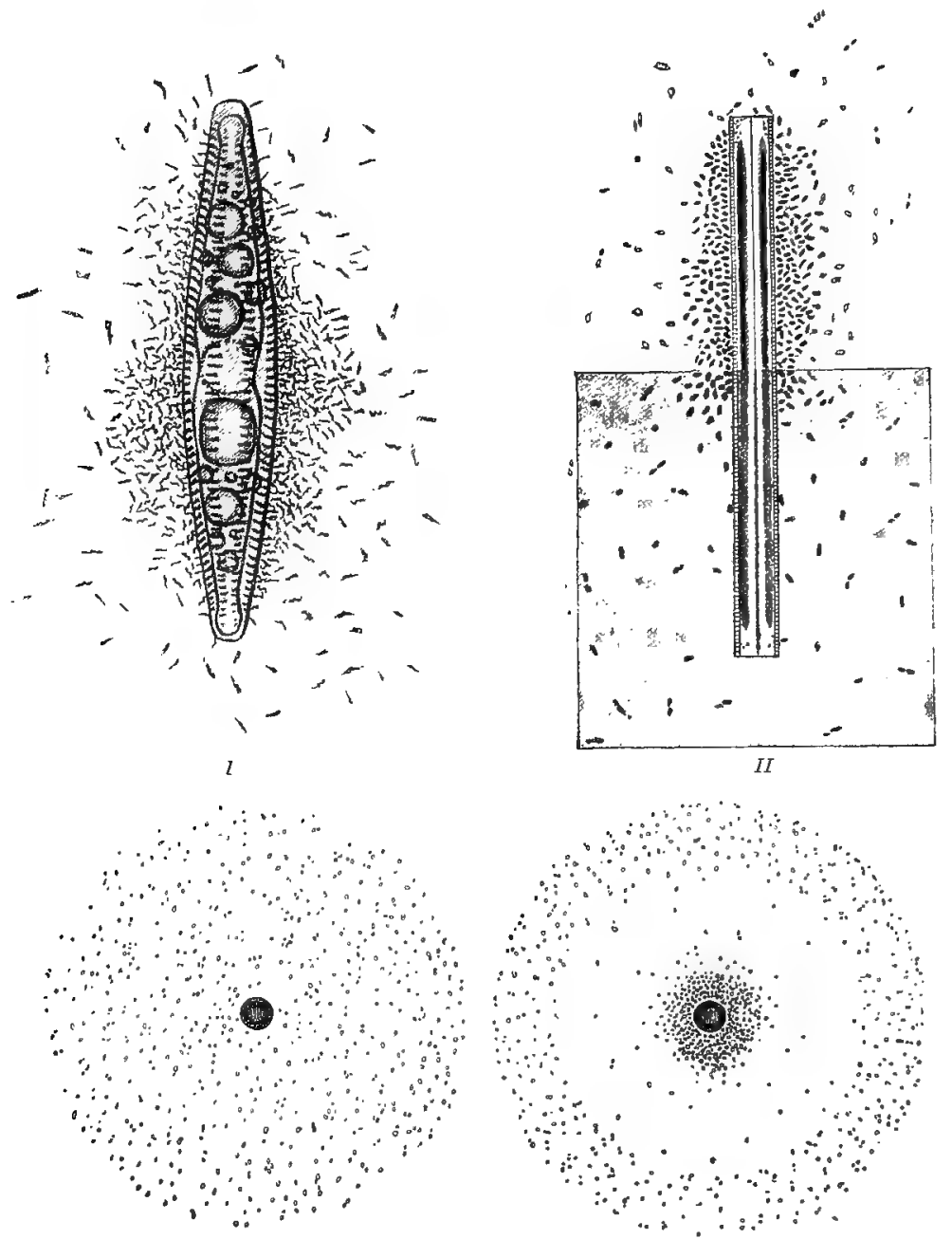

III

FIG. 211.-Chemotaxis of Bacteria toward oxygen which is produced by Alga-cells in the light. I, Diatom evolving oxygen in sunlight and surrounded by Spirilla. II, Diatom half-shaded, half-illuminated. The Bacteria have collected in the illuminated half, where oxygen is being evolved. III, An Alga-cell surrounded by Bacteria; $A$, in the dark, $B$, in the light. (II and $I I I$ after Engelmann.)

plants. The spermatozoon seeks the ovum, and almost everywhere in the living world is led in the right path by the chemotactic action which the metabolic products of the egg-cell exert upon the 
freely-moving sperm-cell (Fig. 212). The fact, which must otherwise appear very wonderful, that among the innumerable swarm of spermatozoa of the various marine animals every species finds the proper ovum, is in a great majority of cases a direct result of chemotaxis, and is explained very simply by the further fact that every species of spermatozoon is chemotactic to the specific substances that characterise the ovum of the corresponding species. We have here an adaptive phenomenon of the simplest kind, which gives us anew an idea of how extraordinarily deeply the phenomena of chemotaxis reach into life-relations.

Pfeffer's experiment was as follows: He filled a capillary tube, sealed at one end, with a solution of $c .0 .05$ per cent. malic acid and placed it in a drop that contained a great number of the spermatozoids of a fern; the malic acid gradually diffused from the opening of the tube into the drop, and thus became the source
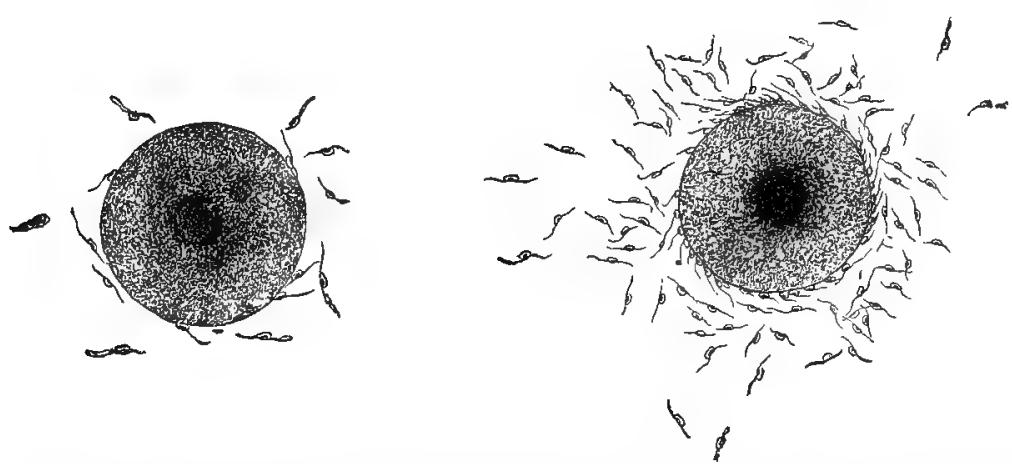

FIg. 212.-Two ova of the plant, with spermatozoids swarming about them. (After Strasburger.)

of a stimulus acting unilaterally. Microscopic examination showed that the spermatozoids immediately began to steer toward the opening of the tube and to swim into it. After a half-minute some 60 spermatozoids had entered the capillary, and in some cases after five minutes approximately 600 . In one experiment with 24 spermatozoids, after twelve minutes all except one, which lay at rest outside, had collected in the capillary. Hence malic acid exerts a very strong chemotactic action upon the spermatozoids of ferns; toward all the other substances that Pfeffer tested with respect to their chemotactic power, the spermatozoids behaved with complete indifference. This suggested strongly the supposition that in the archegonium that holds the ovum it is malic acid that causes the spermatozoids to approach and enter. On account of the minuteness of the objects and the lack of micro-chemical methods, Pfeffer was not able to demonstrate malic acid in the contents of the archegonia themselves, but 
he succeeded macro-chemically in establishing the presence of that acid in all parts of the plant that contain sexual products; hence the above supposition obtains a degree of probability that borders upon certainty. The spermatozoids of mosses behaved indifferently toward malic acid, but were remarkably chemotactic toward weak solutions of cane-sugar.

Later, Pfeffer extended his investigations to a large number of Bacteria and flagellate Infusoria, and obtained a series of results that are interesting in the highest degree. These show that very different substances act very differently upon different microorganisms. Substances to which one species reacts prove ineffective with another. Many substances induce only positive chemotaxis, others only negative. In the latter case the organism turns away from the source of the stimulus, and the capillary tube remains empty. The threshold of stimulation, i.e., that degree of concentration at which the substances just begin to exert their chemotactic effect, is very different for different substances and different organisms. But the most interesting fact is that many substances that induce positive chemotaxis in weak solution, induce

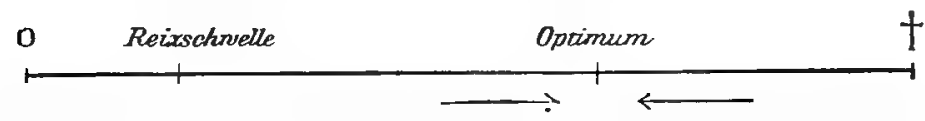

FIG. 213.-Scheme of chemotactic reaction. The concentration increases from the left toward the right; at 0 the zero-point of concentration, at $t$ the death-point. The arrows indicate the direction of movement.

negative chemotaxis in the same organisms in strong solution. There exists, therefore, a stimulus-optimum toward which the organisms strive from both sides, from the weaker as well as from the stronger solution. If the solution becomes too strong, death naturally results. Hence, four important grades of concentration may be established: the zero-point, where the substance in question is wholly wanting; the threshold of stimulation, where its concentration is such that the substance is just effective; the optimum-point, toward which the organisms strive from all degrees of concentration above the threshold; and the death-point, at which the concentration is too great to permit life (Fig. 213). With the same substance the optimum-point for different organisms usually exists at different grades of concentration. Massart ("91) found a beautiful example of this in the different behaviour toward oxygen of a bacterium, Spirillum, and a ciliate infusorian, Anophrys. If the two species of organisms were under the cover-glass in great numbers, both congregated like a wall at the edge of the glass or around air-bubbles, but not immediately at the boundary between the air and the water; each kept its own distance from the source of oxygen, the Anophrys ncarer, the Spirillum somewhat farther. 
The oxygen-optimum of each species was represented very distinctly by the distance of the individuals from the source of oxygen (Fig. 214, $I$ and $I I$ ).

Among ciliate Infusoric chemotactic phenomena have thus far become little known; nevertheless, for a few species Massart has been able to demonstrate chemotactic properties toward various substances. The negative chemotaxis of the infusorian already mentioned, Anophrys, toward common salt may be cited; this may be demonstrated in a very simple way. Massart laid at the edge
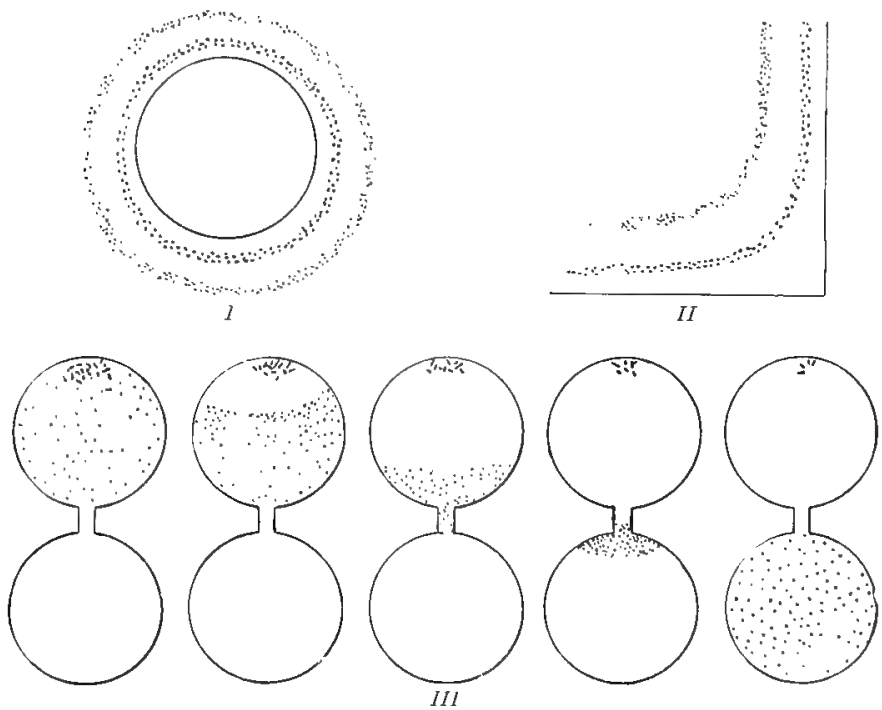

Wra. 214.-Chemotaxis of Bacteria (Spirillum) and Infusoria (Anophrys). I, Bubble of air under the cover-glass, surrounded by two zones, the nearer of which consists of Anopkrys, the farther of Spirillum. II, Edge of the cover.glass. Anorphrys and sprillum form similar zones. III, Two drops of water united by a bridge of water. In the upper drop is common salt. The Infusoria (Anophr/s) in this drop wander over into the drop of pure water, the more the salt dissolves. (After Massart.)

of a drop in which numerous specimens of Anophrys existed a few small crystals of common salt, and connected the opposite side of the drop by a narrow bridge of water with a similar drop of distilled water (Fig. 214, III). The result was that the Infiusoria fled from the spot where the salt lay the more, the more the salt dissolved and diffused into the neighbourhood, until finally they had all passed over the narrow connection into the other drop.

Jennings ${ }^{1}$ has recently made very comprehensive and systematic investigations of the chemotixis of I'crumerium, and has employed a method that possesses in many respects great advantages.

${ }^{1}$ H. s. Jennings: "Studies of reactions to stinuli in unicellular organisms. I. ete.,"Joum. of Physiol., 1897, xxi., p. 25s. 
Jennings performs his experiments upon the slide under a large cover-glass which is supported by two glass rods, so that a pretty thick layer of water containing Paramoecia lies between the slide and the cover. In this layer, which must be free from all admixtures, he places carefully by means of a pipette drawn out into a capillary, a drop of the solution the chemotactic action of which is to be investigated (Fig. 215). The substances in this solution diffuse at once into the surrounding liquid, in which the Paramacia are scattered uniformly and in motion. Then, according to the mode of action of the substances in question, very characteristic effects are produced. If the substances are ineffective, as, e.g., solutions of sugar, the Paramocia swim undisturbed into the drop, and after a few seconds are again spread uniformly under the cover-glass. If the drop induces negatively chemotactic properties, as, e.g., alkalies, a circle that is completely free from Paramoecia, forms at the place (Fig. 215, $A$ ). But if the drop induces positive chemotaxis, as e.g., most acids, all Paramacia that are present under the cover-glass swim into it (Fig. 215, B). If the concentration of the effective substance is above the optimum, the Infusoria accumulate about the drop in a circular zone (Fig. 215, $C$ ). It is remarkable that Paramocica are positively chemotactic toward carbonic acid, as toward other acids. If a bubble of chemically pure carbonic acid and at the same time, for control, one of ordinary air be placed under the cover-glass, the Paramcecia, leaving the air, congregate in a dense mass about the carbonic acid (Fig. 215, D). But, in proportion as the latter diffuses into the water and accumulates in a concentration above the optimum, they retreat in a closed circle from the bubble, because they are negatively chemotactic toward strong solutions of carbonic acid. Thus very characteristic figures appear (Fig. 215, E). Further, since Paramacia, like all other organisms, produce carbonic acid, constantly more individuals. become attracted to the place where for any reason a number have assembled. We have here a very interesting case of the formation of an assemblage simply by reason of positive chemotaxis. As Fig. 215, $B$ shows, by the transference of a drop of water from a group to another cover-glass preparation containing Paramocict a chemotactic assemblage may be produced.

Finally, chemotactic phenomena afford a means of forming an approximate conception of how extremely slight may be the stimuli that are able to exert a visible effect upon living substance. In his experiments Pfeffer found that the spermatozoids of ferns. exhibited distinct chemotaxis when he employed a capillary tube containing a solution of 0.001 per cent. malic acid. If it be borne in mind that the malic acid must first diffuse into the drop in order to exercise its power, it follows that the quantity that acts upon the spermatozoids must be exceedingly small. ut this is not all. In orcler to produce a chemotactic effect it is 
not sufficient that a certain quantity of the substance in question be uniformly distributed in the vicinity of the organism, but a

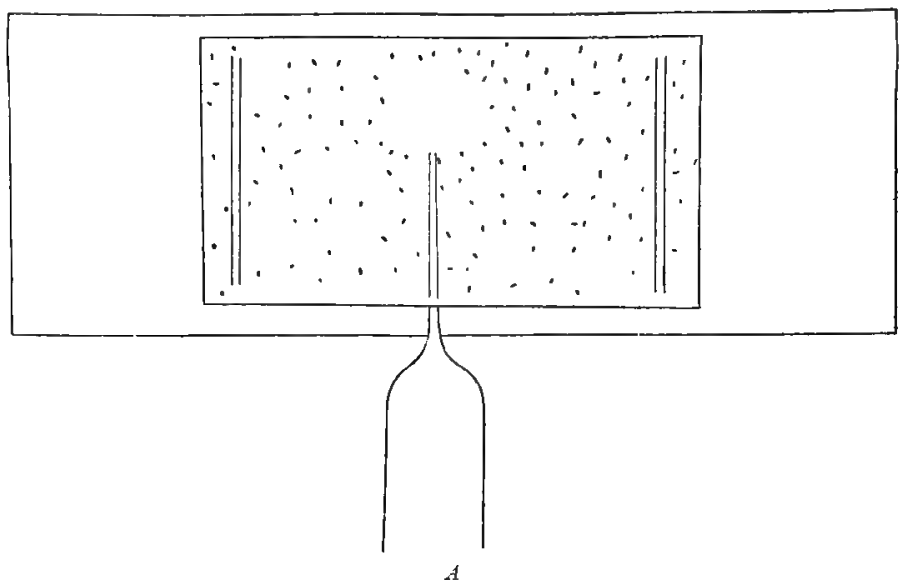

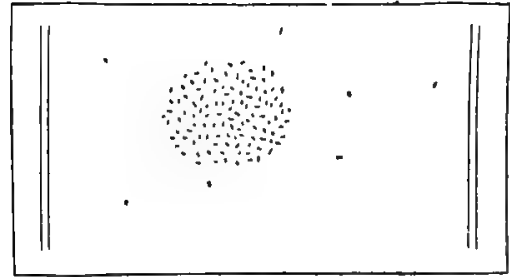

$B$

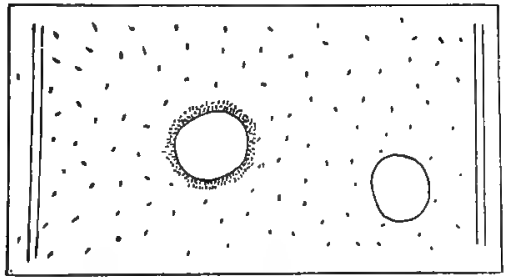

$D$

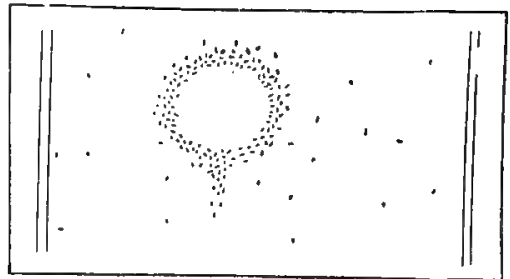

$c$

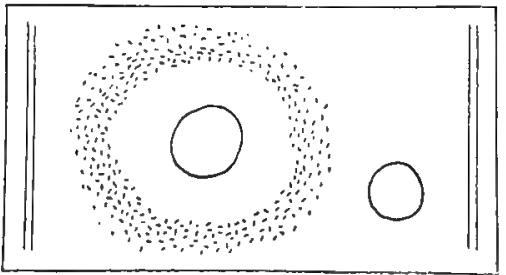

$E$

Fro. 215.-Chematoxis of Paramcecium aurelia. A, Chemotactic cover-glass preparation; a drop of liquid that induces negatively chemotactic properties has been introduced under the coverglass by means of a capillary pipette. $B$, Positively chemotactic assemblage. $C$, The same with too strong a solution; the Paramecia have congregated in a circle at the optimum-point of concentration. $D, A$ bubble of carbonic acid and one of air are under the cover-glass; the former (at the left) induces positively chemotactic properties; the latter is indifferent. $B$, The same preparation a few minutes later; the carbonic acid has diffused into the surrounding water and by its too high concentration has driven the Parancecia to the place where they find their optimum of carbonic acid. (After Jennings.)

decrease in concentration must take place on one side. In other words, it is the amount of the difference in concentration at the 
two ends of the spermatozoid that determines the appearance of the chemotactic effect. Since the spermatozoid possesses only the minute length of $0.015 \mathrm{~mm}$., we can form an approximate idea of how extraordinarily small must be the difference in concentration at its two poles, and therewith the amount of the stimulus that calls out a chemotactic effect. Thus, chemotactic phenomena and, as we shall see, analogous phenomena caused by other stimuli, give us a better idea than all other reactions of how excessively teeble stimuli produce a remarkable effect upon living substance. Living substance responds to extremely delicate influences. When homcopathy affirms the effectiveness of extremely small quantities of certain medicines, its claim in this respect is fairly justified, however much superstition in other respects may attach to the homœopathic doctrine.

\section{Barotaxis}

All mechanical stimulation of living substance consists in a change of the pressure-relations under which it exis.ts. Every degree of pressure can act as a stimulus, from crushing or cutting, which destroys the continuity of the substance, down to the slightest touch and the most delicate change in the pressure of the air or the water that surrounds the organism. Under the unilateral action of pressure-stimuli-in other words, in all cases where differences of pressure exist upon two different parts of the body of an organismphenomena appear that correspond to those of chemotaxis. Since these possess in common the one characteristic of being called forth by pressure ( $\beta a$ pos) acting unequally on different sides, they may be designated by the term barotaxis. Various kinds of barotaxis can be distinguished according to the kind of pressure; and it can be positive or negative, according as the organism turns toward the side of the higher or the lower pressure.

Under thigmotaxis, all those cases of barotaxis can be grouped in which the phenomena are caused by the more or less strong contact of living substance with more solid bodies. Naked protoplasmic masses, such as rhizopods and leucocytes, exhibit the simplest form of this. These afford, indeed, striking examples of how feeble contact calls out positive thigmotaxis, strong contact negative, and how, analogously to the case of chemotaxis, differences in the intensity of the stimulus are of essential importance. If, e.g., a marine rhizopod, such as the often-mentioned Orbitolites (Fig. 98, p. 238), be left quiet in a glass vessel containing sea-water, after some time pseudopodia begin to be put out from the small openings in the calcareous shell. Consisting at first of very short fibrils, they lloat freely in the water. Soon, becoming longer and heavier, their ends sink to the bottom and become fixed there by means of a delicate secretion, and the protoplasm begins 
actively to stream along the bottom without rising again freely into the water. In other words, upon slight contact with the bottom the living substance of the rhizopods behaves positively thigmotactically and turns toward the object. Except in the free-swimming Radiolaria, Heliozoa, etc., extension and wide expansion of the pseudopodia take place always in contact with some body, whether it be the bottom, the cover-glass, the surface of the water, or objects in the water. On the other hand, by strong mechanical stimulation of the tip of an extended pseudopodium of Orbitolites, best by press-

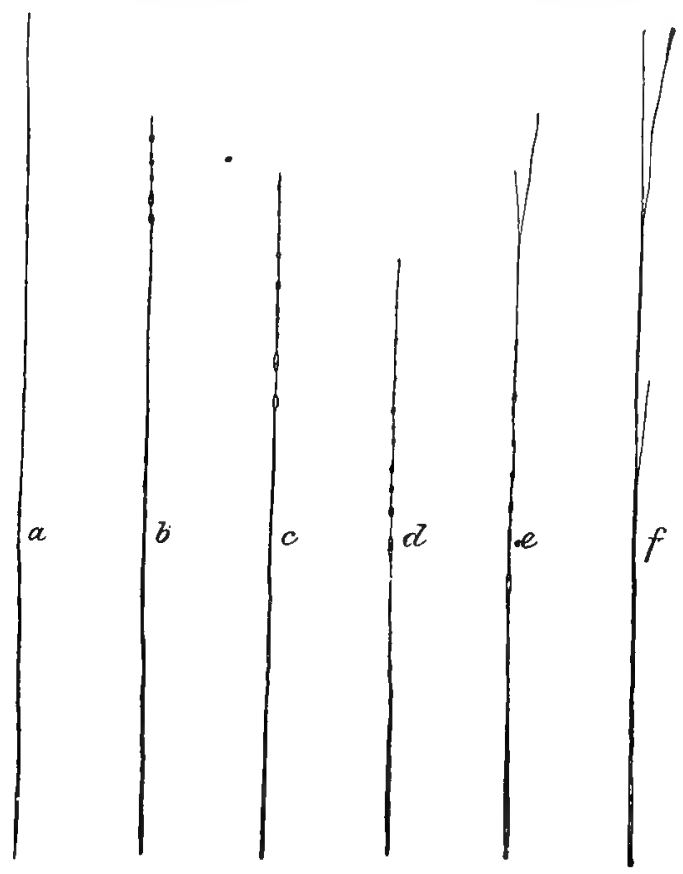

FIa. 216.-Psendopodium of Orbitolites, in a cut across at *; $b, c, d, e, f$, succossivo stages of the reaction.

ing upon it with a needle or cutting it across with a scalpel, it is possible to call out negative thigmotaxis of its living substance, the protoplasm at the place of stimulation drawing together into small globules and spindles, and streaming away (Fig. 216). ${ }^{1}$ The same phenomenon may be observed still more distinctly in a rapidly reacting fresh-water rhizopod, the shell-bearing Cyphoderic ; here the protoplasm of the pseudopodia withdraws from the place of stimulation with great rapidity (Fig. 217).

Thigmotactic phenomena are wide-spread. Among plants they ${ }^{1}$ Cf. Verworn $(' 92,1)$. 
are best known in the creeping plants and climbers, whose tendrils. and twining shoots turn toward the objects which they touch and grow in constant contact with them (Fig. 218). Here the structural relations are so complex that the behaviour of the living substance within the single cellulose-capsule cannot be directly

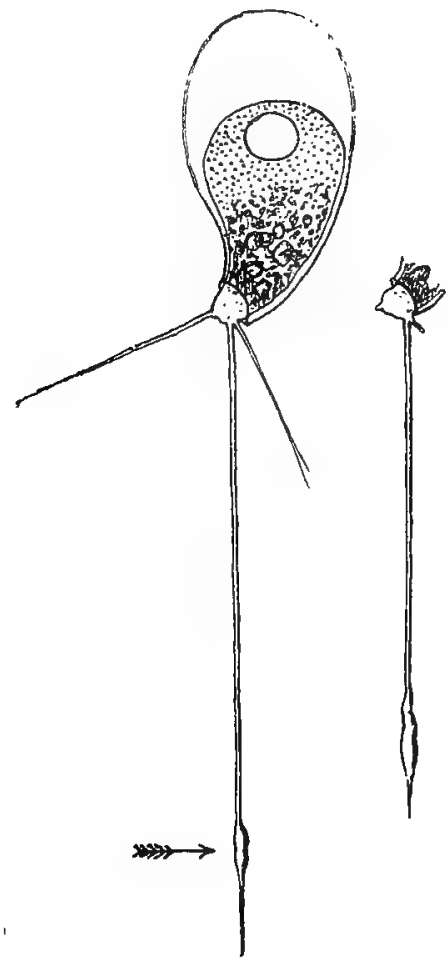

FIG. 217.-Cyphoderia, with extended pseudopodia, stimulated at $\longrightarrow$. The protoplasm is flowing away from the place of stimulation. observed; hence, thus far it is not known with certainty in what way the individual cell shares: in the thigmotactic twining.

Dewitz ('86) found positive thigmotaxis in the spermatozoa of the cockroach (Periplanetce orientalis). If the spermatozoa be brought into a 0.6 per cent. solution of common salt between the slide and the coverglass, after a short time all the individuals collect partly upon the lower surface of the cover-glass, and partly on the upper surface of the slide, and in these places describe circles with their flagella, the direction of which, without exception, is opposite to that of the hands of a watch. The greater portion of the liquid remains completely free from spermatozoa, the latter not leaving the surface of the glass after having once reached it. If a ball be placed in the drop, its surface is at once sought by them. If a solution of common salt, containing spermatozoa, be placed in the cavity of a ball, after a short time the whole inner surface of the latter is covered, and the liquid in the middle is completely deserted. The pronounced thigmotaxis of these spermatozoa, like the positive chemotaxis of many others, is of the greatest importance for the fertilisation of the ova.

A contrast to this behaviour of the spermatozoa of Periplaneta is afforded by the following observation upon a genus of ciliate Infusoriu, Oxytricha. 'Their flat, yielding bodies are beset upon 
their under side with cilia, which the animals, like woodlice, use as legs, with which to creep about upon objects in the water. These Infusoric are always seen creeping about busily and restlessly upon the slide, the cover-glass, or particles of mud lying in the water, without ever of themselves losing contact with the objects. The following episode from the life of an Oxytricha illustrates this positive thigmotaxis particularly well. In a flat dish containing river-water and an Oxytricha, there lay some spherical eggs of the river-mussel Anodonta. When the contents were poured into the dish, the Oxytricha in some manner came into contact with one of the eggs. It ran about unremittingly for hours upon the spherical surface without being able to leave it, since the egg rested with one point only upon the level bottom (Fig. 219, $C^{\prime}$ ). The organism must have travelled an enormous distance. After four hours it was able to forsake its enforced retreat by means of a particle of mud which came to the isolated egg. Experiments which artificially imitated with other Oxytrichae essentially the same conditions give wholly analogous results.

Jennings ${ }^{1}$ has recently discovered in Paramcecium another typical case of positive thigmotaxis. If a piece of filterpaper, or any other substance provided with a rough surface, be placed under a cover-glass under which are numerous Paramcecic distributed uniformly through the water, after some time the piece is beset with a thick coating of the Infusoria, which touch it with their cilia without moving from their place. By employing high powers it is shown that those cilia that are in direct contact with the foreign body stand straight out and perfectly still (Fig. 220,A), and that the activity of

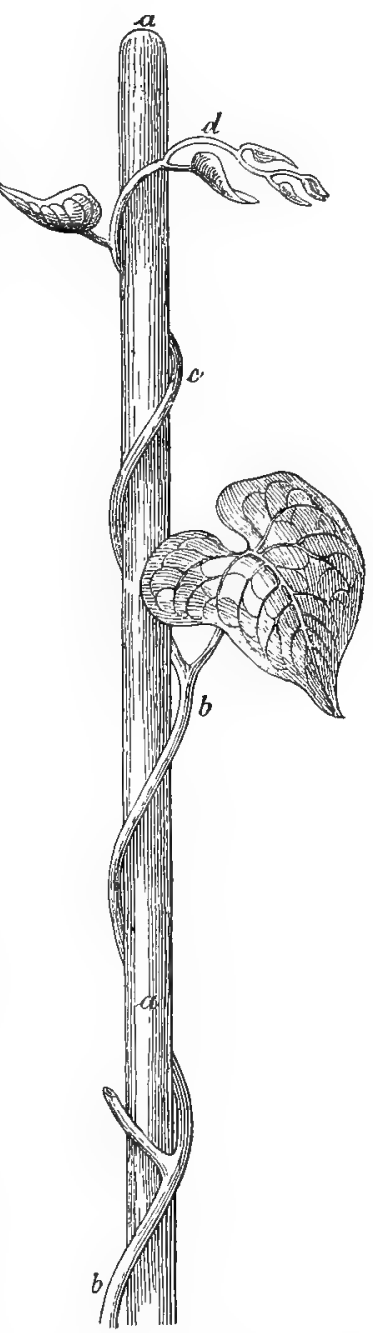

FIG, 218.-Pusitive thigmotaxis of $a$ plant. $a, a$, Rod; $b, b, c, d$, twining shoot. (After Sachs.) the cilia over all the rest of the body is greatly depressed and eventually wholly stopped. There is here a very pronounced thigmotaxis. In connection with this it is noteworthy that the thigmotactic assemblage of Paramacia con- 
stantly attracts new individuals chemotactically by its production of carbonic acid; thus all the individuals in the drop accumulate finally about the foreign body (usually in the course of 5-10 minutes), although, since it is surrounded by an impenetrable wall of individuals held thigmotactically, most of them cannot come into direct contact with it (Fig. 220, B). Thigmotaxis, which causes the individuals that swim by chance to the foreign body to remain, is merely the first cause of the assemblage; chemotaxis toward the carbonic acid produced by them completes it.

A second form of barotaxis, in which the stimulus is produced, not as in thigmotaxis by contact with a solid body, but by a gentle current of slowly flowing water, is rheotaxis, which was discovered by Schleicher and carefully investigated by Stahl ('84). This is
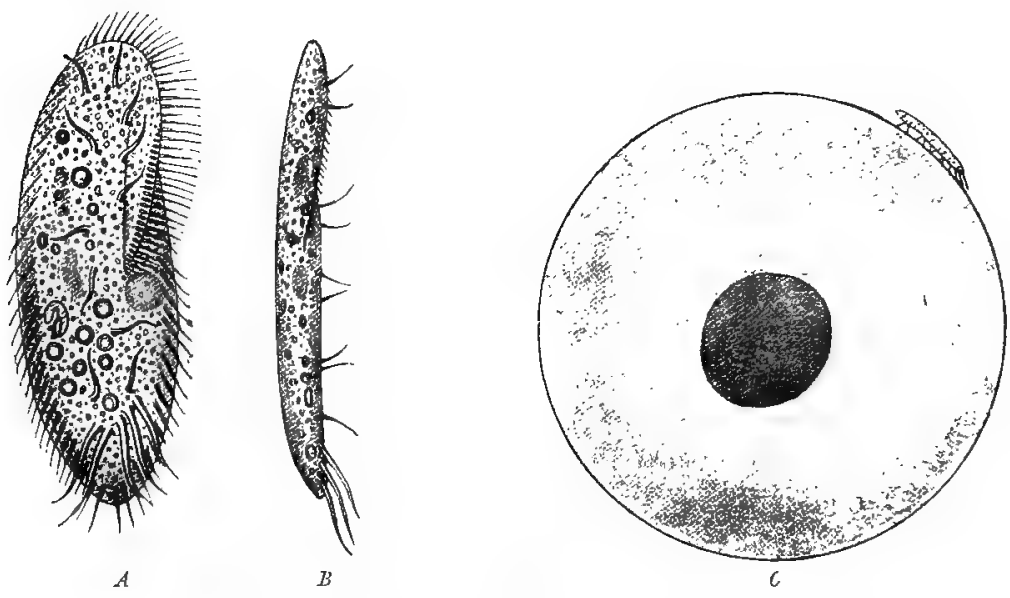

F19. 219.-Oxytricha, a ciliate infusorian. A, Seen from below; $B$, seen from the side ; $C$, creeping about over the egg of a mussel.

the peculiarity belonging to certain organisms, of taking toward flowing water a direction of motion opposed to the direction of the current. Since these organisms thus turn toward a pressurestimulus, rheotaxis is merely a special form of positive barotaxis. Thus far rheotaxis is known in a few organisms only. Stahl demonstrated it best in the plasmodia of Myxomycetes in Aethalium. septicum, by the following experiment. He suspended a narrow strip of filter-paper in a beaker filled with water, and somewhat elevated, in such a manner that one end of the strip dipped into the water, while the other end hung far down over the edge of the beaker. In such a strip there is a continuous slow current of water directed toward the end that hangs down, as is proved by placing upon it a coloured mark. Stahl laid this end upon a mass of tan, in which the plasmodia of Acthalium live. The result was 
that the plasmodia slowly crept from the tan upward in the strip over the edge of the beaker and downward upon the inner side of the glass, until they spread themselves out upon the surface of the water. By proper control-experiments it was possible to determine with certainty that it was only the streaming water which afforded the stimulus.

Unfortunately the rheotactic properties of other organisms have been little investigated. It is, however, very probable that rheotaxis is wide-spread. Among other cases, it is easy to assume that the human spermatozoa are rheotactic and find their way to the egg-cell by means of this property. When the spermatozoa come into the uterus, they meet a current of mucous liquid coming toward them, since the cilia of the epithelium lining the uterine
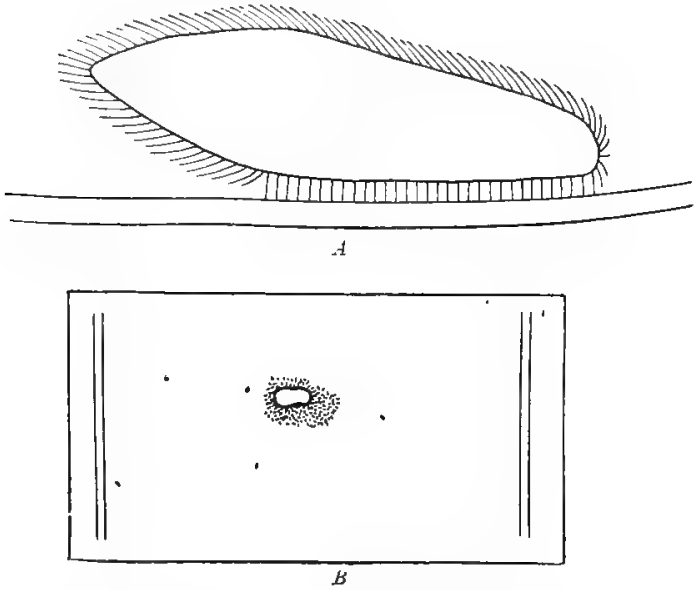

FIG. 220,-Thigmotaxis of Paranaciun. $A$, An individual in contact with a fibre of filter-paper; the cilia that touch the fibre directly are still. $B$, Assemblage of Paramacia about a bit of filter-paper under the cover-glass. (After Jennings.)

cavity have a direction of stroke toward the os, and hence produce a current toward the outside. That it is chemotaxis of the spermatozoa toward the ovum which points out the path to them becomes very improbable when it is remembered that the spermatozoa wander upward in the uterus before the ovum has left the ovarian follicle. As a matter of fact Roth ('93) has succeeded in showing experimentally that spermatozoa and likewise certain Bacteria are rheotactic, by producing under the cover-glass a feeble continuous current and observing that these unicellular organisms move in opposition to it.

As a third form of barotaxis we have to consider finally geotaxis, i.e., the phenomenon that certain organisms place themselves and move with their median axis in a very definite direction toward 
the centre of the earth. In this case the stimulus is afforded by the minimal differences of pressure that exist at points of different height, both in the water and in the air.

These phenomena have been known longest in botany, for all plants are geotactic in a pronounced manner. The roots grow toward the centre of the earth and, therefore, are positively geotactic; the branches and the stems grow away from the centre of the earth and, therefore, are negatively geotactic. Further, in the behaviour of the leaves and in many cases the branches, which grow essentially tangential to the earth's surface, a third sort, transverse geotaxis, is seen.

In free-living cells geotactic properties have been recognised, especially by Schwarz ('84, 1), Aderhold ('88), Massart ('91), and Jensen $(93,1)$, who have found that of Infusorix and Bacteria in closed vessels containing water, some rise

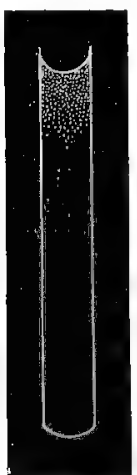

Iisc. 221. - Glass tube containing Paramacia, which as a result of negative geotaxis have collected at the upper end. (After Jonsen.) ences in pressure at different heights. As is well known, the hydrostatic pressure in a column of water is considerably less at the top than at the bottom. The higher pressure operates as a stimulus and causes the organisms to leave the place where it is present and seek the places of lowest pressure. As all consideration at once shows, no other differences exist between the upper and the lower portions of the column of liquid in the vertical glass tube. An unprejudiced observer must, therefore, recognise in geotactic phenomena a pressureeffect. But that they are this actually, Jensen was able to show by experiment upon the disc of "a centrifuge. In tubes placed horizontal and hence in the line of the radius of the disc, in which under ordinary circumstances no geotactic ac- 
cumulation of Paramcecic can take place, he increased by rotation the pressure at the peripheral end in comparison with the central end, and thus artificially imitated the conditions which, according to the laws of the earth's gravity, prevail in a vertical tube. The result was that with not too rapid rotation of the disc the Paramcecia collected at the places of lower pressure, i.e., at the central end of the tube, a phenomenon which Jensen puts beside geotaxis as centrotaxis. With a proper rate of rotation they frequently accumulated with greater certainty than in the upright tube. If they were centrifugalised too rapidly, naturally they were thrown out passively toward the periphery like heavy bodies.

Accordingly, geotaxis, which has occupied a peculiar position so long in botany, must be regarded as a special case of barotaxis.

\section{Phototaxis}

A ray of light extends through space from a source of light in a straight direction, and diminishes in intensity with the distance. Hence, any two points in the line of the ray possess different intensities; the point that is nearer the source has the greater, that which is farther away has the less intensity. A ray of light, therefore, fulfils very completely the conditions that are necessary to the appearance of unilateral stimulation-in fact, it is extremely difficult to establish conditions under which an organism is stimulated by light uniformly upon all sides. As a result of this, stimulation by light calls out very pronounced directive effects, which have been termed phenomena of phototaxis ${ }^{1}$ and form a complete analogy to those of chemotaxis and barotaxis.

The phenomena of phototaxis have been known longest in plants; as a matter of fact, plant physiology, on account of the less complexity of its objects of study, was able to develop in general into systematic completeness much earlier than animal physiology. Every one who cultivates plants in a room has the fact of positive phototaxis daily before his eyes. He sees that the growing parts turn constantly toward the light; and, in order to make a plant grow straight upward, he must turn the pot about from time to time so that any phototactic curving may be compensated. Many plants are so extremely phototactic that in bright sunshine in a garden they follow the course of the sun in their curving. For example, in a bed of blue gentians, all the plants turn the broad

1 Formerly a distinction was made between heliotropism and phototaxis, the former word signifying the attitude, bending, and turning of fixed organisms, the latter the movement of motile organisms, with reference to the source of the light. This distinction is not only superfluous, but it introduces the false idea that the phenomena in the two cases are depenrlent upon different causes. A double terminology for processes that are based upon the same principle should be avoided. The processes are now understood better than at first, and the old distinction, which arose from purely external points of view, should be discarded as unscientific. Many authors have already done this. 
open surface of their gorgeous blossoms to the sun, and in this position follow its slow movement throughout the day; at evening their blossoms have a direction almost the opposite of that in the morning. In many plants, as Stahl ('85) has shown in the horsetails, the direction of growth is influenced by light in a very interesting way even in the spore; in the division of the spore-cell the first division-wall, which divides the cell into two parts, is formed at right angles to the direction of the incident rays of light. A characteristic difference in the kind of phototaxis of the two halves is noticeable, such that the rhizoid-cell, from which the roots develop later, is always turned away from the source of light, and the prothallium-cell, from which the parts above the earth are derived, toward the source (Fig. 222).

Among animals the investigations of Loeb ('90) and Driesch ('90) in recent times have likewise demonstrated wide-spread
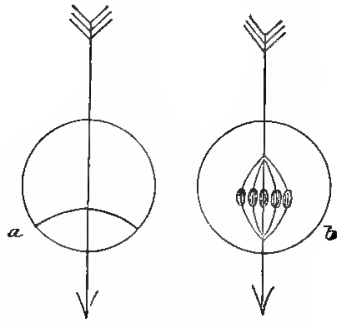

IG. 222.-Division of the sporecell of a horse-tail under the influence of light. The arrow indicates the direction of the rays. $a$, Position of the division-wall, $b$, direction of the maitotic figure. (After Stahl.) phototactic phenomena. But, although it is not altogether easy to obtain a correct view of these phenomena in the cell-community of the plant, it is much more difficult in the complex community of the animal body, on account of the varied share taken by the sense-organs, the nervous system, the motile organs, etc. Hence it is advantageous here to turn our attention primarily to the simplest relations, such as exist in the free-living cell.

The phototactic phenomena of unicellular organisms, observed by Priestley and Ehrenberg, were followed out more. fully by Nägeli, Hofmeister, Baranetzky, Stahl, Klebs, Cohn, and other botanists, but the fundamental labours of Strasburger first gave an exact picture of the laws of the phenomena.

Strasburger ('78) made his investigations chiefly upon swarmspores of various chlorophyllaceous $A l g c e$, and observed their behaviour toward light falling from a window upon one side of a suspended drop. Essentially the same phenomena were shown by flagellated swarm-spores of very different species. The behaviour of the swarm-spores of Ulothrix may serve as a type. In diffused daylight of slight intensity these small flagellated cells hasten in straight paths to the edge of the drop that is turned toward the light, and collect there in great crowds. If the intensity of the light be increased, which Strasburger accomplished by bringing the preparation nearer the window or employing direct sunlight, with a certain intensity the swarm-spores begin to leave the positive side of the drop, i.e., the side that is turned 
toward the source of light, and betake themselves to the opposite or negative side; by further increase of the intensity all collect at the latter side. There exists, therefore, a point in the intensity, toward which the swarm-spores rush, going toward it from both higher and lower intensities-a phenomenon that Strasburger termed photometry. There is here a complete analogy to chemotaxis; the latter is positive up to a certain concentration of the effective substance, but from there on with increasing concentration is negative, so that the term chemometry is justified. Quite analogous to the behaviour of the swarm-spores of Ulothrix is that of the swarm-spores of Chcetomorpha, Ulva, Hcematococcus, and some other Algoe, as well as the flagellate infusorian Chilomonas Paramacium, and the colourless swarm-spores of the Chytridice, all of which are positively phototactic with feeble intensity of light, and negatively phototactic with stronger intensity. There are forms-e.g., the swarmspores of Botrydium granulatum-which show positive phototaxis in all intensities.

Next to these researches of Strasburger comes a whole series of observations by other investigators, who have been able to find phototactic phenomena in all sorts of micro-organisms. Thus, Stahl ('84) investigated the phototaxis of plasmodia of Myxomycetes, previously observed by Hofmeister and Baranetzky, and found that young plasmodia of Aethalium septicum are

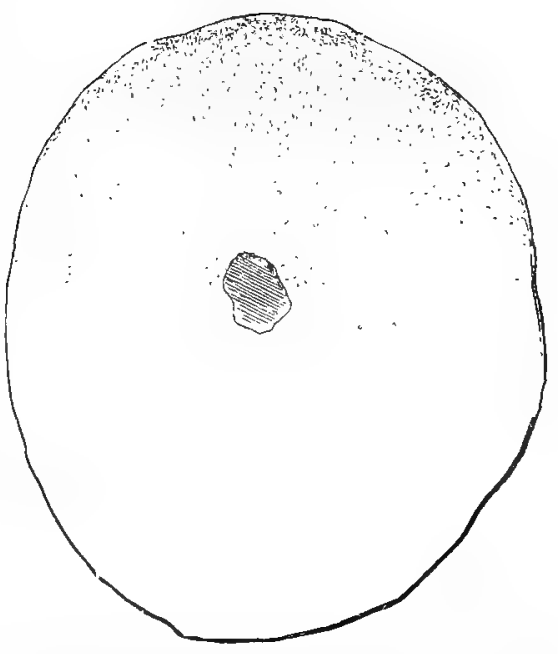

FIa. 223.-Photolasis of Diatomer. A particle of mud which was thickly surrounded by Diatomea lies in the middle of the drop. The organisms have all crept toward the edge turned toward the sun.

positively phototactic in half-darkness, and creep upon the surface of $\tan$, but with stronger illumination they become negatively phototactic, and flow back again into the interior of the mass. Further, Engelmann ('81, 3; '83) found in Bacterium chlorinum and Bacterium photometricum two forms that possess phototactic properties and collect together in the light. Engelmann ('s2), Stahl ('80), Aderhold ('88), and others ${ }^{1}$ discovered phototactic phenomena also in the Diatomece and the Oscillarie, which behave exactly as the swarmspores of Alyce and form very pronounced issemblages (Fig. 223). Finally, Stahl (l.c.), Klebs ('85), and Aderhold (l.c.) demonstrated ${ }^{1} \mathrm{Cf}$. Verworn ('89, 1). 
phototactic movements in the Desmidiaceo, and showed that these alga-cells place themselves with their long axis parallel to the light-rays, and in this position, by the extrusion of their secretion, move along the bottom in their peculiar manner toward the source of light, or with greater intensity away from the source (Fig. 224). In a preparation containing living Closteria ${ }^{1}$ or Pleurotoenioe all individuals place themselves with their long axis parallel to one another and to the direction of the incident light-rays. Thus, we find that among unicellular organisms, so far as they are irritable at all to light, phototaxis is a wide-spread phenomenon.

After phototactic phenomena had been discovered, the question necessarily arose as to whether the different rays of the spectrum are phototactically effective in an equal degree; this was decided very easily by the introduction of coloured glasses and solutions between the source of light and the object. The media employed were so chosen that they let through only rays of a certain portion of the spectrum, so that only rays of certain wave-lengths were

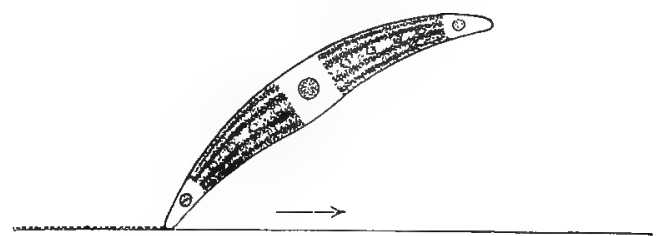

Fra. 224.-Phototaxis of Closterizm. The light comes from the right side. The arrow indicates the direction of movement of the Closterium.

allowed to fall upon the organism (Fig. 225). In this way Cohn, and later Strasburger, established the fact that in general the rays possessing a short wave-length, in other words, the blue and the violet especially, are more effective than those having a greater wave-length, viz., the red; with not too high degrees of intensity the latter act like complete darkness.

One point more deserves mention in the discussion of phototactic phenomena. From the preceding consideration and by analogy with the directive effects of other stimuli it is evident that only the difference in the intensity of the light upon different parts of the body can produce a directive effect; where the stimulus acts upon the surface of the body from all sides with equal intensity, the reason for a definite axial position disappears, as is to be observed most clearly in the action of chemical stimuli upon all sides. Although this is obvious, some investigators, such as Sachs and Loeb, have believed that the direction of the rays is more responsible for the manifestation of phototactic phenomena than are differences in intensity. It is difficult to 
conceive this, for, since the assumption of an axial direction is possible only when differences exist at two different points of the surface of the body, it is wholly mystical how the direction of the rays, which is the same upon all sides of the body, can produce such an effect. In nature, under ordinary conditions, the decrease in intensity coincides with the direction of the rays, and hence we always see the phototactic movement take place in this direction. But the decrease in intensity can very easily be experimentally separated from the direction of transmission of the rays. Oltmanns ('92), making use of an idea already employed by Strasburger, devised a very excellent contrivance for this purpose. $\mathrm{He}$ made a wedge of two glass plates, which were inclined toward one

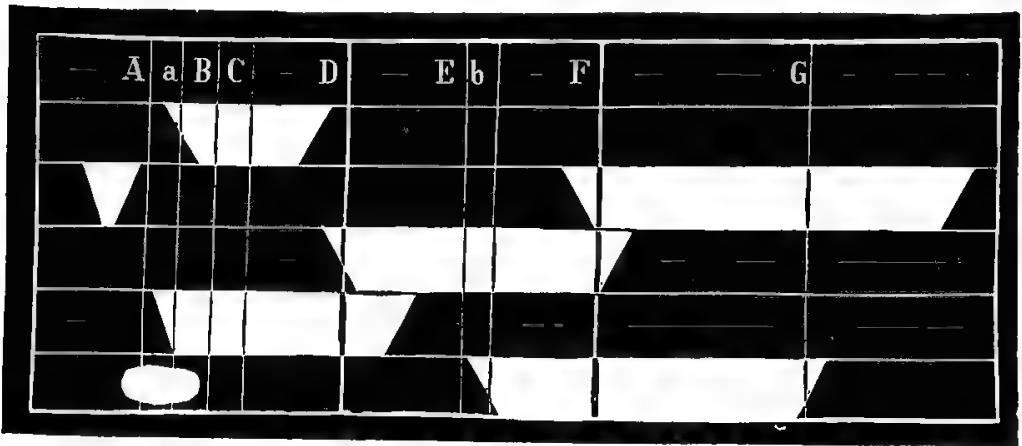

FIG. 225.-Spectra of varinus media; 1, of a red glass ; 2, of a cobalt glass; 3 , of a groon glass ; 4 , of a solution of potassium bichromate; 5 , of an ammoniacal solution of a cupric salt.

another at an angle of $2^{\circ}$, and filled the space between the plates with gelatine clouded with India ink. This wedge let through nearly all the light at its thin end, while at its thick end, where the gelatine was darkest, it absorbed much light. If, therefore, the light fell perpendicularly upon the surface of the plates, the greatest decrease of intensity for objects within a dark box behind the wodge lay at right angles to the direction of the incident rays. By means of these plates and the employment of the proper intensities of light it may actually be proved experimentally that it is not direction of ray, but solely difference in intensity upon different portions of the surface of the body, that produces phototactic phenomena.

\section{Thermotaxis}

Huat, like light, can be employed very easily for unilateral stimulation, since, whether transmitted by conduction or radiation, it alwily'i decreases with the distance from its source, and hence 
differences of temperature always exist at two different points in the medium in the same direction from the source.

The first observation of thermutactic properties was made by Stahl ('84) in plasmodia of Aethalium septicum. He placed two beakers side by side, one of which was filled with water at a temperature of $7^{\circ}$, the other with water at $30^{\circ}$; he then laid over their edges a strip of filter-paper, upon which the plasmodium had spread itself out, in such a manner that one end of the plasmodium dipped into the colder, the other into the warmer water. The protoplasm of the plasmodial network at once began to stream out of the former toward the latter, although before the experiment the opposite direction had been followed. The whole protoplasmic

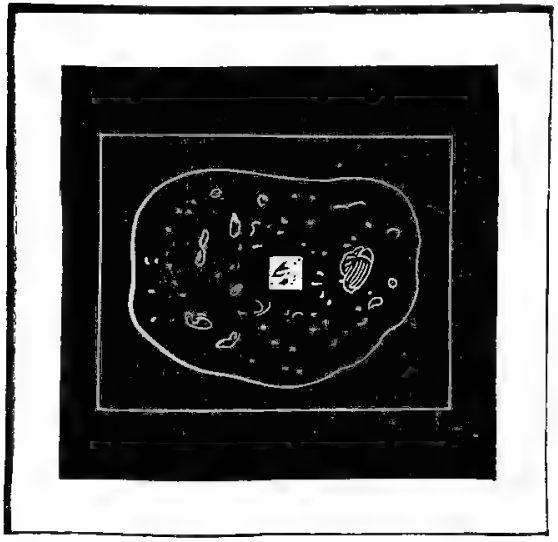

$I$

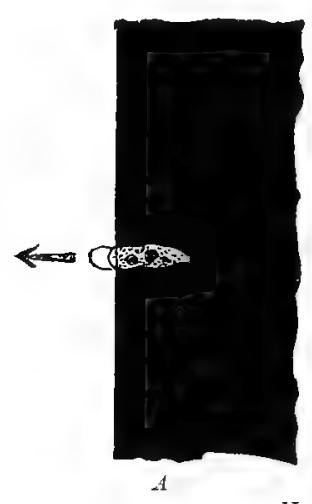

II

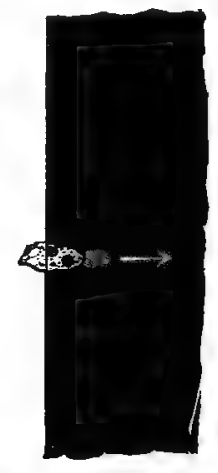

B

FIG. 226. - Negative thermotaxis of Anceba. I, A drop of water containing many amoevae lies upon a large cover-glass. The cover-glass lies upon a black ground, which has in the middle a sharp, square opening. By shoving the cover-glass an amoeba can be so placed that it creeps over the edge of the hole, $I I, A$. If concentrated sunlight is then let through the opening from the nirror of the microscope. the amceba creeps back immediately in to the cool darkncss, $I I, B$. The arrows indicate the direction of the movement.

mass finally passed over to the warm water. This is a case of positive thermotaxis.

Negative thermotaxis can be observed in $A$ moba, ${ }^{1}$ when a temperature of at least $35^{\circ} \mathrm{C}$. is allowed to act upon one part of the body while the rest of the protoplasm is at a lower temperature. This can hardly be accomplished by means of conducted heat. Radiating heat and the following arrangement should be employed. A large drop of water, containing many individuals of Amabe limax, is placed upon a large thin cover-glass and the latter is laid upon a glass plate cemented to black paper and placed upon the stage of the microscope. In the middle of the paper is a suall hole with very sharp edges. The concave mirrur of the

$$
{ }^{1}(y) \text { Verworn }(s 9,1) \text {. }
$$


microscope is so placed that it receives bright sunlight and reflects it through the diaphragm. After the introduction of an opaque plate between the stage and the mirror, an Amoba is so placed by the aid of direct light that, its direction of motion remaining constant, it must creep beyond the edge of the black paper. As soon as the anterior end of the Amoba has passed over the edge of the opening, the opaque plate between the mirror and the stage is suddenly removed, so that the concentrated rays of the sun fall upon that end, while the posterior end is still in the shade of the paper. The result is that the Amoba immediately changes its direction and flows back into the shade (Fig. 226). That this is a pure heat-effect of the sun's rays and not a light-effect can be decided at once by excluding either the chemically acting lightrays by the introduction of an absorbing solution of iodine in carbon bi-sulphide, or the heat-rays by the introduction of plates of ice or alum. In the former case the thermotactic effect is as

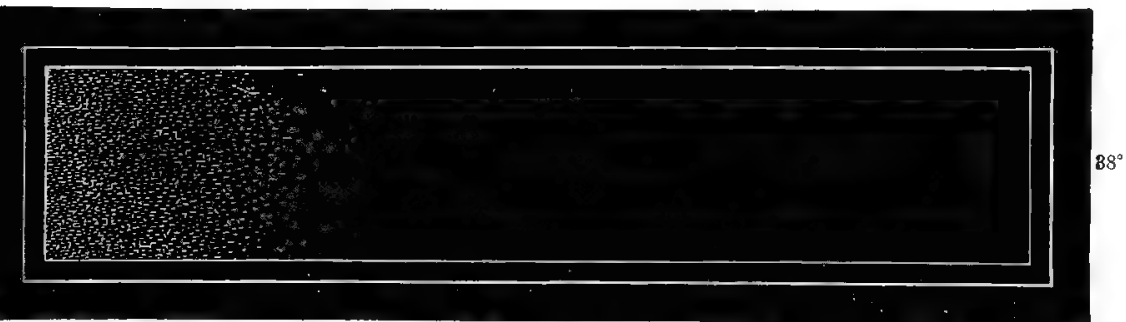

F1G. 227.-Thermotaxis of Paramaecium. In a black ebonite trough, $10 \mathrm{~cm}$. in length, ar? numerous Paramacia; upon unilateral warming of the trough to $24^{\circ}-28^{\circ}$ C. they move toward the cooler side. (After Mendelssohn.)

distinct as in pure sunlight, in the latter it is wanting in spite of the great illumination. Careful tests show that Amcebce is not at all irritable to light. But thermometric measurement of the temperature in the drop directly over the opening in the black paper shows that at least a temperature of $35^{\circ} \mathrm{C}$. must be reached, if the effect is to appear.

The thermotactic action of different degrees of temperature may be studied best in cilate Infusoria, like Parnmacium, which can be bred in great numbers. If a small ebonite trough be placed upon a metallic plate, and liquid containing Paramacia be placed in it, by warming or cooling differences in the temperature which can be measured by a thermometer can be obtained at the two ends of the liquid. These differences have a pronounced thermotactic effect (Fig. 227). The accompanying apparatus, constructed by Mendelssohn ('95), allows heating or cooling with hot or cold water (Fig. 228). With this it is shown that Paramocia at temperatures of more than $24^{\circ} \mathrm{C}$. to $28^{\circ} \mathrm{C}$. are negatively thermo- 
tactic, i.e., swim in crowds away from the warmer side, while with temperatures below this limit they show positive thermotaxis, since they leave the cooler side. There is here a phenomenon completely analogous to chemotaxis and phototaxis, in which the organisms likewise turn from both sides toward a certain degree of intensity of the stimulus. A simple calculation shows how small the difference in temperature can be at the two poles of the body of the Paramacium, and still produce a thermotactic effect. The length of the surface of the liquid, the smallest just effective

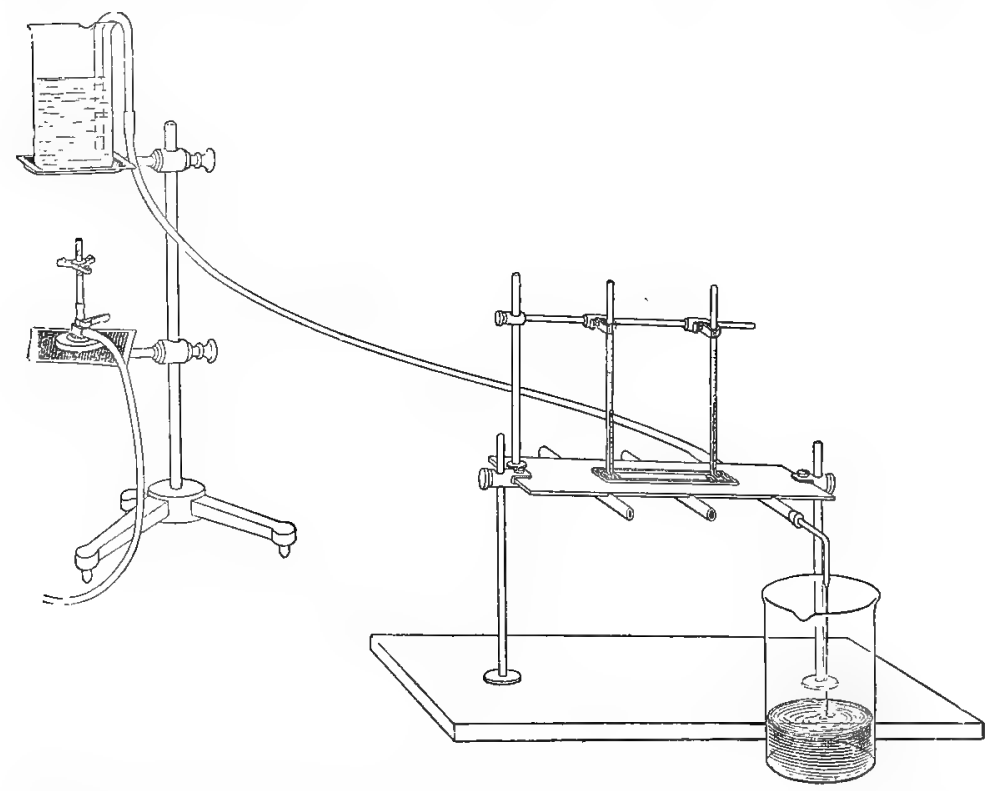

FIG. 228.-Apparatus for the investigation of thermotaxis. A flat trough of black ebunite (Fig. 227), in which is liquid containing Paramecia, rests in a depression upon a metallic plate. The plate has three tubes, through which from a beaker, by means of a tube, water of a desired temperature call be passed. Above the trough thermometers are attacled to a stand, which dip into the liquid containing the Paranceia and at any moment allow the temperature prevailing in different places to be read off. (After Mendelssohn.)

differences in temperature at its two ends, and the length of the body of the I'uramocium must be known. In such a calculation, which, of course, can give only approximate values, Jensen found that Parumcecia are still thermotactic when at the two ends of their body, $0.2 \mathrm{~mm}$. in length, a difference of temperature of $0.01^{\circ} \mathrm{C}$. prevails. There is here expressed a delicacy of distinction in the intensity of stimuli, which finds analogies both in the data obtained by Pfeffer for chemotaxis and in the slight differences of stimulus effective in phototaxis, but which leave the differential capacity of the human consciousness far behind. 


\section{Galvanotaxis}

It is characteristic of the galvanic current that it always calls out phenomena of polar excitation. As a result of this, stimulation by the constant current is especially well fitted to exercise directive effects. Since, further, the current can be graduated in intensity very delicately, and its direction can be readily controlled, it affords a very perfect means of producing experimentally directive reactions in their most exact form, and with the certainty of physical phenomena. The galvanotactic phenomena of motile organisms remind one of the effects of the magnet upon iron particles.

The galvanotactic phenomena of animals were first discovered by Hermann ('85), in the larvæ of frogs and the embryos of fishes. $\mathrm{He}$ ubserved that when a galvanic current was conducted through a vessel containing these animals, upon the making of the current all placed themselves with their long axis parallel with the curved lines of flow of the current, so that their heads were directed toward the anode and their tails toward the kathode. In this position they remained. Analogous effects have been observed more recently and upon various other higher animals by Nagel ('92,'93, '95), Blasius and Schweizer ('93), and latest by Loeb ('96, 2, 3, 4; '97, 1, 2).

Galvanotactic phenomena have also been found in plants, especially the root-tips of many plants; when the constant current is sent through them for a considerable time, the tips bend toward the kathode.

But most striking, and theoretically most interesting, are the phenomena in free-living unicellular organisms, such as Rhizopoda, leucocytes, Infusoria, etc. ${ }^{1}$

In order to investigate the galvanotaxis of these organisms, we can best employ the above-

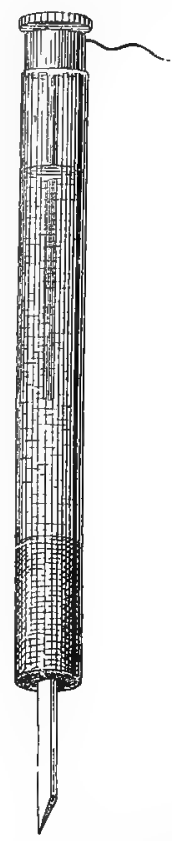

FIa. 229. - Non-polarisable electrode, which, instead of the camel'shair brush has a tip made of fired clay. described slide with non-polarisable clay-electrodes, or non-polarisable electrodes that are arranged like camel's-hair brush-electrodes but, instead of the brush, have tips made of fired clay which can be dipped into the liquid through which the current is to be sent (Fig. 229).

If a few drops of water containing many Paramoecia be placed on the slide between the parallel pieces of clay that serve as ${ }^{1} C f$. Verworn ('89, 2,3 ;; '92, 2 ;'96, 4) and Ludloff ('95). 
electrodes (Fig. 230), and a constant current be passed through the liquid from two brush-electrodes laid upon the clay pieces, at the moment of making all the Paramocia place themselves with the anterior poles of their bodies toward the kathode, and swim freely toward the latter in a dense crowd. In a few seconds the anode is wholly deserted, and at the kathode there is a dense swarm,

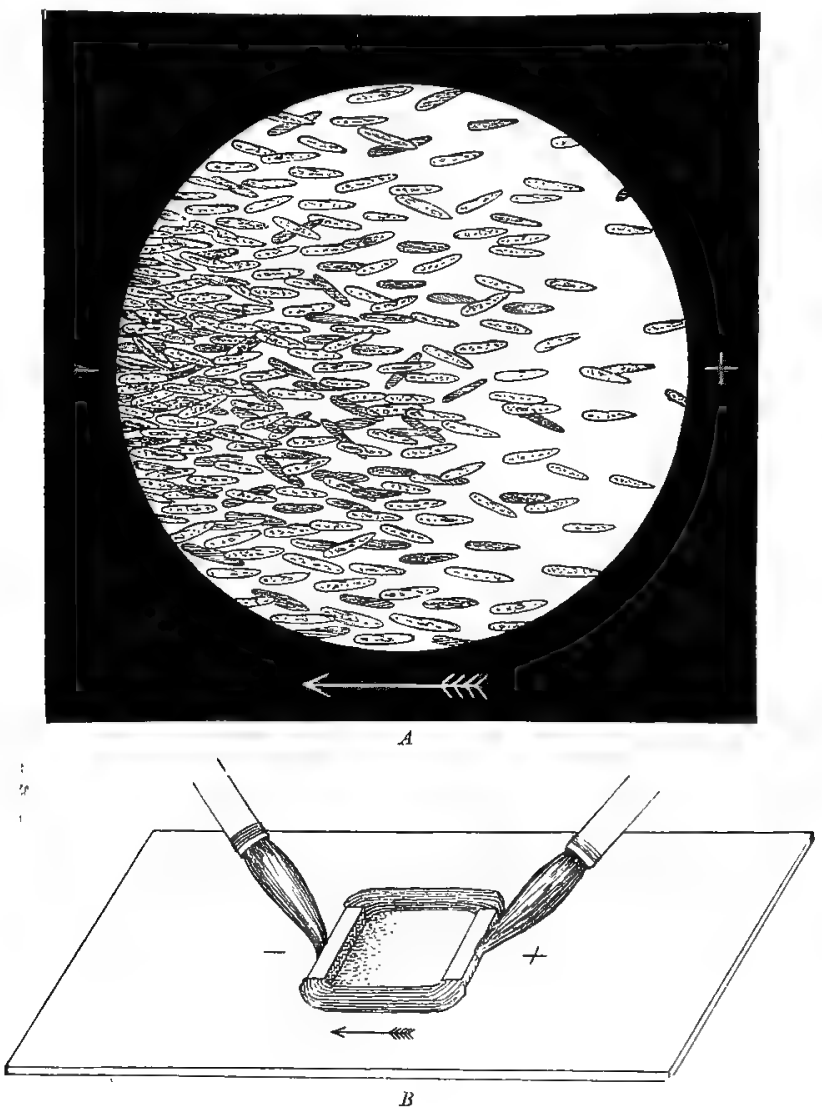

FIG. 230.-Galvanotaxis of Parancecium. The arrow indicates the direction in which the Paramacia are swimming; in $B$ all have collected at the kathode. $A$ Microscopic, $B$, macroscopic picture.

which remains as long as the circuit is closed If now the current be reversed, so that what was before the anode becomes the kathode, and vice versa, the whole swarm rushes over in one mass to the opposite side, and collects, as before, at the kathode. This experiment, which because of the great exactness of the reaction is very fascinating to the observer, can be repeated as often as desired 
If the current be broken, the assemblage disappears from the kathode, and the Paramocia scatter themselves again uniformly throughout the liquid. If the Paramoecia be put into a large drop upon a glass plate, and the pointed electrodes be dipped into the drop, upon making the current the infusorians arrange themselves in the direction of the curved lines of flow of the current like iron filings above a magnet, and swim in this direction (Fig. 231) until they have reached the kathode, behind which they collect in a dense swarm. If the kathodic electrode be made movable, so that its position in the drop can be changed at will, it is possible to direct the Paramoecia with the point of the electrode wherever one wishes, just as tin-fishes may be directed in water

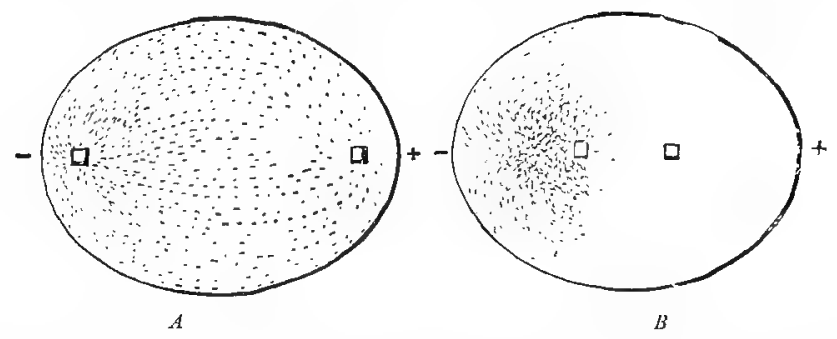

FIc. 231. -Galvanotactic curves of swinming Paramacia, pointed clectrodes being used in the drop of water. $A$, Beginning of the effect; $B$, completed assemblage.

with a magnet. Since the motion of the Paramcecia is directed toward the kathode, this case may be termed kathodic galvanotaxis.

Like Paramacium, the majority of the ciliate Infusoria are kathodically galvanotactic. Among other Protista that show the same phenomenon, Amoba alone may be mentioned. Amoba limax, when the current is made, abandons its original direction; its pseudopodia flow forward toward the kathode, the whole protoplasmic mass streams after, and the body assumes the typical extended creeping form, in which it flows unerringly to the kathode. Other forms of Amoba, such as Amoba proteus (Fig. 232), Amoba verrucosa, and Amaba diffuens (Fig. 233), behave in all respects similarly.

Many flagellate Infusoria show a behaviour opposite to that of the above-mentioned organisms. If, e.g., a constant current be passed through a drop in which is a large number of individuals of the small egg-shaped species, Polytoma vvella, which move through the water, revolving continually about their axis, by means of their two flagella (Fig. 234), upon making the current all individuals immediately turn their anterior flagellated ends toward the anode, and freely swim in their usual manner straight to this pole, where 
they collect in dense crowds. After the breaking of the current they scatter again uniformly throughout the drop. Polytoma, therefore, behaves toward the two electrodes exactly the reverse of Paramacium; in contrast to the latter it is anodically galvanotactic.
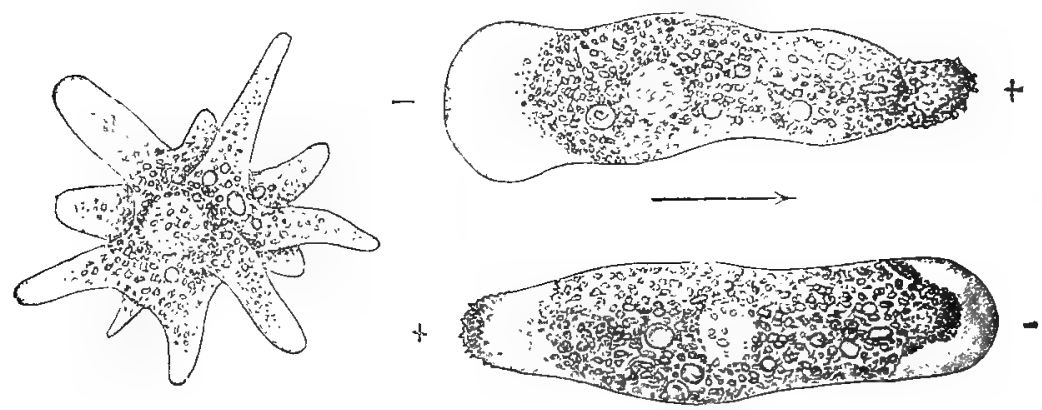

FIG. 232.-Galvanotaxis of Amola proteus. At the left unstimulated and possessing numerous pseudopodia. At the right, above, after making the current; below, after reversal of the current. The arrows indicate the direction in which the animal is creeping.

A very fascinating spectacle results from exposing to the influence of the current, at the same time, anodically galvanotactic Infusoria, e.g., a flagellate form, such as Polytoma, and kathodically galvanotactic forms, e.g., a small ciliate genus, such as Halteria or Pleuronema. The previously inextricable intermingling of the
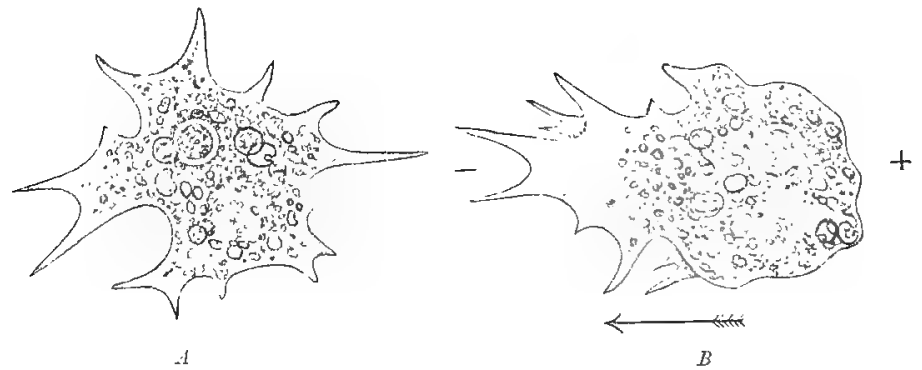

F1G. 233.-Galvanotaxis of Amabc dipluchs. A, Unstimulated, creeping; $B$, after making the constant eurjent. The arrow indicates the direction of the motion.

two forms ceases at once after the making of the current. The Clicata collect at the kathode, the Flayellate at the anode. After a short time the liquid is entirely deserted in the middle, and the two assemblages are sharply separated from one another. If now 
the current be reversed, so that the previous anode becomes the kathode, and vice versa, the two crowds of Infusoria rush toward one another like two hostile armies, cross and again assemble at the opposite poles. There are few physiological experiments that
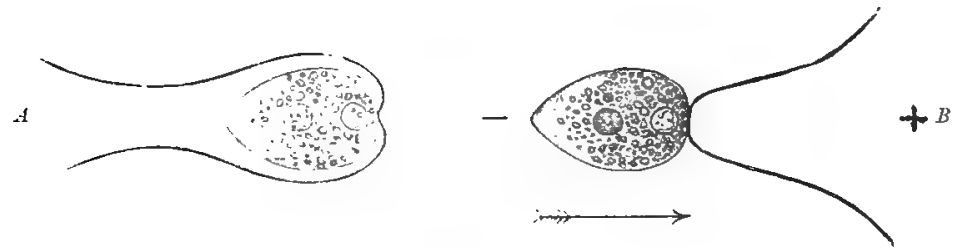

FIG. 234.-Galvanotaxis of Polntoma uvella. A, Resting quietly; $B$, swimming toward the anode after the making of the constant current.

are so pleasing and graceful as the galvanotactic dance of the Infusoria.

A third form of galvanotaxis is shown by the ciliate infusorian Spirostomum ambiguum. ${ }^{1}$ If these clongated Infusoria, which can be perceived even with the naked eye as small white fibres $c .2 \mathrm{~mm}$. in length, be placed in water between parallel clay-electrodes, it is seen that upon the making of the constant current they draw together suddenly by the sudden contraction of their myoid-fibres, but do not, as might perhaps be expected, swim toward one or the other pole. Instead of this, by means of their ciliary motion accompanied by much bending of the body, they gradually turn so

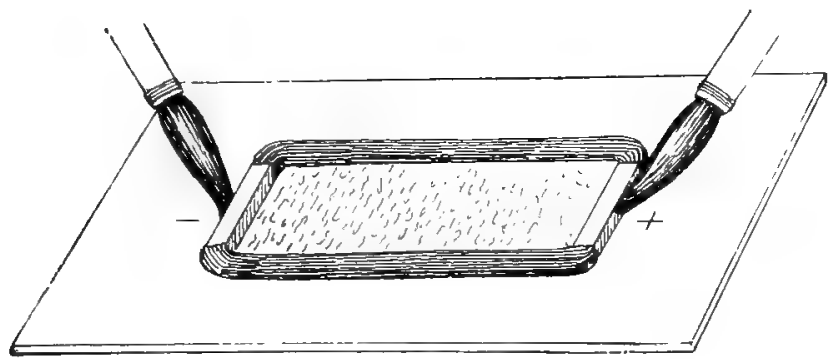

FIG. 235.-Galvanotaxis of spirostomum ambiguum. After the making of the current the lunoricu place themselves with their long axis at right angles to the direction of the current

that their long axes are at right angles to the direction of the current, and maintain this position, although constantly bending and twitching their long bodies (Fig. 235). This form of galvanotaxis may be termed trunsverse. In other organisms trans${ }^{1}(f)$ Verworn (9?, 96$)$. 
verse galvanotaxis has not been observed thus far, although it is scarcely doubtful that it will yet be found to occur in other unicellular organisms.

\section{THE PHENOMENA OF OVER-STIMULATION}

When the Athenians, under the leadership of Miltiades, had gained the victory of Marathon, one of the soldiers named Eukles, still hot from the struggle, hastened from the battle-field to Athens in order to be the first to bring to his countrymen the news of the victory. Plutarch ${ }^{1}$ who has given us the anecdote, tells of the dramatic fate of this runner of Marathon. When Eukles entered Athens exhausted by the effort of the long run, he still had power to call out to his countrymen the news of the victory

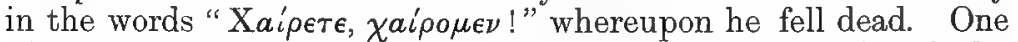
of our modern sculptors, Max Kruse, has illustrated this tale by his figure of the runner of Marathon now in the National Gallery at Berlin, and has given striking expression to the physiological phenomena of total exhaustion.

The cause of the tragic end of Eukles was his excessive muscular exertion. Under the influence of long duration or great intensity of stimuli, changes gradually appear in the living substance which, when they have reached a certain extent, lead to death. In the following pages we will examine somewhat in detail the phenomena resulting from over-stimulation.

\section{Fatigue and Exhaustion}

If a living object be stimulated by long-continued, oft-repeated, or very strong stimuli, after some time it passes into the condition of fatigue. The general characteristic of fatigue is a gradual decrease of the irritability of the living substance. This is expressed especially in the fact that with increasing fatigue, the intensity of the stimulus remaining the same, the result of the stimulation becomes constantly less.

We have already become acquainted with some examples of this fact in considering galvanic stimulation. ${ }^{2}$ If a constant current of average strength be passed through an Actinosphourium, at the moment of making there begin to appear at the anode marked phenomena of contraction. The protoplasm of the pseudopodia flows centripetally until the latter are drawn in. Then the walls of the vacuoles break; and a granular disintegration of the protoplasm results, which proceeds constantly farther from the kathode during the passage of the current. This dis-

${ }^{1} C f$. bibliography.

${ }^{2} C f$. pp. 422 and 423. 
integration, beginning with great energy, becomes slower and less extensive the longer the current flows, and after some time is at a complete standstill. This means that the living substance of the Actinosphorium becomes fatigued in the course of the continual stimulation, and decreases in irritability; hence the stimulus, which at first induced pronounced phenomena of disintegration, later produces no reaction at all. Pelomyxa. is fatigued still more rapidly than Actinosphcerinm. Stimulation for a few seconds is sufficient to make individuals of this genus wholly non-irritable to currents of equal intensity; a much greater intensity is then required to call out the same reaction.

In contrast to these forms of living substance which become fatigued very rapidly, nerves seem to be incapable of fatigue: thus far it has been impossible by continual stimulation to demonstrate in them fatigue phenomena. That nerve is really incapable of fatigue is in the highest degrec improbable. Since, like all living substance, it has a metabolism so long as it lives, and since its irritability is extinguished with its life, it must be supposed that its irritability is associated with its metabolism, and that every excitation produces a change in its metabolism. Possibly these changes are so slight that fatigue cannot be demonstrated at all by the methods that have been used heretofore. To conclude, therefore, from the apparent incapability of' fatigue that the function of nerve is entirely independent of metabolism, and is like the capacity of copper wire to conduct galvanic currents, is quite unjustified. Nevertheless, it would be important to investigate the question, whether in nerves the changes of metabolism produced by stimulation are not perhaps compensated by the metabolism as soon as they appear, so that within a limited time no phenomena of fatigue become noticeable externally. 'That such a condition is very easily possible is shown by the behaviour of another object-viz., cardiac muscle. Although from long before birth up to death the heart-muscle labours uninterruptedly, under normal conditions it does not become fatigued, because the changes resulting from its activity become compensated in its metabolism. Nevertheless, it is capable of fatigue, when for any reason it is obliged to make excessive efforts. This is the case in certain diseases. The phenomena of fatigue become then apparent, not at once, but in the conrse of long spaces of time, and even the substance of the muscle changers profoundly, until its movements wholly cease. Then death by paralysis of the heart results.

While cardiac muscle is thus capable of fatigue only exceptionally, in the tissue of skeletal muscles fatigue phenomena are very crasily induced. Fatigue has been studied most fully and most 
frequently in the cross-striated skeletal muscles of vertebrates. Since by means of the graphic method muscular movement can be recorded and its individual factors made visible, the progressive fatigue of the muscle can be studied very conveniently in the change undergone by the curve that the contracting muscle records. Mosso ('91) has done this in the living man by means of his ergograph, and has presented the results in his excellent and fascinating book entitled "La Fatica." The ergograph is a small apparatus in which the arm of a man is fastened by means of a holder, while one finger is free to move. This finger is connected by a cord with a writing-lever, which records upon a rotating drum all the movements of the finger that take place, either voluntarily or

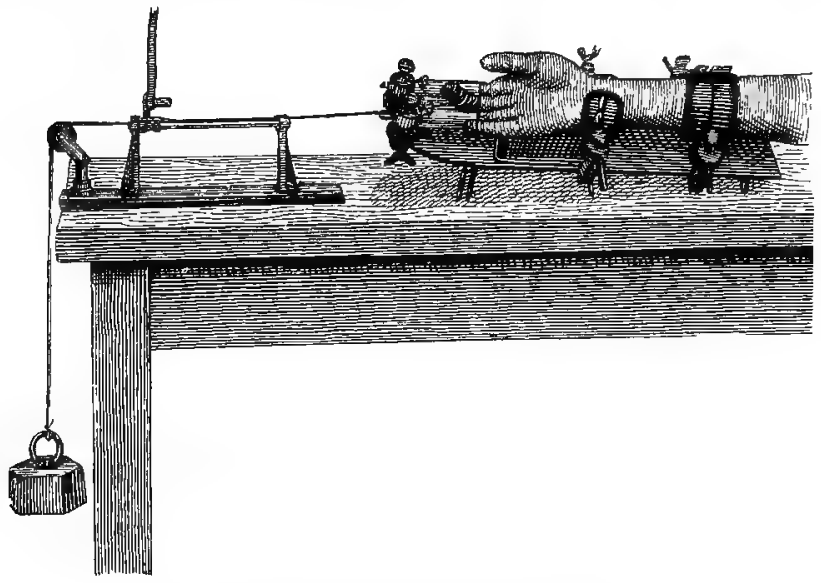

FIG, 236.-Mosso'suergograph, (After Mosso.)

involuntarily as the result of electrical stimulation. A weight can be hung upon the cord, and thus the work performed by the flexor muscles of the finger can be changed at will (Fig. 236). By means of this apparatus it can be shown very clearly that, with the stimulating induction-shocks remaining constant in intensity and following each other at equal intervals, the work performed by the muscles constantly decreases, and finally becomes equal to zero. This is expressed in the curve of contraction, which gives only the extent of the contraction, by a constant decrease in the height of the lift (Fig. 237). After a course of contractions it requires considerably stronger stimulation to produce further contraction of the fatigued muscles equal in height to that at the beginning. The details of the changes are more readily visible when the successive contraction-curves of a frog's leg are fecorded over one another upon a myograph from the beginning of the 
series on, as Marey ('68) did a long time ago. Then it is found that, as Helmholtz discovered, with increasing fatigue not only does the curve decrease in height, but it becomes more extended. its descending limb especially undergoing a lengthening. In other words, the work done by the muscle becomes less while the duration of the contraction increases. The latter phenomenon depends chiefly upon the increasing duration of the stage of expansion. The fatigued muscle needs more time to extend to its complete length.

The phenomena of fatigue appear, perhaps, still more clearly upon stimulation by the tetanizing current than by single induction-shocks. If the curve of tetanus of a frog's gastrocnemius muscle, not too strong and weighted, be recorded upon a rotating drum, it is seen that it continues at its original height for a long time, and follows a straight line (Fig. 238). But after some time it begins slowly to fall, and, not rarely at the same time, small irregularities in its course become visible, which are due to the fact that the muscle begins to tremble. The curve continues to fall gradually. If the stimulation be interrupted, the curve usually does not sink at once to the level of its startingpoint, but remains some distance above the latter, and only in the course of a considerable time returns to it. Hence there is a

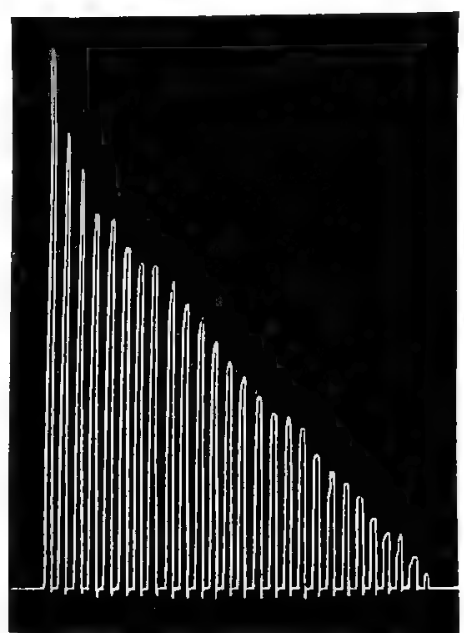

Fig. 237.-Curve of fatigue; decrease of the height of the curves with numerous successive contractions of the flexor muscles of the fingers. (After Mosso.) considerable contraction-remainder in the fatigued muscle after the end of stimulation, and the muscle assumes its original length only very slowly.

It is of great interest that microscopic changes have been observed in fatigued muscle. Of a number of wholly similar blue-bottle flies (Musca vomitoria) H. M. Bernard ('94) kept some in continual motion by constantly exciting them, until they fell to the ground completely exhausted. The fatigued flies were at once killed simultaneously with the others, which, in the meantime, had remained at rest. The two kinds of specimens were then subjected to the same treatment. A marked difference appeared between them. While in the resting flies the musclefibrillæ showed distinct cross-striation and the various dises of the individual segments showed differences in staining-capacity, in the 
fatigued specimens only Dobie's line was to be seen clearly, 'and the whole contents of the segments stained uniformly without any differentiation of the dises being noticeable (Fig. 239). But the granules, or sarcosmmes, lying in the sarcoplasm between the

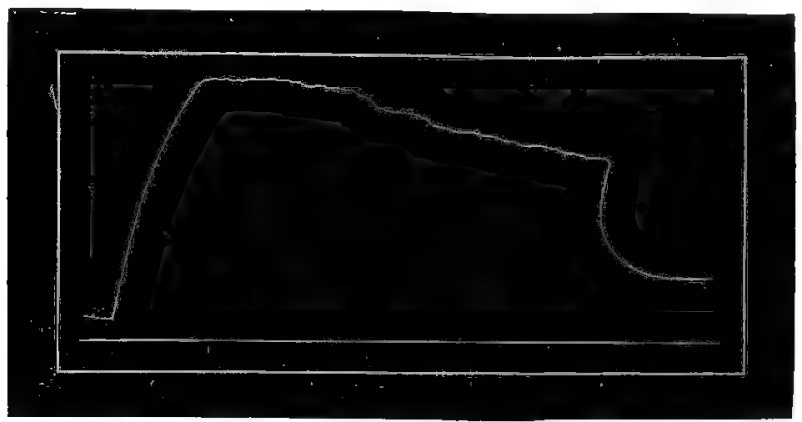

FIG. 238.-Curve of tetanus of a fatigued muscle of a frog.

individual fibrillæ were enormously enlarged in the fatigued, in comparison with the resting, muscle. It would lead us too far to consider in detail the significance of these changes. Hodge ('92), G. Mann ('94), and Lugaro ('95), have recently made known distinct mirroscopic phenomena of fatigue in the ganglion-cells of mammals,
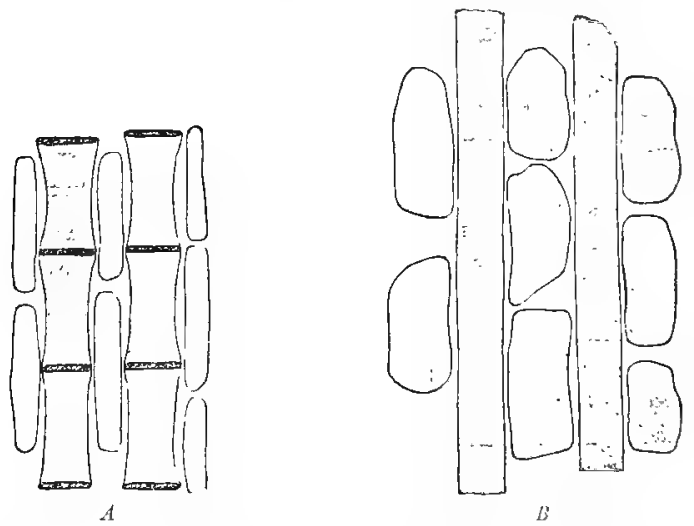

FIG. 239.-Wing-muscles of a blue-bottle fly (Muscu romitoria). A, At rest; $B$, fatigued. The division of the muscle-segments into dises has become invisible and the sarcosomes between the fibrille are cnormously onlarged. (After H. M. Beruard.)

birls, and insects, especially in their nuclei. Thus, according to IIolgce, in the sparrow, in the morning, after resting, the cells of the him hial ganglia, which innervate the wing-muscles, have clear, round, wevicular nuclei (Fig. 241, 1), while in the evening, after 
the exertion of the day, they have an indented contour (Fig. $241, B)$. Likewise in the cat, after stimulation for several hours, the nuclei of the ganglion-cells, which previously were vesicular and round, are shrunken and have an irregular contour, while the arrangement of the contents has changed materially (Fig. 240). According to Mann, and also Lugaro, the change of the ganglioncell during its activity consists essentially in a turgescence of the protoplasm and the nucleus, while during rest a diminution in volume takes place. During work the nuclens becomes poorer in chromatin, and, as Lugaro found, by fatigue the nucleolus can

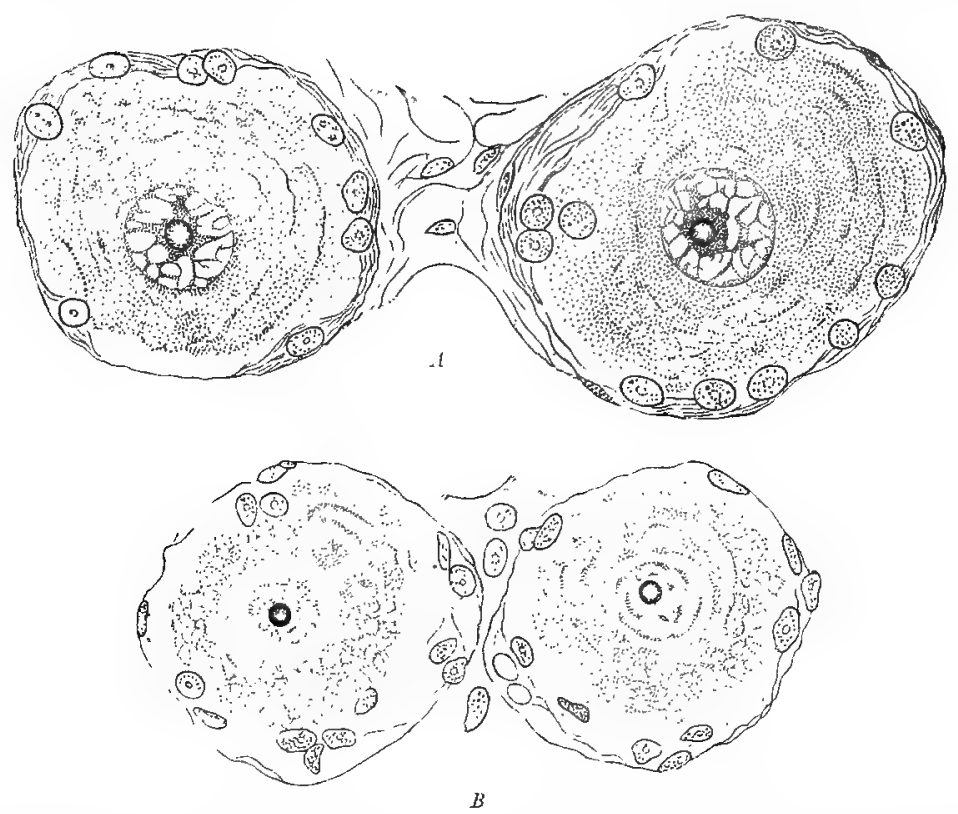

FIG. 240.-Ganglion-cells of the cat. $A$, In the normal condition; $B$, after five hours' stimulation. (After Hodge.)

be made completely to disappear. Here belong, also, the fatiguechanges which Heidenhain ('83) observed a long time ago in salivary glands after stimulation, the cell-nuclei of which, in rest, put out pseudopodium-like processes, but after stimulation assume the spherical form (Fig. 242).

The fatigued muscles recover as soon as the stimulation ceases, and the more rapidly, the less was the degree of fatigue. In recovery the irritability gradually increases; the various phenomena of fatigue, which can be seen in the curve of contraction, gradually pass away, and, finally, the muscles are in the same condition as before. 
That which appears especially interesting is the fact, discovered by Valentin ('47), and Eduard Weber ('46), that excised muscles also are capable of recovery. This, also, can best be seen by the aid of the graphic record of the muscular movement. If an isolated gastrocnemius of a frog be fatigued by being alternately tetanized for perhaps five seconds and allowed to rest for five seconds, after some time, the intensity of the stimulus remaining constant, the curve begins to fall, until, finally, the stimulation no longer produces any contraction, and the muscle remains at rest in a slightly contracted condition, determined by the contractionremainder. If, then, the stimulation be interrupted and the muscle be left to itself for a considerable time, protected from
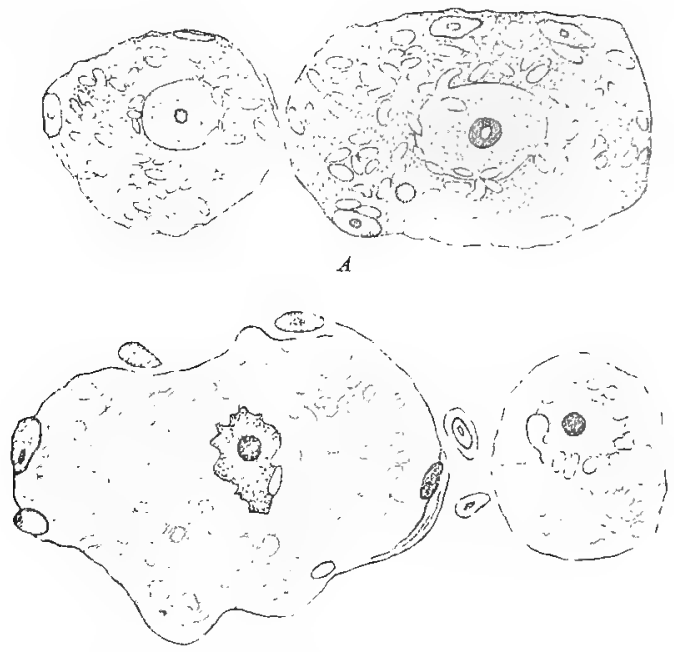

$B$

FIa. 241,-Ganglion-cells of the sparrow. A, Morning; $B$, evening. (After Hodge.)

drying, contractions nearly equal to those before the fatigue can be induced anew with the same strength of stimulus. The muscle now becomes fatigued more rapidly than before. One factor in the recovery, which has recently been established in Richet's laboratory by J. Joteyko ('96), is of interest. This is found in the fact that excised muscle recovers only when oxygen is available; with the exclusion of oxygen after complete fatigue the muscle cannot be put again into activity. Hence oxygen is absolutely necessary for the restoration of the irritability of muscle. But the fact that after great fatigue excised muscle is able to recover in a medium containing oxygen proves that the musclesubstance, while it can perform contractions for a considerable time independently of the circulating blood, must possess in itself, 
also independently of the blood-current which biings in food-stuffs and takes out excretory matters, the factors which, in union with oxygen, are necessary to the restoration of irritability.

If we turn from the phenomena of fatigue that are externally visible in the muscle itself to those that develop secondarily in the body as results of very strong muscular effort, we meet with certain facts which bring us a step farther in the knowledge of fatigue.

If we observe the phenomena that develop in our body in the course of strong muscular effort, we notice first a considerable acceleration and deepening of the respiration. At the same time the frequency of the heart-beat becomes increased. The production of heat which is increased by the muscular activity, is essentially compensated reflexly by the outpouring of perspiration, the evaporation of which lowers the temperature. If
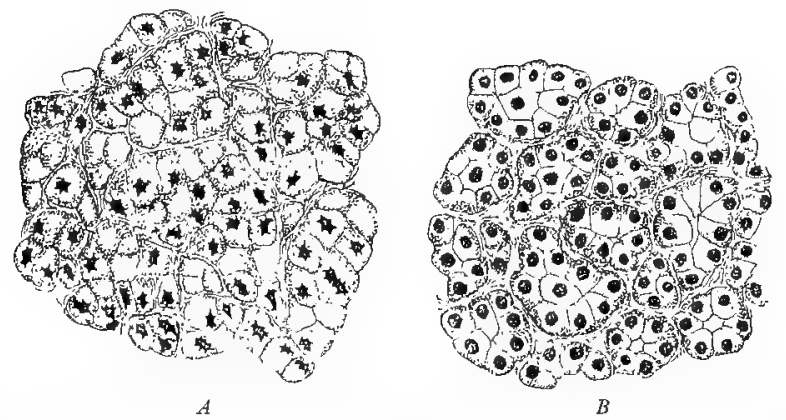

FIa. 242.-Parotid of the rabbit. $A$, During rest ; the cell-nuclei are indented. $B$, After stimula. tion through the sympathetic; the nuclei have become round. (After Heidenhain.)

the activity has been very considerable, not rarely a slight fever appears, especially when the body has made no muscular effort for a considerable time previously. The temperature rises, there are attacks of shivering, and a certain increase in irritability of the central nervous system is noticeable. This fact is so well known that there is recognised a "gymnast's fever," which appears in gymnastic work after too strong exertion. This fever of fatigue is also very frequently observed after very exhausting mountain tours and after long riding. Among the subjective symptoms that manifest themselves as a result of very strong muscular exertion, the best known are excitement appearing during the stage of the fever, c.g., in the evening after an exhaustive march, sleeplessness, lack of appetite, and intense muscle pains, which appear usually upon the next day or even later.

These ph'nomena together present an interesting complex of syınptoms, which remind the physician very strongly of the 
picture of events in acute infectious diseases. The conjecture is strongly suggested that all these symptoms that appear as a result of muscular fatigue appear also as the characteristic complex of symptoms of infectious diseases. Concerning the latter, it is known from the later bacteriological investigations that they are the result of poisoning by certain poisonous metabolic products, the so-called toxines, ${ }^{1}$ which are excreted by invading bacteria. But, like bacteria, a great variety of other forms of living substance excrete poisonous substances in their metabolism, and hence the assumption is not unjustified that the muscles also produce such toxines, which in the quantity usually present produce no effects, but which, as soon as they accumulate in the body in greater quantity as the result of excessive muscular activity, give rise to phenomena of genuine poisoning. Various experiments have proved directly that this conjecture is correct.

The first important experiments were those of Ranke ('65), who found that he could make a fatigued muscle again capable of performing work by washing it out with a dilute solution of common salt which, as is well known, is completely indifferent to living tissue. Hence there must have arisen and accumulated in the muscle as the result of activity certain fatigue-substances, which act to paralyse the muscle-substance itself, but after the removal of which the muscle regains its capacity for work. Ranke was able actually to confirm this by the following experiment. $\mathrm{He}$ made a watery extract of muscles that had been strongly fatigued, and injected it through the blood-vessels into a fresh muscle. The result was that the muscle immediately lost its working capacity and behaved exactly like a fatigued muscle. It is proved by this experiment that phenomena of fatigue are caused by the accumulation of certain metabolic products in the muscle, and can be set aside by the washing-out of the latter. More recently Mosso ('91) performed upon a dog an experiment analogous to Ranke's. When he injected into a narcotized dog blood from a normal dog, the former continued completely normal. But if, instead of this, he used for injection blood from a fatigued dog, whose muscles had been kept in violent contraction by tetanization with the electric current for only two minutes, characteristic phenomena of fatigue immediately appeared: the respiration became accelerated and even dyspnoic, and the heart began to beat strongly. Hence the fatigue-substances that are produced in the muscle do not remain there, but are taken up by the blood and thus go to the organs of the whole body. Hence it comes about that after an exhaustive march not only do the muscles of the legs, but also those of the arms, show phenomena of fatigue. The poisonous substances going with the blood to the brain-centres that control respiration and the movement of the heart, there first 
produce an excitation, which results in a powerful increase of the respiration and the activity of the heart, but finally with too great exertion cause a depression, which leads to standstill of the heart and death. The history of the runner of Marathon is a classic example of this course of phenomena.

But in seeking the origin of muscle-fatigue, we ought not to attach too much importance to the appearance and accumulation of fatigue-substances in the muscle, as is not rarely done. Although it is beyond doubt that the phenomena of fatigue can be produced by the accumulation of fatigue-substances, this is not the sole cause. The chief factor in the production of fatigue is the progressive consumption of substances that are necessary to activity. Accordingly, in muscle and probably in all living substance, two different causes of fatigue may be present. Phenomena of fatigue are observed, on the one hand, when certain substances that are necessary to life are consumed during exhaustive activity more rapidly than they are introduced or reformed; and, on the other, when certain substances that arise as decomposition-products during activity accumulate in such quantity that they produce a depressing effect. On account of this fundamental difference in the genesis of the phenomena in question, it seems advantageous to distinguish between the two causes by the use of different terms, and to call the phenomena of depression that result from the consumption of the necessary substances, exhaustion, and those that result from the accumulation of and poisoning by decomposition-products, fatigue. The end-result of the two series of phenomena arising from such different causes is the same. Both are characterised by depression of the irritability and the activity of living substance.

\section{Excitation and Depression}

Let us first bear in mind that excitation and depression are merely quantitative opposites. The two are merely different degrees of one and the same phenomenon, namely, life, excitation being an increase, depression a decrease of the normal intensity of vital phenomena.

It has been seen in a previous section that phenomena of depression can be called out by over-stimulation. This fact is important, for it shows that the same stimuli which with slight intensity or short duration produce excitation, with increased intensity or long duration can produce precisely the opposite effect, namely, depression.

This relation between excitation and depression is very widespread. The phenomena of fatigue are a single example of it. In this respect the effects of anzsthetics form a complete analogy to the phenomena of futigue. It appears to be a general pro- 
perty of these substances that in very small doses or with very brief administration they produce phenomena of excitation, while with increasing action phenomena of depression become more and more noticeable, and apparently are able to lead to a complete standstill of life. ${ }^{1}$ This fact is well known in pharmacology. Morphine in small doses and at the beginning of its action produces always a stage of excitation, in which the patients are restless and excited, are not able to sleep, and are haunted by all sorts of illusions. But if the dose given be greater, and the stage of excitation appearing at the beginning of its action be passed, deep sleep comes with total absence of motion and sensation. The same result is seen also with other narcotics and with single cells. In ciliate Infusoria the ciliary motion is increased to furious rapidity under the influence of the vapour of ether or chloroform in small quantity or with brief duration. The excitation of the cilia is so great that the organisms shoot through the water like arrows. But if the dose or the duration of the influence of the narcotic become only slightly increased, the motion becornes slower and slower until, finally, complete paralysis results, and the cells remain motionless. The same phenomena have been observed with the many different kinds of anæsthetics, and in all sorts of living substance.

Another example of the fact that with increasing intensity of the stimulus excitation is first increased and then after a certain point gives place to depression, is afforded by stimulation by heat. ${ }^{2}$ With increasing temperature up to a certain degree, which is very different for different forms of living substance and for different vital phenomena in the same form, all vital phenomena undergo an augmentation to a maximum. But if this degree be overstepped, excitation decreases rapidly, and gives place to complete paralysis in the form of heat-rigor. The fermentative activity of yeast-cells, the growth and development of ova, and the protoplasmic and ciliary motions of unicellular organisms, afford distinct examples of this. Other varieties of stimuli illustrate the same general principle.

But this relation of excitation and depression holds good only for those stimuli which consist in an increase of the factors that under normal circumstances act upon the organism as vital conditions, as, e.g., increase of the surrounding temperature, or those which consist in an incoming of foreign factors, as, c.g., poisonstimulations. Those stimuli, however, which depend upon the diminution of vital conditions, as, e.g., decrease of the surrounding temperature, appear in general with increasing intensity to depress vital phenomena without previous excitation. With the present condition of our knowledge a law covering these facts cannot be formulated with certainty, for a cautious critic requires 1 Cf. p. 379. ' $C f$. p. 396. 
a larger number of phenomena before generalising. Nevertheless, large number of discoveries speak directly in favour of the idea here expressed.

E.g., with increasing cold the energy of vital phenomena sinks, until at certain low degrees of temperature, which likewise are very different for different objects, apparently complete paralysis results. The experiments of Kühne ('64) on Amaba, in which the protoplasmic motion was at a complete standstill in coldrigor at $0^{\circ} \mathrm{C}$., as well as a number of other phenomena previously spoken of, afford examples of this. Further, with decrease of moisture the intensity of vital phenomena sinks, until the latter come to a complete standstill. The behaviour of dried, apparently dead, organisms illustrates this. Finally, with decrease of food and of oxygen vital phenomena are depressed, and, as is instanced by the protoplasmic movement of Amebce in Kühne's experiments, cease in an atniosphere of pure hydrogen.

The fact cannot be overlooked that thexe are cases in which with falling temperature, as in the regulation of heat by warmblooded animals, or with decrease of the water-contents, as in drying nerve and muscle, or with decrease of oxygen, as in the asphyxiation of warm-blooded animals in a space free from oxygen, phenomena of excitation are apparent. But the mode of occurrence of these phenomena, which can be investigated in the cell-community only with difficulty on account of the complexity of the conditions, is in large part still obscure, and many investigations directed toward this point alone, especially in single cells or simple tissues, are needed, before it shall be known clearly whether the principle observed in so many cases, that with decrease of the various vital conditions a gradual depression of vital phenomena comes in without previous excitation, really has general application. The question whether within the two extreme limits of vital conditions living substance possesses but one maximum of excitation is surely interesting. There are doubtless many cases in which both augmentation and diminution of the vital conditions produce depression, and in which between these two points excitation rises to a single maximum.

\section{Death by Over-stimulation}

The inevitable end-result of continual or strong over-stimulation is death, but the manner in which it develops differs in individual cases according to circumstances.

With continued, not too strong stimulation death develops fairly gradually, and here the stages of the reaction can be followed best. The effect of narcotics may serve as an example. If, e.g., an infusorian cell, such as the ciliate Spirostomum, be exposed to the influence of the vapour of chloroform or ether, there is seen first a 
stage of excitation, in which the ciliary motion becomes strongly accelerated. Gradually with continued action the excitation gives way, and there begins a stage of depression resulting in a complete standstill of the cilia. From this stage by interruption of the stimulus and the re-establishment of the normal vital conditions the organism can be revived. If, however, the action continues still further, this is no longer possible; narcosis passes directly into death. The same thing is seen in human ganglion-cells in morphine poisoning. At the beginning of the action there is a stage of excitation, which soon gives way to a complete paralysis of the ganglion-cells. With too strong a dose the death of the cells results; this is scen in a standstill of the functions dependent upon them (movement of the heart, respiration, etc.). The same sequence of actions is produced by the thermal stimulus with continual increase of its intensity. The protoplasmic motion of $A m c e b a$ increases with increasing warmth up to nearly $35^{\circ} \mathrm{C}$. Here the motion suddenly diminishes; the organism continues in

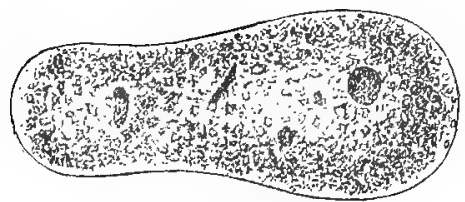

A

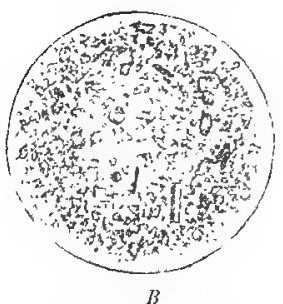

B

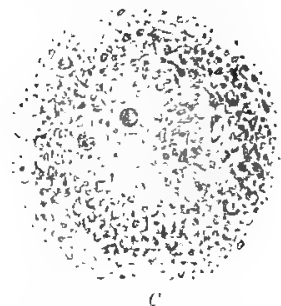

Fra. 243.-Pclonyxa palustris. $A$, Creeping; $B$, contracted as a result of feeble chemical imulation ; $C$, undergoing granular disintegration witl long stimulation.

the stage of contraction and performs at most very feeble motions. With a slightly higher temperature the latter wholly cease. This is the point of heat-rigor. Upon cooling from this point motion returns. But, if the temperature rises above $40^{\circ} \mathrm{C}$., the heatdepression passes over into death. With thermal stimulation the whole sequence of reactions from the minimum of temperature up to the maximum is presented with the greatest clearness: standstill of vital phenomena in cold-rigor, increasing excitation, depression in heat-rigor, and finally death.

The complete series does not always appear. Very frequently one or the other stage is wanting. This depends partly upon the special qualities of the living substance, and partly upon the kind of stimulation. Often under the influence of stimuli of very high intensities all stages are omitted, and death results at once. Sometimes there is a brief stage of excitation, but intense excitation is followed immediately by death. If, while Pelomyxa is creeping quietly, it be stimulated only feebly by acids, alkalies, 
chloroform, or other chemical substances, in a few seconds it draws itself together into a ball (Fig. $243, B$ ), and thus gives the impression of intense excitation of contraction. In the course of a longer, constant action of the stimulus the protoplasmic body begins to undergo granular disintegration from the periphery (Fig. 243, C). If, however, the chemical stimulus be allowed to act in greater intensity upon the resting, extended body, the stage of excitation has no time for its development. The body begins immediately, without first contracting into a ball, to undergo granular disintegration in the form which it had at the moment of stimulation (Fig. 244, B). Here death appears immediately as a result of stimulation, while the other stages of the reaction have not time to develop externally. The same is seen in galvanic stimulation. If Actinosphorium be stimulated by feeble galvanic currents, the typical phenomena of excitation of contraction appear at the anode. The protoplasm of the pseudopodia forms small globules and spindles, and flows centripetally, until the pseudopodia

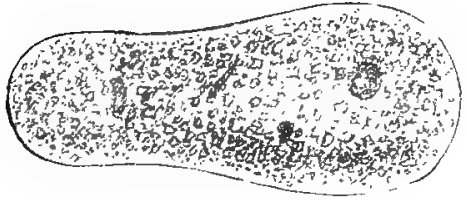

4

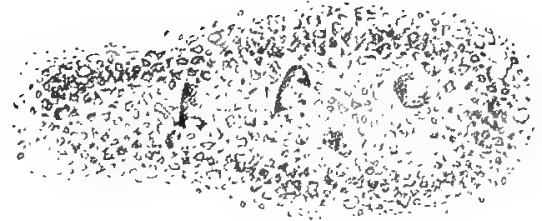

$B$

FIa. 244.-Pelomyra palust ${ }^{2}$ s. A, Creeping; $B$, undergoing granular disintegration as a result of strong chemical stimulation.

are wholly retracted. If, however, a strong galvanic current be applied suddenly, the protoplasm has not time to contract, but immediately undergoes disintegration at the anode.

Granular disintegration of protoplasm as a result of supramaximal stimulation is a valuable aid when, as e.g., in stimulation by galvanic currents, the localisation of the excitation is to be rletermined in objects in which there is no other distinctly visible expression of it. In such cases it is only necessary to employ supramaximal currents, and the place of excitation is recognised at once in the granular disintegration of the protoplasm. Of course this is possible only in forms of living substance which, at the moment of death, show granular disintegration. There are many forms of cells, especially those that are provided with a solid wall, which in dying do not pass into granular disintegration at all. Yeast-cells, c.g., can be killed in various ways by over-stimulation without any disintegration of the body. Their death is indicated only indirectly, by loss of the power of splitting grape-sugar into carbonic acid and alcohol. But we need not here go more in cletail into the different forms in 
which death appcars, since we have previously ${ }^{1}$ become acquainted with them. Over-stimulation, in its most general significance, is nothing but that which has been termed elsewhere external causes of death. The fact does not require special mention that overstimulation, when it consists either in an increase or a decrease of the factors that act as vital conditions, always results finally in death. It has already been seen that overstepping either the minimum or the maximum of vital conditions leads to a fatal outcome.

In a previous chapter we came to regard life as a phenomenon of nature that, like all other phenomena of nature, comes into existence when a certain complex of conditions is fulfilled. If the conditions become changed, the phenomena also change; if the formex wholly disappear, the latter also cease. In stimuli we have become acquainted with such changes of vital conditions. Under the influence of stimuli vital phenomena change, and they wholly cease, when the stimuli overstep a certain limit.

If we except the small number of cases, thus far largely unexplained, such as the metamorphic processes of necrobiosis, where vital phenomena are forced into a perverted path and are qualitatively changed under the influence of stimuli, we observe that within certain limits stimuli cause only a single kind of effect, namely, a gradual, quantitative change of the vital phenomena, either increasing or decreasing the intensity of the latter. Hence in the rast majority of cases stimuli do not call out new phenomena, but produce merely an excitation or depression of those general vital phenomena already existing.

It is here especially to be noticed that the different varieties of stimuli produce in the same object wholly similar reactions. An Amoba may be made to retract its pseudopodia and assume a spherical form by chemical, mechanical, thermal, and galvanic stimuli ; the cells of a ciliated epithelium respond by an acceleration of their ciliary motion to chemical, mechanical, thermal and galvanic stimulation; and by all of these agencies the production of light can be induced in Noctiluer.

This important fact shows that in every form of living substance there must exist an extraordinary inclination toward a specific sequence of processes. 'l'his sequence is continually present in slight degree and finds its expression in the spontaneous vital phenomena; but the slightest stinuli of all kinds augment the discharge of the processes always in the same characteristic sequence for each specific variety of living substance, just as the nitroglycerine molecule can always be made explosively to disintegrate into the same constituents by mechanical, galvanic, or thermal influences. 
The principle of the specific energr of sense-substances in animals provided with sense-urgans, as discurered by Johannes Miiller, ${ }^{1}$ has, therefore, general application. All living substance pussesses specific energy in Miuller's sense: within certain limits wholly different stimuli call forth in the same form of liring substance the same phenomena, while, conversty, the same stimulus in different forms pruduces an ettect wholly different and characteristic for every form. :

${ }^{1}$ Cf. pp. 21 and 45 .

$\because r$ Hering ( $\because t)$. 


\section{CHAPTER VI}

\section{THE MECHANISM OF LIFE}

THE principle which the early civilised races with their mythical ideas poetically personified and represented as the cause of all life in the world, lies at the foundation of all vital phenomena according to the scientific knowledge of to-day. Among most people this principle has found expression in its original form in the allegory of the shifting contest between two hostile forces. These forces are life and death, which the ancient Egyptian personified in the forms of Horus and Typhon; bloom and decay, which the German clothed in the legends of Baldur and Loki; Ahriman struggling with Ormuzd, by which the Persian represented the interchange of the good and the evil in life; God striving with the Devil, in which the Christian of the middle ages perceived the all-creating positive element in its opposition to the all-destroying, "ever-denying spirit" ; and, finally, they are recognised in the ever-alternating processes of becoming and passing away, of building up and breaking down, which control every living being and every vital event.

We have already recognised in the continual construction and destruction of living substance or, in brief, in unbroken metabolism, the real vital process, upon which the physical phenomena of life are based. We have become acquainted with these phenomena, have investigated the conditions under which they make their appearance, and have determined the changes that they experience under external influences. We must now endeavour to construct a bridge between the vital phenomena and the vital process, and, so far as the present condition of our knowledge allows, derive the former mechanically from the latter; the investigation of the mechanism of life forms the nucleus of the science that deals with the physical phenomena of life. 


\section{The Vital Process}

As previous treatment of this subject has shown, ${ }^{1}$ our knowledge of the individual events in the metabolism of living substance is unfortunately thus far very meagre. Investigation of the mechanism of the physical phenomena of life is necessarily still far from complete, and progress can be made only slowly. An essential advance in this direction can be expected only from the detailed study of the processes in the cell, for the cell is the place where the vital process itself has its seat, and where all vital phenomena occur in their simplest form. Not until the physiology of organs, which is able to explain only the gross performances of the complex cell-community, develops into cell-physiology, can we hope essentially to enlarge our knowledge of the more delicate mechanism of life. Thus far only the first steps have been taken in this direction.

If, therefore, we attempt to form, so far as possible upon the basis of our present knowledge, a picture of the vital process in living substance, it can be only a sketch in which the most general elements are indicated in gross outline. Notwithstanding this, some kind of a picture of the vital process is necessary for further systematic investigation.

\section{A. THE METABOLISM OF BIOGENS}

\section{Biogens}

It has been seen in a previous chapter that, in general, the characteristic of living organisms in comparison with those dead or apparently dead consists in their metabolism, the expression of which constitutes the vital phenomena. It is necessary to go a step beyond this general fact.

It will be recalled that in the determination of the chemical compounds that constitute living substance investigation deals exclusively with the dead cell. For the completion of a picture of living substance two questions now remain to be answered, viz. : first, do the chemical compounds which are found in the deal cell occur as such in the living cell? and, second, are there in the living cell still other compounds which are not present in the dead cell, which, in other words, are bound up inseparably with the life of the cell?

The first of these questions is relatively easy of answer. A careful comparison especially of the solid bodies that may be found as reserve-substances for a time unchanged in the living cell, with the corresponding substances of the dead cell shows that there

${ }^{1} C f$. p. 157. 
occur in the living cell proteids, carbohydrates and fats, in other words, the three chief groups of organic compounds, and likewise the products of their decomposition; in brief, there occur all the essential substances that are found in the dead cell.

There remains only the question whether, in addition, compounds exist in the living substance which are destroyed at death and hence are not to be found in the dead cell. A comparison of the chemical behaviour of living and dead cell-substance forces us to assume the existence of such compounds. Physiological chemistry has shown that between the two kinds of substance very essential chemical differences exist, which prove that living substance experiences in dying pronounced chemical changes. A wide-spread difference between the two consists in their reaction. The reaction of living substance is almost without exception alkaline or neutral and with death changes usually to acid. Further, certain proteids that are in solution in living cell-substance, as, e.g., the myosin of muscle, experience very remarkable changes. In death they coagulate and pass into the solid state, which is very unfit for further chemical transformations. Physiological chemistry has shown similar changes in death in great number. All these facts prove that in the death of living cell-substance certain chemical compounds undergo transformations; hence substances exist in it which are not to be found in dead cell-substance.

The fact that these chemical compounds are only present in the living substance and are decomposed with death necessitates the conclusion that the vital process is associated very closely with their existence. At all events an important property belonging to them is their great inclination toward transformation, which is for life an indispensable element. When it is borne in mind how few causes are able to produce death, how almost all chemical substances that are at all soluble in water enter into chemical relations with living cell-substance, while dead cell-substance usually behaves wholly indifferently to the same influences, it must be said that the substances that distinguish living from dead cell-substance possess a very loose constitution.

This conclusion is still more obvious when the fact of metabolism is considered. Metabolism shows that the living cell-substance is being continually broken down and reformed, this process being made possible by the continual giving-off and taking-in of material. In contrast to this, under favourable conditions, dead cell-substance is capable of preservation for an extraordinarily long time without its excreting more than a trace of the material that living cell-substance gives off continually. Hence, in contrast to the former, the latter must be distinguished by the possession of complexes of atoms that have very great tendency toward chemical transformations and are continually undergoing self-decomposition. The currat lability of these complexes depends upon the fact that 
their transformation can be considerably augmented by slight influcnces from the outside, as the excitation of metabolism by stimuli clearly shows. Since, how' (rer, metabolism constitutes the roul vital process, it is seen at once that life depends directly upon the existence of these labile complexes of atoms. We are, theretore, justified in examining these significant substances more in d.tail and investigating their nature somewhat further.

In searching after them we can best sturt from the decompositionprochets excreted in metabolism. It is here found thit among other substances, such as carbonic aciul, water, and lactic acid, which contain only the elements carbon, hydrogen and oxygen, compounclis also occur that contain nitrogen. The non-nitrogenous decomposition-products may possibly be derived from the decomposition of carbohydrates, fits, ete.; but those containing nitrogen can come only from the transformation of proteids or their durivatives, for these are the sole bodies containing nitrogen that are present in all living substance. This important fact directs attention first to the proteids.

That this is the right path becomes at once clear when the facts concerning the proteids are recalled that have been mentioned in the course of the previous considerations. These facts show without doubt that the proteids stand at the centre of all organic life.

It is an important fact that in all cases where large quantities of rescre-substances, such as fat, starch, and glycogen, are not accumulated in cells, the proteids constitute by far the largest part of the organic compounds of living substance. This mroves that they must play a significant role in the life of the cell. The dominant position of the proteids among the chemical compounds of living substance, however, is at onee attested by the fact that they wre the only substances that can be found in every cell without exception. It is a further fact that of all the more important substances in the cell the proteids and their compounds present the highest complexity in chemical compusition, they comprise the largest number and variety of atoms in their molecules. The known chemical relations of the non-nitrogenous organic substances, especially the carbohydrates and fats, to the proteids are in harmony with this dominant position of the latter in living substance; for, so far as their history is known, those substances either are consumed in building up the proteid molecule, or are derived from the transformations of the latter. The former is, of course, shown most clearly by plants, in which all organic compounds are manufiutured synthetically out of simpler inorganic substances. In the edls of the green plant occurs the synthesis of the first organic product, stareh, ont of carbonic acid and water. This arbohydrate constitutes the organic basis from which the proteid molecule is dereloped syrtheticilly in a complex and still partly unknown manner with the help of nitrogemous and sulphur- 
containing salts taken from the earth. Regarding fat, it is known that it can serve for the construction of carbohydrate by transformations in the plant; the carbohydrate then gives off in turn the material for the formation of proteid, for in the seeds of Pconia, which are filled with fatty oils, all oil disappears, e.g., after long exposure to the air, and starch appears in its place. It is thus seen most clearly in the plant how different substances serve for the construction of the proteid molecule; but the animal demonstrates best the fact that the most important non-nitrogenous groups of atoms in living substance, especially carbohydrates and fats, can be derived from the decomposition of the proteid molecule. ${ }^{1}$ Thus, the fact that fat can be derived from proteid has been demonstrated by Leo in his experiments on phosphorus poisoning in frogs, and by Franz Hofmann in his experiments on the nutrition of the larvæ of flies with blood freed from fat. Further, Claude Bernard and recently Mering have proved upon dogs whose bodies were freed from glycogen by fasting, that after the feeding of proteid glycogen is again manufactured in great quantity, in other words, that this carbohydrate can be derived from the transformation of proteid. Finally, Gaglio has established the fact that the lactic acid in the body is derived from the transformation of the proteid molecule, since the quantity of it in the blood is dependent solely upon the quantity of proteid that is eaten. Regarding the nitrogenous excretory products of the body, it is evident that they can be derived only from the transformation of proteids and their compounds, since no other nitrogenous bodies are present among the essential organic compounds of living substance. But the most striking proof of the fact that all substances, both non-nitrogenous and nitrogenous, that are essential to the life of the cell, can be derived by chemical transformation from proteids, is afforded by one of the most significant facts of physiology, namely, the possibility that carnivora are capable of maintaining their life upon pure proteid and, as Pfluger ('91) has recently shown, possess great capacity for doing work. Nothing demonstrates better than this fact the controlling position of the proteid molecule in the vital process.

Hence, not only does it follow from the fact of metabolism that very labile complexes of atoms exist in living substance, with the presence of which life is inseparably associated, but it is the proteids whose presence constitutes the general, essential condition and focus of life. If we endeavour to harmonize these two facts, the unavoidable necessity arises of assuming in living cell-substance, besides the known proteids that occur also in dead substance, certain other proteids or compounds of proteids, that are present in life only and terminate life with their decomposition.

Dearl proteid, as it is found in the dead egg of the fowl, or as it ${ }^{1}$ Cf. p. 163. 
is stored in quantity in living egg-cells in the form of vitellins, is able to exist for an extraordinarily long time without undergoing the slightest decomposition, if protected from bacteria. Certain proteids or proteid compounds of living substance, however, are continually undergoing spontaneous decomposition, even when the living substance is under wholly normal conditions, and, as is shown by the products that are given off, the slightest action of stimuli increases the decomposition. A long time ago Pfliger ('75,1), as has been seen elsewhere, ${ }^{1}$ called attention to this important difference between the proteid in dead and that in living cellsubstance in his valuable work upon oxidation in living substance, and distinguished clearly between living proteid and dead proteid. The fundamental difference between the two consists in the fact that the atoms of the dead proteid molecule are in a condition of stable equilibrium, while the living proteid molecule possesses a very labile constitution.

Pfliiger's assumption of living proteid, which distinguishes living cell-substance from dead and in the loose constitution of which lies the essence of life, is necessitated. But this substance must be of essentially different composition from dead proteid, although, as follows from the character of its decomposition-products, certain characteristic atomic groups of the proteids are contained in it. The great lability that distinguishes it from other proteids, can be conditioned only by an essentially different constitution. Further, critics will rightly object to the terming of this hypothetical compound a "living proteid molecule," for there is a certain contradiction in calling a molecule living. The word "living" can be applied only to something that exhibits vital phenomena. Hence, the expression "living substance" is well justified, for vital phenomena may be observed in living substance as a whole. But a molecule cannot exhibit vital phenomena, at least as long as it exists as such; for if any changes appear in it it is no longer the original molecule; and, if it continues unchanged, vital phenomena are not present in it. The latter, which are based upon chemical processes, can be associated only with the construction or the destruction of the molecule in question; and thus the application of another name to the compound that is at the focus of life is doubly justified. In order to distinguish this body, therefore, from dead proteid and to indicate its high significance in the occurrence of vital phenomena, it appears fitting to replace the term "living proteid" with that of biugen. The expressions "plasma molecule," "plasson molecule," "plastidule," etc., which Elsberg. ('74) and Haeckel ('76) have employed, and the conceptions of which are comprised approximately in the expression "biogen raolecule," are less fitting in so far as they easily give the impression that protoplasm is a chemically unitary body, which consists of 1 Cf. p. 304. 
wholly similar molecules; such a view must be expressly rejected. Protoplasm is a morphological, not a chemical conception. ${ }^{1}$

Extremely little is known concerning biogens, and this fact should not be concealed. Since the constitution of the proteids themselves, i.e., substances that can be investigated chemically at any moment, is not at all known, it is readily understood that we possess much less knowledge concerning the biogens, the composition of which can only be inferred from their decompositionproducts. It can be maintained of them only that they are extraordinarily labile, and this property gives to them a certain similarity to explosive bodies. Pfluger $(75,1)$ has einployed certain facts in a most ingenious manner for the purpose of obtaining conclusions regarding certain characteristics of biogens, which make intelligible the great lability of the biogen molecule in comparison with the molecule of dead proteid.

The starting-point of Pfliger's discussion is a comparison of the decomposition-products that arise spontaneously and continually in the oxidation of living proteid, such as in respiration, with those that are obtained by the artificial oxidation of dead proteid. This demonstrates the important fact that the non-nitrogenous decomposition-products in the two cases agree essentially, while the nitrogenous products possess not the slightest similarity. "It follows from this that, as regards its hydrocarbon radicals, living proteid is not essentially different from the proteid of food." The important difference between the two consists rather in the arrangement of the nitrogenous groups of atoms. If, however, the nitrogenous decomposition-products of living proteid be examined, such as urea, uric acid, creatin, etc., as well as the nuclein bases, adenin, hypoxanthin, guanin and xanthin, it is found that, in contrast to the nitrogenous products that appear in the oxidation of dead proteid, some can be artificially prepared from cyanogen compounds, while others contain cyanogen (CN) as a radical. Hence it is highly probable that the carbon and the nitrogen are combined in the biogen molecule into cyanogen, a radical that is wanting in dead proteids.

Thus there is presented a very fundamental difference in the constitution of biogens and that of dead proteids; this explains also the great lability of the biogen molecule, for cyanngen is a radical that contains a great quantity of internal energy, all its compounds possessing strong inclination toward decomposition. This fact enables us to understand the process of respiration, for when in the biogen molecule two atoms of oxygen come into the vicinity of the very labile cyanogen radical, by reason of the active intramolecular vibrations of the carbon and nitrogen atoms in cranogen the carbon atom will unite with the oxygen to form the vary stible molecnle of carbonic acid. In fact, cyanogen is very

$$
{ }^{1} \text { Cf. p. } 80 \text {. }
$$


easily combustible, and in its combustion yields carbonic acid. Thus, Pflugger believes that the continual taking-in of oxygen and giving-out of carbonic acid on the part of living substance depends upon the presence of the cyanogen radical, and that the intramolecular oxygen is the essential condition of the tendency of living substance to decompose.

In these considerations we find a basis for an idea of the manner in which the formation of a biogen molecule takes place in an animal cell out of the ingested food. By the co-operation of the biogens already present, the atoms of the dead proteid molecule introduced in the food undergo in the cell a rearrangement, in such a manner that an atom of nitrogen always unites with an atom of carbon to form the cyanogen radical with the loss of water. The changes that necessarily appear at the sarne time in the other groups of the proteid molecule are for the present wholly unknown, but, if we may judge from the essential agreement in the non-nitrogenous decomposition-products of the living and of the dead proteid, they do not appear to be of fundamental importance. By the intramolecular addition of inspired oxygen the biogen molecule finally arrives at the maximum of its power of decomposition, so that only very slight impulses are required to bring about the union of the atoms of oxygen with the carbon in the cyanogen. The material of the non-nitrogenous groups of atoms afforded by the explosive decomposition of the biogen molecule can easily be regenerated by the residue of the biogen molecule from the carbohydrates and fats that are present in the living substance and contain such groups; in fact, it has been seen that these substances are consumed in the building-up of proteid. "Probably this is the essential significance of these satellites of the proteid molecule," as Pflüger very fittingly terms the carbohydrates and fats. If, finally, the living substance dies, the labile cyanogen-like compound of nitrogen passes over again into the more stable condition of the ammonia radical with the absorption of water, the nitrogen uniting with the hydrogen of the water. Thus we have again the stable compounds of dead proteid, such as serve for food. These are, in brief, some of the essential features of the abbreviated path followed by the food in the construction of the biogen molecule in the animal cell. The much longer path, which in the plant cell leads from the ingestion of the simplest inorganic compounds through the synthesis of the first carbohydrate and on to the construction of the biogens, is for the present much more obscure.

Notwithstanding the facts that the views here developed have been confirmed by experiment only in part, and that they contain many large gaps, which can be filled only slowly, they afford at least a basis for an understanding of the fundamental processes in living substance. The metabolism of living substance, upon which all 
life is based, is conditioned by the existence of certain very labile compounds, which stand next to the proteids and on account of their elementary significance in life are best termed biogens. To a certain degree the biogens are continually undergoing spontaneous decomposition, just as is the case with other organic bodies, e.g., prussic acid. But this decomposition is much more extensive, If even slight external stimuli act upon the living substance. We must imagine that by reason of the extremely active intramolecular vibration of the atoms, which is the cause of the labile condition, certain atoms, partly spontaneously and partly as a result of external commotions, come under the influence of others for which they possess greater affinity than for their original neighbours, and in this manner more stable groupings of atoms arise as independent compounds. In this respect the biogens can be compared to explosive substances, the atoms of which possess likewise very labile equilibrium and which upon receiving violent shocks explode, i.e., rearrange their atoms into more stable compounds; e.g., nitroglycerine or trinitrate of glyceryl, which is employed for making dynamite, is decomposed by mechanical impulses or electric shocks into water, carbonic acid, nitrogen and oxygen : $2 \mathrm{C}_{3} \mathrm{H}_{5}(\mathrm{O}$ $\left.\mathrm{NO}_{2}\right)_{3}=5 \mathrm{H}_{2} \mathrm{O}+6 \mathrm{CO}_{3}+6 \mathrm{~N}+\mathrm{O}$. But, in contrast to other explosive bodies, we must evidently ascribe to the biogens the peculiarity that in decomposition the whole molecule is not destroyed, but that certain groups of atoms, which are formed by rearrangement, are split off, while the residue is again built up into a complete biogen molecule at the expense of the materials found in its vicinity, just as in the manufacture of concentrated sulphuric acid ${ }^{1}$ the nitrous acid formed from nitric acid by the withdrawal of oxygen is rebuilt into nitric acid with the aid of the oxygen of the air. The substances still present in the living substance in addition to the biogens are merely "satellites" of the biogen molecule, and either serve for its construction or are derived from its transformations. Thus far no substances have been made known in living matter, which can stand in any nearer or more remote relations to the biogens. Nevertheless, from the variety in the decomposition-products that are excreted by different kinds of cells in metabolism, it must be concluded with great probability that biogen molecules have not in all cells exactly the same chemical composition, but that there are various biogen bodies, and even that the biogens not only of different cells, but of the various differentiations of the same cell, such as exoplasm, myoids or contractile fibres, musclefibrillæ, cilia, etc., have different constitutions, although they agree in essential structure. The biogens, therefore, are the real bearers of life. Their continual decomposition and reformation constitutes the life-process, which is expressed in the manifold vital phenomena. 


\section{Bintonus}

Now that we have become acquainted with the simplest schematic expression of the elementary vital process in the construction and destruction of biogens, we must consider more in detail certain metabolic relations that result from these, and we must define certain conceptions which are important in clarifying our ideas upon metabolism.

It will be recalled that two phases are distinguished in metabolism, assimilation and dissimilation. By assimilation is understood the capacity of living substance to construct its like continually from the ingested food-stuffs; by dissimilation, the capacity to decompose continually into the products excreted by it. In accordance with the above considerations, this conception can be formulated more exactly as follows : assimilation comprises all those transformations that lead up to the construction of biogens, dissimilation all those that extend from the decomposition of biogens down to the complete formation of the excretion-products.

Such an exact definition of these two fundamental conceptions of the theory of metabolism is necessary, for, when we glance at the history of the theory, we find that they have been employed with very different meanings. Assimilation, which originally signified in a very general sense the formation of living substance in the organism out of non-living food, has been employed by botanists in a very special way. Plant physiology in large part still means by assimilation exclusively the synthesis of starch from water and carbonic acid in the chlorophyll-bodies of the green plant-cell. This narrow conception has gradually been widened in animal physiology, and the term has been employed not only for the synthesis of the first organic product, but also for the construction out of the ingested food-stuffs of the more complex compounds of living substance, especially those that are characteristic of every form of cell, the proteids. In contrast to this latter use, Ewald Hering ('88) has conceived the word in a narrow sense, and in a small but suggestive work has sharply separated assimilation from growth. By the former he understands only the qualitative chemical change of particles already present; in other words, the completion of the particles up to the maximum of their constitution; under growth, on the other hand, he includes not qualitative changes, but only a quantitative increase of the particles present. In addition to this Hering has created the conception of dissimilation and placed it, beside that of assimilation, finding between dissimilation and atrophy a difference corresponding to that between assimilation and growth; the qualitative change associated with the separation of certain substances from the particles present he terms dissimilation, and the quantitative diminution of the particles, atrophy. 
But this sharp separation of assimilation and dissimilation on the one side, and growth and atrophy on the other, can scarcely be maintained, at least in so far as the former are conceived to be based upon purely qualitative, the latter upon purely quantitative changes of living substance. The formation of living substance takes place only with the help of living substance already present. Only where such substance already exists can new masses of it be formed. This is true even of the plant-cell, in which the living substance is produced in great measure from purely inorganic materials. It must be concluded from this that in growth the biogen molecule attracts to itself from the food the elements necessary for the formation of living substance and combines them chemically, and, therefore, it is changed qualitatively in growth. The general tendency of proteids, and likewise of the cyanogen-containing groups of atoms hypothetically present in the biogen molecule, to polymerisation, as Pfluger has already emphasised, allows us to understand this growth by chemical union. On the other hand, atrophy is only conceivable as taking place by means of chemical decomposition, that is, by a qualitative change of the living particles. But even if we can, and must, distinguish the regeneration of certain parts of the biogen molecule from the reformation of whole biogen molecules, and, likewise, the separation of single groups of atoms from the complete decomposition of the molecule, chemical changes are always present, which are directed to either the construction or the destruction of complete biogen molecules. Regeneration is only a part of the process of the formation of a new biogen molecule, and, likewise, the splittingoff of certain groups of atoms is only a part of the phenomenon of decomposition. In an hypothesis upon the nature of assimilation, Hatschek ('94) has also established a relation between this process and growth. He assumes that in growth the simple molecule of living proteid continually attracts elements to itself from the food until it has become a polymeric molecule; it then breaks down into simple molecules, and the latter gradually develop chemically anew into a polymeric molecule by the union of the necessary atoms and the groups of atoms, and so on. In other words, Hatschek likewise sees in growth a chemical process, which does not differ fundamentally from regeneration. After all these considerations it appears advantageous to employ the conceptions of assimilation and dissimilation in the more general sense, including therein the formation of new and the disappearance of old molecules, and to give to them the above exact wording:

Assimilation commises all those transformations that lead up to the construction of biogens, dissimilation all thrse that extend from the decomposition of biogens doun to the complete formation of the excretion-products.

It is, however important to examine somewhat more in detail 
the relation of these two processes. Living substance is continually performing both. Hering believes that these processes, which constitute the metabolism of living substance, "take place simultaneously in all the most minute parts of the latter." Hatschek has expressed a view differing from this, and emphasises the difficulty of the idea " that the proteid molecule simultaneously receives and gives off carbon." When only a single particle is considered, it is very difficult to conceive this process, for the splitting-off and the regeneration of any groups of atoms by a molecule exclude each other chronologically, and, when considered strictly, although instantaneous, they are only able to take place in succession, unless it is assumed that corresponding groups of atoms, separated from the molecule at one place, are added to it at another place. This latter idea Hering himself rejects, since he emphasises the following: "We ought not to be misled into picturing living substance as a mass that is at rest internally, while being consumed upon one side and built up upon the other." If we are unable to conceive the dissimilation and assimilation of the minutest individual particle or biogen molecule as absolutely simultaneous, within a larger quantity of living substance these two processes can take place at the same time. In this latter case there are always different molecules that are destroyed and rebuilt at the same moment, for only the residue of the biogens already present is capable of regeneration, and, vice versa, only the complete biogen molecules already present are capable of decomposition.

If we consider the quantitative relation of assimilation to dissimilation in a considerable mass of living substance, for example such as is contained in a cell, we find it very variable, and even without the influence of stimuli it changes within wide limits. This relation of the two processes in the unit of time, which can be expressed by the fraction $\frac{A}{D}$ and will be termed, in brief, biotonus, is of fundamental importance for the various phenomena of life. The variations in the value of the fraction effect all changes in the vital manifestations of every organism.

The fraction $\frac{A}{D}$ is merely a general form of the expression of biotonus. In reality, assimilation and dissimilation are not simple processes; on the contrary, the events that lead to the construction of the biogen molecule and the formation of the decomposition-products are very complex and consist of many processes closely interwoven. Hence, if we would express biotonus in a specialised way, we must give the fraction the form $\frac{a+a_{1}+a_{2}+a_{3}+\ldots}{d+d_{1}+d_{2}+d_{3}+\ldots}$ in which $a, a_{1}, a_{2}, a_{3}$, etc., and $d, d_{1}, d_{2}, d_{3}$, etc., represent the partial processes that combine to form the whole. 
With our extremely slight knowledge of the more special transformations that take place in living substance, it is at present impossible even approximately to review the manifold possibilities resulting from changes of the individual components of the biotonous quotient. Therefore, we shall here refer only to some of the more important of the known cases.

If the sum of all the members of series $A$ is equal to the sum of series $D$, i.e., if assimilation and dissimilation are equal in the unit of time, the fraction $\frac{A}{D}=1$. This case is realised in the condition termed metabolic equilibrium. That is, in the unit of time the sum of the excreted substances of every kind is eyual to the sum of the ingested substances.

If the individual members of series $A$ increase in a constant relation to one another, while the members of series $D$ remain equal or decrease, so that in the unit of time the sum of the members of $A$ is greater than that of the members of $D$, then the metabolic quotient $\frac{A}{D}>1$. This case is realised in growth, where the formation of living substance surpasses its destruction.

If, rice versa, the members of series $D$ grow proportionately to one another, while those of series $A$ remain unchanged or become smaller, biotonus $\frac{A}{D}<1$. This condition is the basis of atrophy and leads finally to death.

But it is wholly unrecessary that all members of the one or the other series change always simultaneously and proportionately; individual members can also increase or decrease independently of the others. Thus, the metabolism of carbon in an organism may be augmented without that of nitrogen experiencing a corresponding increase. In this way occur the formation and accumulation of reserve-substances, which are consumed later. Upon such changes of the individual members of the two series depend all the phenomena that appear in an organism in the course of derelopment. In many cases, as is shown best by the changes appearing during development, there exists a certain mutual independence of the individual members of the metabolic series. On the other hand, there are very many cases in which not only the individual members of each series, but also the two series, are dependent upon one another in such a manner that the change of the one results in a similar change of the other. E.g., if there is metabolic equilibrium and the numerator of the fraction increases, the denominator increases equally: if the denominator decreases, the numerator does the same; in other words, every increase of assimilation results in a corresponding increase of dissimilation. In this manner the metabulic quotient $\frac{A}{D}$ remains always equal 
to 1, i.e., rnetabolic equilibrium continues to exist in spite of the absolute change in the extent of metabolism. Hering very fittingly terms this maintenance of equilibrium " the internal selfregulation of the metabolism of living substance." Such a selfregulation of metabolism within definite limits is realised in man in the behaviour of the borly troward ingestred nitrogen. With a definite quantity of ingested proteid, which Voit has found to be approximately $118 \mathrm{gr}$. in the labouring man, nitrogenous equilibrium continues to be maintained; i.e., the more nitrogen is introduced in the proteid, the more is cxcreted in the urine, a sign that the dissimilation of proteid increases in the same proportion as the assimilation.

This last example leads us to the action of stimuli upon biotonus, and we must consider this in some detail.

\section{B. THE ACTION OF STIMULI UPON THE IETABOLISM ()F BIONENS}

\section{Changes of Biotonus upon Total Stimulation}

It has been seen that biogens are very labile compounds containing much intramolecular heat; in other words, the atoms of their molecules are in active vibration. As a result of this, certain atoms come occasionally into the sphere of attraction of others, and becoming united with them into a more fixerl combination, separate off as an independent molecule. In this way the spontaneous dissimilation of the biogen molecule results. But the chemical affinities made available by the withdrawal of the separated groups of atoms have in the constituents of the food that is taken in and transformed in manifold ways, an opportune possibility of combining again, so that the residue of the biogen can be rebuilt into a whole biogen molecule. Thus spontaneous assimilation of the biogen molecule follows its spontaneous dissimilation.

Since the dissimilation of the biogens is conditioned by the intramolecular vibrations of the atoms, it is evident that all factors that increase such vibrations must assist the procesm of dissimilation. In this way is explained the increased decomposition of living substance that can take place under the influence of chemical, mechanical, thermal, photic, and galvanic stimuli. If the external influences are so strong that a profound decomposition of the molecule takes place, and no residuc capable of r'generation is left, there results a decrease of the living substance, and with overstimulation death. On the other hand, the prucess of dissimilation is depressed by all factors that diminish the intramolecular vibrations of the atoms in the biogen molecule, such as cooling and the action of substances that fixate single atoms in a definite position by chemical attraction. All of thes stimuli that either excite 
or depress the process of dissimilation, we shall term dissimilatory stimuli.

Upon the other side, it is clear that assimilation also can be promoted by external influences. This process depends upon the union of chemical affinities belonging to both the residue of the biogens and the complete biogen molecules themselves, the participation of the latter following from their inclination to polymerisation. Hence all those factors can increase assimilation, which procure and put into proper form the substances that are necessary for the union of the existing affinities. The increased introduction of food-material and oxygen is more than all else efficient in this direction. Examples of other agencies are light in the cells of green plants, which is necessary to split up carbonic acid and make carbon available, and all stimuli that incite the production of ferments, which are needed to make solid food-stuffs soluble. But, on the other hand, there are factors that depress the process of assimilation. Special examples of these are lack of food and oxygen, in the plantcell lack of light, and the absence of ferments. We shall term all these factors that either excite or depress the process of assimilation, assimilatory stimuli.

Four important cases of reactions can thus be distinguished. Stimuli are able to produce:-

1. Excitation of dissimilation.

2. Depression of dissimilation.

3. Excitation of assimilation.

4. Depression of assimilation.

But the possibilities are not yet exhausted. For the individual events in living substance are in extremely close correlation with one another, and, as has been seen, in certain cases a complete internal self-regulation of metabolism is thereby occasioned, so that, e.g., every change of assimilation results in an equal change of dissimilation. Hence it is possible that a stimulus can call forth simultaneously excitation or depression of both dissimilation and assimilation. The following must, therefore, be added to the four cases above:-
5. Total excitation.
6. Total depression.

In connection with these it is to be noticed that different parts of the metabolic series can be excited or depressed in unequal degrees.

But still other possibilities are conceivable. Internal selfregulation of metabolism does not exist everywhere, and where it exists it is confined within certain limits; for, if it were effective at all times and in all places, continual metabolic equilibrium would exist, and growth, development, and atrophy would be impossible. Hence, cases are conceivable in which a stimulus produces simultineously excitation of assimilation and depression of dissimilation, 
or, vice irst, clepression of assimilation and excitation of dissimilation. There would thus be added to the above six cases of ractions the last conceivable ones as follows:-

7 . Excitation of assimilation + depression of dissimilation.

$\therefore$. Depression of assimilation + excitation of dissimilation.

Thesc various possible effects of stimulation, which Hering ('Si') has fully truated in his short dissertation upon the events occurring in living substance, give us an idea of the manifold ways in which biotonus can change under the influence of different stimuli. But in reality the relations are much more complex. Whon we rumember that the numcrator as well as the denominator of the fraction $\frac{A}{D}$ represents a whole serics of single nuembers, and that these mombers are able to change in a certain degree independently of one another, we obtain an approximate picture of the uxtrardinary variety of effects which stimuli are able to produce in living substance.

In a jrevions chapter it was found possible to arrange the reactions to stimuli in the living cell according to their external appearances in a f'w groups. It was found that the changes underune by spontaneous vital phenomona as the result of stimulation are either quantitative or qualitative. The quantitative changes were termed excitation wh'n they consisted of an augmentation of the vital phenomena, and depression when characterised by a diminution of the latter. In accordance with the foregoing considerations, we now obtain an approximate idea of the great complexity of the events the externil expression of which was termed briefly excitation and deprossion. But the areme of the complexity is to be seen in those reactions that are at the basis of the qualitative changes of the normal vital phenomena. The metamorphic processes of necrobiosis, typified by amyloid metamorphosis, show clearly that here indiridual mombers of series 1 and series $D$ must slowly and gradually change independently of one another, otherwise accumulitions of individual substanecs that normally do not occur in the coll can not take place. Motamorphic processes constitute a stimulation-phenomenon that is conditioned by changes of biotonus anlogous to those conditioning the phenomena that occur spontaniously in derclopment. The differentiation of glandcolls, musch-colls, Jerve-cells, etc, from the ovum must depend upon changes in the individual members of serice $A$ and $D$ that are ind 'pendent of one another ; but these changes oc'ur spontaneously in the contrs of development, while in amyloid medamorphosis and analogous phenomena they are produced by external inHuences.

Beamse of 
the two metabolic series, it is evident that at present we are quite unable to review even approximately the special changes that biotonus experiences in concrete cases under the action of a stimulus. For the present it is only possible to analyse step by step the outward expression of these changes, which have been termed schematically phenomena of excitation, depression and metamorphosis. Physiology will draw nearer to the solution of this problem the more the methods of cell-investigation are developed.

\section{The Interference of Reactions}

The question of the effects of the interference of two different stimuli is of special interest with reference to a group of very important phenomena belonging to the special physiology of vertebrates. Unfortunately up to the present time there has been no systematic treatment of this subject, and it is only possible to present a few intimations of its connection with certain facts from widely separate physiological fields.

Since biotonus can be influenced very differently by different stimuli, according as this one or that one of its components is excited or depressed, in a systematic investigation of the effects of the interference of two stimuli the manner of action of each must form the starting-point. In order to understand any such effect it must first be decided whether or not the two stimuli act in the same manner, i.e., to excite or depress, and to what component of biotonus their action extends, assimilation or dissimilation. The general laws of interference-effects can be discovered only by answering these questions.

If two stimuli of medium intensity produce effects of the same kind, for example an excitation, and act upon the same components of biotonus; for example upon dissimilation, the general result will be a summation of the excitations. The details of this cannot be predetermined, because the intensity of the stimuli, the varying extent to which the individual components are influenced, the duration of the stimuli, the fact of the self-regulation of metabolism, etc., are factors which, under the circurnstances, are capable of playing important rôles in bringing abont the final result. Here belongs, for example, the whole variety of phenomena that we have become acquainted with in nerve and muscle physiology as cases of increase of irritability. 'Through the action of an exciting stimulus, such as a chemical or thermal stimulus upon a nerve, the irritability of the latter toward a second, such as a galvanic stimulus, is incroased, and the latter causes a greater reaction than if it had berrn cimployed alone.

A contrast to this is afforded by the phenomena that result when living substance is acted upon by two stimuli that work in opposite senses upon like components of biotonus, one depressing and 
the other exciting. The usual result is a decrease of irritability. For example, if a narcotic be allowed to act upon a cell, or if a cell be depressed by over-stimulation, every exciting stimulus will produce a smaller reaction than if it had acted alone; under certain circumstances the cell will be completely inexcitable.

But nuch more interesting are the phenomena that result when two stimuli have the same kind of effect, for example, an excitation, but act upon different, and especially upon antagonistic, components of biotonus, that is, one pre-eminently upon dissimilation, the other upon assimilation. In such a case the one stimulus inhibits, opposes, restrains the other. ${ }^{1}$ A striking example of this is afforded by the polar action of the galvanic current upon contractile substances, for example, Amoba. The current acts antagonistically at the two poles, exciting the amoba-cell to contraction at the anode, and to expansion at the kathode. This fact can be confirmed with surprising clearness in fairly large freshwater Amocbce. If a constant current be passed through an amœba that has been made to contract into a ball by means of strong stimuli, at the moment of making the current the contraction begins to give way at the kathode, and phenomena of expansion begin to appear, i.e., a large pseudopodium projects; while at the opposite pole the phenomena of contraction become still more distinct. A sudden reversal of the current suffices to put an immediate end to the processes at the two ends of the body of the Amoba, and to supplant expansion by contraction and contraction by expansion. Analogous phenomena, except with the poles reversed, are exhibited by muscle. We can observe subjectively in the eye the interesting results of excitation of antagonistic metabolic processes. According to Hering's theory of colour vision, the perception of colours is the psychical expression of metabolic processes taking place in the visual substance, each pair of complementary colours corresponding to antagonistic phases of metabolism. Hence, if two complementary colours be mixed upon the rotating disc of the colour-top, the effect of each ceases, and the whirling disc appears a colourless grey. These facts show that two mutually interfering excitations of antagonistic links in the metabolic chain are able to inhibit or arrest their external effects. In other words, there are two wholly different ways in which the suppression, the inhibition, of a vital phenomenon can be accomplished: on the one hand, by the depression of those components of biotonus upon which it depends; on the other hand, by the excitation of antagonistic components.

Finally, it is conceirable that two stimuli will interfere when they act upon antagonistic components of biotonus in opposite senses-i.e., one to excite, the other to depress. The outward result of this would be an augmentation of those vital phenomena

$$
1 \text { ( }) \text { : Verworn ('96, 2). }
$$


that correspond to the excited components of biotonus. But it is questionable whether this case is actually realised in nature.

Among the various cases of interference between two stimuli there is a very great variety of phenomena which have not yet been analysed at all, but which ought to receive new light from the foregoing reflections. One group, particularly, which pertain to the functions of the central nervous system, and thus far have been among the most obscure phenomena of nerve-physiology, will be elucidated; these are the so-called phenomena of "inhibition" [Hernmung].

Heretofore there have been considerable difficulties in the way of a physiological explariation of the simple fact of the voluntary interruption of a movement, for example, the simple letting-down of a raised arm. The lack of clearness in the problems of inhibition depends chiefly upon insufficient sharpness in distinguishing the conceptions. Inhibition [Hemmung] and depression [Lähmung] have often been confused with each other upon the ground of purely external features; yet, as has been seen, an inhibitory reaction in the cell is not necessarily due to depression, but may be caused by the excitation of processes that oppose existing ones. The stopping or retarding of a muscular movement by a motor ganglion-cell can be the expression of two very different processes. According to the general view, as is well known, the contraction of a muscle is caused by an excitation of dissimilation in its motor ganglion-cells. An expansion can, therefore, originate in the ganglion-cell in two ways: first, by a depression of dissimilation, and, second, by an excitation of assimilation. As regards the muscle, the two have the same result. Hence it is necessary to decide in any one case what processes are taking place in the participating ganglion-cells. Among the manifold phenomena of the inhibition of motion doubtless both cases are present. The frog whose hinder extremities will not perform reflex movements with the strongest stimuli for some time after the upper part of its spinal cord is severed, has evidently experienced a temporary depression of the ganglion-cells of its cord through over-stimulation, just as in surgical shock as a result of a severe operation the nervous system is depressed. But the voluntary relaxation of a contracted muscle can hardly be due to such depression. In this case there must be an inhibition of contraction caused by the excitation of antagonistic, i.e., expansory processes.

The fact that the expression of an excitation can be stopped by the excitation of antagonistic metabolic processes appears to play a very important rôle in the life of ganglion-cells, and to afford a very important factor in the explanation of many processes in the central nervous system.

Esprecially the phenomena of sleep and hypnosis in animals and 
men may be explained, as to their essential factors, by the inhibition of an existing excitation through antagonistic metabolic pro-
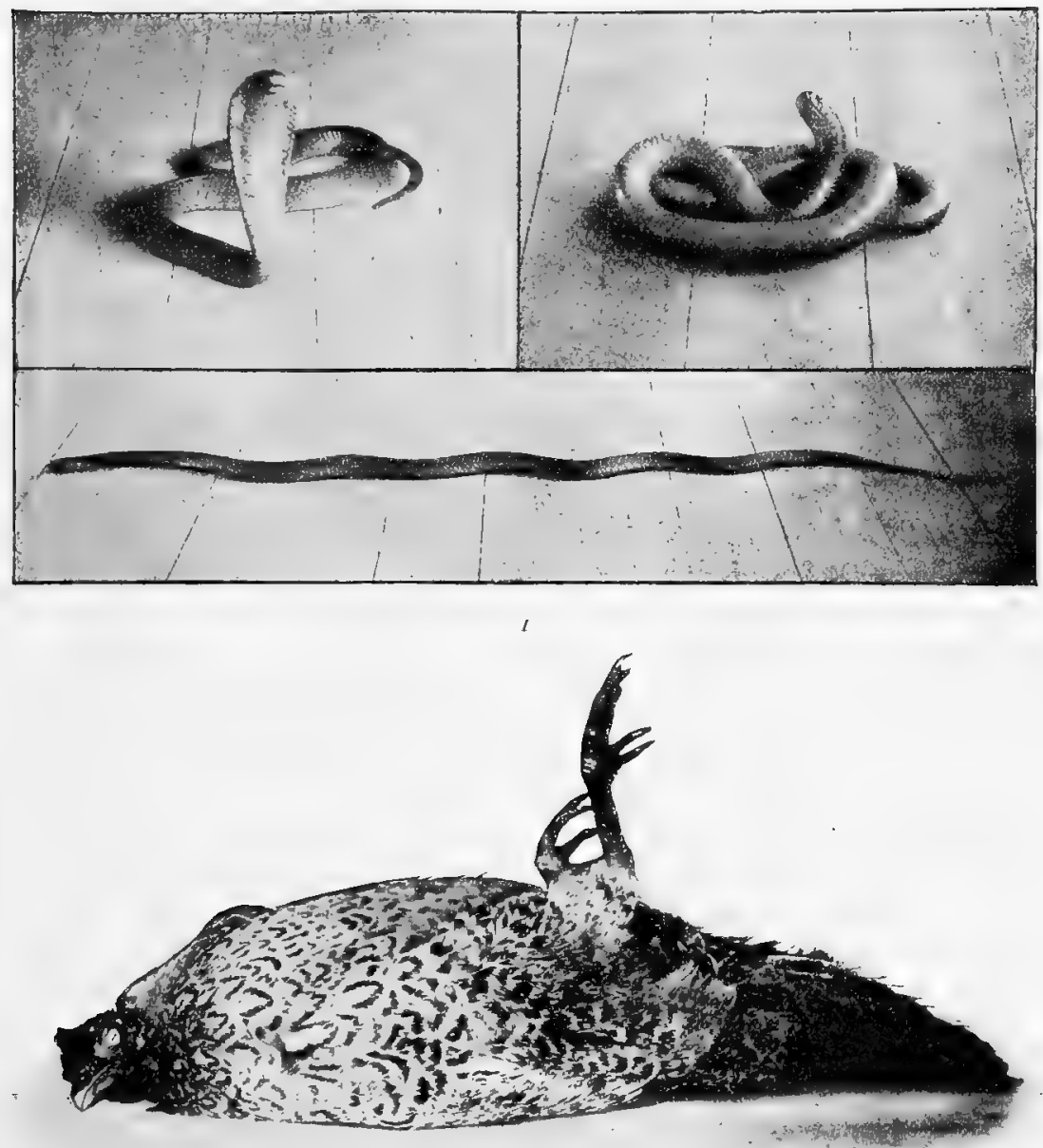

II

FIG. 245.-I, Naja haje (Egyptian hooded snake or asp). Experiment of the Egyptian snakccharmers. At the left, above, the asp is in the excited position of attack (shicld-position). At the right, above, the animal has been made motionless by pressure in the neck-region and has been laid upon its back. Below, it is in a similar condition, extended, and lying upon its belly. II, Fowl made motionless by being firnly held and laid upon its back. Evperimentum mirabile of Father Kircher.

cesses. It may suffice to recall a few well-known phenomena. The ancient experiments of the Egyptian snake-charmers, which 
Moses and Aaron performed before the Egyptian Pharaoh more than three thousand years ago, belong in this category. By.slight pressure in the neck-region it is possible to make a wildly excited, hissing, erect asp (hooded snake) suddenly motionless, so that the dangerous creature can be put into any desired position without fear of its fatal bite (Fig. 245, I). The well-known experimentum mirabile de imaginatione gallinae of Father Kircher depends.upon the same canses. If an excited fowl be seized suddenly with a firm grip and laid carefully upon its back, after a few brief attempts to escape it lies motionless (Fig. 245, II). Guinea-pigs (Fig. 151, p. 358), rabbits, pigeons, frogs, lizards, crabs and numerous other animals behave similarly. The hypnosis of human beings depends likewise essentially upon inhibition of the activity of the ganglioncells in the cerebral cortex, in common language upon an inhibition of the will, and in sleep the inhibition of the activity of all the higher brain-centres is evident. These cases do not depend upon depression; the stimuli that act as causes of them are too feeble. We must recognise in them, as in numerous other phenomena of inhibition, the other case, i.e., the inhibition of an existing excitation by the excitation of antagonistic components of biotonus in the participating ganglion-cells.

It will be a promising task of the future to investigate systematically interference-reactions and to ascertain their relations to the interesting processes in the central nervous system.

\section{Polar Changes of Biotonus and the Mechanism of Axial Orientation upon Unitateral Stimulation}

Thus far we have considered merely the changes of biotonus that are caused by general stimulation of living substance. But the changes that result from local stimulation are worthy of attention, because in certain cases they give rise to very characteristic external effects. These are the directive effects of stimuli upon motile organisms, which we have become acquainted with as chemotaxis, barotaxis, thermotaxis, phototaxis and galvanotaxis. These interesting phenomena are called out, as has been seen, by the unilateral, or unequal, action of stimuli upon the activity of contractile elements. In other words, all these cases of stimulation depend upon changes in those members of the biotonic series $A$ and $D$, that mediate the contraction and expansion of contractile elements. A movement in a definite direction can take place only where differences as regards contraction or expansion exist in two different parts of the cell-body. Since, as regards the motor effect, contraction (c) and expansion (c) are two antagonistic phases of the movement, we can express the mutual relation of these two nembers of biotonus by a fraction in a manner analogous to that of the expression of biotomis itself, without, however, at the same 
time expressing to which of the two biotonic series $c$ and $\varepsilon$ belong. The conditions that exist in a resting cell at the two different parts of the body can then be represented as follows:

\begin{tabular}{ll}
$c$ & $c$ \\
\hline$e$ & $e$
\end{tabular}

in which ${ }^{c}$ expresses the relation of contraction to expansion that prevails at the two opposite poles of the organism.

In a cell in which $c$ and $e$ are equal and an equal tendency toward contraction and expansion exists upon all sides, no movement can take place in any direction. But this is at once changed, when differences in biotonus appear at two points upon the surface, when $c$ or $c$ under the influence of a stimulus acting unilaterally becomes greater or smaller at one pole than at the other. Then a cuuse is afforded for a unilateral movement.

Since the remarkable phenomena of chemotaxis, barotaxis, themotaxis, phototixis and galvanotaxis are even now often considered as mysterious "attractions" and "repulsions" of unicellular organisms proceeding from the source of the stimulus, the origin of which thus far it has not been possible to explain mcchanicully, it is of great interest to see how their mechanism follows with absolute necessity from the special kinds of motion of each form of cell as the result of polar differences in the biotonus. Such a fact is of more interest because many of the phenomena mentioned, especially the chemotaxis of Bacteria and leucocytes, are of far-reaching significance in the pathology of the human body.

If the following three factors be considered, namely, the special modes of motion of any organism (protoplasmic, flagellar, ciliary motion, etc.), the change of this motion under the influence of stimuli, and the part of the body in which with unilateral stimulation the effect in each case is localized, the mechanism of these tactic phenomena, impressive because of their exactness, will appear very simple to any one who is accustomed to the study of motile mechanisms.

Let us imagine a unicellular organism, which is longitudinally differentiated, moving undisturbed in a desired direction through the medium in which it sxists, and then suddenly affected upon one side by a stimulus. It is a general rule that all uniaxially differentiated organisms move in the direction of their long axis. Hence, in order to approach toward or remove from the source of the stimulus, it would be nucissury for the stimulated organism first to assume a definite position with reference to its longitudinal axis, so that it would direct its anterior or its posterior pole toward the source of the stimulus. If this axial position be once assumed, a movement towarl or away from the source of the stimulus must at once take place by the usual method of locomotion, while the further action of the stimulus prevents or corrects occasional deria- 
tions from this direction, which are produced by spontaneous impulses. The essential factor in all directive reactions is, therefore, the assumption of an axial position by the cell-body, and the explanation of this is the key to an understanding of the mechanics of these phenomena. We will now study the mechanism by which the axial position is assumed in various types of free-living cells.

The simplest and clearest relations are, as always, in naked protoplasmic masses, such as Amoba and leucocytes. Let us imagine an $A$ moba in a spherical form about to move, and an excitation of contraction to appear at one spot as a result of a stimulus acting unilaterally (Fig. 246, $a$ ). The excitation would be least at the portion of the surface of the sphere opposite the place of stimulation. The protoplasm there would flow out unhindered, while upon the stimulated side the strong contraction would allow no bulging. The protoplasm would, therefore, form a pseudopodium toward the unstimulated side (Fig. 246, b). Thus an Amœba, which under conditions that are equal upon all sides extends its pseudopodia in all directions and creeps sometimes here and sometimes there, would now assume an axially differentiated form (Fig.
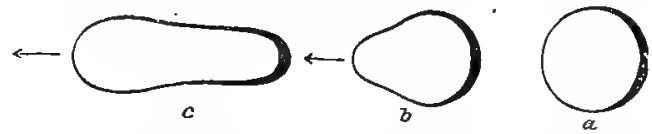

Fic. 246.-Scheme of axial orientation in an Amceba resulting from an excitation of contraction upon the right side. The thickness of the contour indicates the excitation. The arrows indicate the direction of the creeping.

$246, c)$, as is the case in that variety called Amoba liman. Under these circumstances with continual stimulation upon the same side the Amoba would necessarily creep gradually away from the source of the stimulus, as is the case in the negative chemotaxis and thermotaxis of Amoba, Myxomycetes, leucocytes, etc. Vice versa, if the spherical Amceba were to be acted upon on one side by a stimulus that produces a local excitation of expansion, the protoplasm would flow out most strongly toward that side, so that with continual stimulation the Amceba must necessarily approach the source of the stimulus. The positive chemotaxis of leucocytes, Amcoba, Myxomycetes, and other naked protoplasmic masses is thus explained. If a stimulation of expansion acts upon one side of an Amceba, and a stimulation of contraction upon the other side, the results of the two must naturally be expressed in like senses, i.e., the Amoba must creep away from the latter side and toward the former. The galvanotaxis of $A$ moba affords an unusually clear proof of this (Cf. Fig. 232, p. 458).

The mechanism of axial orientation is less complex in those microscopic organisms that do not consist of protoplasmic masses 
that are constantly changing their form, but, like Bacteria and Infusoria, possess an axially differentiated body of a constant form, which moves through the water by means of special organoids of motion, flagella and cilia. The bodies of these organisms are driven through the water after the manner of a row-boat by the rhythmic stroke of the flagella or cilia. The analogy of the motion to that of a boat moved by oars is complete, and may be recognised even in details. Exactly the same means are used to turn and direct the movements of the rowed boat, and the movements of the free-swimming ciliated cell, and we can represent the peculiar behaviour of Bacteria and Infusoria in their axial orientation upon unilateral stimulation no better than by means of this simile. Among the various organisms that propel their extended bodies through the water by means of flagella or cilia, three types can be distinguished as most important, according as they move by means of a single flagellum, two flagella, or several or very many cilia, corresponding to a boat propelled by one, two, or many oars.

We will examine, first, the forms that possess one flagellum, such as many Bacteria and flagellate Infusoria, and will select as representative the delicate, green, flagellate-infusorian Euglena, which, in summer, by means of its countless numbers, changes the water of standing pools into a deep green. The flagellum of the Flagellata is upon the anterior pole of the body and moves through the water in a screw-like path. For the sake of simplicity its motion may be considered as taking place in a single plane. It is then seen that it oscillates about the straight middle position (Fig. 247, $\iota$ ) by Ineans of alternate rhythmic contractions toward the right $(b)$ and toward the left $\left(b_{1}\right)$; the swing out of the middle position $(a)$ into one of the two extreme positions $\left(b\right.$ or $\left.b_{1}\right)$ represents the phase of contraction, the return from one of the extreme positions into the middle position the phase of expansion. The flagellum works, therefore, like an oar that is moved alternately to the right and to the left at the bow of a boat. It is evident that, while undisturbed and having equal conditions upon all sides, the infusorian body must move forward in a straight line, if the flagellum beats equally strongly toward the right and toward the left, i.e., if contraction and expansion occur with equal rapidity toward the two sides. But if a contractile stimulus acts upon the flagellate suddenly from one side, and if the long axis of the body is not already turned in the direction of the stimulus with the posterior pole toward its source, such a position is assumed by means of a few strokes of the flagellum; for with every oblique or 
transverse position of the long axis the flagellum is stimulated to contract more strongly upon the side upon which the stimulus falls than upon the opposite side, it makes stronger strokes toward the former than toward the latter side, and the result is that the anterior part of the body is turned away from the source of the stimulus (Fig. 248). Exactly the same relations exist here as in a boat moved by a single oar. The bow of the boat also turns toward the opposite side when the boat is propelled more strongly upon one side than the other. The unequal strength of the flagellar stroke in the two directions continues, and the anterior part of the body is turned constantly more away from the source of the stimulus, until the body has placed its long axis in the direction of the incident stimulus (Fig. 248, d). Then both sides of the flagellum become equally stimulated and the protist swims in a straight line, so long as the stimulus continues. Thus, negative chemotaxis, phototaxis, etc., appear in uniflagellated
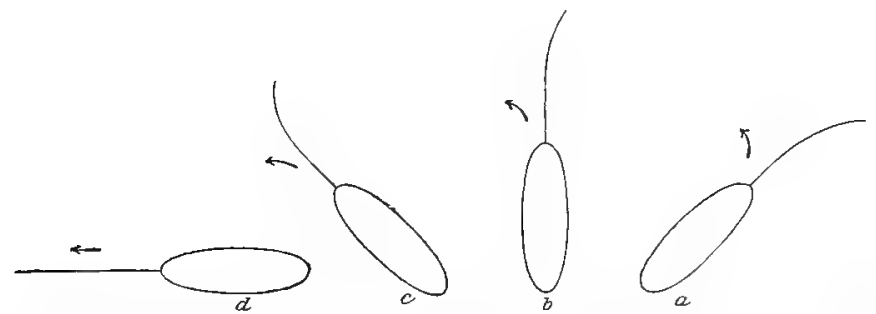

Fia. 248.- Scheme of axinl orientation of a flagellate-infusorian cell, resulting from an excitation of contraction upon the right side. The side toward which the concavity of the flagellum is directed is the stimulated side. The arrows indicate the direction of movement.

Bacteria and Flagellata as a necessary result of a unilateral excitation of contraction in the flagellum.

It is now very easy to imagine the relations as regards axial direction in forms possessing two flagella, such as the flagellate infusorian Polytoma (Fig. 249). If two flagella are present upon the anterior end of the cell, the latter corresponds to a boat that is moved by two oars at the bow. If the strokes of the two oars are equal, the boat moves in a straight line. It is the same with the flagellated cell. If one oar moves more strongly, the bow of the boat is turned toward the opposite side. The same will occur with the cell possessing two flagella, when a contractile stimulus acts upon one side, causing one flagellum to beat more strongly than the other (Fig. $249, \alpha, b, c$ ). The anterior end of the cell must then be turned away from the source of the stimulus, until the long axis is turned in the direction of the latter, with the posterior end nearer it. In this direction the two flagella are equally stimulated (Fig. $249, d$ ), and as a result the 
flagellate swims straight away from the source of the stimulus. In this way negative chemotaxis, etc., occur in the forms possessing two flagella, by reason of unilateral excitation of contraction.

The same thing that occurs in Polytome and other forms as the result of the activity of two flagella, occurs in ciliate Infusorica by means of the beat of numerous cilia. The movements of Paramocizm are analogous to the movements of a long boat possessing many oars. If all the oars upon the two sides move with exactly equal force, the boat moves straight forward; if the stroke of the oars is stronger upon one side than upon the other, the boat turns toward the opposite side. The same is true of the ciliary movement in Paramecinum. If the cilia beat with equal strength upon the two sides, the infusorian swims forward in a straight line; if, however, a contractile stimulus acts upon one side, so that the cilia upon that side are made to beat more strongly than upon the other (Fig. 250,a), the anterior end of the body must turn away
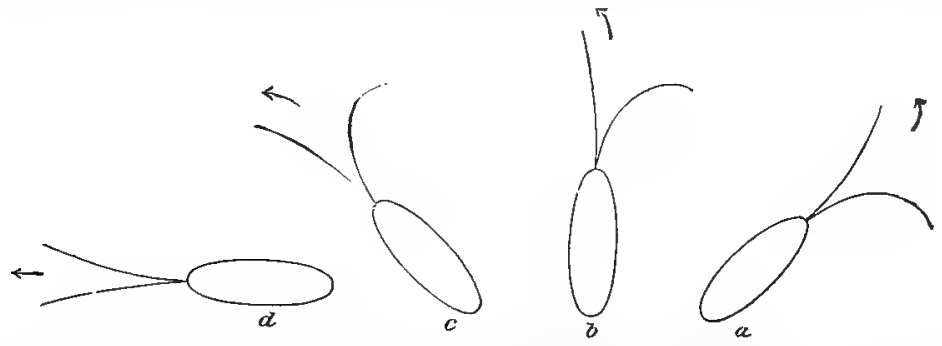

Fro. 249, - Scheme of axial orientation of a bifiagellated infusorian cell, resulting from an excitation of contraction upon the right side. The greater concavity of one flagellum indicates the stronger excitation. The arrows indicate the direction of movement.

from the source of the stimulus until its long axis is placed in the direction of the latter. The cilia then become stimulated equally upon corresponding points of the two sides of the body, and the cell swims forward in a straight direction away from the source of the stimulus. In this way negative chemotaxis, barotaxis, thermotaxis, and phototaxis occur in ciliate Infusoric from unilateral excitation of contraction.

The mechanism of axial orientation in the positively tactic movements of ciliated cells is likewise simple. Orientation in these cases can be called forth by a unilateral excitation of expansion. If such a stimulus acts upon one side, the expansion of the ciliary stroke, i.e., the return of the fligellum or the cilium to the resting-position, will then take place more energetically upon this side of the body than upon the opposite side. The result will be the reverse of that when the contraction is more energetic, i.e., the anterior end of the body will be turned toward the side of the incident stimulus, until the long axis is 
placed in the direction of the stimulus. It depends then upon the relative extent of the phases of contraction and expansion of the cilia, whether the motor effect will be so directed that the cell in taking the axial position will move toward the source of the stimulus or away from it. The axial position must always be taken, however, whether the cell moves by means of one cilium, by two, or by many (Fig. 251).

If, finally, an excitation of contraction occurs upon one side of the infusorian cell and an excitation of expansion upon the other
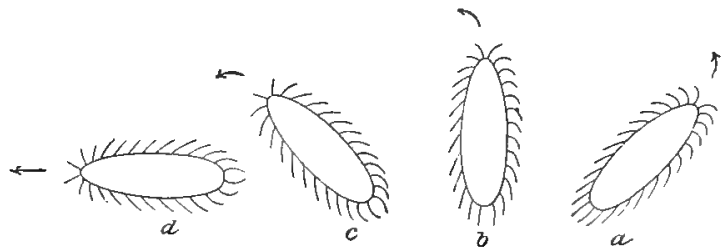

FIG. 250.-Scheme of axial orientation of a ciliate infusorian, resulting from an excitation of contraction upon the right side. The greater concavity of the cilia backwards indicates the stronger excitation. The arrows indicate the direction of the movement and are placed at the anterior pole of the body

side, it is evident that as regards the axial position of the body, the two must act in the same sense, i.e., so that the anterior end is directed toward the side of the expansory stimulus. Whether in this position a movement in one or the other direction or a standstill takes place, depends wholly upon the extent and
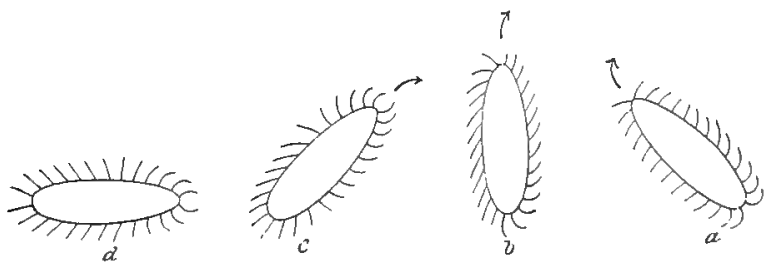

FIg. 251.-Scheme of axial orientation of a ciliate infusorian, resulting from an excitation of expunsion upon the right side. The arrows are at the anterior pole of the body and indicate the direction of movement. The greater concavity of the cilia forward indicates the excitation.

direction of the motor effect exerted by the ciliary stroke at the two ends of the body. That all these three possibilities are realised, is shown most beautifully by galvanotaxis, in which, according to the intensity of the galvanic current, swimming forward or backward, or standstill, can be obtained.

From the foregoing considerations the mechanism of axial orientation resulting from a depression of contraction or expansion upon wne side of the body may be at once understood. In such a case there exists a difference in the activity of the organoids of 
movement on the two sides of the body, and, according to known principles, this must cause a rotation upon the axis until the difference is balanced.

If we add to our first scheme the various axial positions that a cell body, differentiated as regards its poles either temporarily or permanently, may assume by reason of an excitation or depression of contraction or expansion at one pole, we obtain the following cases, in which the points of the arrows indicate the position of the anterior and of the body:

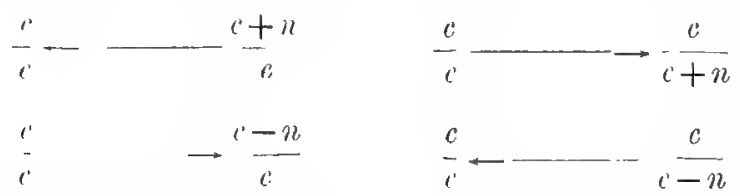

Expressad in words, this means: the anterior pole of the body turns away from the source of the stimulus with excitation of contraction or depression of expansion upon one side; and toward the source of the stimulus with depression of contraction or excitation of expansion upon one side.

Whether the cell in axial orientation moves forward or backwarl, or stands still, depends in a given case upon the relation, as regards intensity, of the phase of contraction to that of expansion in the whole ceil.

Thus the phenomena of positive and negative chemotaxis, barotaxis, thermotaxis, phototaxis and galvanotaxis which are so highly interesting and important in all organic life, follow with mechinical necessity as the simple results of differences in biotonus, which are produced by the action of stimuli at two different poles of the free-living cell.

Wo recognised with PHiger as the essential part of metabolism the continual construction and destruction of certain proteidlike compounds of rery labile constitution. Although at present any chemical characterisation of these is unsatisfactory, we termed them, in brief, biogens because of their great significance for life, and we defined the vital process in the simple schematic form: the sum of all the processes that are associated with the construction and destruction of biogens.

The non-living matter that 'nters into the living substance from outside is manufictured continually into living matter by complex transformations in the living substince; it also dies continually and is excreted as non-living matter. Thus, life consists of an eternal process of becoming alive and dying, which go on in all living substance at wery moment side by side and minterruptedly.

The sum of all processes assuciated with the construction of living substance forms the phise of assimilation, the sum of all processes 
associated with the destruction of living substance forms the phase of dissimilation. Assimilation and dissimilation are the basis of all life. Their relation to one another, which was termed biotonus, controls vital phenonema. From the beginning of development to death biotonus is constantly changing by reason of individual members of the series of assimilation or dissimilation assuming different values, and thus the details of the vital phenomena likewise change. Biotonus likewise changes if stimuli act upon the living substance, and, accordingly, vital phenomena change under the influence of stimuli. Thus, vital phenomena are determined by the individual links in the long chain of metabolism, which together form the vital process.

\section{The Mechanics of Cell-Life}

After having recognised metabolism as the elementary vital process, our present task is to derive mechanically from netabolism the vital phenomena, which must be regarded as the expression of the vital process.

It has been seen that all living substance that now inhabits the earth's surface possesses the form of cells : hence the cell is the proper seat of the vital process. In the cell the general vital phenomena are found in their elementary form. If, therefore, their mechanical analysis is not to stop half-way, the cell must be made the object of investigation. Before we can expect to derive the rarious vital phenomena of the cell mechanically from its metabolism, the question must be answered, how the metabolism of living substance, which thus far we have schematically conceived to take place in a uniform substratum, goes on in the cell which possesses the characteristic differentiations of its contents. Although with our very slight knowledge of the individual chemical processes in the cell, we are quite unable to picture in detail its more delicate metabolic mechanism, the investigations of the last decade have afforded sufficient material to give an idea of its general metabolic relations. These investigations have revealed a large number of facts, which allow a definite conclusion to be drawn regarding the much-discussed significance of the two essential cell-constitients, the nucleus. and the protoplasm, as well as regarding the nature of their relation to one another.

A. THE RÔLE OF THE NUCleUS AND THE PROTOPLAș IN THE LIFE OF THE CELL

\section{The Theory of the Dominance of the Nucleus in the Cell}

The classical researches of the earlier investigators of protoplasm, among whom may be named Dujardin and Max Schultze, were directil toward establishing the protoplasm as the bearer of all vital activities. In the older doctrine of the cell all perceptible. 
vital phenomena were regarded as taking place in the protoplasin, and wo one knew what to do with the nucleus; hence the latter was consirlered unessential and recoived little attention.

It is psychologically interesting and a characteristic phenomenom in the history of human thought that the knowlergge of the truth swings to either side of the middle point before it comes to a standstill at the latter. An wexeme view, which in the course of time proves to be untenable, cause's a swing to the opposite (xatrence, and the true medium is found only gradually by meins of a healthy reaction. Thus it wits with the cell-eloctrine.

After it was found that the nucleus undergees profound changes, esperially in the remuluetion of the coll by clivision and in the fertilisation of the orum, while the protoplasm remains apparently quict, the original idea of the dominant rile of the protoplasm changed to the opposite one of control by the nucleus. The latter regarded the nucleus as the wsential bearer of the coll-life, and the protoplasm as performing urerely an accessory function. What in the earlier cell-doetrine was ascribed exclusively to the protoplasm, in the later one was ascribed (xielusively to the nucleus. During the last few years a healthy reaction against this swing to the other extreme is beginning to make itself felt.

It is not possible to 'xamine all the individual facts, relative to the function of the nucleus and the protoplasm, that have recently been brought together. It will sutfiec to indicate some of the more important observations and experiments that have led to important deductions.

The idea that the nucleus pliys a dominant rile in the cell has obtained at the present time wide accuptance and has been expressed in various forms. One prominent view is defended by eminent investigators, such as 11 remann, Hertwig, Boveri and others, and takes special account of the remarkably complex and regular changes that the newer morphology has demonstrated in the nucleus in comneretion with the phenomena of fertilisation and division of the orum. The "ssence of this view is that the nucleus is the bearer of certain substances called hereditury sulstonces, that hereditary transmission takes place by means of a transference of these substances to the descendants, and that the protoplasm contains no substances necessary to heredity.

The fact that in the fertilisation of the ovum by the spermatozoon only a very small quintity of protoplasm is transferred by the latter to the deserndiants, since the spermatozoon comsists in by far the greater part of nuelear sulswance, indued biologists to neglect completely this smitl quantity of protoplitsm, and to ascribe the transference of the patermal charateristics to the descendants exclusively to the nuclens of the spermatozoon. This assumption appeared the more probable beciuluse the small mass of protoplasm in the spermatozoon, which is contained chiefly in the flagellum, 
cannot be distinguished from the protoplasm of the ovum after it has appeared in the latter, while the characteristic and profound changes caused by fertilisation appear in the nucleus alone. To the more critical minds, the weakness of the arguments, upon which rested the theory of the dominance of the nucleus in heredity, was painfully evident, and they sought after unequivocal proofs of the theory.

The fundamental fact, which Nussbaum ('84, '86) established in Infusoria, that non-nucleated pieces of a cell after some time invariably die, while nucleated pieces are regenerated into complete cells and continue to reproduce by cell-division, was experimentally confirmed in other Infusoria by Gruber ('85), and brought forward as a direct proof of the dominance of the nucleus. Gruber ('86, 1) says: "By a purely empirical method we are here placed before the undeniable fact that the nucleus is the most important, the species-maintaining, constituent of the cell, and that to it is rightly ascribed the highest significance in the processes of fertilisation and hereditary transmission." But Gruber forgets that, in order that the nucleus may be established as the sole species-maintaining constituent of the cell, the reverse experiment must also be made, namely, the investigation of the nucleus without the protoplasm. If the nucleus then continues to live, if it regenerates a new protoplasmic body and forms a complete individual, the experiment would be, in fact, undeniable proof of the all-important significance of the nucleus. But if it perishes without regeneration, like the protoplasm deprived of its nucleus, no reason then exists for ascribing more to the nucleus than to the protoplasm; with equal right the protoplasm could then be spoken of as the species-maintaining constituent of the cell. Such an experiment has been performed and shows that the nucleus deprived of its protoplasm perishes like non-nucleated protoplasm. In the large radiolarian Thalassicolla (Fig. 171, p. 380) the nucleus, which is visible to the naked eye, can by a skilful operation with delicate instruments be removed uninjured from the protoplasm of the central capsule, and be observed isolated. The result is that, even when it is protected from all injury, after some time it invariably dies without a trace of regenerative phenomena being seen. ${ }^{1}$ The same may be observed in Infusoria. Such a result breaks the force of Gruber's argument.

Another experiment, which is claimed to support the theory of the dominance of the nucleus, was performed by Boveri ('89) upon the eggs of the sca-urchin. In connection with the fact observed by the brothers Hertwig ('87), that non-nucleated pieces of protoplasm of the ova of sea-urchins are capable of being fertilised by spermatozoa, Boveri found that these fertilised pieces develop into dwarf larval forms, which, apart from their small size, are 
wholly like normal larvæ. Boveri employed this fact in experiments upon the cross-fertilisation of non-nucleated pieces of ova of one species of sea-urchin with spermatozoa of another species, especially non-nucleated pieces of ova of Sphoerechinus granularis with spermatozoa of Echinus microtuberculatus. He shook a number of eggs of Sphocrechinus in a test-tube, by which treatment non-nucleated pieces of protoplasm were broken off, and fertilised the shaken liquid with sperm of Echinus. It was not possible to fertilise isolated non-nucleated pieces, since cross-fertilisation between the two forms takes place relatively very seldom. Among the larvæ obtained by fertilisation were the following:

a. Bastards, such as were always obtained by the crossing of the two species.

b. Dwarf bastards, obtained by the fertilisation of nucleated pieces.

c. Dwarfs possessing genuine Echinus characters, obtained by the fertilisation of non-nucleated pieces.

According to Boveri, the occurrence of the last-mentioned larval forms is a direct proof of the theory of nuclear dominance; for, since from one species of sea-urchin only non-nucleated protoplasm from the ovum was transmitted, while from the other the nucleus of the spermatozoon, the result, namely, larvæ of the paternal form, proves that the nucleus alone can be the bearer of the hereditary substances. Critical examination, however, shows that this experiment, which thus far has been considered by many as the strongest support of the theory of dominance, appears as such inadequate in more than one respect. In the first place, the derivation of dwarf larvæ of the type of the paternal species can be doubted. Since the fertilisation of non-nucleated pieces of ova of one species with spermatozoa of the other species was not carried on isolated, it is very questionable whether the larvæ in question were really derived from such a fertilisation. It is conceivable that larvæ pre-eminently of the paternal form can develop from the fertilisation of nucleated pieces of ova or whole ova of one form with spermatozoa of the other; we see that very frequently the characteristics of either the father or the mother are transmitted pre-eminently to the offspring. But the various larval forms that Boveri obtained possess in the developmental stage in question so few distinguishing marks, that from their presence conclusions ought not to be drawn with certainty regarding the one-sided derivation. Yet, even if the explanation that Boveri gives of the derivation of the larvæ is to be accepted, the experiment is far from conclusive. It will be conclusive only when the nucleus alone, not the whole spermatozoon, unites with the non-nucleated protoplasm of the ovum. If in this case larvæ of the character of the father appear, we shall be obliged to acknowledge that the nucleus alone is the bearer of hereditary 
characters. Since, however, the spermatozoon is a complete cell possessing nucleus and protoplasm, nothing in Boveri's experiment has proved that the protoplasm also does not take part in hereditary transmission. The fact that pre-eminently or exclusively paternal characters are observed in the larvæ ought hardly to cause surprise, since upon the paternal side a whole cell enters into the fertilisation, but upon the maternal side only a bit of protoplasm, which, as is well known, is destined to die because of the loss of its nucleus, and is no longer able to preserve its characteristic features, and consequently not able to transmit them. Hence, in the light of the fundamental fact of the infallible death of non-nucleated protoplasmic masses, Boveri's view, that in his experiment maternal characteristics would necessarily have been transmitted also, if the protoplasm were to take part in heredity like the nucleus, appears unsupported. After all the above we cannot help considering Boveri's experiment wholly indifferent as regards the decision of the question whether the features of the cell that determine its character are contained in the nucleus alone.

Another form of the supremacy theory is expressed in the view of Eimer ('88), Hofer ('89-'90), and others, that the nucleus controls the vital phenomena of the cell, especially the movements of the protoplasm, after the manner of a central nervous organ. Eimer supports his view by various morphological observations, not wholly undisputed, upon the ending of nerve-fibres in the nuclei and even in the nucleoli of cells. But, even if these should really be confirmed, there would be no ground in them for ascribing to the nucleus alone the regulation of the movements of the protoplasm. Hofer believes that from experiments on Amoba he can draw the conclusion that "the nucleus is a regulatingcentre for movement." He cut the bodies of large Amœbo into nucleated and non-nucleated parts. While the nucleated parts continued to behave exactly like complete Amobce, the non-nucleated pieces showed normal behaviour for 15-20 minutes only. Then the movements became irregular, the formation of pseudopodia taking place abnormally, and finally wholly ceased. From this Hofer concludes that the protoplasm possesses the power of movement, but that the nucleus is a centre which regulates the movements. That this view cannot be held follows from the striking experiments of Balbiani ('88), who observed that under favourable conditions non-nucleated pieces of Infusoria continue to live for many days with completely unchanged movements. Finally, exhaustive experiments upon valious $R$ hisopoda and Infusoria, ${ }^{1}$ especially upon ciliate Infusoric that perform very complex and characteristic movements, hare been directed particularly toward this question, whether the nucleus ought to be regarded as

$$
{ }^{1} C f \text {. Verworn }(' 89,1) \text {. }
$$


the centre of motion in the same sense as the centres of the central nervous system in higher animals are regarded. We can present the result of these best by means of a vivisection-experiment upon Lacrymaria.

Lacrymarice olor belongs to the holotrichous ciliata and is distinguished by its very characteristic movements; from these it proves to be an exceptionally favourable object for experiment upon the influence of the nucleus upon movement. In the condition of moderate contraction it is flask-shaped and presents a trunk, neck, and head (Fig. 252, $a$ and $b$ ). When undisturbed, it is in restless motion, every portion of the cell-body taking part in its peculiar activity. The trunk undergoes constant changes of form of a peristaltic character. At times the neck extends into an extremely long and slender thread, the anterior end of which lengthens, shortens, bends about and gropes here and there between the particles of mud (Fig. 252, a), and at times it suddenly draws together like a stretched rubber cord, soon to begin its play anew. The head, provided with long oral cilia, gropes about in all directions upon objects in the water, the cilia seeming to run over them like little feet. In this way the whole protist twitches constantly forward and backward by the alternate direction of the strokes of the cilia, so that it moves very little from one place and is engaged chiefly in searching about with its long neck and head with restless eagerness. If it be stimulated, it suddenly contracts and swims in the condition of moderate contraction some distance backward, then takes a forward direction and whirls forward through the water at a furious rate, constantly turning about its axis.

The macro-nucleus with the closely applied micro-nucleus lies in the middle section of the trunk.

It is possible with some patience to separate, under the microscope, by sharp cuts the individual parts of the body, in which the head, the neck, and the posterior end-piece of the trunk are always non-nucleated, while the trunk itself contains the two kinds of nuclei. The result of the cross-section is that in every piece the ciliary motion is very much accelerated. All the pieces whirl about their axis and through the water with furious rapidity in the contracted condition. The enormous augmentation of ciliary activity gradually passes away, and then every piece behaves exactly as it behaved when in connection with the whole organism. The nucleated trunk continues its metabolic movements, twitching now forward, now backward, by changing the direction of the ciliary stroke; the neck at times stretches far out (Fig. 252, d) and gropes restlessly about, although it possesses neither head nor trunk, and at times contracts like a rubber cord (Fig. 252, c); the head, being now free from the trunk, runs about over the particles of mud in the water like an independent individual by means of exactly the same ciliary motions as in the 
uninjured organism (Fig. 252,e). In short, as regards its movements, every piece behaves exactly as when in connection with the body of the normal Lacrymaria. Upon stimulation, contraction of the myoids and acceleration of the ciliary stroke take place in

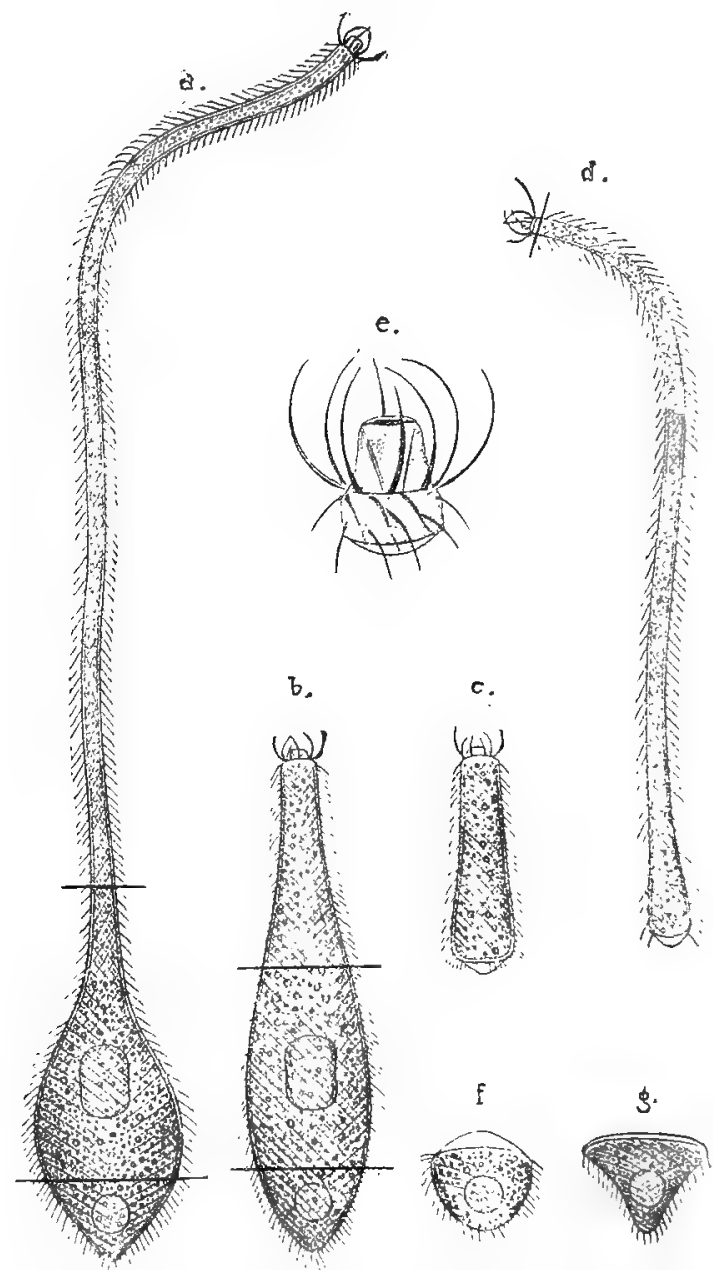

Fra. 252.-Section of Lacrymaria olor. The black lines indicate the cuts.

all pieces, the cilia causing the rotation upon the axis, exactly as happens upon stimulation in the uninjured protist. In the nonnucleated pieces, this normal motion continues, as a rule, for nearly a day. Then the difference between the non-nucleated and the 
nucleated pieces, appears, the former perishing, while the latter regenerate themselves into complete individuals.

The behaviour of non-nucleated pieces of cells may, therefore, be summarised in the statement, that, after the passage of a stage of excitation caused by the stimulation of the operation, every piece continues to carry out the movements peculiar to it in the uninjured organism and to react to stimuli in the same manner as before the operation. The normal character of the movements is not changed until the appearance of the phenomena of necrobiosis, which affect the non-nucleated protoplasm and lead to death.

It appears in all such experiments that, after the passage of a stage of excitation caused by the operation, the movements of nonnucleated pieces of protoplasm continue for a long time, frequently for several days, completely unchanged; they undergo disturbances only in the course of the necrobiosis of the piece and finally cease. The facts discovered by Hofer agree completely with this. If, however, the normal movement of the protoplasm continues for days after the removal of the nucleus, the nucleus cannot be a regulating centre for the movement, and thus the theory falls.

\section{Nucleus and Protoplasm as Links in the Metabalic Chain of the Cell}

It appears from the above discussion that the later views upon the dominance of the nucleus in the cell, in whatever form they are presented, are as little justified as the earlier ideas, which recognised the protoplasm alone as the essential bearer of life. Everything suggests that the truth lies between the two, i.e., that neither the nucleus nor the protoplasm alone plays the chief rôle in the life of the cell, but that the two are concerned equally in the inauguration of vital phenomena.

All the experiments and observations so far upon the relations of the nucleus and the protoplasm show that this view is the correct one. It would lead too far to present all the facts bearing upon this question; only the more important ones will here be noticed.

The first and most significant one is the phenomenon, already mentioned and confirmed by all past vivisection-experiments upon a great variety of cells, that after a longer or shorter time nonnucleated protoplasmic masses invariably perish, just as do nuclei deprived of protoplasm. Unquestionable proof is thus afforded that the vital phenomena of the cell come about only through the undisturbed correlation of the two parts of the cell. That this correlation is a metabolic correlation is a priori evident, since vital phenomena are merely the expression of cell-metabolism. But this fact is proved by special facts relative to phenomena that occur up to the time of death in protoplasmic masses deprived of 
a nucleus. During the frequently long time that elapses between the removal of the nucleus and the death of the enucleated protoplasmic mass, certain metabolic phenomena gradually disappear, while many activities continue even until the last moment before death. The disappearance of phenomena shows at once that by the removal of the nucleus the meta'oolism of the protoplasm has undergone a disturbance.

One group of phenomena that disappear relates to the treatment of ingested food, and may be especially well observed in the naked protoplasm of Rhizopoda. If in a Polystomella, whose delicate, snail-like, calcareous shell is filled with a protoplasmic body that is usually uninucleated, a piece of the shell containing nonnucleated protoplasm be skilfully cut off, after some time the protoplasm forms again wholly normal pseudopodia, and for some days behaves like an uninjured Polystomella. Small Infusoria, which serve the organism as food, are still caught by the pseudopodia, which latter are covered with a delicate viscous secretion; and under certain circumstances these Infusoric can even be killed by the action of the pseudopodial protoplasm surrounding them; but no digestion takes place. ${ }^{1}$ The same observation can be made readily upon large Radiolaria, like Thalassicolla, which can with ease be deprived of its central capsule containing the nucleus. After this operation the large non-nucleated protoplasmic body behaves like a complete Thalassicolla. The pseudopodia hold fast the swimming food-Infusoria and surround them with their protoplasm. The Infusoric are killed and sometimes even altered in form, but complete digestion never takes place. ${ }^{2}$ Hofer ('89-'90) observed the same thing in large specimens of Amoba. When he divided under the microscope Amobce that had devoured Infusoria, so that the latter were present in the nucleated as well as in the non-nucleated half of the protoplasm, those in the latter half underwent feeble digestion at first and then ceased to be affected, while those in the nucleated half were completely digested, as in an uninjured Amoba. It follows from all these experiments that the assimilation of ingested food ceases in the protoplasm after the extrusion of the nucleus.

As with the consumption, so the production of certain substances by the protoplasm ceases after removal of the nucleus. A nonnucleated protoplasmic mass of Polystomella no longer excretes calcium carbonate to complete its calcareous shell, while nucleated pieces repair an imperfection in the shell immediately by laying down new calcareous masses at the wounded place. ${ }^{3}$ The secretion of slime by the naked protoplasm of $A m c b a$, as Hofer ('89-'90) has shown, is not observed in non-nucleated masses; hence after enucleation such pieces float in the water, while nucleated pieces, like uninjured Amator, immediately attach themselves again to
${ }^{1}$ ('f. Verworn ('88).
${ }^{2} \mathrm{Cf}$. Verworn ('91).
${ }^{3} C f$. Verworn ('88). 
the bottom by means of the delicate layer of slime, and continue creeping. In non-nucleated pseudopodia of Diffugia at first a secretion of slime takes place, but it soon ceases, and after a few hours these protoplasmic masses likewise lose the power of attaching themselves. ${ }^{1}$ Finally, the loss of the power of producing cellulose in the formation of a cell-wall, which Klebs ('87) observed in plantcells, is a very characteristic phenomenon. In his experiments Klebs made use of the fact that harmless solutions of substances that extract water cause the protoplasmic body of the plant-cell to contract and to break up into separate protoplasmic globules, a phenomenon that is termed by botanists "plasmolysis." If he put threads of Zygnence or Spirogyra into a 16 per cent. solution of cane-sugar, the protoplasmic body of the cell in many cases broke into two or more globules, of which one contained the single nucleus. Both nucleated and non-nucleated pieces continued to live, in many cases the latter eren for six weeks. But during this time a profound difference in the two was shown: the nucleated pieces immediately surrounded themselves with a new cellulose membrane, while the non-nucleated pieces always remained naked. It follows from this experiment that the nucleus with its metabolism takes an essential part in the formation of cellulose. But the experiment is especially interesting from the fact that very recently it has received a desirable completion by another experiment, which Demoor ('95) has performed upon the cells of Spyrogyra. In a manner analogous to that in which by means of vivisectionoperations the influence of the nucleus upon the protoplasm was excluded, Demoor succeeded by means of the suitable application of various agents, such as chloroform, hydrogen, cold, etc., in bringing to a standstill the life of the protoplasm while the nucleus remained still active; in other words, the activity of the protoplasm was excluded. The result was that the nucleus remained living for a considerable time undisturbed, just as after the exclusion of the nucleus the protoplasm shows normal vital phenomena for a considerable time. In Demoor's experiments the vital activity of the nucleus expressed itself just as in the normal life of the cell, pre-eminently by the phenomena of nuclear division. The nucleus proceeded to divide as normally, and to form the well-known complex mitotic figures, and soon two nuclei appeared and separated from une another. ${ }^{2}$ While, however, in the undisturbed cell upon the separation of the two nuclei in the protoplasm a new cellulose membrane is always formed immediately, completing the division of the whole cell into two daughter-cells, in Demoor's experiments the formation of such a membrane was invariably absent, although the nucleus still con-

1 Cf. Verworn ('90, 1).

" [Cf. hereupon Loeb and Hardesty ('95), Loeb (95; '96, 1), Morgan (96), and Norman ('96). ] 
tinued to show its normal vital phenomena. While, therefore, the experiments of Klebs prove that the nucleus is necessary for the formation of cellulose, those of Demoor show that the protoplasm also takes part in its production. In other words, cellulose can be formed only by the combined action of nucleus and protoplasm.

Besides these experimental results, a considerable number of morphological observations upon very different kinds of cells exist, all pointing toward an active exchinge of substance between nuclens and protoplasm. Of great interest are the positions of the nucleus relative to certain substances that are produced or taken in by the cell, which have been demonstrated by Haberlandt upon plant-cells and by Korschelt upon animal-cells. Haberlandt's investigations ('87, '89) have reference to the phenomena of growth of the cell-membrane. In a wide range of material he has established the fact that in certain cases the nucleus exists at the place at which growth-processes are localized. Such cases are the following: where local growth of the cell-wall is necessary to its final form, such as in the thickening on the

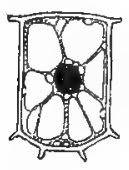

$A$

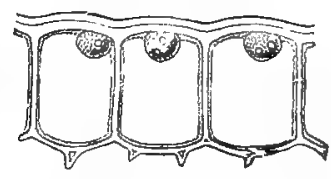

$B$

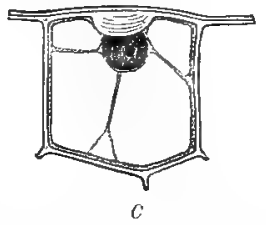

Fıa. 253.-A, An epidermis-cell of a foliage Ieaf of Lvzula maxima. The nucleus lies in the middle of the cell. $B$, Epidermis-cells of a leaf of Cypripedium insigne. The upper cell-wall is thickening; the nuclei lie upon it. C, Epidermis-cell of a leaf of Aloë verrucosc. A swelling is being formed upon the upper cell-wall; upon it lies the nucleus. (After Elaberlandt.)

outer side of epidermal cells, in the formation of ridges upon the guard-cells of stomata, and in the rudiments of root-hairs that develop by apical growth at the growing point of roots; and where regeneration of an artificially injured cell-wall takes place; in brief, wherever a special development of material for the cellwall takes place (Figs. 253 and 254). But before the beginning and after the cessation of these various phenomena of growth the nucleus takes no definite position in the cell (Fig. 253, A).

These comprehensive observations of Haberlandt are paralleled by the striking zoological researches of Korschelt ('89). Korschelt has studied chiefly the ova and secreting-cells of insects. In the egg-tubes of the ovaries of Dytiscus marginalis, a large water-beetle, the ova are arranged in succession like a string of pearls and separated from one another by a so-called nutrient chamber. This chamber consists of cells which produce and give off nutrient material to the ova. The behaviour and the position of the nuclei of the ova toward this nutrient material is very characteristic (Fig. 255). From the chamber the nutrient material extends into the 
ovum in the form of a granular mass and there disposes itself in such a manner that it comes into very close contact with the nucleus. But the most interesting fact is that which makes the activity of the nucleus toward the nutrient material apparent, namely, that the former sends pointed, pseudopodium-like processes into the granular mass where the latter touches it, and only in this direction, and thus very greatly increases its surface at the place of contact with the nutrient material. If the latter completely surrounds the nucleus, the whole surface shows pseudopodium-like processes. Korschelt describes a similar phenomenon, especially as regards the nucleus, in a whole series of arthropod and colenterate ova. The interesting behaviour of the nuclei in secreting - cells toward the secreted substances forms a counterpart to these phenomena of the ingestion of substance on the part of the nucleus. Here certiain relations exist toward the substances produced, which are wholly analogous to those existing in ova toward ingested substances. In the eggs of certain waterbugs, Nepa and Ranatra, there occur peculiar chitinous appendages, the so-called eggrays, which are formed by cells especially differentiated for this purpose. These cells, of which each two unite into a single cell with two nuclei, termed by Korschelt a double cell,

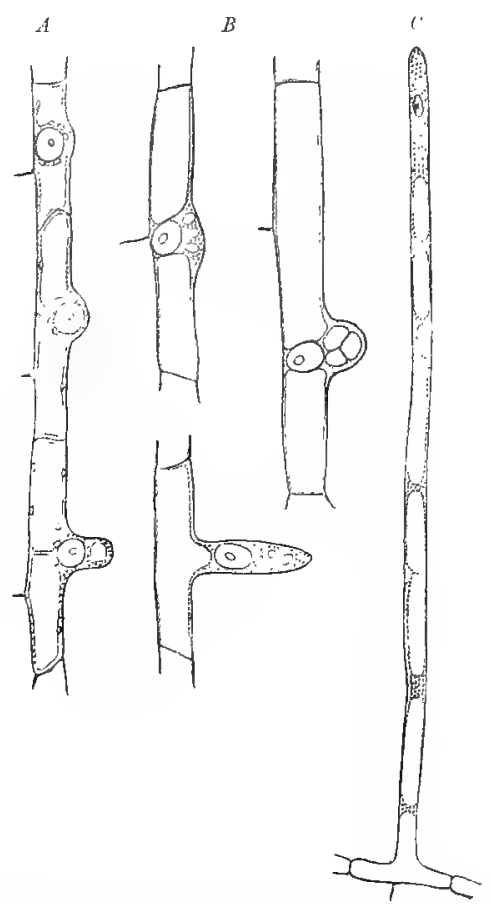

FIG. 254.-A, A row of cells from a root of Pisum sativum. Upon the right side three stages in the formation of a root-hair are shown; the nucleus lies at the places in question. $B$, Three cells from the root of Cucurbita pepo. A root-hair is beginning to form upon each cell ; the nucleus lies at the place where the hair projects. $C$, Root-hair of Cannabis sativa. The nucleus lies at the tip of the hair, where the growth is taking place. (After Haber. landt.) assume a considerable size and secrete within their body the mass of chitin. The behaviour of the two nuclei in this process is very characteristic (Fig. $256, I)$. They send out toward the middle, where the secretion is taking place, numerous, frequently branched, pseudopodium-like processes, which increase the nuclear surface upon this side very considerably, while the rest of the surface remains smooth. Such enlargements of the surface of nuclei are wide-spread in the secreting- 
cells of insects (Fig. 256,II), and show that the exchange of substance between protoplasm and nucleus in secretion must be very active. Corresponding to this is the fact observed by Heidenhain ('83), that the nuclei of the cells of the salivary glands behave essentially differently in the state of rest and that of extreme secretion; in rest they send out pointed processes into the surrounding protoplasm, while after continued stimulation they are spherical and possess a smooth surface (Fig. 257). Further, Baum ('86) has found that the nuclei of resting gland-cells stain much more deeply with nuclear stains than the nuclei of gland-cells that have secreted strongly - a sign that the chromatic nuclein must be destroyed in secretion. Lily Huie ("97) has also recently discovered very profound changes in the

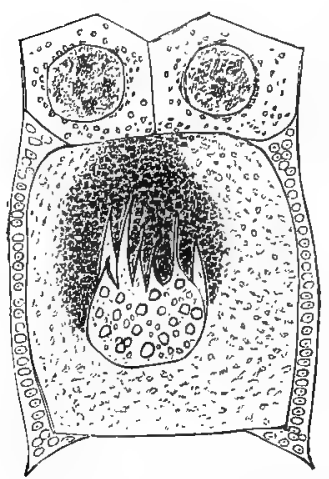

FIG. 255.-Ovum of Dytiscus marginalis with two nutrient cells above it. Nutrient material is passing from the nutrient cells into the ovum, and the nucleus of the latter is sending out pointed pseudo. podia toward this material. (After Korschelt.) nucleus during increased activity of the cell in the secreting-cells of the insecteating marsh-plant Drosera, when the latter is fed with egg-albumin. Greenwood ('96) has likewise observed in the colonial infusorian Carchesium nuclear changes which develop parallel with the nutrition of the cell. The phenomena of fatigue which Hodge, Lugaro, Mann and others have observed in ganglion-cells during excessive activity and with which we have already become acquainted, ${ }^{1}$ belong in the same category; and the same is true of O. Hertwig's observation ('84) that in eggs that are rich in yolk, the nucleus always moves toward the place where there is the greatest accumulation of protoplasm. Finally, in a wide variety of cells a remarkable change in the size of the nucleus during cell-life ${ }^{2}$ can be observed, which can be brought about only by the nucleus receiving substances from the protoplasm and giving off others to it.

Usually, on account of the existence of a nuclear membrane, an exchange of liquid substances only is possible between nucleus and protoplasm. But in many cases where because of the failure or disappearance of such a membrane solid masses may be exchanged, many observers, such as Frommann, Auerbach, Leydig, Brass, Stuhlmann, and others, have observed on the part of the nucleus a direct ingestion or extrusion of granules and flakes. In certain stages in the course of development of many cells there even occurs regularly a disintegration of the nucleus into many small particles, which are resorbed by the protoplasm. Thus, we recall the be-

$$
{ }^{1}(\% \text {. p. } 464 .
$$$$
\text { " ( } f \text {. Sichwarz }(' 84,2) \text {. }
$$ 
haviour of the nuclei observed br R. Hertwig ('88-'89) in the conjugation of ciliate Infusoria : ${ }^{\text {T }}$ the fairly large macronucleus breaks up into numerous separate particles and becomes completely re-
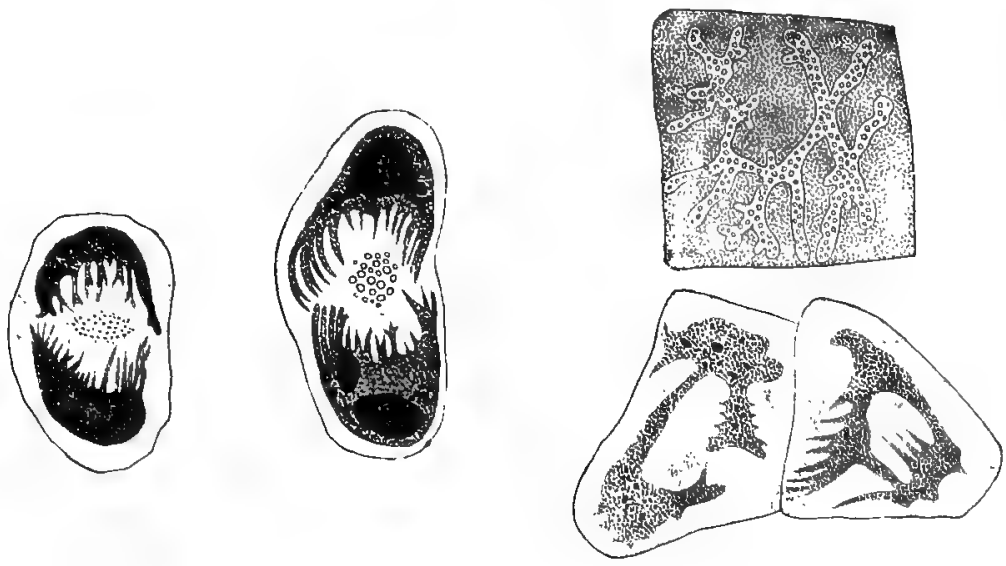

$I$

II

Fra. sin.-Form of the nucleus in secroting-cells. I, Double cells with two nuclei from the eggfollicles of Nepa rinerer. Between tho two nuclei a mass of chitin is being secreted for the formation of an egg-ray; the nuclei have extended pseudopodia toward this side, so that the secreted (gravular) mass is circularly onclosed by the nuclear pseudopodia. $I I$, Secreting*cells from the spinning glands of caterpillar's. (After Korschelt.)

sorbed; the micronucleus grows by the ingestion of substance, differentiates, divides, and gives rise to a new micronucleus and a
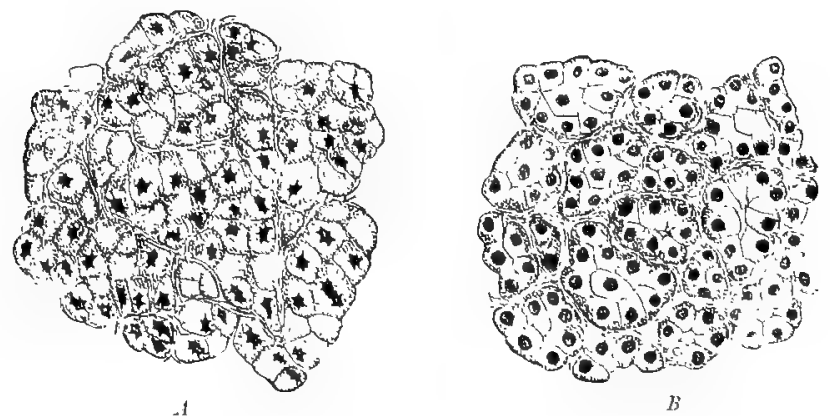

Fra. 25T,-Cells of snlivary glands. $-t$, At rest. The nucloi are star-shaped. $b$, After stimulation of the glind. The nuclei are round. (After Hoidenbain.)

new macronucleus, which undergo a rery considerable increase in size.

It is unnecessary to cxtend still farther the enumeration of facts. ${ }^{1}(j)$ p. 2011 . 
From the experiments and observations presented it is evident that between the protoplasm and the nucleus a mutual exchange of substance takes place, without which neither of the two parts of the cell can continue to exist. In other words, both nucleus and protoplasm take part in the metabolism of the whole cell and are indispensable to its continuance.

\section{B. DERIVATION OF THE ELEMENTARY VITAL PHENOMENA FROM THE METABOLISM OF THE CELL}

\section{The Mechanics of Cell-metabolism}

\section{a. Scheme of Cell-metabolism}

With the inclusion of the nucleus and the protoplasm in the metabolic chain the mechanics of cell-metabolism assumes extraordinary complexity. With our lack of knowledge of the special chemical processes in living substance we can at present form no idea of the metabolism of the biogens in the type of cell possessing a differentiated nucleus and protoplasm, nor of the share taken by the two parts of the cell in the various components of this metabolism; nor do we even know whether we must not assume the existence of a large number of different kinds of biogens in the nucleus and in the protoplasm, whose metabolism is closely interwoven with one another. Although we must leave the solution of all these questions to the future, upon the basis of our present knowledge we can at least form a picture of the great complexity of the metabolic machinery by means of a graphic scheme showing the mutual relations of the surrounding medium, the protoplasm and the nucleus.

For this purpose it is necessary first to become acquainted with certain new facts. Although the non-appearance of certain phenomena in non-nucleated protoplasmic masses on the one hand, and in nucleated cells with the protoplasm excluded on the other, shows that as regards many metabolic processes the nucleus and the protoplasm are greatly dependent upon each other, with reference to many other phenomena there appears from some experiments a certain independence of the two. For example, certain processes continue in the protoplasm for a considerable time even after the removal of the nucleus. Among these processes two groups may be distinguished. First, since the nucleus is continually giving off substances to the protoplasm, there always occur in the latter certain quantities of those substances, which we shall term in brief nuclear substances, which camnot be removed with the removal of the nucleus. Hence certain processes, for the occurrence of which the nuclear substances are absolutely neccssary, are still able to continue at the expense of those present in the protoplasm since a time previous to the enucleation. 
They cease only with the consumption of these substances. These processes constitute the first group. Those constituting the second group are not immediately dependent upon the presence of nuclear substances. That such processes exist is shown by the observations made by Klebs ('87) in plasmolysed cells of Spirogyra, which Gerassimoff ('92) has completely confirmed. W'hen by plasmolysis in a 16 per cent. solution of cane-sugar Klebs caused the cells of a thread of Spirogyra to break up into protoplasmic lumps, bits of protoplasm frequently appeared that possessed shreds of the chlorophyll-band but no nucleus. By employing narcotics upon cells of Spirogyr'c that were undergoing division Gerassimoff ('97) has recently obtained cells completely destitute of a nucleus. Under favourable conditions these non-nucleated masses of protoplasm in Klebs's experiments continued to live for weeks. As has already been seen, in contrast with nucleated masses they had lost the power of forming a new cellulosemembrane. But they exhibited other vital phenomena unchanged. For example, when put into the dark, they consumed completely the starch that was contained in them, and, when in the light, they formed new starch in case they still possessed chlorophyll. In other words, the synthesis of starch from carbonic acid and water, and the further consumption of starch is in a certain degree independent of the influence of the nucleus. We say " in a certain degree," for, if the non-appearance of other phenomena caused by the removal of the nucleus has reached a certain extent, evidently the starch-building chlorophyll-bodies will share in the disorder; they will experience changes, will form starch no longer, and will finally perish. In the case above mentioned this came in relatively late, frequently not until after several weeks. In so far, therefore, as the metabolism of the chlorophyll-bodies is disturbed along with the disturbance of the whole metabolism by the removal of the nucleus, the formation of starch is in a certain sense, but only indirectly, dependent upon the nucleus.

While non-nucleated masses of protoplasm in plant-cells, in case they still possess chlorophyll, split up carbonic acid and produce starch synthetically, they are also able to respire for a long time. The proof of the fact that respiration continues in such pieces to the same extent as in nucleated pieces or whole cells, is afforded by the following experiment. ${ }^{1}$ We place in a pendent drop in Engelmann's gas-chamber" a number of nucleated and non-nucleated pieces of Inflewria together with uninjured individuals, and let a stream of washed hydrogen pass through the chamber from a Kipp's apparatus (Fig. 258); in a short time this forces out the air contained in the chamber. As a rule after five or ten minutes we see the non-nucleated and the nucleated pieces and the uninjured Infusoria begin to undergo granular disinI Cf. Verworn ('91).

2 ( $f$. p. p. 283. 
tegration. If then the hydrogen be very rapidly displaced by fresh air, it is frequently possible to prevent complete disintegration, which otherwise invariably appears after a short time. It follows from this that in non-nucleated protoplasmic masses, oxidation processes take place as in nucleated masses and uninjured cells. Respiration, therefore, is in a certain degree independent of the influence of the nucleus. This is completely confirmed by the experiments of Demoor ('95), who put the cells of Spirogyra into pure hydrogen and found that the protoplasm soon suspended ail

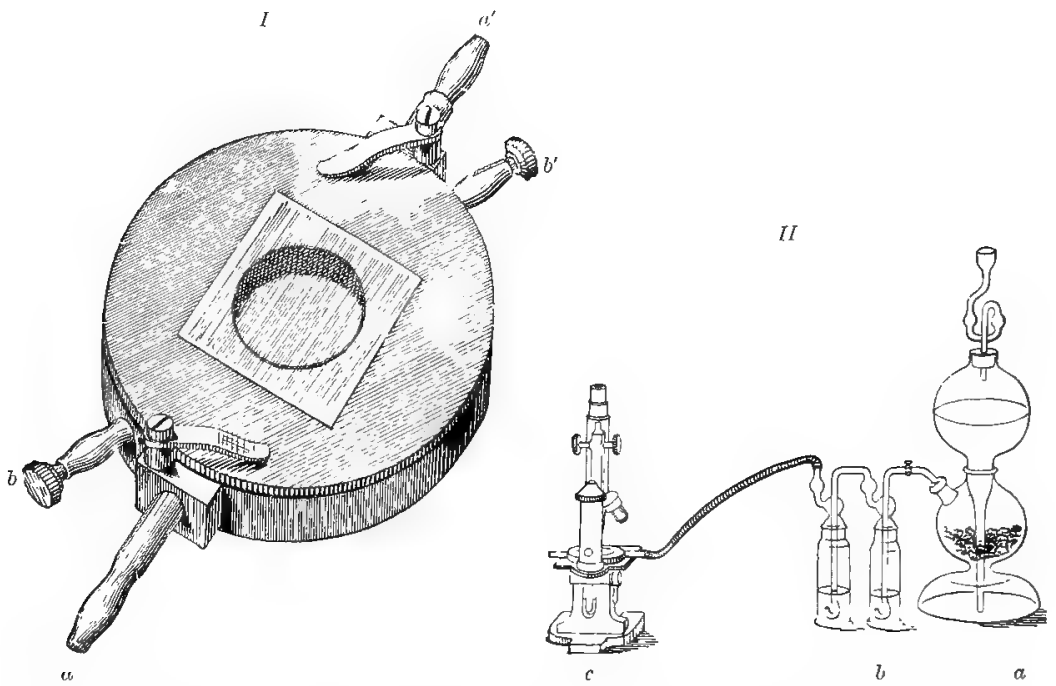

Fig. 258.-I, Engelmann's gas-chamber. An annular space is closed below hr a glass plate and above by a metal cover, the latter having in its middle a cover-glass for the examination of a pendent drop; $a a^{\prime}$ are tubes which open into the cavity of the ring and serve for heating the latter by conveying warm water through it; $b b^{\prime}$ are tubes which open into the glasscovered chamber and serve for the passage of gases; the drop suspended upon the coverglass with its living contents is bathed by the gas in the chamber. 11 , Arrangement for investigation in puxe hydrogen. $a$, Kipp's apparatus for the production of h5drogen; $b$, two wash-bottles for purifying the hydrogen; $c$, microscope upon which is the gas-chamber containing the pendent drop.

vital phenomena, while the nucleus showed no disturbance and quietly proceeded to divide. It appears, accordingly, as if respiration were localized exclusive ${ }^{\mathrm{l}} \mathrm{y}$ in the protoplasm, as if the nucleus took no direct part in the oxidation-processes.

In view of these discoveries in non-nucleated masses of protoplasm, it would be desirable to perform analogous experiments upon nuclei deprived of protoplasm, in order to tind out whether certain metabolic processes continue undisturbed after the exclusion of the latter. But the decision of this question is beset with great difficulties, for the simple reason that it is not easy to find 
in the nucleus any outwardly visible evidences of its metabolism. Nevertheless, it follows clearly from Demoor's experiments that the nucleus also exhibits vital phenomena after the exclusion of the protoplasm. Demoor narcotized cells of Spirogyra with chloroform so that the protoplasm was completely paralysed, and found that, notwithstanding, the nucleus went through all the stages of division undisturbed, and showed the characteristic changes that it exhibits in an uninjured cell in division. In the leucocytes of the frog the nucleus has the power of amœboid motion, and Demoor was able to paralyse the protoplasm by the use of chloroform without interrupting the movements of the nucleus (Fig. 259). These discoveries show that individual processes take place in the nucleus in a certain measure independent of the influence of protoplasm. Naturally it cannot be decided at present whether these processes continue only because after the exclusion of the protoplasm there are still contained in the nucleus protoplasmic substances which must be consumed before the processes in question cease, or whether the latter are not directly dependent upon protoplasmic substances. Possibly both

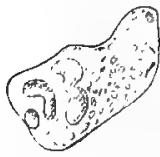

a.

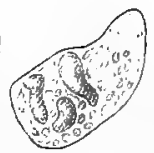

b

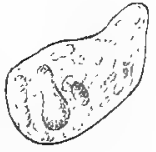

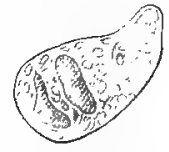

r

FIG. 259.-Leucocyte from the frog in a state of chloroforn. narcosis; the protoplasm is completely paralysed, while the nucleus still makes amoboid movements. $C f . a, b, c$, and $d$. (After Demoor.)

cases are here realized; this appears very probable when it is borne in mind that the nucleus has direct metabolic relations with the external medium, without the mediation of the protoplasm. Without doubt there are substances that pass from the external medium through the protoplasm unchanged into the nucleus, to be employed there for metabolism. This is certainly the case with water in a cortain quantity, which is absolutely necessary to every vital process. The water is able to diffuse continually through the cell-membrane into the protoplasm and through the nuclear membrane into the nucleus. It is possible that along with the water many substances which are dissolved in it also come from the outside into the nucleus to engage there in chemical transformation.

Finally, it may be assumed that all the substances that the nucleus gives off are not employed by the protoplasm for transformations, but that some pass unused through the protoplasm and are transferred to the outside.

In order to obtain a clear idea of how closely and firmly the nucleus is interwoven into the metabolism of the cell, and what 
complications are caused in the latter by the introduction of the nucleus into the chain, it will be advantageous to bring together the experimental facts in a schematic form, such as is shown in the accompanying Fig. 260. ${ }^{1}$ This represents a cell containing a nucleus, and each arrow signifies a quantity of subsiances upon their pathway through the metabolic circulation.

The cell receives certain substances from the outside; of these some (a), upon meeting substances already present in the protoplasm, undergo decomposition and syntheses. Of the substances resulting from these transformations some $(b)$ are at once excreted as useless, others $(c)$ remain in the protoplasm and are there

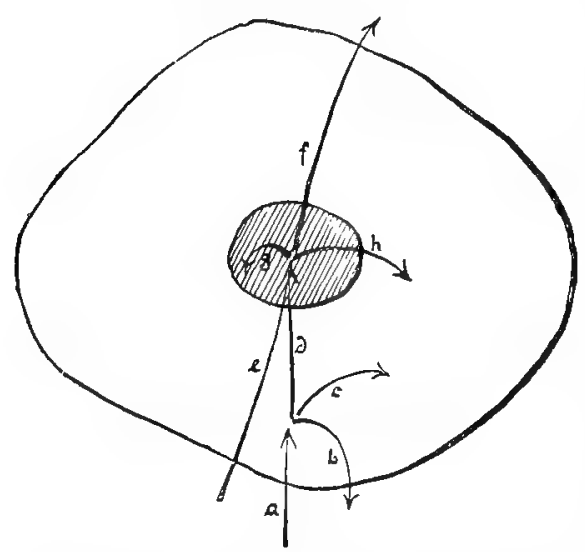

FIG. 260.-Scheme of cell-metabolism. The arrows indicate the direction of movement of substance. employed further, while a third class $(d)$ is passed on to thenucleus. Thenucleus; moreover, obtains a portion of the substances (e) received from the outside and passed on unchanged through the protoplasm. The substances $(d+e)$ entering into the nucleus there undergo on their part certain transformations, from which again substances result; these in part $(f)$ are given off to the outside without being changed by the protoplasm, in part $(h)$ pass to the protoplasm to find there further employment, and in part $(g)$ remain in the nucleus itself.

If, now, we realize that every arrow represents a sum of substances, that the substances passing from the nucleus to the protoplasm undergo transformations as well as those entering from the outside, and that the substances arising from these transformations are in part conveyed again to the nucleus, we obtain an approximate idea of how close the metabolic connection of the nucleus with the protoplasm is.

Further, it should be remembered that in all the above considerations, nucleus and protoplasm represent a great sum of different, in many cases even morphologically different, bodies, that in the conception "nucleus" are comprised all forms termed accessory nuclei, micronuclei, etc., and that by "protoplasm" there is understood the whole sum of the various products of differentiation, even chlorophyll-bodies. Only when we consider that all the various constituents of nuclear substance and, likewise, the 
granules, chlorophyll-bodies, etc., of protoplasm share, at least temporarily, in the metabolism, do we obtain an approximate idea of the complexity of the cell-metabolism and of the endlessly multiform relations in which nucleus and protoplasm are united.

A far-reaching correlation between the individual elements of the cell, especially between the nucleus and the protoplasm, follows from these close metabolic connections. The one is conditioned by the other. One is dependent upon the substances that the other produces. Thus the profound changes are explained that the life of the cell experiences when the individual links of the great metabolic chain are changed, whether spontaneously in the course of development or as a result of the action of external stimuli. Every change of one biotonic link brings about a change of many others and, if for any reason one drops out, the metabolic chain is broken, and necrobiosis, which finally ends in death, begins.

\section{b. The Mechanics of the Ingestion and Output of Substances}

There now remains the question of the mechanics of this involved process of cell-metabolism. Since the metabolic relations between the nucleus and the protoplasm, like those between the whole cell and the medium, are based upon the ingestion and output of substances, this question may be simply comprised in the problem of the mechanics of these processes on the part of the cell.

It is advantageous to consider separately the cells that receive and give off substances in solution only, and those that receive and give off solid substances also.

For a long time the processes of exchange of dissolved substances between the cell and the surrounding medium, both resorption and secretion, were regarded as conforming directly to the laws of filtration and diffusion. But recently attention has been directed to various facts which prove that in most cases filtration plays no rôle at all in these processes, and also that diffusion or osmosis alone is not sufficient to explain them. Especially from the later observations of Heidenhain ('94) it is known that the vital process in the cell itself plays the most important rôle in the exchange; diffusion alone is unable to explain, c.g., the propelling power with which the secretion is extruded in many cases from glandcells, or the considerable energy with which certain food-stuffs are taken up by the intestinal epithelium-cells. Hence, in explaining the mechanics of resorption and secretion the two factors, diffusion and the chemism of the cell, must be taken into consideration.

By diffusion or osmosis is understood, as is well known, the fact that two different gases or liquicls which are miscible will mix with one another spontaneously into a homogeneous mass, when they are brought into contact. As a rule, the word diffusion is 
employed when the gases or liquids are in direct contact, and osmosis, when they are separated from one another by an organic membrane. We have already become acquainted with this process. If we recall the experiment that illustrated osmosis (p. 104) and vary it somewhat, we can at the same time make clear the great importance which the second factor, the chemism of the cell, possesses in the presence of diffusion or osmosis. If in the larger vessel (Fig. 261) there is a diffusible salt solution, and in the cylinder the solution of a substance that does not diffuse, a certain quantity of salt will diffuse out of its solution into the liquid of the cylinder, while no substance can pass from the cylinder into the larger vessel. If, however, the substance in the cylinder has a chemical affinity for the salt, the salt diffusing into the cylinder goes into chemical combination at once. If the chemical compound thus arising be continually removed and replaced by new solution of the same kind

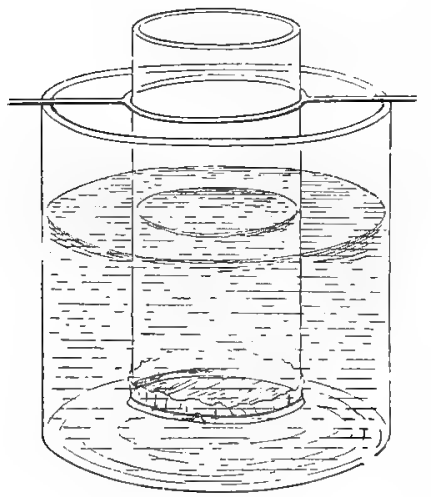

FIG. 261.-Dialyzer. as before, the salt solution in the larger vessel will become constantly weaker and weaker, until finally all the salt has diffused into the cylinder, has become combined and is removed, so that in the large vessel there is nothing but water.

This case is realized in the process of taking in gaseous and dissolved substances by living substance. The living substance is capable of mixing with the gaseous and dissolved food-stuffs, for it has a chemical affinity for them. The cell-membrane, if such be present, represents the membrane of the cylinder; the cell-contents, the contents of the cylinder; and the gaseous or dissolved substances, the salt solution of the larger vessel. These substances must be diffusible, if they are to be taken in; nevertheless, the living substance cannot diffuse through the cell-membrane, since the proteids, etc., belong to the so-called colloid substances. Hence the food-stuffs will pass into the cell, but the living substance cannot pass out. Since the latter has a chemical affinity for the food-stuffs, it must enter into combination with them immediately after their entrance into the cell. But it is continually decomposing, giving off substances to the outside, and reforming; in other words, the food-substances taken in are constantly being consumed, so that a continual balance between the inside and the outside can never take place, and new masses must constantly diffuse in. The output of substances must take place in an analogous manner. Let us, then, imagine a cell, surrounded by a membrane, existing in 
a mediuı that contains food-stuffs, such as a bacterium in a nutrient liquid. By means of osmosis and the chemism of the cell the constituents of the nutrient liquid that are miscible with the cell-contents and do not possess too large molecules must pass through the cell-wall into the cell-substance and, vice versa, the constituents of the cell-substance that are miscible with the nutrient liquid and are able to pass through the cell-wall, must go out of the cell into the nutrient liquid. This exchange goes on in so far as the substances in question are not held fast by other forces upon the one or the other side. Theoretically, it will necessarily continue until a balance as regards the substances capable of transportation is struck between the cell-contents and the medium, when the metabolism will necessarily cease. But in the living cell such a condition is never reached, since compounds there exist which are continually decomposing and building themselves up anew. On the one hand, the substances received by the cell from the medium are always consumed at once and transformed into other compounds; and, on the other hand, those that the cell gives off to the medium are constantly being formed. Hence the exchange between cell and medium must continue as long as the cell is still capable of taking up food-stuffs in sufficient quantity from the medium and of giving off excretory substances in sufficient quantity to the medium. If, therefore, the mass and character of the medium are fixed, and not changed from the outside, after some time the cell must perish; this will occur either when the food-stuffs contained in the medium are consumed, or when the latter is so saturated with excretory substances, that the output of them by the cell has diminished or ceased. It is very easy to produce both cases experimentally in cultures of Bacteria. The bacteria die either from lack of food or from the accumulation of the products of their own metabolism, because the osmotic exchange of substance between bacterium-cell and nutrient liquid gradually ceases through the gradual equalisation of the substances belonging to the two.

In many cases the mechanism of exchange between cell and medium is more complex. If, for example, the nutrient substances in the surrounding medium are not present in a diffusible form, i.e., if they are either solid or possess so large molecules that they are unable to pass through the pores of the cell-wall, they must be made soluble and diffusible. This is performed through the action of ferments which the cell produces and in many cases gives off to the outside. In contact with these ferments, the polymeric molecules of proteid, of gelatine, of starch, etc, and solid masses of these substances become split up and brought into solution, and they are then able to diffuse into the interior of the cell. This process may be followed very easily in bacterial cells. If a bacterial cell be placed upon a glass plate covered with solid nutrient. 
gelatine, the cell begins gradually to liquefy the gelatine in its vicinity, i.e., to bring the solid substance into solution, and from the accumulation of liquid thus arising and surrounding the bacterium, the dissolved nutrient substances are able to diffuse into the cell. In cells possessing a naked protoplasmic surface and no cell-wall extracellular digestion is naturally not required, because the food-stuffs, even when they cannot diffuse, are able to come into chemical relation with the surface of the protoplasm directly.

These considerations allow us to form a general idea of the mechanics of the process by which resorption and secretion in the living cell are able to go on continually and automatically. The great quantity of energy that is involved in the two can also be understood, if the chemism of the living cell be taken into sufficient consideration; for, if very strong chemical affinities for foodstuffs exist in a cell, and if a very active transformation of substance takes place, it is then very easily conceivable that the great amount of chemical energy can lead to the performance of very considerable work. Special cases are still puzzling; but the solution of these cases belongs elsewhere.

While, as may be supposed, the exchange of dissolved substances between cell and medium is based upon the same principle in all cases, that of solid substances is performed in very different ways in different cases. The only thing common to all these latter is the fact that the exchange is mediated solely by active movements of the cell in question, but in different cases these movements are influenced in very different ways by the food. The ingestion and output of solid substances is not wide-spread and occurs, indeed, only in naked protoplasmic masses, such as Rhizopoda and leucocytes, and in Infusoria in so far as they possess a special mouthopening. In many Infusoria and especially in those that lead a sessile life, such as Stentor and Vorticella, the ingestion of food appears to be left solely to chance, which occasionally leads small free-swimming food-particles, such as Alga-cells, swarm-spores, Bacteria, etc., into the region of the lively whirlpool that is produced by the circlet of cilia upon the peristome. This whirlpool, while capable of being influenced in direction by changes in the action of the cilia, is so regulated at the time of food-ingestion that it leads directly into the mouth-opening of the cell-body. Free-swimming Infusoria and most naked protoplasmic masses seek solid food. Either they are attracted from a distance by chemical stimuli which go out from the masses of food by the diffusion of certain substances, or they are led to take up the food by mechanical stimulation through direct contact with the food-masses. In the former case the ingestion of food is the result of a positive chemotaxis, since the cell moves toward the source of the chemical stimulus, and its protoplasm comes into very close connection 
with the substance in question; in the latter case a positive thigmotaxis exists, since the cell endeavours to extend as much as possible the surface of contact with the food-body and surrounds the latter with its protoplasm. These two factors are very frequently united. But the ball of food is always surrounded upon all sides by the protoplasm, if it has come into contact with the latter, whether upon the surface of a naked protoplasmic body, or in the mouthopening of an infusorian cell. The process of surrounding it is explained very simply from the expansory effect which the stimulus of the food-ball exercises upon the protoplasm; if the surface of the latter rises up around it, it must finally be surrounded by the protoplasm. The ingestion of solid food, therefore, finds its explanation in the mechanism of chemotactic and thigmotactic reactions; we have already become acquainted with these in detail elsewhere. ${ }^{1}$ How solid substances are given off is still little understood. This appears to be left more or less to chance. At least this is the impression obtained from Amoba. As a rule the solid substances lie in vacuoles, and, if by the continual movement of the protoplasm the racuole is brought close to the surface, the thin wall that separates it from the surrounding medium occasionally breaks, and the contents are set free. But perhaps stimuli of some sort coming from the excreted particle are necessary for this rupture of the wall. The questions whether the removal of the excretion through the anal opening, which occurs in infusorian cells, likewise depends upon stimulation, must be left until the process has been studied more in detail.

A remarkable phenomenon, which has often been cited as affording special difficulties to a mechanical explanation, is the so-called selection of food on the part of certain cells, i.e., the fact that certain cells take up only certain substances among all those available. ${ }^{2}$ Thus, regarding the seeking of Spirogyra-threads by Vampyrella Spirogyrae and the selection of fat-droplets from the intestinal contents by the intestinal epithelium-cells, Bunge ('94) says: "No chemical explanation of these phenomena is conceivable." But why this should be so is not easily understood. If in these apparently isolated phenomena the fact upon which they are based is clearly understood, i.e., that every cell takes up certain substances and not others, the action of the cells is self-evident. Every cell has its characteristic composition and its own peculiar metabolism. Is it not then comprehensible that only those substances are drawn from the medium into the metabolic circulation of the cell that have chemical relations with the constituents of the cell-body and are necessary to the maintenance of metabolism, while others which have no such relations with the living substance and are indifferent to the cell, are not taken up and, when free locomotion is possible, are not sought out? The principle upon which this phenomenon
1 Cf. p. 498.
${ }^{2} C f$. p. 146. 
is based is evidently the same as that which controls in general atoms and molecules, namely, affinity. It is surely no less wonderful that an atom of phosphorus unites very easily with an atom of oxygen, but not with an atom of platinum, than that an intestinal epithelium-cell takes up fat-droplets but never pigment-granules. And it is no less comprehensible that a Vampyrella surrounds with its body-protoplasm and digests only Spirogyra threads and no. other bodies, than that a drop of rancid oil, as Cad ('78) has shown, sends out amoeboid processes to an alkaline liquid and uses the alkali for the manufacture of soap, but is inactive toward an acid liquid. But the behaviour of the Vampyrella and the intestinal epithelium-cell is by no means peculiar, every living cell behaves. similarly. Every tissue-cell in the human body takes up from the common nutrient liquid, the blood, certain substances only, as is evident from the fact that gland-, muscle-, and cartilage-cells, produce wholly different and characteristic substances. In this respect, as Haeckel ('66) has already emphasised, the cell behaves. exactly like a crystal, for example a crystal of alum, which out of a. mother liquor containing numerous salts in solution always selects alum molecules only, in order to employ them for its growth, or, if it has been injured, for its regeneration. Thus the mystical obscurity that some investigators have endeavoured to wrap about the so-called selection of food-stuffs on the part of the individual cell does not really exist. What has been called by the anthropomorphic term the "selection of food" by the cell is an absolutely necessary consequence of the fact that the living substance of every cell possesses its own specific composition and its own characteristic metabolism.

Thus, the phenomena of cell-metabolism may all be referred to chemical and physical principles, as they are found in inorganic nature, and although at present we are unable to trace in individual cases the finer details of the special metabolic processes, we are certain that the whole metabolism comes about in a purely mechanical manner, and that phenomena are never met with that cannot be explained mechanically. There can evidently be no exception to the conclusion that everything that consists of matter must obey the laws of matter.

\section{The Mechanics of Changes of Cell-form}

Although in the present condition of our knowledge of cellprocesses, we do not know what special share in the whole metabolism is taken by the individual constituents of the cell, with what chemical processes in the history of the biogens the nucleus and the protoplasm with their specific constituents are associated, our discoveries so far regarding the general metabolic relations in the cell are sufficient to enable us to recognise that the phenomena 
of change of form, i.e., the phenomena of growth, reproduction, development, and hereditary transmission, may also be derived as mechanical consequences from these metabolic relations.

\section{a. Growth as the Fundamental Phenomenon of Change of Form}

Growth constitutes the fundamental phenomenon of changes of form in organic nature, for not only is growth of the cell the simplest case of change of form in general, but at the same time, as the following considerations will at once show, it contains the internal causes of the more complex phenomena of cell-reproduction and development. We have referred elsewhere ${ }^{1}$ to the mode of growth of living substance. We know that there are molecules in living substance, that possess an extraordinary tendency toward polymerisation, i.e., under given conditions, by continually taking on similar groups of atoms, they endeavour to enlarge and to form chains of many similar links. We have become acquainted with such polymeric molecules in the native proteids. It is, a priori, probable that the so-called living proteids, or biogens, likewise possess this property, and the more probable because there is reason to assume with Pflüger, that the radical cyanogen, which tends strongly towards polymerisation, is contained in the biogen molecule. Moreover, the fact of growth requires the assumption of polymerisation in the biogen molecule. Growth can be conceived only as a process in which a biogen molecule attaches to itself little by little similar groups of atoms frorn the materials of the environment (food-stuffs); these groups then proceed in the same manner to attract to themselves certain atoms from the environment and place them in similar positions, and so on.

This process, which is here pictured in a uniform substratum, goes on in a much more complex manner in the cell whose living substance and whose metabolism are very widely differentiated. In the cell the constituents of both the nucleus and the protoplasm with all their special differentiations share more or less closely in the formation and the growth of the biogen molecule. But with this close relationship and dependence of the individual constituents of the cell upon one another, it is easily understood that the growth of certain biogens of the protoplasm by polymerisation is only possible when at the same time other constituents of the protoplasm or of the nucleus increase in a definite measure; in other words, whenever a single substance of the protoplasm or of the nucleus grows, other substances also will grow.

It is important to consider somewhat fully the relations that, with this close correlation of the individual parts of the cell, are developed by growth. For example, let us imagine a free-living, spherical cell which has at its disposal in the surrounding medium

${ }^{3} C f$. pp. 30.7 and 486 . 
in sufficient quantity all substances necessary to its life, and let us assume that the cell grows. With the increasing size of the cell the relation of surface to mass will gradually change; according to known mathematical laws the former will grow in comparison with the latter in the proportion of the square to the cube. In other words, the smaller the cell, the greater is the surface in proportion to the mass; and the more the cell grows, the less does the surface grow in proportion to the mass.

This simple fact is of fundamental importance. This becomes at once clear, when it is realised that the individual parts of the cell-body are in close metabolic relations to one another and to the external world. As regards the food-stuffs and the oxygen received from the outside, the more the cell grows, the more a disproportion between the external and the internal layers of the cell-body will come about; for, since the surface through which the food is taken in increases less than the mass of the cell-body, the time will come when the ingested food is no longer sufficient for the whole body, and the result of this must become evident in the fact that the internal cell-layers are too little nourished in comparison with the external. While nutrition goes on rapidly and richly in the latter, in the former it proceeds more slowly and more sparingly. This will affect not only the protoplasm, but also the nucleus. The nucleus will receive fewer substances from the outside, if the protoplasmic layer surrounding it becomes gradually thicker, than if it is thin. But, vice versa, the external layers of the cell will become provided with nuclear substances much less richly than the internal. In brief, with the close relationship of the individual parts of the cell the metabolism must undergo profound changes, which increase the more, the more the cell grows. Hence, so long as the cell continues to grow, at no time is its metabolism exactly the same as at the preceding and the following intervals.

This important consequence from the fact of growth contains within itself the principle of all development, i.e., with the close metabolic relations that exist between the individual constituents of the cell and of the medium, the fact of growth is alone completely sufficient to lead and must lead to all the changes that are termed "development."

It follows from these considerations that the cell can never surpass a certain size; for, if the disturbance of metabolism that arises because of the increasing disproportion between the more superficial and the deeper layers, has reached a certain extent, the cell can no longer continue living in its existing form. Thus the remarkable fact is explained very simply, that no cells of constant form are known that are larger than a few millimetres in diameter; and thus we are made to understand why the development of large organisms is only possible by the arrangement of the living substance into an aggregate of small cells, instead of into a single 
cell, for example of the size of a man. At the same time it is comprehensible that under certain circumstances cells whose surface is considerably increased in proportion to their mass, such as the flat leaves of Caulerpa, or those cells whose protoplasm is in continual circulation between the surface and the interior, such as the plasmodia of Myxomycetes, are capable of reaching a rery considerable size, especially when by multiplication the nuclear substance presents a considerable increase. In these cases the difference between the outer and the inner layers of the cell-body cannot develop in the same degree as in compact cells. But where the cell-body is a compact mass, where there is no active streaming of the contents toward the surface, and where only one nucleus is present in the protoplasm, the cell cannot surpass a certain size. If, therefore, the living substance of such a cell is not to perish by growth, at some period in its growth a correction of this disproportion between mass and surface and of the disturbance of metabolism conditioned by it must come in; such a connection is realised in the reproduction of the cell by division.

The reproduction of the cell by division is, accordingly, to be considered merely as a result of growth, and the morphologists for a long time have rightly termed reproduction a continuation of growth, "a growth beyond the measure of the individual." Unfortunately our knowledge of the special mechanics of the process of cell-division is thus far limited; but it is to be expected that a comparative physiological investigation of the well-known morphological facts, when especially directed to the mechanical conditions, as they are realised in various ways in different forms of cells, will yield gratifying results. It is especially important always to keep in mind and to select as the starting-point of the investigations the metabolic relations of the individual parts of the cell. The mechanical results of the metabolic relations between the individual parts of the cell and the medium, are fitted to throw some light upon the processes of cell-division, many of which appear wonderfully complex. The most important factor in the explanation of the mechanics of the characteristic figures of celland nuclear division is probably to be found in the mechanical movements caused by the chemical relations between the individual parts of the cell; among these, diffusion-processes and changes of the cohesion and surface-tension of different cell-elements play a prominent rôle. Some time ago Bütschli ('76) expressed the view that the radiating figure that is formed in the protoplasm about the centrosome in nuclear divison is an expression of diffusion-processes arising between the centrosome and the protoplasm; and later $(' 92,3)$ he showed that, when warm getatine-foams poured upon a glass plate dry and coagulate, radiation-phenomena, exactly like those of the karyokinetic figure, are caused by the traction of the contracting air about the air-bubbles (Fig. 262). It may, 
therefore, be supposed that the radiation that forms about the centrosome likewise has its origin in the traction existing between the centrosome and the foamy protoplasm, and that this traction is derived from the chemical relations and diffusion-processes that develop between the two cell-constituents, But only a systematic and comparative investigation of these processes will be able to make this supposition a certainty. The mechanical theories of cell- and nuclear division, which M. Heidenhain ('94,'95,'96), Driner ('94), Rhumbler ('96,' 97), and others have very recently put forward, are so contradictory, incomplete and full of hypotheses, that at present it is quite impossible to say anything certain, except of the most general nature, concerning the mechanics of

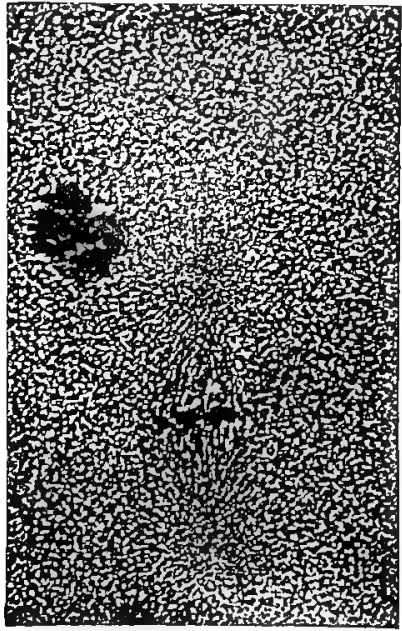

$I$

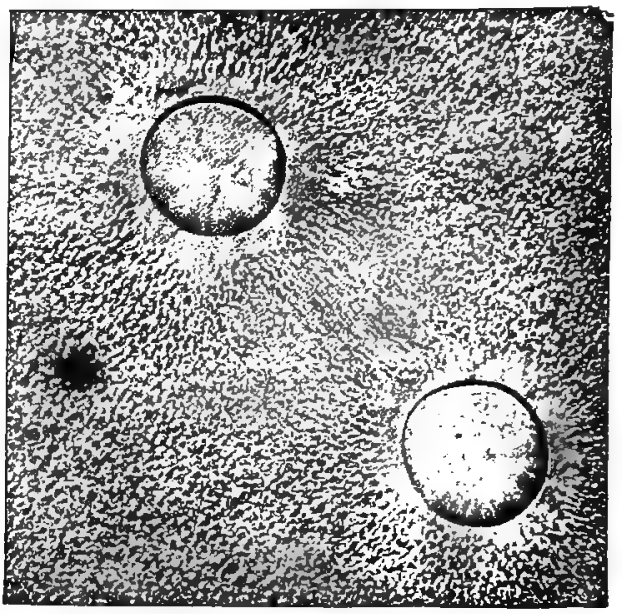

II

FIG. 262.-Photographic reproduction of radiating figures. I, Nuclear radiation from a cephalopod embryo. II, Radiation about two air-bubbles in a gelatine foam which was coagulated by means of chromic acid. (After photographs by Butschli.)

these complex events. Before all else, as R. Fick ('97) very correctly emphasises, their molecular-physical relations must be given much more careful attention; thus far Rhumbler alone has done this properly and to a considerable extent. Further, it will be of essential importance in such an investigation to start from the simplest forms of nuclear division, i.e., from the so-called direct nuclear division, in which there are no complicated figures. The lengthening and simple constriction of the nucleus giving rise to two nuclei is a simple mode of increase of the nuclear surface, the mass remaining the same; and the subsequent constriction of the protoplasm has the same significance for the cell-body. It is the simplest form of a correction of the disproportion arising between 
surface and mass with continued growth, and ought to offer relatively the least difficulties to a mechanical explanation.

With the division of the cell into two independent cells, the relation of surface to mass in the two latter is very different from that existing in the large cell before division. The result is that the metabolic relations will again change, and each cell will assume the same condition which the mother-cell had when it arose by division and began to grow into an independent individual. Thus, from one cell-division to another the same cycle of changes, which is conditioned by the growth of the cell-body and the disturbance in the metabolism caused thereby, repeats itself. If these changes are slight, they will not be especially noticeable outwardly, except in an increase in size. Most cells are like this, since they simply grow and, when they have reached a certain size, divide. Where, however, the disturbances in metabolism are considerable, they will be expressed also in a change of the external form of the cell-body, constituting a typical development. A large number of free-living unicellular organisms show this, especially those whose cell-body in division breaks up, not into two halves, but into a larger number of parts, or spores. The difference in size between the spore and the adult infusorian is, indeed, very considerable. Hence the differences in the metabolism must also be very considerable, and a somewhat long development is required before the spore becomes an adult.

Thus the development of the cell, the periodic return of one and the same cycle of form-changes from one cell-division to another, from one spore-formation to another, is seen to be a simple expression of changes in the cell-metabolism that are caused by growth. During growth with the close correlation of all parts of the cell with one another and the constituents of the medium, innumerable other factors, both chemical and physical, must appear, and these must combine with one another to assist and promote the form-changes. But as the fundamental cause of all these changes no other factor than growth need be assumed. It alone suffices to explain their periodic character.

\section{b. Developmental Mechanics}

A question that has reference to the development of the multicellular organism from the ovum by continued division has recently become the focus of active discussion. It is this:-How does the division of a cell into two unequal parts, a circumstance that forms the fundamental condition of the development of every differentiated cell-community, come about? This question, which is fundamentally important in an understanding of the development of all higher organisms, is answered in two very different ways. The view of one class of investigators follows the theory of His 
('74) regarding "organ-forming germ-regions." Roux ('95) and Weismann ('92, 2) are the latest defenders of this view, which affirms that different areas are present in the egg, which in continued division are transferred to different cells, and each one of which affords the material for the development of very definite tissues and organs. In other words, the rudiments of the different parts of the body of the adult organism exist, separate from one another, in different parts of the egg. The chief supports of this view are the results of the experiments that Roux has performed upon the frog's egg, in which he observed that after artificial destruction of one of the first two cleavage-cells, from the other at first only halfembryos developed, i.e., embryos in which one half of the body was wholly wanting, this half being capable of development later by "post-generation," as Roux expresses it. In contrast to this is the view of another class of experimenters, especially Pflüger ('83, '84), O. Hertwig ('92, '93), and Driesch ('92,93), who deny the existence of organ-forming germ-regions, and believe that the differentiation of the homogenous egg-cell into the various kinds of cells is brought about solely by the influence of external factors upon the various substances contained in the egg. Thus, in eggs like that of the frog, which contain substances of different specific gravities-in the frog's egg there is a white substance that is richer in yolk and a pigmented substance that is richer in protoplasm-gravity acts in such a manner as to lead to polar differentiation, so that the heavier substance comes to lie below, the lighter above, and, when the egg is turned, the substances move correspondingly. At the first division of the frog's egg, the polardifferentiated cell becomes divided by a vertical groove into two equal halves, each of which contains white substance and black substance equally. But when Pfliger put frogs' eggs into an abnormal position and fixed them there, the eggs in cleavage frequently segmented into two unequal parts, one of which contained pre-eminently the light, the other the dark mass; nevertheless, normal larvæ developed. The contents of the egg can, therefore, not be so differentiated beforehand, that from each part certain organs only can develop; on the contrary, the different areas in the egg must be wholly similar as regards development. The fact observed by Hertwig speaks a priori in favour of this view, namely, that even single small pieces of the ovum, if they are capable of life and are fertilized, develop into whole individuals. Moreover, in opposition to the observations of Roux, Driesch discovered in the eggs of sea-urchins that from each of the first two, four or eight cleavage-cells, when he had isolated them from one another by shaking, complete individuals always developed, which were distinguished from normal ones by their small size only; this fact has since been confirmed by numerous observers upon various species of animals, among others by O. Hertwig upon 
Roux's own object, the frog's egg.

That definite parts or organs of the embryo do not develop from single cleavage-cells arising by the division of the ovum, Driesch and Hertwig were able to show by continuing in a different way the experiment performed by Pfluiger. They, like Pfliger, clamped frogs' eggs between two glass plates in such a way that the cells arising in division were able to arrange themselves in one plane only instead of in a spherical mass, so that they were abnormally placed with reference to one another. Notwithstanding this, completely normal embryos developed from them. From this fact the conclusion must necessarily be drawn that the individual cells arising in cleavage do not represent definite rudiments of organs, and that no organforming germ-regions can be present in the ovum.

To summarise briefly the contrasts that are expressed in these two wholly opposed theories, the idea of Weismann and Roux is essentially the old, more or less clearly expressed doctrine of preformation of the time of Haller, in somewhat modern garb, while the view of Pfluger, Hertwig, and Driesch represents the standpoint of the doctrine of epigenesis of Caspar Friedrich Wolff, which Haeckel, more than all others, has constantly maintained with great persistence in the later embryology. As thus contrasted, the two doctrines are incompatible with one another. There can be no doubt that the facts are adverse to such a very minute preformation of organ-forming germ-regions in the egg, as especially Weismann and De Vries (' 89 ) have assumed. The two facts, first, that small pieces of an egg-cell and isolated halves and quarters, formed in cleavage, produce a normal, complete organism of a correspondingly small size; and, secondly, that, when the cleavage-cells are displaced, animals develop with their organs in a completely normal position,- these facts prove that the different parts of the egg-cell must be of absolutely equal value in the production of the cells, tissues and organs proceeding from them, and that we are not justified in speaking of a localized preformation of definite rudiments in the egg; it makes no difference whether we assume 10,100 , or 1000 rudiments, as Roux does, or several billions. ${ }^{1}$ While, further, the

1 Since Roux has protested against being reckoned among the preformationists, where he has been placed not only by myself, but by many (I think most) investigators belonging to his special field, in order to do hin no injustice I ought not to leave his protest unmentioned. But, at the same time, I must say that upon the basis of his own work I have not been able to alter my foregoing judgment and to accept his explanation. Since, as Roux himself acknowledges, "there are at present few anthors who know my [his] views clearly," in oriler to give the reader the opportunity of an independent judgment concerning them, I will guote his own words, in which his standpoint is statel. In Virchow's Arhiv, vol. cxiv, 1888, as well as in the Verhandlungen der anatomischen Gesellsthaft auf der sechsten Versammlung in Wien, 1892, Roux summarises the results of his experiments and speculations, and explains that "cleavage divides quantitatively the part of the germinal material that accomplishes the direct development of the individual, especially the nuclear materiul, and, by means of the arrangement thus made of the various separate materials, determines at once the position of the later 
theory of Weismann and Roux seeks in the egg the causes of the origin of differentiated daughter-cells from the cleavage, the idea of Pfluger and Hertwig finds the causes pre-eminently in factors acting from outside upon the cells. While, according to the one view, cells divide into unlike products from internal causes, according to the other, external factors essentially produce the unlikeness in continued division. Doubtless both views are correct in this respect, and here is a point where a reconciliation is possible.

From the above-developed idea of the mechanism of the development and reproduction of the individual cell upon the basis of the metabolic changes arising because of growth, it is evident that internal and external causes of form-changes cannot be separated from one another. The whole process of producing and changing form is a compromise, a correlation of factors lying within and without the cell. Because the cell as a result of the characteristic quality of its living substance has the property of taking in substances from the outside and of giving out substances to the outside, the elementary vital process, the metabolism, represents a compromise between the internal and the external factors, without which the life of the cell is impossible. But, since with otherwise uniform external conditions the cell grows as a result of the composition of its living substance, the relations with the external factors become changed, so that the latter now act in a manner different from before. Thus, at every moment of time a different compromise is effected between the cell and the medium, between the internal and the external factors, the expression of which is the change, the development, and finally, the reproduction of the cell. Hence it is clear that the change of the cell, or the variation of the products of its division, is not dependent solely upon its internal character, or the external factors; development and reproduction are an expression of changes in the metabolic relations between cell and medium, conditioned by growth.

The fundamental distinction between a single free-living cell and an egg-cell developing into a cell-community consists wholly in the fact that the daughter-cells arising by the division of the unicellular organism become separated from one another immediately after the division, while in the development of the egg-cell the daughter-cells that arise in segmentation remain in connection with one another. In the unicellular organism, therefore, the correlation between cell and medium always passes through the same short cycle of changes; in the division of the egg-cell, however, this correlation changes in an entirely new way with each of the almost innumerable divisions. Hence it happens that, differentiated organs of the embryo." "His's principle of organ forming germregions is here applicable; it has been here demonstrated that gastrulation is w mosaic work." From this the reader who is familiar with the ideas of preformation and epigenesis will easily be able to decide for himself in how far Roux is a preformationist and in how far not. 
while the unicellular organism needs to undergo either a sarcely perceptible development or only a short "rele of changes, the egg-cell must pass through an exceedingly long series of formchanges up to the derelopment of the multicellular organism. Agrowth in the multicellular organism gradually ceases the cells undergo constantly ferer form-changes, and mant tissue-cells, $\iota . g$. . the ganglion-cells, many of which do not grow at all in the adult organism, remain apparently wholly unchanged, neither dividing nor differentiating further. "In reality, howerer, as has been seen elsewhere, ${ }^{1}$ general derelopment nerer ceases wholly until the time of death, but the later changes occur so rerr slowly and are relatively so slight, that they are perceired only within long interrals of time. In this apparently stationary condition the tissue-cells are similar to those unicellular organisms that have no perceptible derelopment: in both, the correlation between the internal and the external facturs changes imperceptibly, in the tissue-cells proceeding slowly, and in the unicellular organisms beims slight and constantly returning to its starting-point. In neither are essential changes of form obserred.

From these considerations it appears how incorrect it is, from the fact that the small egg is differentiated into a cell-structure of astonishing complexity, to deduce the idea that the living substance of the former in comparison with that of erery other cell. either erery unicellular organism or every tissu-cell, must be distinguished by an inconceirably delicate and complex structure. This idea, which is met with rery frequently, is only an unrecognised relic of the doctrine of preformation and. as has been seen. is both unnecessary and unjustified: for the derelopment and differentiation of the cell-community from the egg are based solely upon the correlation between the liring substance of the cells and the external factors. which is continually changing with the continual growth and division of the cells. Growth is the cause of all derelopment, both of the indiridual cell and of the whole cell-community, and this fundamental fact can scarcely be expressed better than in the words of the old master of embryologr. Karl Ernst ron Baer (28). who thus stated the most general result of his studies upon the emlrrolugg of animals: "The divelopmenter history of the individual is the histry of the gruming indiriduality in all its relations."

A summary of the above considerations regarling the mechanics of derelopment leads to the following viers. The dereloping cell. like ererr cell, represents a drop of living substance. Which is characterised by a very definite metabolism. This metabolism is the expression of the correlation existing between the medium with its individual factors upon the one side, and the cell with its manifold internal differentiations upon the other. Br the growth 
of the cell, the correlation between it and the medium necessarily changes, because the relation of the surface and the mass of the living substance is gradually altered. As a result of this there is also a change in the metabolism. In other words, there is in the growing cell a continual succession of different metabolic conditions forming a very gradual transition, every succeeding condition resulting necessarily from the preceding one. Since here, as everywhere else in the physical world, form is among other things a function of matter, it is thus made clear that with a change of metabolism the form of the cell under certain circumstances will also change, and thus a continual succession of different form-conditions goes hand in hand with the succession of different metabolic conditions, in other words, there is a development. It follows from this that the development of the cell is a real epigenesis in the sense of Caspar Friedrich Wolff, i.e., a succession of constantly new forms, and not a more distinct appearance of already preformed, but hitherto imperceptible, structural differentiations of the living substance. According to the extent of the changes in the correlation between medium and cell the change of form will be expressed in one case less, in another case more strongly; it will be most pronounced where, as in the development of the cell-community of plants and animals from the egg, the cells dividing as a result of growth remain in connection with one another and act upon one another, in other words, where the relations between cell and medium change rapidly and continually.

\section{c. Structure and Liquid}

Special importance has been attached elsewhere to the fact that living substance possesses the essential properties of a liquid. In the production of form another factor, namely, structure, plays an important rôle. Since at first sight it might appear as if structure and a liquid state are mutually exclusive, it will be advantageous to examine briefly this question. ${ }^{1}$

If by structure there is understood a definite mutual relation of the smallest particles of which a substance is composed, the fundamental requisites of the inauguration of structure are the mutual attraction and grouping of definite particles. We can speak of structure only where certain particles attract one another and become grouped. This requirement is fulfilled not merely in solid bodies, but in a certain measure in liquids, for in liquids also, as cohesion shows, the individual parts attract one another. The difference in the structure of liquids and solids is in reality a gradual one, depending wholly upon the degree of consistency; imperceptible transitions exist between the two states. The difference between them consists essentially in the fact that ${ }^{3} C f$. Verworn ('97). 
the more solid a body is, the less its molecules are in motion. Motion is least in the hardest bodies, and greatest in the thinnest liquids; in the latter the intensity of the motion is almost equal to that in gases, which, as is well known, is so great that the molecules repel one another. Between the two limits of very thin liquids and very hard bodies the solidity of the structure varies, becoming greater the harder the body is. There is, in fact, a certain molecular structure in every simple solution. If, e.g., a crystal of pure salt be put into a vessel containing distilled water, after some time it dissolves, and the molecules of the salt become scattered by diffusion uniformly throughout the liquid, so that in every volume of the latter, even the most minute, the same percentage of salt molecules is contained. In other words, an attraction between the molecules of the salt and those of the liquid takes place, and about every one of the former is grouped a certain number of the latter. The only difference as regards this grouping or structure between the mobile liquid and the solid body is that, during the active molecular motion in the liquid, molecules are continually being drawn away from their groups and replaced by others, so that the structure is continually being destroyed and reformed; while in the solid body, where the motion of the molecules is slight, the structure can exist for a long time undisturbed. This continual reformation of structure in the liquid is, however, of fundamental importance to living substance, for only where there is a possibility of continual outgo and income of molecules can a metabolism exist, and without this living substance is inconceivable. But this continual change of molecules does not hinder the continual appearance of differentiations of form in certain places resulting from molecular and atomic groupings in the living substance. Just as a stream of water or a gas flame can maintain a very definite form, although at no two successive moments do the same molecules produce that form, so living substance, in spite of its liquid nature, can show certain continual differentiations of form, which exist so long as the causes for the definite grouping of the molecules and atoms remain the sane.

This consideration is of great importance, for it enables us to understand the general phenomena of the construction of form in living substance. The apparent paradox that living substance, although its components are undergoing continual change, can possess in many cases a constant and often extraordinarily complex form, is at once explained. Let us imagine a cell that possesses various kinds of differentiations, for example, the flagellate infusorian Poteriodendron, which besides its nucleus is provided with a flagellum and a contractile myoid-fibre (Fig. 263). In each of its individual differentiations the particles are arranged in a specific manner, in the nucleus differing from that upon the surface of the protoplasm, in the flagellum differing from that in the myoid-fibre. Never- 
theless, from all these individual differentiations atoms and groups of atoms pass out continually in definite directions, and into them new atoms and molecules enter continually, so that the structure is continually being destroyed and rebuilt. There is, therefore, a continual stream of matter which ramifies into the various differentiations in an extremely complicated way and possesses a very different composition in its different parts. This stream of matter is the expression of the complex metabolic relations between the individual parts of the cell-body, and it is the direct condition of the very definite and peculiar form of the cell in question. The structure is able constantly to re-establish and maintain itself, only when certain atoms are at the necessary place at the right time. If the stream of matter ceases, the molecules disintegrate, and the definite grouping is dissolved. So long, however, as the stream of matter is uninterrupted, the individual molecules and atoms take up by attraction

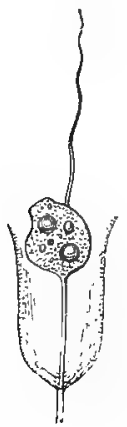

FIG. 263. - Poteriodendror. A single individual of a colony. The cell-body, fastened upon a myoid-fibre, sits in a bellshaped cup and contracts its flagellum. the necessary particles, and the structure continues to exist. If the stream of matter changes in the direction and composition of its particles, the form of the cell and its differentiations must change also, and there is a development.

We have already found the comparison of vital phenomena with a flame very pertinent in many respects. This simile is also adapted to make clear in an especially striking manner, the relation between form-construction and metabolism. The butterfly figure of a gas-flame has a very characteristic differentiation of form. At the bottom immediately above the slit in the burner, there is complete darkness; above it there is a blue zone, only feebly luminous; and above that upon each side the bright luminous surface is extended out on both sides like the wings of id butterfly. This peculiar form with its characteristic differentiations, which continues to exist so long as the position of the gas-cock and the surrounding conditions are not changed, depends solely upon the fact that in the individual parts of the flame, the grouping of the molecules of illuminating gas and oxygen is very definite, although the molecules themselves change at every interval. At the bottom of the flame the molecules of illuminating gas are pressed together so closely that the oxygen necessary for their combustion cannot come in between them, and as a result of this there is here complete darkness. In the bluish zone some molecules of oxygen are combined with those of illuminating gas, and the result is a feeble light. In the large 
flat flame, however, the molecules of illuminating gas are in such a numerical relation to those of the oxygen of the air that active combustion takes place. The change of the substance of the flame through the incoming gas and the surrounding air is, however, so regulated, that at the same place the same kinds of molecules constantly come together in the same number. As a result, the same form of flame with its differentiations is maintained continually. But if the stream of matter be altered by letting less gas pass out, the form of the flame also changes, because now the mutual position of the molecules of illuminating
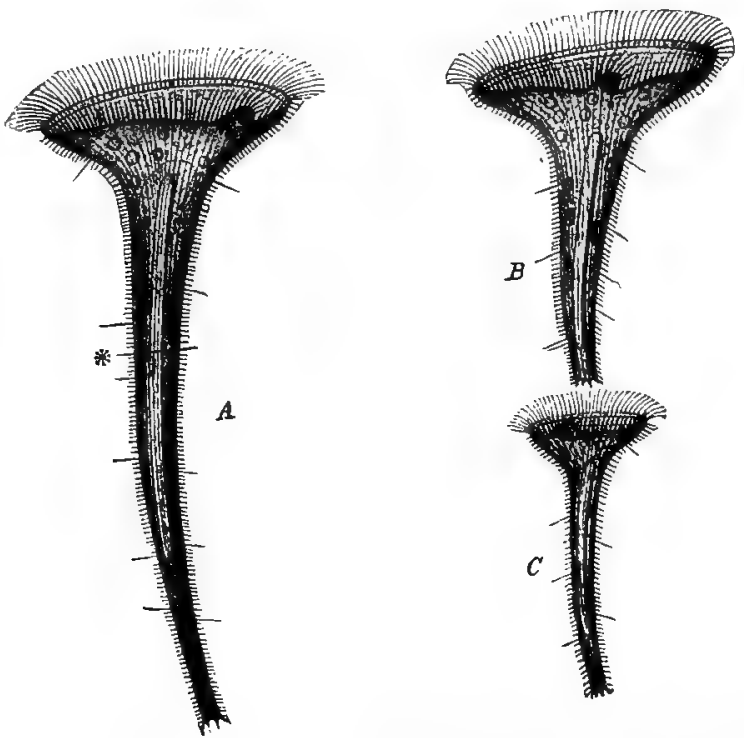

F10. 264.-Stentor Reselii, $A$, Cutacross at*, $B$ and $C$, The two pieces have become regenerated into complete Stentors. The clear extended mass in the interior is the nucleus.

gas and of oxygen is changed. It is thus seen that such a fiame, even in its details, presents exactly the same conditions that we have found to be important in the construction of the cell-form.

Another interesting group of phenomena of form-construction is at once clear from this point of view, namely, the phenomena of regeneration. If a cell-best an infusorian cell that is provided with very characteristic differentiations of its surface, such as the delicate Stentor Rasclii-be cut into two pieces, so that each contains a part of the nuclens and hence possesses the value of a cell, in a short time, as has been seen elsewhere, ${ }^{1}$ each of the two 
pieces regenerates the parts lacking in it. The wounds close, and the lower part of the cell at once arranges it substance so that a new peristome appears with the characteristic spiral of cilia and a mouth-opening, while the upper part becomes extended so that a new foot-piece is developed, with which the new Stentor attaches itself. Thus, in a short time by the deposition of particles
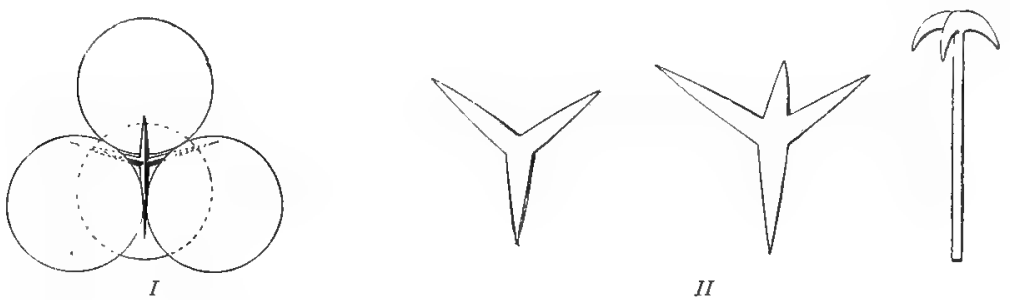

II

FIG. 205.-Silicious spicules of sponges. I, Scheme of the production of a quadriradiate spicule between four vascular spaces. (After F. E. Schulze.) II, Various forms of silicious spicules.

from the interior of the body at the place of the wound, a complete Stentor is developed from each piece (Fig. 264). This fact of regeneration is now very easily understood. Since in the process of differentiating organised cell-forms every particle attracts and holds fast other specific particles and upon the withdrawal of the latter in metabolism, at once attracts and holds corresponding particles again, so in regeneration the particles existing at the wound, which are separated from their neighbours by the cut,

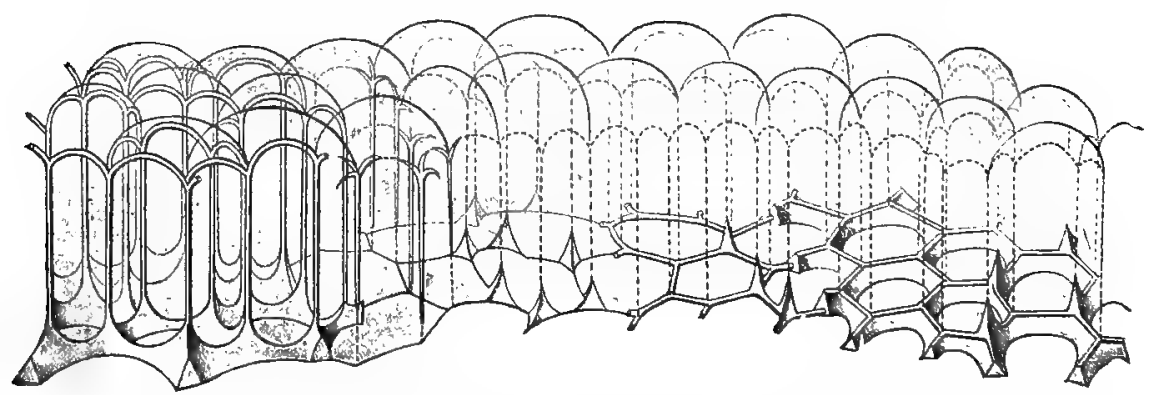

[1G. 26ib. - Scheme of the production of various skcletal forms hy the cxcretion of skeletal substance into the walls of a vacuolur system. (After Drejer.)

must immediately attract and attach to themselves corresponding particles, if they are obtainable. Since, however, in such a division of the body metabolism experiences no fatal disturbance, the necessary particles are still, as formerly, brought in by the stream of matter, and can attach themselves to the others as the peculiar quality of each one demands. But if the metabolism has 
been irremediably injured in the division, regeneration is no longer possible, because the necessary molecules and atoms are no longer produced and brought to the necessary place. Hence it is a universal and fundamental fact that non-nucleated pieces of a cell, i.e., pieces into which a fatal disturbance of metabolism has come, do not regenerate lost parts, although under certain circumstances they are able to live for days.

One phenomenon, which some years ago appeared very puzzling, is approximately explained by the fact of structures in the cellprotoplasm. This is the formation of very regular silicious and calcareous skeletons, especially in the delicate Rudiolarir, Foraminifera, and sponges. F. E. Schulze, ('87) called attention to the fact that the formation of triradiate and quadriradiate spicules

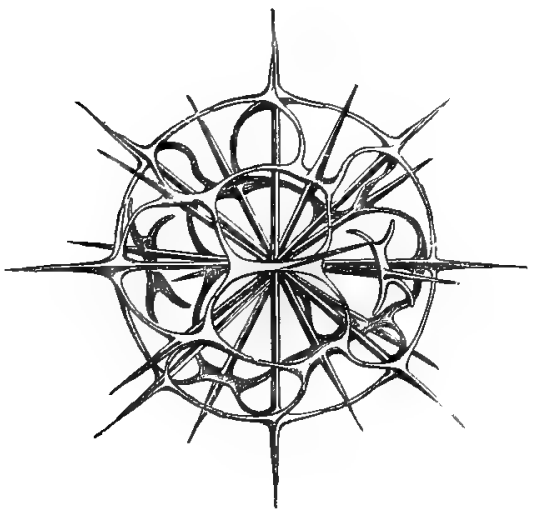

$A$

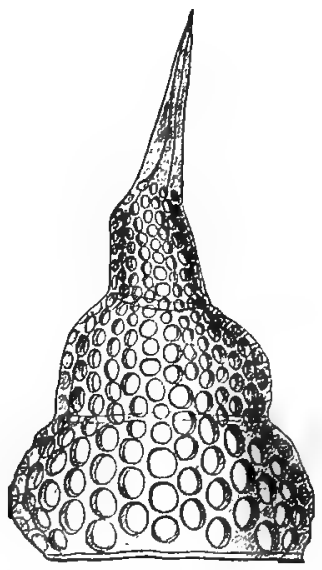

$B$

Fis. 267.-Silicious skeletons of Radiolaria. (After Haeckel.) A, Dorataspis. B, Theoconus.

(Fig. 265, II), which play so great a rôle in the silicious and calcareous skeletons of sponges, must take place when several spherical bodies are in contact with one another and a skeletogenous substance, such as calcium carbonate or silicic acid, is excreted into the fine spaces between them (Fig. 265, I). Lately Dreyer ('92) has extended the same idea to several special examples, and has shown how various and often extremely complex skeletal parts, especially in the Radiolario, may easily be traced to the excretion of skeletogenous substance in the protoplasmic walls of a vacuolar layer (Fig. 266). Thus, according to the form of the vacuoles, the thickness of their walls, the place at which the secreted skeletal substance is deposited, and its quantity, a great variety of skeletal forms must result, and are actually realised in the richly varied forms of the radiolarian 
skeleton. Thus the fact, which previously seemed so wonderful, that the simple protoplasmic masses of rhizopod cells, while continually engaged in streaming and forming pseudopodia, are able to construct such astonishingly regular, complicated, and delicate skeletons, is at once understood from the fact that the protoplasm of these cells possesses in a certain body-zone a vacuolar or honeycomb structure. According to the form, the position and the extent of this vacuolar layer and its vacuoles, the effusions, which result from the excretion of skeletal substance between the vacuoles and form the skeleton, must vary extraordinarily (Fig. 267). Doubtless a rôle similar to this of the vacuolar structure of the protoplasm in the formation of many radiolarian skeletons is played by the structure of protoplasm, as well as by the form and the mutual pressure of the individual cells, in the formation of the skeleton in other organisms.

\section{d. The Mechanics of Hereditary Transmission}

There finally remains a brief examination of the mechanics of hereditary transmission. The conditions of hereditary transmission are simplest in the lowest unicellular organisms; for example, in Amceba, apart from an increase in the size of the body, no distinct development is observable. Here, where the reproduction of the organism takes place simply by the division of the cell into two halves, the process of the transmission of all the characteristics of the mother-cell to the two daughter-cells is at once comprehensible. The living substance of the mothercell with its characteristic metabolism and its peculiar vital phenomena, continues to live independently in the daughter-cells; it is no wonder, therefore, that the separate pieces, when living under the same external conditions, possess exactly the same characteristics that the undivided cell possessed. But this. simplest case of inheritance exhibits very clearly the essential factors of the phenomenon, just as all vital phenomena in general are to be seen and understood most clearly where they appear in their simplest form, i.e., in the simplest cells. It is seen here that the transference of the characteristics of the ancestors to the descendants, takes place by the transference of substance which possesses the characteristics of the ancestors. In order that this substance may possess all the characteristics of the latter, it must be a complete cell with all the essential cellconstituents. The characteristic peculiarities of the mother-cell are the expression of its metabolism. If, therefore, the peculiarities of the mother-cell are to be transmitted to the daughtercells, its whole metabolism must betransmitted. But this is possible only when a certain quantity of all the essential constituents, i.c.. of the protoplasm and nucleus of the mother-cell, passes orer to. 
the danghter-coll, for otherwise the metabolism of the latter would not be able to continue, and the cell would necessarily perish. In fact, it is seen not only in unicellular organisms, but everywher in organic nature, that hereditary transmission takes place without exception by means of the transference of a complete cell with nucleus and protoplasm.

If by hereditary transmission there is understood the transference of the peculiarities of the ancestors to the descendants, and if the peculiarities of an organism are merely the expression of its phrsical relations to the external world, the conclusion is absolutely unavoidable, that in hereditary transmission the living substance, with its peculiar metabolic relations, must be transferred. But this is only pussible when all the essential parts of the metabolic chain are transferred, the protoplasm as well as the nuclear substanee, in other words a whole cell.

However logical and obrious this simple conclusion is, and however completely it is confirmed by actual facts, it has really never been clearly drawn on the part of morphology, which thus far has been almost the sole department of biology to deal with the problem of heredity. As has been seen, among the morphologists, especially in connection with the views of $\mathrm{O}$. Hertwig, Strasburger, Weismann, Boveri and others, the view has become very wide-spread, that the hereditary transmission of parental characteristics to the offspring is mediated by the transference of nuclear substance only, by means of egg- and sperm-cells, and the nuclein of the cell-nucleus has been specially termed the "hereditary substance." Only a few morphologists, like Rauber, Bergh and Haacke, have thus fur expressed themselves against this riew; but, as our previous presentation of the subject has shown, ${ }^{1}$ the grounds upon which it rests, are not able to withstand rigid criticism. For the physiologists, moreover, the view is conceived somewhat too morphologically, for it takes no account of the most essential factor of life, metabolism. The physiological mode of thought will hardly be able to adapt itself to the idea of a single hereditary substance, which is localised somewhere in the cell and transferred in reproduction. A substance that is to convey the characteristics of a cell to its descendants, before all else must be capable of life, i.e., must have a metabolism, and this is impossible without a connection with other substances necessary to cellmetabolism, i.c., without the integrity of all essential cell-constituents. The designation of a single cell-constituent as the specially differentiated bearer of heredity is wholly unjustified, the coll-protoplasm is of exintly the sime value in this respect as the nucleus, and we must constantly return to the fact that in all living nature no instance is known in which il complete cell I (. f. p. . T(1.). 
possessing nucleus and protoplasm does not always mediate hereditary transmission.

To summarise, the character of every cell is determined by its peculiar metabolism. Hence, if the peculiarities of a cell are to be transmitted, its characteristic metabolism must be transmitted; this is only conceivable when nuclear substance and protoplasm with their metabolic relations are transferred to the daughter-cells. This is true of the sexual reproduction of the higher animals, as well as of the asexual reproduction of unicellular organisms; in the former, however, the metabolism of one cell, the spermatozoon, is by the process of fertilisation combined with that of another cell, the ovum, into a single resultant, the metabolism of theoffspring that arises from the fertilised ovum; the offspring hence possesses the characters of the two parents.

\section{The Mechanics of the Transformation of Energy by the Cell}

The third aspect in which the changes of a body make themselves manifest, besides those of substance and of form, is that of transformation of energy. The three aspects are inseparable and are the expression of all that happens in the physical world. Given one of the three in all its details, the other two would be known. This is true of both living and lifeless bodies, for both are physical systems and must obey the strict laws of all matter.

\section{a. The Circulation of Energy in the Organic World}

Unfortunately, as regards the transformation of energy in the living organism, our knowledge at the present time is but fragmentary. The beginning and the end are known, but between the two is the complex series of events in which the energy in its passage through the living substance takes part, and thus far only a few of these events have been discovered. But so much is evident: the changes of energy are just as manifold in their details as are the changes of substance and of form, and every kind of cell is characterised as much by the former as by the latter. It has been seen that the green plant-cell is that form of living substance which in a certain sense is the basis of all life now existing upon the earth's surface, in so far as it is the laboratory in which from inorganic materials organic compounds, which are the necessary vital condition of all other organisms, are manufactured. Hence, in outlining a scheme of the general circulation of energy in living nature, attention must be given to the green plant as the starting-point for the entrance of energy into the living physical world.

That form in which energy is introduced into the green plant- 
cell is pre-eminently the energy of sunlight. Almost no chemical energy is introduced into the plant; the chemical substances from which the plant constructs its living substance, namely, carbonic acid, water and the salts dissolved in it, are compounds which in this form contain almost no chemical potential. These compounds are transferred to substances possessing chemical potential only by the introduction of light mediated by the activity of chlorophyll in the green plant-cell. The affinities of carbon and oxygen, e.g., can be made available only by the splittingup of carbonic acid, $\mathrm{CO}_{2}$, into carbon and oxygen. Energy is consumed in this process, and the amount required for it is supplied from the energy introduced by light into the plant-cell. It has, therefore, been said that all life is derived in direct descent from sunlight; and thus in a certain sense an exact scientific background is given to the ancient poetic worship of light and the sun by Asiatic and American races. But sober scientific consideration forces us to modify the above statement. If the idea that the sun's light-rays are the energy from which all the energy of the living world in the last instance is derived, may be expressed at all in this general form, it is true only for the conditions now prevailing upon the earth's surface. If we go back to the times when the living substance first appeared upon the earth, we shall doubtless be obliged to turn to chemical energy as that form of energy that was first introduced into the living substance. Of course the living substance of the present day, like all substance, is finally derived, together with its energy, from the sun, for the earth is only a part of the sun's mass thrown off; but light can hardly be considered directly as that form of energy which effected upon the cooling earth the construction of those compounds, containing energy in the potential form, that are termed living substance. In reality, at present it is not the light that directly accomplishes the splitting-up of the carbonic acid and the combination of the atoms of carbon, hydrogen and oxygen into the first product of assimilation, starch. This idea, which perhaps has been suggested by an inexact mode of expression, is wholly incorrect. In reality, it is only the chemical energy of certain compounds of the chlorophyll-bodies in the green plant-cell that does this. The energy of the light-rays alone can never split up carbonic acid, quite apart from the coupling of the atoms of carbon with those of hydrogen and oxygen into starch molecules. Light is indispensable only in so far is it is that form of onergy which fivours the rearrangement of the atoms in certain compounds of the chlorophyll-bodies, so that these atoms are able to enter into chemical relation with those of carbonic acid and thus decompose the latter. The energy of the light-rays, therefore, is first transformed into chemical energy, and it is the latter in the chlorophyll-bodies that effects the splitting-up of carbonic acid and therewith inaugurates 
the endless chain of transformations of energy which characterise the life not only of the plants but also of the animals. The rôle of light is similar to that of the heat that is introduced; heat is indispensable to life in both the plant and the animal body, and serves to increase the intramolecular vibrations of the atoms, so that the latter become inclined to rearrangements. But it is always chemical energy that effects these rearrangements. In other words, in the plant chemical energy must be already present in the chlorophyll-bodies, and only by the introduction and transformation of photic energy is this so increased that it can accomplish the first cleavage of the molecule of carbonic acid, which has such very important consequences. Where living substance with its chemical energy is not already present, the introduction of light cannot produce life. Thus, the chemical energy introduced into the organic world along with the first living substance in prehistoric times, even now continues to work in all living organisms, without ever having undergone a break in continuity. Life cannot be transferred from one organism to another without the transference of living substance containing chemical energy. It is a tiny quantity of both substance and chemical energy that is transferred in the microscopic egg-cell to the offspring, but it is sufficient to ensure the continuity of both. The chemical energy thus transferred makes reproduction possible, gives the impulse to the continual transformation of constantly greater and greater quantities of energy, in a certain sense by ferment-like action, and finally causes a powerful development of force in the adult organism. It is the original capital with which the developing organism begins its dealings, and without which its existence would be impossible. In this sense it can be said: that form of energy from which in the last instance all the work of the crganue world is derived is chemical encrgy. The light and heat introduced act only by making chemical energy available.

It is evident that this is equally true of animals and of plants. Out of the original chemical energy available in the plant not only is the manifold external work of the plant supplied, but a considerable quantity is stored up in the form of chemical energy in the organic compounds of the plant-body. These complex organic compounds afford food for the herbivore, while the flesh of the herbivore does the same for the carnivore. Thus, with plant-food energy comes to animals in chemical form and affords potentials for the performances of the animal body, which are distinguished so characteristically from those of the plants by their powerful extrinsic development of force. In fact, the chemical energy introduced into the animal body with the food forms the sole source of the energy of the animal body, with the exception of the small quantity of heat that acts froni the outside upon all organisms. The jroof of this fact has been afforded in the most 
satisfactory manner by the calorimetric investigations of very recent times, especially by the very exact work of Rubner ('94). If upon the basis of calorimetric combustions the chemical energy of food be expressed in terms of heat, the food will yield just as many calories as an animal affords when all its production of energy is expressed in output of heat. The differences between the quantity of heat that is evolved by the combustion of food to substances lacking chemical energy, and the quantity of heat which the animal produces with like food during rest, are so small in the extremely delicate experiments of Rubner, that they fall wholly within the unavoidable technical limits of error. If it were at all necessary at the present time to prove the validity of the law of the conservation of energy for living nature, the best evidence would be given by Rubner's new calorimetric researches.

The passage of energy through the organic world ends with the output of heat or mechanical work by the animal body. The animal body gives off to the outside no chemical energy that is capable of being used further, with the exception of that adhering to the egg-cell in reproduction. The substances that leave the animal body, such as water, carbonic acid, etc., are compounds that possess in their existing form no more chemical potential, and the introduction of light into the green plant-cell is necessary to enable the latter to create available chemical energy out of these substances. Thus, the circle of the changes of energy between living and lifeless nature is a closed one. Light makes available chemical energy in the plant-cell. Out of this chemical energy are derived all the chemical, mechanical, and thermal activities of the plant in a complex series. The herbivore takes into its body with its food the chemical energy that is stored up in the organic compounds of the plant, and with the materials of its own body-substance becomes to the carnivore the indispensable source of chemical energy; from the latter is derived all the thermal, mechanical, and in special cases also the photic and electrical energy which the animal body gives off to the outside as heat, as mechanical energy of muscular movement, and as light and electricity. Out of the substances that leave the animal body, poor in mechanical energy, carbonic acid and water, the plant-cell under the influence of the light-rays creates anew chemical energy, and thus the endless circulation begins again.

\section{b. The Principle of the Transformation of Chemical Energy in the Cell}

However clear the main outlines of the organic change of energy appear, its details are obscure. This is true partly because of our lack of knowledge of the metabolism of living substance, but largely because of the extremely slight development of the general theory of energy in physics and chemistry. The transformations of energy 
involved in events the material basis of which is known in fullest detail, are still wholly unknown. Thus, concerning the work performed in many chemical transformations, we do not know at all whether the mechanical energy thus set free is derived directly from the transformation of chemical energy, or after passing through other forms, such as heat, or electricity. The direct transformation of chemical energy into heat and electricity has been investigated exactly and in detail, but that of chemical into mechanical energy has thus far scarcely been studied. This circumstance, indeed, has frequently led to the mistaken belief that chemical energy can pass over into mechanical energy never directly, but only through the mediation of heat, an idea that is wholly without foundation. To make the subject still more difficult to understand there is the added fact that the conceptions of the individual forms of energy are not at all fixed, that, e.g., the expressions, molecular energy, mechanical energy, etc., are employed in very different senses, which results from the fact that the relations obtaining between the various forms are thus far not at all cleared up. Notwithstanding, it must be assumed that such relations, and even very close genetic ones, exist. It is, accordingly, evident that the more special energetics of living substance is at present one of the most obscure fields of physiology, only isolated and disconnected facts being known.

The general fact must be regarded as established, that all the work of the organism is based finally upon chemical energy. So far as is known at present, most of it is directly dependent upon this source at the moment of its occurrence. But the energy of many actions comes in a roundabout way. Pfeffer ('93) has recently made this fact especially clear for plants. Thus, it happens very frequently that in metabolism chemical energy first passes over into potential mechanical energy and is stored up as tension, to be transferred at the proper opportunity into the kinetic energy of mechanical work. Jumping-fruits and seeds of certain plants furnish examples of this. Here the chemical energy of growth first accumulates in the form of mechanical tension, and, when the fruit is touched, this passes uver into vital motion, the fruit bursts open and scatters its seeds with great force. Analogous cases of the indirect derivation of work from chemical energy occur frequently among both plants and animals.

The chief forms in which the energy evolved by the cell is expressed, are mechanical energy and heat. The evolution of light and electricity is much more limited.

In order to obtain an insight into the energetics of the cell, the chief principle that controls the transformation of energy in chemical changes must be recalled. This was formulated as follows: if in a chemical process affinities become united rather than scparated, energy is liberated; if affinities become separated 
rather than united, energy is absorbed. ${ }^{1}$ In the light of this, the fundamental principles of organic transformations of energy follow clearly from the well-known facts of metabolism. There exist in living substance certain compounds possessing strong chemical affinities. Other chemical affinities are introduced from outside into the living cell along with food and oxygen. These relatively simple substances that are introduced are employed for the construction of much more complex compounds, which we have termed living proteids or biogens. In this process the chemical energy that is introduced passes over into the complex compounds in the form of potential energy, and helps to loosen their structure.

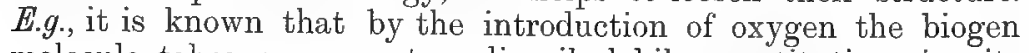
molecule takes on an extraordinarily labile constitution, i.e., its intramolecular heat becomes very great. As a result of this the molecule tends toward decomposition, and explodes, partly spontaneously and partly upon slight external stimulation. This explosive decomposition depends upon a re-arrangement of the atoms, whereby, as in all explosive bodies, within single atomic groups stronger affinities become united than were previously united in the labile molecule. Therefore, as a whole, dissimilatory processes must be associated with a considerable production of energy. The compounds that are derived from this decomposition of the biogens and leave the body, such as carbonic acid, water, etc., contain scarcely perceptible quantities of chemical potential, while the compounds that remain in the body, the residue of the biogens, again possess chemical affinities for food-stuffs and oxygen, and employ them in uniting with the latter. The energy thereby made available is employed again for loosening the biogen molecule, and thus the chain ends. The principle upon which it is based accordingly appears clear: there is a continual storing up of potential chemical energy and a transference of it into other forms; the source of it is the food and the oxygen; the original capital is the chemical energy that every minute droplet of living substance has carried over from its ancestors; and the result is expressed in the work accomplished by the living substance.

The relations as regards energy that develop in living substance under the influence of stimuli are comprehensible, in their general outlines, upon the basis of these facts. Those cases are simplest in which the stimulus causes an excitation of dissimilation. As has already been seen, ${ }^{2}$ this process consists of an augmentation of spontaneous processes. The potential energy that is stored in the labile biogen molecules is to a certain extent spontaneously transformed into actual energy, the atoms being rearranged and combined with one another by stronger affinities, and an explosive decomposition simultaneously occurring. It is easily comprehensible that certain stimuli may increase directly the intramolecular

$$
{ }^{1} \text { Cf. p. } 215 .
$$$$
{ }^{2} C f . \text { p. } 474 .
$$ 
motion of the atoms in the biogen molecule, and thereby give greater opportunity for rearrangement and explosive decomposition ; the augmentation of the processes under the influence of certain stimuli needs no further explanation. Those cases of reactions where there is a depression of dissimilation also scarcely need further discussion, for all those stimuli that diminish the intramolecular motion of the atoms in the biogen molecule, or hinder the rearrangement and combination of definite atoms in any way, such as cold or narcotics, must evidently diminish the normal work of the cell. But not all the performances of living substance are associated with the dissimilatory phase of metabolism. Many important ones go hand in hand with the assimilatory phase. Hence, stimuli that excite assimilation, such as increased food, will augment such performances, since they afford a greater opportunity for the formation of new biogen masses; and, vice versa, stimuli that depress assimilation will produce the opposite effect. Those vital phenomena that are associated with assimilation and are augmented by stimuli that excite this process, have been greatly neglected by investigators in comparison with the more evident phenomena associated with dissimilation, and they deserve special consideration in the future.

This idea of the action of stimuli does not imply that the energy that is manifested in definite work as the result of a stimulation is always derived directly and solely from the excitation or depression of one or another link in the metabolic chain. According to our idea, the satisfying of the free affinities of the residue of the biogen molecule, i.e., its regeneration, follows directly its explosive decomposition. Hence, under certain circumstances there is contained in the reaction not only the energy set free by the decomposition of the complex compounds, but also the energy that becomes actual in the processes that result directly from the decomposition, and the same is true of other cases of reactions. Thus, all the elements of the energetics of the cell are extraordinarily closely interwoven. This follows necessarily from the facts of metabolism above discussed. The very great difficulty of following in their details the more delicate transformations undergone by the energy in a given work, whether spontaneously or upon stimulation, is evident; and hence, with the extremely few investigations that have been carried on in this field thus far, it is at present impossible to determine with any certainty the energetics of even the more evident performances of the cell, such as the production of light or electricity and the evolution of mechanical encrgy in the various kinds of movement. To study in detail the extremely interesting history of the energy in the various internal and external labours of the living cell will be one of the most stimulating tasks of the physiology of the future. 


\section{c. The Source of the Energy of Musele}

Although thus far but few investigations have been made by physiologists upon the mechanics of the transformation of energy in living substance in general, this is not true of one particular class of these phenomena. This class comprises the movements of contraction and expansion. The mechanics of muscle-contraction especially, in which energy is developed with most remarkable and most astonishing power, has from early times engaged very actively the attention of physiologists, and the number of theories that have been formed regarding the mechanies of muscular movement is only a little smaller than the number of investigators who have studied the problen thoroughly. An interesting chapter in the history of human thought is reflected in these theories from the time of Galen down to the present day, and it is pleasing to one's historic sense to trace these theories from their very naive beginnings. Whoever is interested in this bit of physiological history will find the literature of the older theories down to the preceding century collected by Haller (1762). Hermann ('79) has given the essentials of the later theories in his Flandbuch der Physiologie, and the newest views, so far as they possess interest, are collected and critically examined in a work that has recently appeared, ${ }^{1}$ which considers the old problem from the comparative, cell-physiological side.

Without a doubt, of all the activities of organisms, muscle-work is that in which the greatest transformation of energy takes place in the shortest time. The quantity of energy that is set free in muscle-activity, as is well known, is astonishing. Hence the question presents itself: : of the energy introduced into the body what portion affords the energy that is thus set free, in other words, where is the source of muscle energy to be sought?

It is evident that the source must be chemical energy, for the animal-body performs its labours by means of chemical energy exclusively. But which of the food-stuffs introduced into the body afford by their transformation the chemical energy necessary to muscle activity? Is it the proteids, or is it the carbohydrates and the fats?

An active contest, which very recently has become augmented, has been carried on over this question. The original and very clear theory of Liebig ('57, '70) that proteid as the chief constituent of muscle must be the source of its work, was attacked during its author's lifetime, and it has since been thought for decades that in place of Liebig's view the correct solution of the problem had been found. The argument that led to this idea, which has prevailed to the present time, is irteresting. It was said: if the source of 1 Cf: Verworn $(92,1)$. 
muscle-energy lies in the decomposition of proteid, the latter must be increased by extensive muscular activity. Since, now, it was believed that an absolute measure of the extent of proteid-decomposition in the body is to be found in the excretion of nitrogen in the urine, the question would appear to be decided by a comparison of the nitrogenous contents of the urine during rest and during extreme muscular activity. If the nitrogen were increased during work, the increase could be derived only from increased proteidtransformation; if it remained the same, the source of muscleenergy was not to be sought in proteid but in non-nitrogenous food-stuffs. The problem was, therefore, presented in a very clearcut form, and the decision could not be left to itself. Fick in company with Wislicenus ('65) showed upon himself, and Voit ('70, '81) upon the dog, that the excretion of nitrogen in the urine is not markedly increased by intense muscular activity.

The question thus appeared to be answered very precisely. It was concluded that the decomposition of proteid could not be the sole source of muscle-energy. It was argued that of the non-nitrogenous food-stuffs the carbohydrates especially, and eventually the fats, must come into consideration; and it was known that with intense muscular activity the glycogen stored in the muscle disappears, and accumulates again during rest. The argument appeared wholly unobjectionable, and the view was generally accepted that muscle-energy is afforded chiefly by the decomposition of carbohydrates.

But the view that proteid does not take the chief part in the extreme activity of the muscle-cell necessarily appeared parodoxical to all who were at all familiar with the general vital characteristics of living substance. Proteid is the substance with the formation and decomposition of which life is inseparably associated, and hence it seemed very remarkable that in augmented vital activity, such as is represented by intense muscular movement, the transformation of proteid was the same as during rest. Pfiuger could not sympathise with this view. In a series of striking researches, supported by experiments free from objection, he recently attacked it, and sought to establish proteid-decomposition as the chief source of the energy of muscle. It was already known to Voit that dogs can maintain themselves upon a meat diet alone. Pfliger ('91), therefore, fed a dog for many months exclusively with meat, as pure and free from fat as possible, and made him perform several times every day for weeks very difficult labour. The animal showed continually "very extraordinary strength and elasticity in all his movements." Since the slight traces of carbohydrates and fat contained in the meat need not be considered in nutrition, it was proved that all the energy produced during the hard labour of the dog was derived from the transformation of proteid. But in order to discover whether the proteid served simply as a 
compensatory source of muscle-energy while carbohydrates and fats were lacking in the food, Pfluger ('92) carried on a series of experiments with mixed food. These led to the important result that with a food composed of proteid, carbohydrates, and fats, the quantity of the two latter substances that is destroyed in metabolism depends wholly upon the fact whether much or little proteid is fed. "In general, the quantity of carbohydrate and fat that undergoes destruction is smaller, the greater the income of proteid." The undestroyed carbohydrate and fat are changed into body-fat and accumulate as reserve-material, while, as is well known, the introduced proteid, however much it may be, is destroyed even to an excessively small fraction. It may, therefore, be said : "the need of food is satisfied first by proteid." Proteid is the "primitive food," carbohydrate and fat are simply a "compensatory food" employed during a lack of proteid.

Although in accord with this it is established beyond doubt that muscular work is made possible primarily by the decomposition of proteid, the equally undeniable fact, that the excretion of nitrogen in the urine does not appear to be correspondingly augmented by the most intense muscular activity, must excite surprise. In this connection another experiment of Pflüger deserves attention. Pfluger found that with pure proteid food and with an equal quantity of food during rest and during labour the excretion of nitrogen is increased by muscular activity very inconsiderably, and under certain circumstances not at all. Nevertheless, all the working-power must be derived from the decomposition of proteid alone, since no carbohydrate and fat are fed. With an excess of proteid such a remarkable phenomenon would be directly comprehensible, if we were to bear in mind that even during rest all proteid introduced into the body is decomposed; for if, as has been shown, the energy of muscular work is derived from the proteid decomposed, it might be concluded that an amount of proteid equal to that consumed during activity has been saved elsewhere in the body. This would be supported by the fact that all proteid eaten beyond a certain quantity is a luxus consumption, and is, therefore, available whenever needed. But if it be borne in mind that, as Voit ('60, '66) has shown, in hunger the excretion of nitrogen in the urine of the $\mathrm{dog}$ is increased by labour in the treadmill either not at all or only inconsiderably, this conclusion cannot be drawn, and the above explanation of the non-increase of nitrogen excretion does not suffice.

There still remains one possibility, which Pfluger has only touched upon, namely, that during labour a transformation of proteid takes place in the muscle without the nitrogen of the transformed proteid appearing in the urine.

This idea, to which we are forced by the facts, although directly 
contradicting an old-established physiological dogma, is not so wholly paradoxical as at first sight it appears. The dogma, which has retarded not a little the advance of knowledge of vital processes, and the origin of which is due simply to the fact that investigators devoted themselves so exclusively to the vital phenomena of higher animals, is expressed in the statement that the excretion of nitrogen in the urine is an absolute measure of the proteid-transformation in the body. Such an assumption is quite unproven, at least in this form. ${ }^{1}$ It may be said with a certain amount of justification that the nitrogen excreted in the urine is derived from the decomposition of proteids. But there is absolutely no justification for maintaining, vice versa, that all the nitrogen of the proteid transformed in the body appears in the urine. The fact that all food-proteid beyond a certain quantity is transformed in the body into groups of atoms, the nitrogen of which is excreted in the urine, cannot be generalised, and especially it cannot be applied to the decomposition of organised proteid, the biogens. As is well known, both non-nitrogenous and nitrogenous groups of atoms are derived from the decomposition of the biogen molecule. The non-nitrogenous groups such as carbonic acid, water, lactic acid, etc., leave the body at once. But the assumption is not required that all the nitrogenous groups also leave the body at once. It is conceivable that under certain circumstances the nitrogenous residue becomes regenerated into a complete biogen molecule at the expense of the food-stuffs and the oxygen, or in hunger at the expense of the reserve-substances. There would then be a decomposition of biogens which would result in no excretion of nitrogen in the urine. There is no fact that disputes the view that in muscle-activity the biogen molecule is decomposed, and that, in general, the nitrogenous residue regenerates the lost non-nitrogenous groups of atoms at the expense of the food. Such economy with the costly nitrogen would be wholly in accord with the methods of the organic household.

This idea, which has been here put forward simply as a possibility suggested by the facts, upon more careful consideration seems even probable. Before all else, it is in harmony with our general physiological views upon the nature of the vital process, and it accords with the ideas that must be formed, upon the basis of innumerable facts, regarding the events occurring in living substance. As is well known, the proteids are the chief constituents of living substance, and they are also the sole organic substances by the transformation of which alone the work of the living organism can be maintained. Moreover, as has already been seen, ${ }^{2}$ of all other substances that occur in the cell some serve for the construction of the proteids and biogens, and some are derived from the transformation of them. In other words, there can be no doubt ${ }^{1}$ Cf. p. 175. ${ }^{2} C_{f} . \mathrm{pp} .163$ and 479. 
that life is associated most closely with the construction and destruction of certain highly complex proteids, which for this very reason we have termed biogens. If this be granted, it would be in the highest degree paridoxical, if an increase of the vital process, and so churmous an increase as is expressed in intense muscular activity, should not be necessarily associated with an augmentation of the biogen-transformation in the body. Hence, to the end of his life Liebig, the old master of physiological chemistry, believed indefatigably that we ought to defind the view that the proteids are the substances the decomposition of which constitutes the source of muscle-mergy; and hence also Pfliger, one of the most far-seeing of the physiologists, combats again to-day the view that muscle-activity is able to take place without the decomposition of proteids. But if, during muscular activity, an increased transformation of biogens takes plice, and if, nevertheless, no more nitrogen is then excreted than during rest, the further conclusion is necessiry that the nitrogenous residue is again regenerated int, the complete biogen molecule. Without such a possibility the simplest and most general vital phenomena cannot be understood. How, for axmple, can the fact of growth, the fact that living substance is formed from other living substance only, be otherwise conceived than in accordance with the idea that the biogen molecule is capible of uniting to itself definite atoms and groups of atoms, and thus gradually grows into a polymeric molceule? Polmmerisation depends upon the successive introduction of groups of atoms. Further, regeneration rests in principle upon the same processic's as growth. The tact of complete recovery after total fatigue, also, and many other fundamental phenomena of living substance, presuppose unconditionally the regenerating: power of the biogen molecule.

It is, however, esinecially importint that the idea here developed is in accord with the two apparently irreconcilable views upon the source of muscle-energy. According to this idea, both the proteids and the carbohydrates of the food may serves as the source of this (nergy. If nuscular arivity is focussed in the decomposition and reconstruction of the biogen molecule, and if in its decomposition only non-nitrogenous groups of atoms leave it, it is evidunt that only non-nitrogenous groups of atoms also are employed for its reconstruction. The facts prove that the proteids as well as the carbohydrates of the food cin serve for this, although, as Pfliger has shown, with mixud ford and sufficient proteid the proteid is preferred. Thus the incontestable fact that in muscular activity the proteid food cin be protected to a certain degree by the carbohydrates, is comprehensible: and the clime that the carbohydrates on the one hand, and the proteids on the other, alre the source of musch-energy, are chually justiticl. The two substances cam play the same role, hewcrer, only by placing at the disposal of the 
biogen-residue non-nitrogenous groups of atoms. The vital process in muscle is the same, whether it draws its material from the proteid of the food or from the carbohydrate and fat.

The general validity of the idea here presented of the source of muscle-energy will be more apparent after the problem of the mechanics of the special changes of energy in contractile movements has been examined in detail.

\section{d. Theory of the Movements of Contraction and Expansion}

Without considering singly the almost innumerable theories that have been put forward regarding the mechanism of musclecontraction, we can distinguish among the more important views expressed in the later physiology two essentially different groups. There is general unanimity in the belief that muscle-energy has its source in chemical energy, and, if the ideas here expressed upon the vital process be accepted, there can be no doubt about this. But, while according to some physiologists the mechanical energy of musclework comes directly from the transformation of chemical energy, according to others it comes by a roundabout way through heat. The former view is defended by Pflïger ('75, 1), Fick ('82, '93, 1) and others, ${ }^{1}$ the latter especially by Engelmann ('93). A theory by the Göttingen philosopher, Elias Muiller ('91), which derives the one kind of energy from the other indirectly, first through heat and then through pyro-electricity, has not yet appeared in a complete form.

For the starting-point in our consideration we may best choose Engelmann's thermodynamic theory of contraction. Engelmann sees a difficulty in the direct derivation of muscle-work from chemical energy in the following circumstances. If from the amount of energy produced by muscle, upon the assumption that this is afforded by the combustion of carbohydrates, and upon the basis of a combustion heat of 4,000 calories per gram of carbohydrates, the quantity of substance be computed that is necessary to the work performed by the muscle in a single contraction, it is found that this quantity is surprisingly small in proportion to the mass of the muscle. Engelmann finds that approximately only one four-millionth of the whole mass can be considered as yielding the energy afforded in a single contraction. With the great amount of water in the muscle, which he assumes at approximately $70-80$ per cent., he regards it as incomprehensible how such an enormous, passive mass can be put into motion by the direct effect of the chemical energy of such a small quantity of effective substance working locally. He regards this as possible only when the chemical energy is first transformed into heat, which can be distributerl everywhere, and, therefore, is not limited in its effect to

${ }^{1}$ Cf. Verworn (92, 1). 
the place of its origin. According to him the transformation of a part of the heat into mechanical energy comes about by the shortening, as the result of being heated, of elements that are capable of swelling. In the last assumption he relies upon the two facts, that all positive, uniaxial, doubly-refracting substances, if capable of swelling, upon doing so shorten in the direction of their optical axis, and that bodies that are capable of swelling do so more when they are heated. According to Engelmann's investigations there exist in the anisotropic substance of muscle positive, uniaxial, doubly-refracting elements; and, as Engelmann likewise has shown, in the contraction of muscle liquid substance passes over from the isotropic, more liquid mass of the muscle-segment into the more solid mass of the anisotropic disk, so that the latter increases in volume. Engelmann supposes, therefore, that in excitation of muscle the elements of the anisotropic muscle-substance, which he terms "inotagmata." swell as a result of the heat derived from chemical energy and shorten, so that a contraction of the muscle results. Engelmann endeavours to make his idea especially clear by an experiment, in which the contraction of muscle is imitated, according to the thermodynamic principle, by the thermal swelling and shortening of catgut. In a beaker filled with water there is a stretched violin-string which is surrounded by a coil of wire and is connected with a writing-lever. By the making of an electric current the coil can be heated, so that heat is communicated to the string. The result is that the string swells and shortens and performs a certain amount of work by raising a weight. Upon the breaking of the current and cooling of the coil the string is extended again. Through its ingenious simplicity this experiment makes Engelmann's view extremely clear, and it is not to be doubted that at first sight it prepossesses one in favour of the thermodynamic theory. Nevertheless, there are many arguments against the latter, and various weighty objections to it have been brought forward, especially by Fick ('93, 1, 2).

Unfortunately it is impossible to discuss here the various difficulties that lie in the way of accepting Engelmann's theory. One only may be mentioned briefly, because its consideration leads to another view which, upon the basis of microscopic facts, is connected with the chemical theories of muscle-contraction. It must be demanded of a theory of muscle-contraction that its principle shall hold good for the explanation not only of muscular novement, but also of all other forms of contractile phenomena, i.c., for protoplasmic and ciliary movements also. "Since these are united by close transitions with one another and with muscular movement, the same explanatory principle must be able to find employment in all." But the above theory does not wholly correspond to this first and foremost requisite, which Engelmann himself puts forward. It is not able, e.g., to explain the motile phenomena of amœboirl 
protoplasmic masses. These simplest of all contractile phenomena offer insuperable difficulties to Engelmann's view. In order to bring the phenomena of amoboid movement into harmony with his theory, Engelmann $(79,1)$ is forced to the assumption that in amœboid protoplasm also the contractile elements have an elongated form and are capable of swelling so as to become spherical. But this assumption made $a d$ hoc is not only not based upon facts, but is unable really to explain the phenomena. In spite of careful investigation Engelmann has not succeeded in finding in amœboid protoplasm doubly-refracting elements similar to the fibrous structures of muscular substance. The observation that in Actinosphorium the pseudopodia have a doubly-refracting axial strand, is not applicable, because this axial strand has nothing whatever to do with contraction; it is simply a track upon which the contractile protoplasm can flow, and hence is analogous to the rays of the radiolarian skeleton, which are very wide-spread, especially in the Acanthometridce. But, even if the contractile protoplasm of Rhizopoda consists of numerous elongated elements that become spherical upon swelling, the extension of the extraordinarily long and slender thread-like pseudopodia that characterise most Foraminiferc and Radiolaria and numerous fresh-water Rhizopoda, would be wholly inconceivable upon this assumption. These varieties of pseudopodia are formed simply by the extension of the shorter, blunt or incised processes of an Amceba or a leucocyte. Even the formation of these latter pseudopodia cannot be explained according to Engelmann's view. How is the occurrence of even a moderate change of form of the Amoba body to be imagined through the simple extension of numerous elements which are of a size far below the limit of perceptibility and, as Engelmann himself assumes, lie irregularly among one another pointing in all directions? These difficulties are insurmountable.

We have here arrived at the point where the problem of the movements of contraction can first be taken into consideration with reference to the result. In the amoboid cell there is the most primitive form of contractile substance; here the relations are undeniably much simpler than in the fibrous forms with their complex differentiations. Moreover, the phenomena exhibited by the living object can be investigated experimentally with incomparably more ease in the free-living and relatively large protoplasmic masses of amœboid cells, than in the very small constituents of the muscle, which, separated from continuity with their neighbours, invariably perish in a very short time.

Hence, we will consider, first, the amœboid movement of naked protoplasmic masses. ${ }^{1}$ As has already been seen, ${ }^{2}$ the element common to all phenomona of contraction is the alternation of two opposed phases, one of contraction in which the surface is diminished

$$
1 \text { ( } f \text {. Verworn }(92,1) \text {. }
$$

$2 C y$. pp. 233 and 252 . 
in proportion to the mass, and one of expansion, in which the surface is increased. In amoboid movement expansion is expressed in the extension of pseudopodia, and contraction in their retraction and the endeavour to assume a spherical form (Fig. 268). The interchanges between the two constitute the whole phenomenon of amcboid movement. As is well known, a naked protoplasmic drop, for example an $A$ moeba-cell, behaves physically like a liquid. Its movements must, therefore, obey the general laws of liquids, as Berthold ('86) especially has consistently applied them to numerous special cases. Physically considered, every movement of a drop of liquid is the expression of changes of surface-tension, i.e., of the energy of cohesion with which the individual particles in a freely-suspended drop attract one another. If the surface-tension is equal at all points, the drop assumes a spherical form. If for any reason it is diminished in one place, there occurs there as the result of pressure from the other sides a protuberance which increases until equilibrium is again established. If the surface-tension at the same place becomes greater, the protuberance diminishes correspondingly. Hence, the spherical form of an amoboid cell is the expression of a surface-tension equal at all points; the extension of pseudopodia at localised places is the index of a diminution of surface-

A
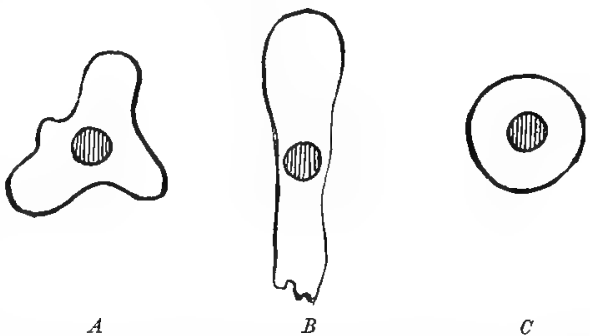

Fig. 26s.-Amoeba in outline; the nucleus lies in the interior. $A$, Extending pseudopodia in various directions; $B$, creeping in one direction; $C$, contracted into a ball. tension at those places.

In other words, the problem of amœboid movement thus made clear is contained in the question: what causes, on the one hand, a diminution of surface-tension (extension of pseudopodia), and, on the other, an increase of surface-tension (retraction of pseudopodia and tendency toward a spherical form)?

Regarding the manner of diminution of surface-tension, Kühne's experiments ('64) upon Amceba and Myxomycetes, already spoken of, are decisive. When Kühne placed a drop containing Amcbce in a medium that contained no oxygen, but was indifferent in other respects, as, for example, hydrogen, the amœboid movement gradually ceased, and the Amoebo maintained the forms that they had already assumed. If oxygen were then allowed to enter, the movement began again, new pseudopodia were extended and the Amoboe resumed their creeping: Kiihne's experiments upon the plasmodia of Myxomycetes are equally clear. He put a lump of a dry plasmodium of Didymium in a vessel filled with water containing 
no oxygen. In this condition no pseudopodia were formed for days. When, however, he let a few small bubbles of air into the vessel, the extension of pseudopodia immediately began, and after five hours the lump of protoplasm had extended upon the inner wall of the vessel into a richly-branched network. It is evident from this that it is the chemical affinity of certain portions of the protoplasm for oxygen that diminishes the surface-tension at definite places, and so leads to the formation of pseudopodia. With unilateral action of oxygen this must lead to positive chemotaxis, as has actually been demonstrated by Stahl ('84) in naked protoplasmic masses. As regards the manner in which the chemical affinity of the protoplasmic particles for the oxygen of the medium diminishes the surface-tension of the drop, it may at least be imagined that by the introduction of the oxygen-atoms into the biogen-molecules the cohesion of the latter is diminished.

In non-living nature there is a very striking analogue of the fact that amœboid changes of form and movements are caused in a drop by the chemical affinity of certain constituents of the drop for substances in the surrounding medium. This is afforded by the interesting experiments of Gad ('78) upon the behaviour of oildrops in alkaline media, which later were studied by Quincke ('88). It is well known that rancid fats and oils contain molecules of free fatty, or oily, acids between the molecules of pure fat or oil. Upon contact with alkalies these acids combine with them to form soluble soaps. Hence, if a drop of rancid oil be put into a feebly alkaline liquid, a continual formation of soap takes place at the surface of contact of the two. Thereby the surface-tension is diminished locally here and there, and there results a genuine formation of pseudopodia by the oil-drop. By varying the alkalinity of the medium and the amount of free acids in the oil-drop, a great variety in the forms of the processes can be produced, many of the latter presenting a startling similarity to the forms of pseudopodia of certain Rhizopoda (Fig. 269).

If, thus, by the chemical affinity of certain particles of a drop for substances in the surrounding medium the surface-tension is diminished, vice versa an increase of surface-tension must come about by increased attraction between the particles of the drop. Such an increase of cohesion between the biogen-molecules themselves, or between them and other constituents of the cellbody, is comprehensible when it is borne in mind that the extent of the molecular attraction is influenced by changes in the chemical constitution of the molecules. It has been seen above that the cohesion is diminished by the oxidation of the latter. If now they be decomposed, the idea is strongly suggested that this profound change in their chemical constitution is associated with an increase of cohesion.

Upon the basis of this idea, the following picture may be drawn 
of the mechanism of amœboid protoplasmic motion. Starting from the spherical form of the amcboid cell, the surface-tension would be locally diminished at any desired point of the periphery by the introduction of oxygen into the biogen-molecule; the protoplasm would be bulged out; and sunce new biogen-molecules would thus constantly come into contact with the oxygen of the

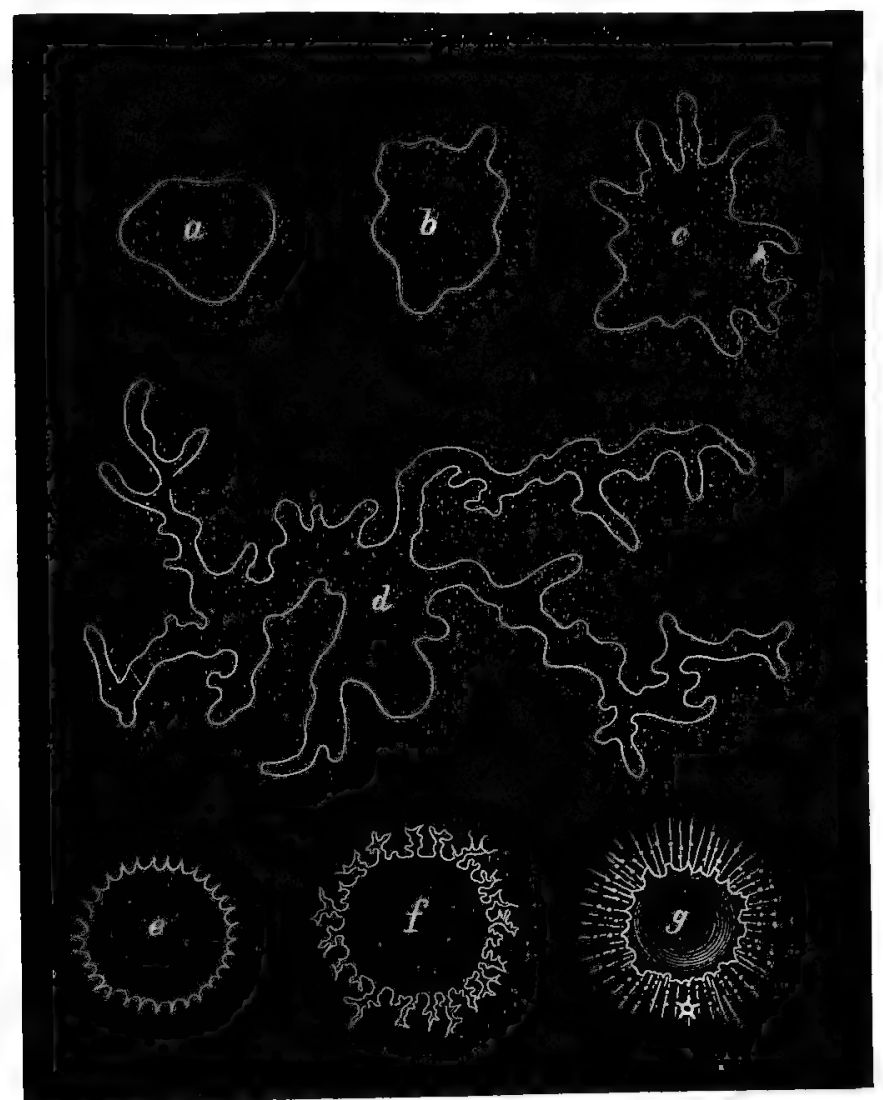

FIG. 269,-Various forms assumed by oil-drops in an alkaline liquid.

surrounding medium, a longer or shorter pseudopodium, according to the peculiar character of the protoplasm, would form. The phase of expansion would thus be mechanically explained. By the introduction of oxygen the biogen-molecules would then have reached the maximum of their labile constitution. They would then become decomposed, to a certain extent spontaneously, but more through the action of stimuli that excite dissimilation. 
With their decomposition the surface-tension would increase, and the stimulated protoplasm would necessarily flow back centripetally, so that the pseudopodia would be retracted-a phenomenon that is called out in a very characteristic manner by all sorts of stimuli. Thus the phase of contraction would be mechanically explained. After their return to the central cell-body the biogen-molecules would have an opportunity to regenerate themselves with the aid of substances produced by the protoplasm and the nucleus, which are absolutely necessary to the intact life of the cell; then after the introduction of oxygen they would begin their course anew.

In accordance with this idea, all the special phenomena exhibited by amœboid protoplasmic masses in their movement may be understood. The necrobiotic phenomena of naked protoplasmic masses especially, which can be followed very beautifully in amputated, non-nucleated, hyaline pseudopodia of Diffugia
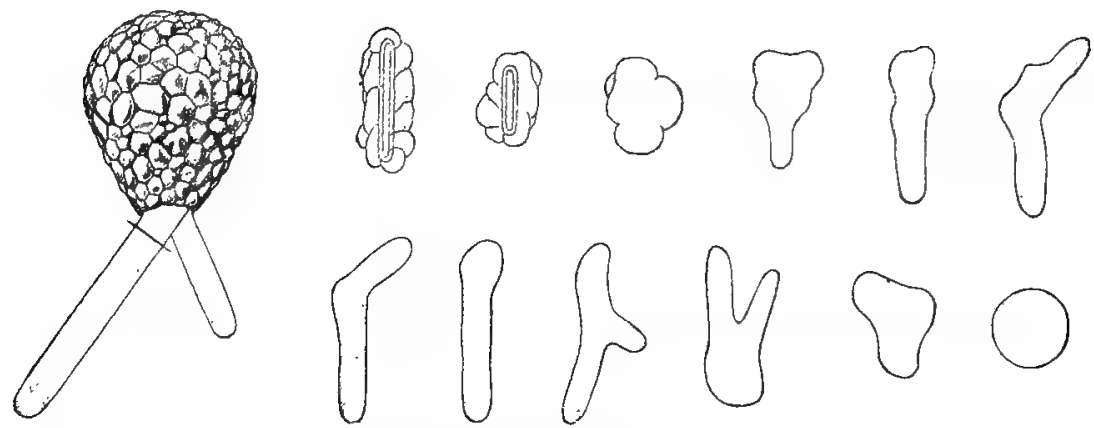

FIG, 270.-Diflugia lobostoma, with two pseudopodia projecting from the sand-capsule, the larger of which is amputated. Beside it, the changes which the separated mass of protoplasm passes through in the course of a few hours are shown from the left above to the right below. At first, normal movement with formation of pseudopodia, finally, death in the spherical form.

(Fig. 270), may be explained at once: the continuation of the amoboid motion at the beginning, the gradual cessation of the formation of pseudopodia, and finally death in the spherical, contracted condition. ${ }^{1}$ At the beginning, immediately after the amputation of the mass, a quantity of nuclear and protoplasmic substances, which the biogen-molecules need for their regeneration, is available in the protoplasm; the extension and amalgamation of the pseudopodia proceed at first normally. But gradually these substances are consumed, the oxydized biogen-molecules become decomposed, the pseudopodia retract, the regeneration of the biogens becomes impossible, and the incomplete biogen-residues are incapable of oxidation. Hence new pseudopodia are no longer formed, and, when all the oxidized molecules are decomposed, the mass perishes without any further change of its spherical form. 
The above-developed idea of the mechanism of amœboid protoplasmic movement has at once the great advantage that, though modified by special conditions in individual cases, its principles nuy be applied to all other phenomena of contraction, to protoplasmic streaming in plant-cells as well as to ciliary and muscular movement. We will here select only the most complicated case, the movement of cross-striated muscles. Since the same processes go on in all the individual muscle-segments, we will limit ourselves to consideration of the single segment. As has already been seen, ${ }^{1}$ the muscle-segment consists of two different substances, the more solid, anisotropic substance lying in the middle, and the isotropic substance lying at the two sides of the latter (Fig. 271). The phenomena that are visible with the microscope during a contraction resulting from stimulation, as Engelmann ${ }^{2}$ and others have established in detail, consists essentially in the flowing of isotropic substance from both sides into the anisotropic; thus the latter substance increases

$I$

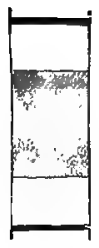

4

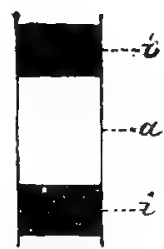

$B$
$I I$

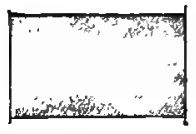

$-4$

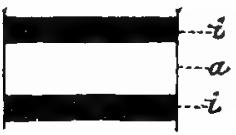

$B$

FIG. 271.-Muscle-segments. $I$, At rest, $I$, in contraction; $A$, in ordinary, $B$, in polarized light. $r$, Anisotropic, $i$, isotropic disks.

in volume and the disk becomes broader, while the length of the whole segment decreases correspondingly. Hence the elementary fundamental phenomenon in muscle-contraction is a mixing of two substances which at rest lie beside one another; constituents of the isotropic, or more mobile substance, force their way into the anisotropic, or fixed substance. In this process the fact, which E. A. Schäfer ( $91,1,2,3$, discovered, is noteworthy, namely, that the anisotropic substance, which does not change its place, offers the greatest possible surface to the entrance of the isotropic substance by means of the system of tubes already mentioned, ${ }^{3}$ so that the intermingling is able to take place very rapidly (Fig. 272). During the explosive decomposition of the biogens, either in the isotropic or the anisotropic substance, which latter is regarded by Engelmann as the specially contractile element, the chemical constitution of the biogen-molecules is so changed that a molecular attraction arises between them and certain
${ }^{1} C f$. p. 213.
Cf. p. 245.
${ }^{3} C f . \mathrm{p} .245$. 
constituents of the other substance. As a result of this, the surface-tension between the two disks must necessarily diminish (or even become zero); i.e, an intermingling, a mutual penetration of the two substances must take place. In this process the isotropic, as the more mobile, substance will necessarily diffuse into the anisotropic, as the more fixed, i.e., the muscle-segment will necessarily decrease in length and increase in breadth. There will thus be in principle the same process as in swelling, except that, as Engelmann assumes, there will be, not a simple admission of water, but a chemical swelling, in which along with the water other chemical substances will enter, especially such as take part in the regeneration of the decomposed biogen-molecules. But in proportion as these molecules are regenerated and by the introduction of oxygen are brought back to the maximum of their labile constitution, a change in the molecular relations occurs, and now, in contrast to what happened previously, a separation of the two substances will take place, which will

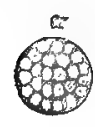

b

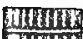
Jinimit

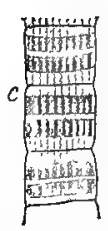

Fro. 272.-Muscle-segment of the wasp containing tubes of anisotropic substance. $a$, Anisotropic disk seen from above; $b$, seen from the side; $c$, three muscle. segments. (After Schäfer.) give to the muscle-segment its original form. Although the processes, which for the present are wholly unknown, may in reality take place very differently, at all events the principle of modification of the molecular attraction by changes in the chemical constitution of the molecules, the same principle that explains amoeboid movement, appears to be able to elucidate in its essential points the obscure phenomenon of muscular movement. Thus, contraction-movements in their most essential points are controlled by the direct interchanges of chemical and mechanical energy without the mediation of another form of energy, such as heat or electricity.

Here consideration of the mechanism of contraction-movements merges with that of the changes of energy in muscle activity, and we arrive again at the view already reached by an entirely different path, namely, that the activity of muscle depends upon the alternation of the decomposition and regeneration of living protoplasmic particles.

We have now reached the end of our inquiry into the mechanics of cell-life. Starting from the idea that in metabolism lies the real vital process, which is expressed in the manifold vital phenomena, we endeavoured to trace back the elementary vital phenomena of the cell to the chain of metabolic processes, by which the individual parts of the cell are united with one another and with the external world. Our last discussion, that of movement in the cell, affords the best example of how the changes of 
form and energy are inseparably connected with the changes of substance, how all three in reality form a single whole, which offers merely different sides for consideration. So far as scientific knowledge renders it possible, an attempt has been made to solve the problem. Naturally many suppositions and many hypotheses have been found necessary to close the wide gaps in our present knowledge, and in spite of this many recognized gaps still remain open. But cell-physiology, aided by the stern necessity of its development, and its great working power, is beginning to give encouragement to the highest expectations.

\section{The Constitutional Relations of the Cell-Community ${ }^{1}$}

So far, in all our investigations, experiments, discussions and theories the individual cell, as the independent elementary organism, has been the chief object of interest. Now, in terminating our long examination of the physiological problem, it remains to examine the mechanism resulting from the association of the cells in a community. The life of the multicellular organism is not a simple summation of the lives of the individual cells which compose it; many special relations are inaugurated by the association of the individual cells, and these are expressed in the vital phenomena of the multicellular organism.

\section{A. INDEPENDENCE AND DEPENDENCE OF THE CELLS}

It has been seen elsewhere ${ }^{2}$ that the size of the individual cell is necessarily limited. From this fact an important consequence follows. A large organism can never be formed by a single cell, it must be constructed from many cells. All large organisms are cell-communities. By the union of the individual cell with. others of its like, relations are presented that influence the life of the former so that its vital phenomena are different from what they are when it lives free. As in the formation of every community. the formation of that composed of cells requires a compromise between individuals. The compromise consists in the fact that every cell gives up a part of its independence for the advantage that it derives from association with other cells. The special form of this compromise between the individual components is very different in different cases. In the cell-communities of the series of organisms we find realised a much greater variety of forms of government than we sce developed in human society, and it would

1 [The word "state," as employed in Political Science, carries with it the idea that the association of individuals thus designated is a self-conscious association. (Cf. Giddings: The Principles of Sociology, New York, 1896.) I have thought, therefore, that in the present book the author's term "Zellenstaat" would preferably be translated, not "cell-state," but "cell-community."-F. S. L.]

${ }^{2}$ Of. pp. 79 and 530 . 
be very profitable to treat modern sociology in the light of these. If this were done, doubtless many schemes regarding social reform would result very differently from at present.

In a cell-community there is nothing living but the cells. The life of the community is merely the expression of the lives of the cells. Hence it is evident that a cell-community can live only when its individual constituents lead suitable lives. The independent vital activity of the individual cell is, therefore, the indispensable prerequisite of the life of the compound organism. But how much of its independence the individual cell gives up in uniting with others is subject to great variation. That it must always give up something is evident when it is borne in mind that by the association of the different cells the external vital conditions of the individual cell become greatly changed. Cells that have permanently exchanged free individual life for life in a cell-community, such as the tissue-cells of the higher plants and animals, usually perish very soon when separated from their associates. The other cells of the community become an external vital condition for the tissue-cell.

This condition of dependence in which the cells of the community stand to one another is less, and the independence of the individual cell is greater, the lower we descend in the series of organisms, the more the individual cells of the community resemble one another.

The simplest relations are found among the Protista. Here we find cell-communities of the primitive type of a genuinely republican form of government, in which every cell is like the others and is capable of existing by itself independently of the others. A Carchesium stalk (Fig. 273, I), a Eudorina colony (Fig. 273, II, A), and a Magosphocra globule (Fig. 273, II, $B$ ), are such true cellrepublics. Sometimes the members of these communities separate themselves from one another and lead an independent life. But, so long as they are united, a certain dependence exists even in the genuinely republican community, in spite of the great independence of the individual cells. The individual Carchesium is influenced by its neighbours. If one of its neighbours suddenly contracts, it is likewise made to contract by the shock. The individual Eudorinaor Magosphorra-cell in its movement is likewise dependent upon the others. The stroke of its cilia does not drive it to the place where it would swim if it had free locomotion, but is only one of the many components from which the movement of the whole spherical colony results.

But the dependence of the cells is much greater in the cellcommunities of the plants and the lowest Colenterata, which stand upon the same social grade with the plants, than in these cellrepublics of the Protiste. The government of plants has also been termerl republican, in contrast to the more monarchical govern- 
ment of animals. This designation is correct, but the government of the cell-communities of plants, sponges, and hydroid polyps is not the primitive form of a republic, which has been seen in the colonies of the Protista. We find here no longer the power of the individual cell to exist by itself apart from association with the

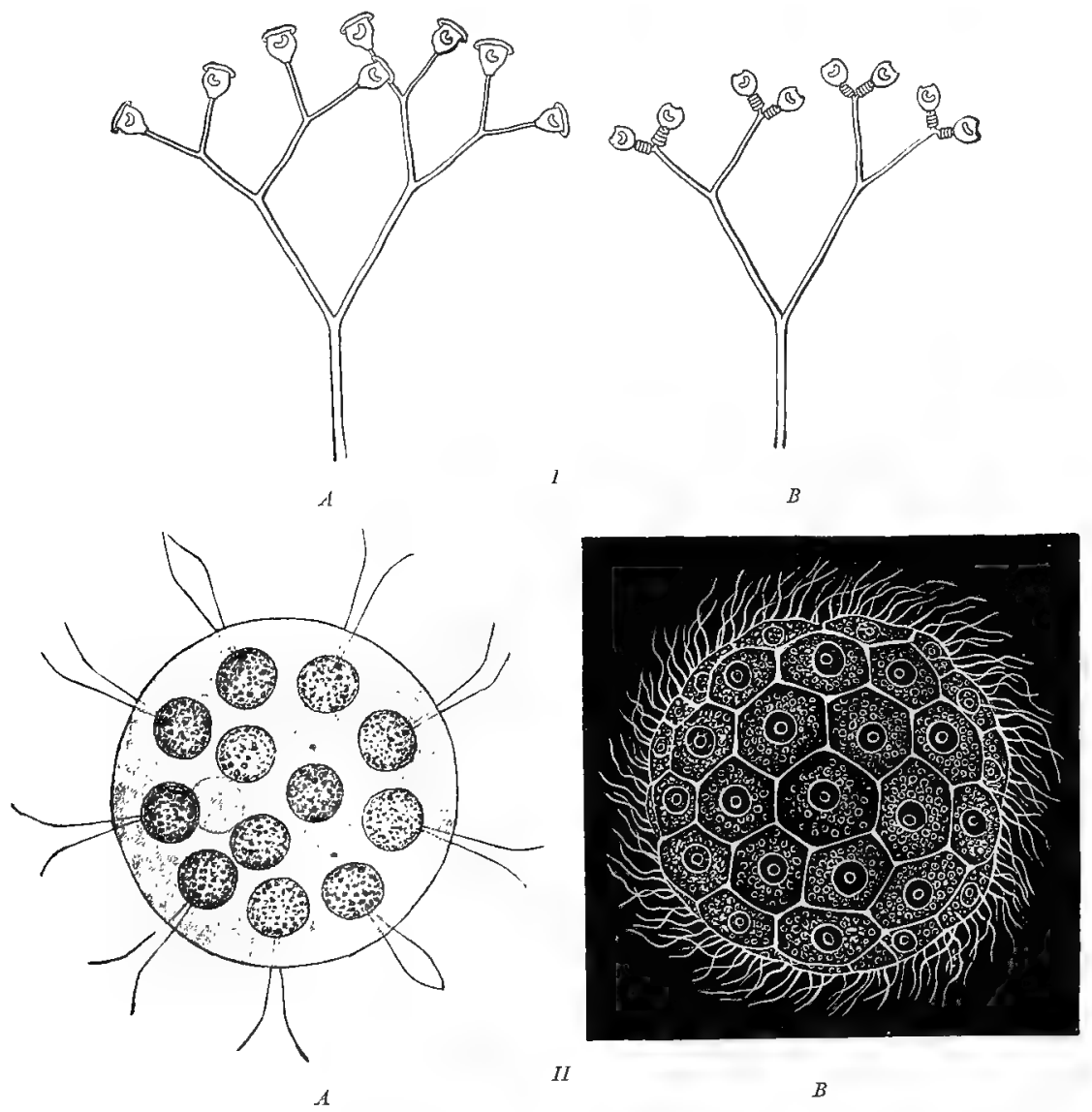

FIG. 273.-I, Carchesium polypinum, a stalk of Ciliata. A, The individuals are extended upon their . a colony of Flagellata; $B$, Magosphera planula, a colony of Ciliata. (After Haeckel.)

others. Dependence upon the other cells is too great, but small groups of cells can maintain themselves and live separately. E.g., as Vöchting ('85) has shown, the leaves of many plants can be cut into minute pieces and from them whole plants can grow, and likewise, as has been seen (Fig. 2, p. 57), every piece of a Hydru that has been cut up is capable of independent life. 
The dependence of the individual cells upon one another in unany tissues of the higher animals is still closer than in the plants and the lowest Colenterata. Here a pronounced despotism prevails. The constitution of ciliated epithelia affords an interesting example. As is well known, a ciliated epithelium consists of many successive rows of ciliated cells arranged one after another in each row, and each cell possessing a number of cilia (Fig. $274, I$ ). The cilia of these cells are in rapid, rhythmic vibration, but it is seen that the ciliary motion of the individual cells of one row is not irregular and independent of the others; there exists a

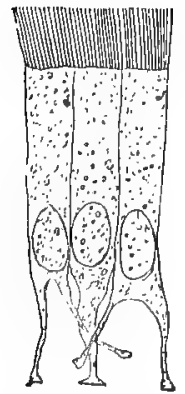

$I$

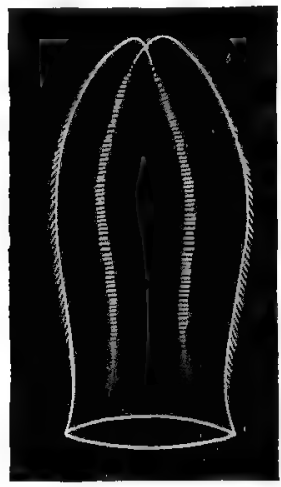

II

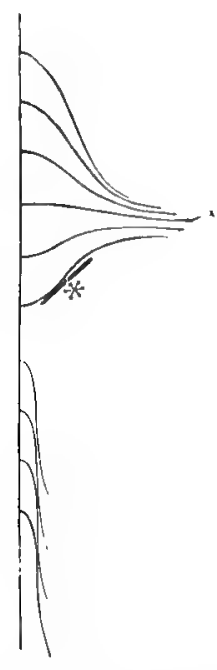

III

I'H:. 274.-Ciliated opitholium. I, Throe ciliated cells connectod with ono another, from the cpididymis. (After Schiefferdocker.) $11, B e r^{*} 0 e$ ora/a, with tho four rows of ciling plates upon ono side. III, Row of cilia of a Beroë seon from the sido. At * a plate is fxed by boing bont back by meang of a small scalpol, so that it cannot contract. $A$ a result of this, the ciliary wavos pass from abovo only to this plate, whilo tho plates below it are at it staud. still.

metachronism in the ciliary stroke ${ }^{1}$ in such a manner that the cilia of all the cells contract in regular succession, beginning with the end-cell of the row. This phenomenon can be observed much better in the rows of ciliary plates in the Ctenophora (Fig. 274,II) than in the microscopic ciliated epithelium of the vertebrates. In the former, where the ciliary plates are: to be seen very distinetly with the naked eye, and where the movement goes on often very slowly, it is readily observed that cvery plate moves only when the precerling one has movesl, and then remains at rest until a new wave comm from the first plate. If' such a row with the under-

$$
1 \text { Cf. p. } 247 .
$$




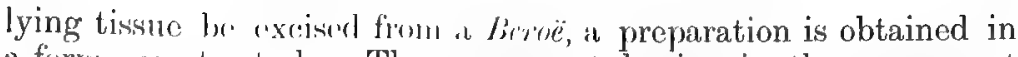
a form casy to study. The movement begins in the uppermost plate and prowerest to all the succeding ones. If the uppermost plate is at rest, so :W all the suceceding ones; a plate in the midkll. of the row nuver contracts while the preceding ones are at rest. If a plate in the middle of the row be held fast, the ciliary waves course from abov: down to this plate only; they stop here, and all the lower plates in the sorics stand still (Fig. 274, III). Thus every plate is in the closint dependence upon the one next ibove, and is never able to mure independently. But the moveinent of all the plates is determined in this manner by the first plate of the series (Fig. 275, A). In spite of this, c:very plate posisesise in potentia is certain independence. If, e.g., the row be cut through, the plate that now stands first in the series underliakes the learl, and controls by its contraction and its rest all the plates standing below it, so that the two separated halves of the
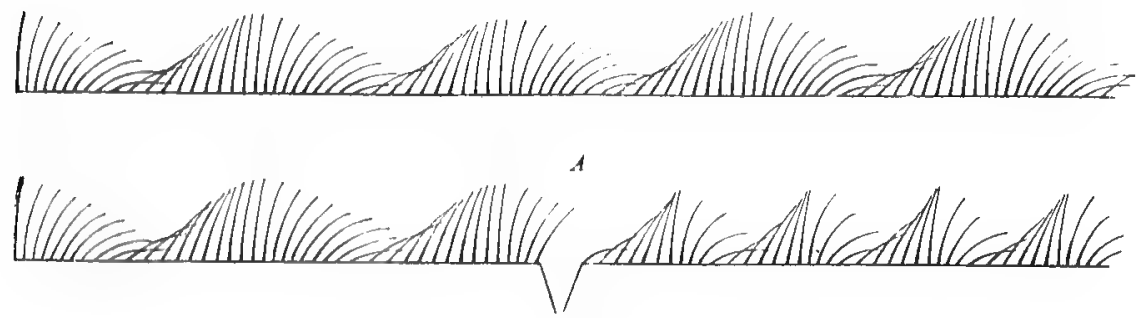

h

1'14. $275,-A$, Intact row of eilin showing nomal motuchronimm of bont. The uppormost (loft) cllium ineugurates the rhy thm and the othors follow at thosamo rato. $B$, lew of cilia dividod in the intallo by a cut. Ioreh half actio with ity own rhythm.

row now act with separats rhythms (Fig. 275, b'). Every individual plate, inded, bik'n out of the series contracts rhythmically, providerl that the cell-body belonging to it is still present. There is here an intoresting casce of completesubordination. Every ciliated cell of an eprithelium, so long as it is living, possesses in itself complete autonomy as regalds its movenont; in union with its like, however, it has wholly given up the independence of its movenumt. This is neressiary if a motachronous motion, which

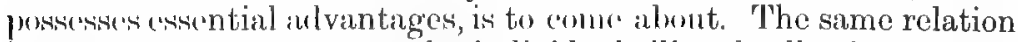
is found not only betweren the individual ciliated eells of an epithelium, but also bitwer'm the individual cilia of at cell. In the latter the simm me maxhronism of beat exists in a long row of eilia, as may

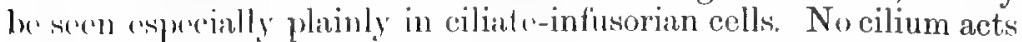
befire the one jureceling it in the row. If the uppermost one rests, the whole soriss is quiol. Novertheless, "vory individual cilium, separated from the others, shows complow independence of movement. If', c.g., in striresstomum the long row of peristome 
cilia be cut into at one place, the two halves are able to act independently of one another. ${ }^{1} \quad$ Even if a single cilium with a droplet of attached protoplasm be cut off from the cell-body, it acts rhythmically and spontaneously until it perishes. It must, therefore, be assumed that the complete dependence of the individual cilium and the individual ciliated cell upon the one next it, is conditioned by some kind of mechanism in the basal protoplasm, which hinders all independent movement, and mediates only impulses from that side. ${ }^{2}$ But this is only possible when in the ciliated epithelium an unbroken continuity of the basal protoplasm exists throughout the whole row of cells. It is known that protoplasmic connections between the individual cells in the cellcommunity are wide-spread in both plants and animals.

Finally, the most thorough-going despotism exists in the higher animal in the dominion of the nerve-cells over the cells of all kinds of tissues. The higher we go in the animal series, the more we see the tendency of the nerve-cells to extend their dominion to all the tissues of the body. The loss of independence thus resulting goes so far in many tissue-cells, that their vital activity sinks to a minimum so long as it is not stimulated by impulses from the nervecells. Spontaneity is apparently wholly lost. A skeletal muscle in the vertebrates never performs a contraction spontaneously; the ganglion-cells of the central nervous system alone by their impulses are able to put it into contraction. We ought not to be misled by this lack of spontaneous contractions into believing that the metabolic processes that characterise muscular activity are at a complete standstill during rest. This is only apparently the case. As a comparison of the arterial blood streaming to the muscle with the venous blood coming from it teaches, the same metabolic processes go on in the muscle during rest as during activity, but in so slight extent and so uniformly that a contraction is not thus brought about. But if by nervous influence they undergo a sudden augmentation, the contraction appears. Wholly analogous to the dependence of the muscle-cells is the relation of many other tissue-cells, e.g., gland-cells, to the central nervous system; and even the relation of the ganglion-cells to one another is partly of the same kind.

The general principle upon which is based the formation of the cell-community, and with it the formation of a more or less close dependence of the individual cells upon one another, is the principle that controls all development. It is the principle of utility. The fact that the cells remain together after division and thus form a community consisting of several like components, which occurs in the Protista, secures the advantage of greater protection for the individual cell. But, as has been seen, a certain dependence of the individual cells upon one another is conditioned by this simple

$$
1 C f \text {. Verworn ('89, I). }
$$$$
2 C f \text {. Verworn ('90, 2). }
$$ 
fact. That this dependence, the higher we ascend in the developmental series of organisms, becomes closer and more fixed, depends again solcly upon utility, for the greater the unity in the government of the whole community, the surer and greater is the work of the whole, and the greater also is the advantage that the individual cell receives from the common life. Unity in the government of the cell-community is, however, determined essentially by the dependent relation of the individual cell to the other cells. Darwin's theory of selection, which contains a general explanation of adaptation in the organic world, has made it clear how such adaptative arrangements must be developed in a natural manner. Of course, the immediate mechanical causes are to be sought in each individual case.

\section{B. DIFFERENTIATION AND DIVISION OF LABOUR AMONG THE CELLS}

In the evolution of mutually dependent relations between the cells in the origin of the cell-community we have become acquainted with only one result of the common life of the cells. It is the sole result, so long as the community does not surpass certain dimensions. If, however, the community becomes larger, if it develops into a compact mass, another necessary mechanical result of the association is observed, namely, the differentiation of and division of labour among the cells.

The differentiation of cells consists, as is well known, in the assumption by the cells of different characters, so that the community is no longer composed of like cells, but of cells and cell-groups of different kinds. Therewith there appear not only morphological, but also physiological differences between the individual cells, i.e., the performances of certain cells or cell-groups become different from those of others, and a division of labour between them takes place. Differentiation and division of labour are inseparable from one another.

The mechanical causes of cell-differentiation in the cell-community are fairly evident. All the properties of an organism, morphological as well as physiological, are the expression of the interaction of two factors, namely, the relations between its internal and its external vital conditions. ${ }^{1}$ If one of these two factors changes, there is a change of the properties of the organism. If, therefore, a cell divides into many like offspring, and if all these offspring remain together and form a cell-community, all the constituents of this community will remain alike, so long as the external conditions surrounding each cell are the same as those surrounding all the others. We have become acquainted with such cell-communities among the Protista. But such a community is only possible when all the cells are arranged beside one another

$$
1 \text { C t p. }=207 .
$$


to form a row or a surface. This is the case here. The largest cell-communities composed of like cells that are known among the Protistc are those that are related to the Algoe among the plants. They are either fibrous, such as the Confervoe (Fig. 276), or foliaceous, such as the large Ulvaceo. In the latter, cell is attached to cell to form a flat surface, so that the part of the cell-surface that is free, and the part that borders upon its neighbours are the same in every cell; thus all cells are under like external conditions. But, if the cells proceeding from the division of one cell do not all remain under like external conditions, and if the cells do not perish, in time differences must appear. This condition is realised in the formation of every cell-community the component cells of which are not arranged in a flat surface, but are distributed in all direc-

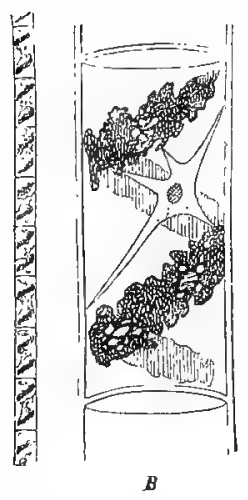

FIG. 276...Spirogyra, a multicellular, fresh-water Alga. $A$, Piece of a multicellular thread. $B$, Single cell. In every cell the chlorophyllbody winds spirally along the inside of the wall. tions as solid complexes. Here the cells that lie in the interior of the community are under wholly different external vital conditions from those at the surface. As a result of this they must form a contrast to the latter, both morphologically and physiologically; in other words, differentiation and division of labour result. The simplest examples of this are likewise met with in certain forms of the Protista, which form such an extremely interesting transitionstage to the cell-communities of the plants and the animals. Such an organism is the Protospongic Hockiliz (Fig. 277), a colony of flagellate Infusoria, which as regards histological structure has a certain similarity to the lowest sponges. Upon the surface of a gelatirous mass sit numerous, cup-shaped, flagellated cells, while in the interior of the mass there are many amoboid cells without flagella. Here, therefore, is a differentiation of the cells living in the interior as compared with those living upon the surface, which is extremely marked and the cause of which is at once evident. It is especially interesting in connection with this organism that the differentiation exists only so long as the causes exist. The amceboid cells of the interior, for example, have the power of wandering to the surface, and in this case they likewise develop into cup-shaped flagellated cells. In these lowest forms of the differentiated cell-community, therefore, the individual cells still possess in the highest degree the capacity of changing into other forms.

Differentiation of the cells by adaptation to the external conditions afforded by different positions, which is only barely indicated in the Protiste, is the fundamental principle in the construction of 
the animal and plant cell-community. This principle is here realised most completely and in the smallest details, and finally leads to the construction of so complex an organism as the cellcommunity of the human body. The whole development of the most complex animal body with all its differentiations depends solely upon the principle that the farther cell-increase proceeds upon the simple mechanical basis of the different relative positions of the cells and cell-groups arising from the continued division of the ovum, the more various must be the mutual relations and the external vital conditions of these cells and cell-groups, so that, by adaptation to the constantly changing external conditions, the cells and cell-groups finally diverge and become gradually differentiated as regards all their characteristics. As is known from the fundamental law of biogenesis, the mechanics of ontogenetic development, in so far as special adaptations do not come into play, pursues essentially the same course that development has pursued in thephylogenetic series. The mechanical causes of the differentiation of the cells in the formation of every cell-community are evidently the same in their most essential features in

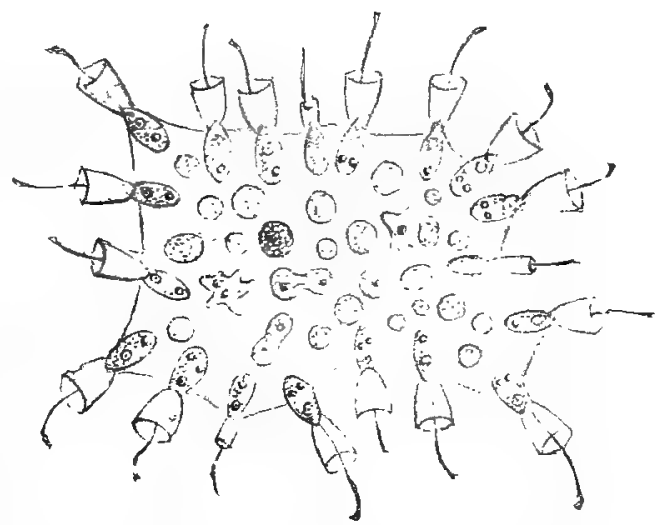

Fio. 2T.-Protospongia Hecelelii. (After Lang.) the ontogeny as in the phylogeny. It remains for the embryology of the future to discover in detail the very manifold special relations, which are as different as the organisms themselves.

While the mechanical causes of cell-differentiation in the complicated cell-community must be sought in changes of its relations with the environment, which for every cell and cell-generation are due to continued cell-division, division of labour among the cells is based upon the development of the cell-community itself. ${ }^{1}$ The work of every multicellular organism is the expression of the activity of its individual cells. If the cells are different, they contribute in a different manner to the whole labour of the organism. That this combined labour must become harmonious and advantageous follows from the principle of selection, which controls all organic development, phylogenetic as well as ontogenetic. Only those cell-communities continue to live, in which the cell-generations

$$
1 \text { Cf. p. } 536 .
$$


arising from the continued division of the egg-cell are in harmony with the special conditions under which they appear. All in which this is not the case must perish in the struggle for existence through the action of selection. But the most complete harmony is reached when the individual labours of the different cells so fit into one another that, although every cell or cell-group has developed a different labour for its own specialty, this labour is for the good of all the other cells, is, indeed, necessary to all the others. Thus, the extraordinarily far-reaching differentiation and surprisingly detailed division of labour of the individual cells and tissues in the cell-community become comprehensible.

As a result of the division of labour, every kind of cell, every tissue, every organ in the multicellular community undertakes a special task, and since early times physiology has termed this task the "physiological function" of the cell-complex in question. All elementary vital phenomena which, in the lowest organisms, take place in the individual cell, in multicellular organisms are developed in a special degree as specific functions of definite cell-groups and become adapted very perfectly to specific purposes. Thus, in the higher animals, by the special development of contractility, movement becomes the specific function of the muscle-cells. The capacity of appreciating stimuli is developed in an especially high degree as the function of the sense-organs. The capacity of conducting stimuli is augmented to a surprising extent as the function of nerves. Secretion undergoes its greatest perfection in the function of gland-cells. Every kind of cell retains all the elementary vital phenomena, but the one becomes preeminently developed as its specialty. The more the specialties of the individual cells and cell-groups come to act for the good of all cells and assist their vital processes, the more highly evolved does the cell-community become. It represents a mechanism in which, in spite of its extraordinary extent and its excessive complication, as exhibited especially in the bodies of the higher animals, all its parts co-operate as a unit.

\section{CENTRALISATION OF ADMINISTRATION}

If the last point, namely, the development of a unity in the cooperation of the cells and tissues of the cell-community be developed more in detail, it is found that in addition to the principles of dependence and cell-differentiation, a third principle comes into consideration, namely, that of centralisation of administration. This principle is connected very closely with the two others; considered from the point of view of natural selection, it is in a certain sense a necessary result of those, and it is developed pari passu with them.

The farther the differentiation of the cells goes and the closer 
becomes the dependence of the cells upon one another, the stronger is the necessity of bringing into relation with one another the more outlying cells, tissues and organs of the cell-community, in order that unified co-operation may take place; selection must make this relation constantly more intimate, the more complex the structure of the cell-community becomes. Along with this there arises in the community a tendency toward centralisation.

The first step in the direction of centralisation is really taken by means of the division of labour, when certain cellgroups or organs undertake a definite function for the whole community. Thus the function in question becomes centralised for the whole body in one place, and as many centres arise as there are organs differentiated for definite functions. This first step toward centralisation of administration is met with in the cell-community of the plant. Here the synthesis of starch, upon which the nutrition of the whole plant depends, is centralised in the green cells of the leaf. Further, the function of taking up water, without which life cannot continue to exist, is localised in the roots alone. Corresponding localisations are present in the animal cell-community. Thus, in the higher animals the nutrition and respiration of the individual tissue-cells are centralised in the heart, which drives the blood, rich in food and oxygen, to all the cells of the various tissues and organs (Fig. 278).

In the animal cell-community the second important step toward centralisation is taken, namely, the union of all individual centres or organs of function with one another by the appearance of

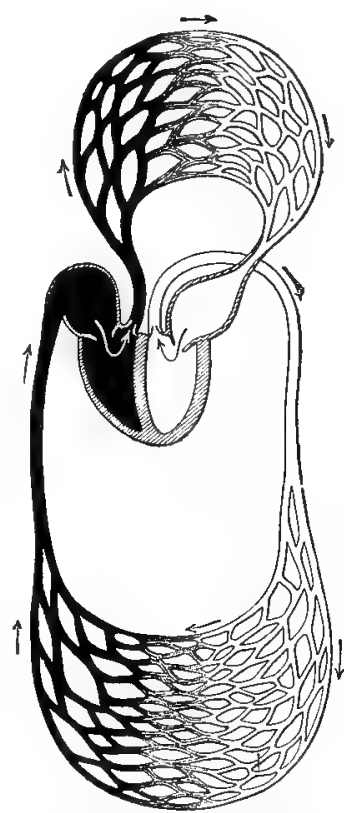

FIG. 278.-Scherne of the circulation of bloud in man. Centralisation of the nutrition of all cells in the blood-current. The darker half is the venous, the lighter the arterial system. The two are united by the capillary network of the lungs (above) and of the other tissues (below). In the capillaries the blood - current bathes all the tissues, the cells of which take food from it and give off to it their useless substances. (From Ranke.) a central nervous system with its paths of conduction. This principle, in greater and greater perfection, leads finally in the animal series to a far-reaching centralisation, such as is met with in the complex cell-community of the vertebrates, and especially of man. We have in the central nervous system a central organ which alone has the function of uniting cells, tissues, and organs with one another, su that an advantageous co-operation of them becomes possible; and the farther we ascend in the animal series, 
the more we find the tendency of the central nervous system to extend its authority toward a unified control of all cells and cellcomplexes of the animal body. In order to make graphic the principle upon which the mechanics of the central nervous system is based, it will be advantageous to consider the simplest form in which the function of the latter is expressed, namely, the reflex action.

The essence of the reflex action consists in the fact that an element that appreciates stimuli and an element that reacts to stimuli are so put into relation with one another by a central bond, that every stimulus acting upon the appreciating element is conducted first to the centre, and thence, as an impulse to a reaction, to the reacting element. Such a mechanism, in which every stimulus acting upon the sensory end calls out with machine-like certainty a reaction at the other end, is a reflex arc. The most primitive
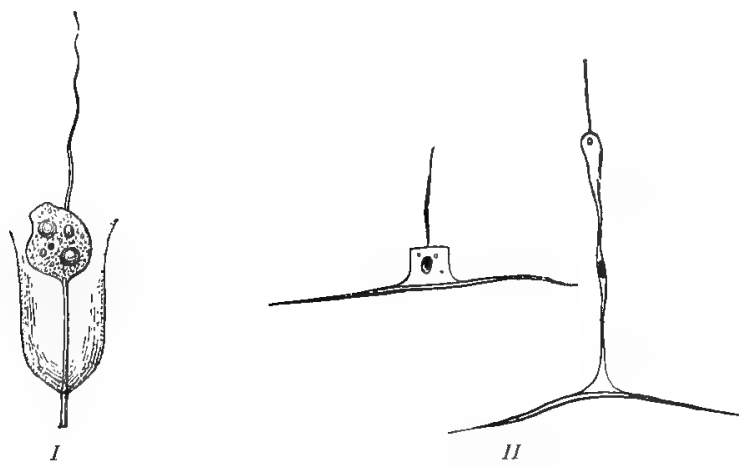

Fro. 279.-Primitive reflex are in a single cell. $I$, Poteriodenrion, a flagellated cell fixed in it cup-shaped sheath upon a myoid-fibre. $I I$, Neuro-muscular cells fron an actinian. (II, after Hertwig.)

form of a reflex arc exists in unicellular organisms, the cell-body of which possesses both the sensory and the motor elements, and even functions also as the central bond for the two. A single Poteriodendron represents a reflex arc of the simplest kind (Fig. 279, I). The cell-body, fixed upon a myoid-fibre at the bottom of a delicate, cup-shaped sheath, bears a flagellum which is extremely sensitive. The slightest stimulus which acts upon the latter is conducted centripetally to the cell-body, and from there centrifugally to the myoid-fibre, and the action of the stimulus upon the flagellum is followed at once by the contraction of the fibre. Wholly analogous to this is the behaviour of Vorticella, except that in the latter the sinsory elements are present chiefly. in the form of the cilia of the peristome. The same relations, further, exist in the so-called neuro-muscular cells of the Coelen- 
lirma (Fig. 279, II). Her', likewise, a cell possesses, upon the one side, a sensory (lenlent, and, upon the other, a contractile fibre, which contracts as soon as the sensory end-organoid is stimulaterl. What in all these cases is differentiated within a single cell, is in the nervins system of animals distributed to several cells. In the simplest cilsw of the latter, three different cells art concerned. One cell, the sensory cell, receives the'stimulus; from this il centripetal nerve-path conducts to a central cell, the ganglioncell, and from here a centrifugal nerve-path conducts to il cell that performs the raiction, the notor end-cell (Fig. 280, A). But this form of rettex are is realised perhaps only in the invertebrates. In vertebrates, so far as the conditions are known, a fourth cell at least is interpolated in the are, since in place of one ganglion-cell at least two ar' present, one of which receives the stimulus from the sensory-cell and conducts it to the other, while the other transfers the impulse to the motor end-cell (Fig. $280, B)$. In a given ("ise the end-cell of the ecintrifugial path may be either motor or sucretory, or may produce light or clectricity. 'Thus rettexly by the gang'liom-cells parts of the collcommunity, wholly diftirent and far removed from one another, sure put into union and activity by inpulse's from the central nervous systim.

If we start fiom the scheme of the rettex arc, the further fictors that

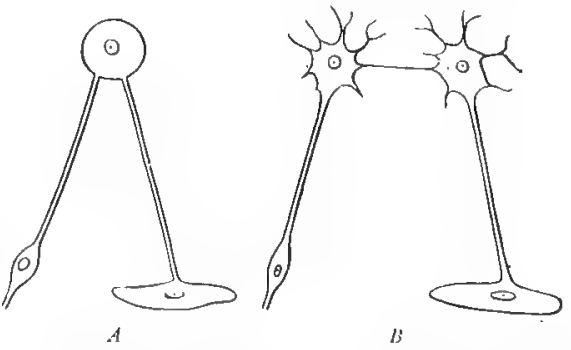

Fiu. Zsti--Schomos of the retlox ire. A, Simple rehome of rollex are. At the left, bolow, a sensory ('ell; in the middle, above, a central ganglion.c'll; at tho right, below, a muscle-cell. $B$, Schene of it roflox are in vertobratos. At the left, below, in mensury cell, at tho left, above, a sensory ganglionecli. $\Lambda$ the right, abovo, a motor ganglion-cell, nt tho right, buluw, a muscle-coll. (After Gegenbitur.)

come into consideration in the mechanism of the central nerrous system ir' viry simple. They consist only in the facts that, wpon the ane hand, betwe'n the sensory and the motor endorgan more than two ganglion-cells possessing diffirent functions are interpolated, and, upon the other hand, certain ganglioncolls arc innervated not simply from one side, by a single other ganglion-cell, but by several, and under certain circumstances by many other's. Thus, by means of their nerve-fibres very complex and intricute connections are formed between the ganglion-cells and the individual systems of ganglion-cells, which latter are the contres of definite vital processes and hence the seat of definite impulse's. A network of ganglion-cells and uniting nerve-fibres results, which is apparently inextricable, but in reality insures a very definite and unified co-operation of the various parts of the organism that it binds together. By the proper innervation of all 
kinds of cells, tissues and organs of the cell-community by the central nervous system, the cells of which form in vertebrates the brain and the spinal cord together with the sympathetic nervous system, a central system of administration for the whole cellcommunity is inaugurated,

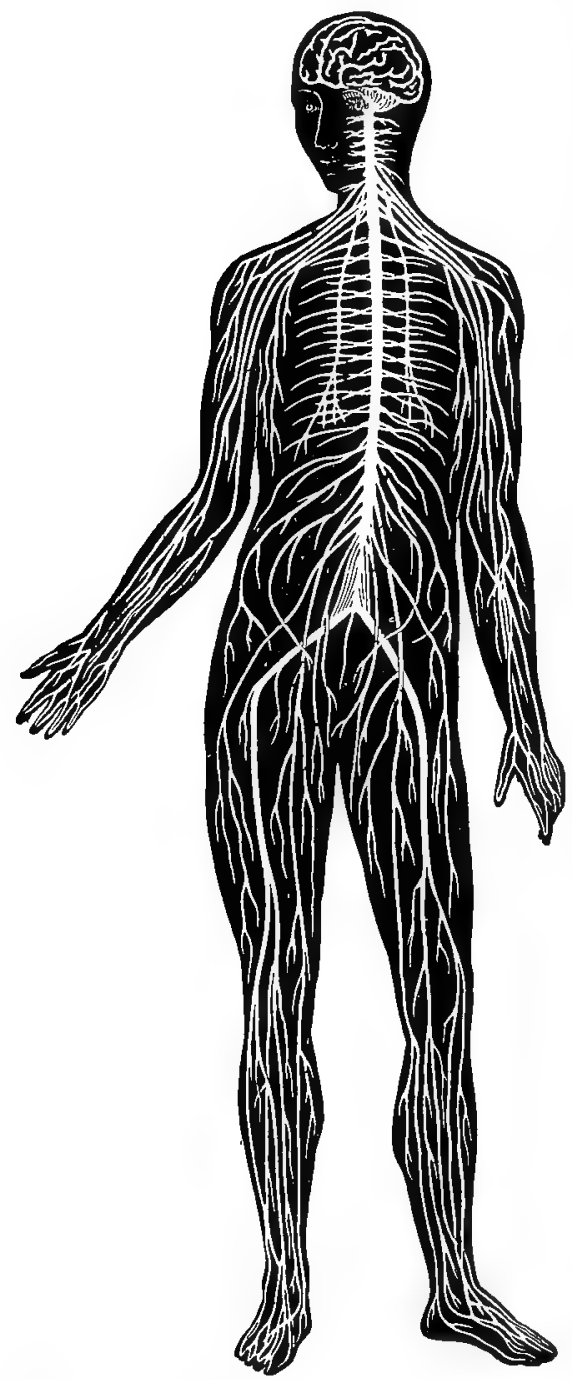

Fick 281,-Nervous system of man. The nerve-trunks, which contain centrifugal and centripetal paths of cunduction, pass from the brain and spinal cord to all parts of the body, and thus unite the latter through the central nervous system into a unified whole. (After lanke.) which from the brain and spinal cord by means of their long paths of conduction brings even most distant parts of the community under a unified control (Fig. 281). Hence the nervous system has been compared very graphically to a telegraphic net,work, the wires of which put the most distant regions of a country into connection with a central place of government. The comparison of a central nervous system to a great telegraph station and the nerve-fibres to the telegraph wires is very fitting with respect to the principle of centralisation upon which the two are based. But, as has sometimes happened, such a comparison ought not to be carried too far ; for example, the nerves should not be regarded simply as conducting-wires for electricity. In reality, nerves are extensions of ganglion-cells, and, like these, consist of living substance, i.e., they have a metabolism with which their life and, therefore, their function are inseparably connected. This follows directly from the fact that the nerve invariably perishes, like any non-nucleated protoplasmic mass, after being cut off from the ganglioncell to which it belongs. 
The manner in which the elements of the nervous system are united with one another anatomically and functionally deserves special attention, since the later researches upon the finer structure of the central nervous system, which have been made possible by the extraordinary development of the microscopic technique, especially by Golgi, Weigert, Ehrlich, and others, have led to the discovery of very peculiar but fixed relations. The element of the central nervous system is the ganglion-cell, but the ganglioncell with its characteristic differentiations. From the body of the cell there extend processes, more or less numerous according to the function of the cell, among which two kinds may be distinguished sharply from one another. Some form a more or less richly branched structure, and are, therefore, appropriately termed dendrites. The older histologists termed these protoplasnic processes. The others are the nerve-processes. So far as we now know, as regards the number of the latter there are only two varieties of ganglion-cells : unipolar (previously called multipolar on account of the numerous dendrites), provided with only one nerve-process, and bipolar, with two nerve-processes. These nerveprocesses are simply the beginning of the nerve-fibres, which not rarely reach a length of one metre and more. The conducting nerve puts even the most distant cells of the animal body into physical connection with the ganglion-cells, and transmits the impulses that go out from the bodies of the ganglion-cells to the tissue-cells, or in specific cases to other ganglion-cells. In its course from the body of the ganglion-cell to the cell that it innervates, the nerve-process appears different at different points. It sends off here and there collateral branches, and a little beyond its origin is surrounded by a sheath consisting of myelin, the medullary sheath. The latter is divided into segments by the so-called nodes of Ranvier, and disappears shortly before the cell which the nerve supplies is reached. The medullary sheath, in which the nerve-fibre runs as the axis-cylinder, is itself usually surrounded by a membranous sheath, the neurilemma. The end of the nerve shows very characteristic differentiations according to the kind of cell which it innervates. Such a complete cell, i.e., a ganglion-cell with all its appendages, represents the elementary constituent of the nervous system, and can fittingly be termed with Waldeyer a neuron (Fig. 282). The combination of the innumerable neurons with one another constitutes the nervous system of the animal. According to the Iater researches of Golgi, Kölliker, His, Ramon y Cajal, and others, the connection of the neurons with one another appears to be everywhere of such a kind that the dendrites of the ganglion-cells receive the stimulating impulses, while the nerve-process transmits them from one ganglion-cell to the dendrites of another. The bipolar ganglion-cells, which are contained chiefly in the spinal ganglia lying at the two sides of the spinal 


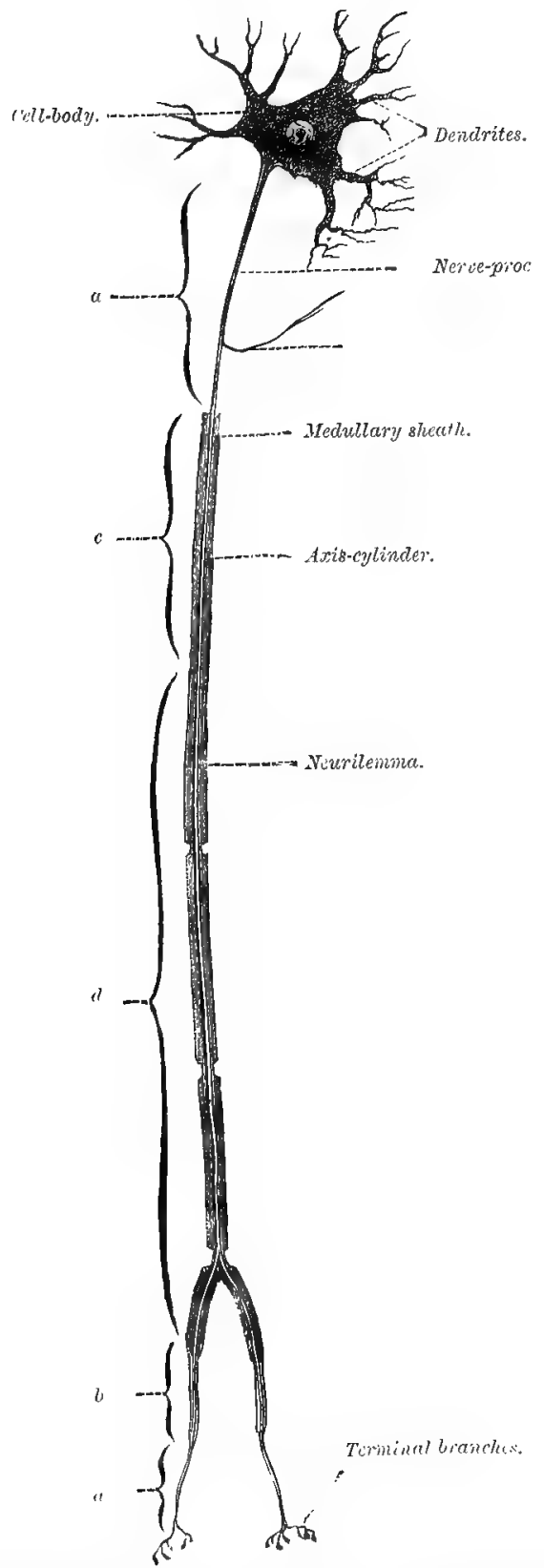

Frr. 282.-Scheme of a neuron; $a$, free axis-cylinder; $b$, axis-cylinder surrounded by neurilemma alone; $r$, axis-cylinder surrounded by the modullary sheath alone; $d$, axis-cylinder surrounded l,y the medullary sheath and the neurilemma, and divided intosegments by the nodes of Ranvier. (From Stïhr.) 
cord, alone possess in their one nerve-process a sensory path, which receives impulses from the periphery in the form of external stimuli and transmits them to the cell-bodies; thence the impulses are continued through the other nerve-process to other neurons. Hence, as regards the body of the ganglion-cell to which they belong, the dendrites conduct always centripetally, the nerveprocesses in the unipolar ganglion-cells always centrifugally. The greater or smaller number of the dendrites of a ganglion-cell

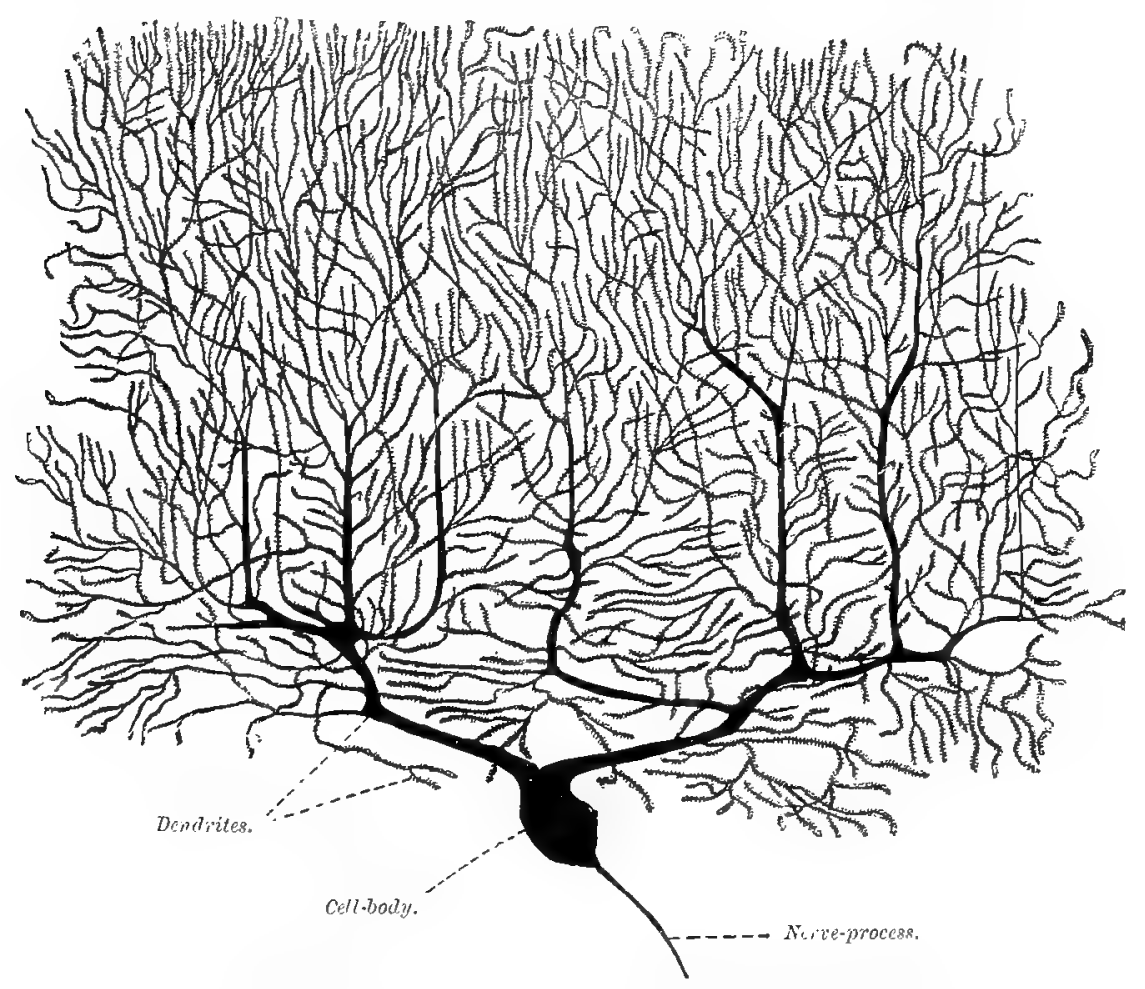

Firi. 283.-Cell of Purkinje from the grey cortical layur of the brain. (From Stijhr.)

appears to depend upon the question, with how many other neurons the ganglion-cell is in connection. Thus, the cells of Purkinje in the grey cortical layer of the brain, in which the most complex psychic processes are believed to be localised, have an extraordinarily richly developed system of dendrites (Figs. 283 and 284). The nerve-fibres of one ganglion-cell pass to the dendrites of another ganglion-cell. It is here a noteworthy fact, that, according to the later investigations, the connection between the two takes place not by direct continuity of their substance, or, as 
is said,"per" continuitatem," but through simple contact, "per" contiguitotem." The end of a nerve-fibre and the end of a dendrite join at their tips, but a piece that does not consist of nerve-substance is intercalated between them. It must be assumed that this intercalated piece, which is to be seen only with very strong magnifying powers, consists also of living substance, else it would be difficult to understand how it is able to conduct the excitation from the nerveprocess to the dendrites.

While there is great unanimity in the mode of union of the neurons with one another, the kind of transition of the nervefibres into the end-cells, which they innervate, or from which they

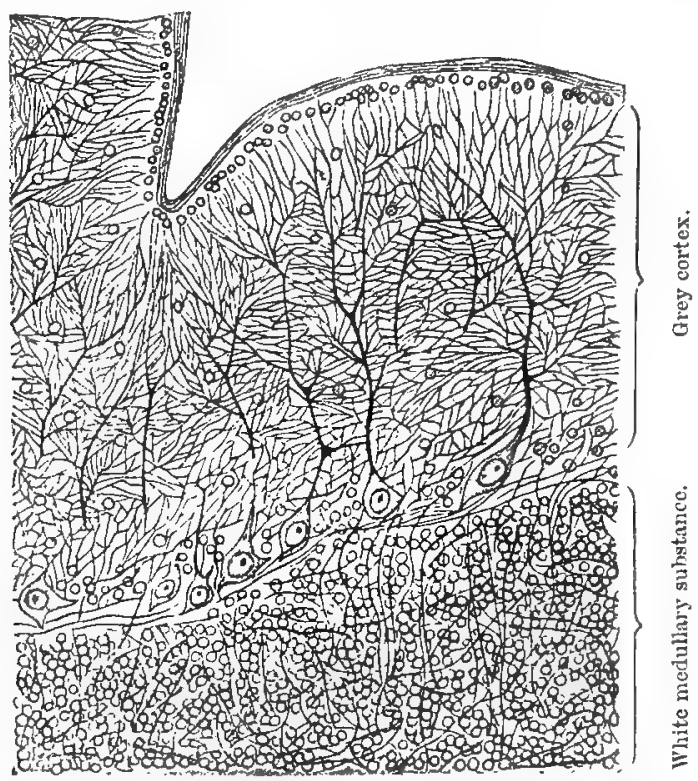

Fid. 284.-Section through the cortex of the cerebellum of a ealf, The large, branched cells are Purkinje's cells. (After Schiefferdecker.)

spring, is very various. The nerves (sensory) that conduct centripetally from the periphery of the body, as well as those (motor, secretory, electric, etc.) that conduct centrifugally to the periphery vary according to the organ in which they end. Among the former there are some that end free in the skin in the form of an end-bulb, without being in connection with a sense-cell (Fig. 285, II). The others appear to go out directly from a sense-cell, which is specially developed for the reception of the stimulus, as, e.g., the rods and cones of the eye, the hair-cells of the ear, the olfactory cells of the nose (Fig. 285, 1), etc. Among the endings 
of centrifugal nerves those of the motor nerves in cross-striated muscles are most characteristic. Here the transition of the nervefibre into the muscle-substance is mediated by a specially differentiated end-organ, the motor end-plate, a flat or branched extension of the axis-cylinder in the sarcoplasm. The latter, which in this place is very granular and is characterised by many nuclei, is covered by the sarcolemma of the muscle-fibre; the sarcolemma here passes over directly into the neurilemma of the nerve (Fig. $285, I I I)$. The manner of ending of the centrifugal nerves in other organs, such as smooth muscle-cells, gland-cells, photo-
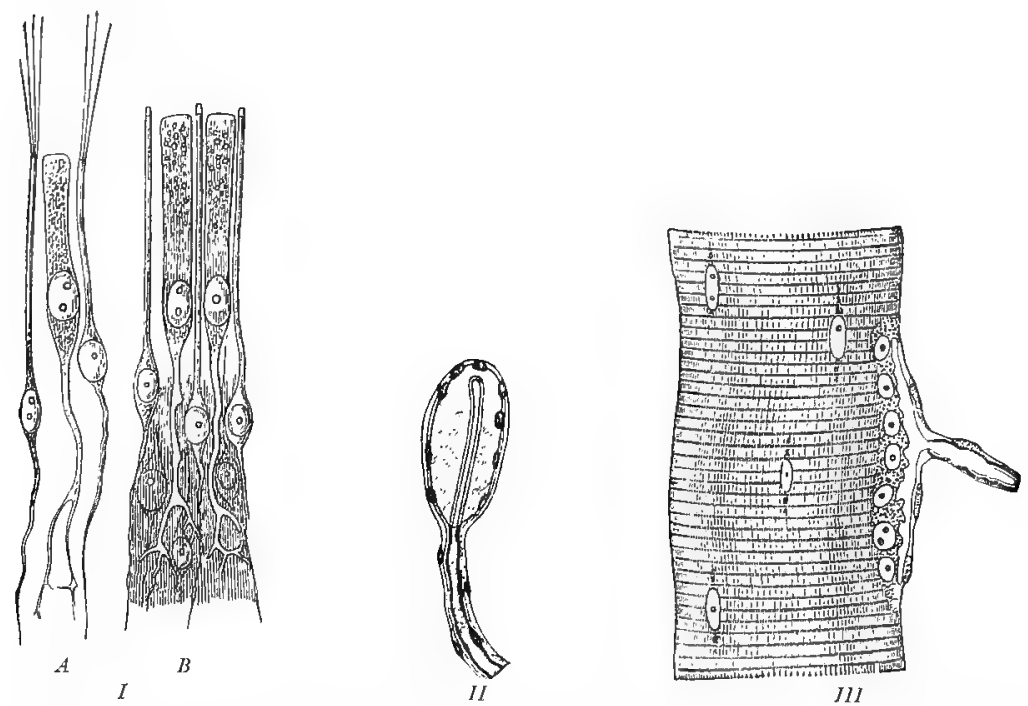

FIG. 285.-Nerve-endings. $I$, Olfactory cells ; $A$, from the frog, $B$, from man. The slender, spindleshaped cells are the olfactory cells; to these the nerve goes; the broad cells, branched below, are epithelial supporting-cells. (After Frey.) II, Nerve end-plate from the conjunctiva of a calf. (After Schiefferdecker.) III, Motor end-plate in cross-striated muscle, secn from the side. (From Lang.)

genic cells, etc., appears to be inuch less complicated; but these relations need more careful investigation.

Only through the central control of all functions of the whole organism by the nervous system is it possible for the cellcommunity of the animal body to be differentiated so extensively as it is. Only when at the proper moment this or that organ is put into activity or remains at rest, only when this or that organ reacts appropriately to an influence in this or that part of the body, only when the cells, tissues and organs work together in the most perfect harmony, can so complicated a mechanism be developed, as exists in the cell-community of the vertebrates and especially of man. 
Here general physiology passes into the special physiology of the animal or plant cell-community and its various forms. It is the task of special physiology to investigate individually the special mechanisms that result from the associated life of the cells in the community, and their co-operation. The sphere of general physiology extends only to those vital phenomena that are common to all organisms. The cell is the element of living substance. All living substance exists in cells, and all the functions of living substance originate in the elementary vital phenomena of the cells. Hence, if the task of physiology lies in the explanation of vital phenomena, general physiology can be only cell-physiology. 


\section{BIBLIOGRAPHY}




\section{BIBLIOGRAPHY}

Throughout the text references to the literature are indicated by placing the abbreviated year of publication after the author's name. If more than one article published during the year is referred to, a numeral is added to indicate the number of the article. In the following bibliographical list each reference is similarly headed. The heading is then followed successively by the title of the article, the title of the journal, the year, the volume, and the page. In the case of books, the title, and the place and date of publication are given. F. S. L.

Aderhold, R., '88 : Beitrag zur Kenntniss richtender Kräfte bei der Bewegung niederer Organismen. Jen. Zeitsch. f. Naturwiss., 1888, N. F. XV, p. 310.

Altmann, R.,'89 : Ueber Nucleïnsäuren. Arch. f. Anat. u. Physiol., Physiol. Abth., 1889, p. 524 ; Id., '90 : Die Elementarorganismen und ihre Beziehungen zu den Zellen. Leipzig, 1890.

v. BAER, C. E., '28: Ueber Entwickelungsgeschichte der Thiere. Beobachtung und Reflexion. Erster Theil. Königsberg, 1828.

Balbiani, E. G., '61: Recherches sur les phénomènes sexuels des infusoires. Journ. de la Physiol., 1861, IV, p. 465 ; Id., '88 : Recherches expérimentales sur la mérotomie des infusoires cilliés. Contribution à l'étude du rôle physiologique du noyau cellulaire. Ix'e partie. Recueil zoologique Suisse, 1888, $\nabla$, p. 1 .

Ballowitz, E., '90: Das Retzius'sche Endstïck der Säugetier-Spermatozoen. Internat. Monatsschr. f. Anat. u. Physiol., 1890, VII, p. 2]1; Id., '9l . Weitere Beobachtungen über den feineren Bau der Säugethierspermatozoen. Zeitschr. f. wiss. Zool., 1891, LII, p. 217.

Dł BaRY, A., '87: Vorlesungen über Bakterien. 2te Aufl. Leipzig, 1887.

BAUM, H., '86 : Die morphologisch-histologischen Veränderungen in den ruhenden und thätigen Leberzellen. Deutsch. Zeitschr. f. Thiermed. u. vergl. Pathol, 1886, XII.

YAN BENEDEN, E., '83 : Recherches sur la maturation de l'œuf et la fécondation. Arch. de Biol., 1883, IV, p. 265.

van BENEDEN, E., and NEXT, A., '87: Nouvelles recherches sur la fécondation et. la division mitosique chez l'Ascaride mégalocéphale. Bull. acad. roy. d. sci. de Belg., Bruxelles, 1887, 3 ser., XIV, p. 215.

Bernard, Claude, '78: Leçons sur les phénomènes de la vie communs aux animaux et aux végétaux. I. Paris, 1878; Id., '79 : Tbid., II. Paris, 1879.

BERNARD, H. M., '94: On the relations of the isotropous to the anisotropous layers in striped muscles. Zool. Jahrb., Abth. f. Anat., 1894, VII, p. 533.

Bert, P., '73: Recherches expérimentales sur l'influence que les changements dans la pression barométrique exercent sur les phénomènes de la vie. Compt. rend. de l'acad. d. sciences, 1873, LXXPI, pp. 443, 578, 1276, and 1493; LXXVII, p. 531.

BERTHOLD, G., '86 : Studien über Protoplasmamechanik. Leipzig, 1886.

VON BEZOLD, A., '61 : Untersuchungen ïber die elektrische Erregung der Nerven und Muskeln. Leipzig, 1861.

Biedermaxs, W., '79-'85: Beiträge zur allgemeinen Nerven- und Muskelphysiologie. Sitzungsber. d. kais. Akar,. d. Wiss. Wien., Math.-nat. Cl., III Abth., 1879 , LXXIX, p. 289 ; 1880 , LXXXIT, p. 257 ; 1883 , LXXXYII, pp. 66, 115 ; 1884, LXXXIX, p. 19; 1885, XCI, p. 29 ; XCII, p. 142; Id., '90, 1: Zuv 
Physiologie der glatten Muskeln. Arch. f. d. ges. Physiol., 1890, XLYI, p. 398; Id., '90, 2 : Zur Lehre von der elektrischen Erregung quergestreifter Muskeln. Ibid., 1890, XLVII, p. 243; Id., '95 : Electrophysiologie. Jena, 1895; authorised Eng. trans. by F. A. 'Welby : Electrophysiology. I, London, 1896. In this book all the literature is reviewed, and the whole subject is systematically summarised from the later points of view.

Bısz, C., '67: Ueber die Einwirkung des C'hinin auf die Protoplasma-Bewegungen. Arch. f. mik. Anat., 1867, II, p. 383.

Blasits, E., and ScHWErzER, F., '93: Electrotropismus und rerwandte Erscheinungen. Arch. f. d. ges. Physiol., 1893, LIII, p. 493.

Di Bois-Reriusd, E., '48-'84: Untersuchungen über thierische Elektricität. Berlin, 1848, 1849, 1860, 1884; Id. '59: Gedächtnissrede auf Johannes Miiller. AbhandI. d. kais. Akad. d. Wiss. zu ‘Berlin, 1859; also reprinted, Berlin, 1860; Id., '84:' Ueber die Grenzen des Naturerkennens. Leipzig, 1884, also in Reden, erste Folge, Leipzig, 1886.

boveri, Тн., '87, '88, '90: Zellenstudien. Jen. Zeitsch. f. Naturwiss., 1887, N. F. XIV, p. 423 ; 1888 , N. F. XY, p. 685 ; 1890, N. F. XVII, p. 314; Id., '89: Ein geschlechtlich erzeugter Organismus ohne muitterliche Eigenschaften. Sitzungsber. d. Ges. f. Norphol. u. Physiol. zu München, 1889, V. Eng. trans: The Ámerican Naturalist, 1893, XXVII, p. 222 ; Id., '95 : Ueber das Verhalten der Centrosomen bei der Befruchtung des Seeigeleies, nebst allgemeinen Bemerkungen iber Centrosomen und Terwandtes. Verhandl. d. physik.-med. Ges. zu Würzburg. 1895, N. F. XXIX.

BraID, JAMES : For a list of Braid's works see article by J. M. Bramwell ; James Braid, Surgeon and Hypnotist. Brain, 1896, XIX, p. 90 ; Id., '50 : Obserrations on Trance; or, Human Hybernation. London and Edinburgh, 1850.

BrandT, K., '85 : Die koloneibildenden Radiolarien des Golfes ron Neapel, etc. Fauna und Flora des Golfes von Neapel; Herausgegeben von der zoologischen Station zu Neapel, Berlin, 1885 ; Id., '95 : Biologische und faunistische Untersuchungen an Radiolarien und anderen pelagischen Thieren. 1. Untersuchungen über die hydrostatischen Apparat von Thallassicollen und coloniebildenden Radiolarien. Zool. Jahrb., Abth. f. Syst. etc., 1895, LX, p. 2-.

Brater, A., '93, 1: Zur Kenntniss der Herkunft des Centrosomas. Biol. Centralb., 1893, XIII, p. 285; Id., '93, 2: Zur Kenntniss der Spermatogenese von Ascaris megalocephala. Arch. f. mik. Anat., 1893, XLII, p. 153.

Brieger, L., '85-'86 : Ueber Ptomaine. Theil I, II, and II, Berlin, 1885, 1886.

Brieger, L., and Fränkel, C. '90 : Untersuchungen ïber Bakteriengifte. Berl. klin. Wochenschr., 1890, XITII, pp. 241, 268.

Browx, R., '33: Obserrations on the organs and mode of fecundation in Orchidex and Asclepiadee. Trans. of the Linn. Soc., London, 1833.

BRÜCKE, E., '61 : Die Elementarorganismen. Sitzungsber. d. kais. Akad. d. Wiss. Wien., 1861, XLIV, 2 Abth., p. 381.

Buchxer, H., '90: Die chemische Reizbarkeit der Leukocyten und deren Beziehung zur Entzïndung und Eiterung. Berl, klin. Wochenschr., 1890, XYTTI. p. 1084 .

Вётsсн土, O., '76: Studien über die ersten Entwicklungsrorgänge der Eizelle, die Zelltheilung und die Conjugation der Infusorien; Abhandl. d. Senckenberg. naturforsch. Ges., 1876, X; Id., '90: Ueber den Bau der Bakterien und verwandter Organismen. Leipzig, 1890 : Id., "92, 1 : Untersuchungen iiber mikroskopische Schäume und das Protoplasma. Leipzig, 1892 ; authorised Eng. trans. by E. A. Minchin : Inrestigations on microscopic foans and on protoplasm. London, 1894. The bibliography of the subject is also given here. Id., '92, 2 : Die Bewegung der Diatomeen. Terhandl. d. naturhist.-med. Ver. zu Heidelberg, 1892, N. F. IV, Heft 5 ; Id. '92, 3 : Ueber die künstliche Nachahmung der karyokinetschen Figuren. Mid., 1892, N. F. V.

Buxce, G., '83: Ueber das Sauerstoff bedürfniss der Darmparasiten. Zeitschr. f. physiol. Chem., 1883, TIII, p. 48; Id., '94: Lehrbuch der physiologischen und pathologischen Chemie. 3te Aut,, Leipzig, 1894.

'CHosiva, C., '43 : Recherches expérimentales sur l'inanition. Mlémoires présentés par divers sarant à l'acad. roy. des sci. de l'inst. de France, 1843, VIII, p. 438.

Crevkowsin, L., '65: Beitrïge zur Kenntniss der Monaden. Arch. f. mik. Anat., 1865, I, p. 203. 
Clatsics, R., 'it: Ueber die Elektricitiatsleitung in Elektrolyten. Poggendorff's Annal. «. Physik. u. Chem., 18.T. CI, p. 338.

Сонмнег, J. '7-'80 : Torlesungen über allgemeine Pathologie. 2te Aufl. Berlin, $187-1880$; Eng. trans. by A. B. HeKee, from 2nd Germ. ed. . Lectures on General Pathology. London, New Sydenham rociety. 1889.

DARIIN, C., 59: On the origin of species by means of natural selection, or the preservation of favoured races in the struggle for life. London, 1859.

DAresport, C. B., and NeAL, H. T., "96: Studies in morphogenesis. $V$. On the acclimatisation of organisms to poisonous chemical substances. Arch. f. Entwicklungsmech., 1896, II, p. 564.

Dexoor, J., '95 : Contribution à l'étude de la physiologie de la cellule (indépendance fonctionelle du protoplasme et du no5au). Arch. de Biol., 1895, XIII, p. 163 ; Il., '96 : La plasticité morphologique des neurones cérébraux. Ibid., 1896, XIV, p. 723 .

Dewiтz, J., 86: Ueber Gesetzmässigkeit in der Ortsveränderung der Spermatozoen und in der Vereinigung derselbed mit dem Ei. I. Arch. f. d. ges. Physiol., 1886, XXXVIII, p. 358.

Dreyer, Fr., (2) : Die Principien der Gerüstbildung bei Rhizopoden, spongien und Echinodermen. Ein Tersuch zur mechanischen Erklärung organischer Gebilde. Jen. Zeitsch. f. Naturw, 1892, N. F. XIX, p. 204.

Driesch, H., '90: Heliotropismus bei Hydroidpolypen. Zool. Jahrb., Abth. f. Syst., ete., 1890, V, p. 147; Id. '92: Entwicklungsmechanische Studien. I. Der Werth der beiden ersten Furchungszellen in derEchinodermenentwicklung. Experimentelle Erzeugung von Theil- und Doppelbildung. Zeits. f. wiss. Zool., 1892, LIII, p. 160; Id., '93: Zur Verlagerung der Blastomeren des Echinideneies. Anat. Anzeig., 1893, TIII, p. 348.

Drixer, L.. '94: Zur Morphologie der Centralspindel. Jen. Zeitschr. f. Naturw., 1894, N. F. XXI, p. 469.

Dгвог, R., 9: Anatomie et physiologie comparées de la Pholade dactyle. Annales de l'Universití de Lyon, I892, II.

Dujardix, F., 41 . Histoire naturelle des zoophytes: infusoires. Paris, 1841.

EARenbert, C. $4 ., 38$ : Die Infusionsthierchen als vollkommene Organismen. Leipzig, 1838; Id., "5s: Erläuterung einer ungarischen aus Kiesselorganismen bestehenden Felsart durch die Wirkung heisser Quellen auf Ischia. Monatsber. d. Akal. ג. Wiss. zu Berlin, 1858, p. 488.

Emer, G. H. Tн., 'S8: Die Entstehung der Arten auf Grund ron Tererben erworbener Eigenschaften nach den Gesetzen organischen Wachsens. Jena, 1858; Eng. trans. by J. T. Cunningham: Organic evolution as the result of the inheritance of acquired characters according to the laws of organic growth. London, 1890.

Elsberg, 74: Proceedings of the American Association for the Advancement of Science, Hartford, 1sit.

Excieluat, Th. IT., 68 : Ueber die Flimmerbewegung. Jen. Zeitschr. f. Naturw., 1868, IV, p. 321 ; Id., '69 : Berträge zur Physiologie des Protoplasma. Arch. f. d. ges. Physiol., 1869 , II, p. 307 ; Id., '70 : Beiträge zur allgemeinen Muskel- und Nervenphysiologie. Ibid., is70, III, p. 247; Id., '73 : Mikroskopische Untersuchungen uiber die quergestreifte Muskelsubstanz. I. II. Ibid., 1873, TII, pp. 33, 155 ; Id., '75: Contractilität und Doppelbrechung. Ibid., 1875, XI, p. 432; Id., 78; Neue Untersuchungen uiber die mikroskopischen Vorgänge bei der Muskelcontraction. Mid., 1878, X TIII, p. 1.; Id.. '79, 1 : Physiologie der Protoplasma- und Flimmerbewegung. Hermann': Handbuch der Phrsiologie. I, Leipzig, 1879, p. 34l ; Id., 79, 2 : Ueber Reizung contractilen Protoplasmas durch plotzliche Beleuchtung. Arch. f. d. ges. Physiol., 1879, XIX, p. 1; Id., '81, 1 : Neme Methode zur Untersuchung' der Sauerstoffausscheidung pflanzlicher und thierischer Oryanismen. Ibid., 1881 , XXV, p. 285 ; Id., 'S1, "2: Ueber den fasigeren Bau der contractilen Substanzen, mit besonderer Berücksichtigung der glatten und doppelt schräggestreiften Nuskelfasern. Ibid., 1881, XXT, p. 738; Id. "81, 3: Zur Biologie der Schyzomyceten. Ibid., 1881, XXVI, p. 537 ; Id., 82 : Ueber Licht- und Farbenperception niederster Organismen. Ibid., 1882, XXIX, p. 387 ; Id., '83 : Bacterium photometricum. Ein Beitrag zur vergleichenden Physiologie des Licht- und Farbensinns. Tbid., 1883, XXX, p. 95 : Id., '93: 
Ueber den Ursprung der Muskelkraft. 2te Aufl. Leipzig, 1893; Id., '94 : Die Erscheinungsweise der Sauerstoffausscheidung chromophyllhaltiger Zellen im Licht bei Anwendung der Bacterienmethode. Verhand1. d. kon. Akad. van Wetensch., Amsterdam, 1894, 2 Sect., III. ; also Onderz. Physiol. Lab., Utrecht, 1895, IV Reeks, III Deel. The other works of Engelmann upon this subject are here cited.

FabRe, '55 : Recherches sur la cause de la phosphorescence de l'agaric de l'olivier Compt. rend. de l'acad. de sciences, 1855, XLI, p. 1245.

Frcr, A., '82 : Mechanische Arbeit und Wärmeentwickelung bei der Muskelthätigkeit. Intemat. wiss. Bibliothek, LI, Leipzig, 1882; Id., '93, I : Einige Bemerkungen zu Engelmann's Abhandlung über den Úrsprung der Muskelkraft. Arch. f. d. ges. Physiol., 1893, LIII, p. 606; Id., '93, 2: Noch einige Bemerkungen zu Engelmann's Schrift iiber den Ursprung der Muskelkraft. Ibid., 1893, LIV, p. 313.

Fick and WisLicenus, '65: Ueher die Entstehung deŕ Muskelkraft. Vierteljahrsschr. d. Züricher naturforsch. Ges., 1865, X, p. 317.

Fick, R., '97 : Bemerkungen zu M. Heidenhain's Spannungsgesetz. Arch. f. Anat. u. Physiol., Anat. Abth., 1897, p. 97.

Fremmina, W., "82 : Zellsubstanz, Kern und Zelltheilung. Leipzig, 1882.

Fol, H., '91 : Le quadrille des centres. Un épisode nouveau dans l'histoire de la fécondation. Arch. de sci. phys. et nat., 1891, XXV.

GAD, J., '78 : Zur Lehre von der Fettresorption. Arch. f. Anat. u. Physiol., Physiol. Abth., 1878, p. 157.

Gaglio, G., '86 : Die Milchsäure des Blutes und ihre Ursprungsstätten. Arch. f. Arat. u. Physiol., Physiol. Abth., 1886, p. 400.

Gerassimorr, '92 : Ueber die kemlosen Zellen bei einigen Conjugaten. Bull. de la soc. impér. des naturalistes de Moscou, 1892; Id., '97. Ueber ein Verfahren kernlose Zellen zu erhalten. Ibid., 1897.

Golubew, A., '68: Ueber die Erscheinungen welche elektrische Schläge an den sogenannten farblosen Bestandtheilen des Blutes hervorbringen. Sitzungsber. d. kais. Akad. d. Wiss. Wien., Math.-naturw. Cl., 1868, LVII, II Abth., p. 555 .

Gratam, T., '61 : Liquid diffusion applied to analysis. Phil. Trans. of the Roy. Soc. of London, 1861, CLI, Part I, p. 183.

GREENwOOD, M., '86, '87: On the digestive process in some Rhizopods. I. Journ. of Physiol., 1886, VII, p. 253 ; II. Ibid., 1887, VIII, p. 263 ; Id., '94: On the constitution and mode of formation of "food vacuoles" in Infusoria, as illustrated by the history of the processes of digestion in Carchesium polypinum. Phil. Trans. of the Roy. Soc. of London, B., 1894, CLXXXV, Part I, p. 355; Id., '96: On structural change in the resting nuclei of Protozoa. Part I. The macronucleus of Carchesium polypinum. Journ. of Physiol., 1896, XX, p. 427.

GRuBer, A., '85: Ueber künstiiche Theilung der Infusorien. Biol. Centralb., 1885, IV, p. 717; V, p. 137; Id., '86, 1 : Beiträge zur Kenntniss der Physiologieund Biologie der Protozoen. Ber. d. naturf. Ges. z.u Freiburg i. B., 1886, I ; Id., '86, 2 : Der Conjugationsprocess bei Paramæecium aurelia. Ibjd., 1887, II ; Id., '88 : Ueber einige Rhizopoden aus dem Genueser Hafen. Bbid., 1888, IV ; Id., '89: Biologische Studien an Protozoen. Biol. Centralb., 1889, IX, p. 14 .

GRÜBLER, G., '81 : Ueber ein krystallinisches Eiweiss der Kürbissamen. Journ. f. prakt. Chemie., 1881, N. F. XXIII, p. 97.

GompREсHт, '96: Leukocytenzerfall im Blute bei Leukämie und bei schweren. Anämien. Deutsch. Arch. f. klin. Med., 1896, LVII, p. 523.

Haberland, G., '87 - Ueber die Beziehungen zwischen Function und Lage des Zellkernes bei den Pflanzen. Jena, 1887; Id., '89: Ueber Einkapselung des Protoplasmas mit Rücksicht auf die Function des Zellkerns. Sitzungsber. d. kais. Akad. d. Wiss. Wien., Math.-naturw. Cl., 1889, XCVIII, Abth. I., p. 190 .

HAECKFL, E., '62: Die Radiolarien. Berlin, 1862 ; Id., ‘66 : Generelle Morphologie der Organismen. Berlin, 1866; Id., 70: Biologische Studien. I. Studien über Moneren und andere Protisten. Leipzig, 1870 ; Id., '75: Ziele und Wege der hëutigen Entwickelungsgeschichte. Jena, 1875; Id., '76: 
Die Perigenesis der Plasticlule octer die Wellenzengung «ler Lebenstheilchen. Berlin, 1876; Id., '91: Anthropogenie oder Entwickelungsgeschichte des Menschen. 4te Aufl. Leipzig, 1891.

v. Haller, A., 1762: Elementa physiologiae corporis humani. IV. Lausanne, 1762.

y. Hanstein, J., '80: Das Protoplasma als Träger der thierischen und pflanzlichen Lebensverrichtungen. Heirielherg, 1880.

Hatschez, B., '94: Hypothese ïber das Wesen der' Assinilation, eine vorläufige Mittheilung. Lotos, 1894, N. F. XIV.

Hetdexhain, M., '94: Neue Untersuchungen ïber die Centralkörper und ihre Beziehungen zum Kern- und Zellenprotoplasma. Arch. f. mik. Anat., 1894, XLIII, p. 423 ; Id., '9.5 : Crtomechanische Studien. Arch. f. Entwicklungsmech., 1895, I, p. 473 ; Ić., '96 : Ein neues Modell zum Spannungsgesetz der centrirten Systeme. Verhandl. d. anat. Ges. zu Berlin, 1896, p. 67 ; Anat. Anz., 1896, XII.

Heidenhain, R., '8:3: Physiologie der Absonderungsvorgänge. Hermann's Handbuch d. Physiol. V, Leipzig, 1883, p. 1, ; Id., "94 : Neue Versuche über die Aufsaugnng im Dünndarm. Arch. f. d. ges. Physiol., 1894, LVI, p. 579.

v. Hexmoltz, H., '84 : Ueber die Entstehung des Planetensystems. Vorträge und Reden. II, Braunschweig, 1884.

Hering, E., '84 : Ueber die specifischen Energieen des Nervensystems. Lotos, 1884, N. F. V. : Id., '88: Zur Theorie der Vorgänge in der lebendigen Substanz. Lotos, 1888, N. F. IX.

Hermann, L., '67, '68 : Untersuchungen zur Physiologie der Muskeln und Nerven. I-III. Berlin, 1867, 1868 ; Id., '79 : Handbuch der Physiologie. I. Leipzig, 1879 ; Id., '85: Eine Wirkung galranischer Ströme auf Organismen. Arch. f. d. ges. Physiol., 1885, XXXVII, p. 457.

Hertwig, 0 ., '76, '77, "78 : Beiträge zur Kenntniss der Bildung, Befruchtung und Theilung des thierischen Eies. Morphol. Jahrb., 1876, I, p. 347 ; 1877, III, p. 1; 1878, IV, p. 156 ; Id., '84: WeIchen Einfluss ibt die Schwerkraft auf die Theilung der Zelle? Jena, 1884 ; Id., '90 : Lehrbuch der Entwicklungsgeschichte des Menschen und der Wirbelthiere. 3te Aufl. Jena, 1890; Id., '92: Die Zelle und die Gewebe. Jena, 1892 ; authorised Eng. trans. by M.' and H. J. Campbell: The Cell : Outlines of General Anatomy and Physiology. London and New York, 1895; Id., '93: Ueber den Werth der ersten Furchungzellen für die Organbildung des Embryo. Experimentelle Studien am Frosch- und Tritonei. Arch. f. mik. Anat., 1893, XLII, p. 662.

Hertwig, O. and R., '87 : Ueber den Befruchtungs- und Theilungsvorgang des thierischen Eies unter dem Einfluss äusserer Agentien. Jen. Zeitschr. f. Naturwiss., 1887, N. F. XIII, pp. 120, 477.

Hertwis, R., '88-'89: Ueber die Conjugation der Infusorien. Abhandl. d. Math. physik. Cl. d. Kön. bayr. Akad. d. Wiss., 1888-89, XVII, p. $15 \mathrm{l}$.

His, W., '74: Unsere Körperform und das physiologische Problem ihre: Entstehung. Briefe an einen befreundeten Naturforscher. Leipzig, 1874.

HodGe, C. F., '92; A microscopical study of changes due to functional activity in nerve cells. Journ. of Morphol., 1892, VII, p. 95.

HoFER, B., '89-90: Experimentelle Untersuchungen uiber den Einfluss des Kerns auf das Protoplasmd. Jen. Zeitschr. f. Naturw., 1889-90, N. F. XVII, p. 105.

Hofmaxy, F., '72: Der Uebergang von Nahrungsfett in die Zellen des Thierkörpers. Zeitschr. f. Biol., 1872, VIII, p. 153.

-Hofmeister, F., '82: Zur Lehre vom Pepton. V. Das Verhalten des Peptons in der Magenschleimhaut. Zeitschr. f. physiol. Chem., 1882, VI, p. 69.

Hoppe-Seyler, F., '77: Physiologische Uhemie. Theil I. Berlin, $187 \%$.

Honvath, A., '78: Ueber den Einfluss der Ruhe und der Bewegung auf das Leben. Arch. f. ג. ges. Physiol., 1878, XVII, p. 125.

Hore, L., ' 97 : Changes in the cell-organs of Drosera rotundifolia, produced by feeding with egg-albumen. Quart. Journ. of Mic. Sici., 1897, N. S. XXXIX, p. 387.

IsRAeL, O., '97, 1. Biologische Stulien mit Riicksicht auf die Pathologie. III. Oligodynamische Erscheinungen (v. Nägeli) an pflanzlichen und thierischen Zellen. Virchow's Arch. f. pathol. Anat. u. Physiol. etc., 1897, CXLVIT, p. 293; Id., '97, 2: Ueber den 'Tod der Zelle. Berl. klin. Wochenschr. 1897. 
Jensen, P., '93, 1 : Ueber den Geotropismus nierlerer Urganismen. Arch. f. d. ges. Physiol., 1893, LIII, p. 428.; Id., '93, 2. . Die absolute Kraft einer Flimmerzelle. Ibid., 1893, LIV, p. 537.

Jотеуко, J., '96 : La fatigue et la respiration élementaire du muscle. Paris, 1896.

Kerner $\checkmark$. Marilaun, A., '87: Pflanzenleben. Allgemeine Naturkunde. I., Leipzig, 1887.

KLEBs, G., '85: Ueber Bewegung und Schleimbildung der Desmidiaceen. Biol. Centralb., 1885, V, p. 353; Id., '87: Ueber den Einfluss des Kernes in der Zelle. Ibid., 1887, VII, p. 161.

Kochs, W., '90: Kann die Continuität der Lebensvorgänge zeitweilig völlig unterbrochen werten? Ibid., 1890, X, p. 673.

KorotnefF, A., '92: Histolyse und Histogense des Muskelgewebes bei der Metamorphose der Insecten. Ibid., 1892, XII, p. 261.

Konschelt, E., '89 : Beiträge zur Morphologie und Physiologie des Zellkernes. Zool. Jahrb., Abth. f. Anat., 1889, IV, p. 1.

Korschelt, E. and Heider, '90: Lehrbuch der vergleichenden Entwicklungsgeschichte der wirbellosen Thiere. Jena, 1890.

Kosset, A., '91 : Ueber die chemische Zusammensetzung der Zelle. Arch. f. Anat. u. Physiol., Physiol. Abth., 1891, p. 181.

Kowalevsky, A., "85: Beiträge zur nachembryonalen Entwicklung der Musciden. Zool. Anz., 1885, VIII, pp. 98, 123, 153; Id., '87. : Beiträge zur Kenntniss der nachembryonalen Entwicklung der Musciden. I. Zeitschr. f. wiss. Zool., 1887, XLV, p. 542.

Kraft, H., '90: Zur P'lysiologie des Flimmerepithels bei Wirbelthieren. Arch. f. d. ges. Physiol., 1890 , XLVII, p. 196.

KRUkenbert, C. F. W., '82: Die Schutzdecken der Echinodermen. Tergleich. physiol. Stud., 2te Reihe, I. Abth., Heidelberg, 1882.

Kühne, W., '64: Untersuchungen über das Protoplasma und die Contractilität. Leipzig, 1864.

LANGJEY and VERY, ' 90 ; On the gheapest form of light; From studies at the Alleghany Observatory. Amer. Jüurn. of Sci., 1890, 3rd series, XL, p. 97.

LA OTERBorn, R., '94: Zur Frage nach der Ortsbewegung der Diatomeen. Ber. r. deutsch. bot. Ges., 1894, XII, p. 73.

LeBER, Th., '88 : Ueber die Entstehung der Entzündung und die Wirkung der entzïndungserregenden Schädlichkeiten. Fortschr. Q. Med., 1888, VI, p. 460 ; also, Leipzig, 1891.

LEe, F. S., '94: The scope of modern physiology. The American Naturalist, 1894, XXVIJI, pp. $380,473$.

LEEUWENHOEK, A., 1719 ; Epistolæ ad societatem regiam Anglicam et alios illustres viros seu continuatio mirandorum arcanorum natura detectorum. Lugdun. Batav., 1719.

LEo, H., '85 : Fettbildung und Fetttransport bei Phosphorintoxication. Zeitschr. f. physiol. Chem., 1885, IX, p. 469.

v. Limbia, J., '57 : Chemische Briefe, 1857 ; Id., '70 : Ueber Gährung, über Quelle der Muskelkraft und Ernährung. Leipzig and Heidelberg, 1870.

Lilienfeld, L., and Monti, A., '93 : Ueber die mikrochemische Localization des Phosphors in den Geweben. Zeitschr. f. physiol. Chem., 1893, XVII, p. 410.

IILIIE, F. R., '96: On the smallest parts of Stentor capable of regeneration: A contribution on the limits of divisibility of living matter. Journ. of Morphol, 1896, XII, p. 241.

LoEB, J., '90: Der Heliotropismus der Thiere und seine Uebereinstimmung mit den Heliotropismus der Pflanzen. Würzburg, 1890. ; Id., 95: Untersuchungen über die physiologischen Wirkungen des Sauerstoffmangels. Arch. f. d. ges. Physiol., 1896, LXII, p. 249 ; Id., '96, 1. : Ueber Kerntheilung ohne Zelltheilung. Arch. f. Entwicklungsmech., 1896, II, p. 298; Id., '96, 2, 3, 4; '97, 1, 2 : Zur Theorie des Galvanotropismus. I (with S. S. Maxwell). Arch. f. d. ges. Physiol., 1896, LXIII, p. 121 ; II (with W. E. Gerry). Ibid., 1897, LXV, p. 41 ; III. Ibid., 1897, LXV, p. 308 ; IV (with S. P. Budgettl. Ibid., 1897, LXV, p. 518 ; V. Ibid., 1897, LXVII, p. 483.

Loeb, J., and HaRdesty, I.,'95: Ueber die Localisation der Athmung in der Zelle. Ibid., 1895, LXXT, p. 583.

Löfrter, F. '90: Del' gegenwartige Stand der Frage nach der Entstehung der Diphtherie. Deutsch. med. TTochenschr., 1890, XVI, pp. 81,108. 
Looss, A., '89: Ueber Degenerations-Erscheinungen im 'Theirreich, besonder's über die Reduction des Froschlarvenschwanzes und die in Verlaufe derselben auftretenden histolytischen Processe. Preisschriften der Fürstlich Jablonowski'schen Gesellschaft zu Leipzig, 1889.

Luctani, L., '90: Das Hungern. Studien und Experimente am Menschen. Deutsch von M. O. Fränkel. Hamburg and Leipzig, 1890.

LUDLOFF, K., '9.' : Untersuchungen üljer den Galvanotropismus. Arch. f. d. ges. Physiol., 1895, LIX, p. $52 \%$.

Luciako, '95: Sulle modificazioni delle cellule nervose nei diversi stati funzionali. Lo sperim. giornalo medico, An. 1895, XLIV, sec. Biol., F. 11.

MACH, F., '86 : Beiträge zur Analyse der Empfindungen. Jena, 1886.

Malfatt, H., '9l, '92: Zu' Chemie des Zellkerns. Ber. d. naturw.-med. Verein zu Innsbruck, 1891-92, XX.

Mann, G., '94: Histological changes induced in sympathetic, motor and sensory nerve-cells by functional activity. Journ. of Anat. and Physiol., 1894, XXIX, p. 100 .

Marey, E. J., '68: Du mouvement dans les fonctions de la vie. Paris, 1868.

Massart, J., 91 : Recherches sur les organismes inférieurs. Bull de l'acad. roy. de Belgique, 1891, 3me sér., XXII ; Id., '93 : Sur l'irritabilité des Noctiluques. Bull. scient. de la France et de la Belgique, 1893, XXV.

Massart, J. and Bortet, C., '90; Recherches sur l'irritabilité des leucocytes et sur l'intervention de cette irritabilité dans la nutrition des cellules et dans l'inflammation. Journal publié par la soc. roy. des sci. med. et nat. de Bruxelles, 1890.

MaUpas, E.,' '88 : Recherches expérimentales sur la multiplication das infusoires ciliés. Arch. de zool. expér. et, gén., 1888, 2me. ser., VI, p. 165.

MEAD, A. D., '95: Some observations on maturation and fecundation in Chaetopterus pergamentacens, Cuvier. Journal of Morphol., 1895, X, p. 313.

Meissner, M., '88 : Beiträge zur Ernährungsphysiologie der Protozoen. Zeitschr. f. wiss. Zool., 1888, XYYT, p. 498.

IIfltzek, S. J., '94: Ueber die fundamentale Bedeutung der Erchütterung fur die lebende Materie. Zeitschr. f. Biol., 1894, N. F. XII, p. 466.

Mendelssohn, M., '95: Ueber den Thermotropismus einzelliger Organismen. Arch. f. d. ges. Physiol., 1895, LX, p. 1.

v. Mering, '77: Zur Glyeogenbildung in der Leber. Ibid. 1877, XIV, p. 274.

IEtscenikoff, E., '83: Untersuchungen ïber die intracelluläre Verdaumn bei wirbellosen Thieren. Arb. aus. d. Zool. Inst. d. Univ. Wien., 1883, V.; Id., '84: Ueber die Beziehung der Phagocyten zu Milzbrandbacillen. Virchow's Arch. f. pathol. Anat. und Physiol., etc., 1884, XCVII, p. 502 ; Id., '92: Leçons sur la pathologie comparée de l'inflammation. Paris, 1892.

Mrescher, '74: Verhandl. d. naturforsch. Ges. i. Basel, 1874.

Mrescher-Resch, '80: Antl. Ber. über die internat. Fischereiausstellung in Berlin, 1880 ; Wissensch. Abth.

Minkowski, O.,'86: Ueber den Einfluss der Leberexstirpation anf den Stoffwechsel. Arch. f. exp. Pathol. u. Pharmacol., 1886, XXI, p. 41.

Minot, C. S., '90: On certain phenomena of growing old. Proc. of the Am. Assoc. for the Advancement of Science, 1890, XXXIX, p. 271 ; Id., '91 : Senescence and rejuvenation. Journ. of Physiol., 1891, XII, p. 97.

MorGan, T. H. ' ' 96 : The production of artificial astrosphaeres. Arch. f. Entwickelungsmech. d. Organismen, 1896, III, p. 339.

Mosso, A., '91 : La F'atica. Milano, 1891; German trans. ly I. Glinzer: Dic Ermüdung. Leipzig, 1892.

MüLteR, F., '64: Für Darwin. Leipzig, 1864; Eng. trans. by W. S. Dallas : Facts and arguments for Darwin. London, 1869.

MÜLler, G. E., '9l : Theorie der Muskelcontraktion. I. Theil. Leipzig, 1891.

IIÚLJeR, J., '44: Handbuch der Physiologie der Menschen für Vorlesungen. I. 4 te Aufl. Coblenz, 1844.

Müller, O., '93, '94, '96, '97: Die Ortsbewegung der Bacillariaceen, Ber. d. deutsch. hot. Ges, 1893, XI, p. 571 ; 1894, XII, p. 136; 1896, XIV, pp. 54, 111 ; $1897, \mathrm{XV}, \mathrm{p} .70$.

Müllek, W., 58 : Beiträge zur Theorie der Respiration. Nitzungsber. d. kais. Akad. d. Wiss. Wien. Math.-naturw. Cl., 1858, XXXIII, p. 99.

.Iunk, I., '84: Zur Lehre von der Resorption, Bildung und Ablagerung der Fette 
im Thierkörper. Virchow's Arch. f. pathol. Anat. u. Physiol. etc., 1884, $\mathrm{XCV}$, p. 407.

v. NäGel, C., '60: Die Bewegung im Pflanzenreiche. Beiträge zur wiss Botanik, 1860, Heft 2, p. 1; Id., '77: Die Schranken der naturwissenschaftlichen Erkenntniss. Tageblatt der fünfrigsten Versamml. deutsch. Naturforsch. u. Aerzte in München, 1877; Id., '84: Mechanisch-physiologische Theorie der Abstammungslehre. München and Leipzig, 1884.

NAGEL, W., '92:' Beobachtungen iiber das Verhalten einiger wirbelloser Thiere gegen galvanische und faradische Reizung. Arch. f. d. ges., Physiol., 1892, LI, p. 624; Id., '93: Fortgesetzte Beobachtungen über polare galvanische Reizung bei Wasserthieren. Ibid., 1893, LIII, p. 332 ; Id, '95: Ueber Galvanotaxis. Ibid., 1895, LIX, p. 603.

Nedmeister, R., '90: Zur Physiologie der Eiweissresorption und zur Lehre von den Peptonen. Zeitschr. f. Biol., 1890, N. F. IX, p. 309 ; Id., '93 : Lehrbuch der physiologisehen Chemie. I. Theil. Jena, 1893; 2te Auf., 1897.

Norman, W. W., '96: Segmentation of the nucleus without segmentation of the protoplasm. Arch. f. Entwickelungsmech. d. Organismen, 1896, III, p. 106.

NusSBAUM,'84: Ueber spontane und kïnstliche Theilung von Infusorien. Verhandl. d. naturhist. Ver. d. preuss. Rheinlande. Bonn, 1884; Id., '86 : Ueber die Theilbarkeit der lebendigen Materie. I. Mittheilung. Die spontane und kïnstliche Theilung der Infusorien. Arch. f. mik. Anat., 1886, XXVI, p: 485.

Oltmanss, F., '92: Ueber die photometrischen Bewegungen der Pflanzen. Flora, 1892 , p. 183.

Pfeffer, W., '84 : Locomotorische Richtungslyewegungen durch chemische Reize. Unters. aus $d$. bot. Inst. zu Tübingen, 1884, I, p. 363; Id., '88: Ueber chemotactische Bewegungen von Bakterien, Flagellaten und Volvocineen; Ibid., 1888, II, p. 582 ; Id., '93 : Studien zur Energetik der Pflanze. Abhandl. d. math. -phys. Cl. d. sächs. Ges. d. wiss. Leipzig, 1893, XVIII, p. 149.

Pflüger, E., '59: Untersuchungen über die Physiologie des Electrotonus. Berlin, 1859; Id., '75, 1 : Ueber die physiologische Verbrennung in den lebendigen Organismen. Arch. f. d. ges. Physiol., 1875, X, p. 251 ; Id., '75, 2 : Ueber die Phosphorescenz verwesender Organismen. Ibid., 1875, XI, p. 222 ; Id.,'78: Ueber Wärme und Oxydation der lebendigen Materie. Ibid., 1878, XVIII, p. 247 ; Id., '83, '84 : Ueber den Einfluss der Schwerkraft auf die Theilung der Zellen. Ibid., 1883, XXXI, p. 311 ; 1883, XXXII, p. 1; 1884, XXXIV, p. 607 ; Id., '91 : Die Quelle der Muskelkraft. Ibid., 1891, L, p. 98 ; Id., '92: Ueber Fleisch- und Fettmästung. Ibid., 1892, LII, p. 1; Id.,'93 : Ueber einige Gesetze des Eiweissstoffwechsels. Ibid., 1893, LIV, p. 333.

Pictet, Raoul, '93 : La vie et les basses températures. Rev. scient., 1893, LII, p. 577.

Plotakch : Opera moralia. Ed. Didot. I. p. 425. Paris, 1885.

Preyer, W.,'66: De hæmoglobino observationes et experimenta, (Dissertation). Bonn, 1866 ; Id., ' 80 : Die Hypothesen über den Ursprung des Lebens. Naturwissenschaftliche Thatsachen und Probleme. Berlin, 1880; Id., '91, 1. Die organischen Elemente und ihre Stellung im System. Wiesbaden, 1891; Id., '91, 2 ;'92: Das genetische System der chemischen Elemente. Naturwiss. Wochenschr., 1891, VI, No. 52 ; 1892, VII, Nos. 1, 2, 3 ; also reprint, Berlin, 1893.

Preyer, W., and Wendt, G., '91 - Ueber den Chemismus im lebendigen Protoplasma. I. Mittheilung. Himmel und Erde : Illustrirte Monatsschr. herausg. v. d. Ges. Urania., 1891, IV, p. 15.

Qrincke, G., '88: Ueber periodische Ausbreitung an Flüssigkeits-Oherflächen und dadurch hervorgerufene Bewegungserscheinungen. Sitzungsber. d. kgl. preuss. Akad. d. wiss. zu Berlin, 1888, p. 791.

Radziszewski, B., '80 : Ueber die Phosphorescenz der organischen und organisirten Körper. Liebig's Annalen d. Chemie, 1880, CCIII, p. 305.

Ranke, J., '65: Tetanus : Eine physiologische Ntudie. Leipzig, 1865.

Reinke, J., '80: Ueber den Einfluss mechanischer Erschütterung auf die Entwicklung der Spaltpilze. Arch. f. d. ges. Physiol., 1880, XXIII, p. 434.

Pemak, R., 44 : Neurologische Erläuterungen. Arch. f. Anat., Physiol. u. wiss. Mecl., 1844, p. 463.

Ruumber, L., 88 : Die verschiedenen Cystenbildungen und die Entwicklungsgeschichte der holotrichen Infusoriengattung Colpoda. Zeitschr. f. wiss. Zool., 1888, XLVI, p. 549; Id., 96 : Fersuch einer mechanischen Erklärung 
der indirecten Zell- und Kerntheilung. I. Theil : Die Cytokinese. Arch. f. Entwickelungsmech.; 1896, III, p. 527; Id., '97: Stemmen die Strahlen der Astrophäre oder ziehen sie? Ibid., 1897, IV, p. 659 .

Richter, H. E., '65 : Zur Darwin'schen Lehre.' Schmidt's Jahrh. d. ges. Med., 1865, CXXVI, p. 243 ; Id., "70 : Bericht itber medicinische Meteorologie und Klimatologie. Ibid., 1870, CXLVIII, p. 57 ; Id., '71 ; Die nevern Kenntnisse von den krankmachenden Schmarotzerpilzen. Ibid., 1871, CLI, p. 313.

ฯ. Rindfleisch, E.,'88: Aerztliche Philosophie. Festrede zur Feier,des dreihundert und sechsten Stiftungstages der königl. Julius-Maximilians-Universität. Würzburg, 1888 ; Id., '95 ; Neovitalismus. Verhandl. d. Ges, deutsch Nat. v. Aerzt. 67 Versamml. zu Lübeck, 1895, I. Theil. Leipzig, 1895, p. 111.

Rossmach, '7l. Die rhythmischen Bewegungsercheinungen der einfachsten Organismen und ihr Verhalten gegen physikalische Agentien und Arzeneimittel. 1871 ; Arb. d. zool.-zoot. Inst. zu Würzburg, 1874.

Kort, A., '93 : Ueber das Verbalten beweglicher Mikroorganismen in strömender Flüissigkeit. Deutsch. med. Wochenschr., 1893, XIX, p. 351.

Roox, W.,' '95: Gesammelte Abhandlungen tiber Entwicklungsmechanik der Organismen. Leipzig, 1895.

Rubrer, M.,'94: Die Quelle der thierischen Wärme. Zeitschr. f. Biol., 1894, N. F. XII, p. 73.

Sachs, J., : Ueber den Einfluss der Lufttemperatur und des Tageslichts auf die stündlichen und täglichen Aenderungen des Längenwachsthums der Internodien. Arb. d. bot. Inst. in Wïrburg, I. ; Id., '82 : Vorlesungen ïber Pflanzen-Physiologie, Leipzig, 1882.

Salvioli, G., '80. . Eine neue Methode für die Untersuchung der Functionen des Dïnndarms. Arch. f. Anat. u. Physiol., Physiol. Abth., Supplement, 1880, p.95.

ScHÄFER, E. A., '91, 1 : On the structure of amoboid protoplasm, with a comparison between the nature of the contractile process in amoboid cells and in muscular tissue, and a suggestion regarding the mechanism of ciliary action. Proc. Roy. Soc. of London, 1891, XLIX, p. 193; Id., '91, 2 : On the minute structure of the muscle-columns or sarcostyles which form the wing muscles of insects. Preliminary note. Ibid., 1891, XLIX, p. 280 ; Id., ' 91,3 : On the structure of cross-striated muscle. Internat. Monatsschr. f. Anat. u. Physiol., 1891, VIII, p. 177.

Schleiden, M. J., '38 : Beiträge zur Phytogenesis. Arch. f. Anat., Physiol. u. wiss. Med., 1838 , p. 137.

SCHMANKEWITCH, W., '77: Zur Kenntniss des Einflusses der äusseren Lebensbedingungen a die Organisation der Thiere. Zeitsehr. f. wiss. Zool., 1877, XXIX, p. 429.

SchösDorfF, B., '93: In welcher Weise beeinflust die Eiweissnahrung den Eiweissstoffwechsel der thierischen Zelle? Arch. f. d. ges. Physiol., 1893, LIV, p. 420.

Schultze, M., '54: Jer Organismus der Polythalamien. Leipzig, 1854; Id., '61 : Ueber Muskelkörperchen, und das was man eine Zelle zu nennen habe. Arch. f. Anat., Physiol. u. wiss. Med., 1861, p. 1 ; Id., '63 : Das Protoplasma der Rhizopoden und der Pflanzenzellen. Leipzig, 1863; Id., '65: Zur Kenntniss der Leuchtorgane von Lampyris splendidula. Arch. f. mik. Anat., 1865, I, p. 124; Id., '71, 72: Allgemeines über die Structurelemente des Nervensystems. Stricker's Handbuch der Lehre von den Geweben des Menschen und der Thiere. Leipzig, 1871-2; Eng. trans. . A Manual of Histology, by S. Stricker. New York, 1872.

Schulze, F. E., '87: Zur Stammesgeschichte der Hexactinelliden. Abhandl. d. kgl. preuss. Akad. d. Wiss. zu Berlin, 1887.

SuHWaN, Тн., '39 : Mikroskopische Untersuchungen über die Uebereinstimmung in der Structur und dem Wachsthum der Thiere und Pflanzen. Berlin, 1839; Eng. trans. by H. Smith: Microscopical researches into the accordance in the structure and growth of animals and plants. London, Sydenham Soc., 1847.

Schwarz, F. '84,1 : Der Einfluss der Sihwerkraft auf die Bewegungsrichtung von Chlamydomonas und Euglena. Ber. d. deutsch. bot. Ges., I884, II, p. 51 ; Id., '84,-2 : Beitrag zur Entwicklungsgeschich te des pflanzlichen Zellkerns nach der. Theilung. Beitr. 2. Biol. d. Pflanzen von Ferd. Cohn. 
Breslau, 1884, Heft IV, p. 79 ; Id., '87: Jie morploologische und chemische Zusammensetzung des Protoplasmas. Breslau, 1887.

Senon, R., '87 : Beiträge zur Naturgeschichte der Synaptiden des Mittelmeers. Mittheil. d. zool. Stat. zu Neapel, 1887, VII, p. 272.

SEnper, K., '68 : Reisen im Archipel der 1'hilippinen. Theil I. I. Holothurien. Leipzig, 1868.

v. SichERTR, O., '96: Chemotaxis der Warmbliitel-Leukocyten ausserhalb des Körpers. Münch. med. Wochenschr., 1896, XLIII, p. 976.

Sorncke, L., '88: Die Entslehung des Stromes in der galvanischen Kette. Sitzungsber. d. math.-physik. Cl. d. kön. bayr. Akad. d. Wiss., 1888, Heft III.

SPEncer, H., "93: The inarlequacy of natural selection. Contemporary Review, 1893, LXIIJ, pp. 153,439 .

S'тAнL, E., ' 80 : Ueber den Einfluss von Richtung und Stärke der Beleuchtung auf einige Bewegungserscheinungen im Pflanzenreiche. Bot. Zeitung., 1880, XXXVIII, pp. 297, 321, 345, 361, 377, 393, 409; Id., '84: Zur Biologie der Iyxomyceten. Ibid., 1884, XLII, pp. 14.5, 161, 187; Id., '85 : Finfluss der Beleuchtungsrichtung auf die Theilung der Exuisetumsporen. Ber. d. cleutseh. bot. Ges., 1885, III, p. 334; Id., "88 : Pflanzen und Schnecken. Eine biologische Sturlie ïber die Schutzmittel der Pflanzen gegen Schneckenfrass. Jen. Zeitschr. f. Naturw., 1888, N.F. XV, p. 5ir.

Steinach, E., '92 : Untersuchungen zur vergleichenden Physiologie der Tris. Arch. f. d. ges. Physiol., 1892, LII, p. 495.

Strrasburger, E., '78: Wirkung des Lichtes und aler Warme anf Schwärmsporen. Jen. Zeitschr, f. Naturw., 1878, XII, p. 451 ; Id. '80: Zellbildung und Zelltheilung. Jena, 1880 ; Id. '88: Histologische Beitraige. Heft I : Ueber Kern- und Zelltheilung im Pflanzenreiche. Jena, 1888.

v. Thanhoffer, L. '74: Beiträge zur Fettresorption und histologischen Structur der Diinndarmzutten. Arch. f. d. ges. Physiol., 1874, VIII, p. 391.

Valientin, G. G., '47-'50: Lehrbuch der Physiologie des Menschen. 2te Aufl. Braunschweig, $1847-50$.

Verworn, M.,'88: Biologische Protistenstudien. I. Zeitschr. f. wiss. Zool., 1888, XLVI, p. 455 ; Id., '89, 1 . Psychophysiologische Protistenstudien. Experimentelle Untersuchungen. Jena, 1889; Id., '89, 2, 3: Die polare Erregung der Protisten durch den galvanischen Strom. Arch. f. d. ges. Physiol., 1889, XLV, p. 1 ; 1890, XLVI, p. 267 ; Id., '90, 1 : Biologische Protistenstudien.II. Zeitschr. f. wiss. Zool., 1890, L, p. 443; Id., 90, 2: Studien zur Physiologie der Flimmerbewegung. Arch. f. d. ges. Physiol., 1891, XLVIII, p. 149; Id. ,'91: Die physiologische Bedeutung des Zellkerns. Thid, 1892, LI, p. 1 ;Id., '92, 1 : Die Bewegung der lebendigen Substanz. Eine vergleichend-physiologische Untersuchung der Contractionserscheinungen. Jena, 1892 ; Id., '92, 2 : Untersuchungen iiber die polare Erregung der lebencligen Substanz durch den constanten Strom. III. Ber. d. zweit. internat. Physiol.Congress. in Liittich, 1892 ; also Arch. f. d. ges. Physiol., 1896, LXII, p. 415 ; Id., '93 : Ueber die Fähigkeit der Zelle activ ihr specifisches Gewicht zu verändern. Arch. f. d. ges. Physiol, 1893, LIII, p. 140; Id., '94: Modern Physiology. The Monist, 1894, IV, p. 355; Id., '96, 1 : Der körnige Zerfall. Ein Beitrag zur Physiologie des Todes. Ibit., 1896, LXIII, p. 253; Id., '96, ? : Erregung und Lähmung. Verhandl. A. Ges. deutsch. Naturf. u. Aerzte zu Frankfurt a. M., I. Theil., 1896, p. 73 ; also Deutsch. med. Wochenschr., 1896, XXII, p. 640 ; It., '96, 3: Zellphysiologische Studien am rothen Meer. Sitzungsber. d. kgl. preuss. Akad. d. wiss. zu Berlin, 1896, p. 1243; Id., '96, t: Die polare Erregung der lebendigen Substanz durch den constanten Strom. IV. Arch. f. d. ges. Physiol., 1897, LXV, p. 47 : It., '96, 5 : Tonische Reflexe. Ibid., 1897, LXV, p. 63; Id., '97: On the relation between the form and the metabolism of the cell. Science Progress, 1897, N.s., I, No. 3.

Vrronow, R., '58 : Die Cellularpathologie in ihrer' Begriindung auf physiologische und pathologische Gewebelehre. Ite, Aufl. Berlin, 1858 ; Id., '71 : Ibid. 4te Aufl. Berlin, 1871.

Vöchtinc, H., '85: Ueber die Regeneration der Marchantien. Jahrb. f. wiss. Bot., 1885 , XVI, p. 367.

v. Vort, C., '60: Untersuchungen über den Einfluss des Kochsalzes, des Kaffees und der Muskelbewegnug anf ien Ntoffwechsel. Wunchen, 1860; Id., '66: 
Ueber die Verschiedenheiten her Eiweisszersetzung beim Hungern. Zeitschr. f. Biol., 1866, II, p. 307 ; Id., '70: Ueher rlie Entwicklung der Lehre von der Quelle cler Muskelkraft und einiger Theile der Ernährung seit .5.5 Jahren. Ibid., 1870, VI, p. 305 ; Id., 'il : Physiologie des allgemeinen Stoffwechsels und der Ernährung. Hermann's Handbuch der Physiologie, 1881, VI.

DE Vrifs, H., '89 : Intracellulare Pangenesis. Jena, 1889.

WEeER, E., ' 16 : Muskelbewegung. Wagner's Handwörterbuch del' Physiologie, III, 1846.

Weirart, C., '75: Ueber pockeniihnliche Gebilde in parenchymätosen Organen und deren Beziehungen zu Bakteriencolonieen. Breslau, 1875; Icl., '77, '78: Ueber Cromp und Diphteritis. Ein experimenteller und anatomischer Beitrag zur Patholngie der specifischer, Entzuindungsfor'men. Virchow's Arch. f. pathol. Anat. u. Physiol, etc., 1877, LXX, p. 461; 1878, LXXII, p. 218 ; Id., '80: Ueber' die pathologischen Gerimnungsvorgänge: Ibid., 1880, LXXIX', p. 87.

Wetsmaxy, A., '82: Ueber die Dauer des Lebens. Jena, 1862; authorized Eng. trans. in Essays upon Heredity. I. Oxford, 1889 ; Id., ' 84 : 'Ueber Leben und Tod. Jena, 1884; authorized Eng. trans. in Essays upon Heredity. I. Oxford, 1889 ; Id., '92, 1 : Aufsätze über Vererbung und verwandte biologische Fragen. Jena, 1892 ; authorized Eng. trans. : Essays upon heretity and kindred biological problems. 2 vols., Oxford, 1889, 1892. This contains all the works of Weismann upon heredity from 1881 to date. Id., '92, 2: Das Keimplasma. Eine Theorie der Vererbung. Jena, 1892; authorized Eng. trans. by W. N. Parker and $\mathbf{H}$. Rönnfeldt: The gem-plasm: it theory of heredity. New York, 1893.

WENDT, G., '91 : Die Entwicklung der Elemente. Berlin, 1891.

WTFNer, C., '63 : Erklärung des atomistischen Wesens des tropfbar flüssigen Körperzustandes, etc. Poggendorff's Annal. d. Physik u. Chen., 1863, CXVIII, p. 79.

Wilson, E.B. and Matjews, A. P., '95: Maturation, fertilization and polarity in the echinoderm egg. New light on the "quadrille of the centers." Journ. of Morphol., 1895, X, p. 319.

Winogradsky, S., '88 : Ueber Eisenbakterien. Bot. Zeitung., 1888, XLVI, p. 261 ; Id., '90: Recherches sur les organismes de la nitrification. Annal. de l'inst. Pasteur., 1890, IV, pp. 213, 2.57, 760.

Wöhler, F., '28 : Ueber künstliche Bildung des Harnstoffs. Poggendorff's Annal. d. Physik. u. Chem., 1828, XII, p. 253.

ZACHARTAS, O, ' 81 -' 87 : Varions articles in Bot. Zeitung, 1881, 188:2, 1883, 1885, 1887; Id.,' '85 : Experimentelle Untersuchungen ïber Psendopodienbildung. Biol. Centralb., 1885, V, p. 259.

ZingleR, E., '95: Lehrbuch der allgemeinen Pathologie und der pathologischen Anatomie. 9te Aufl. in progress, Jena, 189.5.

Zinoffsky, O., '85, Ueber die Grösse des Hämoglobinmoleciuls. Zeitschr. f. physiol. Chem., 1885, X, p. 16.

Zuntz, 92: Die Frnährung des Herzens und ihre Beziehung zu seiner Arbeitsleistung. Berl. klin. Wochenschr., 1892, XXIX, p. 366. 
INDEX 



\section{INDEX}

A canthocystis, myoporlia of, 2.:2

Accessory disc, 243

Achromatic nuclear substance, 91

Actinosphorium, adaptation of. to stimulus, 357

chemical stimulation of, 367

double refraction of, 99,560

fatigue of, 460

galvanic stimulation of, 420,473

mechanical stimulation of, 379,382 , 386

polar excitation of, 420,473

Adaptation, 179, 182, 208

individual, 183

phyletic, 185

to stimulus, 357

Adenin, 108, 109, 162, 175, 305, 482

Athalium septicum, chemotaxis of, 430 phototaxis of, 449

plasmodium of, 72

rheotaxis of, 444

thermotaxis of, 452

Agaricus, luminosity of, 255

Albuminoids, 108

Albumins, 107

Albumoses, 152

Alchemy, 55

Aleurone-grains, $83,105,108,172$

Algar, 316

A mblystoma, polar excitation of, $4 \mathrm{ls}$

A maeba, action of cold upon, 289,394

action of heat upon, 291

action of solutions upon, 184

artificial division of, 295, 508

as organism without organs, 119

axial orientation of, 498

behaviour of non-nucleaterl pieces of, 508,512

chemical stimulation of, 367

development of, 204

effect of withdrawal of oxygen from, 284,561

electrical stimulation of, $419,423,493$

excretion by, 169

food-vacuoles of, 144, 169

galvanotaxis of, 457
Amaba, ingestion of food by, 143

inheritance in, 544

mechanical stimulation of, 382,386

movements of, 235, 561

narcosis of, 375

polar excitation of, 419,493

reproduction of, 192

spherical form of, in death, 327

thermal stimulation of, 392,472

thermotaxis of, 452

Amoboid movement, 234, 284,560

Amphimixis, 317

Amphipyrenin, 92

Amyloid metamorphosis, 491

Amyloid substance, 334

Anabiosis, 130

Anaërobia, 287

Anresthetics, action of, $337,371,469$

A nastatica, swelling movements of, 223

Anatomy, comparative, 313

Anaximander, 8

Angionperma, 316

Animat 12,16

Animals, assimilation in, 160 cold-blooded, 217, 257, 288 genealogical tree of, 316 metabolism of, 164,173 nutrition of, 138,274

warm-blooded, 217,257

Animistic system of Stahl, 16

Anisotropic substance, 243, 245

Anode, 405

Anophrys, chemotaxis of, 436

Apoplexy, 335

Apposition, growth by, 122, 168

Arcella, specific gravity of 98,229

Areheopteryx, 314

Arrher, van Helmont's doctrine concerning, 13

Aretaus, 9

Argentieri, 12

Aristotle, 9

A roülece, procluction of heat $1, y, 2.5$

Artemire Writhrusenï, 183

selina, 183

Arthropoda, 317 
Ascaris, centrosome of spernatozoa of, 70

fertilisation in, 202

Asp, 496

Asphyxia, 282

Assimilation, I57, 485

in animals, 160

in plants, 158, 287

Astronomical knowledge, 33

Atavism, 180

Athenæus, 9

Atmosphere, composition of, $26: 2$

Atom, 33, 209

Atrophy, 321, 488

and dissimilation, 48.;

from disuse, 323,352

nomal, 321

pathological, 323

pigment, 336

senile, 323,338

Avicenna, 11

Axial orientation, 496

Bacillus butyricus, 112

Bacon, 13

Bacteria, action of temperature upon growth of, 391

anaërobic, 287

chemotaxis of, 432

geotaxis of, 446

iron-, 278

mechanical stimulation of, 388

multiplication of, 366

nitrogen-, 140, 274

nucleus of, 67

nutrition of, 140

of cholera, 287

of symptomatic anthrax, 28 ;

of tetanus, 287

phototaxis of, 449

resistance of, to high temperatures, 291

rheotaxis of, $\neq 45$

spore-formation of, 281

structure of, 67

sulphur-, 278

Barterizm larticum, 112 photometricum, 400

Batyli, 7

Ballooning, effects of, 293

Barotaxis, $\mathbf{4 4 0}$

Barthez, 18

Bear-animalcules, desiccation of, 129

Bees, production of heat by, 2 is

Bell, Charles, 19

Bell's law, 19

Bernard, Claude, 23

Bioblasts, 63

Biogens, 481

decomposition of, 484

formation of, $48: 3$

growth of, 529

polymcrism of, 486,520
Biogenesis, law of, $30,207,208,315$, 575

Biotonus, 487

action of general stimuli upon, 489

action of local stimuli upon, 496

Blisters, 325

Blood, circulation of, 57

discovery of circulation of, 11, 12, 13 streaming of, in capillaries, 219

Boerhaave, 16

du Bois-Reymond, 2:3, 263

Bone, 168

Bordeu, 18

Borelli, 14

Brain, 580

Branchipus stagnalis, 183

Brown, John, 18

Brownian movement, 4, 220

Butyric acid as decomposition-product of carbohydrate, 113

C.ALCIFICATIOr, 335

Calcium guanin, 83, 109, 176

Calcium oxalate in plant-cells, 83

Calorie, 21:2, 260

Calorimeter, 260

Calorimetry, 44, 260, 549

Cane-sugar, 112

Carbohydrates, 110

as product of proteid-decomposition, 164

as source of muscle energy,, 554

combinations of, 108,113

fate of ingested, 161

localisation of, in cell, 116

origin of, from fat, 480

Carbon-equilibrium, 275

Carbonic acid as constituent of living substance, $11 \tilde{5}$

as excretion, 173

as product of carbohydrate-decomposition, 113

as product of proteid-decomposition, 110,164

cleavage of, in plunt-cell, 158, 217, 366,399

Carchexium as cell-republic, 568

digestion in, $15^{\circ}$ ?

Carmicora, 140, 141, 276

Cartilage, 168

Casein, 108

Catalytic action, 1.กั.

Crulerpa, 7.2

Causality, craving for, $5,32,35$

Cell, amoboid, 75

artificial, 167

as constituent of organism, 27,60

as elementary organism, $\mathbf{5 6}$

as object of physiological study, $4 \mathrm{~s}$

chemical compounds of, 102

ciliated, 77,247

definition of, 68

development of, $: 2(14,530$ 
Cell, flagellated, 247

form of, 74

growth of, $5: 29$

multinucleate, 71,89

reproduction of, 531

size of, 78

specific gravity of, 97,229

turgor of, 226

('ell-community, centralisation in, 57 (

constitutional relations of, 567

differentiation in, 573

rlivision of labour in, $57: 3$

Cell-constituents, general and special, 64

('ell-differentiation, 573

Cell-division, action of narcotics upon, 374

direct, 191

equal, 195

forms of, 191

indirect, 193

mechanics of, 531,533

multiple, 196

partial, 196

reducing, 197

total, 195

unequal, 195

Cell-life, mechanics of, 504

Cell-membrane, 65

growth of, 168

movement by swelling of, 222

Cell-metabolism, mechanics of, 518 scheme of, 522

Cell-republics, 568

Cell-sap, 225

Cell-theory, 27, 65

Cell-turgor, 226

Cellulose, 112

as secretion, 173

Cenogeny, 207

Centralisation in cell-community, 576

Central nervous system, 577

Centrosome, 69

in cell-division, 194

quadrille of, 203

Centrotaxis, 447

Cesalpino, 12

Chaussier, 18

Chemical energv, introduction of, into organism, 212

Chemometry, 44!

Chemotaxis, $4: 29$

Chemotropism, 429

Chitin, 173

Chlorophyll-bodies, 81

cleavage of carbonic acid in, 217

Cholera, vibrios of, 287

Cholesterins, 110

Chondrin, 173

Chromatic nuclear substance, 91

Chymosin, 171

Cilia, chemical stimulation of, 368 electrical stimulation of, $418,423,425$
Cilia, mechanical stimulation of, 357 , 570

narcosis of, 375

optical properties of, 99

polar excitation of, 418

thermal stimulation of, 393

work of, 252

( 'iliary movement, 247

in absence of oxygen, $\mathbf{2 8 6}$

metachronism of, 247,570

Cilicta, galvanotaxis of, 458

Ciliater cells, 77,247

Circulation of blcod, 577

of energy, 546

of protoplasm, 239

Cleavage, riscoidal, 196 superficial, 197

Climbing-plants, thigmotaxis of, 442

Clostcrium, Brownian movement in, 220

locomotion of, 231

phototaxis of, $\mathbf{4 5 0}$

Coagulation of proteids, 106

of silicic acid, 106

-necrosis, 324

Cockroach, thigmotaxis of spermatozoa of, $44^{2}$

Collenterata, 317

('old, physiological effect of, 288

Cold-blooded animals, 217, 257, 24

Cold-centre, 391

Cold-rigour, 394

Coleps, food-ingestion by, 146

Collagen, 109

Colloids, 105

Colombo, 12

Colony, 59

Colour-vision, Hering's theor'y of, 493

Community, 59

Conchiolin, 109

Condensations, 43

Conduction, 360

Conjugation, 92, 200, 517 of Infusoria, 343

Connective substances, 173

Consciousness, 33

Contact-action, 155

Contraction, $233,244,558$

Engelmann's theory of, 558

relation of varieties of, 252

(Yornein, 109

Corpulency, 331

Correlation of parts in organism, $18 \mathrm{I}$

Cosmology of Ionic philosophers. 7

Cosmozoa, theory of, $300,302,317$

(reatin, $109,162,163,175,305,480$

Creatinin, 109, 163

Crystalloids, 105

(tinophora, ciliary motion in, 249,

('yanic acid, 306

(yanogen, 315, 311, 482

Cyciode, 68

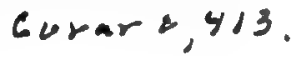


DAY-FLIES, life of, 340

Death and life, 133

apparent, 126,133

as adaptation, 342

by over-stimulation, 471

causes of, 336

development of, 134, 319, 471

external causes of, 337

history of, 134,319

internal causes of, 338

moment of, 134

natural, 339

of man, 133

of cell, 134

-stiffening, 133, 324, 329

Decay, 326

Degeneration, hyaline, 336 waxy, 325

Dendrites, 581

Depression, 356, 357, 469, 490

and inhibition, 494

by chemical stimuli, $37 \mathrm{I}$

by electrical stimuli, 427

by mechanical stimuli, 388

by photic stimuli, 403

by thermal stinudi, 394

Descartes, 13

Descent, theory of, $8,28,178,312$

Desert-organisms, 279

Desmirls, movement of, 231 phototaxis of, 450

Desiccation of organisms, 129

Developnsent, 178, 204

as perfecting process, 318

germinal, 178, 187, 204, 207, 314, 575

mechanics of, 533

of $A m \mathrm{~m}-b \alpha, 204$

of Colpoda, 205

of the cell, 204,530

of the multicellular organism, 20106

of the unicellular organism, 204

racial, 178, 207, 313, 575

Dextrose, 110,112

Diabetes mellitus, 164

Dialyzer, 104, 524

Diastase, 1.54

Diatoms, locomotion of, 232

nechanical stimulation of, 389

photic stimulation of, 402

phototaxis of, 449

Didymizm, action of oxygen upon, 369

Differential theory, Hermann's, 267

Inifferentiation of cells in cell-community, 573

Difflugia, behaviour of non-nucleated pieces of, 513

capsule of, 148

conduction in, 362

conjugation of, 92,200

mechanical stimulation of, 382

necrobiosis of, 564

specific gravity of, !s, 2.29
Uigestion, 150

Diphtheria, bacilli of, 176

Direction-corpuscles, 196, 199, 202

Disaccharids, 112

Discharge, conception of, 355

Disintegration, granular, 326

Dissimilation, 161, 485

Division of labour, 573

Dobie's line, 243

Dübereiner's lamp, 43

Jrosera, changes in nuclei of cells of, 516

peptonising ferment of, 171

Dualism of borly and mind, 7, 14, 16, 38,40

Dumas, 18

Dytiscus, nuclei in eggs of, 514

Echinodermata, 317

Ectoderm, 317

Egg-albumin, 10\%

Elastin, 109

Elaters of spores of horse-tail, 224

Electric fishes, 268

organs, 269

Electrical induction, 410 resistance, 407

Electricity, animal, 263

as cause of death, 337

origin of, 265

production of, 262

therapeutic use of, 352

Electrodes, non-polarisable, 268, 406, 455 ,

Electromotive force, 407

Electrotonus, 414,421

Elements, chemical, 99

galvanic, 265,404

organic, 99

thermo-electric, 258

Empedocles, 8

Endoplasm, 235

Energy, action of stimuli upon transformation of, 5 s 1

chemical, 547

circulation of, 546

forms of, 209

introduction of, into organism, 21르

kinetic, 26, 211, 355

law of conservation of, 26, 210, 211

mechanics of transformation of, 546

modifications of, 211

potential, 26, 211, 355

theory of specific, $21,45,475$

transformation of, in chemical proces. ses, 213

Entoderm, 317

Fnzymes, 109, 151, 156, 171

Epigenesis, 17, 535, 538

Equilibrium, carbon-, 275

dynamical, 44, 123

metabolic, 275,488

nitrogen-, 275, 365 
Erasistratus, 8

Ergograph, 462

Esters, 113

Eurlorine, 60 as cell-republic, 568

Euglena, axial orientation of, 499

Excitability, 18

Excitation, 356, 357, 469, 490

by chemical stimuli, 365

by electrical stimuli, 412

by mechanical stimuli, 379

by photic stimuli, 399

by thernal stimuli, 389

polar, 414

Excretion by $A$ moba, 169 of dissolved substances, 166 of gaseous substances, $166^{\circ}$ of solid substances, 166

Exeretions, 166, 173 gaseous, 173

liquid, 174 solid, 176

Exhaustion, 469

Exoplasm, 235

Expansion, 233 of muscle, 246

Experimentum mirabile, 496

Extra current, 410

FAKIRs, Indian, 126

Fat, 113

decrease of, in hunger, 277

digestion of, 155

fate of ingested, 161

localisation of, in cell, 117

origin of, from carbohydrate, 159

origin of, from proteid, 110, 163, 332, 480

Fat-droplets, 83, 95, 172

ingestion of, 144

Fat-metamorphosis, 331

Fatigue, 460, 469

causes of, 469

Fatigue-curve of muscle, 463

Fatigue-substances, 468

Fattening, 275, 331

Fatty acids, 113, 155

Fatty infiltration, 331

Fermentation, alcoholic, 111, 157

butyric acid, 112

lactic acid, 112

Fermentation-tube, 111, 372

Ferment-organisms, 156

Ferments, 155

van Helmont's doctrine of, 13,15 organised, 156

unorganised, 109, 15l, 156, 171

Fernelius, 12

Forns, chemotaxis of spermatozoids of, 435

Fertilisation, 190, 198

Hibrin, 107

Fibrinogen, 107
Filirincer, 316

Fish embryos, galvanotaxis of, $4 \pi, \pi$

Fission, 191

Fleegellata, galvanotaxis of, 4.5

Flayellated cell, 247

Fly-larve, histolysis of tissue in, 33:2 origin of fat from proteid in, 164

Food as general vital condition, 274 compensatory, $141,161,555$

ingestion of, 142

primitive, 141, 555

selection of, 146,527

Food-bodies in cells, $8: 2$

Food-stuffs, 138

Food-racuoles, 144, 146, 169, 万.2.7

Force, :2us

Force hyperméchanique 18, 45

Form, changes of, 177

Fowl, experimentum mirabile, 4!li

Frog, reflex tone of, 3.5

Fruit-sugar, 110

Function, change of, 270 physiological, 576

Functions, physical (natural), 11 psychical (animal), ll sphygmical (vital), 11

Fingi, 316 nutrition of, 140,274

GALEx, 9

Galvani, 19, 263

Galvanic current, 264,4114 polar excitation by, 414

Galvanic element, 265, 404

Galvanic key, 406

Galranism, 263

Galvanometer, 259

Galvanotaxis, 455

Ganglion-cell, 581

action of narcotics upon, 376

calcification of, 335

changes in, during activity, 464,516

depression of, 494

excitation of, 494

fatigue of, 464

inhibition of, 494

( trangrene, dry, 32t

moist, 326

('as-chamber, Engelmann's, 284, 519

Gases as constituents of living sulv. stance, 115

Gas-fiame, comparison of vital phenomena with, 5411

Gasmeada, 317

Gastrula, 317

Gelatine foams, radiation-phenomend in, 531

Gemmation, 191, 196

Genealogical tree of organisms, 178, 316

Geotaxis, $4+5$

Germ-regions, organ-forming, 534

Girtanner, 19

Glisson, 15, 17 
Globulins, 107

Glucose, 110

Glutin, 108, 173

Glycerine, 113, 155

Glycogen, 83, 112, 116

as product of proteid-decomposition, $110,164,480$

transformation of grape-sugar into, 161

Glyco-proteids, 108

Granular disintegration, 473

Granular streaming, 94, 220, 237

Granules, Altmann's hypothesis concerning, 63,88

in protoplasm, 83

Grape-sugar, 110

as product of proteid-decomposition, 110,164

transformation of, into glycogen, 161

Graphic method, 23

Gregarince, locomotion of, 233

Growth, action of narcotics upon, 373 action of temperature upon, 391 and assimilation, 485

and reproduction, $188,488,531$

by apposition, 122, 168

by intussusception, 122, 168

mechanics of, 529

movements by, 233

Guanin, 108, 109, 162, 175, 176, 305, 482

Guinea pig, tonic excitation of, 358

Gymnast's fever, 467

Gymnospermo, 316

HAMOGLOBIN, 103, 105, 108

in muscle, 286

Haller, 16

Harvey, 13

Heart-muscle, work of, 246

Heat, introduction of, into organism, 217

physiological effect of, 291

production of, 256

Heat-centre, 391

Heat-equivalent, 212

Heat-rigour, 396

Heliotropism, 429, 447

van Helmont, 13

Henson's disc, 243

Heraclitus, 8

Herbivora, 140, 276

Hereditary substance, 505, 545

Heredity, see Inheritance

Herophilus, 8

Hibernation, 128

Hippocrates, 8

Hippuric acid, 109, 163, 175

Histolysis, 321

Hoffmann, 16

Holothurians, mucous metamorphosis of, 170,333
Homothermal animals, 217, 257

heat.regulation in, 390

Horse-tails, elaters of spores of, 224 phototaxis of spores of, 448

von Humboldt, 19

Hunger, 275

Hyalopus, graular disintegration of, 326

Hydra, regeneration of, 57

Hydrotaxis, 430

Hypnosis, 349, 496

Hypoxanthin, 108, 109, 162, 175, 305, 482

IATROCHEMICAL school, 15

Iatromathematical schuol, 15

Iatromechanical school, 15

Iatrophysical school, 15

Ignorabimus, du Bois-Reymond's, 34, 47

Inmortality, physical, 341

Immunity, 359

Impatiens, movements of seeds of, 233

Inanition, 275

Incasement, theory of, 17, 535

Individuals, conception of organic, 56 definition of organic, 58

real and virtual, 62

varieties of, 62

Individuality as idea, 37

Induced current, 410

Infusoria, chemical stimulation of, 368 conjugation of, 92, 200,343, 517

death of, 343

digestion in, 152

discovery of 15,298

granular disintegration of, 326

myoids of, 241

narcosis of, 375

old age of, 343

thermal stimulation of, 394

Ingenhouss, 19

Ingestion of food, 142

Inheritance, 179, 207, 318

and metabolism, 545

mechanics of, $\check{5} 44$

nucleus as medium of, 505

of acquired characteristics, 180,318

Inhibition, 494

Inorganic constituents of living sub. stance, 114

Inotagmata, 559

Intestinal epithelium, resorption through, 144, 527

Intussusception, growth by, 122, 168

Inversion of disaccharids, 112

Invertin, 157

Investigation, conception of, 5 goal of, 40

Ionic philosophers, cosmology of, 7

Iron-bacteria, 278

Irritability, 124, 348, 353

theory of, $15,17,18$

Isotropic substance, 243,245 
KАтกОIN, 405

Koratin, 109,141

Kiclney, excretion by cells of, 1660

Knowlelge, astronomical, 33 conception of, 31,34

Knowleilge of nature, du Bors-Reymond's definition of, 31

limits of, 31

Kuihne, 23

Kymograph, invention of, :2:3

Larrymaria, movement of non-nucle. ated pioces of, 509

Lactic acid as excretion, 174

as product of carbohyllrate-ilecompo. sition, 112,113

as product of proteid-rlecomposition, 110,164

in synthesis of uric acisl, 17.5

Lactose, 112

Livvulose, 110, 112

Lavoisier, 19

Law, biogenetic, 30, 207, 208, 315, 575 of conservation of energy, 26, 2111,211 of conservation of matter, 26 of polar excitation, 414

Ohm's, 407

Lecithins, 109

Leeuwenhoek, 15

Liucocytes, action of quinine upon, 375 behaviour of nuclens in narcosis of, 521

chemotaxis of, 430

degeneration of, 323

food-ingestion by, 144

food-selection by, 148

in necrobiosis, 327

rible of, in histolysis, $: 322,432$

Lieberkühnia, ingestion and digestion of food by, 152

Liebig, 23

Life, actual, 132

und apparent death, 120

and death, 133

as proteid-metabolism, 136, 3111

conception of, $2,303,309$

oxternal conditions of, 273,274

general and special conditions of, 27,3

internal conditions of, $273,2.94$

latent, 132

origin of, $297,307,311$

I'fliger's idea of origin of , 304

potential, 132

primitive ileas concerning, 3,7

theory of continuity of, 121, 3112, 309

Light, action of, in plant-cell, lis, 217 , 547

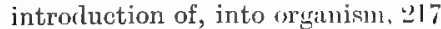

nature of organic, 254

production of, 25.3

Lightning-bug, luminosity of, $23,4,2.5$

Linin, 92

Liquefaction, 325
Living and lifeless, 4, 118, 481

Living substance, chemical properties of, 99

comprosition of, ...5

formation of, 483

galvanic eurrent of, 260

inorganic cunstituents of, 114

optical properties of, 98

organisation of, 93,119

physical properties of, 93

specific gravity of, 97

Lurlwig, 23

Luminosity of chemical sulustances, 250 organic, 253

MACRONUCHALTS, 92, 201, 34:3, 51T

Magrenrlie, 每;

Magnetism, animal, :3+9

Mroyosyhere as cell-republic, 568

Malpighi, 1.;

Man, fasting of, 276

development of, 339

Marey, 23

Mass-effect, 43

Matter and mind, 34

Matter, law of conselvation of, 26 nature of, 33,35

Maturation of ovum, 199, 2012

Maximum of excitation, 471

of stimulus, 350

of temperature, 288, 291, 396

of vital conditions, 349

Mayow, I5

Mechanico-rlynamical system of Hoffman, 16

Mechanical energy, production of, 219

Mechanical equivalent, $: 212$

$.1 /$, s mbryanthemum, water in, 279

Netabolism, 137, 483

action of heat upon, 390

action of stimuli upon, 489

and oxygen, 366

as characteristic of living organism, $125,131,311,477$

curve of, 177

in manufacture of sulphuric acid, 125

in tetanus, 388

of animals, 164, 173

of plants, 164, 173

scheme of, in cell, 522

self-regulation of, 489

without nucleus, 518

Metachronism of ciliary motion, 247 , 50

Metamorphosis, 330, 491

antyloid, 334, 491

colloid, 336

fat, 331

mucons, 332

Metaphysics and natural science, 39

. Vetaphyta, 316

Heteror, 316

Micronucleus, 92, 201, 343, 517 
Microscope, invention of, 15

Milk, formation of, 331

Milk-sugar, 112

Mimosa, electrical stimulation of, 426 mechanical stimulation of, 380 movement of, 227 narcosis of, 374

Mind and matter, 34 Egyptian doctrine of, 7

Minimum of stimulus, 350 of temperature, 288,395 of vital conditions, 349

Mitosis, 193

Molecule, 209

Mollusca, 317

Monera, 66, 300, 312, 316

Monism, 34, 38, 40, 41

Monosaccharide, 110

Movement, ama-boid, 234, 284, 560 as characteristic of life, 4,7 Brownian, 4, 220 by change of cell-turgor, 225

by change of specific gravity, 229 by contraction and expansion, 233 by growth, 233

by secretion, 231

by swelling of cell-wall, 222

ciliary, 247,570

kinds of, 219

muscular, 240

passive, 219

Moulds, adaptation of, to salt solutions, 184

Mucigen,170, 333

Mucin, 108, 113, 171, 333

Mucous cells, 170,333 metanorphosis, 332

Mucus, 171, 332 secretion of, 169

Müller, Johannes, 20

Multiplier, 258

Mummification, 324

Mummy wheat, 130

II usca, muscle fatigue of, 463 origin of fat in larve of, 164

Truscinea, 316

Iuscle, action current of, 427 chemical stimulation of, 368 contraction of, $244,558,565$

contraction of, in absence of oxygen, 286

electrical stimulation of, 413,415 , 420,427

expansion of, 246

fatigue of, 461

galvanic current of, 267

hemoglobin in, 286

irritability of, 15,17

mechanical stimulation of, 384,385

metabolism of, 427

metalolism of tetanised, 348

narcosis of, 375

photic stimulation of, 4112
Muscle, polar excitation of, 415,420 production of heat by, 427 recovery of fatigued, 465

rigor mort is of, 133

secondary results of fatigue of, 467

source of energy of, 5.53

thermal stimulation of, 394

work of, 246

Muscle-albumin, $10 \%$

-fibre, 240

histolysis of, 322

optical properties of, $98,243,245$, 559

structure of erosa-striatel, $24 \cdot 2$

structure of smooth, 241

Muscle-fibrilla, 241

Muscle-segment, 24;3

Myograph, 124

Myoid, 241

Myoporia, 252

Myosin, 107, 324

Myxomyretes, 72

action of oxygen mpon, 369

chemotaxis of, 4.30

ilerelopment of, 74

movement of, in absence of oxygen, 284

phototaxis of, 449

protoplasmic streaming in, 239

rheotaxis of, 444

thermotaxis of, 452

NARCoTtCs, 337, 371, 469

Natural science and metaphrsies, 39 purpose of, 1

Nature, philosophy of, 20,140

Necrobiosis, 135, 319

Necrosis, $320,324,398$

Neef's hammer, 411

Neovitalism, $31,44,45,46$

Nerve, centripetal and centrifugal, $\pi,-9$, .584

chemical stimulation of, 371

conduction in, 360

electrical stimulation of, 41,5

fatigue of, 461

galvanic current of, 267

polar excitation of, 415,421

pressure-paralysis of, 389

sensibility of, 18

Nerve-endings, 584

Nerve-fibre, 581

Neuro-muscular cell, its

Neuron, 581

Niwns formations, 19

Nitella, action of temperature upon, 393

Nitrogen, excretion of, in urine, 17 r

Nitrogen-bacteria, $140,-74$

Nitrogen-equilibrium, $27 \overline{5}, 365$

Nitroglycerine, decomposition of, 124 , 215,484

Nitromonas, 140 
Noctiluca, chemical stimulation of, 370

electrical stimulation of, 427

mechanical stimulation of, 388

narcosis of, 376

Nuclear membrane, 91

Nuclear sap, 91

Nucleic acid, $10 s$

Nuclein bases, 108, 109, 16: 163, 17. 305,482

Nucleins, 92, 108

in secretion, 516

localisation of, in cell, 116

Nucleo-albumins, 108

Nucleolus, 91

Nucleo-proteids, 108

Nucleus, 88

achromatic substance of, 91

and protoplasm, 296,511

and respiration, 519

chroniatic substance of, 91

direct division of, 192

discovery of, 27,66

form of, 88

function of, $5(14,511$

in Bacteria, 67

indirect or mitotic djvision of, 193

in fatigue, 465

in growth, 514

in IIonera, 66

in secretion, 515

mechanics of division of, 531

position of, in cell, 514

resting-stage of, 193

structure of, 92

substance of, 90

theory of dominance of, 3it

Nutrient solution for plants, 138

Nutrition of animals and plants, 138, 274

of Burteria, 140

of Fungi, 140, 274

OHN"s law, 407

Oil-drops, amoboid processes of, 528, 562

Oil-foans, structure of, 86

Ontogeny, 178, 157, :204, 207, 314, 375

Opalina, 72,188

Optimum of vital conditions, 350

Orlitolitcs, concluction in, 363

mechanical stimulation of, $386, \pm 1$

necrobiosis of non-nucleated picces of, 135

protoplasmic streaming in, 239

thigmotaxis of, $4+10$

Organ, 59

() granisation of living substance, 93, 119

() $)$ ganisms and inorganic bodies, 11 s

Organisms, form-changes in, 177

gentalogicul tree of, 176,316
Orrillurie, locomotion of, 232

mechanical stimulation of, 389

phototaxis of, 449

Over-stimulation, 460,471

Orum, 190

fertilisation of pieces of, 506

maturation of, 196, 199, 202

types of segmentation of, 195

Uxalic acicl as decomposition-product of proteid, 110

Oxygen as excretion, 17:3

as general rital condition, 141,281

discovery of, 11, 19, 281

in atmosphere, 242

ingestion of, 142, 174

intramolecular, $4 \$ 3$

partial pressure of, $24 \cdot 2$

results of removal of, $2 \times 3$

Oxytricha, thigmotaxis of, 442

Palmontologr, 31:

Palingeny, 207

Paracelsus, 12

Paralinin, 92

Paramuecum, axial orientation of, 501 chemotaxis of, 437

conjugation of, $92,200,517$

galvanic stimulation of, 419

galvanotaxis of, 455

geotaxis of, 446

mechanical stimulation of, 383

multiplication of, 366

polar excitation of, 419

specific gravity of, 97

thermotaxis of, 453

thigmotaxis of, 443

work of, 's.'?

Paramuclein, 92

Parthenogenesis, 190, ․14

Pathology, 320

cellular, 25

Peas, production of heat in growth of, 258

Pelomyxa, chemical stimulation of, 472 fatigue of, 461

nuclei of, 89

photic stimulation of, 399

polar excitation of, 418

Pepsin, 109, 151, 171

Peptone, 105, 152

fate of, 160

Percumer, electrical stimulation of, 423

mechanical stimulation of, 383

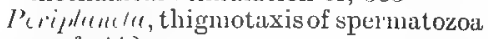
of, $44^{\circ}$

Perpetual motion, problem of, 38

Per'son, 59

Platgreytes, 143, 144, 148, 3:2, 430, 4,32

Philosophy, bacon's nonistic, I4

Descartes's, 14

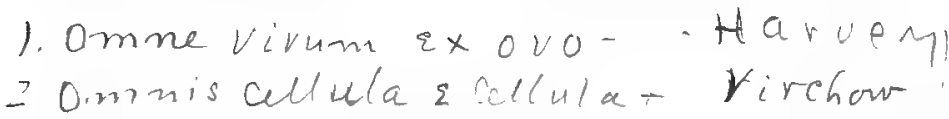


Phosphorescence, animal anıl plant, 254 of chemical substances, 256

Phosphorus poisoning, 163, 332

Photometry, 449

Phototaxis, 447

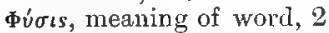

Phylogeny, 178, 207, 313, 575

Physical world and mind, 34 as idea, 36

Physiology, achievements of, 29

and psychology, 21, 31

cell-, 46, 477, 586

chemical tendency of, 23

comparative, 22,50

general, 25

history of, 6

meaning of word, 2

methods of, 28,53

of organs, 49,477

physical tendency of, 23

position of modern, 47

psychological tendency of, 25 task of, 2

Phytogenesis, Schleiden's theory of, 66

Pigeons, fasting of, 277

Pigment-atropliy, 336

Pigment-granules, 83,172

Plankton, 239, 253

Plants, assimilation in, 158, 287

carnivorous, 171

galvanotaxis of roots of, 4.55

genealogical tree of, 316

geotaxis of, 446

in absence of oxygen, 286

metalolism of, 164,173

nutrition of, 138,274

phototaxis of, 447

respiration of, 287

Plant-albumin, 107

Plant-cells, narcosis of, 372

photic stinulation of, 399

Plant-globulin, 108

Plant-physiolosy, 26

Plant-seerls, absence of metalolism in drierl, 132,279

action of temperature upon germinating, 391

germination of dried, 130

origin of carbohydrate from fat in, 480

origin of fat from carlsolyylrate in, 159

Plasmodiun, 7:2

I'lasmolysis, 226

l'leuroncme, mechanical stimulation of, 383

photic stimulation of, 401

Pneunil, doctrine of, $8,9,10,19$

Poikiluthermal animals, $217,297,265$

Poisons, 337

Polar borles, 196, 199, 2015:2

l'olarisat ion curlent, 268 , 41.5 products, $268,411.5$
Polymerism of biogen molecule, 486, 529 of proteid molecule, 106 of silicic acid molecule, 106

Polysaccharids, 112

Polystomella, hehaviour of nonnucleated pieces of, 512

Polytoma, axial orientation of, 500 galvanotaxis of, 457

Post-generation, 534

Poteriodendron, as reflex arc, 578 differentiation in, 539

Precipitation-membrane, 167

Preformation, theory of, 17, 535

Pressure as general vital condition, 292

Priestley, 19

Primordial utricle, 225, 239

Principium nervosum, 16

Proteid, 103

as characteristic of the organism, $125,310,479$

as primitive food, $I 41,555$

as source of muscle energy, 553

chemical formule of, 104

circulating, 160

classification of, 107

coagulation of, 106

combinerl, 108

crystallising, 105

decomposition-products of, 109,162 , $175,305,482$

digestion of. 151

living and dead, 304, 481

localisation of, in cell, 116

non-diffusibility of, 104

polymerism of, 106

reserve-, 161

self-rlecomposition of living, 304,478

tests for, 107

tissue-, 160

Proteus, in hunger, 276

sensitiveness of, to light, 397

Protista, 51, 178, 316

immortality of, 341

Protophyta, 316

Protoplism, 27, 65, 80

alveolar structure of, 86

and nucleus, 296, 511

as emulsion, 96

circulation of, 239

conception of, 80,481

consistency of, 93

contents of, 81

fibrillar structure of, $\mathrm{sij}$

granular disintegration of, 473

ground-substance of, 8.j

hyaline, 8.7

rélicular structure of, 85

rotation of, 239

solid constituents of. $\mathrm{sl}$

spurifire gravity of, 97

splicrical form of, 94, 9. 3 .

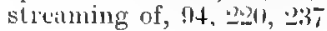


Protonpongin Heckelii, 574

Protozoa, 316

Pseudopodia, 75, 235

Psychology and physiology, 21, 31 task of, 39

Psycho-monism, 38

Ptomaines, 175

Ptyalin, 109, 154, 171, 172

Purposefulness in nature, 182

Pyrenin, 92

Pyrophorus, luminosity of, 254, 255

Pythagoras, 7

\section{Raja, 270}

Reactions, 355

and stimulus, 353

duration of, 357

interference of, 492

Reflex action, 578

Reflex arc, 578

Regeneration, 486, 541 of crystals, 528

Reil, 18

Relationship, natural, 178

Rennet-ferment, 171

Rueproduction, 120 and growth, 188, 488, 531 asexual, 190, 191

by division, $189,191,531$

by fission, 191

by gemmation, 191 sexual, 190

Reserve-substances, 172, 275

Resorption, 142, 151, 523

Respiration, 14l, 174, 482 Mayow's theory of, $15,19,281$

Resurrection-plants, 2.33

Reversion. 180

Rheochord,

Rheotaxis, 444

Rhizoplasma, aciion of temperature upon, 393

in absence of oxygen, 284

Rhizopoda, granular disintegration of, $326,47 \cdot 2$

ingestion and digestion of fool by, 152

narcosis of, 378

Rhythm of cilary motion, 247

Rigor mortis, $133,324,329$

Ritter, 19

Rose of Jericho, 22:3

Rotation of protoplasin, 239

Rotatoria, desiccation of, 129

\section{Saccharomyces, 111, 156}

production of heat by, 258

See least-cells

Saccharose, 112

Salivary corpuscles, Brownian move. ment in, 220
Salivary glands, cells of, 465,516

Salmon, fasting of, 277

Salting-out, 107

Salts, 115, 142

Sarcode, 65

Sarcolactic acid, 174

Sarcolemma, 243

Sarcoplasm, 241

Sarcosomes, 464

Sarkin, 108, 109, 162, 175, 305, 482

Schleiden, 27

Sichwann, 27

Sea-urchin, fertilisation in, 203

Sebum, 172

Secretion, mechanics of, 523

Secretions, 166,171 protective, 172

Selaginella, swelling movements of, 223

Selection, artificial, 186

Darwin's theory of, 28, 185, 317, 573

Empedocles's theory of, 8, 28

natural, $8,28,185,317$

Self-regulation of metabolism, 489

Serum-albumin, 107

Serum-globulin, 107

Serveto, 12

Short circuit, 408

Silicic acid, coagulation of, 106 polymerism of, 106

Siphonophora, 59

Skeletal substances, 108, 172

excretion of, 166, 543

Sledge-inductorium, 411

Snake-charming, 495

Snake-poison, 176

Soaps, 114, 155

Spallanzani, 19

Sparrow, fatigue of ganglion-cells of, 464

Specialisation, danger of, 1

Specific energy, 21, 45, 475

Specific gravity, of cell, 97,229 of living substance, 97

Spermatozoa, 190

chemotaxis of, 433,438

development of, 197

discovery of, 15, 198

forms of, 199

rheotaxis of, 44.5

structure of, 199

Sperm-cells, see Spermatozoa

Sperm nother'cells, 197

Sphorechinus, cross-fertilisation of, 507

Spinal cord, isil

Spinning glanils, nuclei of cells of, 89 , 516

spirillam chemotaxis of, 436 nucleus of, 67

Spirits, animal, 8, 10, 102

doctrine of the, $8,9,10$

natural, 10

vital, 8,10 
Spirogyra, arrangement of cells in, 574 behaviour of non-nucleated pieces of, 513,519

narcosis of, 372,521

respiration of, 520

Spirostomum, ciliary motion in, 571

galvanotaxis of, 459

granular disintegration of, 326 narcosis of, 471

Sponge-spicules, 169, 172

formation of, 543

Spongin, 109

Spontaneity, 347

Spontaneous generation, doctrine of, 15 , $20,298,302,309,311$

Spore-formation, 197, 205, 281, 344

Spores of bacteria, 281, 291

of Colpoda, 205

of horse-tail, 224,448

of Myxomycetes, 74

Splenic fever, resistance of bacilli of, 291

Spring-myograph, 361

Squaring of circle, problem of, 38

Stahl, 16

Staphylococcus, 431

Starch, 112, 172

digestion of, 154

formation of, by plant, 159

in chlorophyll-bodies, 159

localisation of, in cell, 116

Starch-grains, 83, 112

digestion of, 154

Steam engine, comparison of with organism, 123

Steapsin, 155, 171

Stentor, food-ingestion by, 526

mechanical stimulation of, 384

myoids of, 241

nareosis of, 375

regeneration of, 61,541

stimulation and vital conditions, 349

diagram of, 350

phenomena of, 364

threshold of, 351,436

unilateral, 428

Stimulus, 474

action of chemical, 365

netion of electrical, 403

action of mechanical, 379

action of photic, 397

uction of thermal, 389,470

arlaptation to, 357

infter-effect of, 358

and reaction, 353

issimilatory, 490

conduction of, 360

definition of, 348,357

dissimilatory, 490

intensity of, 349

interferunce of, 402

trophic, $35 \mathrm{l}$

varieties of, 348
Structure and liquid, 538

conception of, 538

Struggle for existence, 8, 28, 185, 317

Sulphur-bacteria, 278

Sulphuric acid as decomposition-product of proteid, 110

manufacture of, $125,156,484$

Sûsruta, 7

Swammerdamm, 15

Swarm-spores, phototaxis of, 448

Swimming-plates of Ctenophora, 249, 570

Sylvius, 15

Symbiotic organisms, 84

Symptomatic anthrax, bacilli of, 287

Syncytium, 74

TACTIC movements, mechanism of, 497

Tadpole, galvanotaxis of, 455 histolysis of tail of, 321

Tardigrada, desiccation of, 129

Teleology, 182, 318

Temperature as general vital condition, 288

determination of, 258

maximum of, $288,291,396$

minimum of, 288,395

of organism, $25 i$

Temperature-sense, 391

Tetanus, analysis of, 423

bacteria of, 287

closing, 414

mechanical, 385

metabolism in, 388

Thalassicolla, behaviour of non-nucleated pieces of, $51: 2$

chemical stimulation of, 370

mechanical stimulation of, 379

rising and sinking of, 230

spore-formation of, 344

Theoria generationis of Wolf, 17

'T'hermo-chemical equivalent, 214

Thermo-electric element, 258

Thermotaxis, 451

Thigmotaxis, $4+0$

Threshold of stimulation, 351, 436

T'issue, 60

Tone, muscular, 358

Torpedo, 269

Toxalbumins, 176

'T'oxallumoses, 176

Toxines, 175,468

Tradescantia, electrical stimulation of, 423

protoplasmic streaning in, 2399,393

'Lianspiration, 17t

'T'reviranus, 19

Trophic nerves, 353 stimuli, 351

'Trophotaxis, 430

thypsin, 109, 1.74, 171

T'tuberculin, 176

'Tuberculosis, growth of batuilli of, 391 
Tumours, 367

Tunicata, 317

UREA, 109, 162, 175, 305, 48: artificial synthesis of, 23,43 derivation of, 162

Uric acid, 109, 162, 175, 305, 45.2 derivation of, 162

VACUOJ.RS, 81 contractile, 82, 166

Vamlyrella, food-selection by, 148,527

Variability, individual, 185, 317

Vuncherix, formation of globules ly plotoplasm of, 94

I'ermes, 317

Vertebrata, 317

Vie latente. 132

Vital conditions, diagram of, 350

Vital force, doctrine of, $17,18,21,31$, 41,123

Vitalism, mechanical, 44 psychical, 45

Vitellins, 108

Volta, 263

rorticella, as reflex arc, 578 chemical stimulation of, 368 food-ingestion by, 145,526
Vorficella, mechanical stimulation of, 384,388 myoid of, 242

WAGNER's hammer, 411

Warm-blooded animals, $21 \%, 25$ heat-regulation in, 390

Warm stage, 392

Water as constituent of living substance, 114

as general vital condition, 279 excretion of, 166,174

Theel-animalcules, desiccation of, 129

Wïhler, 23

Wolff, 17

World-formula, du Bois-Reymond's, 32

Xanthin, 108, 109, 162, 175, 305, 482

YAJURVEDA, 7

Yeast-cells, 111, 156

action of temperature upon, 390 narcosis of, 371

production of heat by, 258

See Saccharomyces

Zoöchlorellow, 84

Zoöxanthellop, 84

Zygnema, behaviour of non-nucleated pieces of, 513

THE END 
- 


$$
\text { - }
$$




